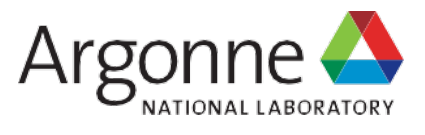

\title{
Independent Review of Paducah Gaseous Diffusion Plant Freon Release Authorized Limits and Evaluation of Corresponding Radiological Doses
}

Environmental Science Division 


\begin{abstract}
About Argonne National Laboratory
Argonne is a U.S. Department of Energy laboratory managed by UChicago Argonne, LLC under contract DE-AC02-06CH11357. The Laboratory's main facility is outside Chicago, at 9700 South Cass Avenue, Lemont, Illinois 60439. For information about Argonne and its pioneering science and technology programs, see www.anl.gov.
\end{abstract}

\title{
DOCUMENT AVAILABILITY
}

Online Access: U.S. Department of Energy (DOE) reports produced after 1991 and a growing number of pre-1991 documents are available free at OSTI.GOV (http://www.osti.gov/),

a service of the US Dept. of Energy's Office of Scientific and Technical Information.

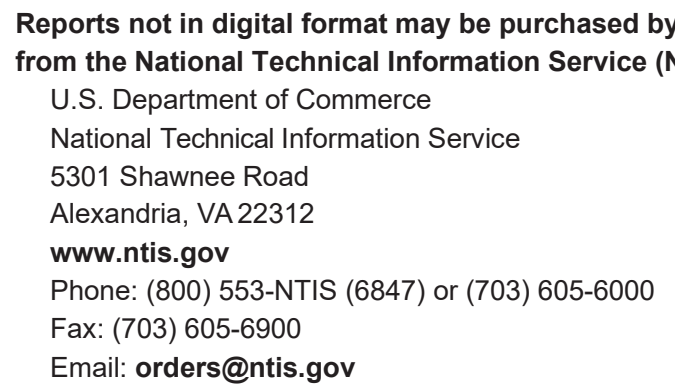

Reports not in digital format are available to DOE and DOE contractors from the Office of Scientific and Technical Information (OSTI):

U.S. Department of Energy

Office of Scientific and Technical Information

P.O. Box 62

Oak Ridge, TN 37831-0062

www.osti.gov

Phone: (865) 576-8401

Fax: (865) 576-5728

Email: reports@osti.gov

\begin{abstract}
Disclaimer
This report was prepared as an account of work sponsored by an agency of the United States Government. Neither the United States Government nor any agency thereof, nor UChicago Argonne, LLC, nor any of their employees or officers, makes any warranty, express or implied, or assumes any legal liability or responsibility for the accuracy, completeness, or usefulness of any information, apparatus, product, or process disclosed, or represents that its use would not infringe privately owned rights. Reference herein to any specific commercial product, process, or service by trade name, trademark, manufacturer, or otherwise, does not necessarily constitute or imply its endorsement, recommendation, or favoring by the United States Government or any agency thereof. The views and opinions of document authors expressed herein do not necessarily state or reflect those of the United States Government or any agency thereof, Argonne National Laboratory, or UChicago Argonne, LLC.
\end{abstract}




\section{Independent Review of Paducah Gaseous Diffusion Plant Freon Release Authorized Limits and Evaluation of Corresponding Radiological Doses}

by

Sunita Kamboj and Charley Yu

Environmental Science Division

Argonne National Laboratory

June 12, 2020 



\section{CONTENTS}

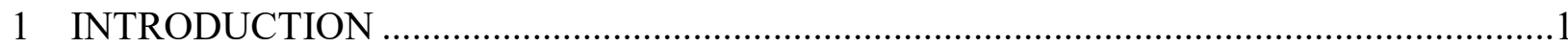

2 DOSE ESTIMATION FOR DIFFERENT ACTIVITIES ASSOCIATED WITH

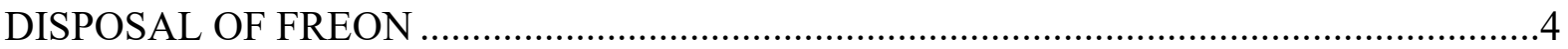

2.1 Shipping Freon in ISO Containers to Incinerators...................................................4

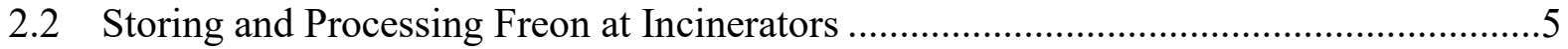

2.2.1 Managing Ashes Generated from Incineration of Freon .................................... 7

2.3 Exposure to Non-involved Workers and General Public..............................................8

2.3.1 Scenario 1: Radionuclides Exhausted from the Incinerator Stack, thereby

Exposing MEI (PPPO 2020, Section 5.2.4) ..................................................... 8

2.3.2 Scenario 2: All radionuclides Are Captured in the Emission Controls or

Collected in the Incinerator Ash and Subsequently Disposed of in a

Landfill, Resulting in Exposure to a Landfill Neighbor (PPPO 2020,

Section 5.2.4)

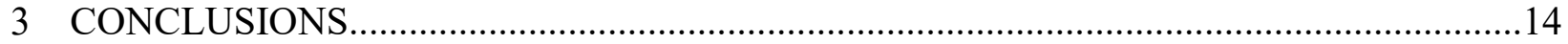

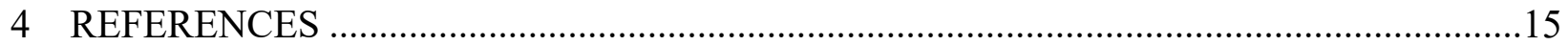

APPENDIX A: RESRAD-BUILD RUN RESULTS FOR TRUCK DRIVER, INTERNATIONAL ORGANIZATION FOR STANDARDIZATION CONTAINER HANDLING WORKER, AND BAGHOUSE WORKER SCENARIOS ......................... A-1

A.1 RESRAD-BUILD Results for Truck Driver Scenario ............................................ A-1

A.2 RESRAD-BUILD Results for ISO Container Handling Worker Scenario ................ A-9

A.3 RESRAD-BUILD Results for Incinerator Baghouse Worker/Processor Scenario.... A-15

APPENDIX B: RESRAD-OFFSITE RUN RESULTS FOR NON-INVOLVED

WORKER, INCINERATOR NEIGHBOR, AND LANDFILL NEIGHBOR

SCENARIOS

B.1 RESRAD-OFFSITE Results for the Incinerator Neighbor Scenario...........................

B.2 RESRAD-OFFSITE Results for Incinerator Neighbor Scenario................................6-63

B.3 RESRAD-OFFSITE Results for Landfill Neighbor Scenario ................................. 125

\section{FIGURES}

1 ISO Tank Container and Receptor Locations 4 


\section{TABLES}

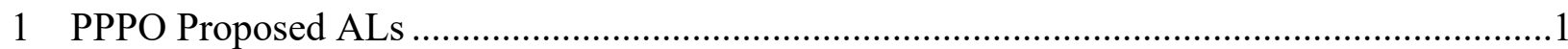

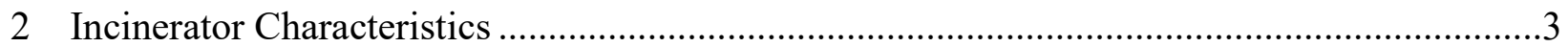

3 Direct External Dose for Truck Driver Transporting Freon from Paducah to Veolia North America Incinerato

4 Total Dose for ISO Container Handling and Processing Worker at Incinerator Facility .........6

5 Incinerator Baghouse Worker/Processor Pathway Doses .................................................

6 Non-involved Incinerator Worker Inhalation Dose from Air Effluent Release at Aragonite

7 Incinerator (El Dorado SN-01) Neighbor Dose Estimation................................................ 11

8 Landfill Neighbor Scenario Dose for Different Exposure Pathways..................................13 


\section{INTRODUCTION}

The Portsmouth/Paducah Project Office (PPPO) proposes to use the American National Standard Institute (ANSI)/Health Physics Society (HPS) N13.12 (ANSI 2013) volumetric screening levels (Table 1) as Authorized Limits (ALs) for the release of cryofluorane R-114 (Freon) at the Paducah Gaseous Diffusion Plant (PGDP) in Paducah, Kentucky (PPPO 2020). A review of the proposed ALs document (PPPO 2020) indicated that potential exposure scenarios from the release of Freon are adequately discussed therein.

However, questions arose regarding the assumptions made for the ANSI N13.12 clearance values, and whether or not all pathway doses were adequately considered in the Authorized Limits Request (PPPO 2020). For example, PPPO (2020) used the inverse square rule to estimate dose to the truck driver. A more appropriate evaluation would have considered the material as a cylindrical source for this pathway. In addition, the inverse square rule was inadequately applied to the inhalation and ingestion pathways. Furthermore, while the ANSI N13.12 volumetric clearance values are used in the ALs, questions arose concerning whether the values are appropriate for materials of greater than 1 metric ton, and whether or not the ANSI N13.12 standard could be used for liquids that are sent to an incinerator.

For these reasons, dose modeling was conducted to demonstrate that using ANSI/HPS N13.12 screening levels as ALs for release of PGDP Freon meets the dose criteria set in U.S. Department of Energy (DOE) Order 458.1. This report provides a bounding dose analysis using scenarios described in PPPO (2020) document. Realistic parameters, such as the incinerator characteristics data, are used when available.

TABLE 1 PPPO Proposed ALs

\begin{tabular}{lc}
\hline Radionuclide & $\begin{array}{c}\text { Volumetric } \\
\text { concentration } \\
\text { (pCi/g) }\end{array}$ \\
\hline Am-241 & 3 \\
Cs-137 & 3 \\
Np-237 & 30 \\
Pu-238 & 3 \\
Pu-239 & 3 \\
Pu-240 & 3 \\
Tc-99 & 30 \\
Th-228 & 3 \\
Th-230 & 3 \\
Th-232 & 3 \\
U-234 & 30 \\
U-235 & 30 \\
U-238 & 30 \\
\hline
\end{tabular}

Reference: Table 8 in PPPO (2020). 
Approximately 8.5 million pounds of Freon is currently stored at the PGDP (PPPO 2020). The PPPO (2020) document identified three vendors (Clean Harbors Aragonite, Clean Harbors El Dorado, and Veolia North America/Port Arthur) that have the capability to incinerate the total quantity of Freon within the allotted 10-year period (PPPO 2020). Table 2 lists the characteristics of these incinerators.

Radiological doses are calculated for 13 radionuclides (listed in Table 1) for multiple receptors involved in different activities associated with the disposal of Freon. The activities considered include shipping Freon in International Organization for Standardization (ISO) containers to incinerators, storing and processing Freon at incinerators, handling dust/waste generated at incinerators, and disposing of dust/waste at a landfill. Many people may receive exposure as a result of different activities, but doses are estimated for a maximally exposed individual (MEI) who is either directly involved in the activity or indirectly exposed. The directly involved individuals could include truck drivers transporting Freon in ISO containers to incinerators, workers involved in storing and processing Freon at incinerators, workers involved in handling dust/waste at incinerators, and workers involved in disposing of dust/waste at a landfill. The non-involved individuals include other workers at the incinerators who are not directly involved in handling or processing Freon, members of the general public (incinerator neighbor) who may be exposed to air released from incinerators, and members of the general public (landfill neighbor) who live near the landfill. 


\section{TABLE 2 Incinerator Characteristics}

\begin{tabular}{|c|c|c|c|c|c|c|c|c|}
\hline Incinerator & $\begin{array}{l}\text { Flow rate, } \mathrm{ft}^{3} / \mathrm{min} \\
\left(\mathrm{m}^{3} / \mathrm{s}\right)\end{array}$ & $\begin{array}{l}\text { Stack height, } \mathrm{ft} \\
(\mathrm{m})\end{array}$ & $\begin{array}{l}\text { Particulate } \\
\text { matter } \\
\text { emission, } \\
\mathrm{g} / \mathrm{m}^{3}\end{array}$ & $\begin{array}{c}\text { Average } \\
\text { uptime } \\
(\%)\end{array}$ & $\begin{array}{c}\text { Driver } \\
\text { exposure } \\
\text { time, } \mathrm{h} / \mathrm{yr}\end{array}$ & $\begin{array}{c}\text { Nearest } \\
\text { neighbor, } \\
\text { miles }\end{array}$ & $\begin{array}{c}\text { Average } \\
\text { discharge } \\
\text { rate, } \mathrm{g} / \mathrm{s}\end{array}$ & $\begin{array}{c}\text { Total } \\
\text { yearly } \\
\text { discharge } \\
\text { rate, } \mathrm{g} / \mathrm{yr}\end{array}$ \\
\hline Clean Harbors Aragonite & $77,800(36.72)$ & $149(45.42)$ & 0.028 & 95 & $1,162.5$ & 16 & 0.98 & $3.08 \mathrm{E}+07$ \\
\hline $\begin{array}{l}\text { Clean Harbors El Dorado- } \\
\text { SN-44 }\end{array}$ & $89,000(42.0)$ & $199(60.66)$ & 0.028 & 90 & 1,100 & 3 & 1.06 & $3.34 \mathrm{E}+07$ \\
\hline $\begin{array}{l}\text { Clean Harbors El Dorado- } \\
\text { SN-01 }\end{array}$ & $100,568(47.46)$ & $195(59.44)$ & 0.028 & 90 & 1,100 & 3 & 1.20 & $3.77 \mathrm{E}+07$ \\
\hline Veolia North America & $39,248(18.52)$ & $130(39.62)$ & 0.0034 & 85.85 & $1,212.5$ & 3.5 & 0.05 & $1.71 \mathrm{E}+06$ \\
\hline
\end{tabular}

References: PPPO (2020), Whitehurst (2020a-d). 


\section{DOSE ESTIMATION FOR DIFFERENT ACTIVITIES ASSOCIATED WITH DISPOSAL OF FREON}

The potential exposure to various activities involved in transporting, processing, incinerating, and disposal of Freon are analyzed. Four exposure scenarios are discussed below: (1) shipping Freon in ISO containers to incinerators, (2) storing and processing Freon at the incinerators, (3) managing ashes generated from incineration of Freon, and (4) exposure to noninvolved workers and public from incinerating Freon.

\subsection{SHIPPING FREON IN ISO CONTAINERS TO INCINERATORS}

The truck driver transports Freon in ISO containers to incinerators. The ISO container dimensions are shown in Figure 1; it is $2.43 \mathrm{~m}$ wide, $2.59 \mathrm{~m}$ high, and $6.09 \mathrm{~m}$ long (Dihel 2020). The driver is assumed to be $3 \mathrm{~m}$ away from the containers while driving the truck. They are assumed to be $1 \mathrm{~m}$ away on the side of the truck when they are near the vehicle checking the truck and inspecting ISO containers (PPPO 2020). The driver exposure time is highest for an individual who drives from Paducah to Veolia North America/Port Arthur incinerator; this would involve 1,112.5 hours of driving time and 100 hours near the vehicle while it is parked (total 1,212.5 hours) according to Section 5.2.2 in PPPO (2020). It is assumed 1.5 million pounds of Freon are shipped to an incinerator in 1 year.

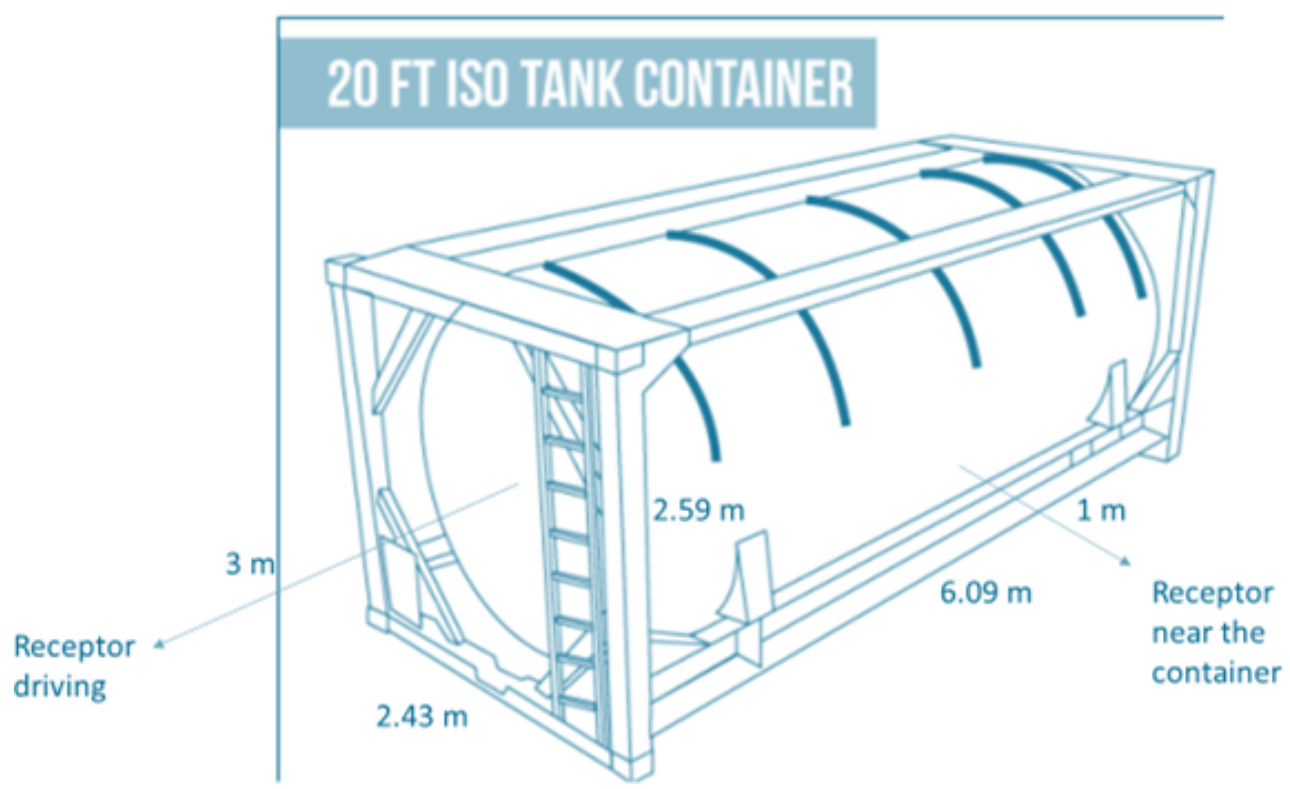

FIGURE 1 ISO Tank Container and Receptor Locations (Source: Dihel 2020) 
The dose to the truck driver is calculated at the proposed ALs (Table 1) using the RESRAD-BUILD code (Yu et al. 2003) for two source receptor configurations, as shown in Figure 1. It is assumed that the source is a volume source with the dimensions of the ISO container, and that it is filled with Freon at a density of $1.5 \mathrm{~g} / \mathrm{cm}^{3}$. It is also assumed that there is no leakage of Freon from the ISO containers when it is being transported from Paducah to the incinerators. Therefore, the truck driver would only be exposed to external radiation dose from radionuclides contained in the ISO containers. The results for transporting Freon to Veolia North America Incinerator (i.e., the longest distance and travel time among three incinerators) are shown in Table 3. The results indicate that the calculated dose to the truck driver from each individual radionuclide is less than $1 \mathrm{mrem} / \mathrm{yr}$; the highest dose is $0.484 \mathrm{mrem} / \mathrm{yr}$, for $\mathrm{Np}-237$. The output of RESRAD-BUILD run results is included in Appendix A. The output also includes a list of all the input parameter values used.

TABLE 3 Direct External Dose for Truck Driver Transporting Freon from Paducah to Veolia North America Incinerator ${ }^{a}$

\begin{tabular}{|c|c|c|c|c|c|c|}
\hline \multirow[b]{2}{*}{ Radionuclide } & \multicolumn{3}{|c|}{$\begin{array}{l}\text { Truck driver dose for one trip } \\
\text { (mrem) }\end{array}$} & \multicolumn{3}{|c|}{$\begin{array}{c}\text { Truck driver dose for } 50 \text { trips in a } \\
\text { year (mrem) }\end{array}$} \\
\hline & $\begin{array}{c}3 \text { m away } \\
\text { while } \\
\text { driving } \\
\end{array}$ & $\begin{array}{c}1 \mathrm{~m} \text { away } \\
\text { near the } \\
\text { vehicle }\end{array}$ & Total & $\begin{array}{c}3 \text { m away } \\
\text { while } \\
\text { driving } \\
\end{array}$ & $\begin{array}{c}1 \mathrm{~m} \text { away } \\
\text { near the } \\
\text { vehicle }\end{array}$ & Total \\
\hline Am-241 & $1.98 \mathrm{E}-05$ & $1.04 \mathrm{E}-05$ & $3.02 \mathrm{E}-05$ & $9.89 \mathrm{E}-04$ & $5.21 \mathrm{E}-04$ & $1.51 \mathrm{E}-03$ \\
\hline Cs- 137 & $1.98 \mathrm{E}-03$ & $1.05 \mathrm{E}-03$ & $3.04 \mathrm{E}-03$ & $9.92 \mathrm{E}-02$ & $5.26 \mathrm{E}-02$ & $1.52 \mathrm{E}-01$ \\
\hline Np-237 & $6.32 \mathrm{E}-03$ & $3.36 \mathrm{E}-03$ & $9.68 \mathrm{E}-03$ & $3.16 \mathrm{E}-01$ & $1.68 \mathrm{E}-01$ & $4.84 \mathrm{E}-01$ \\
\hline $\mathrm{Pu}-238$ & $6.05 \mathrm{E}-08$ & $3.74 \mathrm{E}-08$ & $9.79 \mathrm{E}-08$ & $3.02 \mathrm{E}-06$ & $1.87 \mathrm{E}-06$ & $4.89 \mathrm{E}-06$ \\
\hline $\mathrm{Pu}-239$ & $1.52 \mathrm{E}-07$ & $8.81 \mathrm{E}-08$ & $2.40 \mathrm{E}-07$ & $7.59 \mathrm{E}-06$ & $4.41 \mathrm{E}-06$ & $1.20 \mathrm{E}-05$ \\
\hline $\mathrm{Pu}-240$ & $5.87 \mathrm{E}-08$ & $3.58 \mathrm{E}-08$ & $9.45 \mathrm{E}-08$ & $2.93 \mathrm{E}-06$ & $1.79 \mathrm{E}-06$ & $4.73 \mathrm{E}-06$ \\
\hline Tc-99 & $6.22 \mathrm{E}-07$ & $3.26 \mathrm{E}-07$ & $9.49 \mathrm{E}-07$ & $3.11 \mathrm{E}-05$ & $1.63 \mathrm{E}-05$ & $4.74 \mathrm{E}-05$ \\
\hline Th-228 & $4.75 \mathrm{E}-03$ & $2.53 \mathrm{E}-03$ & $7.28 \mathrm{E}-03$ & $2.37 \mathrm{E}-01$ & $1.27 \mathrm{E}-01$ & $3.64 \mathrm{E}-01$ \\
\hline Th-230 & $1.99 \mathrm{E}-06$ & $1.05 \mathrm{E}-06$ & $3.04 \mathrm{E}-06$ & $9.93 \mathrm{E}-05$ & $5.26 \mathrm{E}-05$ & $1.52 \mathrm{E}-04$ \\
\hline Th-232 & $2.39 \mathrm{E}-04$ & $1.27 \mathrm{E}-04$ & $3.66 \mathrm{E}-04$ & $1.20 \mathrm{E}-02$ & $6.36 \mathrm{E}-03$ & $1.83 \mathrm{E}-02$ \\
\hline $\mathrm{U}-234$ & $1.85 \mathrm{E}-06$ & $1.01 \mathrm{E}-06$ & $2.87 \mathrm{E}-06$ & $9.27 \mathrm{E}-05$ & $5.06 \mathrm{E}-05$ & $1.43 \mathrm{E}-04$ \\
\hline U-235 & $4.22 \mathrm{E}-03$ & $2.22 \mathrm{E}-03$ & $6.44 \mathrm{E}-03$ & $2.11 \mathrm{E}-01$ & $1.11 \mathrm{E}-01$ & $3.22 \mathrm{E}-01$ \\
\hline U-238 & $9.37 \mathrm{E}-04$ & 4.95E-04 & $1.43 \mathrm{E}-03$ & 4.69E-02 & $2.48 \mathrm{E}-02$ & $7.16 \mathrm{E}-02$ \\
\hline
\end{tabular}

a Truck driver is potentially exposed to radionuclides contained in Freon transported in ISO containers driving for 1,112.5 hours (22.25 hours/trip) and 100 hours ( 2 hours/trip) near the ISO container in 1 year (50 trips).

\subsection{STORING AND PROCESSING FREON AT INCINERATORS}

Dose is calculated for a worker involved in different activities at the incinerators (PPPO 2020, Section 5.2.3). It is assumed that the worker is near the container (1 m away) and spends 127 hours/year in these activities. The dose for 13 radionuclides with concentrations listed in Table 1 is calculated using the RESRAD-BUILD code. The ISO container dimension 
and receptor locations are the same as shown in Figure 1. Some key (sensitive) parameters are conservatively assumed for bounding calculations. These include mass loading of $1.0 \times 10^{-4} \mathrm{~g} / \mathrm{m}^{3}$ for the inhalation pathway, and a transfer rate of $0.01 \mathrm{~g} / \mathrm{hr}$ for the ingestion pathway. For calculating air concentration, it is conservatively assumed that the radionuclide concentration in dust particulates is the same as the radionuclide concentration in Freon. In reality, radionuclide concentration in particulates could be higher than in Freon, but a small fraction of the dust in the air may be due to particulates released from Freon; this means that there is a lower concentration of radionuclides in the air than in Freon. The dose results are shown in Table 4. The dose calculated for each radionuclide is less than $1 \mathrm{mrem}$ for handling and processing the ISO container, for a total time of 127 hours in a year. The highest dose is $0.237 \mathrm{mrem} / \mathrm{yr}$ for Np-237. The output of the RESRAD-BUILD run results is included in Appendix A. The output also includes a list of all input parameter values used.

TABLE 4 Total Dose (mrem) for ISO Container Handling and Processing Worker at Incinerator Facility ${ }^{\mathrm{a}}$

\begin{tabular}{|c|c|c|c|c|}
\hline Radionuclide & $\begin{array}{c}\text { External } \\
\text { exposure } \\
\text { (mrem) }\end{array}$ & $\begin{array}{c}\text { Inhalation } \\
\text { (mrem) }\end{array}$ & $\begin{array}{c}\text { Ingestion } \\
\text { (mrem) }\end{array}$ & $\begin{array}{c}\text { Total Dose } \\
\text { (mrem) }\end{array}$ \\
\hline Am-241 & $6.61 \mathrm{E}-04$ & $1.62 \mathrm{E}-02$ & $2.82 \mathrm{E}-03$ & $1.97 \mathrm{E}-02$ \\
\hline Cs-137 & $6.68 \mathrm{E}$ & $6.60 \mathrm{E}-06$ & $1.83 \mathrm{E}-04$ & $6.70 \mathrm{E}-02$ \\
\hline $\mathrm{Np}-237$ & $2.13 \mathrm{E}-01$ & $8.46 \mathrm{E}$ & $1.55 \mathrm{E}$ & $2.37 \mathrm{E}-01$ \\
\hline $\mathrm{Pu}-238$ & 2.381 & $1.86 \mathrm{E}$ & $3.24 \mathrm{E}$ & 2.19E-02 \\
\hline $\mathrm{Pu}-2$ & 5.601 & 2.03 & 3.52 & $2.38 \mathrm{E}-02$ \\
\hline $\mathrm{Pu}-240$ & 2.28 & 2.03 & 3.52 & $2.38 \mathrm{E}-02$ \\
\hline Tc-99 & 2.07 & 2.2( & 9.02 & $1.13 \mathrm{E}-04$ \\
\hline Th-2 & 1 & & 1 & 01 \\
\hline Th-2 & 6 & 1.6 & 2 & -02 \\
\hline Th-232 & 3 & 1.8 & 3.2 & -02 \\
\hline $\mathrm{U}-2$ & & 1. & 6.9 & 03 \\
\hline U-235 & 1 & 1.4 & 6.6 & -01 \\
\hline U-238 & $3.15 \mathrm{E}-02$ & $1.35 \mathrm{E}-03$ & $6.34 \mathrm{E}-03$ & $3.92 \mathrm{E}-02$ \\
\hline \multicolumn{5}{|c|}{$\begin{array}{l}\text { External dose calculations use dose coefficients based on the } \\
\text { International Commission on Radiation Protection (ICRP) (1991) } \\
\text { methodology from Federal Guidance Report (FGR) } 12 \text { (Eckerman } \\
\text { and Ryman 1993); internal dose calculations use those from ICRP } \\
\text { (1996) for an adult member of the public. External dose is calculated } \\
\text { using RESRAD-BUILD. The receptor spends a total of } 127 \text { hours } \\
\text { (Section 5.2.3 of PPPO 2020) handling and processing ISO } \\
\text { container at the incinerator. An inhalation rate of } 1.2 \mathrm{~m}^{3} / \mathrm{hr} \text { and a } \\
\text { mass loading of } 1 \mathrm{E}-4 \mathrm{~g} / \mathrm{m}^{3} \text { are used to estimate inhalation dose. An } \\
\text { ingestion transfer rate of } 0.01 \mathrm{~g} / \mathrm{hr} \text { is used to estimate ingestion dose. } \\
\text { Mass loading, inhalation rate, and ingestion transfer rates are taken } \\
\text { from ANSI N13.12 2013 Standard (ANSI 2013), and ANSI N13.12 } \\
\text { 1999 Standard (ANSI 1999). }\end{array}$} \\
\hline
\end{tabular}




\subsubsection{Managing Ashes Generated from Incineration of Freon}

Dose is calculated for the worker involved in activities related to processing the ashes generated at the incinerator. We assume that the baghouse worker/processor loads the dust/ash bags and transports and disposes of them at a landfill. The dust bags are changed every year and it is conservatively assumed that all radionuclides contained in Freon are all captured in the dust/ash bags. To estimate external dose from the baghouse, it is assumed that the dust/ash bag is cylindrical in shape with a length of $100 \mathrm{~cm}$ and a radius of $34 \mathrm{~cm}$, and that the material density of the dust collected is $1.4 \mathrm{~g} / \mathrm{cm}^{3}$ (Cheng et al. 2000). Dose coefficients based on the ICRP (1991) methodology from FGR 12 (Eckerman and Ryman 1993) are used in external dose calculations and those from ICRP (1996) for an adult member of the public are used for internal dose calculations. External dose is calculated using the RESRAD-BUILD computer code. It assumes that the worker is $2 \mathrm{~m}$ away from the dust/ash bag and spends 18 hours ( 6 hours per cell $\times 3$ cells) in 1 year handling bags at the El Dorado facility (Whitehurst 2020a). For inhalation dose calculations, it assumed that inhalation rate is $1.2 \mathrm{~m}^{3} / \mathrm{hr}$ and mass loading is $1 \times 10^{-4} \mathrm{~g} / \mathrm{m}^{3}$, and airborne particulate filtration efficiency is $99.97 \%$ (i.e., the dust/ash bag handlers wear fullface air-purifying respirators with MERSORB-P100 cartridges; Whitehurst 2020b). The inhaled air concentration is reduced to $0.03 \%$ and no ingestion dose is calculated because workers use personal protective equipment (PPE). The parameter values for inhalation rate and mass loading used are from ANSI N13.12 (ANSI 2013), 1999 Standard (ANSI 1999). The doses to the baghouse worker/processor are listed in Table 5. The total dose to the baghouse worker/processor from each radionuclide is less than $1 \mathrm{mrem} / \mathrm{yr}$. The highest dose is $0.986 \mathrm{mrem} / \mathrm{yr}$ for $\mathrm{Np}-237$. The output of RESRAD-BUILD run results is included in Appendix A. The output also includes a list of all input parameter values used.

TABLE 5 Incinerator Baghouse Worker/Processor Pathway Doses ${ }^{\mathrm{a}}$

\begin{tabular}{lccccc}
\hline Radionuclide & $\begin{array}{c}\text { Concentration } \\
\text { in the baghouse } \\
(\mathrm{pCi} / \mathrm{g})\end{array}$ & $\begin{array}{c}\text { Baghouse } \\
\text { worker air } \\
\text { concentration } \\
\left(\mathrm{pCi} / \mathrm{m}^{3}\right)\end{array}$ & $\begin{array}{c}\text { Inhalation } \\
\text { dose } \\
(\mathrm{mrem} / \mathrm{yr})\end{array}$ & $\begin{array}{c}\text { External } \\
\text { dose } \\
(\mathrm{mrem} / \mathrm{yr})\end{array}$ & $\begin{array}{c}\text { Total dose } \\
(\mathrm{mrem} / \mathrm{yr})\end{array}$ \\
\hline Am-241 & $4.01 \mathrm{E}+03$ & $1.20 \mathrm{E}-04$ & $1.85 \mathrm{E}-03$ & $3.19 \mathrm{E}-03$ & $5.04 \mathrm{E}-03$ \\
$\mathrm{Cs}-137$ & $4.01 \mathrm{E}+03$ & $1.20 \mathrm{E}-04$ & $7.50 \mathrm{E}-07$ & $3.00 \mathrm{E}-01$ & $3.00 \mathrm{E}-01$ \\
Np-237 & $4.01 \mathrm{E}+04$ & $1.20 \mathrm{E}-03$ & $9.62 \mathrm{E}-03$ & $9.76 \mathrm{E}-01$ & $9.86 \mathrm{E}-01$ \\
Pu-238 & $4.01 \mathrm{E}+03$ & $1.20 \mathrm{E}-04$ & $2.12 \mathrm{E}-03$ & $1.10 \mathrm{E}-05$ & $2.13 \mathrm{E}-03$ \\
Pu-239 & $4.01 \mathrm{E}+03$ & $1.20 \mathrm{E}-04$ & $2.31 \mathrm{E}-03$ & $2.52 \mathrm{E}-05$ & $2.33 \mathrm{E}-03$ \\
Pu-240 & $4.01 \mathrm{E}+03$ & $1.20 \mathrm{E}-04$ & $2.31 \mathrm{E}-03$ & $1.06 \mathrm{E}-05$ & $2.32 \mathrm{E}-03$ \\
Tc-99 & $4.01 \mathrm{E}+04$ & $1.20 \mathrm{E}-03$ & $2.50 \mathrm{E}-06$ & $9.77 \mathrm{E}-05$ & $1.00 \mathrm{E}-04$ \\
Th-228 & $4.01 \mathrm{E}+03$ & $1.20 \mathrm{E}-04$ & $7.70 \mathrm{E}-04$ & $7.21 \mathrm{E}-01$ & $7.22 \mathrm{E}-01$ \\
Th-230 & $4.01 \mathrm{E}+03$ & $1.20 \mathrm{E}-04$ & $1.92 \mathrm{E}-03$ & $3.03 \mathrm{E}-04$ & $2.23 \mathrm{E}-03$ \\
Th-232 & $4.01 \mathrm{E}+03$ & $1.20 \mathrm{E}-04$ & $2.12 \mathrm{E}-03$ & $3.61 \mathrm{E}-02$ & $3.82 \mathrm{E}-02$ \\
U-234 & $4.01 \mathrm{E}+04$ & $1.20 \mathrm{E}-03$ & $1.81 \mathrm{E}-03$ & $3.05 \mathrm{E}-04$ & $2.11 \mathrm{E}-03$ \\
U-235 & $4.01 \mathrm{E}+04$ & $1.20 \mathrm{E}-03$ & $1.64 \mathrm{E}-03$ & $6.54 \mathrm{E}-01$ & $6.56 \mathrm{E}-01$ \\
U-238 & $4.01 \mathrm{E}+04$ & $1.20 \mathrm{E}-03$ & $1.54 \mathrm{E}-03$ & $1.43 \mathrm{E}-01$ & $1.45 \mathrm{E}-01$ \\
\hline
\end{tabular}

Footnote continued on next page. 


\section{TABLE 5 (Cont.)}

a It is assumed that baghouses are changed every year and that the radionuclides in Freon processed during this time are captured in the baghouses. Baghouse characteristics: cylindrical in shape with length $=100 \mathrm{~cm}$, radius $=34 \mathrm{~cm}$, and material density of the dust collected $=1.4 \mathrm{~g} / \mathrm{cm}^{3}$ (data taken from RESRAD-RECYCLE Manual, Cheng et al. 2000). Dose coefficients are based on ICRP (1991) methodology from FGR 12 (Eckerman and Ryman 1993) for external dose calculations; those from ICRP (1996) for an adult member of the public are used for internal dose calculations. External and internal doses are calculated using RESRAD-BUILD computer code. Receptor is $2 \mathrm{~m}$ away from the dust/ash bags and spends 18 hours ( 6 hours per cell $\times 3$ cells) in 1 year handling baghouse at the El Dorado facility (Whitehurst 2020a). Inhalation rate $=1.2 \mathrm{~m}^{3} / \mathrm{hr}$ and mass loading $=1 \times 10^{-4} \mathrm{~g} / \mathrm{m}^{3}$, airborne particulate filtration efficiency is $99.97 \%$ (employees wear full-face air-purifying respirators with MERSORB-P100 cartridges; Whitehurst 2020b) are used for inhalation dose estimation. There is no ingestion dose because workers use PPE. Mass loading is from ANSI N13.12 (ANSI 2013), 1999 Standard (ANSI 1999).

\subsection{EXPOSURE TO NON-INVOLVED WORKERS AND GENERAL PUBLIC}

Three potential scenarios/receptors are considered: (1) non-involved incinerator worker or general public near the incinerator, (2) general public near the disposal facility where the incinerator ashes/sludge are disposed of, and (3) general public accidentally drinking effluent water from incinerator emission system washing/cleaning. For scenario/receptor 3 , at all three incinerators that Freon is going to be shipped to, emission system effluent water is not directly released to waterways. It is disposed of eventually to ground or landfill. Therefore, we do not analyze the effects of directly drinking effluent water. If the effluent water containing all radionuclides is disposed of in a landfill, it is covered in scenario 2 because that scenario assumes all radionuclides from the incinerator are deposited at the landfill. The dose estimations for scenarios 1 and 2 are discussed below.

\subsubsection{Scenario 1: Radionuclides Exhausted from the Incinerator Stack, thereby Exposing MEI (PPPO 2020, Section 5.2.4)}

For scenario 1, dose is estimated for two receptors: a non-involved worker at the incinerator who is not directly involved in incineration of Freon, and a member of the public near the incinerator facility.

To calculate the bounding potential inhalation dose for the non-involved worker at an incinerator, we use the Aragonite Incinerator because the particulate emission from this incinerator results in the maximum MEI dose. It is assumed that a non-involved worker is located 100-1,000 $\mathrm{m}$ away from the air release point (stack). The worker is present at that location for 2,000 hours in 1 year (PPPO 2020, Section 5.2.4). 
It is assumed that 1.5 million pounds of Freon at the ALs (Table 1) is processed each year for 6 years at the Aragonite incinerator. To estimate the average particulate release rate, it is assumed that particulate emission occurs from the incinerator at the incinerator's permit limit for particulates: $2.8 \times 10^{-2} \mathrm{~g} / \mathrm{m}^{3}$ from a stack at a height of 149 feet $(\sim 45 \mathrm{~m})$. Flow rate is $77,840 \mathrm{acfm}$ (actual cubic feet per minute). The particulate released mass is $<5 \%$ of the processed mass at the incinerator (processed Freon mass is 680 million g/yr and particulate released mass is 30.8 million $\mathrm{g} / \mathrm{yr}$ from the Aragonite stack). For calculating radionuclide concentrations in the released particulates no reliable data is available; it is conservatively assumed that released particulates have the same radionuclide concentrations as in the Freon fed to the incinerator. Released mass per unit time is converted to activity released per unit time using the proposed ALs for each radionuclide. The conversion used is $1 \mathrm{ft}^{3}=0.0283168 \mathrm{~m}^{3}$. Table 6 shows the yearly radionuclide release rate.

Release of radionuclides from incinerators to the atmosphere will undergo downwind transport and mixing processes. They will be removed by deposition (both wet and dry) on the ground and by radioactive decay.

The RESRAD-OFFSITE code is used to calculate the non-involved worker dose. The dose is estimated from direct external exposure from deposited material, inhalation pathway, and soil ingestion of deposited particulates in soil at a receptor location ( $900 \mathrm{~m}$ away from the stack) that gives the maximum dose. The location that results in maximum dose is found by multiple runs of RESRAD-OFFSITE code. To model stack release, primary contaminated area is set at $1 \mathrm{~m}^{2}$ and release height is set at $45 \mathrm{~m}$ (equal to the stack height). Wind with a speed of 0.89 $\mathrm{m} / \mathrm{s}$ in neutral stability Class $\mathrm{D}$ is assumed to blow toward the receptor, and the receptor is present at that location for 2,000 hours.

The dose results for the non-involved incinerator worker are listed in Table 6. The calculated dose to this worker from each individual radionuclide is less than $1 \mathrm{mrem} / \mathrm{yr}$. The highest dose is $0.196 \mathrm{mrem} / \mathrm{yr}$ for $\mathrm{Np}$-237. The output of the RESRAD-OFFSITE run results is included in Appendix B. The output also includes a list of all input parameter values used.

TABLE 6 Non-involved Incinerator Worker Inhalation Dose from Air Effluent Release at Aragonite ${ }^{a}$

\begin{tabular}{lccccc}
\hline & $\begin{array}{c}\text { Yearly } \\
\text { radionuclide } \\
\text { release rate } \\
(\mathrm{pCi} / \mathrm{yr})\end{array}$ & $\begin{array}{c}\text { Ground } \\
\text { external } \\
(\mathrm{mrem} / \mathrm{yr})\end{array}$ & $\begin{array}{c}\text { Inhalation } \\
(\mathrm{mrem} / \mathrm{yr})\end{array}$ & $\begin{array}{c}\text { Soil } \\
\text { ingestion } \\
(\mathrm{mrem} / \mathrm{yr})\end{array}$ & $\begin{array}{c}\text { Total } \\
(\mathrm{mrem} / \mathrm{yr})\end{array}$ \\
\hline & & & & & \\
$\mathrm{Am}-241$ & $9.24 \mathrm{E}+07$ & $2.81 \mathrm{E}-07$ & $3.76 \mathrm{E}-02$ & $2.20 \mathrm{E}-07$ & $3.76 \mathrm{E}-02$ \\
$\mathrm{Cs}-137$ & $9.24 \mathrm{E}+07$ & $2.86 \mathrm{E}-05$ & $1.53 \mathrm{E}-05$ & $1.87 \mathrm{E}-08$ & $4.39 \mathrm{E}-05$ \\
$\mathrm{~Np}-237$ & $9.24 \mathrm{E}+08$ & $1.01 \mathrm{E}-04$ & $1.96 \mathrm{E}-01$ & $1.67 \mathrm{E}-06$ & $1.96 \mathrm{E}-01$ \\
$\mathrm{Pu}-238$ & $9.24 \mathrm{E}+07$ & $1.26 \mathrm{E}-09$ & $4.31 \mathrm{E}-02$ & $3.47 \mathrm{E}-07$ & $4.31 \mathrm{E}-02$ \\
$\mathrm{Pu}-239$ & $9.24 \mathrm{E}+07$ & $2.76 \mathrm{E}-09$ & $4.70 \mathrm{E}-02$ & $3.87 \mathrm{E}-07$ & $4.70 \mathrm{E}-02$ \\
$\mathrm{Pu}-240$ & $9.24 \mathrm{E}+07$ & $1.25 \mathrm{E}-09$ & $4.70 \mathrm{E}-02$ & $3.87 \mathrm{E}-07$ & $4.70 \mathrm{E}-02$ \\
\hline
\end{tabular}


TABLE 6 (Cont.)

\begin{tabular}{lccccc}
\hline & $\begin{array}{c}\text { Yearly } \\
\text { radionuclide } \\
\text { release rate } \\
\text { RCi/yr) }\end{array}$ & $\begin{array}{c}\text { Ground } \\
\text { external } \\
(\mathrm{mrem} / \mathrm{yr})\end{array}$ & $\begin{array}{c}\text { Inhalation } \\
(\mathrm{mrem} / \mathrm{yr})\end{array}$ & $\begin{array}{c}\text { Soil } \\
\text { ingestion } \\
(\mathrm{mrem} / \mathrm{yr})\end{array}$ & $\begin{array}{c}\text { Total } \\
(\mathrm{mrem} / \mathrm{yr})\end{array}$ \\
\hline Tc-99 & $9.24 \mathrm{E}+08$ & $1.64 \mathrm{E}-10$ & $5.09 \mathrm{E}-05$ & $1.38 \mathrm{E}-10$ & $5.09 \mathrm{E}-05$ \\
Th-228 & $9.24 \mathrm{E}+07$ & $3.26 \mathrm{E}-05$ & $1.71 \mathrm{E}-02$ & $8.51 \mathrm{E}-08$ & $1.71 \mathrm{E}-02$ \\
Th-230 & $9.24 \mathrm{E}+07$ & $1.41 \mathrm{E}-07$ & $3.92 \mathrm{E}-02$ & $3.27 \mathrm{E}-07$ & $3.92 \mathrm{E}-02$ \\
Th-232 & $9.24 \mathrm{E}+07$ & $2.81 \mathrm{E}-05$ & $4.31 \mathrm{E}-02$ & $7.02 \mathrm{E}-07$ & $4.31 \mathrm{E}-02$ \\
$\mathrm{U}-234$ & $9.24 \mathrm{E}+08$ & $3.24 \mathrm{E}-08$ & $3.68 \mathrm{E}-02$ & $6.60 \mathrm{E}-07$ & $3.68 \mathrm{E}-02$ \\
$\mathrm{U}-235$ & $9.24 \mathrm{E}+08$ & $6.31 \mathrm{E}-05$ & $3.33 \mathrm{E}-02$ & $6.38 \mathrm{E}-07$ & $3.34 \mathrm{E}-02$ \\
$\mathrm{U}-238$ & $9.24 \mathrm{E}+08$ & $1.33 \mathrm{E}-05$ & $3.14 \mathrm{E}-02$ & $6.52 \mathrm{E}-07$ & $3.14 \mathrm{E}-02$ \\
\hline
\end{tabular}

a It is assumed that a non-involved worker is located at a distance $(\sim 900 \mathrm{~m})$ where ground air concentration reaches its maximum value from the stack release at a height of $45 \mathrm{~m}$. The receptor spends 2,000 hours outdoors and is exposed to deposited material on the ground and the contamination in the air. RESRADOFFSITE code is used. To model stack release in RESRAD-OFFSITE, it is assumed that the primary contaminated area $=1 \mathrm{~m}^{2}$, and release height is equal to the stack height, $45 \mathrm{~m}$. To estimate the yearly average radionuclide release rate, it is assumed particulate emission occurs from the incinerator at $2.8 \times 10^{-2} \mathrm{~g} / \mathrm{m}^{3}$. The incinerator is operational $95 \%$ of the time. Flow rate is $77,840 \mathrm{acfm}$. Conversions used $1 \mathrm{ft}^{3}=0.0283168 \mathrm{~m}^{3}$. The inhalation rate in RESRAD-OFFSITE code is increased to $10,512 \mathrm{~m}^{3} / \mathrm{yr}$ to match $1.2 \mathrm{~m}^{3} / \mathrm{h}$ inhalation rate for the worker.

Dose is also calculated for a member of the public located $5 \mathrm{~km}$ (about 3 miles) away from the incinerator (see Table 2). It is assumed that 1.5 million lb. of Freon at the ALs (Table 1) is processed each year for 6 years at the El Dorado incinerator. The released activity is $<6 \%$ of the processed activity at the incinerator (processed freon mass is 680 million $\mathrm{g} / \mathrm{yr}$ and particulate released mass is 37.7 million $\mathrm{g} / \mathrm{yr}$ from the El Dorado SN-01 stack). For calculating radionuclide concentrations in the released particulates, because no reliable data are available, it is conservatively assumed that the released particulates have the same radionuclide concentrations as in the Freon fed to the incinerator.

RESRAD-OFFSITE code (Yu et al. 2019) is used in this dose estimation. The dose is estimated from direct external exposure from deposited material, inhalation pathway, and incidental ingestion of deposited particulates in soil. To model stack release, the RESRADOFFSITE input for the primary contaminated area is set at $1 \mathrm{~m}^{2}$ and the release height is set at $59.44 \mathrm{~m}$ (i.e., the stack height).

Table 7 shows the estimated maximum yearly dose for a member of the public located $5 \mathrm{~km}$ (about 3 miles) from the incinerator (incinerator neighbor). Results are shown for the El Dorado-SN-01 stack release because releases there are the highest among three incinerators that Freon is planned to be shipped to, and it has the nearest resident. The highest dose is 0.0463 mrem/yr for Np-237. The output of RESRAD-OFFSITE run results is included in Appendix B. The output also includes a list of all input parameter values used. 
TABLE 7 Incinerator (EI Dorado SN-01) Neighbor Dose Estimation ${ }^{\text {a }}$

\begin{tabular}{|c|c|c|c|c|c|}
\hline \multirow[b]{2}{*}{ Radionuclide } & \multirow[b]{2}{*}{$\begin{array}{c}\text { Yearly } \\
\text { radionuclide } \\
\text { release rate } \\
(\mathrm{pCi} / \mathrm{yr})\end{array}$} & \multicolumn{4}{|c|}{ Dose by Pathways ${ }^{\mathrm{a}}$} \\
\hline & & $\begin{array}{l}\text { Ground } \\
\text { external } \\
\text { (mrem/yr) }\end{array}$ & $\begin{array}{l}\text { Inhalation } \\
(\mathrm{mrem} / \mathrm{yr})\end{array}$ & $\begin{array}{c}\text { Soil } \\
\text { ingestion } \\
(\mathrm{mrem} / \mathrm{yr})\end{array}$ & $\begin{array}{c}\text { Total } \\
\text { (mrem/yr) }\end{array}$ \\
\hline Am-241 & $1.13 \mathrm{E}+08$ & $8.77 \mathrm{E}-08$ & $8.88 \mathrm{E}-03$ & $8.66 \mathrm{E}-08$ & $8.88 \mathrm{E}-03$ \\
\hline Cs-137 & $1.13 \mathrm{E}+08$ & $1.12 \mathrm{E}-05$ & $3.61 \mathrm{E}-06$ & 7.35E-09 & $1.48 \mathrm{E}-05$ \\
\hline Np-237 & $1.13 \mathrm{E}+09$ & $3.90 \mathrm{E}-05$ & $4.63 \mathrm{E}-02$ & $6.58 \mathrm{E}-07$ & 4.63E-02 \\
\hline Pu-238 & $1.13 \mathrm{E}+08$ & $3.96 \mathrm{E}-10$ & $1.02 \mathrm{E}-02$ & $1.36 \mathrm{E}-07$ & $1.02 \mathrm{E}-02$ \\
\hline Pu-239 & $1.13 \mathrm{E}+08$ & $8.64 \mathrm{E}-10$ & $1.11 \mathrm{E}-02$ & $1.52 \mathrm{E}-07$ & $1.11 \mathrm{E}-02$ \\
\hline Pu-240 & $1.13 \mathrm{E}+08$ & $3.94 \mathrm{E}-10$ & $1.11 \mathrm{E}-02$ & $1.52 \mathrm{E}-07$ & $1.11 \mathrm{E}-02$ \\
\hline Tc-99 & $1.13 \mathrm{E}+09$ & $5.13 \mathrm{E}-11$ & $1.20 \mathrm{E}-05$ & $5.41 \mathrm{E}-11$ & $1.20 \mathrm{E}-05$ \\
\hline Th-228 & $1.13 \mathrm{E}+08$ & $1.27 \mathrm{E}-05$ & $4.04 \mathrm{E}-03$ & $3.35 \mathrm{E}-08$ & $4.05 \mathrm{E}-03$ \\
\hline Th-230 & $1.13 \mathrm{E}+08$ & $5.43 \mathrm{E}-08$ & $9.25 \mathrm{E}-03$ & $1.29 \mathrm{E}-07$ & $9.25 \mathrm{E}-03$ \\
\hline Th-232 & $1.13 \mathrm{E}+08$ & $1.10 \mathrm{E}-05$ & $1.02 \mathrm{E}-02$ & $2.76 \mathrm{E}-07$ & $1.02 \mathrm{E}-02$ \\
\hline U-234 & $1.13 \mathrm{E}+09$ & $1.02 \mathrm{E}-08$ & $8.70 \mathrm{E}-03$ & $2.60 \mathrm{E}-07$ & $8.70 \mathrm{E}-03$ \\
\hline U-235 & $1.13 \mathrm{E}+09$ & $2.00 \mathrm{E}-05$ & 7.87E-03 & $2.51 \mathrm{E}-07$ & $7.89 \mathrm{E}-03$ \\
\hline U-238 & $1.13 \mathrm{E}+09$ & $5.18 \mathrm{E}-06$ & $7.41 \mathrm{E}-03$ & $2.56 \mathrm{E}-07$ & $7.41 \mathrm{E}-03$ \\
\hline
\end{tabular}

${ }^{\text {a }}$ It is assumed that the incinerator neighbor is located $5 \mathrm{~km}$ away from the incinerator and is exposed to effluent release. The receptor spends $50 \%$ of their time inside a dwelling and $25 \%$ time outdoors and is exposed to deposited material on the ground and the contamination in the air. RESRAD-OFFSITE code is used. To estimate the yearly average radionuclide release rate, we assume that particulate emission occurs from the incinerator at $2.8 \times 10^{-2} \mathrm{~g} / \mathrm{m}^{3}$. The incinerator is operational $90 \%$ of the time. Flow rate is $100,568 \mathrm{acfm}$. Conversions used $1 \mathrm{ft}^{3}=0.0283168 \mathrm{~m}^{3}$.

\subsubsection{Scenario 2: All radionuclides Are Captured in the Emission Controls or Collected in the Incinerator Ash and Subsequently Disposed of in a Landfill, Resulting in Exposure to a Landfill Neighbor (PPPO 2020, Section 5.2.4)}

Dose is calculated for a member of the public living in a dwelling $50 \mathrm{~m}$ away from the landfill. It is assumed, based on professional judgement, that the landfill area is $40,000 \mathrm{~m}^{2}$ with a depth of $2 \mathrm{~m}$; disposal will occur over a period of 6 years and may be at different areas and at different depths at a landfill; baghouse dust, ash, slag, or any other waste generated at the incinerator may get mixed with other materials deposited at a landfill. RESRAD-OFFSITE is used in this dose estimation. The total dose from all relevant exposure pathways in the operational phase of the landfill is calculated for a 50 -year time frame and after the operational phase (1,000-year time frame). The landfill may have liners that would reduce water infiltration. Because there is a lack of data for any specific landfill, all RESRAD-OFFSITE defaults are used except for the site layout. When the landfill is closed, cover material may be placed on top, further reducing water infiltration and radionuclide leaching. Neither liners nor covers are included in these calculations. 
Table 8 shows the maximum estimated dose in the time frame of 1,000 years, for the landfill neighbor scenario (a person living in a dwelling $50 \mathrm{~m}$ away from the landfill) by

pathways and total. The calculated total dose for each radionuclide is less than $1 \mathrm{mrem} / \mathrm{yr}$. The highest dose is $0.468 \mathrm{mrem} / \mathrm{yr}$ for Tc-99. 
TABLE 8 Landfill Neighbor Scenario Dose for Different Exposure Pathways ${ }^{a}$

Dose by Pathways (mrem/yr)

\begin{tabular}{|c|c|c|c|c|c|c|c|c|c|c|}
\hline Radionuclide & $\begin{array}{c}\text { Time of } \\
\text { peak } \\
\text { total dose } \\
(\mathrm{yr})\end{array}$ & $\begin{array}{c}\text { Direct } \\
\text { radiation }\end{array}$ & Inhalation & $\begin{array}{l}\text { Ingestion } \\
\text { of fish }\end{array}$ & Plant & Meat & Milk & Soil & $\begin{array}{c}\text { Drinking } \\
\text { water }\end{array}$ & Total \\
\hline Am-241 & 778 & $1.53 \mathrm{E}-05$ & $1.08 \mathrm{E}-04$ & $6.88 \mathrm{E}-09$ & $1.05 \mathrm{E}-02$ & $7.58 \mathrm{E}-05$ & $1.09 \mathrm{E}-05$ & $1.41 \mathrm{E}-05$ & $2.55 \mathrm{E}-02$ & $3.62 \mathrm{E}-02$ \\
\hline Cs-137 & 0 & $2.11 \mathrm{E}-03$ & $2.86 \mathrm{E}-07$ & $2.61 \mathrm{E}-07$ & $8.85 \mathrm{E}-08$ & $1.12 \mathrm{E}-07$ & $6.91 \mathrm{E}-08$ & $4.65 \mathrm{E}-11$ & $0.00 \mathrm{E}+00$ & $2.11 \mathrm{E}-03$ \\
\hline Np-237 & 2 & $5.99 \mathrm{E}-03$ & $3.71 \mathrm{E}-03$ & $3.54 \mathrm{E}-05$ & 8.06E-06 & $3.44 \mathrm{E}-07$ & 3.83E-09 & $1.99 \mathrm{E}-08$ & $0.00 \mathrm{E}+00$ & $9.74 \mathrm{E}-03$ \\
\hline $\mathrm{Pu}-238$ & 0 & $1.83 \mathrm{E}-08$ & 8.13E-04 & $2.40 \mathrm{E}-07$ & $1.52 \mathrm{E}-06$ & $6.50 \mathrm{E}-09$ & $1.52 \mathrm{E}-10$ & $8.31 \mathrm{E}-10$ & $0.00 \mathrm{E}+00$ & 8.15E-04 \\
\hline $\mathrm{Pu}-239$ & 14 & $1.41 \mathrm{E}-07$ & 8.89E-04 & $3.57 \mathrm{E}-06$ & $1.70 \mathrm{E}-06$ & 8.36E-09 & $1.80 \mathrm{E}-10$ & 2.61E-08 & $0.00 \mathrm{E}+00$ & 8.94E-04 \\
\hline $\mathrm{Pu}-240$ & 9 & $2.41 \mathrm{E}-08$ & 8.89E-04 & $3.01 \mathrm{E}-06$ & 1.69E-06 & $7.90 \mathrm{E}-09$ & $1.75 \mathrm{E}-10$ & $1.72 \mathrm{E}-08$ & $0.00 \mathrm{E}+00$ & 8.94E-04 \\
\hline Tc-99 & 17 & $1.54 \mathrm{E}-06$ & 4.94E-07 & $6.81 \mathrm{E}-14$ & $1.31 \mathrm{E}-01$ & $1.71 \mathrm{E}-03$ & $5.98 \mathrm{E}-02$ & $1.53 \mathrm{E}-06$ & $2.76 \mathrm{E}-01$ & $4.68 \mathrm{E}-01$ \\
\hline Th-228 & 0 & $4.62 \mathrm{E}-03$ & $2.72 \mathrm{E}-04$ & $1.60 \mathrm{E}-08$ & $8.00 \mathrm{E}-07$ & $3.42 \mathrm{E}-09$ & $4.00 \mathrm{E}-10$ & $4.00 \mathrm{E}-10$ & $0.00 \mathrm{E}+00$ & $4.89 \mathrm{E}-03$ \\
\hline Th-230 & 1,000 & $9.19 \mathrm{E}-04$ & 7.64E-04 & $3.25 \mathrm{E}-04$ & $1.06 \mathrm{E}-05$ & $8.61 \mathrm{E}-07$ & $5.41 \mathrm{E}-07$ & $1.68 \mathrm{E}-06$ & $1.44 \mathrm{E}-06$ & $2.02 \mathrm{E}-03$ \\
\hline Th-232 & 1,000 & $9.19 \mathrm{E}-03$ & $1.26 \mathrm{E}-03$ & $3.19 \mathrm{E}-04$ & $2.85 \mathrm{E}-04$ & $1.10 \mathrm{E}-05$ & $1.09 \mathrm{E}-05$ & $6.30 \mathrm{E}-06$ & $8.62 \mathrm{E}-40$ & $1.11 \mathrm{E}-02$ \\
\hline U-234 & 1,000 & 2.19E-05 & $6.93 \mathrm{E}-05$ & 7.63E-06 & 2.79E-03 & $1.37 \mathrm{E}-04$ & 8.63E-04 & 7.95E-06 & $6.70 \mathrm{E}-03$ & $1.06 \mathrm{E}-02$ \\
\hline U-235 & 1,000 & $8.04 \mathrm{E}-04$ & $9.38 \mathrm{E}-05$ & $7.02 \mathrm{E}-07$ & $3.67 \mathrm{E}-03$ & $7.59 \mathrm{E}-04$ & 8.39E-04 & $1.10 \mathrm{E}-05$ & $8.72 \mathrm{E}-03$ & $1.49 \mathrm{E}-02$ \\
\hline U-238 & 1,000 & $1.93 \mathrm{E}-04$ & $4.21 \mathrm{E}-05$ & $1.34 \mathrm{E}-07$ & $2.77 \mathrm{E}-03$ & $1.36 \mathrm{E}-04$ & $8.57 \mathrm{E}-04$ & 7.83E-06 & $6.66 \mathrm{E}-03$ & $1.07 \mathrm{E}-02$ \\
\hline
\end{tabular}

${ }^{a}$ Pathway doses are listed at the time when the maximum total dose occurs. 


\section{CONCLUSIONS}

The PPPO request for ALs used simplistic calculations to determine public exposure. This independent review performed a more detailed analysis of the exposure pathways associated with the incineration of the Freon for the same receptors and scenarios described in PPPO (2020). Bounding doses were calculated to verify compliance with DOE O 458.1 dose criteria. Radiological doses were calculated for 13 radionuclides at the proposed AL concentrations for multiple receptors involved in different activities associated with the disposal of Freon. The dose was estimated for a maximally exposed truck driver transporting Freon in ISO containers to incinerators, maximally exposed workers involved in storing and processing Freon at the incinerator, maximally exposed workers involved in handling dust/waste at the incinerator, and workers disposing of dust/waste at a landfill. The doses were also estimated for the maximally exposed non-involved workers at the incinerator not directly involved in handling or processing Freon, a member of the general public (incinerator neighbor) who may be exposed to the air release from the incinerator, and a member of the general public (landfill neighbor) who may be exposed to activity disposed in a landfill. The estimated doses were all less than $1 \mathrm{mrem} / \mathrm{yr}$ for each radionuclide at the proposed ALs, meeting the DOE O 458.1 dose requirements. Therefore, setting the ALs at the ANSI N13.12 (ANSI 2013) concentration limits for these 13 radionuclides as shown in Table 1 is reasonable and recommended. 


\section{REFERENCES}

ANSI (American National Standards Institute, Inc.), 2013, American National StandardSurface and Volume Radioactivity Standards for Clearance, ANSI/HPS N13.12-2013. Approved May 6.

ANSI, 1999, American National Standard - Surface and Volume Radioactivity Standards for Clearance, ANSI/HPS N13.12-1999. Approved August 31, 1999.

Cheng, J.J., B. Kassas, C. Yu, D. LePoire, J. Arnish, E.S. Dovel, S. Y. Chen, W.A. Williams, A. Wallo, and H. Peterson, 2000, RESRAD-RECYCLE: A Computer Model for Analyzing the Radiological Doses and Risks Resulting from the Recycling of Radioactive Scrap Metals and the Reuse of Surface-Contaminated Material and Equipment, ANL/EAD-3, November.

Dihel, D., 2020, personal communication from Dihel (Enterprise Technical Assistance Services) to C. Yu (Argonne National Laboratory), April 22.

Eckerman, K.F., and J.C. Ryman, 1993, External Exposure to Radionuclides in Air, Water, and Soil, Exposure to Dose Coefficients for General Application, Based on the 1987 Federal Radiation Protection Guidance, EPA 402-R-93-076, Federal Guidance Report 12, prepared by Oak Ridge National Laboratory, Oak Ridge, Tenn., for U.S. Environmental Protection Agency, Office of Radiation and Indoor Air, Washington, D.C.

ICRP (International Commission on Radiological Protection) 1991, 1990 Recommendations of the International Commission on Radiological Protection, Publication 60, Annals of the ICRP, 21(1-3), Pergamon Press, New York, N.Y.

ICRP, 1996, Age-Dependent Doses to Members of the Public from Intake of Radionuclides: Part 5-Compilation of Ingestion and Inhalation Dose Coefficients, Publication 72, Annals of the ICRP, Vol. 26(1), Pergamon Press, New York, N.Y.

PPPO (Portsmouth/Paducah Project Office), 2020, Authorized limits request for volumetric release limits for R-114 (Freon) at the Paducah Gaseous Diffusion Plant Paducah, Kentucky, U.S. Department of Energy, Lexington, Kentucky, March.

Whitehurst, G., 2020a, personal communication from Whitehurst (Portsmouth/Paducah Project Office) to C. Yu (Argonne National Laboratory), May 5.

Whitehurst, G., 2020b, personal communication from Whitehurst (Portsmouth/Paducah Project Office) to C. Yu (Argonne National Laboratory), May 6.

Whitehurst, G., 2020c, personal communication from Whitehurst (Portsmouth/Paducah Project Office) to C. Yu (Argonne National Laboratory), May 8. 
Whitehurst, G., 2020d, personal communication from Whitehurst (Portsmouth/Paducah Project Office) to C. Yu (Argonne National Laboratory), May 12.

Yu, C., D.J. LePoire, J.-J. Cheng, E. Gnanapragasam, S. Kamboj, J. Arnish, B.M. Biwer, A.J. Zielen, W.A. Williams, A. Wallo III, and H.T. Peterson, Jr., 2003, User's Manual for RESRADBUILD Version 3, ANL/EAD/03-1, June.

Yu, C., E. Gnanapragasam, J.-J. Cheng, D. LePoire, S. Kamboj, and C. Wang 2020, User's Manual for RESRAD-OFFSITE Code Version 4, NUREG/CR-7268, ANL/EVS/TM-19/2, February. 


\section{APPENDIX A: \\ RESRAD-BUILD RUN RESULTS FOR TRUCK DRIVER, INTERNATIONAL ORGANIZATION FOR STANDARDIZATION CONTAINER HANDLING WORKER, AND BAGHOUSE WORKER SCENARIOS}

RESRAD-BUILD code was used to analyze the truck driver, International Organization for Standardization (ISO) container handling worker, and baghouse worker scenarios. The results are provided in this appendix in the form of reports generated from different scenario runs from RESRAD-BUILD code.

\section{A.1 RESRAD-BUILD RESULTS FOR TRUCK DRIVER SCENARIO}

** RESRAD-BUILD Dose Program Output, Version 3.8.7.0 Beta 06/05/20 09:56:34 Page: 1 ** Title : RESRAD-BUILD Truck Driver (S1R1 and S2R2

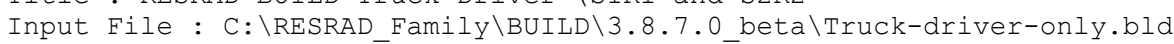
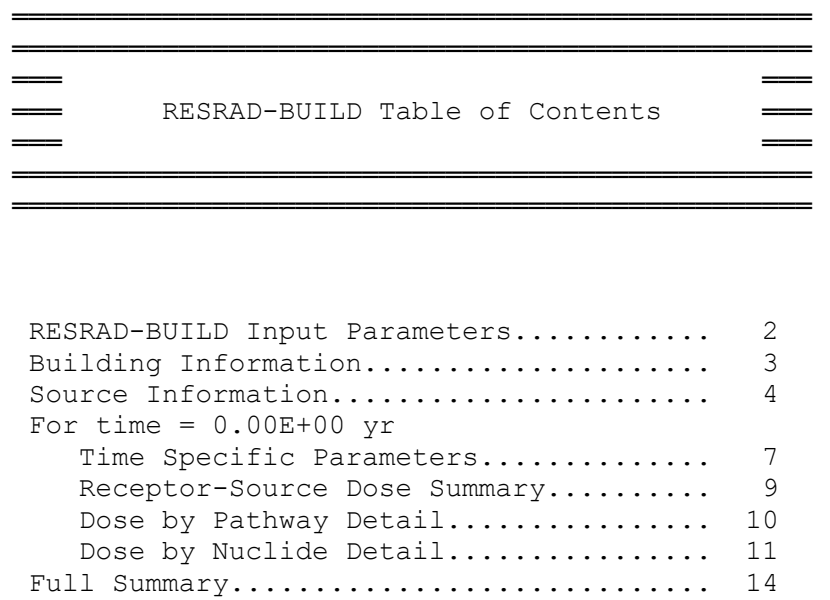

** RESRAD-BUILD Dose Program Output, Version 3.8.7.0 Beta 06/05/20 09:56:34 Page: 2 ** Title : RESRAD-BUILD Truck Driver (S1R1 and S2R2

Input File : C: \RESRAD_Family \BUILD 3.8.7.0_beta\Truck-driver-only.bld
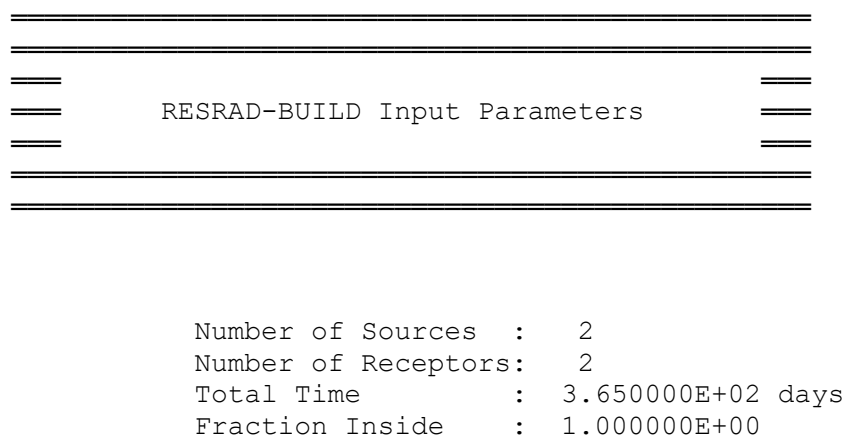

Total Time : $3.650000 \mathrm{E}+02$ days

$1.000000 \mathrm{E}+00$ 


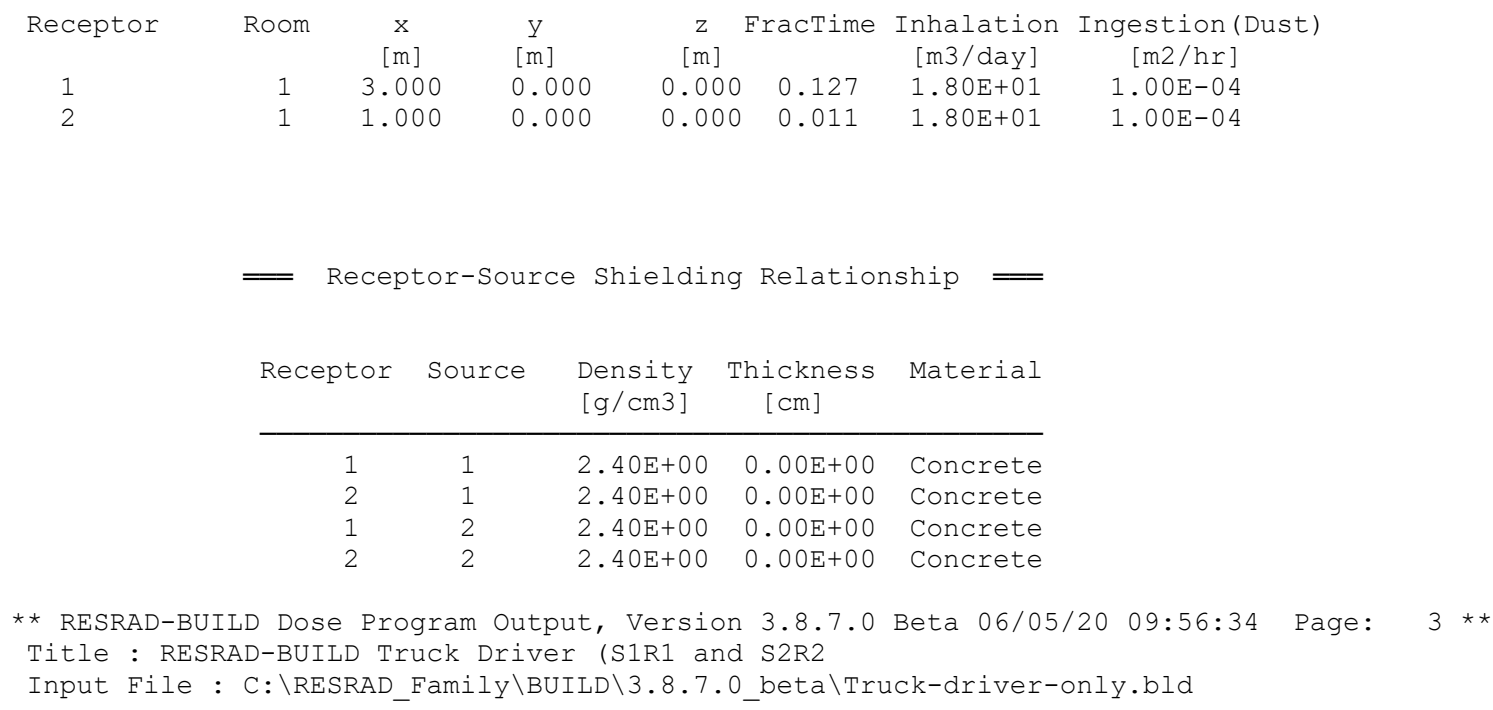


Porosity $\quad: 1.00 \mathrm{E}-01$

Eff. Diffusion $[\mathrm{m} 2 / \mathrm{s}]: 2.00 \mathrm{E}-05$

Emanation Fractions (1):0.00E+00

(2) $: 0.00 \mathrm{E}+00$

Contamination: :

Nuclide Concentration

\begin{tabular}{|c|c|c|c|c|c|}
\hline & {$[\mathrm{pCi} / \mathrm{g}]$} & $\begin{array}{l}\text { Ingestion } \\
{[\mathrm{mrem} / \mathrm{pCi}]}\end{array}$ & $\begin{array}{l}\text { Inhalation } \\
\text { [mrem/pCi] }\end{array}$ & $\begin{array}{l}\text { Submersion } \\
{[\mathrm{mrem} / \mathrm{yr} /} \\
(\mathrm{pCi} / \mathrm{m} 3)]\end{array}$ & $\begin{array}{c}\text { External } \\
{[\mathrm{mrem} / \mathrm{yr} /} \\
{[\mathrm{pCi} / \mathrm{g}]}\end{array}$ \\
\hline PU-238 & $3.000 \mathrm{E}+00$ & $8.510 \mathrm{E}-04$ & $4.070 E-01$ & $4.088 E-07$ & $1.166 \mathrm{E}-04$ \\
\hline $\mathrm{AM}-241$ & $3.000 \mathrm{E}+00$ & $7.400 \mathrm{E}-04$ & $3.552 \mathrm{E}-01$ & $7.872 E-05$ & $3.719 \mathrm{E}-02$ \\
\hline $\mathrm{PU}-240$ & $3.000 \mathrm{E}+00$ & $9.250 \mathrm{E}-04$ & $4.440 \mathrm{E}-01$ & $3.995 \mathrm{E}-07$ & $1.129 \mathrm{E}-04$ \\
\hline PU-239 & $3.000 \mathrm{E}+00$ & $9.250 \mathrm{E}-04$ & $4.440 \mathrm{E}-01$ & $4.065 E-07$ & $2.635 \mathrm{E}-04$ \\
\hline $\mathrm{U}-238$ & $3.000 \mathrm{E}+01$ & $1.791 \mathrm{E}-04$ & $2.963 \mathrm{E}-02$ & $2.091 E-04$ & $1.557 \mathrm{E}-01$ \\
\hline$N P-237$ & $3.000 \mathrm{E}+01$ & $4.102 \mathrm{E}-04$ & $1.850 \mathrm{E}-01$ & $1.101 \mathrm{E}-03$ & $1.012 \mathrm{E}+00$ \\
\hline $\mathrm{U}-234$ & $3.000 \mathrm{E}+01$ & $1.813 \mathrm{E}-04$ & $3.478 \mathrm{E}-02$ & $7.136 \mathrm{E}-07$ & $3.439 \mathrm{E}-04$ \\
\hline $\mathrm{U}-236$ & $0.000 \mathrm{E}+00$ & $1.739 \mathrm{E}-04$ & $3.219 \mathrm{E}-02$ & $4.508 E-07$ & $1.781 \mathrm{E}-04$ \\
\hline $\mathrm{U}-235$ & $3.000 \mathrm{E}+01$ & $1.752 \mathrm{E}-04$ & $3.145 \mathrm{E}-02$ & $8.074 \mathrm{E}-04$ & $6.918 \mathrm{E}-01$ \\
\hline $\mathrm{TH}-230$ & $3.000 \mathrm{E}+00$ & $7.770 \mathrm{E}-04$ & $3.700 \mathrm{E}-01$ & $1.729 \mathrm{E}-06$ & $1.071 \mathrm{E}-03$ \\
\hline $\mathrm{U}-233$ & $0.000 \mathrm{E}+00$ & $1.887 \mathrm{E}-04$ & $3.552 \mathrm{E}-02$ & $1.659 \mathrm{E}-06$ & $1.265 \mathrm{E}-03$ \\
\hline$P A-231$ & $0.000 \mathrm{E}+00$ & $2.627 \mathrm{E}-03$ & $5.180 \mathrm{E}-01$ & $1.834 \mathrm{E}-04$ & $1.762 \mathrm{E}-01$ \\
\hline $\mathrm{TH}-232$ & $3.000 \mathrm{E}+00$ & $8.510 E-04$ & $4.070 \mathrm{E}-01$ & $8.456 E-07$ & $4.560 \mathrm{E}-04$ \\
\hline$R A-226$ & $0.000 \mathrm{E}+00$ & $1.037 \mathrm{E}-03$ & $3.526 \mathrm{E}-02$ & $9.769 \mathrm{E}-03$ & $1.060 \mathrm{E}+01$ \\
\hline TH-229 & $0.000 \mathrm{E}+00$ & $2.269 \mathrm{E}-03$ & $9.481 \mathrm{E}-01$ & $1.624 \mathrm{E}-03$ & $1.480 \mathrm{E}+00$ \\
\hline $\mathrm{RA}-228$ & $0.000 \mathrm{E}+00$ & $2.555 \mathrm{E}-03$ & $5.929 \mathrm{E}-02$ & $5.240 \mathrm{E}-03$ & $5.662 \mathrm{E}+00$ \\
\hline$A C-227$ & $0.000 \mathrm{E}+00$ & $4.473 E-03$ & $2.104 \mathrm{E}+00$ & $2.034 \mathrm{E}-03$ & $1.869 \mathrm{E}+00$ \\
\hline TH-228 & $3.000 \mathrm{E}+00$ & $5.301 \mathrm{E}-04$ & $1.614 \mathrm{E}-01$ & $8.921 E-03$ & $9.672 \mathrm{E}+00$ \\
\hline PB-210 & $0.000 \mathrm{E}+00$ & $2.558 \mathrm{E}-03$ & $2.106 \mathrm{E}-02$ & $3.534 \mathrm{E}-05$ & $7.457 \mathrm{E}-03$ \\
\hline $\mathrm{PO}-210$ & $0.000 \mathrm{E}+00$ & $4.440 \mathrm{E}-03$ & $1.591 \mathrm{E}-02$ & $4.544 \mathrm{E}-08$ & $4.934 \mathrm{E}-05$ \\
\hline
\end{tabular}

** RESRAD-BUILD Dose Program Output, Version 3.8.7.0 Beta 06/05/20 09:56:34 Page: 5 ** Title : RESRAD-BUILD Truck Driver (S1R1 and S2R2

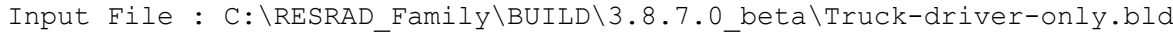

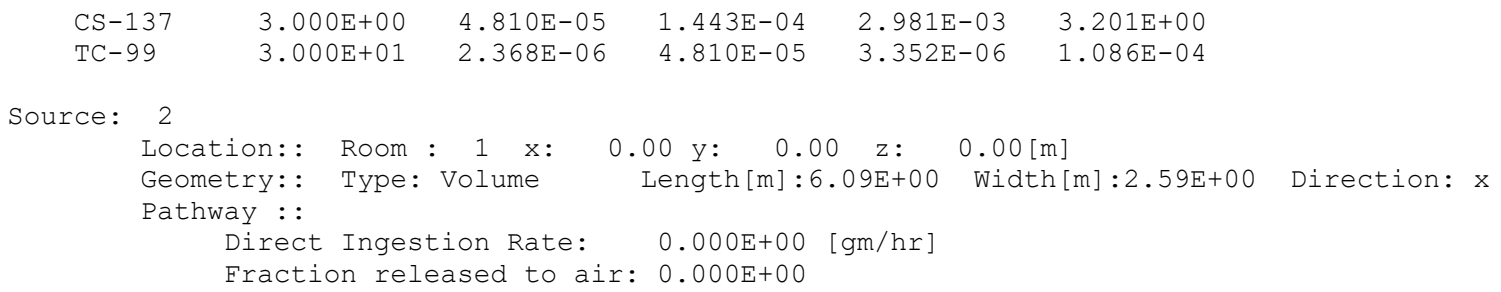

Contamination: :

Nuclide Concentration Dose Conversion Factor (Library: ICRP 72 (Adult))

\begin{tabular}{|c|c|c|c|c|c|}
\hline PUI-238 & [pCi/g] & $\begin{array}{l}\text { Ingestion } \\
\text { [mrem/pCi] }\end{array}$ & $\begin{array}{l}\text { Inhalation } \\
\text { [mrem/pCi] }\end{array}$ & $\begin{array}{l}\text { Submersion } \\
{[\mathrm{mrem} / \mathrm{yr} /} \\
(\mathrm{pCi} / \mathrm{m} 3)]\end{array}$ & $\begin{array}{c}\text { External } \\
\text { [mrem/yr/ } \\
{[\mathrm{pCi} / \mathrm{g}]}\end{array}$ \\
\hline
\end{tabular}




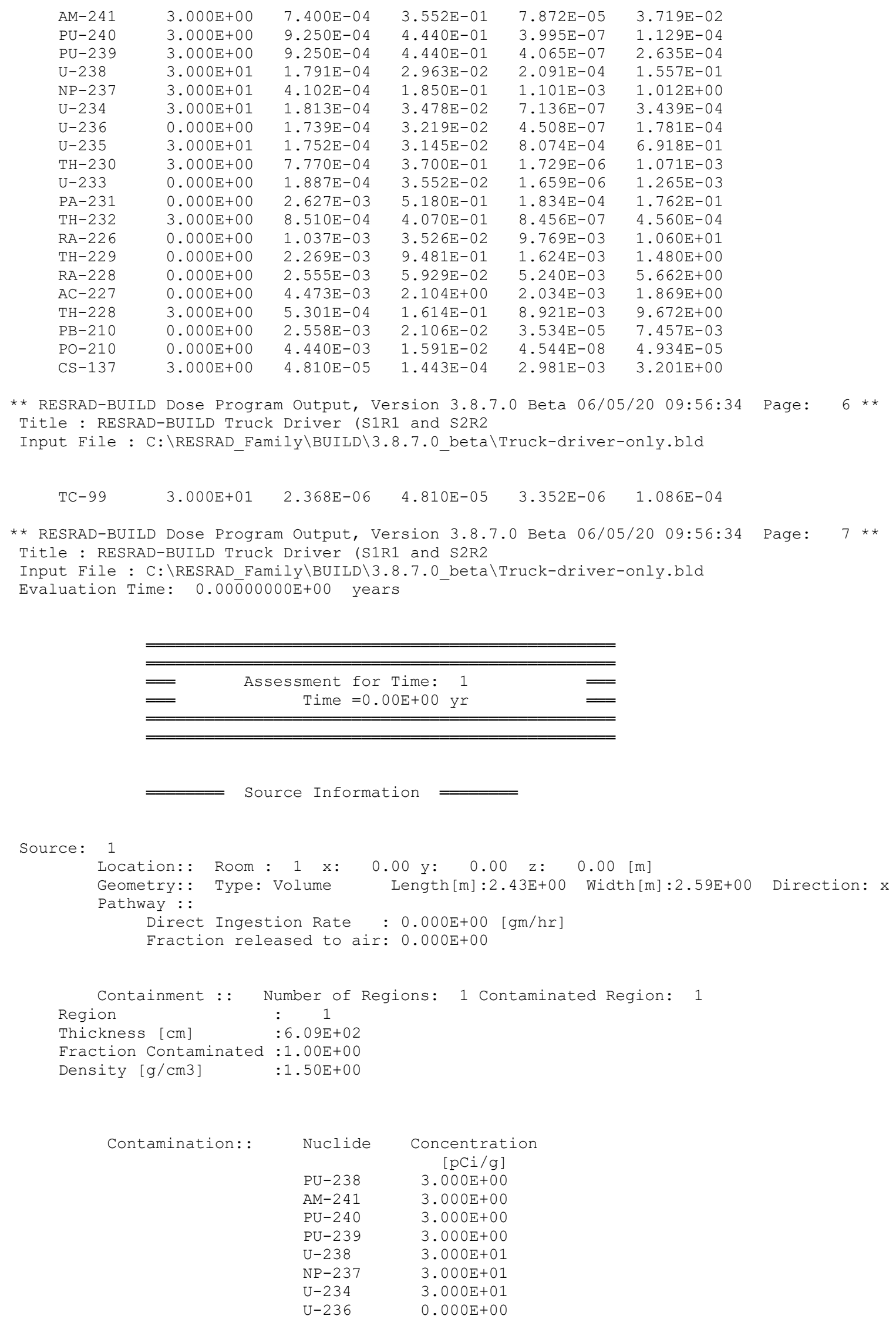




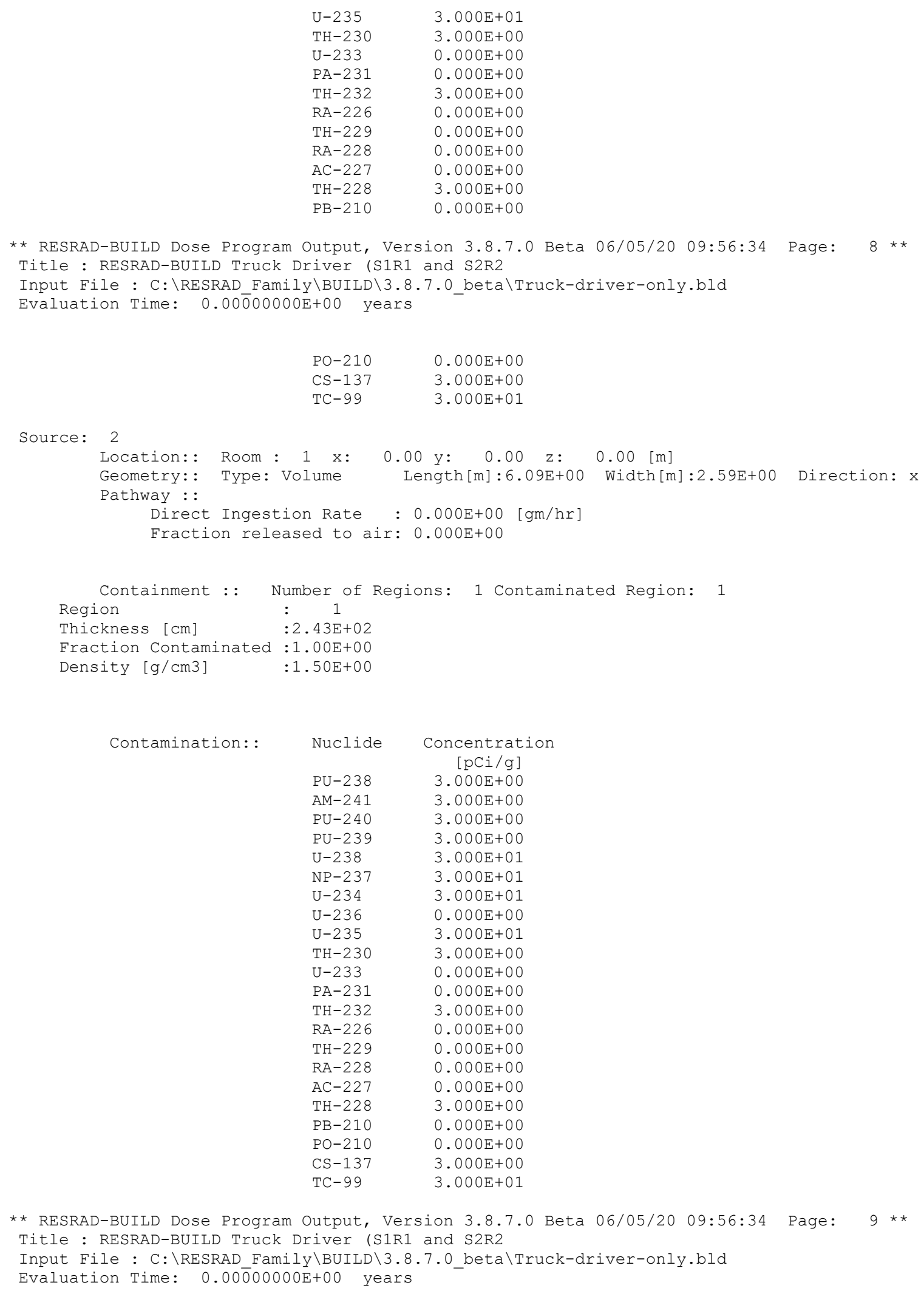



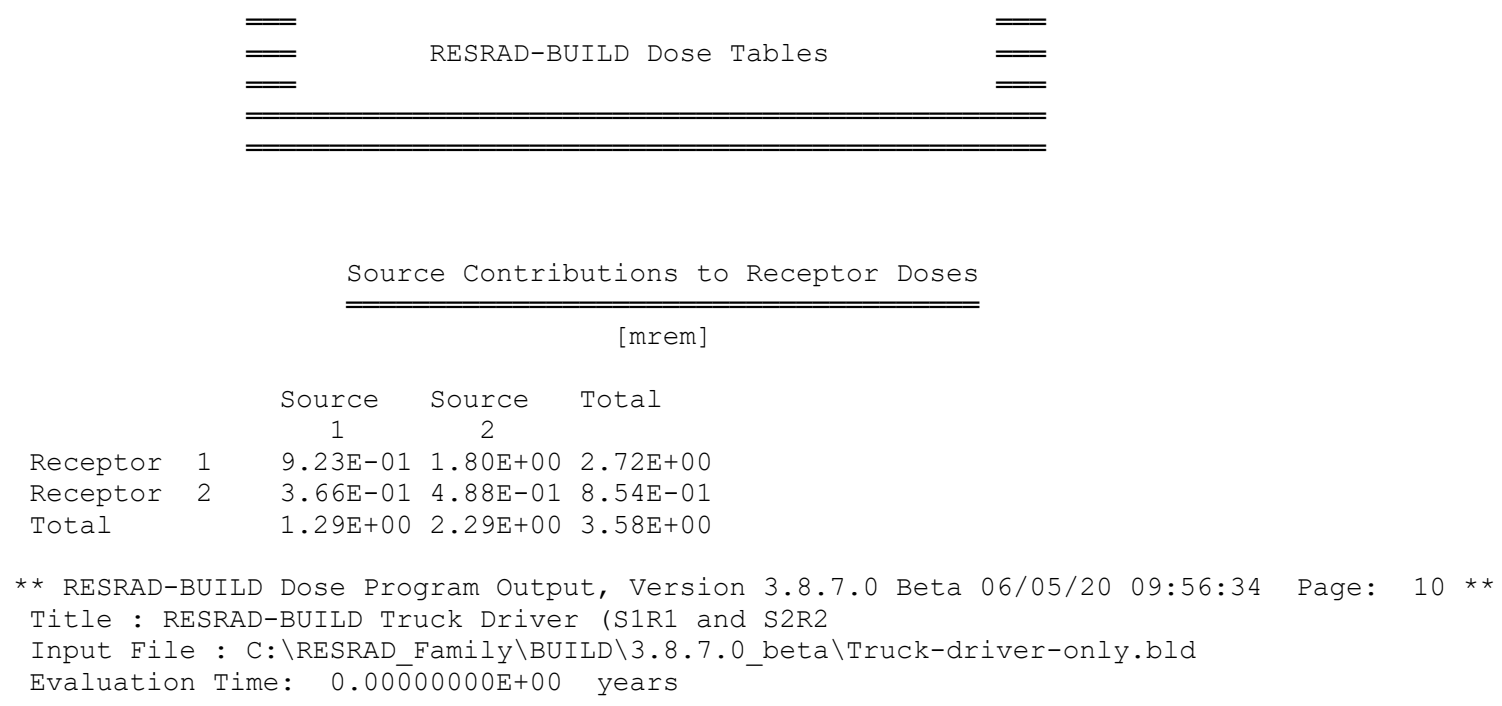

** RESRAD-BUILD Dose Program Output, Version 3.8.7.0 Beta 06/05/20 09:56:34 Page: 11 ** Title : RESRAD-BUILD Truck Driver (S1R1 and S2R2

Input File : C: \RESRAD Family \BUILD $\backslash 3.8 .7 .0$ beta $\backslash$ Truck-driver-only.bld

Evaluation Time: $0.00 \overline{0} 00000 \mathrm{E}+00$ years

Nuclide Detail of Doses

[mrem]

Source: 1

$\begin{array}{lccc}\text { Nuclide } & \begin{array}{c}\text { Receptor } \\ 1\end{array} & \begin{array}{c}\text { Receptor } \\ 2\end{array} & \text { Total } \\ \text { PU-238 } & 3.03 \mathrm{E}-06 & 1.48 \mathrm{E}-06 & 4.51 \mathrm{E}-06 \\ \mathrm{U}-234 & 1.31 \mathrm{E}-11 & 5.51 \mathrm{E}-12 & 1.86 \mathrm{E}-11 \\ \mathrm{TH}-230 & 1.25 \mathrm{E}-16 & 5.05 \mathrm{E}-17 & 1.75 \mathrm{E}-16 \\ \mathrm{RA}-226 & 1.48 \mathrm{E}-16 & 5.81 \mathrm{E}-17 & 2.06 \mathrm{E}-16 \\ \mathrm{~PB}-210 & 5.42 \mathrm{E}-22 & 2.22 \mathrm{E}-22 & 7.65 \mathrm{E}-22 \\ \mathrm{PO}-210 & 1.04 \mathrm{E}-24 & 4.12 \mathrm{E}-25 & 1.45 \mathrm{E}-24 \\ & & & \\ \mathrm{AM}-241 & 9.89 \mathrm{E}-04 & 4.01 \mathrm{E}-04 & 1.39 \mathrm{E}-03 \\ \mathrm{NP}-237 & 5.12 \mathrm{E}-09 & 2.04 \mathrm{E}-09 & 7.16 \mathrm{E}-09 \\ \mathrm{U}-233 & 8.98 \mathrm{E}-18 & 3.61 \mathrm{E}-18 & 1.26 \mathrm{E}-17 \\ \mathrm{TH}-229 & 2.51 \mathrm{E}-19 & 9.96 \mathrm{E}-20 & 3.51 \mathrm{E}-19 \\ & & & \\ \mathrm{PU}-240 & 2.93 \mathrm{E}-06 & 1.42 \mathrm{E}-06 & 4.35 \mathrm{E}-06\end{array}$




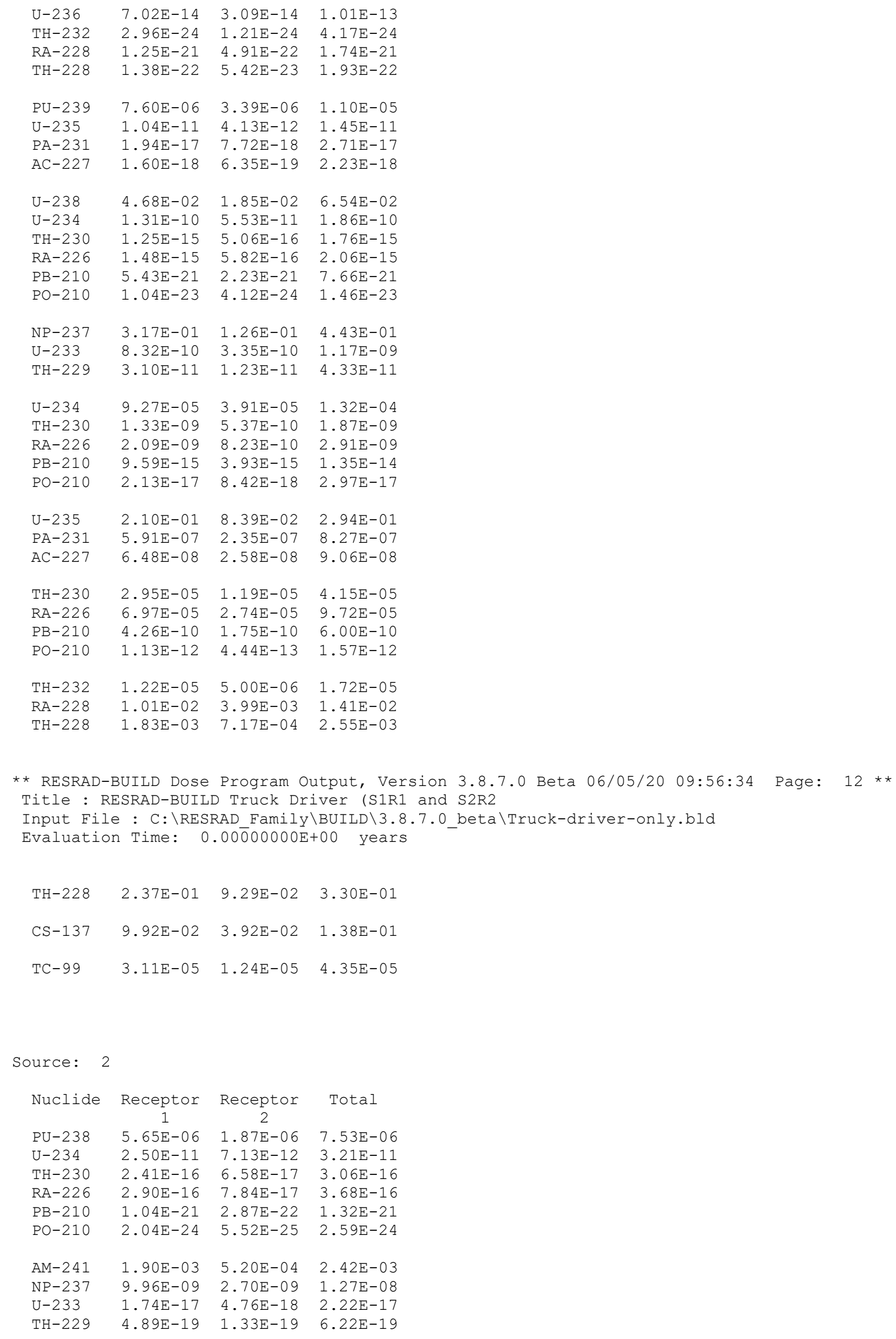



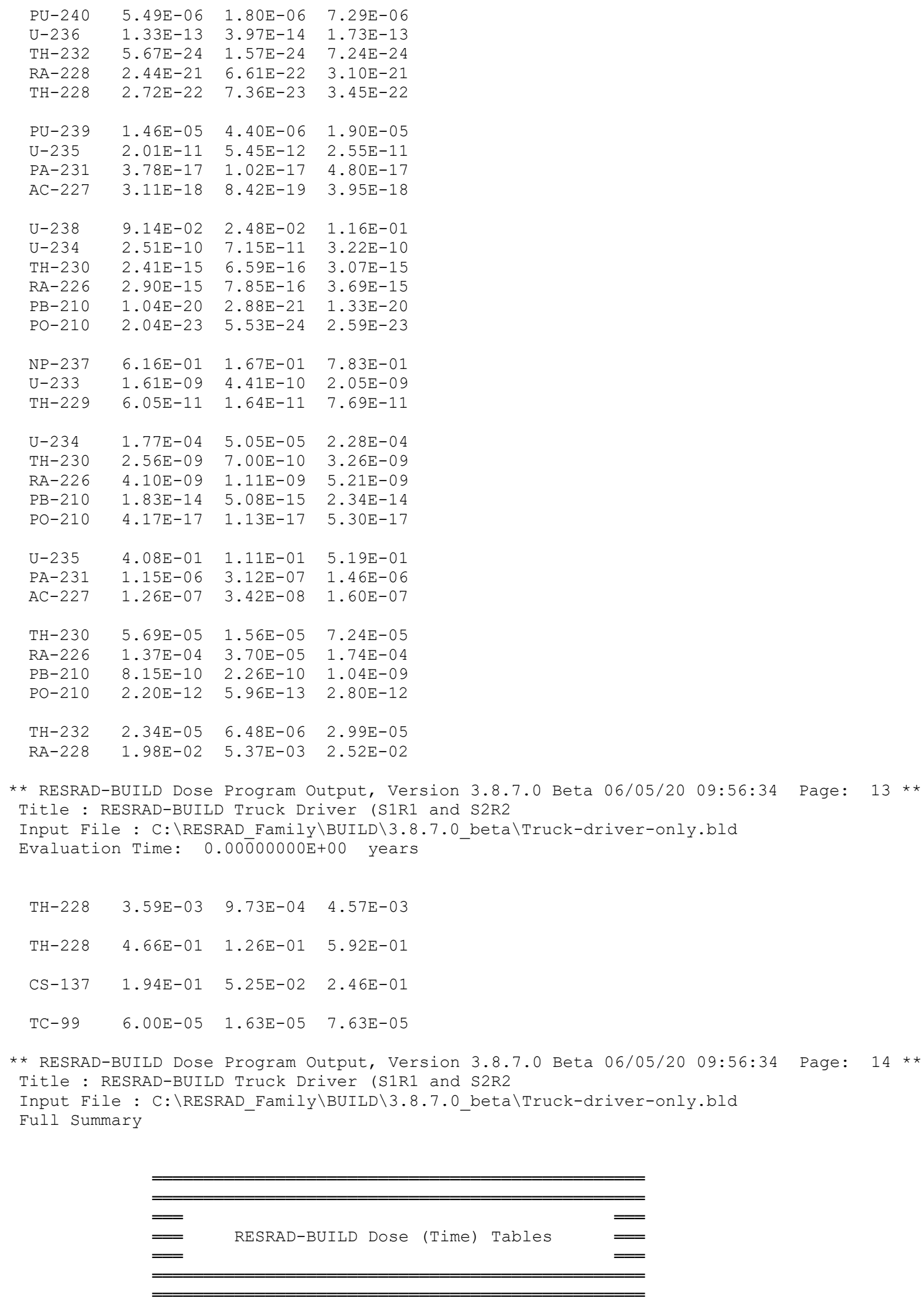


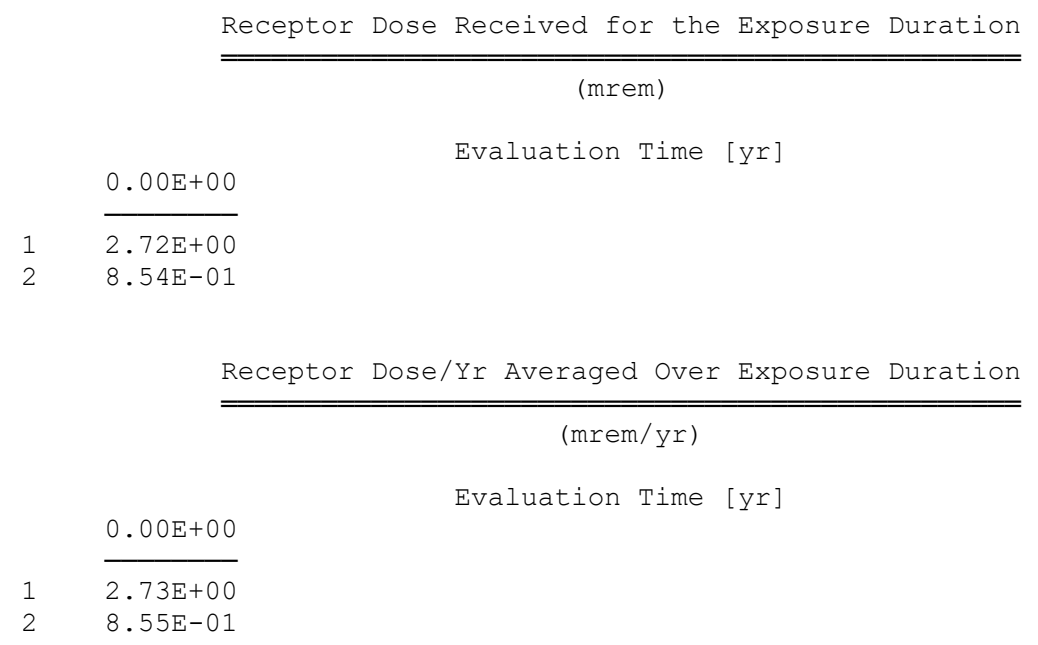

Note: The Receptor 1 dose for an individual radionuclide from Source 1 gives the truck driver dose in a year while driving (Table 3 in the main report). The Receptor 2 dose for an individual radionuclide from Source 2 gives the truck driver dose in a year while $1 \mathrm{~m}$ away from the vehicle (Table 3 in the main report).

\section{A.2 RESRAD-BUILD RESULTS FOR ISO CONTAINER HANDLING WORKER SCENARIO}

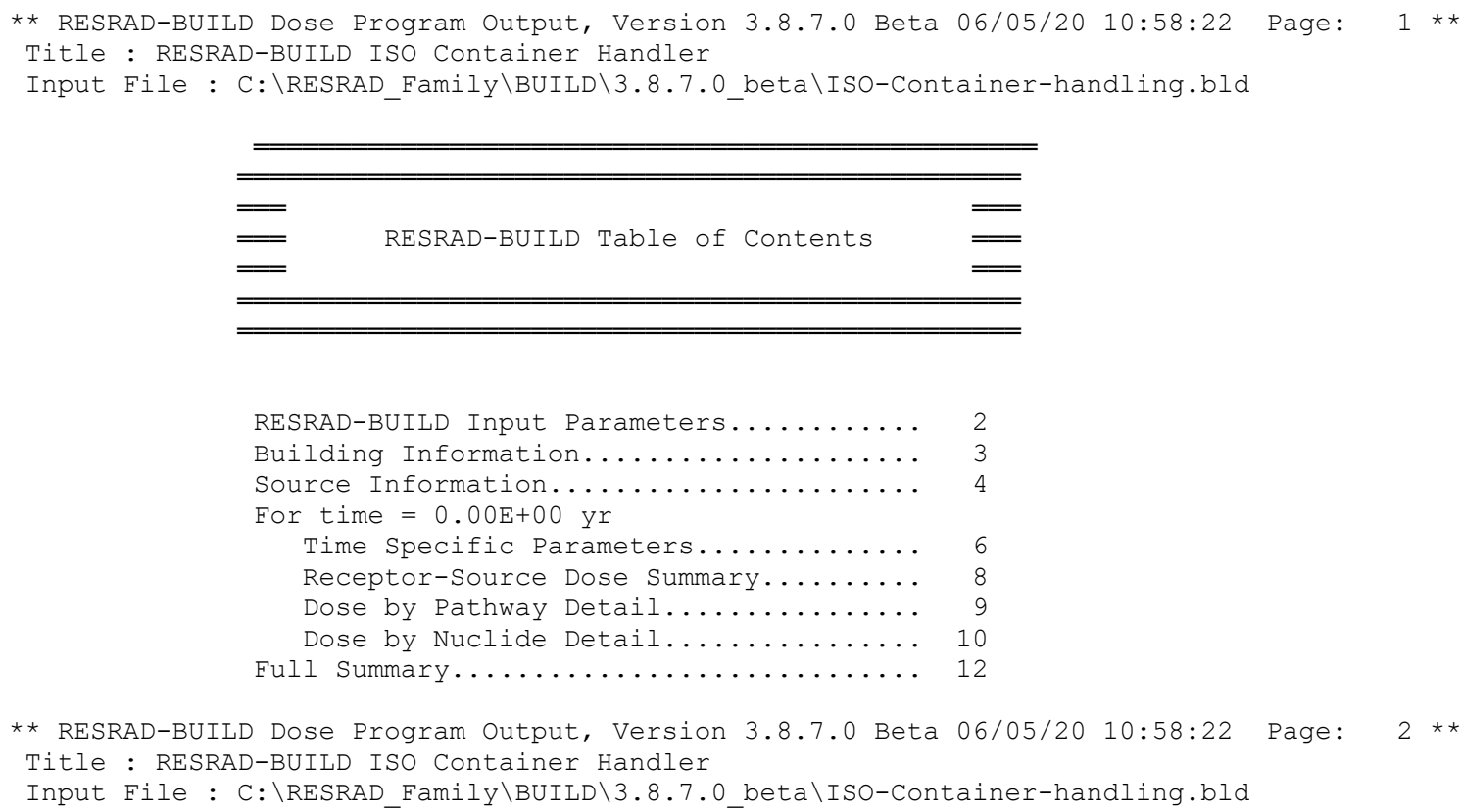

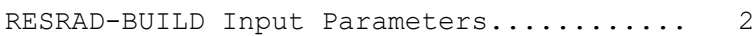

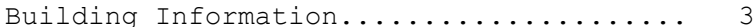

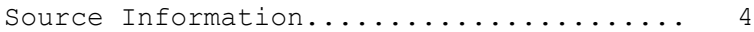

For time $=0.00 \mathrm{E}+00 \mathrm{yr}$

Time Specific Parameters........... 6

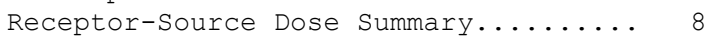

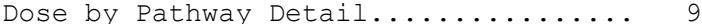

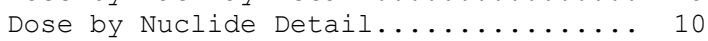

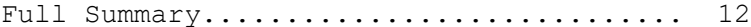

* RESRAD-BUILD Dose Program Output, Version 3.8.7.0 Beta 06/05/20 10:58:22 Page: 2 **

Title : RESRAD-BUILD ISO Container Handler

Input File : C: \RESRAD_Family $\backslash$ BUILD \3.8.7.0_beta\ISO-Container-handling.bld

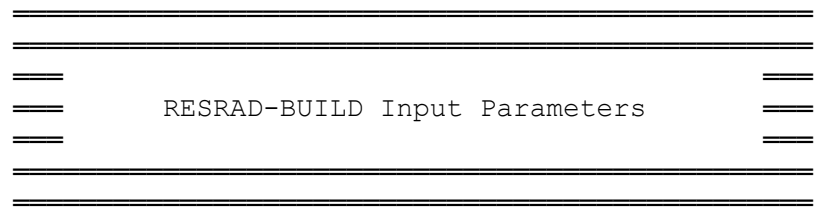




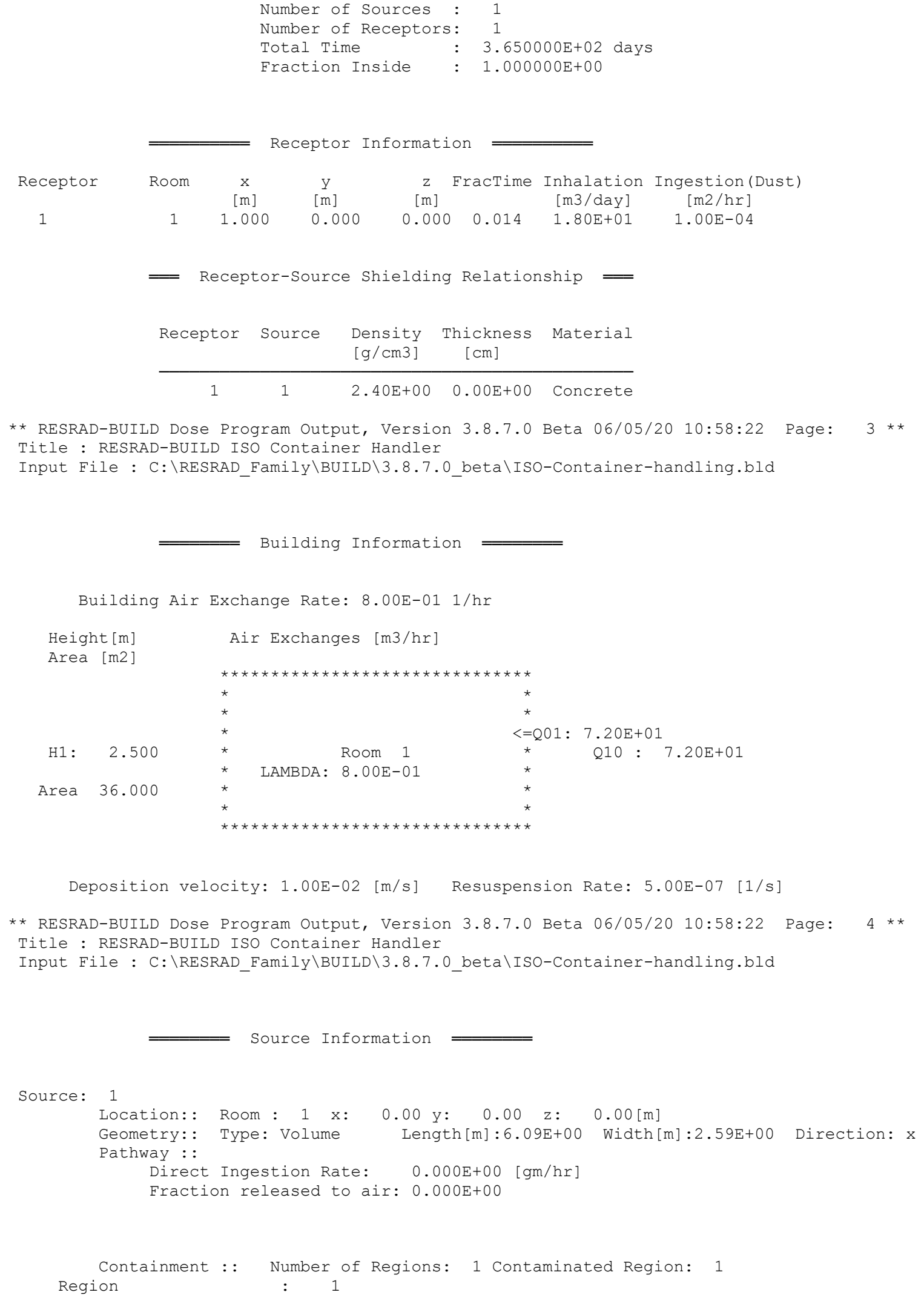


$\begin{array}{ll}\text { Thickness [cm] } & : 2.43 \mathrm{E}+02 \\ \text { Density [g/cm3] } & : 1.50 \mathrm{E}+00 \\ \text { Material } & : \text { Concrete } \\ \text { Erosion Rate [cm/day] } & : 0.00 \mathrm{E}+00 \\ \text { Porosity } & : 1.00 \mathrm{E}-01 \\ \text { Eff. Diffusion [m2/s] } & : 2.00 \mathrm{E}-05 \\ \text { Emanation Fractions (1) }: 0.00 \mathrm{E}+00\end{array}$

(2) : $0.00 \mathrm{E}+00$

Contamination: :

Nuclide Concentration Dose Conversion Factor (Library: ICRP 72 (Adult))

\begin{tabular}{|c|c|c|c|c|c|}
\hline & {$[\mathrm{pCi} / \mathrm{g}]$} & $\begin{array}{l}\text { Ingestion } \\
{[\mathrm{mrem} / \mathrm{pCi}]}\end{array}$ & $\begin{array}{l}\text { Inhalation } \\
\text { [mrem/pCi] }\end{array}$ & $\begin{array}{l}\text { Submersion } \\
\text { [mrem/yr/ } \\
\text { (pCi/m3)] }\end{array}$ & $\begin{array}{c}\text { External } \\
\text { [mrem/yr } \\
{[\mathrm{pCi} / \mathrm{g}]}\end{array}$ \\
\hline PU-238 & $3.000 \mathrm{E}+00$ & $8.510 E-04$ & $4.070 E-01$ & $4.088 E-07$ & $1.166 \mathrm{E}-04$ \\
\hline $\mathrm{AM}-241$ & $3.000 \mathrm{E}+00$ & $7.400 \mathrm{E}-04$ & $3.552 \mathrm{E}-01$ & $7.872 \mathrm{E}-05$ & $3.719 \mathrm{E}-02$ \\
\hline $\mathrm{PU}-240$ & $3.000 \mathrm{E}+00$ & $9.250 \mathrm{E}-04$ & $4.440 E-01$ & $3.995 E-07$ & $1.129 \mathrm{E}-04$ \\
\hline $\mathrm{PU}-239$ & $3.000 \mathrm{E}+00$ & $9.250 \mathrm{E}-04$ & $4.440 E-01$ & $4.065 E-07$ & $2.635 E-04$ \\
\hline$U-238$ & $3.000 \mathrm{E}+01$ & $1.791 \mathrm{E}-04$ & $2.963 E-02$ & $2.091 E-04$ & $1.557 \mathrm{E}-01$ \\
\hline$N P-237$ & $3.000 \mathrm{E}+01$ & $4.102 \mathrm{E}-04$ & $1.850 E-01$ & $1.101 \mathrm{E}-03$ & $1.012 \mathrm{E}+00$ \\
\hline $\mathrm{U}-234$ & $3.000 \mathrm{E}+01$ & $1.813 E-04$ & $3.478 E-02$ & $7.136 \mathrm{E}-07$ & $3.439 \mathrm{E}-04$ \\
\hline$U-236$ & $0.000 \mathrm{E}+00$ & $1.739 \mathrm{E}-04$ & $3.219 \mathrm{E}-02$ & $4.508 \mathrm{E}-07$ & $1.781 \mathrm{E}-04$ \\
\hline$U-235$ & $3.000 \mathrm{E}+01$ & $1.752 \mathrm{E}-04$ & $3.145 \mathrm{E}-02$ & $8.074 \mathrm{E}-04$ & $6.918 E-01$ \\
\hline $\mathrm{TH}-230$ & $3.000 \mathrm{E}+00$ & $7.770 E-04$ & $3.700 \mathrm{E}-01$ & $1.729 \mathrm{E}-06$ & $1.071 \mathrm{E}-03$ \\
\hline$U-233$ & $0.000 \mathrm{E}+00$ & $1.887 \mathrm{E}-04$ & $3.552 \mathrm{E}-02$ & $1.659 \mathrm{E}-06$ & $1.265 E-03$ \\
\hline$P A-231$ & $0.000 E+00$ & $2.627 E-03$ & $5.180 \mathrm{E}-01$ & $1.834 \mathrm{E}-04$ & $1.762 \mathrm{E}-01$ \\
\hline $\mathrm{TH}-232$ & $3.000 \mathrm{E}+00$ & $8.510 E-04$ & $4.070 E-01$ & $8.456 \mathrm{E}-07$ & $4.560 \mathrm{E}-04$ \\
\hline$R A-226$ & $0.000 \mathrm{E}+00$ & $1.037 \mathrm{E}-03$ & $3.526 \mathrm{E}-02$ & $9.769 \mathrm{E}-03$ & $1.060 \mathrm{E}+01$ \\
\hline $\mathrm{TH}-229$ & $0.000 \mathrm{E}+00$ & $2.269 E-03$ & $9.481 E-01$ & $1.624 \mathrm{E}-03$ & $1.480 \mathrm{E}+00$ \\
\hline$R A-228$ & $0.000 \mathrm{E}+00$ & $2.555 E-03$ & $5.929 E-02$ & $5.240 E-03$ & $5.662 \mathrm{E}+00$ \\
\hline$A C-227$ & $0.000 \mathrm{E}+00$ & $4.473 E-03$ & $2.104 \mathrm{E}+00$ & $2.034 \mathrm{E}-03$ & $1.869 \mathrm{E}+00$ \\
\hline $\mathrm{TH}-228$ & $3.000 \mathrm{E}+00$ & $5.301 E-04$ & $1.614 \mathrm{E}-01$ & $8.921 E-03$ & $9.672 \mathrm{E}+00$ \\
\hline$P B-210$ & $0.000 \mathrm{E}+00$ & $2.558 \mathrm{E}-03$ & $2.106 \mathrm{E}-02$ & $3.534 \mathrm{E}-05$ & $7.457 \mathrm{E}-03$ \\
\hline $\mathrm{PO}-210$ & $0.000 \mathrm{E}+00$ & $4.440 E-03$ & $1.591 \mathrm{E}-02$ & $4.544 E-08$ & $4.934 E-05$ \\
\hline
\end{tabular}

** RESRAD-BUILD Dose Program Output, Version 3.8.7.0 Beta 06/05/20 10:58:22 Page: 5 ** Title : RESRAD-BUILD ISO Container Handler

Input File : C: \RESRAD_Family \BUILD \3.8.7.0_beta\ISO-Container-handling.bld

$\begin{array}{llllll}\mathrm{CS}-137 & 3.000 \mathrm{E}+00 & 4.810 \mathrm{E}-05 & 1.443 \mathrm{E}-04 & 2.981 \mathrm{E}-03 & 3.201 \mathrm{E}+00 \\ \mathrm{TC}-99 & 3.000 \mathrm{E}+01 & 2.368 \mathrm{E}-06 & 4.810 \mathrm{E}-05 & 3.352 \mathrm{E}-06 & 1.086 \mathrm{E}-04\end{array}$

** RESRAD-BUILD Dose Program Output, Version 3.8.7.0 Beta 06/05/20 10:58:22 Page: 6 ** Title : RESRAD-BUILD ISO Container Handler

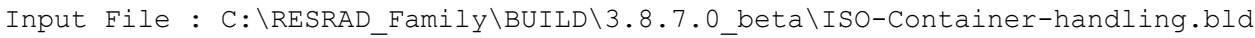
Evaluation Time: $0.00 \overline{0} 00000 \mathrm{E}+00$ years

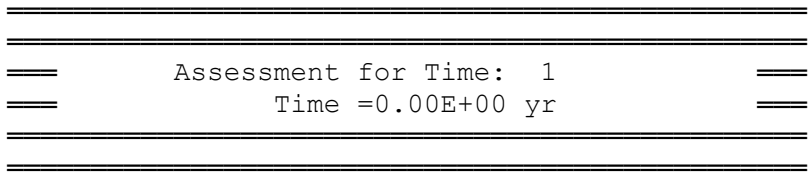

Source Information

Source: 1

Location: : Room : $1 \mathrm{x}: 0.00 \mathrm{y}: 0.00 \mathrm{z}: 0.00$ [m]

Geometry:: Type: Volume Length $[\mathrm{m}]: 6.09 \mathrm{E}+00$ Width $[\mathrm{m}]: 2.59 \mathrm{E}+00$ Direction: $\mathrm{x}$

Pathway : :

Direct Ingestion Rate : 0.000E+00 [gm/hr]

Fraction released to air: 0.000E+00 
Region

Containment :: Number of Regions: 1 Contaminated Region: 1

\section{Thickness}

Eraction Contami

$: \quad 1$

$: 2.43 \mathrm{E}+02$

Density [ $\mathrm{g} / \mathrm{cm} 3]$

$: 1.50 \mathrm{E}+00$
Concentration

$$
[\mathrm{pCi} / \mathrm{g}]
$$

$3.000 \mathrm{E}+00$

$3.000 \mathrm{E}+00$

$3.000 \mathrm{E}+00$

$3.000 \mathrm{E}+00$

$3.000 \mathrm{E}+01$

$3.000 \mathrm{E}+01$

$3.000 \mathrm{E}+01$

$0.000 \mathrm{E}+00$

$3.000 \mathrm{E}+01$

$3.000 \mathrm{E}+00$

$0.000 \mathrm{E}+00$

$0.000 \mathrm{E}+00$

$3.000 \mathrm{E}+00$

$0.000 \mathrm{E}+00$

$0.000 \mathrm{E}+00$

$0.000 \mathrm{E}+00$

$0.000 \mathrm{E}+00$

$3.000 \mathrm{E}+00$

$0.000 \mathrm{E}+00$ 
** RESRAD-BUILD Dose Program Output, Version 3.8.7.0 Beta 06/05/20 10:58:22 Page: 7 ** Title : RESRAD-BUILD ISO Container Handler

Input File : C: \RESRAD Family $\backslash$ BUILD $\backslash 3.8 .7 .0$ beta \ISO-Container-handling.bld

Evaluation Time: $0.00000000 \mathrm{E}+00$ years
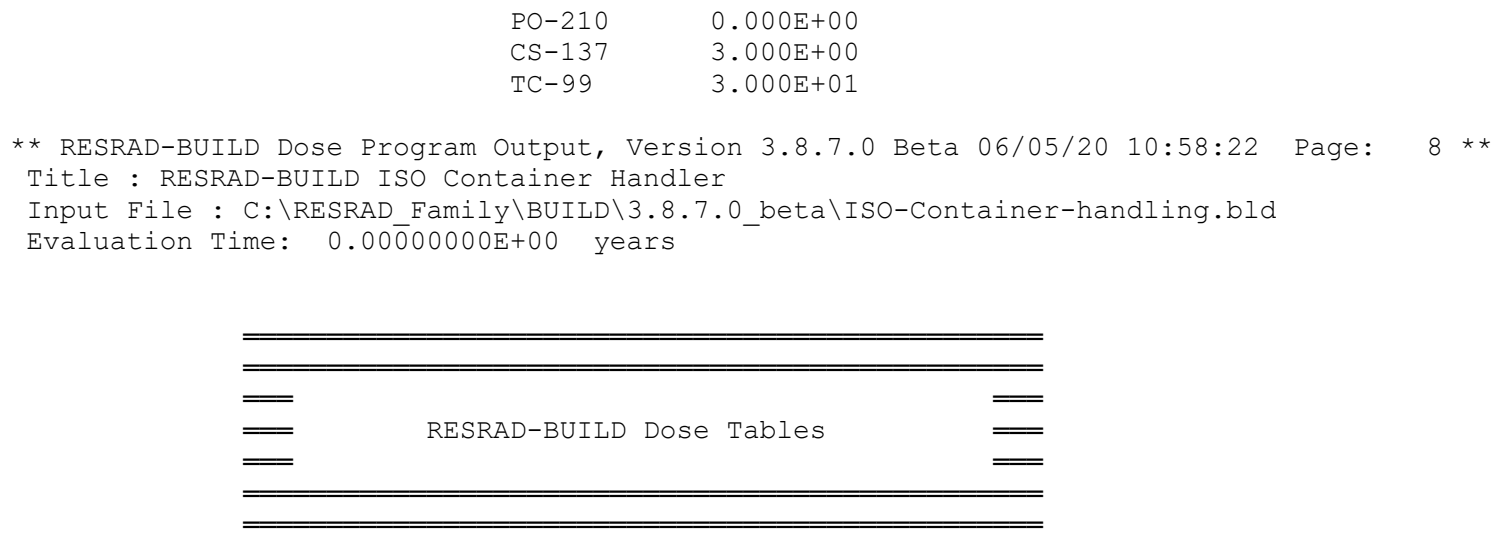

Source Contributions to Receptor Doses

[mrem ]

Source Total

1

Receptor $1 \quad 6.21 \mathrm{E}-016.21 \mathrm{E}-01$

Total 6.21E-01 6.21E-01

** RESRAD-BUILD Dose Program Output, Version 3.8.7.0 Beta 06/05/20 10:58:22 Page: 9 ** Title : RESRAD-BUILD ISO Container Handler

Input File : C: \RESRAD_Family $\backslash$ BUILD \3.8.7.0_beta\ISO-Container-handling.bld

Evaluation Time: $0.00 \overline{0} 00000 \mathrm{E}+00$ years

Pathway Detail of Doses

[mrem]

Source: 1

$\begin{array}{cllllll}\text { Receptor } & \text { External } & \text { Deposition } & \text { Immersion } & \text { Inhalation } & \text { Radon } & \text { Ingestion } \\ 1 & 6.21 \mathrm{E}-01 & 0.00 \mathrm{E}+00 & 0.00 \mathrm{E}+00 & 0.00 \mathrm{E}+00 & 0.00 \mathrm{E}+00 & 0.00 \mathrm{E}+00 \\ \text { Total } & 6.21 \mathrm{E}-01 & 0.00 \mathrm{E}+00 & 0.00 \mathrm{E}+00 & 0.00 \mathrm{E}+00 & 0.00 \mathrm{E}+00 & 0.00 \mathrm{E}+00\end{array}$

* RESRAD-BUILD Dose Program Output, Version 3.8.7.0 Beta 06/05/20 10:58:22 Page: 10 ** Title : RESRAD-BUILD ISO Container Handler

Input File : C: \RESRAD Family \BUILD \3.8.7.0_beta\ISO-Container-handling.bld

Evaluation Time: $0.00000000 \mathrm{E}+00$ years

Nuclide Detail of Doses

[mrem ]

Source: 1

$\begin{array}{lcc}\text { Nuclide } & \begin{array}{c}\text { Receptor } \\ 1\end{array} & \text { Total } \\ \text { PU-238 } & 2.38 \mathrm{E}-06 & 2.38 \mathrm{E}-06 \\ \mathrm{U}-234 & 9.06 \mathrm{E}-12 & 9.06 \mathrm{E}-12 \\ \mathrm{TH}-230 & 8.37 \mathrm{E}-17 & 8.37 \mathrm{E}-17 \\ \mathrm{RA}-226 & 9.97 \mathrm{E}-17 & 9.97 \mathrm{E}-17 \\ \mathrm{~PB}-210 & 3.66 \mathrm{E}-22 & 3.66 \mathrm{E}-22\end{array}$



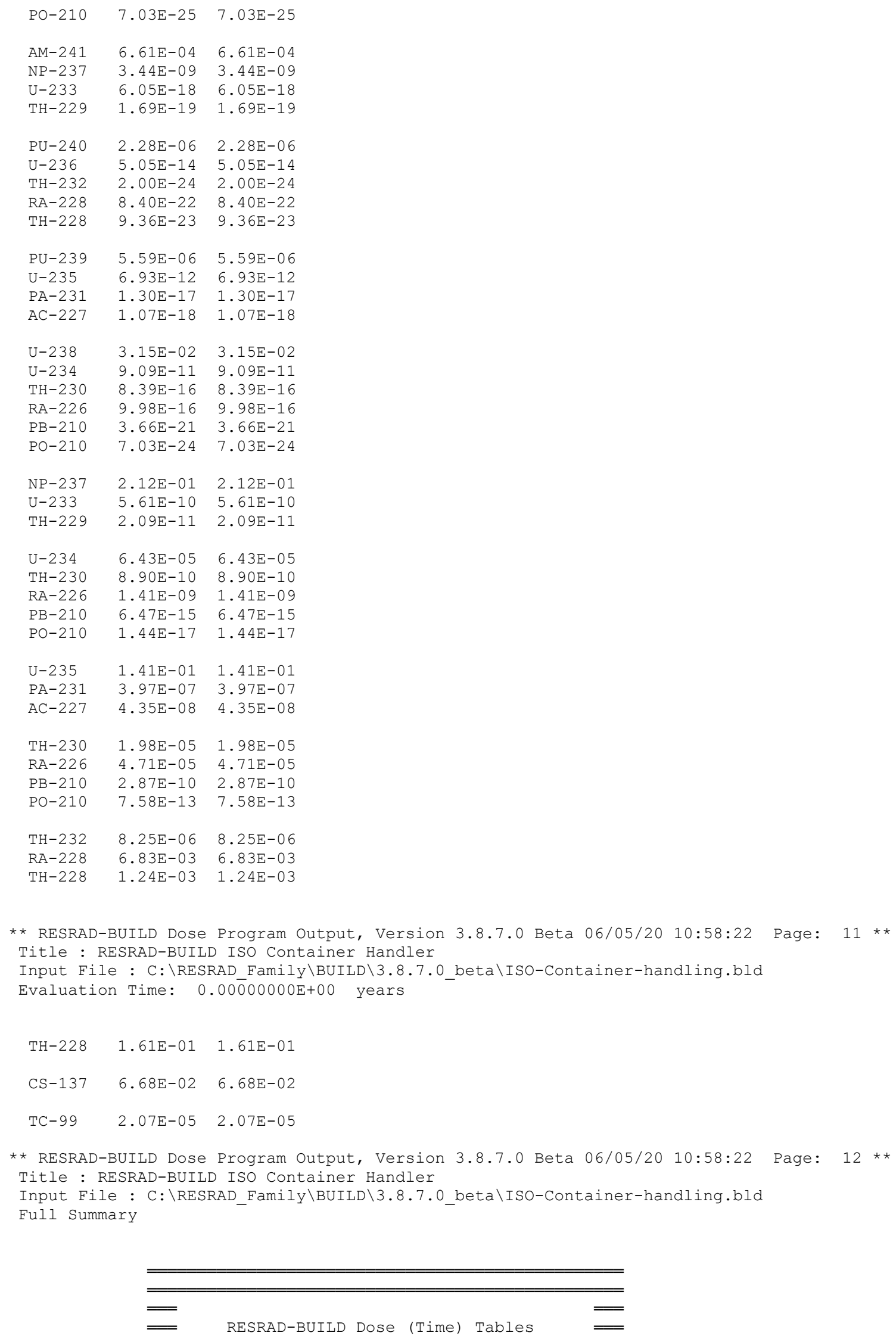


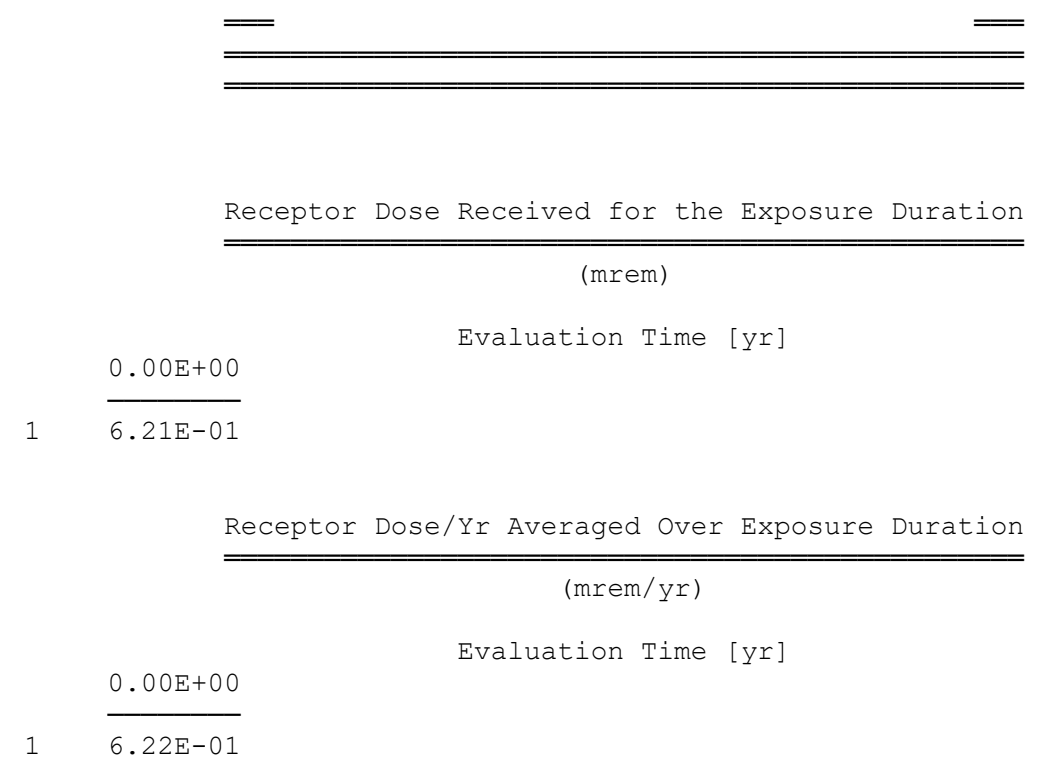

Note: The Receptor 1 dose for an individual radionuclide from Source 1 gives the ISO container handling and processing worker's external dose in a year (Table 4 in the main report).

\section{A.3 RESRAD-BUILD RESULTS FOR INCINERATOR BAGHOUSE WORKER/PROCESSOR SCENARIO}

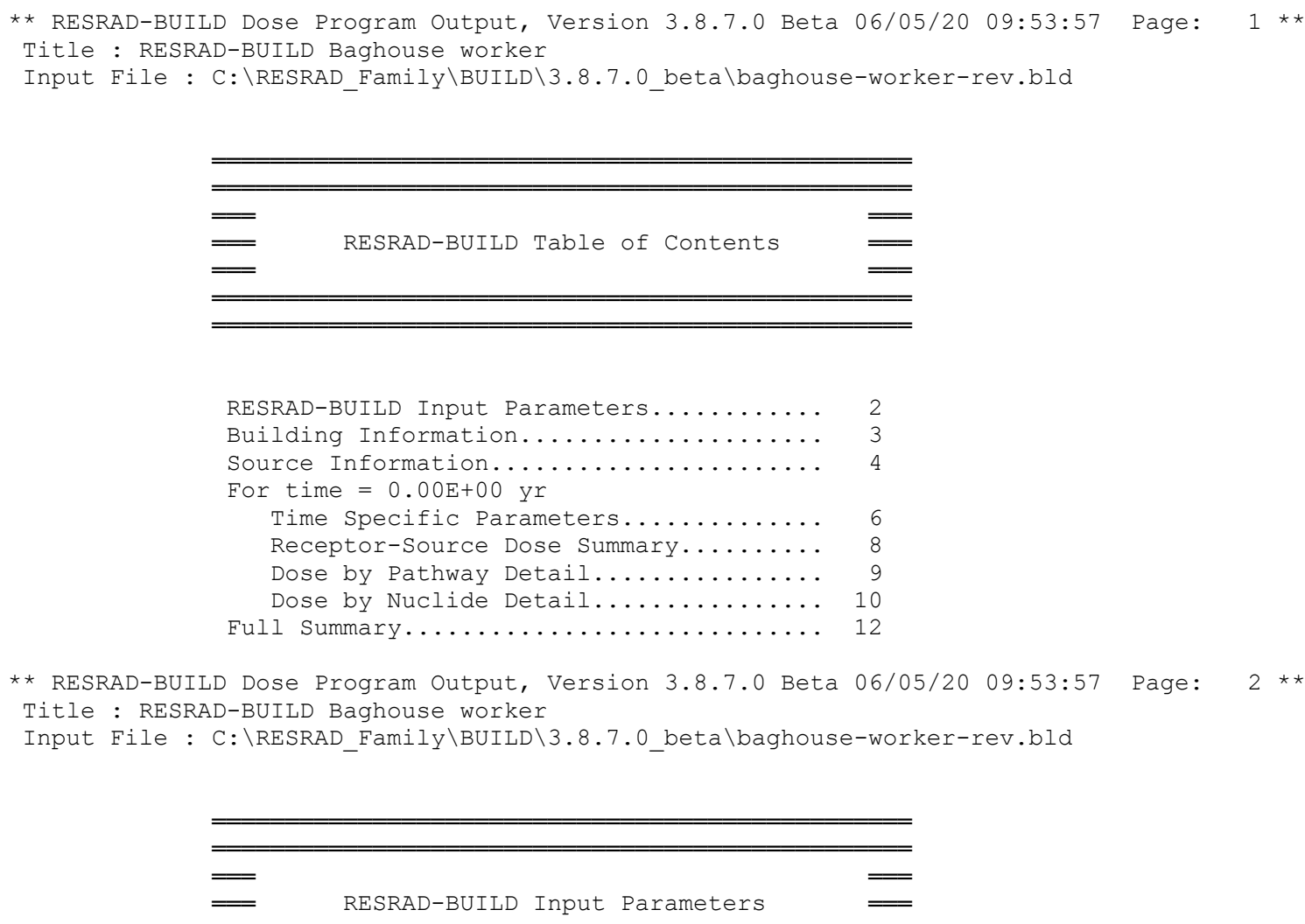

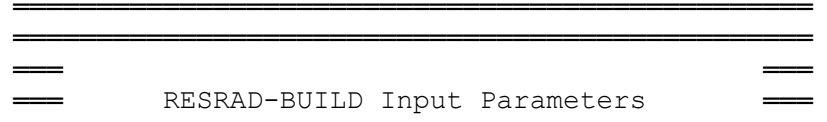




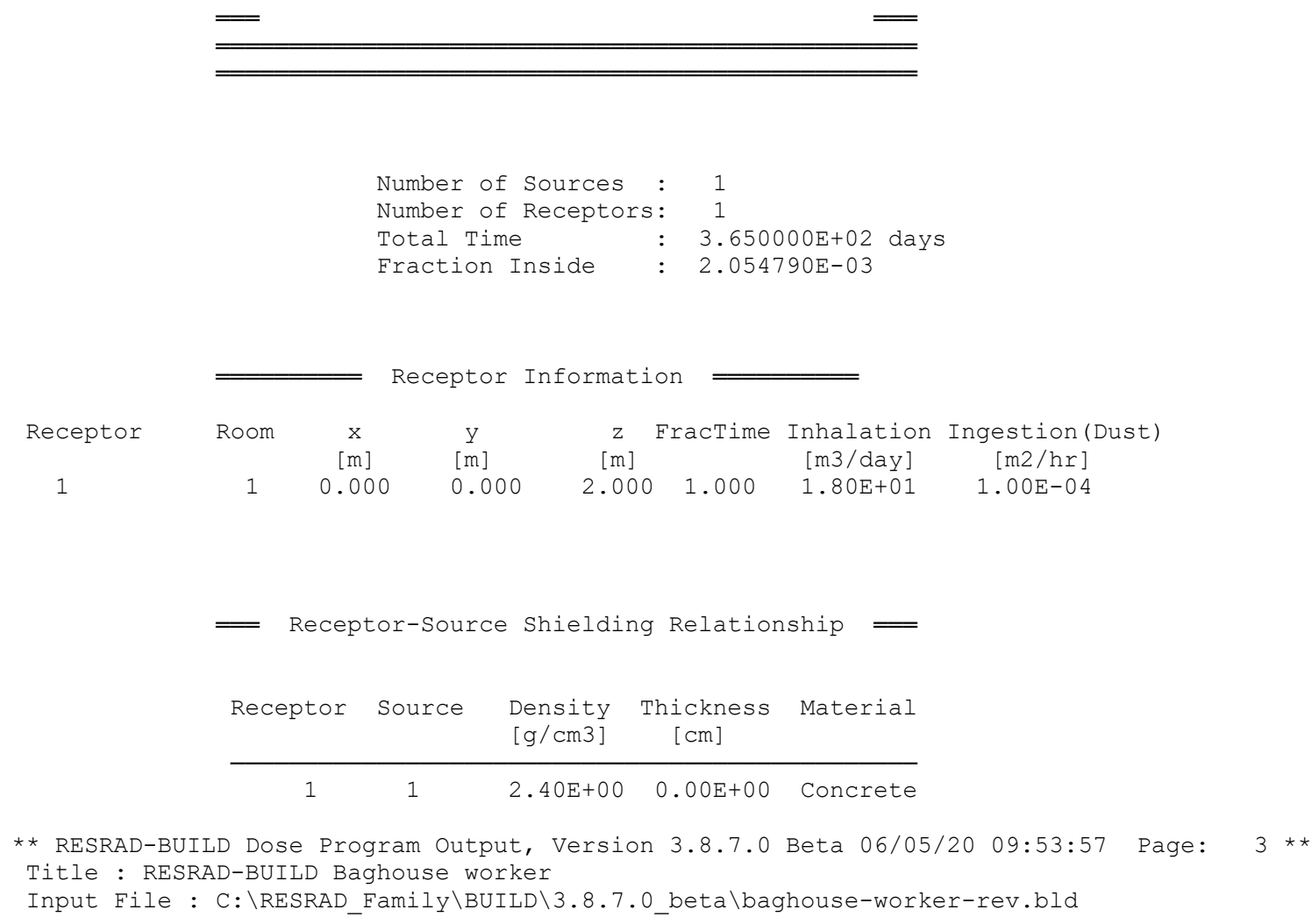


Direct Ingestion Rate: $0.000 \mathrm{E}+00$ [gm/hr]

Fraction released to air: 0.000E+00

\begin{tabular}{|c|c|}
\hline Containment : : & Number of \\
\hline Region & 1 \\
\hline Thickness [ $\mathrm{cm}]$ & $: 1.00 \mathrm{E}+0$ \\
\hline Density $[\mathrm{g} / \mathrm{cm} 3]$ & $40 E$ \\
\hline Material & : Concret \\
\hline Erosion Rate [cm/day] & $: 0.00 \mathrm{E}+$ \\
\hline Porosity & $: 1.00 \mathrm{E}$ \\
\hline Eff. Diffusion [m2/s] & $: 2$ \\
\hline nanation Fractions (1 & 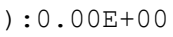 \\
\hline
\end{tabular}

(2) :0.00E+00

Contamination: :

Nuclide Concentration Dose Conversion Factor (Library: ICRP 72 (Adult))

\begin{tabular}{|c|c|c|c|c|c|}
\hline & [pCi/g] & $\begin{array}{l}\text { Ingestion } \\
\text { [mrem/pCi] }\end{array}$ & $\begin{array}{l}\text { Inhalation } \\
\text { [mrem/pCi] }\end{array}$ & $\begin{array}{l}\text { Submersion } \\
\text { [mrem/yr/ } \\
\text { (pCi/m3)] }\end{array}$ & $\begin{array}{c}\text { External } \\
{[\mathrm{mrem} / \mathrm{yr} /} \\
{[\mathrm{pCi} / \mathrm{g}]}\end{array}$ \\
\hline PU-238 & $4.010 \mathrm{E}+03$ & $8.510 \mathrm{E}-04$ & $4.070 \mathrm{E}-01$ & $4.088 E-07$ & $1.166 \mathrm{E}-04$ \\
\hline $\mathrm{AM}-241$ & $4.010 \mathrm{E}+03$ & $7.400 \mathrm{E}-04$ & $3.552 \mathrm{E}-01$ & $7.872 \mathrm{E}-05$ & $3.719 \mathrm{E}-02$ \\
\hline PU-240 & $4.010 \mathrm{E}+03$ & $9.250 \mathrm{E}-04$ & $4.440 \mathrm{E}-01$ & $3.995 \mathrm{E}-07$ & 1.129E-04 \\
\hline PU-239 & $4.010 \mathrm{E}+03$ & $9.250 \mathrm{E}-04$ & $4.440 \mathrm{E}-01$ & $4.065 E-07$ & $2.635 E-04$ \\
\hline$U-238$ & $4.010 E+04$ & $1.791 E-04$ & $2.963 E-02$ & $2.091 E-04$ & $1.557 \mathrm{E}-01$ \\
\hline$N P-237$ & $4.010 \mathrm{E}+04$ & $4.102 \mathrm{E}-04$ & $1.850 \mathrm{E}-01$ & $1.101 \mathrm{E}-03$ & $1.012 \mathrm{E}+00$ \\
\hline$U-234$ & $4.010 E+04$ & $1.813 E-04$ & $3.478 E-02$ & $7.136 \mathrm{E}-07$ & $3.439 E-04$ \\
\hline$U-236$ & $0.000 \mathrm{E}+00$ & $1.739 \mathrm{E}-04$ & $3.219 \mathrm{E}-02$ & $4.508 E-07$ & $1.781 \mathrm{E}-04$ \\
\hline$U-235$ & $4.010 E+04$ & $1.752 \mathrm{E}-04$ & $3.145 E-02$ & $8.074 E-04$ & $6.918 \mathrm{E}-01$ \\
\hline $\mathrm{TH}-230$ & $4.010 \mathrm{E}+03$ & $7.770 E-04$ & $3.700 E-01$ & $1.729 E-06$ & $1.071 E-03$ \\
\hline $\mathrm{U}-233$ & $0.000 \mathrm{E}+00$ & $1.887 \mathrm{E}-04$ & $3.552 E-02$ & $1.659 \mathrm{E}-06$ & $1.265 E-03$ \\
\hline$P A-231$ & $0.000 \mathrm{E}+00$ & $2.627 E-03$ & $5.180 \mathrm{E}-01$ & $1.834 \mathrm{E}-04$ & $1.762 \mathrm{E}-01$ \\
\hline $\mathrm{TH}-232$ & $4.010 E+03$ & $8.510 E-04$ & $4.070 E-01$ & $8.456 \mathrm{E}-07$ & $4.560 E-04$ \\
\hline$R A-226$ & $0.000 \mathrm{E}+00$ & $1.037 E-03$ & $3.526 E-02$ & $9.769 E-03$ & $1.060 \mathrm{E}+01$ \\
\hline $\mathrm{TH}-229$ & $0.000 \mathrm{E}+00$ & $2.269 E-03$ & $9.481 E-01$ & $1.624 E-03$ & $1.480 \mathrm{E}+00$ \\
\hline$R A-228$ & $0.000 \mathrm{E}+00$ & $2.555 E-03$ & $5.929 \mathrm{E}-02$ & $5.240 E-03$ & $5.662 \mathrm{E}+00$ \\
\hline$A C-227$ & $0.000 \mathrm{E}+00$ & $4.473 E-03$ & $2.104 \mathrm{E}+00$ & $2.034 E-03$ & $1.869 \mathrm{E}+00$ \\
\hline $\mathrm{TH}-228$ & $4.010 \mathrm{E}+03$ & $5.301 E-04$ & $1.614 \mathrm{E}-01$ & $8.921 E-03$ & $9.672 \mathrm{E}+00$ \\
\hline$P B-210$ & $0.000 \mathrm{E}+00$ & $2.558 E-03$ & $2.106 \mathrm{E}-02$ & $3.534 \mathrm{E}-05$ & $7.457 \mathrm{E}-03$ \\
\hline $\mathrm{PO}-210$ & $0.000 \mathrm{E}+00$ & $4.440 E-03$ & $1.591 E-02$ & $4.544 E-08$ & $4.934 E-05$ \\
\hline
\end{tabular}

* RESRAD-BUILD Dose Program Output, Version 3.8.7.0 Beta 06/05/20 09:53:57 Page: 5 ** Title : RESRAD-BUILD Baghouse worker

Input File : C: \RESRAD Family BUILD $\backslash 3.8 .7 .0$ beta \baghouse-worker-rev.bld

$\begin{array}{llllll}C S-137 & 4.010 \mathrm{E}+03 & 4.810 \mathrm{E}-05 & 1.443 \mathrm{E}-04 & 2.981 \mathrm{E}-03 & 3.201 \mathrm{E}+00 \\ \mathrm{TC}-99 & 4.010 \mathrm{E}+04 & 2.368 \mathrm{E}-06 & 4.810 \mathrm{E}-05 & 3.352 \mathrm{E}-06 & 1.086 \mathrm{E}-04\end{array}$

* RESRAD-BUILD Dose Program Output, Version 3.8.7.0 Beta 06/05/20 09:53:57 Page: 6 ** Title : RESRAD-BUILD Baghouse worker

Input File : C: \RESRAD_Family \BUILD \3.8.7.0_beta \baghouse-worker-rev.bld

Evaluation Time: $0.00000000 \mathrm{E}+00$ years

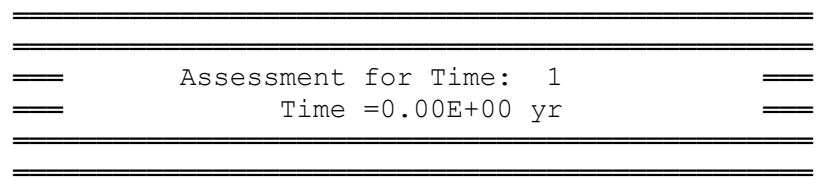

Source Information

Source: 1 


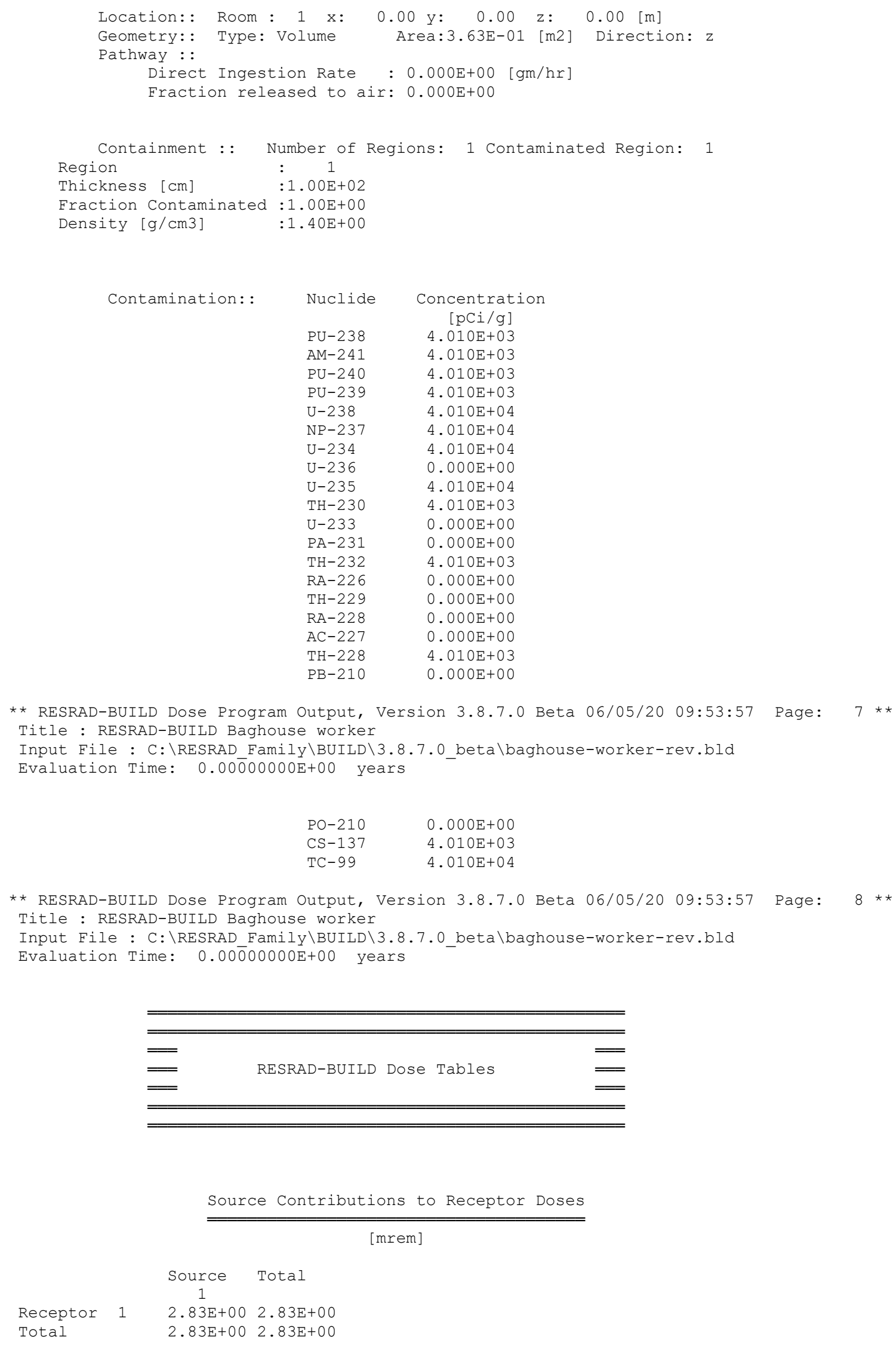

Source Contributions to Receptor Doses

[mrem]

Source Total

1

Receptor $1 \quad 2.83 \mathrm{E}+00 \quad 2.83 \mathrm{E}+00$

Total 2.83E+00 $2.83 \mathrm{E}+00$ 


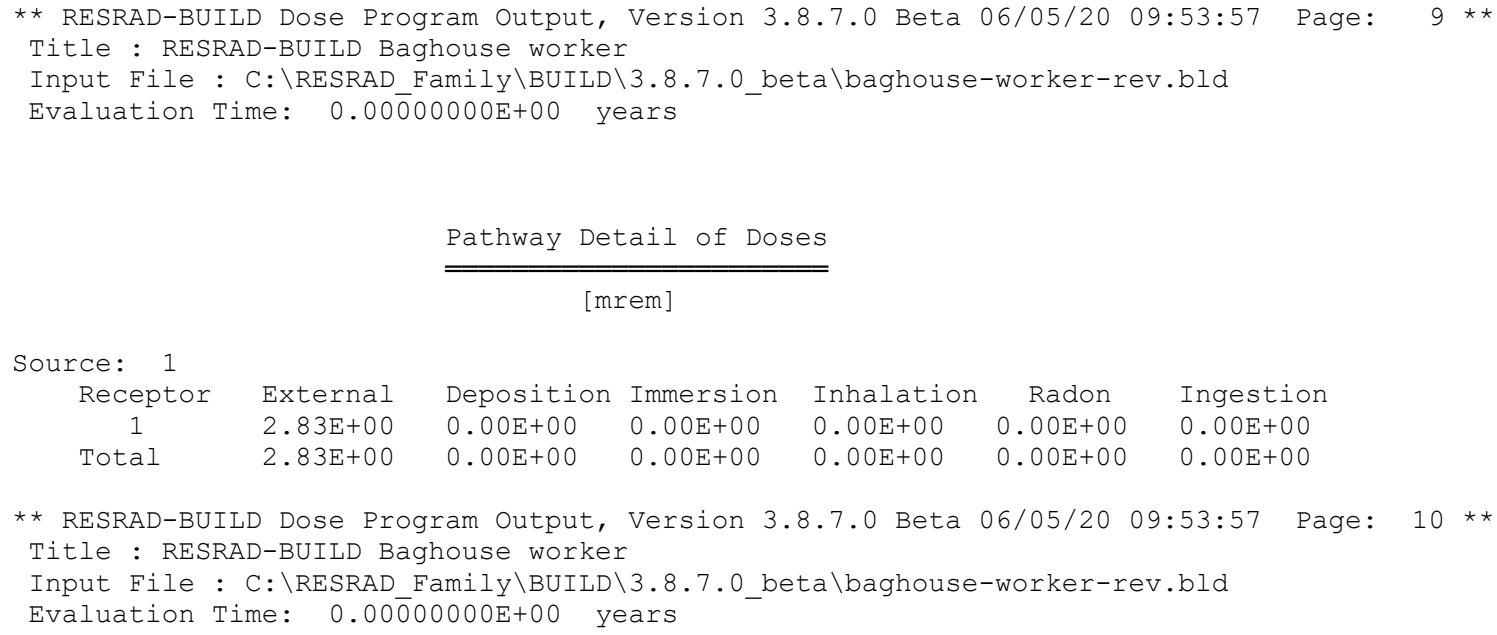

\begin{tabular}{|c|c|c|}
\hline Nuclide & $\begin{array}{c}\text { Receptor } \\
1\end{array}$ & Total \\
\hline$P U-238$ & 1.10E-05 & $1.10 \mathrm{E}-05$ \\
\hline $\mathrm{U}-234$ & 4. 30E-11 & 4. $30 \mathrm{E}-11$ \\
\hline $\mathrm{TH}-230$ & $3.98 E-16$ & 3. $98 E-16$ \\
\hline$R A-226$ & 4. $43 E-16$ & 4. $43 E-16$ \\
\hline$P B-210$ & $1.77 E-21$ & $1.77 E-21$ \\
\hline $\mathrm{PO}-210$ & $3.15 E-24$ & $3.15 E-24$ \\
\hline $\mathrm{AM}-241$ & $3.19 \mathrm{E}-03$ & $3.19 \mathrm{E}-03$ \\
\hline$N P-237$ & 1. $58 \mathrm{E}-08$ & $1.58 \mathrm{E}-08$ \\
\hline$U-233$ & $2.81 \mathrm{E}-17$ & $2.81 \mathrm{E}-17$ \\
\hline $\mathrm{TH}-229$ & $7.69 \mathrm{E}-19$ & 7. $69 \mathrm{E}-19$ \\
\hline PU-240 & $1.06 \mathrm{E}-05$ & $1.06 \mathrm{E}-05$ \\
\hline $\mathrm{U}-2$ & $2.38 E-13$ & 2. $38 E-13$ \\
\hline $\mathrm{TH}-232$ & $9.53 E-24$ & $9.53 E-24$ \\
\hline $\mathrm{RA}-$ & $3.75 E-21$ & $3.75 E-21$ \\
\hline $\mathrm{TH}-228$ & $4.20 E-22$ & 4. $20 \mathrm{E}-22$ \\
\hline $\mathrm{PU}-$ & $2.52 E-05$ & $2.52 E-05$ \\
\hline $\mathrm{U}-2$ & 3. $22 E-11$ & 3. $22 E-11$ \\
\hline $\mathrm{PA}-231$ & $5.97 E-17$ & $5.97 E-17$ \\
\hline$A C-227$ & 4. $91 E-18$ & 4. $91 E-18$ \\
\hline $\mathrm{U}-2$ & 1. $43 \mathrm{E}-01$ & $1.43 E-01$ \\
\hline $\mathrm{U}-2$ & $4.31 E-10$ & 4. $31 E-10$ \\
\hline $\mathrm{TH}-$ & $3.98 E-15$ & $3.98 E-15$ \\
\hline$R A-226$ & $4.43 E-15$ & 4. $43 E-15$ \\
\hline $\mathrm{PB}-$ & 1. $77 \mathrm{E}-20$ & 1. $77 \mathrm{E}-20$ \\
\hline $\mathrm{PO}-210$ & $3.15 E-23$ & $3.15 E-23$ \\
\hline$N P-237$ & $9.76 E-01$ & $9.76 \mathrm{E}-01$ \\
\hline $\mathrm{U}-2$ & $2.60 \mathrm{E}-09$ & $2.60 \mathrm{E}-09$ \\
\hline $\mathrm{TH}-229$ & $9.51 \mathrm{E}-11$ & $9.51 \mathrm{E}-11$ \\
\hline $\mathrm{U}-2$ & $3.05 E-04$ & $3.05 E-04$ \\
\hline $\mathrm{TH}-$ & $4.23 E-09$ & $4.23 E-09$ \\
\hline $\mathrm{RA}-$ & $6.27 E-09$ & $6.27 E-09$ \\
\hline $\mathrm{PB}-$ & $3.12 \mathrm{E}-14$ & $3.12 \mathrm{E}-14$ \\
\hline $\mathrm{PO}-210$ & $6.44 E-17$ & $6.44 \mathrm{E}-1$ \\
\hline
\end{tabular}




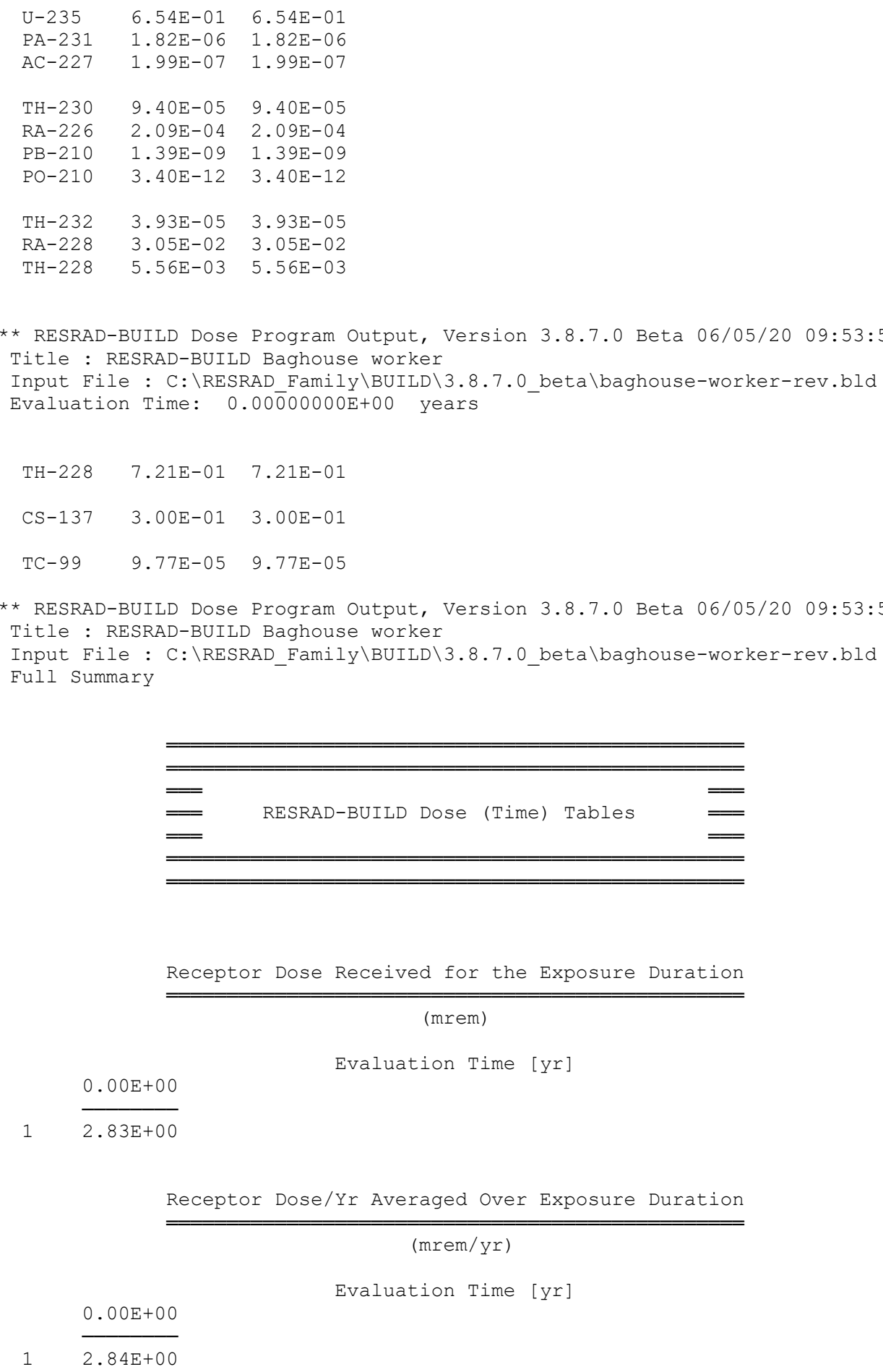

Receptor Dose Received for the Exposure Duration

(mrem)

Evaluation Time [yr]

$1 \quad \overline{2.83 \mathrm{E}+00}$

Receptor Dose/Yr Averaged Over Exposure Duration

$(\mathrm{mrem} / \mathrm{yr})$

$0.00 \mathrm{E}+00$

Evaluation Time [yr]

$12.84 \mathrm{E}+00$

Note: The Receptor 1 dose for an individual radionuclide from Source 1 gives the incinerator baghouse worker/processor external dose in a year (Table 5 in the main report). 


\section{APPENDIX B: \\ RESRAD-OFFSITE RUN RESULTS FOR NON-INVOLVED WORKER, INCINERATOR NEIGHBOR, AND LANDFILL NEIGHBOR SCENARIOS}

RESRAD-OFFSITE code was used to analyze the non-involved worker, incinerator neighbor, and landfill neighbor scenarios. The results are provided in this appendix in the form of reports generated from different scenario runs from RESRAD-OFFSITE code. 


\section{B.1 RESRAD-OFFSITE RESULTS FOR THE INCINERATOR NEIGHBOR SCENARIO}

To run the non-involved worker scenario, the AIRFLUXIN.DAT file was changed to match the estimated radionuclide's yearly release rate from the Aragonite incinerator.

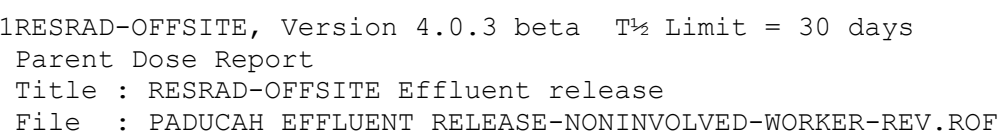

\section{Table of Contents}

Part I: Mixture Sums and Single Radionuclide Guidelines

Dose Conversion Factor (and Related) Parameter Summary ... 2

Site-Specific Parameter Summary $\ldots \ldots \ldots \ldots \ldots \ldots \ldots \ldots \ldots \ldots$

Summary of Pathway Selections ....................... 48

Contaminated Zone and Total Dose Summary ............. 49

Total Dose Components

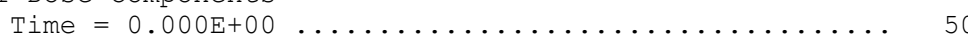

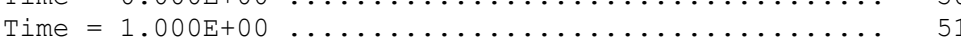

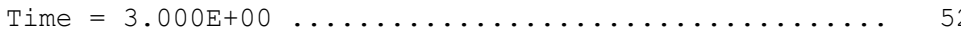

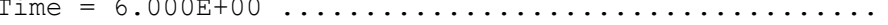

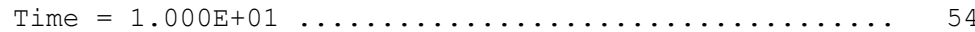

Dose/Source Ratios Summed Over All Pathways ............... 55

Single Radionuclide Soil Guidelines .................................... 57

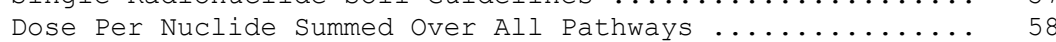

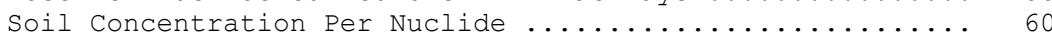

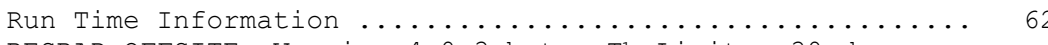

1RESRAD-OFFSITE, Version 4.0 .3 beta $\mathrm{T}^{1 / 2}$ Limit $=30$ days

Parent Dose Report

Title : RESRAD-OFFSITE Effluent release

File : PADUCAH EFFLUENT RELEASE-NONINVOLVED-WORKER-REV.ROF

Dose Conversion Factor (and Related) Parameter Summary Current Library: ICRP 60

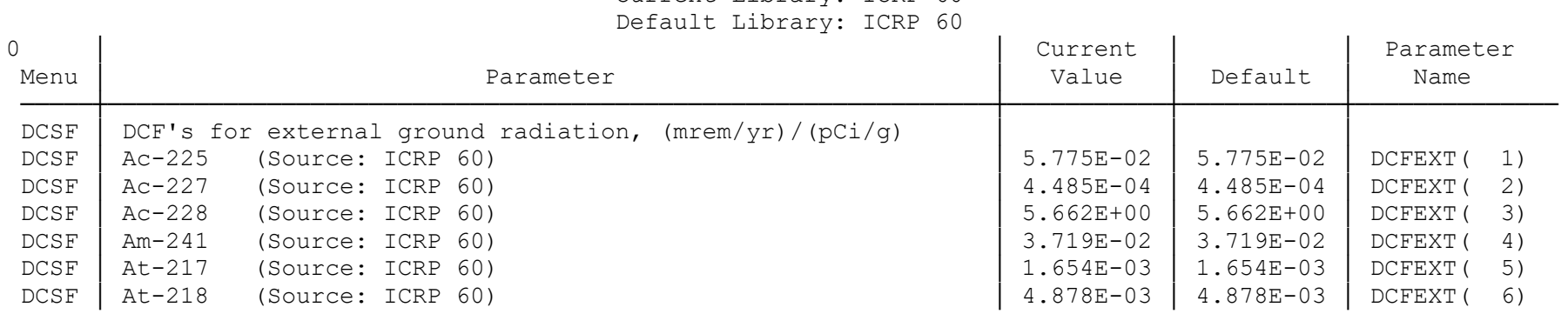




\begin{tabular}{|c|c|c|c|c|}
\hline DCSF & $\mathrm{Ba}-137 \mathrm{~m}$ & (Source: & ICRP & 60) \\
\hline DCSF & $\mathrm{Bi}-210$ & (Source: & ICRP & 60) \\
\hline DCSF & $\mathrm{Bi}-211$ & (Source: & ICRP & 60) \\
\hline DCSF & Bi-212 & (Source: & ICRP & 60) \\
\hline DCSF & $\mathrm{Bi}-213$ & (Source: & ICRP & $60)$ \\
\hline DCSF & Bi-214 & (Source: & ICRP & 60) \\
\hline DCSF & $\mathrm{Cs}-137$ & (Source: & ICRP & $60)$ \\
\hline DCSF & Fr-221 & (Source: & ICRP & 60) \\
\hline CSF & Fr -223 & (Source: & ICRP & $60)$ \\
\hline DCSF & $\mathrm{Np}-237$ & (Source: & ICRP & $60)$ \\
\hline DCSF & $\mathrm{Pa}-231$ & (Source: & ICRP & 60) \\
\hline DCSF & $\mathrm{Pa}-233$ & (Source: & ICRP & 60) \\
\hline DCSF & $\mathrm{Pa}-234$ & (Source: & ICRP & $60)$ \\
\hline DCSF & $\mathrm{Pa}-234 \mathrm{~m}$ & (Source: & ICRP & $60)$ \\
\hline DCSF & $P b-209$ & (Source: & ICRP & 60) \\
\hline DCSF & $\mathrm{Pb}-210$ & (Source: & ICRP & $60)$ \\
\hline DCSF & $\mathrm{Pb}-211$ & (Source: & ICRP & $60)$ \\
\hline DCSF & $\mathrm{Pb}-212$ & (Source: & ICRP & $60)$ \\
\hline DCSF & $\mathrm{Pb}-214$ & (Source: & ICRP & $60)$ \\
\hline DCSF & Po-210 & (Source: & ICRP & 60) \\
\hline DCSF & Po-211 & (Source: & ICRP & 60) \\
\hline DCSF & Po -212 & (Source: & ICRP & $60)$ \\
\hline DCSF & Po -213 & (Source: & ICRP & $60)$ \\
\hline DCSF & Po-214 & (Source: & ICRP & $60)$ \\
\hline DCSF & Po- 215 & (Source: & ICRP & $60)$ \\
\hline DCSF & Po-216 & (Source: & ICRP & $60)$ \\
\hline DCSF & Po-218 & (Source: & ICRP & $60)$ \\
\hline DCSF & $\mathrm{Pu}-238$ & (Source: & ICRP & 60) \\
\hline DCSF & Pu-239 & (Source: & ICRP & $60)$ \\
\hline DCSF & $\mathrm{Pu}-240$ & (Source: & ICRP & 60) \\
\hline DCSF & $\mathrm{Ra}-223$ & (Source: & ICRP & $60)$ \\
\hline DCSF & $R a-224$ & (Source: & ICRP & $60)$ \\
\hline DCSF & $\mathrm{Ra}-225$ & (Source: & ICRP & $60)$ \\
\hline DCSF & $R a-226$ & (Source: & ICRP & $60)$ \\
\hline DCSF & $R a-228$ & (Source: & ICRP & $60)$ \\
\hline DCSF & $R n-219$ & (Source: & ICRP & 60) \\
\hline DCSF & $R n-220$ & (Source: & ICRP & $60)$ \\
\hline DCSF & $R n-222$ & (Source: & ICRP & 60) \\
\hline
\end{tabular}

1RESRAD-OFFSITE, Version 4.0 .3 beta $\mathrm{T}^{\frac{1}{2}} \mathrm{Limit}=30$ days

Parent Dose Report

Title : RESRAD-OFFSITE Effluent release

File : PADUCAH EFFLUENT RELEASE-NONINVOLVED-WORKER-REV.ROF

\begin{tabular}{l|l|l}
$3.383 \mathrm{E}+00$ & $3.383 \mathrm{E}+00$ & $\operatorname{DCFEXT}(7)$ \\
$5.476 \mathrm{E}-03$ & $5.476 \mathrm{E}-03$ & $\operatorname{DCFEXT}(8)$ \\
$2.373 \mathrm{E}-01$ & $2.373 \mathrm{E}-01$ & $\operatorname{DCFEXT}(9)$ \\
$1.114 \mathrm{E}+00$ & $1.114 \mathrm{E}+00$ & $\operatorname{DCFEXT}(10)$ \\
$7.158 \mathrm{E}-01$ & $7.158 \mathrm{E}-01$ & $\operatorname{DCFEXT}(11)$ \\
$9.325 \mathrm{E}+00$ & $9.325 \mathrm{E}+00$ & $\operatorname{DCFEXT}(12)$ \\
$8.372 \mathrm{E}-04$ & $8.372 \mathrm{E}-04$ & $\operatorname{DCFEXT}(13)$ \\
$1.413 \mathrm{E}-01$ & $1.413 \mathrm{E}-01$ & $\operatorname{DCFEXT}(14)$ \\
$1.813 \mathrm{E}-01$ & $1.813 \mathrm{E}-01$ & $\operatorname{DCFEXT}(15)$ \\
$6.971 \mathrm{E}-02$ & $6.971 \mathrm{E}-02$ & $\operatorname{DCFEXT}(16)$ \\
$1.762 \mathrm{E}-01$ & $1.762 \mathrm{E}-01$ & $\operatorname{DCFEXT}(17)$ \\
$9.419 \mathrm{E}-01$ & $9.419 \mathrm{E}-01$ & $\operatorname{DCFEXT}(18)$ \\
$1.088 \mathrm{E}+01$ & $1.088 \mathrm{E}+01$ & $\operatorname{DCFEXT}(19)$ \\
$9.867 \mathrm{E}-02$ & $9.867 \mathrm{E}-02$ & $\operatorname{DCFEXT}(20)$ \\
$7.550 \mathrm{E}-04$ & $7.550 \mathrm{E}-04$ & $\operatorname{DCFEXT}(21)$ \\
$1.981 \mathrm{E}-03$ & $1.981 \mathrm{E}-03$ & $\operatorname{DCFEXT}(22)$ \\
$2.915 \mathrm{E}-01$ & $2.915 \mathrm{E}-01$ & $\operatorname{DCFEXT}(23)$ \\
$6.466 \mathrm{E}-01$ & $6.466 \mathrm{E}-01$ & $\operatorname{DCFEXT}(24)$ \\
$1.243 \mathrm{E}+00$ & $1.243 \mathrm{E}+00$ & $\operatorname{DCFEXT}(25)$ \\
$4.934 \mathrm{E}-05$ & $4.934 \mathrm{E}-05$ & $\operatorname{DCFEXT}(26)$ \\
$4.485 \mathrm{E}-02$ & $4.485 \mathrm{E}-02$ & $\operatorname{DCFEXT}(27)$ \\
$0.000 \mathrm{E}+00$ & $0.000 \mathrm{E}+00$ & $\operatorname{DCFEXT}(28)$ \\
$0.000 \mathrm{E}+00$ & $0.000 \mathrm{E}+00$ & $\operatorname{DCFEXT}(29)$ \\
$4.840 \mathrm{E}-04$ & $4.840 \mathrm{E}-04$ & $\operatorname{DCFEXT}(30)$ \\
$9.456 \mathrm{E}-04$ & $9.456 \mathrm{E}-04$ & $\operatorname{DCFEXT}(31)$ \\
$9.830 \mathrm{E}-05$ & $9.830 \mathrm{E}-05$ & $\operatorname{DCFEXT}(32)$ \\
$5.326 \mathrm{E}-05$ & $5.326 \mathrm{E}-05$ & $\operatorname{DCFEXT}(33)$ \\
$1.166 \mathrm{E}-04$ & $1.166 \mathrm{E}-04$ & $\operatorname{DCFEXT}(34)$ \\
$2.635 \mathrm{E}-04$ & $2.635 \mathrm{E}-04$ & $\operatorname{DCFEXT}(35)$ \\
$1.129 \mathrm{E}-04$ & $1.129 \mathrm{E}-04$ & $\operatorname{DCFEXT}(36)$ \\
$5.532 \mathrm{E}-01$ & $5.532 \mathrm{E}-01$ & $\operatorname{DCFEXT}(37)$ \\
$4.728 \mathrm{E}-02$ & $4.728 \mathrm{E}-02$ & $\operatorname{DCFEXT}(38)$ \\
$8.634 \mathrm{E}-03$ & $8.634 \mathrm{E}-03$ & $\operatorname{DCFEXT}(39)$ \\
$2.915 \mathrm{E}-02$ & $2.915 \mathrm{E}-02$ & $\operatorname{DCFEXT}(40)$ \\
$0.000 \mathrm{E}+00$ & $0.000 \mathrm{E}+00$ & $\operatorname{DCFEXT}(41)$ \\
$2.859 \mathrm{E}-01$ & $2.859 \mathrm{E}-01$ & $\operatorname{DCFEXT}(42)$ \\
$2.130 \mathrm{E}-03$ & $2.130 \mathrm{E}-03$ & $\operatorname{DCFEXT}(43)$ \\
$2.186 \mathrm{E}-03$ & $2.186 \mathrm{E}-03$ & $\operatorname{DCFEXT}(44)$ \\
$12020-11.39$ & $\operatorname{DC}(49)$ &
\end{tabular}

06/11/2020 11:39 Page 3

Dose Conversion Factor (and Related) Parameter Summary (continued)

Current Library: ICRP 60

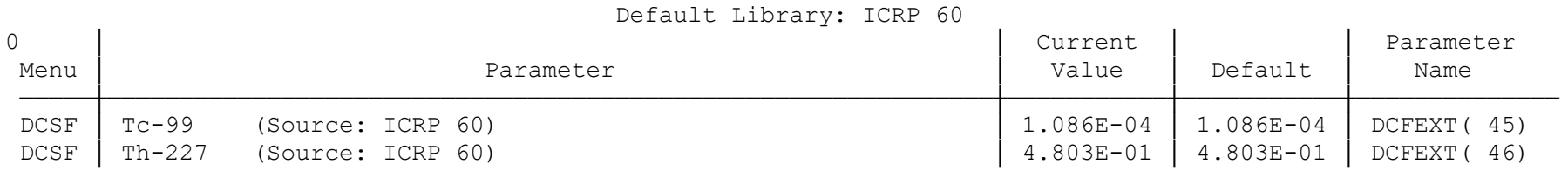




\begin{tabular}{l|ll} 
DCSF & Th-228 & (Source: ICRP 60) \\
DCSF & Th-229 & (Source: ICRP 60) \\
DCSF & Th-230 & (Source: ICRP 60) \\
DCSF & Th-231 & (Source: ICRP 60) \\
DCSF & Th-232 & (Source: ICRP 60) \\
DCSF & Th-234 & (Source: ICRP 60) \\
DCSF & Tl-207 & (Source: ICRP 60) \\
DCSF & Tl-208 & (Source: ICRP 60) \\
DCSF & Tl-209 & (Source: ICRP 60) \\
DCSF & Tl-210 & (Source: ICRP 60) \\
DCSF & U-233 & (Source: ICRP 60) \\
DCSF & U-234 & (Source: ICRP 60) \\
DCSF & U-235 & (Source: ICRP 60) \\
DCSF & U-236 & (Source: ICRP 60) \\
DCSF & U-238 & (Source: ICRP 60)
\end{tabular}

\begin{tabular}{|l|l|l}
$7.176 \mathrm{E}-03$ & $7.176 \mathrm{E}-03$ & $\operatorname{DCFEXT}(47)$ \\
$2.897 \mathrm{E}-01$ & $2.897 \mathrm{E}-01$ & $\operatorname{DCFEXT}(48)$ \\
$1.071 \mathrm{E}-03$ & $1.071 \mathrm{E}-03$ & $\operatorname{DCFEXT}(49)$ \\
$3.214 \mathrm{E}-02$ & $3.214 \mathrm{E}-02$ & $\operatorname{DCFEXT}(50)$ \\
$4.560 \mathrm{E}-04$ & $4.560 \mathrm{E}-04$ & $\operatorname{DCFEXT}(51)$ \\
$2.130 \mathrm{E}-02$ & $2.130 \mathrm{E}-02$ & $\operatorname{DCFEXT}(52)$ \\
$2.299 \mathrm{E}-02$ & $2.299 \mathrm{E}-02$ & $\operatorname{DCFEXT}(53)$ \\
$2.186 \mathrm{E}+01$ & $2.186 \mathrm{E}+01$ & $\operatorname{DCFEXT}(54)$ \\
$1.226 \mathrm{E}+01$ & $1.226 \mathrm{E}+01$ & $\operatorname{DCFEXT}(55)$ \\
$1.661 \mathrm{E}+01$ & $1.661 \mathrm{E}+01$ & $\operatorname{DCFEXT}(56)$ \\
$1.265 \mathrm{E}-03$ & $1.265 \mathrm{E}-03$ & $\operatorname{DCFEXT}(57)$ \\
$3.439 \mathrm{E}-04$ & $3.439 \mathrm{E}-04$ & $\operatorname{DCFEXT}(58)$ \\
$6.597 \mathrm{E}-01$ & $6.597 \mathrm{E}-01$ & $\operatorname{DCFEXT}(59)$ \\
$1.781 \mathrm{E}-04$ & $1.781 \mathrm{E}-04$ & $\operatorname{DCFEXT}(60)$ \\
$7.961 \mathrm{E}-05$ & $7.961 \mathrm{E}-05$ & $\operatorname{DCFEXT}(61)$ \\
& &
\end{tabular}

Current Library: ICRP 72 (Adult)

Default Library: ICRP 72 (Adult)

\begin{tabular}{|c|c|c|c|c|}
\hline $\begin{array}{l}0 \\
\text { Menu }\end{array}$ & Parameter & $\begin{array}{l}\text { Current } \\
\text { Value }\end{array}$ & Default & $\begin{array}{l}\text { Parameter } \\
\text { Name }\end{array}$ \\
\hline DCSF & Dose conversion factors for inhalation, mrem/pCi: & & & \\
\hline DCSF & $A C-227+D$ & $2.104 \mathrm{E}+00$ & $2.104 \mathrm{E}+00$ & $\mathrm{DCF} 2(1)$ \\
\hline DCSF & $\mathrm{Cs}-137+\mathrm{D}$ & $1.443 \mathrm{E}-04$ & $1.443 \mathrm{E}-04$ & $\mathrm{DCF} 2(3)$ \\
\hline DCSF & $\mathrm{Np}-237+\mathrm{D}$ & $1.850 \mathrm{E}-01$ & $1.850 \mathrm{E}-01$ & $\mathrm{DCF} 2(4)$ \\
\hline DCSF & $\mathrm{Pa}-231$ & $5.180 \mathrm{E}-01$ & $5.180 \mathrm{E}-01$ & $\mathrm{DCF} 2(5)$ \\
\hline DCSF & $\mathrm{Pb}-210+\mathrm{D}$ & $2.106 \mathrm{E}-02$ & $2.106 \mathrm{E}-02$ & $\operatorname{DCF} 2(6)$ \\
\hline DCSF & $\mathrm{Pu}-239$ & $4.440 \mathrm{E}-01$ & $4.440 \mathrm{E}-01$ & DCF2 (10) \\
\hline DCSF & $\mathrm{Pu}-240$ & $4.440 \mathrm{E}-01$ & $4.440 \mathrm{E}-01$ & DCF2 (11) \\
\hline DCSF & $\mathrm{Ra}-226+\mathrm{D}$ & $3.526 \mathrm{E}-02$ & $3.526 \mathrm{E}-02$ & DCF2 (13) \\
\hline DCSF & $\mathrm{Ra}-228+\mathrm{D}$ & $5.929 \mathrm{E}-02$ & $5.929 \mathrm{E}-02$ & DCF2 (14) \\
\hline DCSF & $\mathrm{TC}-99$ & $4.810 \mathrm{E}-05$ & $4.810 \mathrm{E}-05$ & DCF2 (15) \\
\hline DCSF & $T h-228+D$ & $1.614 \mathrm{E}-01$ & $1.614 \mathrm{E}-01$ & DCF2 (16) \\
\hline DCSF & $T h-229+D$ & $9.481 \mathrm{E}-01$ & $9.481 \mathrm{E}-01$ & DCF2 (17) \\
\hline DCSF & Th -232 & $4.070 \mathrm{E}-01$ & $4.070 \mathrm{E}-01$ & DCF2 (19) \\
\hline DCSF & $U-233$ & $3.552 \mathrm{E}-02$ & $3.552 \mathrm{E}-02$ & DCF2 (20) \\
\hline DCSF & $\mathrm{U}-234$ & $3.478 \mathrm{E}-02$ & $3.478 \mathrm{E}-02$ & DCF2 (21) \\
\hline
\end{tabular}

TESRAD-OFFSITE, Version 4.0 .3 beta $\mathrm{T}^{1 / 2} \mathrm{Limit}=30$ days

06/11/2020 11:39 Page 4

Parent Dose Report

File: PADUCAH EFFLUENT RELEASE-NONINVOLVED-WORKER-REV.ROF

Dose Conversion Factor (and Related) Parameter Summary (continued) Current Library: ICRP 72 (Adult) Default Library: ICRP 72 (Adult) 


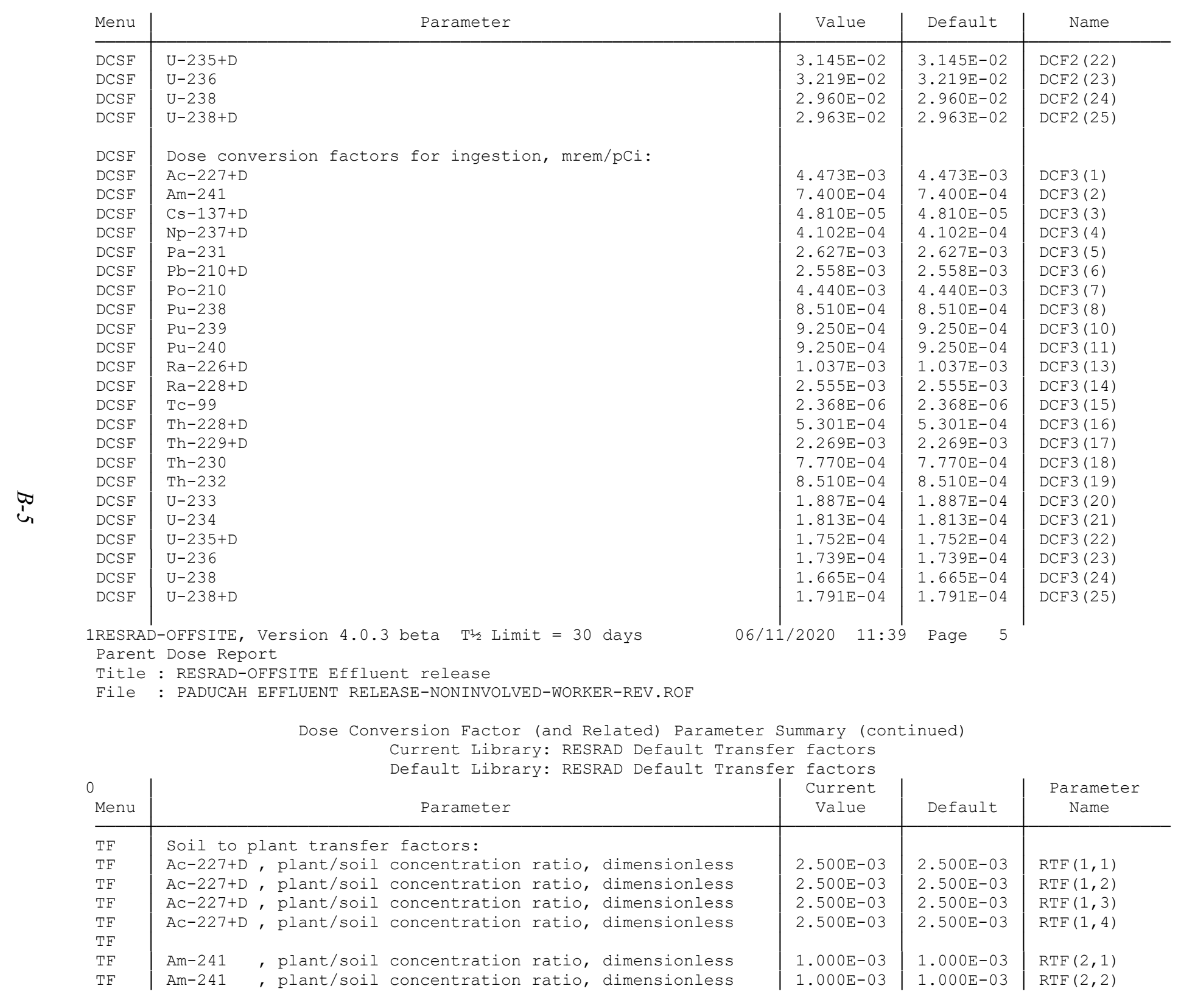




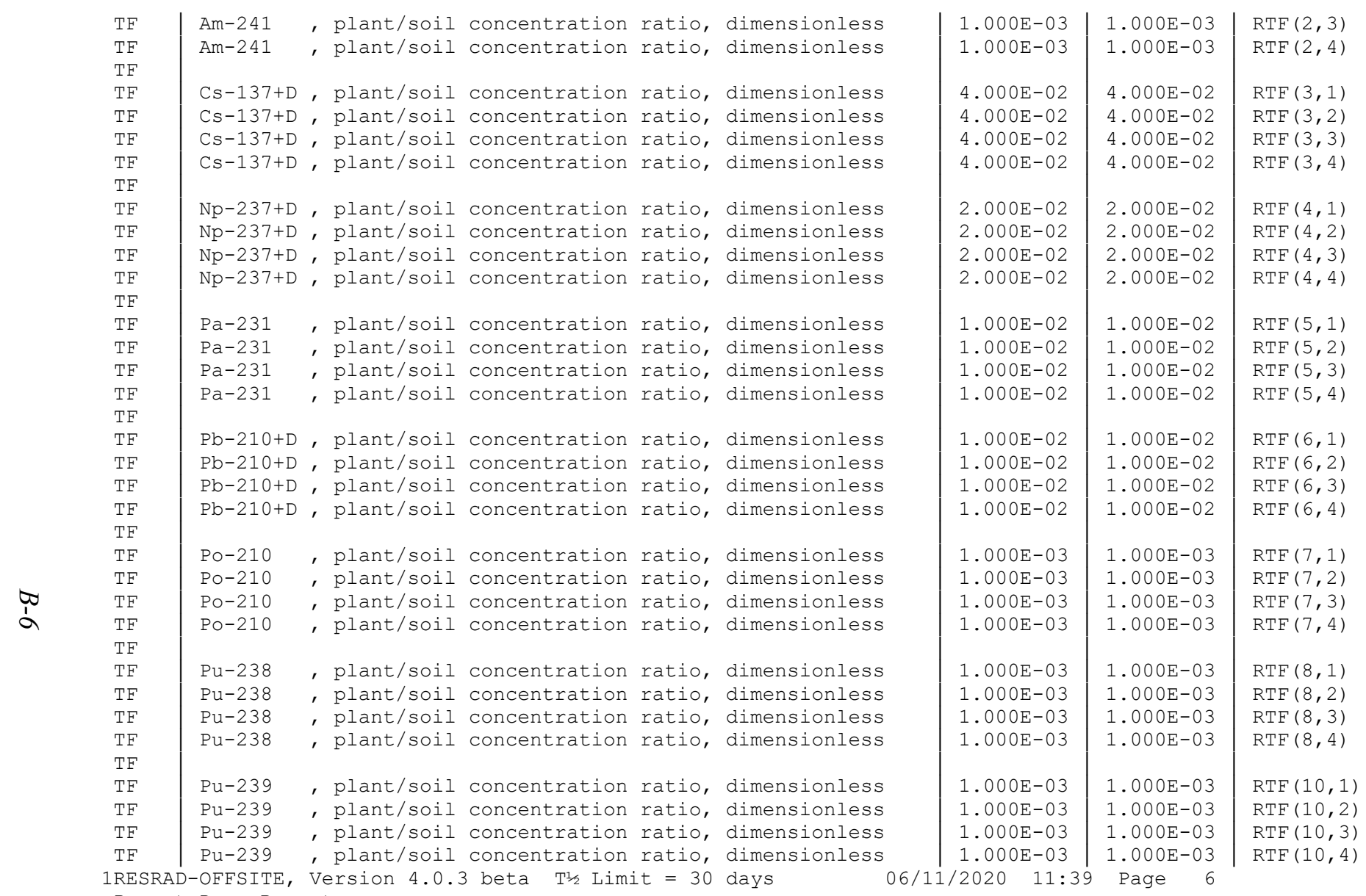

Parent Dose Report

Title : RESRAD-OFFSITE Effluent release

File : PADUCAH EFFLUENT RELEASE-NONINVOLVED-WORKER-REV.ROF

Dose Conversion Factor (and Related) Parameter Summary (continued)

Current Library: RESRAD Default Transfer factors

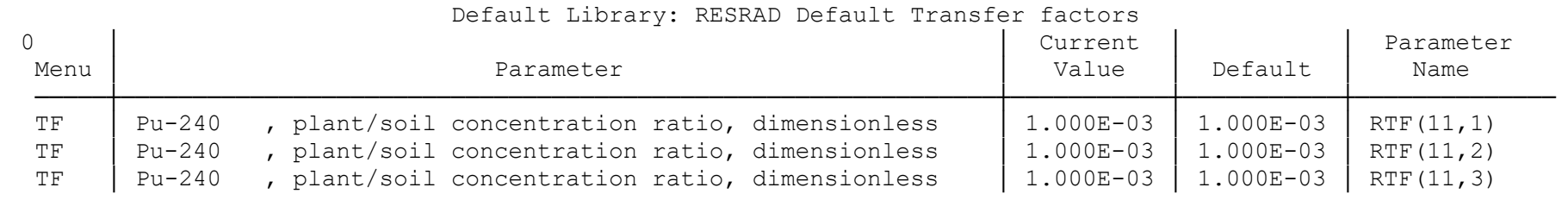




\begin{tabular}{|c|c|c|c|c|c|c|c|c|}
\hline $\begin{array}{l}\text { TF } \\
\text { TF }\end{array}$ & $\mathrm{Pu}-240$ & , plant/soil & concentration & ratio, & dimensionless & $1.000 \mathrm{E}-03$ & $1.000 \mathrm{E}-03$ & $\operatorname{RTF}(11,4)$ \\
\hline TF & $\mathrm{Ra}-226+\mathrm{D}$ & , plant/soil & concentration & ratio, & dimensionless & $4.000 \mathrm{E}-02$ & $4.000 \mathrm{E}-02$ & $\operatorname{RTF}(13,1)$ \\
\hline $\mathrm{TF}$ & $\mathrm{Ra}-226+\mathrm{D}$ & , plant/soil & concentration & ratio, & dimensionless & $4.000 \mathrm{E}-02$ & $4.000 \mathrm{E}-02$ & $\operatorname{RTF}(13,2)$ \\
\hline $\mathrm{TF}$ & $\mathrm{Ra}-226+\mathrm{D}$ & , plant/soil & concentration & ratio, & dimensionless & $4.000 \mathrm{E}-02$ & $4.000 \mathrm{E}-02$ & $\operatorname{RTF}(13,3)$ \\
\hline $\begin{array}{l}\text { TF } \\
\text { TF }\end{array}$ & $R a-226+D$ & , plant/soil & concentration & ratio, & dimensionless & $4.000 \mathrm{E}-02$ & $4.000 \mathrm{E}-02$ & $\operatorname{RTF}(13,4)$ \\
\hline TF & $\mathrm{Ra}-228+\mathrm{D}$ & , plant/soil & concentration & ratio, & dimensionless & $4.000 \mathrm{E}-02$ & $4.000 \mathrm{E}-02$ & $\operatorname{RTF}(14,1)$ \\
\hline TF & $\mathrm{Ra}-228+\mathrm{D}$ & , plant/soil & concentration & ratio, & dimensionless & $4.000 \mathrm{E}-02$ & $4.000 \mathrm{E}-02$ & $\operatorname{RTF}(14,2)$ \\
\hline $\mathrm{TF}$ & $\mathrm{Ra}-228+\mathrm{D}$ & , plant/soil & concentration & ratio, & dimensionless & $4.000 \mathrm{E}-02$ & $4.000 \mathrm{E}-02$ & $\operatorname{RTF}(14,3)$ \\
\hline $\begin{array}{l}\text { TF } \\
\text { TF }\end{array}$ & $\mathrm{Ra}-228+\mathrm{D}$ & , plant/soil & concentration & ratio, & dimensionless & $4.000 \mathrm{E}-02$ & $4.000 \mathrm{E}-02$ & $\operatorname{RTF}(14,4)$ \\
\hline $\mathrm{TF}$ & TC-99 & , plant/soil & concentration & ratio, & dimensionless & $5.000 \mathrm{E}+00$ & $5.000 \mathrm{E}+00$ & $\operatorname{RTF}(15,1)$ \\
\hline $\mathrm{TF}$ & TC-99 & , plant/soil & concentration & ratio, & dimensionless & $5.000 \mathrm{E}+00$ & $5.000 \mathrm{E}+00$ & $\operatorname{RTF}(15,2)$ \\
\hline TF & TC-99 & , plant/soil & concentration & ratio, & dimensionless & $5.000 \mathrm{E}+00$ & $5.000 \mathrm{E}+00$ & $\operatorname{RTF}(15,3)$ \\
\hline $\begin{array}{l}\text { TF } \\
\text { TF }\end{array}$ & TC-99 & , plant/soil & concentration & ratio, & dimensionless & $5.000 \mathrm{E}+00$ & $5.000 \mathrm{E}+00$ & $\operatorname{RTF}(15,4)$ \\
\hline $\mathrm{TF}$ & $T h-228+D$ & , plant/soil & concentration & ratio, & dimensionless & $1.000 \mathrm{E}-03$ & $1.000 \mathrm{E}-03$ & $\operatorname{RTF}(16$, \\
\hline TF & Th $-228+D$ & , plant/soil & concentration & ratio, & dimensionless & $1.000 \mathrm{E}-03$ & $1.000 \mathrm{E}-03$ & $\operatorname{RTF}(16,2)$ \\
\hline $\mathrm{TF}$ & $T h-228+D$ & , plant/soil & concentration & ratio, & dimensionless & $1.000 \mathrm{E}-03$ & $1.000 \mathrm{E}-03$ & $\operatorname{RTF}(16,3)$ \\
\hline $\begin{array}{l}\mathrm{TF} \\
\mathrm{TF}\end{array}$ & Th $-228+D$ & , plant/soil & concentration & ratio, & dimensionless & $1.000 \mathrm{E}-03$ & $1.000 \mathrm{E}-03$ & $\operatorname{RTF}(16,4)$ \\
\hline $\mathrm{TF}$ & $T h-229+D$ & , plant/soil & concentration & ratio, & dimensionless & $1.000 \mathrm{E}-03$ & $1.000 \mathrm{E}-03$ & $\operatorname{RTF}(17,1)$ \\
\hline $\mathrm{TF}$ & $T h-229+D$ & , plant/soil & concentration & ratio, & dimensionless & $1.000 \mathrm{E}-03$ & $1.000 \mathrm{E}-03$ & $\operatorname{RTF}(17,2)$ \\
\hline $\mathrm{TF}$ & $T h-229+D$ & , plant/soil & concentration & ratio, & dimensionless & $1.000 \mathrm{E}-03$ & $1.000 \mathrm{E}-03$ & $\operatorname{RTF}(17,3)$ \\
\hline $\mathrm{TF}$ & $T h-229+D$ & , plant/soil & concentration & ratio, & dimensionless & $1.000 \mathrm{E}-03$ & $1.000 \mathrm{E}-03$ & $\operatorname{RTF}(17,4)$ \\
\hline $\mathrm{TF}$ & Th-230 & , plant/soil & concentration & ratio, & less & $1.000 \mathrm{E}-03$ & $1.000 \mathrm{E}-03$ & $\operatorname{RTF}(18,1)$ \\
\hline $\mathrm{TF}$ & Th-230 & , plant/soil & concentration & ratio, & dimensionless & $1.000 \mathrm{E}-03$ & $1.000 \mathrm{E}-03$ & $\operatorname{RTF}(18,2)$ \\
\hline $\mathrm{TF}$ & Th-230 & , plant/soil & concentration & ratio, & dimensionless & $1.000 \mathrm{E}-03$ & $1.000 \mathrm{E}-03$ & $\operatorname{RTF}(18,3)$ \\
\hline $\begin{array}{l}\text { TF } \\
\text { TF }\end{array}$ & Th -230 & , plant/soil & concentration & ratio, & dimensionless & $1.000 \mathrm{E}-03$ & $1.000 \mathrm{E}-03$ & $\operatorname{RTF}(18,4)$ \\
\hline TF & Th-232 & , plant/soil & concentration & ratio, & dimensionless & $1.000 \mathrm{E}-03$ & $1.000 \mathrm{E}-03$ & $\operatorname{RTF}(19,1)$ \\
\hline $\mathrm{TF}$ & Th-232 & , plant/soil & concentration & ratio, & dimensionless & $1.000 \mathrm{E}-03$ & $1.000 \mathrm{E}-03$ & $\operatorname{RTF}(19,2)$ \\
\hline TF & Th-232 & , plant/soil & concentration & ratio, & dimensionless & $1.000 \mathrm{E}-03$ & $1.000 \mathrm{E}-03$ & $\operatorname{RTF}(19,3)$ \\
\hline $\begin{array}{l}\text { TF } \\
\text { TF }\end{array}$ & $T h-232$ & , plant/soil & concentration & ratio, & dimensionless & $1.000 \mathrm{E}-03$ & $1.000 \mathrm{E}-03$ & $\operatorname{RTF}(19,4)$ \\
\hline TF & $U-233$ & , plant/soil & concentration & ratio & ionless & $2.500 \mathrm{E}-03$ & $2.500 \mathrm{E}-03$ & $\operatorname{RTF}(20,1)$ \\
\hline $\mathrm{TF}$ & $\mathrm{U}-233$ & , plant/soil & concentration & ratio, & dimensionless & $2.500 \mathrm{E}-03$ & $2.500 \mathrm{E}-03$ & $\operatorname{RTF}(20,2)$ \\
\hline $\mathrm{TF}$ & $\mathrm{U}-233$ & , plant/soil & concentration & ratio, & dimensionless & $2.500 \mathrm{E}-03$ & $2.500 \mathrm{E}-03$ & $\operatorname{RTF}(20,3)$ \\
\hline TF & $\mathrm{U}-233$ & , plant/soil & concentration & ratio, & dimensionless & $2.500 \mathrm{E}-03$ & $2.500 \mathrm{E}-03$ & $\operatorname{RTF}(20,4)$ \\
\hline
\end{tabular}

1RESRAD-OFFSITE, Version 4.0 .3 beta T1/2 Limit $=30$ days

06/11/2020 11:39 Page 7

Parent Dose Report
Title: RESRAD-OFFSITE Effluent release

file: PADUCAH EFFLUENT RELEASE-NONINVOLVED-WORKER-REV.ROF

Dose Conversion Factor (and Related) Parameter Summary (continued) Current Library: RESRAD Default Transfer factors Default Library: RESRAD Default Transfer factors 


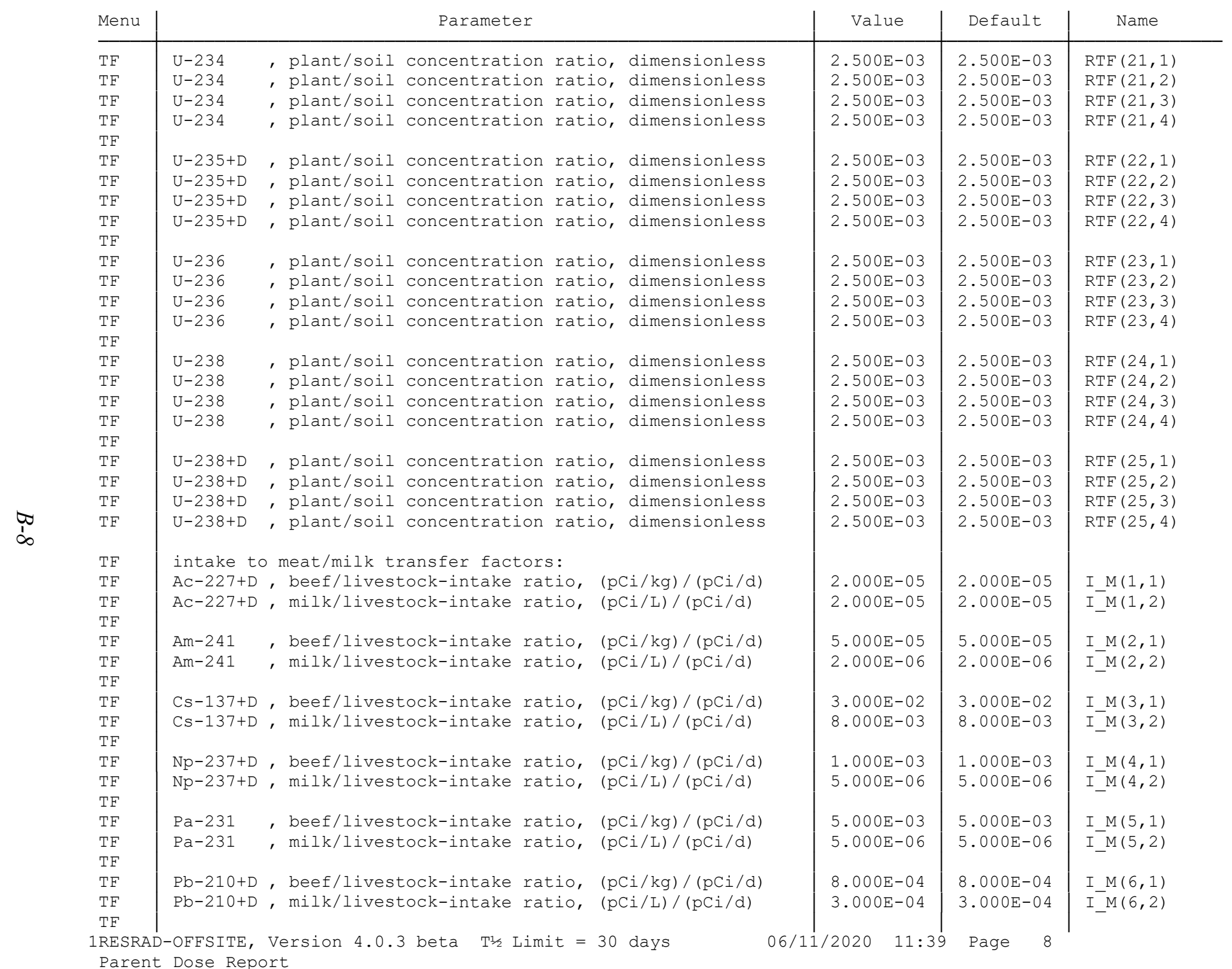

Parent Dose Report

Title : RESRAD-OFFSITE Effluent release

File : PADUCAH EFFLUENT RELEASE-NONINVOLVED-WORKER-REV.ROF 
Dose Conversion Factor (and Related) Parameter Summary (continued)

Current Library: RESRAD Default Transfer factors

Default Library: RESRAD Default Transfer factors

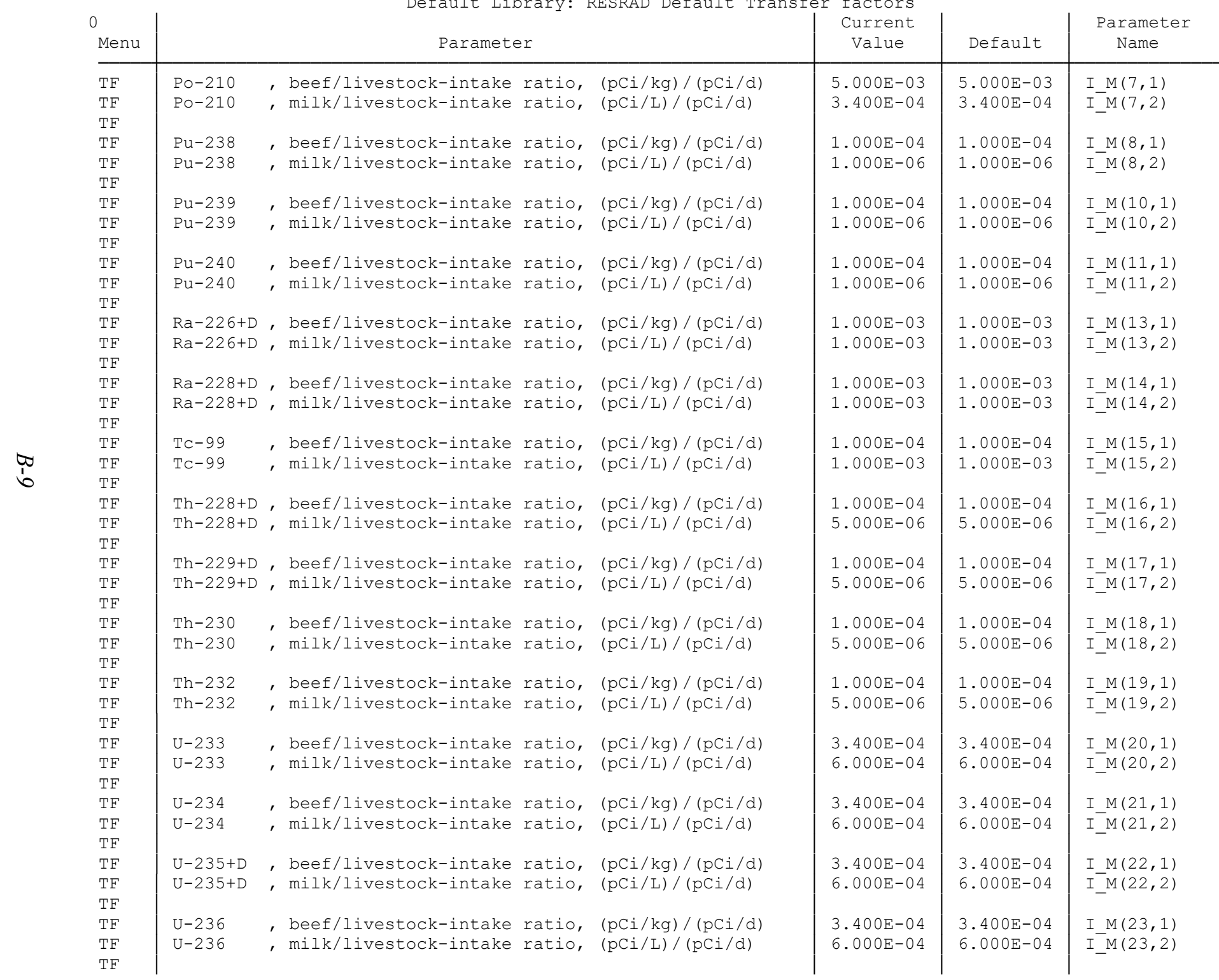


1RESRAD-OFFSITE, Version 4.0 .3 beta $T^{\frac{1}{2}}$ Limit $=30$ days

Parent Dose Report

Title : RESRAD-OFFSITE Effluent release

File : PADUCAH EFFLUENT RELEASE-NONINVOLVED-WORKER-REV.ROF

Dose Conversion Factor (and Related) Parameter Summary (continued) Current Library: RESRAD Default Transfer factors Default Library: RESRAD Default Transfer factors

\begin{tabular}{|c|c|c|c|c|c|}
\hline $\begin{array}{l}0 \\
\text { Menu }\end{array}$ & & Parameter & $\begin{array}{c}\text { Current } \\
\text { Value }\end{array}$ & Default & $\begin{array}{c}\text { Parameter } \\
\text { Name }\end{array}$ \\
\hline $\mathrm{TF}$ & $\mathrm{U}-238$ & , beef/livestock-intake ratio, $(\mathrm{pCi} / \mathrm{kg}) /(\mathrm{pCi} / \mathrm{d})$ & $3.400 \mathrm{E}-04$ & $3.400 \mathrm{E}-04$ & I_M $(24,1)$ \\
\hline $\mathrm{TF}$ & $\mathrm{U}-238$ & , milk/livestock-intake ratio, $(\mathrm{pCi} / \mathrm{L}) /(\mathrm{pCi} / \mathrm{d})$ & $6.000 \mathrm{E}-04$ & $6.000 \mathrm{E}-04$ & $I_{-}^{-} M(24,2)$ \\
\hline$T F$ & & & & & \\
\hline TF & $\mathrm{U}-238+\mathrm{D}$ & , beef/livestock-intake ratio, $(\mathrm{pCi} / \mathrm{kg}) /(\mathrm{pCi} / \mathrm{d})$ & $3.400 \mathrm{E}-04$ & $3.400 \mathrm{E}-04$ & I_M $(25,1)$ \\
\hline $\mathrm{TF}$ & $\mathrm{U}-238+\mathrm{D}$ & , milk/livestock-intake ratio, $(\mathrm{pCi} / \mathrm{L}) /(\mathrm{pCi} / \mathrm{d})$ & $6.000 \mathrm{E}-04$ & $6.000 \mathrm{E}-04$ & $I_{-}^{-} M(25,2)$ \\
\hline $\mathrm{TF}$ & Bioaccumu & ulation factors, fresh water, L/kg: & & & \\
\hline $\mathrm{TF}$ & $A c-227+D$ & , fish & $1.500 \mathrm{E}+01$ & $1.500 \mathrm{E}+01$ & $\operatorname{BIOFA}(1,1)$ \\
\hline $\mathrm{TF}$ & $A c-227+D$ & , crustacea and mollusks & $1.000 \mathrm{E}+03$ & $1.000 \mathrm{E}+03$ & $\operatorname{BIOFA}(1,2)$ \\
\hline $\mathrm{TF}$ & & & & & \\
\hline TF & Am-241 & , fish & $3.000 \mathrm{E}+01$ & $3.000 \mathrm{E}+01$ & $\operatorname{BIOFA}(2,1)$ \\
\hline $\mathrm{TF}$ & $\mathrm{Am}-241$ & , crustacea and mollusks & $1.000 \mathrm{E}+03$ & $1.000 \mathrm{E}+03$ & $\operatorname{BIOFA}(2,2)$ \\
\hline $\mathrm{TF}$ & & & & & \\
\hline $\mathrm{TF}$ & Cs $-137+D$ & , fish & $2.000 \mathrm{E}+03$ & $2.000 \mathrm{E}+03$ & $\operatorname{BIOFA}(3,1)$ \\
\hline $\mathrm{TF}$ & Cs $-137+D$ & , crustacea and mollusks & $1.000 \mathrm{E}+02$ & $1.000 \mathrm{E}+02$ & $\operatorname{BIOFA}(3,2)$ \\
\hline TF & & & & & \\
\hline $\mathrm{TF}$ & Np $-237+D$ & , fish & $3.000 \mathrm{E}+01$ & $3.000 \mathrm{E}+01$ & $\operatorname{BIOFA}(4,1)$ \\
\hline $\mathrm{TF}$ & $\mathrm{Np}-237+\mathrm{D}$ & , crustacea and mollusks & $4.000 \mathrm{E}+02$ & $4.000 \mathrm{E}+02$ & $\operatorname{BIOFA}(4,2)$ \\
\hline $\mathrm{TF}$ & & & & & \\
\hline $\mathrm{TF}$ & $\mathrm{Pa}-231$ & , fish & $1.000 \mathrm{E}+01$ & $1.000 \mathrm{E}+01$ & $\operatorname{BIOFA}(5,1)$ \\
\hline $\mathrm{TF}$ & $\mathrm{Pa}-231$ & , crustacea and mollusks & $1.100 \mathrm{E}+02$ & $1.100 \mathrm{E}+02$ & $\operatorname{BIOFA}(5,2)$ \\
\hline $\mathrm{TF}$ & & & & & \\
\hline $\mathrm{TF}$ & $\mathrm{Pb}-210+\mathrm{D}$ & , fish & $3.000 \mathrm{E}+02$ & $3.000 \mathrm{E}+02$ & $\operatorname{BIOFA}(6,1)$ \\
\hline $\mathrm{TF}$ & $\mathrm{Pb}-210+\mathrm{D}$ & , crustacea and mollusks & $1.000 \mathrm{E}+02$ & $1.000 \mathrm{E}+02$ & $\operatorname{BIOFA}(6,2)$ \\
\hline $\mathrm{TF}$ & & & & & \\
\hline $\mathrm{TF}$ & Po-210 & , fish & $1.000 \mathrm{E}+02$ & $1.000 \mathrm{E}+02$ & $\operatorname{BIOFA}(7,1)$ \\
\hline $\mathrm{TF}$ & Po-210 & , crustacea and mollusks & $2.000 \mathrm{E}+04$ & $2.000 \mathrm{E}+04$ & $\operatorname{BIOFA}(7,2)$ \\
\hline $\mathrm{TF}$ & & & & & \\
\hline $\mathrm{TF}$ & $\mathrm{Pu}-238$ & , fish & $3.000 \mathrm{E}+01$ & $3.000 \mathrm{E}+01$ & $\operatorname{BIOFA}(8,1)$ \\
\hline $\mathrm{TF}$ & $\mathrm{Pu}-238$ & , crustacea and mollusks & $1.000 \mathrm{E}+02$ & $1.000 \mathrm{E}+02$ & $\operatorname{BIOFA}(8,2)$ \\
\hline $\mathrm{TF}$ & & & & & \\
\hline $\mathrm{TF}$ & $\mathrm{Pu}-239$ & , fish & $3.000 \mathrm{E}+01$ & $3.000 \mathrm{E}+01$ & $\operatorname{BIOFA}(10,1)$ \\
\hline $\mathrm{TF}$ & $\mathrm{Pu}-239$ & , crustacea and mollusks & $1.000 \mathrm{E}+02$ & $1.000 \mathrm{E}+02$ & $\operatorname{BIOFA}(10,2)$ \\
\hline $\mathrm{TF}$ & & & & & \\
\hline $\mathrm{TF}$ & $\mathrm{Pu}-240$ & , fish & $3.000 \mathrm{E}+01$ & $3.000 \mathrm{E}+01$ & $\operatorname{BIOFA}(11,1)$ \\
\hline $\mathrm{TF}$ & $\mathrm{Pu}-240$ & , crustacea and mollusks & $1.000 \mathrm{E}+02$ & $1.000 \mathrm{E}+02$ & $\operatorname{BIOFA}(11,2)$ \\
\hline $\mathrm{TF}$ & & & & & \\
\hline $\mathrm{TF}$ & $\mathrm{Ra}-226+\mathrm{D}$ & , fish & $5.000 \mathrm{E}+01$ & $5.000 \mathrm{E}+01$ & $\operatorname{BIOFA}(13,1)$ \\
\hline $\mathrm{TF}$ & $\mathrm{Ra}-226+\mathrm{D}$ & , crustacea and mollusks & $2.500 \mathrm{E}+02$ & $2.500 \mathrm{E}+02$ & $\operatorname{BIOFA}(13,2)$ \\
\hline $\mathrm{TF}$ & & & & & \\
\hline
\end{tabular}




\begin{tabular}{|c|c|c|c|c|c|}
\hline TF & $\mathrm{Ra}-228+\mathrm{D}$ & , fish & $5.000 \mathrm{E}+01$ & $5.000 \mathrm{E}+01$ & $\operatorname{BIOFA}(14,1)$ \\
\hline TF & $\mathrm{Ra}-228+\mathrm{D}$ & , crustacea and mollusks & $2.500 \mathrm{E}+02$ & $2.500 \mathrm{E}+02$ & $\operatorname{BIOFA}(14,2)$ \\
\hline $\begin{array}{l}\mathrm{TF} \\
\mathrm{TF}\end{array}$ & $T_{C}-99$ & fish & $2.000 \mathrm{E}+01$ & $2.000 \mathrm{E}+01$ & $\operatorname{BIOFA}(15,1)$ \\
\hline $\mathrm{TF}$ & Tc-99 & crustacea and mollusks & $5.000 \mathrm{E}+00$ & $5.000 \mathrm{E}+00$ & $\operatorname{BIOFA}(15,2)$ \\
\hline 1RESRAI & D-OFFSITE, & Version 4.0 .3 beta $T^{1 / 2}$ Limit $=30$ days & $/ 2020 \quad 11: 39$ & Page 10 & \\
\hline Parent & t Dose Repc & & & & \\
\hline Title & : RESRAD-C & DFFSITE Effluent release & & & \\
\hline File & : PADUCAH & EFFLUENT RELEASE-NONINVOLVED-WORKER-REV.ROF & & & \\
\hline & & Dose Conversion Factor (and Related) Parameter Su & ummary (cont: & inued) & \\
\hline & & Current Library: RESRAD Default Transfer & r factors & & \\
\hline & & Default Library: RESRAD Default Transfer & $r$ factors & & \\
\hline 0 & & & Current & & Parameter \\
\hline Menu & & Parameter & Value & Default & Name \\
\hline $\mathrm{TF}$ & Th $-228+D$ & , fish & $1.000 \mathrm{E}+02$ & $1.000 \mathrm{E}+02$ & $\operatorname{BIOFA}(16,1)$ \\
\hline $\mathrm{TF}$ & Th $-228+D$ & , crustacea and mollusks & $5.000 \mathrm{E}+02$ & $5.000 \mathrm{E}+02$ & $\operatorname{BIOFA}(16,2)$ \\
\hline TF & & & & & \\
\hline $\mathrm{TF}$ & Th $-229+D$ & , fish & $1.000 \mathrm{E}+02$ & $1.000 \mathrm{E}+02$ & $\operatorname{BIOFA}(17,1)$ \\
\hline TF & Th $-229+D$ & , crustacea and mollusks & $5.000 \mathrm{E}+02$ & $5.000 \mathrm{E}+02$ & $\operatorname{BIOFA}(17,2)$ \\
\hline $\mathrm{TF}$ & & & & & \\
\hline $\mathrm{TF}$ & Th-230 & , fish & $1.000 \mathrm{E}+02$ & $1.000 \mathrm{E}+02$ & $\operatorname{BIOFA}(18,1)$ \\
\hline $\mathrm{TF}$ & Th-230 & , crustacea and mollusks & $5.000 \mathrm{E}+02$ & $5.000 \mathrm{E}+02$ & $\operatorname{BIOFA}(18,2)$ \\
\hline $\mathrm{TF}$ & & & & & \\
\hline $\mathrm{TF}$ & Th-232 & , fish & $1.000 \mathrm{E}+02$ & $1.000 \mathrm{E}+02$ & $\operatorname{BIOFA}(19,1)$ \\
\hline $\mathrm{TF}$ & Th-232 & , crustacea and mollusks & $5.000 \mathrm{E}+02$ & $5.000 \mathrm{E}+02$ & $\operatorname{BIOFA}(19,2)$ \\
\hline TF & & & & & \\
\hline$T F$ & $\mathrm{U}-233$ & , fish & $1.000 \mathrm{E}+01$ & $1.000 \mathrm{E}+01$ & $\operatorname{BIOFA}(20,1)$ \\
\hline TF & $\mathrm{U}-233$ & , crustacea and mollusks & $6.000 \mathrm{E}+01$ & $6.000 \mathrm{E}+01$ & $\operatorname{BIOFA}(20,2)$ \\
\hline$T F$ & & & & & \\
\hline $\mathrm{TF}$ & $\mathrm{U}-234$ & , fish & $1.000 \mathrm{E}+01$ & $1.000 \mathrm{E}+01$ & $\operatorname{BIOFA}(21,1)$ \\
\hline$T F$ & $\mathrm{U}-234$ & , crustacea and mollusks & $6.000 \mathrm{E}+01$ & $6.000 \mathrm{E}+01$ & $\operatorname{BIOFA}(21,2)$ \\
\hline $\mathrm{TF}$ & & & & & \\
\hline $\mathrm{TF}$ & $U-235+D$ & , fish & $1.000 \mathrm{E}+01$ & $1.000 \mathrm{E}+01$ & $\operatorname{BIOFA}(22,1)$ \\
\hline $\mathrm{TF}$ & $\mathrm{U}-235+\mathrm{D}$ & , crustacea and mollusks & $6.000 \mathrm{E}+01$ & $6.000 \mathrm{E}+01$ & $\operatorname{BIOFA}(22,2)$ \\
\hline$T F$ & & & & & \\
\hline $\mathrm{TF}$ & $\mathrm{U}-236$ & , fish & $1.000 \mathrm{E}+01$ & $1.000 \mathrm{E}+01$ & $\operatorname{BIOFA}(23,1)$ \\
\hline $\mathrm{TF}$ & $\mathrm{U}-236$ & , crustacea and mollusks & $6.000 \mathrm{E}+01$ & $6.000 \mathrm{E}+01$ & $\operatorname{BIOFA}(23,2)$ \\
\hline $\mathrm{TF}$ & & & & & \\
\hline $\mathrm{TF}$ & $\mathrm{U}-238$ & , fish & $1.000 \mathrm{E}+01$ & $1.000 \mathrm{E}+01$ & $\operatorname{BIOFA}(24,1)$ \\
\hline $\mathrm{TF}$ & $\mathrm{U}-238$ & , crustacea and mollusks & $6.000 \mathrm{E}+01$ & $6.000 \mathrm{E}+01$ & $\operatorname{BIOFA}(24,2)$ \\
\hline $\mathrm{TF}$ & & & & & \\
\hline$T F$ & $U-238+D$ & , fish & $1.000 \mathrm{E}+01$ & $1.000 \mathrm{E}+01$ & $\operatorname{BIOFA}(25,1)$ \\
\hline $\mathrm{TF}$ & $U-238+D$ & , crustacea and mollusks & $6.000 \mathrm{E}+01$ & $6.000 \mathrm{E}+01$ & $\operatorname{BIOFA}(25,2)$ \\
\hline
\end{tabular}

1RESRAD-OFFSITE, Version 4.0 .3 beta $T^{1 / 2}$ Limit $=30$ days Parent Dose Report

06/11/2020 11:39 Page 11

Title : RESRAD-OFFSITE Effluent release

File : PADUCAH EFFLUENT RELEASE-NONINVOLVED-WORKER-REV.ROF 


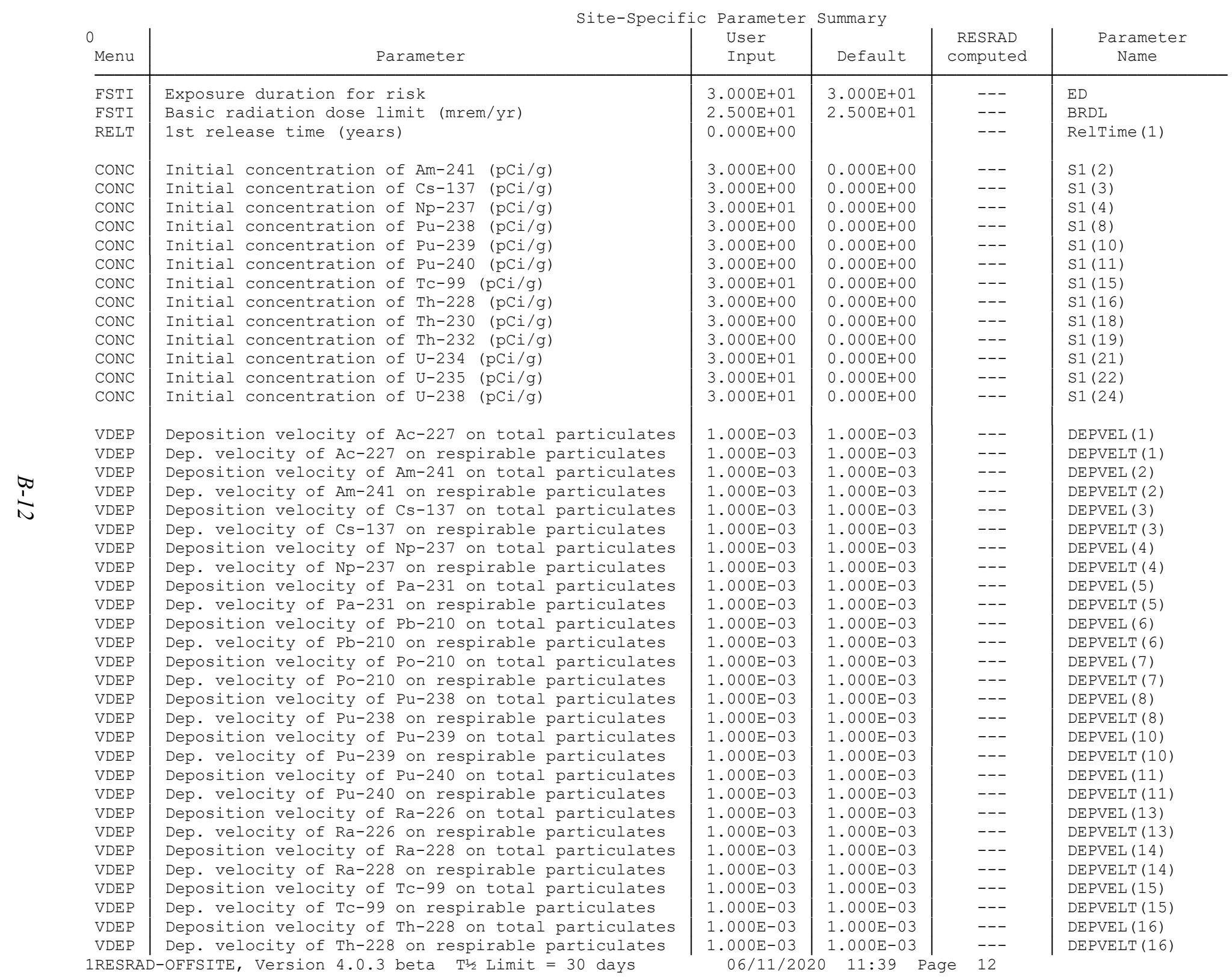


Parent Dose Report

Title : RESRAD-OFFSITE Effluent release

File : PADUCAH EFFLUENT RELEASE-NONINVOLVED-WORKER-REV.ROF

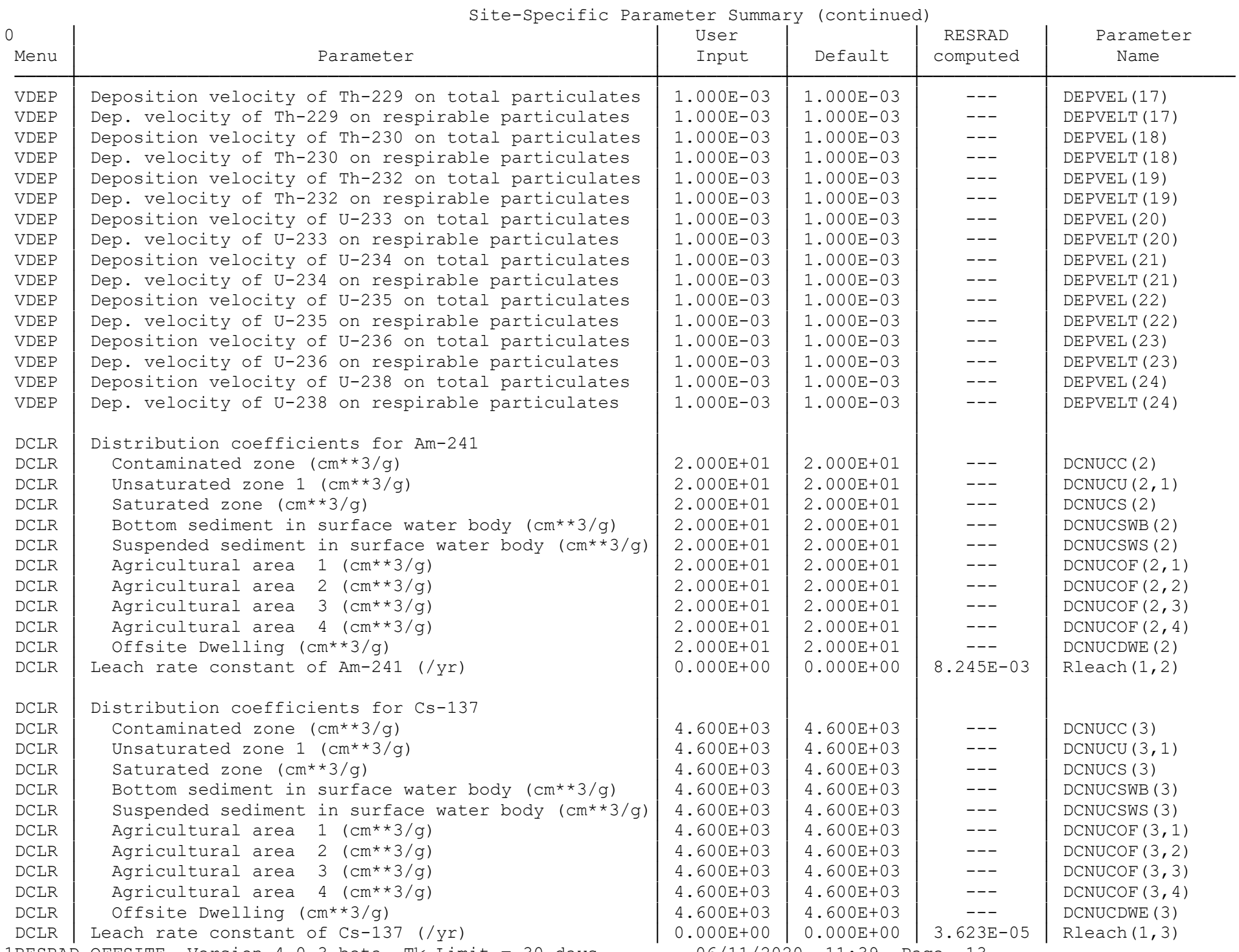

1RESRAD-OFFSITE, Version 4.0 .3 beta $T^{1} / 2$ Limit $=30$ days

06/11/2020 11:39 Page 13 
Parent Dose Report

Title : RESRAD-OFFSITE Effluent release

File : PADUCAH EFFLUENT RELEASE-NONINVOLVED-WORKER-REV.ROF

\begin{tabular}{|c|c|c|c|c|c|}
\hline Menu & Site-Specific Para & $\begin{array}{l}\text { eter Summa } \\
\text { User } \\
\text { Input }\end{array}$ & $\begin{array}{l}\text { (continue } \\
\text { Default }\end{array}$ & $\begin{array}{l}\text { RESRAD } \\
\text { computed }\end{array}$ & $\begin{array}{l}\text { Parameter } \\
\text { Name }\end{array}$ \\
\hline DCLR & Distribution coefficients for Np-237 & & & & \\
\hline DCLR & Unsaturated zone $1(\mathrm{~cm} * * 3 / \mathrm{g})$ & $2.570 \mathrm{E}+02$ & $2.570 \mathrm{E}+02$ & --- & $\operatorname{DCNUCU}(4,1)$ \\
\hline DCLR & Saturated zone $(\mathrm{cm} * \star 3 / \mathrm{g})$ & $2.570 \mathrm{E}+02$ & $2.570 \mathrm{E}+02$ & --- & DCNUCS (4) \\
\hline DCLR & Bottom sediment in surface water body $(\mathrm{cm} * * 3 / \mathrm{g})$ & $2.570 \mathrm{E}+02$ & $2.570 \mathrm{E}+02$ & --- & DCNUCSWB (4) \\
\hline DCLR & Suspended sediment in surface water body $(\mathrm{cm} * * 3 / \mathrm{g})$ & $2.570 \mathrm{E}+02$ & $2.570 \mathrm{E}+02$ & --- & DCNUCSWS (4) \\
\hline DCLR & Agricultural area $3(\mathrm{~cm} * * 3 / \mathrm{g})$ & $2.570 \mathrm{E}+02$ & $2.570 \mathrm{E}+02$ & --- & $\operatorname{DCNUCOF}(4,3)$ \\
\hline DCLR & Agricultural area $4(\mathrm{~cm} * * 3 / \mathrm{g})$ & $2.570 \mathrm{E}+02$ & $2.570 \mathrm{E}+02$ & --- & $\operatorname{DCNUCOF}(4,4)$ \\
\hline DCLR & Offsite Dwelling $(\mathrm{cm} * * 3 / \mathrm{g})$ & $2.570 \mathrm{E}+02$ & $2.570 \mathrm{E}+02$ & --- & DCNUCDWE (4) \\
\hline DCLR & Leach rate constant of $\mathrm{Np}-237$ (/yr) & $0.000 \mathrm{E}+00$ & $0.000 \mathrm{E}+00$ & $6.480 \mathrm{E}-04$ & Rleach $(1,4)$ \\
\hline DCLR & Distribution coefficients for Pu-238 & & & & \\
\hline DCLR & Contaminated zone $(\mathrm{cm} * * 3 / \mathrm{g})$ & $2.000 \mathrm{E}+03$ & $2.000 \mathrm{E}+03$ & --- & DCNUCC (8) \\
\hline DCLR & Agricultural area $2(\mathrm{~cm} * * 3 / \mathrm{g})$ & $2.000 \mathrm{E}+03$ & $2.000 \mathrm{E}+03$ & --- & $\operatorname{DCNUCOF}(8,2)$ \\
\hline DCLR & Agricultural area $3(\mathrm{~cm} * * 3 / \mathrm{g})$ & $2.000 \mathrm{E}+03$ & $2.000 \mathrm{E}+03$ & --- & $\operatorname{DCNUCOF}(8,3)$ \\
\hline DCLR & Agricultural area $4(\mathrm{~cm} * * 3 / \mathrm{g})$ & $2.000 \mathrm{E}+03$ & $2.000 \mathrm{E}+03$ & --- & $\operatorname{DCNUCOF}(8,4)$ \\
\hline DCLR & Offsite Dwelling $\left(\mathrm{cm}^{*} * 3 / \mathrm{g}\right)$ & $2.000 \mathrm{E}+03$ & $2.000 \mathrm{E}+03$ & --- & DCNUCDWE ( 8) \\
\hline DCLR & Leach rate constant of $\mathrm{Pu}-238$ (/yr) & $0.000 \mathrm{E}+00$ & $0.000 \mathrm{E}+00$ & $8.332 E-05$ & Rleach $(1,8)$ \\
\hline DCLR & Distribution coefficients for Pu-239 & & & & \\
\hline DCLR & Contaminated zone $(\mathrm{cm} * * 3 / \mathrm{g})$ & $2.000 \mathrm{E}+03$ & $2.000 \mathrm{E}+03$ & --- & DCNUCC (10) \\
\hline DCLR & Unsaturated zone $1(\mathrm{~cm} * * 3 / \mathrm{g})$ & $2.000 \mathrm{E}+03$ & $2.000 \mathrm{E}+03$ & --- & $\operatorname{DCNUCU}(10,1)$ \\
\hline DCLR & Saturated zone $(\mathrm{cm} * * 3 / \mathrm{g})$ & $2.000 \mathrm{E}+03$ & $2.000 \mathrm{E}+03$ & --- & DCNUCS (10) \\
\hline DCLR & Bottom sediment in surface water body $(\mathrm{cm} * * 3 / \mathrm{g})$ & $2.000 \mathrm{E}+03$ & $2.000 \mathrm{E}+03$ & --- & DCNUCSWB (10) \\
\hline DCLR & Suspended sediment in surface water body $(\mathrm{cm} * * 3 / \mathrm{g})$ & $2.000 \mathrm{E}+03$ & $2.000 \mathrm{E}+03$ & --- & DCNUCSWS (10) \\
\hline DCLR & Agricultural area $1(\mathrm{~cm} * * 3 / \mathrm{g})$ & $2.000 \mathrm{E}+03$ & $2.000 \mathrm{E}+03$ & --- & $\operatorname{DCNUCOF}(10,1)$ \\
\hline DCLR & Agricultural area $2(\mathrm{~cm} * * 3 / \mathrm{g})$ & $2.000 \mathrm{E}+03$ & $2.000 \mathrm{E}+03$ & --- & $\operatorname{DCNUCOF}(10,2)$ \\
\hline
\end{tabular}

Parent Dose Report

Title : RESRAD-OFFSITE Effluent release

File : PADUCAH EFFLUENT RELEASE-NONINVOLVED-WORKER-REV.ROF 


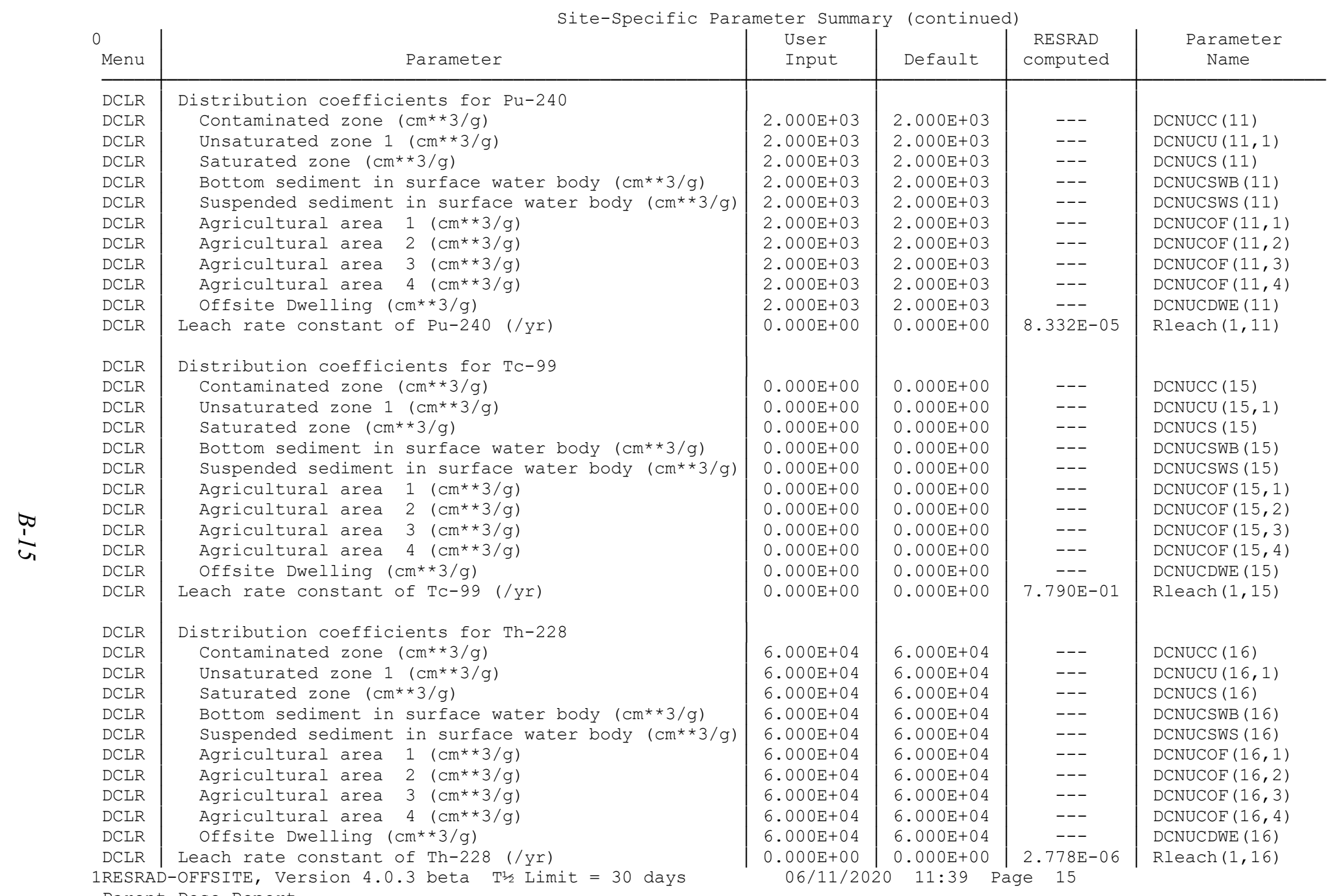

RESRAD-OFFSITE, Version 4.0 .3 beta $T^{1} \frac{1}{2}$ Limit $=30$ days

Title: RESRAD-OFFSITE Effluent release

File : PADUCAH EFFLUENT RELEASE-NONINVOLVED-WORKER-REV.ROF

\begin{tabular}{c|c|c|c|c|c}
0 & \multicolumn{3}{|c}{ Site-Specific Parameter Summary (continued) } \\
Menu & Parameter & $\begin{array}{l}\text { User } \\
\text { Input }\end{array}$ & Default & $\begin{array}{c}\text { RESRAD } \\
\text { computed }\end{array}$ \\
\hline
\end{tabular}




\begin{tabular}{|c|c|c|c|c|c|}
\hline DCLR & Distribution coefficients for Th-230 & & & & \\
\hline DCLR & Contaminated zone $(\mathrm{cm} * * 3 / \mathrm{g})$ & $6.000 \mathrm{E}+04$ & $6.000 \mathrm{E}+04$ & --- & DCNUCC (18) \\
\hline DCLR & Unsaturated zone $1(\mathrm{~cm} * * 3 / \mathrm{g})$ & $6.000 \mathrm{E}+04$ & $6.000 \mathrm{E}+04$ & --- & $\operatorname{DCNUCU}(18,1)$ \\
\hline DCLR & Saturated zone $(\mathrm{cm} * * 3 / \mathrm{g})$ & $6.000 \mathrm{E}+04$ & $6.000 \mathrm{E}+04$ & --- & DCNUCS (18) \\
\hline DCLR & Bottom sediment in surface water body $(\mathrm{cm} * * 3 / \mathrm{g})$ & $6.000 \mathrm{E}+04$ & $6.000 \mathrm{E}+04$ & --- & DCNUCSWB (18) \\
\hline DCLR & Suspended sediment in surface water body $(\mathrm{cm} * * 3 / \mathrm{g})$ & $6.000 \mathrm{E}+04$ & $6.000 \mathrm{E}+04$ & --- & DCNUCSWS (18) \\
\hline DCLR & Agricultural area $1(\mathrm{~cm} * * 3 / \mathrm{g})$ & $6.000 \mathrm{E}+04$ & $6.000 \mathrm{E}+04$ & --- & $\operatorname{DCNUCOF}(18,1)$ \\
\hline DCLR & Agricultural area $2(\mathrm{~cm} * * 3 / \mathrm{g})$ & $6.000 \mathrm{E}+04$ & $6.000 \mathrm{E}+04$ & --- & $\operatorname{DCNUCOF}(18,2)$ \\
\hline DCLR & Agricultural area $3(\mathrm{~cm} * * 3 / \mathrm{g})$ & $6.000 \mathrm{E}+04$ & $6.000 \mathrm{E}+04$ & --- & $\operatorname{DCNUCOF}(18,3)$ \\
\hline DCLR & Agricultural area $4(\mathrm{~cm} * * 3 / \mathrm{g})$ & $6.000 \mathrm{E}+04$ & $6.000 \mathrm{E}+04$ & --- & $\operatorname{DCNUCOF}(18,4)$ \\
\hline DCLR & Offsite Dwelling $\left(\mathrm{cm}^{* * 3 / \mathrm{g})}\right.$ & $6.000 \mathrm{E}+04$ & $6.000 E+04$ & --- & DCNUCDWE (18) \\
\hline DCLR & Leach rate constant of $\mathrm{Th}-230$ (/yr) & $0.000 \mathrm{E}+00$ & $0.000 \mathrm{E}+00$ & $2.778 \mathrm{E}-06$ & Rleach $(1,18)$ \\
\hline DCLR & Distribution coefficients for Th-232 & & & & \\
\hline DCLR & Contaminated zone $(\mathrm{cm} * * 3 / \mathrm{g})$ & $6.000 \mathrm{E}+04$ & $6.000 \mathrm{E}+04$ & --- & $\operatorname{DCNUCC}(19)$ \\
\hline DCLR & Unsaturated zone $1(\mathrm{~cm} * * 3 / \mathrm{g})$ & $6.000 \mathrm{E}+04$ & $6.000 \mathrm{E}+04$ & --- & $\operatorname{DCNUCU}(19,1)$ \\
\hline DCLR & Saturated zone $(\mathrm{cm} * * 3 / \mathrm{g})$ & $6.000 \mathrm{E}+04$ & $6.000 \mathrm{E}+04$ & --- & DCNUCS (19) \\
\hline DCLR & Bottom sediment in surface water body $(\mathrm{cm} * * 3 / \mathrm{g})$ & $6.000 \mathrm{E}+04$ & $6.000 \mathrm{E}+04$ & --- & DCNUCSWB (19) \\
\hline DCLR & Suspended sediment in surface water body $(\mathrm{cm} * * 3 / \mathrm{g})$ & $6.000 \mathrm{E}+04$ & $6.000 \mathrm{E}+04$ & --- & DCNUCSWS (19) \\
\hline DCLR & Agricultural area $1(\mathrm{~cm} * * 3 / \mathrm{g})$ & $6.000 \mathrm{E}+04$ & $6.000 \mathrm{E}+04$ & --- & $\operatorname{DCNUCOF}(19,1)$ \\
\hline DCLR & Agricultural area $2(\mathrm{~cm} * * 3 / \mathrm{g})$ & $6.000 \mathrm{E}+04$ & $6.000 \mathrm{E}+04$ & --- & $\operatorname{DCNUCOF}(19,2)$ \\
\hline DCLR & Agricultural area $3(\mathrm{~cm} * * 3 / \mathrm{g})$ & $6.000 \mathrm{E}+04$ & $6.000 \mathrm{E}+04$ & --- & $\operatorname{DCNUCOF}(19,3)$ \\
\hline DCLR & Agricultural area $4(\mathrm{~cm} * * 3 / \mathrm{g})$ & $6.000 \mathrm{E}+04$ & $6.000 \mathrm{E}+04$ & --- & $\operatorname{DCNUCOF}(19,4)$ \\
\hline DCLR & Offsite Dwelling $\left(\mathrm{cm}^{* * 3 / \mathrm{g})}\right.$ & $6.000 \mathrm{E}+04$ & $6.000 E+04$ & --- & DCNUCDWE (19) \\
\hline DCLR & Leach rate constant of Th-232 (/yr) & $0.000 \mathrm{E}+00$ & $0.000 \mathrm{E}+00$ & $2.778 \mathrm{E}-06$ & Rleach $(1,19)$ \\
\hline DCLR & Distribution coefficients for U-234 & & & & \\
\hline DCLR & Contaminated zone $(\mathrm{cm} * * 3 / \mathrm{g})$ & $5.000 \mathrm{E}+01$ & $5.000 \mathrm{E}+01$ & --- & DCNUCC (21) \\
\hline DCLR & Unsaturated zone $1(\mathrm{~cm} * * 3 / \mathrm{g})$ & $5.000 \mathrm{E}+01$ & $5.000 \mathrm{E}+01$ & --- & $\operatorname{DCNUCU}(21,1)$ \\
\hline DCLR & Saturated zone $(\mathrm{cm} * * 3 / \mathrm{g})$ & $5.000 \mathrm{E}+01$ & $5.000 \mathrm{E}+01$ & --- & DCNUCS (21) \\
\hline DCLR & Bottom sediment in surface water body $(\mathrm{cm} * * 3 / \mathrm{g})$ & $5.000 \mathrm{E}+01$ & $5.000 \mathrm{E}+01$ & --- & DCNUCSWB (21) \\
\hline DCLR & Suspended sediment in surface water body $(\mathrm{cm} * * 3 / \mathrm{g})$ & $5.000 \mathrm{E}+01$ & $5.000 \mathrm{E}+01$ & --- & DCNUCSWS $(21)$ \\
\hline DCLR & Agricultural area $1(\mathrm{~cm} * * 3 / \mathrm{g})$ & $5.000 \mathrm{E}+01$ & $5.000 \mathrm{E}+01$ & --- & $\operatorname{DCNUCOF}(21,1)$ \\
\hline DCLR & Agricultural area $2(\mathrm{~cm} * * 3 / \mathrm{g})$ & $5.000 \mathrm{E}+01$ & $5.000 \mathrm{E}+01$ & --- & $\operatorname{DCNUCOF}(21,2)$ \\
\hline DCLR & Agricultural area $3(\mathrm{~cm} * * 3 / \mathrm{g})$ & $5.000 \mathrm{E}+01$ & $5.000 \mathrm{E}+01$ & --- & $\operatorname{DCNUCOF}(21,3)$ \\
\hline DCLR & Agricultural area $4(\mathrm{~cm} * * 3 / \mathrm{g})$ & $5.000 \mathrm{E}+01$ & $5.000 \mathrm{E}+01$ & --- & $\operatorname{DCNUCOF}(21,4)$ \\
\hline DCLR & Offsite Dwelling $\left(\mathrm{cm}^{* * 3 / \mathrm{g})}\right.$ & $5.000 \mathrm{E}+01$ & $5.000 \mathrm{E}+01$ & --- & DCNUCDWE (21) \\
\hline DCLR & Leach rate constant of $\mathrm{U}-234$ (/yr) & $0.000 \mathrm{E}+00$ & $0.000 \mathrm{E}+00$ & $3.319 \mathrm{E}-03$ & Rleach $(1,21)$ \\
\hline Dente & OFFSITE, Version 4.0 .3 beta $T^{1 / 2}$ Limit $=30$ days & $06 / 11 / 2$ & $0 \quad 11: 39$ & ge 16 & \\
\hline
\end{tabular}

Parent Dose Report

Title : RESRAD-OFFSITE Effluent release

File : PADUCAH EFFLUENT RELEASE-NONINVOLVED-WORKER-REV.ROF

\begin{tabular}{|c|c|c|c|c|c|}
\hline $\begin{array}{l}0 \\
\text { Menu }\end{array}$ & Parameter & $\begin{array}{l}\text { neter Summà } \\
\text { User } \\
\text { Input }\end{array}$ & $\begin{array}{l}\text { (continue } \\
\text { Default }\end{array}$ & $\begin{array}{l}\text { RESRAD } \\
\text { computed }\end{array}$ & $\begin{array}{c}\text { Parameter } \\
\text { Name }\end{array}$ \\
\hline $\begin{array}{l}\text { DCLR } \\
\text { DCLR } \\
\text { DCLR } \\
\text { DCLR }\end{array}$ & $\begin{array}{l}\text { Distribution coefficients for U-235 } \\
\text { Contaminated zone }(\mathrm{cm} * * 3 / \mathrm{g}) \\
\text { Unsaturated zone } 1(\mathrm{~cm} * * 3 / \mathrm{g}) \\
\text { Saturated zone }(\mathrm{cm} * * 3 / \mathrm{g})\end{array}$ & $\begin{array}{l}5.000 \mathrm{E}+01 \\
5.000 \mathrm{E}+01 \\
5.000 \mathrm{E}+01\end{array}$ & $\begin{array}{l}5.000 \mathrm{E}+01 \\
5.000 \mathrm{E}+01 \\
5.000 \mathrm{E}+01\end{array}$ & $\begin{array}{l}--- \\
--- \\
---\end{array}$ & $\begin{array}{l}\operatorname{DCNUCC}(22) \\
\operatorname{DCNUCU}(22,1) \\
\operatorname{DCNUCS}(22)\end{array}$ \\
\hline
\end{tabular}


\begin{tabular}{l|l} 
DCLR & Bottom sediment in surface water body $(\mathrm{cm} * * 3 / \mathrm{g})$ \\
DCLR & Suspended sediment in surface water body $(\mathrm{cm} * * 3 / \mathrm{g})$
\end{tabular}

DCLR

DCLR

DCLR

DCLR

DCTR

DCLR

DCLR

DCLR

DCLR
DCLR

DCLR

DCLR

DCLR

DCLR

DCLR

DCLR

DCLR

DCLR

DCLR

DCLR

DCLR

DCLR

DCLR

DCLR

DCLR

DCLR

Leach rate constant of Ac-227 (/yr)

1RESRAD-OFFSITE, Version 4.0 .3 beta $T \frac{1}{2}$ Limit $=30$ days

Agricultural area 1

Agricultural area $2(\mathrm{~cm} * * 3 / \mathrm{g})$

Agricultural area $3(\mathrm{~cm} * * 3 / \mathrm{g})$

Agricultural area $4(\mathrm{~cm} * * 3 / \mathrm{g})$

Offsite Dwelling $\left(\mathrm{cm}^{* * 3 / \mathrm{g})}\right.$

Leach rate constant of $\mathrm{U}-235$ (/yr)

Distribution coefficients for $\mathrm{U}-238$

Contaminated zone $\left(\mathrm{cm}^{* * 3 / \mathrm{g})}\right.$

Unsaturated zone $1(\mathrm{~cm} * * 3 / \mathrm{g})$

Saturated zone $(\mathrm{cm} * * 3 / \mathrm{g})$

(

uspended sediment in surface water body $\left(\mathrm{cm}^{* * 3 / \mathrm{g})}\right.$

gricultural area $1(\mathrm{~cm} * * 3 / \mathrm{g})$

Aricultural area $2\left(\mathrm{~cm}^{* * 3 / \mathrm{g})}\right.$

Offsite Dwelling $(\mathrm{cm} * * 3 / \mathrm{g})$

Leach rate constant of U-238 (/yr)

Distribution coefficients for progeny Ac-227

Contaminated zone $\left(\mathrm{cm}^{* * 3 / \mathrm{g})}\right.$

Nsaturated zone $1(\mathrm{~cm} * * 3 / \mathrm{g}$

Bottom sediment in surface water body $(\mathrm{cm} * * 3 / \mathrm{g})$

Suspended sediment in surface water body $(\mathrm{cm} * * 3 / \mathrm{g})$

Agricultural area $1(\mathrm{~cm} * * 3 / \mathrm{g})$

Agricultural area $2(\mathrm{~cm} * * 3 / \mathrm{g})$

Agricultural area $3(\mathrm{~cm} * * 3 / \mathrm{g})$

(

Parent Dose Report

Title : RESRAD-OFFSITE Effluent release

File : PADUCAH EFFLUENT RELEASE-NONINVOLVED-WORKER-REV.ROF

\begin{tabular}{|c|c|c|c|c|c|}
\hline $\begin{array}{l}0 \\
\text { Menu }\end{array}$ & Site-Specific Para & $\begin{array}{l}\text { neter Summ } \\
\text { User } \\
\text { Input }\end{array}$ & $\begin{array}{l}\text { y continue } \\
\text { Default }\end{array}$ & $\begin{array}{l}\text { RESRAD } \\
\text { computed }\end{array}$ & $\begin{array}{l}\text { Parameter } \\
\text { Name }\end{array}$ \\
\hline DCLR & Distribution coefficients for progeny $\mathrm{Pa}-231$ & & & & \\
\hline DCLR & Unsaturated zone $1(\mathrm{~cm} * * 3 / \mathrm{g})$ & $5.000 \mathrm{E}+01$ & $5.000 \mathrm{E}+01$ & --- & $\operatorname{DCNUCU}(5,1)$ \\
\hline DCLR & Saturated zone $(\mathrm{cm} * * 3 / \mathrm{g})$ & $5.000 \mathrm{E}+01$ & $5.000 \mathrm{E}+01$ & --- & DCNUCS (5) \\
\hline DCLR & Bottom sediment in surface water body $(\mathrm{cm} * * 3 / \mathrm{g})$ & $5.000 \mathrm{E}+01$ & $5.000 \mathrm{E}+01$ & --- & DCNUCSWB (5) \\
\hline DCLR & Suspended sediment in surface water body $(\mathrm{cm} * * 3 / \mathrm{g})$ & $5.000 \mathrm{E}+01$ & $5.000 \mathrm{E}+01$ & --- & DCNUCSWS (5) \\
\hline
\end{tabular}




\begin{tabular}{|c|c|c|c|c|c|c|}
\hline & DCLR & Agricultural area $3(\mathrm{~cm} * * 3 / \mathrm{g})$ & $5.000 \mathrm{E}+01$ & $5.000 \mathrm{E}+01$ & --- & $\operatorname{DCNUCOF}(5,3)$ \\
\hline & DCLR & Agricultural area $4(\mathrm{~cm} * * 3 / \mathrm{g})$ & $5.000 \mathrm{E}+01$ & $5.000 \mathrm{E}+01$ & --- & $\operatorname{DCNUCOF}(5,4)$ \\
\hline & DCLR & Offsite Dwelling $\left(\mathrm{cm}^{* * 3 / \mathrm{g})}\right.$ & $5.000 \mathrm{E}+01$ & $5.000 \mathrm{E}+01$ & --- & DCNUCDWE (5) \\
\hline & DCLR & Leach rate constant of $\mathrm{Pa}-231$ (/yr) & $0.000 \mathrm{E}+00$ & $0.000 \mathrm{E}+00$ & $3.319 \mathrm{E}-03$ & Rleach $(1,5)$ \\
\hline & DCLR & Distribution coefficients for progeny $\mathrm{Pb}-210$ & & & & \\
\hline & DCLR & Contaminated zone $(\mathrm{cm} * * 3 / \mathrm{g})$ & $1.000 \mathrm{E}+02$ & $1.000 \mathrm{E}+02$ & --- & $\operatorname{DCNUCC}(6)$ \\
\hline & DCLR & Unsaturated zone $1(\mathrm{~cm} * * 3 / \mathrm{g})$ & $1.000 \mathrm{E}+02$ & $1.000 \mathrm{E}+02$ & --- & $\operatorname{DCNUCU}(6,1)$ \\
\hline & DCLR & Saturated zone $(\mathrm{cm} * * 3 / \mathrm{g})$ & $1.000 \mathrm{E}+02$ & $1.000 \mathrm{E}+02$ & --- & DCNUCS (6) \\
\hline & DCLR & Bottom sediment in surface water body $(\mathrm{cm} * * 3 / \mathrm{g})$ & $1.000 \mathrm{E}+02$ & $1.000 \mathrm{E}+02$ & --- & DCNUCSWB (6) \\
\hline & DCLR & Suspended sediment in surface water body $\left(\mathrm{cm}^{* * 3} / \mathrm{g}\right)$ & $1.000 \mathrm{E}+02$ & $1.000 \mathrm{E}+02$ & --- & DCNUCSWS $(6)$ \\
\hline & DCLR & Agricultural area $1(\mathrm{~cm} * * 3 / \mathrm{g})$ & $1.000 \mathrm{E}+02$ & $1.000 \mathrm{E}+02$ & --- & $\operatorname{DCNUCOF}(6,1)$ \\
\hline & DCLR & Agricultural area $2(\mathrm{~cm} * * 3 / \mathrm{g})$ & $1.000 \mathrm{E}+02$ & $1.000 \mathrm{E}+02$ & --- & $\operatorname{DCNUCOF}(6,2)$ \\
\hline & DCLR & Agricultural area $3(\mathrm{~cm} * * 3 / \mathrm{g})$ & $1.000 \mathrm{E}+02$ & $1.000 \mathrm{E}+02$ & --- & $\operatorname{DCNUCOF}(6,3)$ \\
\hline & DCLR & Agricultural area $4(\mathrm{~cm} * * 3 / \mathrm{g})$ & $1.000 \mathrm{E}+02$ & $1.000 \mathrm{E}+02$ & --- & $\operatorname{DCNUCOF}(6,4)$ \\
\hline & DCLR & Offsite Dwelling $(\mathrm{cm} * * 3 / \mathrm{g})$ & $1.000 \mathrm{E}+02$ & $1.000 \mathrm{E}+02$ & --- & DCNUCDWE (6) \\
\hline & DCLR & Leach rate constant of $\mathrm{Pb}-210$ (/yr) & $0.000 \mathrm{E}+00$ & $0.000 \mathrm{E}+00$ & $1.663 \mathrm{E}-03$ & Rleach $(1,6)$ \\
\hline & DCLR & Distribution coefficients for progeny Po-210 & & & & \\
\hline & DCLR & Contaminated zone $(\mathrm{cm} * * 3 / \mathrm{g})$ & $1.000 \mathrm{E}+01$ & $1.000 \mathrm{E}+01$ & --- & $\operatorname{DCNUCC}(7)$ \\
\hline & DCLR & Unsaturated zone $1(\mathrm{~cm} * * 3 / \mathrm{g})$ & $1.000 \mathrm{E}+01$ & $1.000 \mathrm{E}+01$ & --- & $\operatorname{DCNUCU}(7,1)$ \\
\hline & DCLR & Saturated zone $(\mathrm{cm} * * 3 / \mathrm{g})$ & $1.000 \mathrm{E}+01$ & $1.000 \mathrm{E}+01$ & --- & DCNUCS (7) \\
\hline & DCLR & Bottom sediment in surface water body $(\mathrm{cm} * * 3 / \mathrm{g})$ & $1.000 \mathrm{E}+01$ & $1.000 \mathrm{E}+01$ & --- & DCNUCSWB (7) \\
\hline & DCLR & Suspended sediment in surface water body $(\mathrm{cm} * * 3 / \mathrm{g})$ & $1.000 \mathrm{E}+01$ & $1.000 \mathrm{E}+01$ & --- & DCNUCSWS (7) \\
\hline & DCLR & Agricultural area $1(\mathrm{~cm} * * 3 / \mathrm{g})$ & $1.000 \mathrm{E}+01$ & $1.000 \mathrm{E}+01$ & --- & $\operatorname{DCNUCOF}(7,1)$ \\
\hline & DCLR & Agricultural area $2(\mathrm{~cm} * * 3 / \mathrm{g})$ & $1.000 \mathrm{E}+01$ & $1.000 \mathrm{E}+01$ & --- & $\operatorname{DCNUCOF}(7,2)$ \\
\hline & DCLR & Agricultural area $3(\mathrm{~cm} * * 3 / \mathrm{g})$ & $1.000 \mathrm{E}+01$ & $1.000 \mathrm{E}+01$ & --- & $\operatorname{DCNUCOF}(7,3)$ \\
\hline & DCLR & Agricultural area $4(\mathrm{~cm} * * 3 / \mathrm{g})$ & $1.000 \mathrm{E}+01$ & $1.000 \mathrm{E}+01$ & --- & $\operatorname{DCNUCOF}(7,4)$ \\
\hline & DCLR & Offsite Dwelling $(\mathrm{cm} * * 3 / \mathrm{g})$ & $1.000 \mathrm{E}+01$ & $1.000 \mathrm{E}+01$ & --- & DCNUCDWE (7) \\
\hline & DCLR & Leach rate constant of Po-210 (/yr) & $0.000 \mathrm{E}+00$ & $0.000 \mathrm{E}+00$ & $1.632 \mathrm{E}-02$ & Rleach $(1,7)$ \\
\hline & 1 RESRA & -OFFSITE, Version 4.0 .3 beta T1/2 Limi & $06 / 1110$ & $11: 39$ & $=0$ & \\
\hline
\end{tabular}

Parent Dose Report

Title : RESRAD-OFFSITE Effluent release

File : PADUCAH EFFLUENT RELEASE-NONINVOLVED-WORKER-REV.ROF

\begin{tabular}{|c|c|c|c|c|c|}
\hline $\begin{array}{l}0 \\
\text { Menu }\end{array}$ & Site-Specific Par & $\begin{array}{l}\text { eter Summa } \\
\text { User } \\
\text { Input }\end{array}$ & $\begin{array}{l}\text { (continue } \\
\text { Default }\end{array}$ & $\begin{array}{l}\text { RESRAD } \\
\text { computed }\end{array}$ & $\begin{array}{l}\text { Parameter } \\
\text { Name }\end{array}$ \\
\hline DCLR & Distribution coefficients for progeny Ra-226 & & & & \\
\hline DCLR & Contaminated zone $(\mathrm{cm} * * 3 / \mathrm{g})$ & $7.000 \mathrm{E}+01$ & $7.000 \mathrm{E}+01$ & --- & $\operatorname{DCNUCC}(13)$ \\
\hline DCLR & Unsaturated zone $1(\mathrm{~cm} * \star 3 / \mathrm{g})$ & $7.000 \mathrm{E}+01$ & $7.000 \mathrm{E}+01$ & --- & $\operatorname{DCNUCU}(13,1)$ \\
\hline DCLR & Saturated zone $(\mathrm{cm} * * 3 / \mathrm{g})$ & $7.000 \mathrm{E}+01$ & $7.000 \mathrm{E}+01$ & --- & DCNUCS (13) \\
\hline DCLR & Bottom sediment in surface water body $(\mathrm{cm} * * 3 / \mathrm{g})$ & $7.000 \mathrm{E}+01$ & $7.000 \mathrm{E}+01$ & --- & DCNUCSWB (13) \\
\hline DCLR & Suspended sediment in surface water body $(\mathrm{cm} * * 3 / \mathrm{g})$ & $7.000 \mathrm{E}+01$ & $7.000 \mathrm{E}+01$ & --- & DCNUCSWS (13) \\
\hline DCLR & Agricultural area $1(\mathrm{~cm} * * 3 / \mathrm{g})$ & $7.000 \mathrm{E}+01$ & $7.000 \mathrm{E}+01$ & --- & $\operatorname{DCNUCOF}(13,1)$ \\
\hline DCLR & Agricultural area $2(\mathrm{~cm} * * 3 / \mathrm{g})$ & $7.000 \mathrm{E}+01$ & $7.000 \mathrm{E}+01$ & --- & $\operatorname{DCNUCOF}(13,2)$ \\
\hline DCLR & Agricultural area $3(\mathrm{~cm} * * 3 / \mathrm{g})$ & $7.000 \mathrm{E}+01$ & $7.000 \mathrm{E}+01$ & --- & DCNUCOF $(13,3)$ \\
\hline DCLR & Agricultural area $4(\mathrm{~cm} * * 3 / \mathrm{g})$ & $7.000 \mathrm{E}+01$ & $7.000 \mathrm{E}+01$ & --- & $\operatorname{DCNUCOF}(13,4)$ \\
\hline DCLR & Offsite Dwelling $(\mathrm{cm} * * 3 / \mathrm{g})$ & $7.000 \mathrm{E}+01$ & $7.000 \mathrm{E}+01$ & --- & DCNUCDWE (13) \\
\hline DCLR & Leach rate constant of Ra-226 (/yr) & $0.000 \mathrm{E}+00$ & $0.000 \mathrm{E}+00$ & $2.374 \mathrm{E}-03$ & Rleach $(1,13)$ \\
\hline
\end{tabular}




\begin{tabular}{|c|c|c|c|c|c|}
\hline DCLR & Distribution coefficients for progeny Ra-228 & & & & \\
\hline DCLR & Contaminated zone $(\mathrm{cm} * * 3 / \mathrm{g})$ & $7.000 \mathrm{E}+01$ & $7.000 \mathrm{E}+01$ & --- & DCNUCC (14) \\
\hline DCLR & Unsaturated zone $1(\mathrm{~cm} * * 3 / \mathrm{g})$ & $7.000 \mathrm{E}+01$ & $7.000 \mathrm{E}+01$ & --- & $\operatorname{DCNUCU}(14,1)$ \\
\hline DCLR & Saturated zone $(\mathrm{cm} * * 3 / \mathrm{g})$ & $7.000 \mathrm{E}+01$ & $7.000 \mathrm{E}+01$ & --- & DCNUCS (14) \\
\hline DCLR & Bottom sediment in surface water body $(\mathrm{cm} * * 3 / \mathrm{g})$ & $7.000 \mathrm{E}+01$ & $7.000 \mathrm{E}+01$ & --- & DCNUCSWB (14) \\
\hline DCLR & Suspended sediment in surface water body $(\mathrm{cm} * * 3 / \mathrm{g})$ & $7.000 \mathrm{E}+01$ & $7.000 \mathrm{E}+01$ & --- & DCNUCSWS (14) \\
\hline DCLR & Agricultural area $1(\mathrm{~cm} * * 3 / \mathrm{g})$ & $7.000 \mathrm{E}+01$ & $7.000 \mathrm{E}+01$ & --- & $\operatorname{DCNUCOF}(14,1)$ \\
\hline DCLR & Agricultural area $2(\mathrm{~cm} * * 3 / \mathrm{g})$ & $7.000 \mathrm{E}+01$ & $7.000 \mathrm{E}+01$ & --- & $\operatorname{DCNUCOF}(14,2)$ \\
\hline DCLR & Agricultural area $3(\mathrm{~cm} * * 3 / \mathrm{g})$ & $7.000 \mathrm{E}+01$ & $7.000 \mathrm{E}+01$ & --- & $\operatorname{DCNUCOF}(14,3)$ \\
\hline DCLR & Agricultural area $4(\mathrm{~cm} * * 3 / \mathrm{g})$ & $7.000 \mathrm{E}+01$ & $7.000 \mathrm{E}+01$ & --- & $\operatorname{DCNUCOF}(14,4)$ \\
\hline DCLR & Offsite Dwelling $(\mathrm{cm} * * 3 / \mathrm{g})$ & $7.000 \mathrm{E}+01$ & $7.000 \mathrm{E}+01$ & --- & DCNUCDWE (14) \\
\hline DCLR & Leach rate constant of $\mathrm{Ra}-228$ (/yr) & $0.000 \mathrm{E}+00$ & $0.000 \mathrm{E}+00$ & $2.374 \mathrm{E}-03$ & Rleach $(1,14)$ \\
\hline DCLR & Distribution coefficients for progeny Th-229 & & & & \\
\hline DCLR & Contaminated zone $(\mathrm{cm} * * 3 / \mathrm{g})$ & $6.000 \mathrm{E}+04$ & $6.000 \mathrm{E}+04$ & --- & DCNUCC (17) \\
\hline DCLR & Unsaturated zone $1 \quad(\mathrm{~cm} * * 3 / \mathrm{g})$ & $6.000 \mathrm{E}+04$ & $6.000 \mathrm{E}+04$ & --- & $\operatorname{DCNUCU}(17,1)$ \\
\hline DCLR & Saturated zone $(\mathrm{cm} * * 3 / \mathrm{g})$ & $6.000 \mathrm{E}+04$ & $6.000 \mathrm{E}+04$ & --- & DCNUCS (17) \\
\hline DCLR & Bottom sediment in surface water body $(\mathrm{cm} * * 3 / \mathrm{g})$ & $6.000 \mathrm{E}+04$ & $6.000 \mathrm{E}+04$ & --- & DCNUCSWB (17) \\
\hline DCLR & Suspended sediment in surface water body $(\mathrm{cm} * * 3 / \mathrm{g})$ & $6.000 \mathrm{E}+04$ & $6.000 \mathrm{E}+04$ & --- & DCNUCSWS (17) \\
\hline DCLR & Agricultural area $1(\mathrm{~cm} * * 3 / \mathrm{g})$ & $6.000 \mathrm{E}+04$ & $6.000 \mathrm{E}+04$ & --- & $\operatorname{DCNUCOF}(17,1)$ \\
\hline DCLR & Agricultural area $2(\mathrm{~cm} * * 3 / \mathrm{g})$ & $6.000 \mathrm{E}+04$ & $6.000 \mathrm{E}+04$ & --- & $\operatorname{DCNUCOF}(17,2)$ \\
\hline DCLR & Agricultural area $3(\mathrm{~cm} * * 3 / \mathrm{g})$ & $6.000 \mathrm{E}+04$ & $6.000 \mathrm{E}+04$ & --- & $\operatorname{DCNUCOF}(17,3)$ \\
\hline DCLR & Agricultural area $4(\mathrm{~cm} * * 3 / \mathrm{g})$ & $6.000 \mathrm{E}+04$ & $6.000 \mathrm{E}+04$ & --- & $\operatorname{DCNUCOF}(17,4)$ \\
\hline DCLR & Offsite Dwelling $(\mathrm{cm} * * 3 / \mathrm{g})$ & $6.000 \mathrm{E}+04$ & $6.000 \mathrm{E}+04$ & --- & DCNUCDWE (17) \\
\hline DCLR & Leach rate constant of $\mathrm{Th}-229$ (/yr) & $0.000 \mathrm{E}+00$ & $0.000 \mathrm{E}+00$ & $2.778 \mathrm{E}-06$ & Rleach $(1,17)$ \\
\hline 1RESR & -OFFSITE, Version 4.0 .3 beta $\mathrm{T}^{1} \frac{1}{2}$ Limit $=30$ days & $06 / 11 / 20$ & $11: 39$ & ge 19 & \\
\hline
\end{tabular}

Parent Doser Versi

Title: RESRAD-OFFSITE Effluent release

File : PADUCAH EFFLUENT RELEASE-NONINVOLVED-WORKER-REV.ROF

\begin{tabular}{|c|c|c|c|c|c|}
\hline Menu & Site-Specific Par & $\begin{array}{l}\text { neter Summà } \\
\text { User } \\
\text { Input }\end{array}$ & $\begin{array}{l}\text { (continue } \\
\text { Default }\end{array}$ & $\begin{array}{l}\text { RESRAD } \\
\text { computed }\end{array}$ & $\begin{array}{l}\text { Parameter } \\
\text { Name }\end{array}$ \\
\hline DCLR & Distribution coefficients for progeny U-233 & & & & \\
\hline DCLR & Contaminated zone $(\mathrm{cm} * * 3 / \mathrm{g})$ & $5.000 \mathrm{E}+01$ & $5.000 \mathrm{E}+01$ & --- & DCNUCC (20) \\
\hline DCLR & Unsaturated zone $1(\mathrm{~cm} * * 3 / \mathrm{g})$ & $5.000 \mathrm{E}+01$ & $5.000 \mathrm{E}+01$ & --- & $\operatorname{DCNUCU}(20,1)$ \\
\hline DCLR & Saturated zone $(\mathrm{cm} * \star 3 / \mathrm{g})$ & $5.000 \mathrm{E}+01$ & $5.000 \mathrm{E}+01$ & --- & DCNUCS (20) \\
\hline DCLR & Bottom sediment in surface water body $(\mathrm{cm} * * 3 / \mathrm{g})$ & $5.000 \mathrm{E}+01$ & $5.000 \mathrm{E}+01$ & --- & DCNUCSWB (20) \\
\hline DCLR & Suspended sediment in surface water body $(\mathrm{cm} * * 3 / \mathrm{g})$ & $5.000 \mathrm{E}+01$ & $5.000 \mathrm{E}+01$ & --- & DCNUCSWS (20) \\
\hline DCLR & Agricultural area $1(\mathrm{~cm} * * 3 / \mathrm{g})$ & $5.000 \mathrm{E}+01$ & $5.000 \mathrm{E}+01$ & --- & $\operatorname{DCNUCOF}(20,1)$ \\
\hline DCLR & Agricultural area $2(\mathrm{~cm} * * 3 / \mathrm{g})$ & $5.000 \mathrm{E}+01$ & $5.000 \mathrm{E}+01$ & --- & $\operatorname{DCNUCOF}(20,2)$ \\
\hline DCLR & Agricultural area $3(\mathrm{~cm} * * 3 / \mathrm{g})$ & $5.000 \mathrm{E}+01$ & $5.000 \mathrm{E}+01$ & --- & $\operatorname{DCNUCOF}(20,3)$ \\
\hline DCLR & Agricultural area $4(\mathrm{~cm} * * 3 / \mathrm{g})$ & $5.000 \mathrm{E}+01$ & $5.000 \mathrm{E}+01$ & --- & $\operatorname{DCNUCOF}(20,4)$ \\
\hline DCLR & Offsite Dwelling $(\mathrm{cm} * * 3 / \mathrm{g})$ & $5.000 \mathrm{E}+01$ & $5.000 \mathrm{E}+01$ & --- & DCNUCDWE $(20)$ \\
\hline DCLR & Leach rate constant of $\mathrm{U}-233$ (/yr) & $0.000 \mathrm{E}+00$ & $0.000 \mathrm{E}+00$ & $3.319 \mathrm{E}-03$ & Rleach $(1,20)$ \\
\hline DCLR & Distribution coefficients for progeny U-236 & & & & \\
\hline DCLR & Contaminated zone $(\mathrm{cm} * * 3 / \mathrm{g})$ & $5.000 \mathrm{E}+01$ & $5.000 \mathrm{E}+01$ & --- & DCNUCC (23) \\
\hline DCLR & Unsaturated zone $1(\mathrm{~cm} * * 3 / \mathrm{g})$ & $5.000 \mathrm{E}+01$ & $5.000 \mathrm{E}+01$ & --- & $\operatorname{DCNUCU}(23,1)$ \\
\hline
\end{tabular}




\begin{tabular}{|c|c|c|c|c|c|}
\hline DCLR & Saturated zone $(\mathrm{cm} * * 3 / \mathrm{g})$ & $5.000 \mathrm{E}+01$ & $5.000 \mathrm{E}+01$ & --- & DCNUCS (23) \\
\hline DCLR & Bottom sediment in surface water body $(\mathrm{cm} * * 3 / \mathrm{g})$ & $5.000 \mathrm{E}+01$ & $5.000 \mathrm{E}+01$ & --- & DCNUCSWB (23) \\
\hline DCLR & Suspended sediment in surface water body $(\mathrm{cm} * * 3 / \mathrm{g})$ & $5.000 \mathrm{E}+01$ & $5.000 \mathrm{E}+01$ & --- & DCNUCSWS (23) \\
\hline DCLR & Agricultural area $1(\mathrm{~cm} * * 3 / \mathrm{g})$ & $5.000 \mathrm{E}+01$ & $5.000 \mathrm{E}+01$ & --- & $\operatorname{DCNUCOF}(23,1)$ \\
\hline DCLR & Agricultural area $2(\mathrm{~cm} * * 3 / \mathrm{g})$ & $5.000 \mathrm{E}+01$ & $5.000 \mathrm{E}+01$ & --- & $\operatorname{DCNUCOF}(23,2)$ \\
\hline DCLR & Agricultural area $3(\mathrm{~cm} * * 3 / \mathrm{g})$ & $5.000 \mathrm{E}+01$ & $5.000 \mathrm{E}+01$ & --- & $\operatorname{DCNUCOF}(23,3)$ \\
\hline DCLR & Agricultural area $4(\mathrm{~cm} * * 3 / \mathrm{g})$ & $5.000 \mathrm{E}+01$ & $5.000 \mathrm{E}+01$ & --- & $\operatorname{DCNUCOF}(23,4)$ \\
\hline DCLR & Offsite Dwelling $(\mathrm{cm} * * 3 / \mathrm{g})$ & $5.000 \mathrm{E}+01$ & $5.000 \mathrm{E}+01$ & --- & DCNUCDWE (23) \\
\hline DCLR & Leach rate constant of U-236 (/yr) & $0.000 \mathrm{E}+00$ & $0.000 \mathrm{E}+00$ & $3.319 \mathrm{E}-03$ & Rleach $(1,23)$ \\
\hline LYOT & Bearing of $X$ axis (clockwise angle $\mathrm{N}-->X$ in degrees) & $9.000 \mathrm{E}+01$ & $9.000 \mathrm{E}+01$ & --- & DNXBEARING \\
\hline LYOT & Length of Primary contamination in $\mathrm{X}$ Direction & $1.000 \mathrm{E}+00$ & $1.000 \mathrm{E}+02$ & --- & SOURCEXY (1) \\
\hline LYOT & Length of Primary contamination in $\mathrm{Y}$ Direction & $1.000 \mathrm{E}+00$ & $1.000 \mathrm{E}+02$ & --- & SOURCEXY (2) \\
\hline LYOT & Smaller X coordinate of Agricultural Area 1 & $3.438 \mathrm{E}+01$ & $3.438 \mathrm{E}+01$ & --- & $\operatorname{AGRIXY}(1,1)$ \\
\hline LYOT & Larger X coordinate of Agricultural Area 1 & $6.562 \mathrm{E}+01$ & $6.562 \mathrm{E}+01$ & --- & $\operatorname{AGRIXY}(2,1)$ \\
\hline LYOT & Smaller y coordinate of Agricultural Area 1 & $2.340 \mathrm{E}+02$ & $2.340 \mathrm{E}+02$ & --- & $\operatorname{AGRIXY}(3,1)$ \\
\hline LYOT & Larger Y coordinate of Agricultural Area 1 & $2.660 \mathrm{E}+02$ & $2.660 \mathrm{E}+02$ & --- & $\operatorname{AGRIXY}(4,1)$ \\
\hline LYOT & Smaller X coordinate of Agricultural Area 2 & $3.438 \mathrm{E}+01$ & $3.438 \mathrm{E}+01$ & --- & $\operatorname{AGRIXY}(1,2)$ \\
\hline LYOT & Larger $x$ coordinate of Agricultural Area 2 & $6.562 \mathrm{E}+01$ & $6.562 \mathrm{E}+01$ & --- & $\operatorname{AGRIXY}(2,2)$ \\
\hline LYOT & Smaller y coordinate of Agricultural Area 2 & $2.680 \mathrm{E}+02$ & $2.680 \mathrm{E}+02$ & --- & $\operatorname{AGRIXY}(3,2)$ \\
\hline LYOT & Larger y coordinate of Agricultural Area 2 & $3.000 \mathrm{E}+02$ & $3.000 \mathrm{E}+02$ & --- & $\operatorname{AGRIXY}(4,2)$ \\
\hline LYOT & Smaller X coordinate of Agricultural Area 3 & $0.000 \mathrm{E}+00$ & $0.000 \mathrm{E}+00$ & --- & $\operatorname{AGRIXY}(1,3)$ \\
\hline LYOT & Larger $x$ coordinate of Agricultural Area 3 & $1.000 \mathrm{E}+02$ & $1.000 \mathrm{E}+02$ & --- & $\operatorname{AgRIXY}(2,3)$ \\
\hline LYOT & Smaller y coordinate of Agricultural Area 3 & $4.500 \mathrm{E}+02$ & $4.500 \mathrm{E}+02$ & --- & $\operatorname{AGRIXY}(3,3)$ \\
\hline LYOT & Larger Y coordinate of Agricultural Area 3 & $5.500 \mathrm{E}+02$ & $5.500 \mathrm{E}+02$ & --- & $\operatorname{AGRIXY}(4,3)$ \\
\hline LYOT & Smaller X coordinate of Agricultural Area 4 & $0.000 \mathrm{E}+00$ & $0.000 \mathrm{E}+00$ & --- & $\operatorname{AgRIXY}(1,4)$ \\
\hline LYOT & Larger $\mathrm{x}$ coordinate of Agricultural Area 4 & $1.000 \mathrm{E}+02$ & $1.000 \mathrm{E}+02$ & --- & $\operatorname{AGRIXY}(2,4)$ \\
\hline LYOT & Smaller Y coordinate of Agricultural Area 4 & $3.000 \mathrm{E}+02$ & $3.000 \mathrm{E}+02$ & --- & $\operatorname{AGRIXY}(3,4)$ \\
\hline LYOT & Larger y coordinate of Agricultural Area 4 & $4.000 \mathrm{E}+02$ & $4.000 \mathrm{E}+02$ & --- & $\operatorname{AgRIXY}(4,4)$ \\
\hline LYOT & Smaller X coordinate of Dwelling Area & $-1.512 \mathrm{E}+01$ & $3.438 \mathrm{E}+01$ & --- & DWELLXY (1) \\
\hline LYOT & Larger X coordinate of Dwelling Area & $1.612 \mathrm{E}+01$ & $6.562 \mathrm{E}+01$ & --- & DWELLXY (2) \\
\hline $1 \mathrm{RESR}$ & OFFSITE, Version 4.0 .3 beta $T^{1 / 2}$ Limit $=30$ days & $06 / 11 / 20$ & $0 \quad 11: 39$ & 20 & \\
\hline
\end{tabular}

Parent Dose Report
Title : RESRAD-OFFSITE Effluent release

File : PADUCAH EFFLUENT RELEASE-NONINVOLVED-WORKER-REV.ROF

\begin{tabular}{|c|c|c|c|c|c|}
\hline $\begin{array}{l}0 \\
\text { Menu }\end{array}$ & Site-Specific & $\begin{array}{l}\text { meter Summa } \\
\text { User } \\
\text { Input }\end{array}$ & $\begin{array}{l}\text { Cy (continue } \\
\text { Default }\end{array}$ & $\begin{array}{l}\text { RESRAD } \\
\text { computed }\end{array}$ & $\begin{array}{c}\text { Parameter } \\
\text { Name }\end{array}$ \\
\hline LYOT & Smaller Y coordinate of Dwelling Area & $8.500 \mathrm{E}+02$ & $1.340 \mathrm{E}+02$ & --- & DWELLXY (3) \\
\hline LYOT & Larger Y coordinate of Dwelling Area & $9.000 \mathrm{E}+02$ & $1.660 \mathrm{E}+02$ & --- & DWELLXY (4) \\
\hline LYOT & Smaller $X$ coordinate of Surface water body & $-1.000 \mathrm{E}+02$ & $-1.000 \mathrm{E}+02$ & --- & SWXY (1) \\
\hline LYOT & Larger $x$ coordinate of Surface water body & $2.000 \mathrm{E}+02$ & $2.000 \mathrm{E}+02$ & --- & SWXY (2) \\
\hline LYOT & Smaller Y coordinate of Surface water body & $5.500 \mathrm{E}+02$ & $5.500 \mathrm{E}+02$ & --- & SWXY (3) \\
\hline LYOT & Larger y coordinate of Surface water body & $8.500 \mathrm{E}+02$ & $8.500 \mathrm{E}+02$ & --- & SWXY (4) \\
\hline STOR & Storage times of contaminated foodstuffs (days): & & & & \\
\hline STOR & Surface water & $1.000 \mathrm{E}+00$ & $1.000 \mathrm{E}+00$ & --- & STOR T(1) \\
\hline STOR & Well water & $1.000 \mathrm{E}+00$ & $1.000 \mathrm{E}+00$ & --- & STOR_T (2) \\
\hline STOR & Fruits, non-leafy vegetables, and grain & $1.400 \mathrm{E}+01$ & $1.400 \mathrm{E}+01$ & --- & $\mathrm{STOR}^{-} \mathrm{T}(3)$ \\
\hline
\end{tabular}




\begin{tabular}{|c|c|c|c|c|c|}
\hline STOR & Leafy vegetables & $1.000 \mathrm{E}+00$ & $1.000 \mathrm{E}+00$ & --- & STOR_T (4) \\
\hline STOR & Livestock feed - pasture or silage & $1.000 \mathrm{E}+00$ & $1.000 \mathrm{E}+00$ & --- & STOR_T (5) \\
\hline STOR & Livestock feed - grain & $4.500 \mathrm{E}+01$ & $4.500 \mathrm{E}+01$ & --- & STOR_T (6) \\
\hline STOR & Meat and poultry & $2.000 \mathrm{E}+01$ & $2.000 \mathrm{E}+01$ & --- & $\mathrm{STOR}_{-}^{-} \mathrm{T}(7)$ \\
\hline STOR & Milk & $1.000 \mathrm{E}+00$ & $1.000 \mathrm{E}+00$ & --- & STOR_T (8) \\
\hline STOR & Fish & $7.000 \mathrm{E}+00$ & $7.000 \mathrm{E}+00$ & --- & STOR_T (9) \\
\hline STOR & Crustacea and mollusks & $7.000 \mathrm{E}+00$ & $7.000 \mathrm{E}+00$ & --- & STOR_T (10) \\
\hline TIME & Times at which dose/risk are to be reported (yr) & $1.000 \mathrm{E}+00$ & $1.000 \mathrm{E}+00$ & --- & $T(2)$ \\
\hline TIME & Times at which dose/risk are to be reported (yr) & $3.000 \mathrm{E}+00$ & $3.000 \mathrm{E}+00$ & --- & $T(3)$ \\
\hline TIME & Times at which dose/risk are to be reported (yr) & $6.000 \mathrm{E}+00$ & $6.000 \mathrm{E}+00$ & --- & $\mathrm{T}(4)$ \\
\hline TIME & Times at which dose/risk are to be reported (yr) & $1.000 \mathrm{E}+01$ & $1.200 \mathrm{E}+01$ & --- & $T(5)$ \\
\hline TIME & Times at which dose/risk are to be reported (yr) & not used & $3.000 \mathrm{E}+01$ & --- & $\mathrm{T}(6)$ \\
\hline TIME & Times at which dose/risk are to be reported (yr) & not used & $7.500 \mathrm{E}+01$ & --- & $T(7)$ \\
\hline TIME & Times at which dose/risk are to be reported (yr) & not used & $1.750 \mathrm{E}+02$ & --- & $\mathrm{T}(8)$ \\
\hline TIME & Times at which dose/risk are to be reported (yr) & not used & $4.200 \mathrm{E}+02$ & --- & $T(9)$ \\
\hline TIME & Times at which dose/risk are to be reported (yr) & not used & $9.700 \mathrm{E}+02$ & --- & $\mathrm{T}(10)$ \\
\hline SITE & Precipitation (m/yr) & $1.000 \mathrm{E}+00$ & $1.000 \mathrm{E}+00$ & --- & PRECIP \\
\hline SITE & Rainfall Erosion Index & $1.600 \mathrm{E}+02$ & $1.600 \mathrm{E}+02$ & --- & RAINEROS \\
\hline PRCZ & Area of primary contamination $(m * * 2)$ & $1.000 \mathrm{E}+00$ & $1.000 \mathrm{E}+04$ & --- & AREA \\
\hline PRCZ & Length parallel to aquifer flow (m) & $1.000 \mathrm{E}+02$ & $1.000 \mathrm{E}+02$ & --- & LCZPAQ \\
\hline PRCZ & Depth of soil mixing layer (m) & $1.500 \mathrm{E}-01$ & $1.500 \mathrm{E}-01$ & --- & DM \\
\hline PRCZ & Mass loading of all particulates for release $(\mathrm{g} / \mathrm{m} * * 3)$ & $1.000 \mathrm{E}-04$ & $1.000 \mathrm{E}-04$ & --- & MLFD \\
\hline PRCZ & DepositionVelocityofAllParticulates for release $(\mathrm{m} / \mathrm{s})$ & $1.000 \mathrm{E}-03$ & $1.000 \mathrm{E}-03$ & --- & DEPVEL_DUSTT \\
\hline PRCZ & Respirable particulates as a fraction of total & $1.000 \mathrm{E}+00$ & $1.000 \mathrm{E}+00$ & --- & RESPFRĀCPC \\
\hline PRCZ & DepositionVelocityofRespirableParticulatesForRe (m/s) & $1.000 \mathrm{E}-03$ & $1.000 \mathrm{E}-03$ & --- & DEPVEL_DUST \\
\hline PRCZ & Irrigation (m/yr) & $2.000 \mathrm{E}-01$ & $2.000 \mathrm{E}-01$ & --- & RI \\
\hline PRCZ & Evapotranspiration coefficient & $5.000 \mathrm{E}-01$ & $5.000 \mathrm{E}-01$ & --- & EVAPTR \\
\hline PRCZ & Runoff coefficient & $2.000 \mathrm{E}-01$ & $2.000 \mathrm{E}-01$ & --- & RUNOFE \\
\hline PRCZ & Slope-length-steepness factor of prim. contamination & $4.000 \mathrm{E}-01$ & $4.000 \mathrm{E}-01$ & --- & SLPLENSTPPC \\
\hline PRCZ & Cropping-management factor of primary contamination & $3.000 \mathrm{E}-03$ & $3.000 \mathrm{E}-03$ & --- & CRPMANGPC \\
\hline PRCZ & Conservation practice factor of prim. contamination & $1.000 \mathrm{E}+00$ & $1.000 \mathrm{E}+00$ & --- & CONVPRACPC \\
\hline PRCZ & Fraction of primary contamination that is sumberged & $0.000 \mathrm{E}+00$ & $0.000 \mathrm{E}+00$ & --- & SUBMERGEDF \\
\hline PRCZ & Thickness of primary contamination (m) & $2.000 \mathrm{E}+00$ & $2.000 \mathrm{E}+00$ & --- & THICKO \\
\hline & OFFSITE, Version 4.0 .3 beta $T^{1 / 2}$ Limit $=30$ days & $06 / 11 / 20$ & $11: 39$ & 21 & \\
\hline
\end{tabular}

Parent Dose Report

Title : RESRAD-OFFSITE Effluent release

File : PADUCAH EFFLUENT RELEASE-NONINVOLVED-WORKER-REV.ROF

\begin{tabular}{|c|c|c|c|c|c|}
\hline $\begin{array}{l}0 \\
\text { Menu }\end{array}$ & Site-Specific Par & $\begin{array}{l}\text { eter Summ } \\
\text { User } \\
\text { Input }\end{array}$ & $\begin{array}{l}\text { (continue } \\
\text { Default }\end{array}$ & $\begin{array}{l}\text { RESRAD } \\
\text { computed }\end{array}$ & $\begin{array}{l}\text { Parameter } \\
\text { Name }\end{array}$ \\
\hline PRCZ & Soil erodibility factor of contamination (tons/acre) & $4.000 \mathrm{E}-01$ & $4.000 \mathrm{E}-01$ & --- & ERODIBILITYCZ \\
\hline PRCZ & Density of primmary contamination $(\mathrm{g} / \mathrm{cm} * * 3)$ & $1.500 \mathrm{E}+00$ & $1.500 \mathrm{E}+00$ & --- & DENSCZ \\
\hline PRCZ & Computed erosion rate of contamination (m/yr) & $1.147 \mathrm{E}-05$ & $1.147 \mathrm{E}-05$ & --- & $\mathrm{VCZ}$ \\
\hline PRCZ & Total porosity of primary contamination & $4.000 \mathrm{E}-01$ & $4.000 \mathrm{E}-01$ & --- & TPCZ \\
\hline PRCZ & Field capacity of primary contamination & $3.000 \mathrm{E}-01$ & $3.000 \mathrm{E}-01$ & --- & FCCZ \\
\hline PRCZ & Effective porosity of primary contamination & $4.000 \mathrm{E}-01$ & $4.000 \mathrm{E}-01$ & --- & EPCZ \\
\hline
\end{tabular}


\begin{tabular}{l|l} 
PRCZ & Hydraulic conductivity of prime contamination (m/yr) \\
PRCZ & b parameter of primary contamination
\end{tabular} longitudinal dispersivity of prime contamination (m) Cover depth (m)

Soil erodibility factor of cover (tons/acre)

Density of cover material $(\mathrm{g} / \mathrm{cm} * * 3)$

Computed erosion rate of cover material (m/yr)

Total porosity of the cover material

volumetric water content of the cover material

Sediment Delivery Ratio, SDR

from primary contamination to surface water body

from primary contamination to non-leafy veg. field

from primary contamination to leafy veg. field

from primary contamination to pasture

from primary contamination to feed grain field

from primary contamination to surface water body

AGRI Areal extent of Agricultural Area 1 ( $m * * 2)$

AGRI Fraction of Agri. Area 1 directly over the c.z.

AGRI Evapotranspiration coefficient in Agri. Area 1

AGRI Runoff coefficient in Agricultural Area 1

AGRI Mixing depth/plow layer of Agricultural Area 1

AGRI Water filled porosity of soil in Agri. Area 1

AGRI Computed erosion rate of soil in Agri. Area 1

AGRI Dry Bulk Density of soil in Agricultural Area 1

AGRI Soil erodibility factor of Agricultural Area 1

AGRI Slope-length-steepness factor, Agricultural Area 1

AGRI Cropping-management factor of Agricultural Area 1

AGRI Conservation practice factor of Agricultural Area 1

AGRI Total porosity of soil in Agricultural Area 1

AGRI Areal extent of Agricultural Area 2 ( $m * * 2)$

AGRI Fraction of Agri. Area 2 directly over the c.z.

AGRI Evapotranspiration coefficient in Agri. Area 2

AGRI Runoff coefficient in Agricultural Area 2

AGRI Mixing depth/plow layer of Agricultural Area 2

AGRI Water filled porosity of soil in Agri. Area 2

AGRI Computed erosion rate of soil in Agri. Area 2

AGRI Dry Bulk Density of soil in Agricultural Area 2

AGRI Soil erodibility factor of Agricultural Area 2

AGRI Slope-length-steepness factor, Agricultural Area 2

RESRAD-OFFSITE, Version 4.0 .3 beta T1/2 Limit $=30$ days

Parent Dose Report

Title : RESRAD-OFFSITE Effluent release

File : PADUCAH EFFLUENT RELEASE-NONINVOLVED-WORKER-REV.ROF

\begin{tabular}{|c|c|c|c|}
\hline $1.000 \mathrm{E}+01$ & $1.000 \mathrm{E}+01$ & --- & $\mathrm{HCCZ}$ \\
\hline $5.300 \mathrm{E}+00$ & $5.300 \mathrm{E}+00$ & --- & $B C Z$ \\
\hline $5.000 \mathrm{E}-02$ & $5.000 \mathrm{E}-02$ & --- & ALPHALCZ \\
\hline $0.000 \mathrm{E}+00$ & $0.000 \mathrm{E}+00$ & --- & COVERO \\
\hline not used & $4.000 \mathrm{E}-01$ & --- & ERODIBILITYCV \\
\hline not used & $1.500 \mathrm{E}+00$ & --- & DENSCV \\
\hline not used & $1.147 \mathrm{E}-05$ & --- & VCV \\
\hline not used & $4.000 \mathrm{E}-01$ & --- & TPCV \\
\hline not used & $5.000 \mathrm{E}-02$ & --- & PH2OCV \\
\hline $0.000 \mathrm{E}+00$ & $0.000 \mathrm{E}+00$ & --- & SDRDWELL \\
\hline $0.000 \mathrm{E}+00$ & $0.000 \mathrm{E}+00$ & --- & SDROF (1) \\
\hline $0.000 \mathrm{E}+00$ & $0.000 \mathrm{E}+00$ & --- & SDROF (2) \\
\hline $0.000 \mathrm{E}+00$ & $0.000 \mathrm{E}+00$ & --- & SDROF (3) \\
\hline $0.000 \mathrm{E}+00$ & $0.000 \mathrm{E}+00$ & --- & SDROF (4) \\
\hline $1.000 \mathrm{E}+00$ & $1.000 \mathrm{E}+00$ & --- & SDR \\
\hline $1.000 \mathrm{E}+03$ & $1.000 \mathrm{E}+03$ & --- & $\operatorname{AREAO}(1)$ \\
\hline not used & $0.000 \mathrm{E}+00$ & --- & FAREA PLANT (1) \\
\hline $5.000 \mathrm{E}-01$ & $5.000 \mathrm{E}-01$ & --- & EVAPTR $\bar{R}(1)$ \\
\hline $2.000 \mathrm{E}-01$ & $2.000 \mathrm{E}-01$ & --- & RUNOF (1) \\
\hline $1.500 \mathrm{E}-01$ & $1.500 \mathrm{E}-01$ & --- & DPTHMIXG (1) \\
\hline $3.000 \mathrm{E}-01$ & $3.000 \mathrm{E}-01$ & --- & TMOF (1) \\
\hline $1.147 \mathrm{E}-05$ & $1.147 \mathrm{E}-05$ & --- & EROSN (1) \\
\hline $1.500 \mathrm{E}+00$ & $1.500 \mathrm{E}+00$ & --- & RHOB (1) \\
\hline $4.000 \mathrm{E}-01$ & $4.000 \mathrm{E}-01$ & --- & ERODIBILITY (1) \\
\hline $4.000 \mathrm{E}-01$ & $4.000 \mathrm{E}-01$ & --- & SLPLENSTP (1) \\
\hline $3.000 \mathrm{E}-03$ & $3.000 \mathrm{E}-03$ & --- & CRPMANG (1) \\
\hline $1.000 \mathrm{E}+00$ & $1.000 \mathrm{E}+00$ & --- & CONVPRAC (1) \\
\hline not used & $4.000 \mathrm{E}-01$ & --- & TPOF (1) \\
\hline $1.000 \mathrm{E}+03$ & $1.000 \mathrm{E}+03$ & --- & AREAO (2) \\
\hline not used & $0.000 \mathrm{E}+00$ & --- & FAREA PLANT (2) \\
\hline $5.000 \mathrm{E}-01$ & $5.000 \mathrm{E}-01$ & --- & EVAPTR $\bar{R}(2)$ \\
\hline $2.000 \mathrm{E}-01$ & $2.000 \mathrm{E}-01$ & --- & RUNOF (2) \\
\hline $1.500 \mathrm{E}-01$ & $1.500 \mathrm{E}-01$ & --- & DPTHMIXG (2) \\
\hline $3.000 \mathrm{E}-01$ & $3.000 \mathrm{E}-01$ & --- & TMOF (2) \\
\hline $1.147 \mathrm{E}-05$ & $1.147 \mathrm{E}-05$ & --- & EROSN (2) \\
\hline $500 \mathrm{E}+00$ & $1.500 \mathrm{E}+00$ & --- & RHOB (2) \\
\hline-01 & $4.000 \mathrm{E}-01$ & --- & ERODIBILITY (2) \\
\hline $00 E-01$ & $4.000 \mathrm{E}-01$ & --- & SLPLENSTP (2) \\
\hline
\end{tabular}

\begin{tabular}{l|c|c|c|c|c}
0 & \multicolumn{4}{|c}{ Site-Specific Parameter Summary (continued) } \\
Menu & Parameter & User & Input & Default & $\begin{array}{c}\text { RESRAD } \\
\text { computed }\end{array}$ \\
\hline AGRI & Cropping-management factor of Agricultural Area 2 & $3.000 \mathrm{E}-03$ & $3.000 \mathrm{E}-03$ & -- & CRPMANG(2)
\end{tabular}


AGRI | Conservation practice factor of Agricultural Area 2

AGRI Total porosity of soil in Agricultural Area 2

AGRI Areal extent of Agricultural Area $3(m * * 2)$

AGRI Fraction of Agri. Area 3 directly over the c.z.

AGRI Evapotranspiration coefficient in Agri. Area 3

AGRI Runoff coefficient in Agricultural Area 3

AGRI Mixing depth/plow layer of Agricultural Area 3

AGRI Water filled porosity of soil in Agri. Area 3

AGRI Dry Bulk Density of soil in Agricultural Area 3

AGRI Soil erodibility factor of Agricultural Area 3

AGRI Slope-length-steepness factor, Agricultural Area 3

AGRI Cropping-management factor of Agricultural Area 3

AGRI Conservation practice factor of Agricultural Area 3

AGRI Total porosity of soil in Agricultural Area 3

AGRI Areal extent of Agricultural Area 4 ( $m * * 2)$

AGRI Fraction of Agri. Area 4 directly over the c.z.

AGRI Evapotranspiration coefficient in Agri. Area 4

AGRI Runoff coefficient in Agricultural Area 4

AGRI Mixing depth/plow layer of Agricultural Area 4

AGRI Water filled porosity of soil in Agri. Area 4

AGRI Computed erosion rate of soil in Agri. Area 4

AGRI Dry Bulk Density of soil in Agricultural Area 4

AGRI Soil erodibility factor of Agricultural Area 4

AGRI Slope-length-steepness factor, Agricultural Area 4

AGRI Cropping-management factor of Agricultural Area 4

AGRI Conservation practice factor of Agricultural Area 4

AGRI

Total porosity of soil in Agricultural Area 4

DWEL Areal extent of offsite dwelling site $(m * * 2)$

DWEL Evapotranspiration coefficient in dwelling (off) sit

DWEL Runoff coefficient in Offsite dwelling site

DWEL Mixing depth of Offsite dwelling site

DWEL Water filled porosity of soil in offsite Dwelling

DWEI Computed erosion

DWEL Dry Bulk Density of soil in Offsite dwelling site

DWEL Soil erodibility factor of soil in Dwelling site

DWEL Slope-length-steepness factor of Dwelling site

DWEL Cropping-management factor of Dwelling site

DWEL Conservation practice factor of offsite Dwelling sit

DWEL Total porosity of soil in Offsite Dwelling

AIRT Dispersion Coefficients; 1 = Pasquill-Gifford

AIRT Population zone; 1 = Rural

AIRT Release height, (m)

AIRT Heat flux for buoyant plume (cal/s),

1RESRAD-OFFSITE, Version 4.0 .3 beta $T^{1} \frac{1}{2}$ Limit $=30$ days

Parent Dose Report

Title : RESRAD-OFFSITE Effluent release

File : PADUCAH EFFLUENT RELEASE-NONINVOLVED-WORKER-REV.ROF

\begin{tabular}{|c|c|c|c|}
\hline $1.000 \mathrm{E}+00$ & $1.000 \mathrm{E}+00$ & --- & CONVPRAC (2) \\
\hline not used & $4.000 \mathrm{E}-01$ & --- & TPOF (2) \\
\hline $1.000 \mathrm{E}+04$ & $1.000 \mathrm{E}+04$ & --- & AREAO (3) \\
\hline not used & $0.000 \mathrm{E}+00$ & --- & FAREA_PLANT (3) \\
\hline $5.000 \mathrm{E}-01$ & $5.000 \mathrm{E}-01$ & --- & EVAPTR $\bar{R}$ (3) \\
\hline $2.000 \mathrm{E}-01$ & $2.000 \mathrm{E}-01$ & --- & RUNOF (3) \\
\hline $1.500 \mathrm{E}-01$ & $1.500 \mathrm{E}-01$ & --- & DPTHMIXG (3) \\
\hline $3.000 \mathrm{E}-01$ & $3.000 \mathrm{E}-01$ & --- & TMOF (3) \\
\hline $1.147 \mathrm{E}-05$ & $1.147 \mathrm{E}-05$ & --- & EROSN (3) \\
\hline $1.500 \mathrm{E}+00$ & $1.500 \mathrm{E}+00$ & --- & RHOB (3) \\
\hline $4.000 \mathrm{E}-01$ & $4.000 \mathrm{E}-01$ & --- & ERODIBILITY (3) \\
\hline $4.000 \mathrm{E}-01$ & $4.000 \mathrm{E}-01$ & --- & SLPLENSTP (3) \\
\hline $3.000 \mathrm{E}-03$ & $3.000 \mathrm{E}-03$ & --- & CRPMANG (3) \\
\hline $1.000 \mathrm{E}+00$ & $1.000 \mathrm{E}+00$ & --- & CONVPRAC (3) \\
\hline not used & $4.000 \mathrm{E}-01$ & --- & TPOF (3) \\
\hline $1.000 \mathrm{E}+04$ & $1.000 \mathrm{E}+04$ & --- & AREAO (4) \\
\hline not used & $0.000 \mathrm{E}+00$ & --- & FAREA PLANT (4) \\
\hline $5.000 \mathrm{E}-01$ & $5.000 \mathrm{E}-01$ & --- & EVAPTR $\bar{R}$ ( 4) \\
\hline $2.000 \mathrm{E}-01$ & $2.000 \mathrm{E}-01$ & --- & RUNOF ( 4) \\
\hline $1.500 \mathrm{E}-01$ & $1.500 \mathrm{E}-01$ & --- & DPTHMIXG (4) \\
\hline $3.000 \mathrm{E}-01$ & $3.000 \mathrm{E}-01$ & --- & TMOF (4) \\
\hline $1.147 \mathrm{E}-05$ & $1.147 \mathrm{E}-05$ & --- & EROSN ( 4) \\
\hline $1.500 \mathrm{E}+00$ & $1.500 \mathrm{E}+00$ & --- & RHOB (4) \\
\hline $4.000 \mathrm{E}-01$ & $4.000 \mathrm{E}-01$ & --- & ERODIBILITY (4) \\
\hline $4.000 \mathrm{E}-01$ & $4.000 \mathrm{E}-01$ & --- & SLPLENSTP (4) \\
\hline $3.000 \mathrm{E}-03$ & $3.000 \mathrm{E}-03$ & --- & CRPMANG ( 4 ) \\
\hline $1.000 \mathrm{E}+00$ & $1.000 \mathrm{E}+00$ & --- & CONVPRAC (4) \\
\hline not used & $4.000 \mathrm{E}-01$ & --- & TPOF (4) \\
\hline $1.562 \mathrm{E}+03$ & $1.000 \mathrm{E}+03$ & --- & AREAODWELL \\
\hline $5.000 \mathrm{E}-01$ & $5.000 \mathrm{E}-01$ & --- & EVAPTRNDWELL \\
\hline $2.000 \mathrm{E}-01$ & $2.000 \mathrm{E}-01$ & --- & RUNOFDWELL \\
\hline $1.500 \mathrm{E}-01$ & $1.500 \mathrm{E}-01$ & --- & DPTHMIXGDWELL \\
\hline $3.000 \mathrm{E}-01$ & $3.000 \mathrm{E}-01$ & --- & TMOFDWELL \\
\hline $0.000 \mathrm{E}+00$ & $0.000 \mathrm{E}+00$ & --- & EROSNDWELL \\
\hline $1.500 \mathrm{E}+00$ & $1.500 \mathrm{E}+00$ & --- & RHOBDWELL \\
\hline $0.000 \mathrm{E}+00$ & $0.000 \mathrm{E}+00$ & --- & ERODIBILITYDWELL \\
\hline $4.000 \mathrm{E}-01$ & $4.000 \mathrm{E}-01$ & --- & SLPLENSTPDWELL \\
\hline $3.000 \mathrm{E}-03$ & $3.000 \mathrm{E}-03$ & --- & CRPMANGDWELL \\
\hline $1.000 \mathrm{E}+00$ & $1.000 \mathrm{E}+00$ & --- & CONVPRACDWELL \\
\hline not used & $4.000 \mathrm{E}-01$ & --- & TPOFDWELL \\
\hline 1 & 1 & --- & IDISPMOD \\
\hline 1 & 1 & --- & IZONE \\
\hline $4.500 \mathrm{E}+01$ & $1.000 \mathrm{E}+00$ & --- & AIRRELHT \\
\hline $0.000 \mathrm{E}+00$ & $0.000 \mathrm{E}+00$ & --- & HEATELX \\
\hline
\end{tabular}




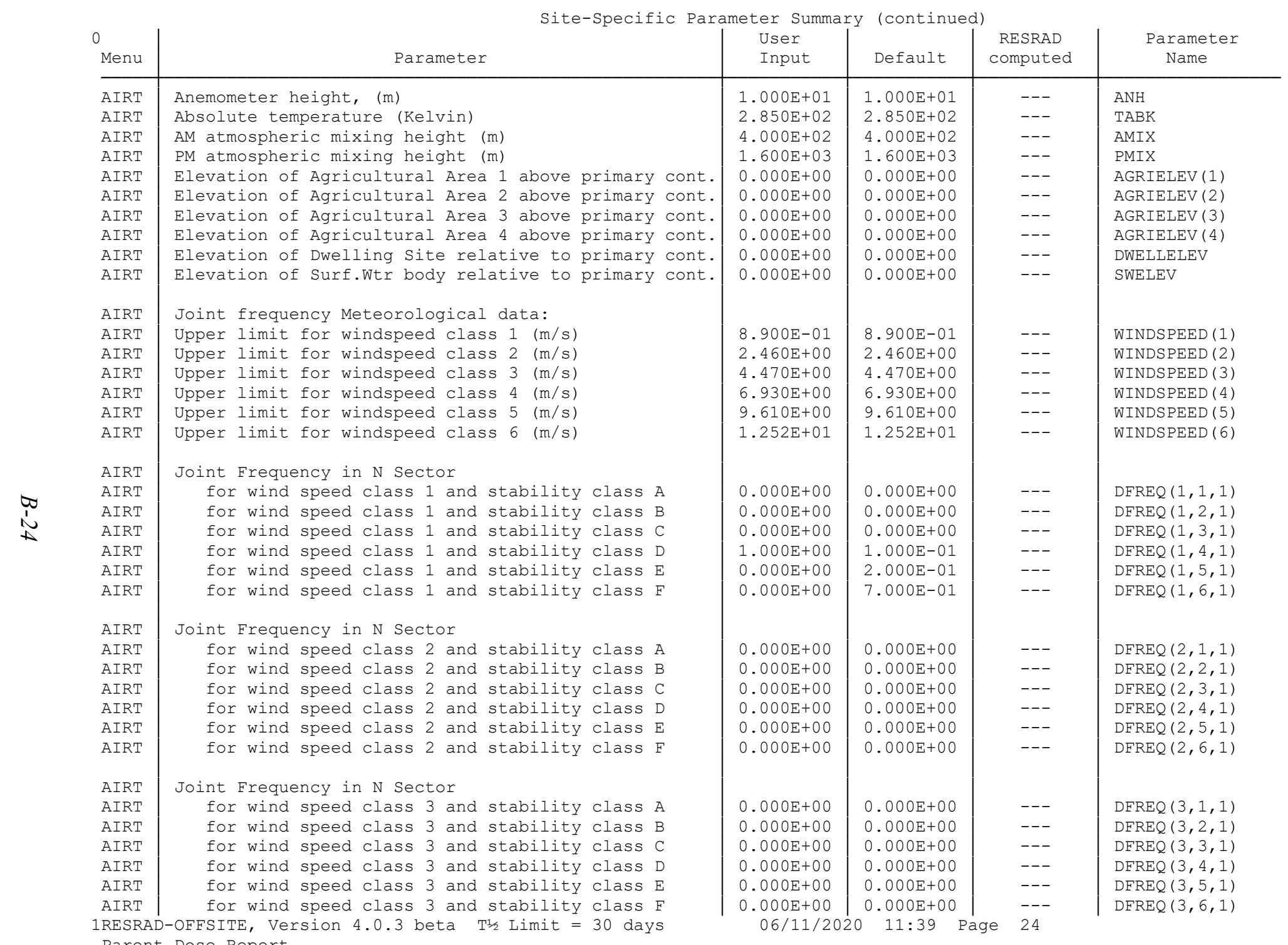

Parent Dose Report

Title : RESRAD-OFFSITE Effluent release

File : PADUCAH EFFLUENT RELEASE-NONINVOLVED-WORKER-REV.ROF 


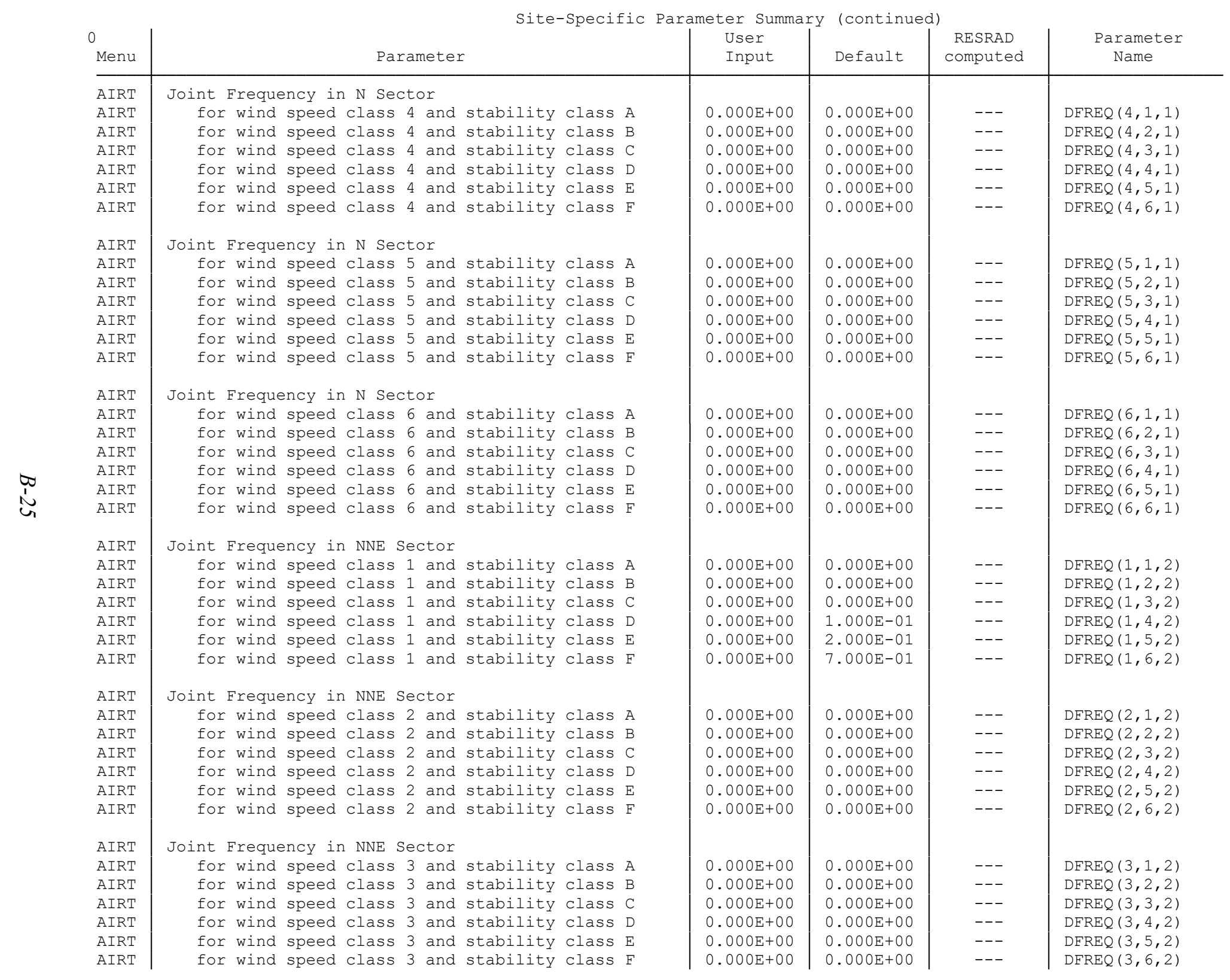


Parent Dose Report

Title: RESRAD-OFFSITE Effluent release

File : PADUCAH EFFLUENT RELEASE-NONINVOLVED-WORKER-REV.ROF

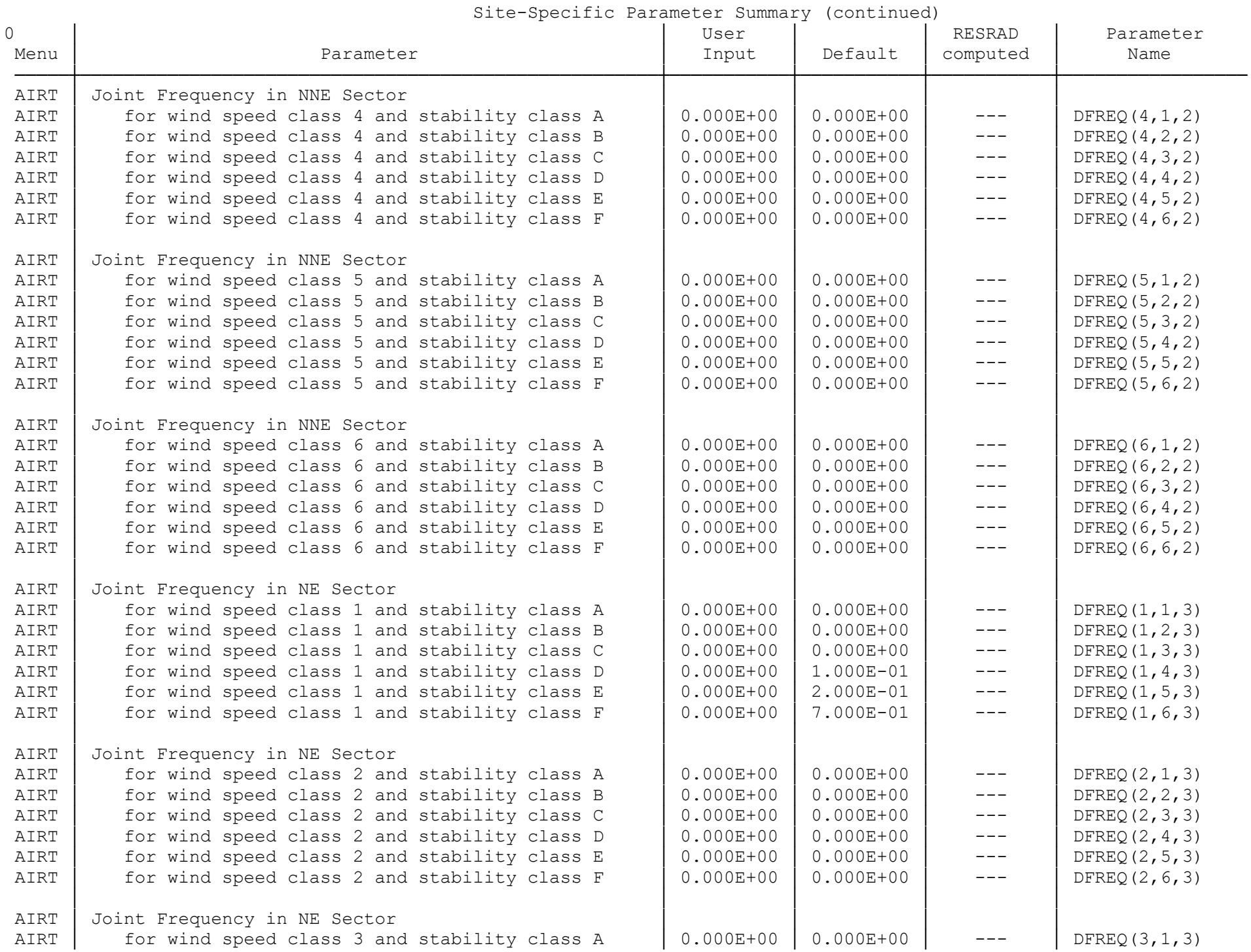


AIRT for wind speed class 3 and stability class B for wind speed class 3 and stability class $C$ for wind speed class 3 and stability class D for wind speed class 3 and stability class F 列 Parent Dose Report

Title : RESRAD-OFFSITE Effluent release

File : PADUCAH EFFLUENT RELEASE-NONINVOLVED-WORKER-REV.ROF

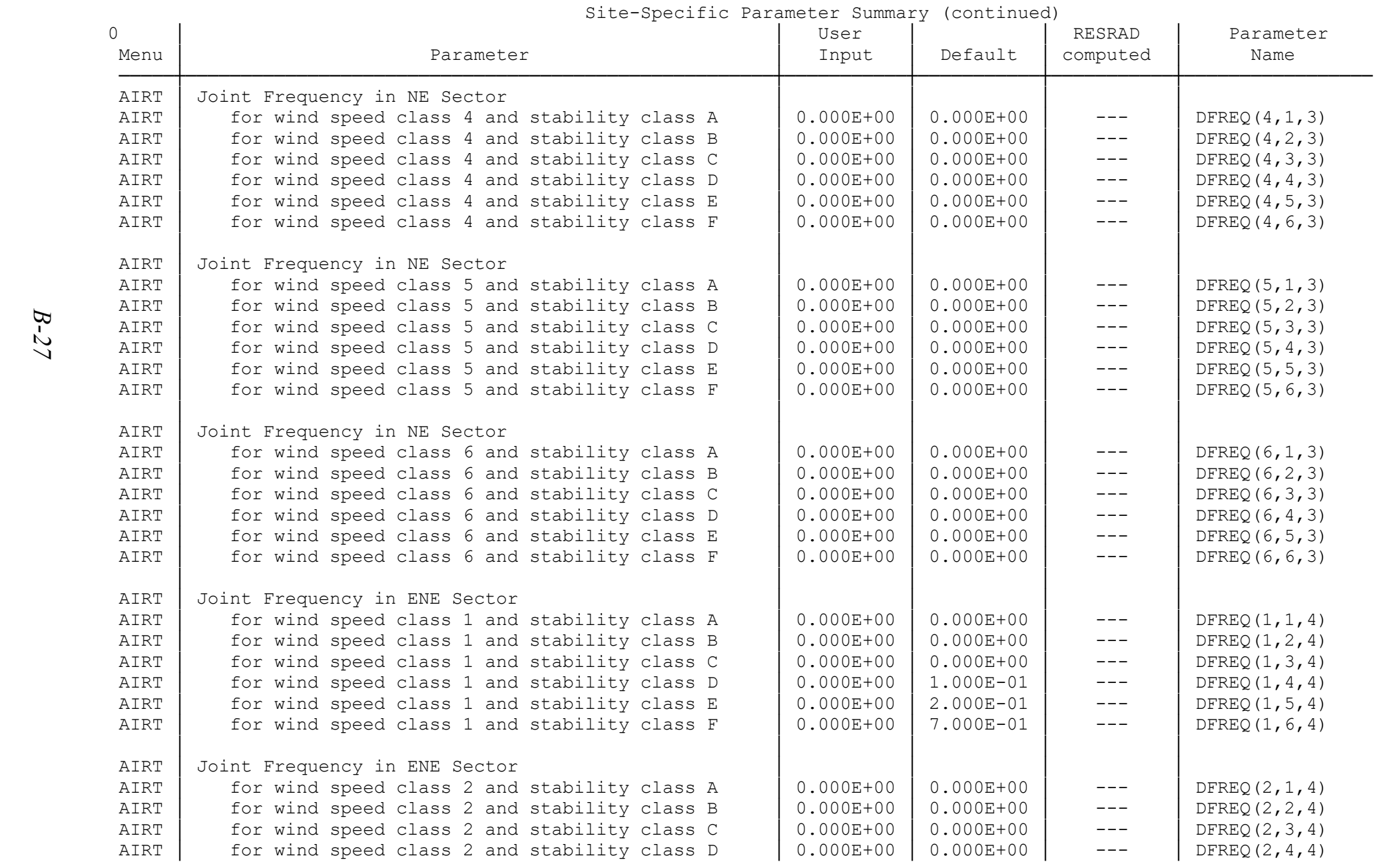


AIRT for wind speed class 2 and stability class E AIRT for wind speed class 2 and stability class F

AIRT Joint Frequency in ENE Sector

AIRT for wind speed class 3 and stability class A

AIRT for wind speed class 3 and stability class B

AIRT for wind speed class 3 and stability class C

AIRT for wind speed class 3 and stability class D

AIRT for wind speed class 3 and stability class E

for wind speed class 3 and stability class $F$

1RESRAD-OFFSITE, Version 4.0 .3 beta $\mathrm{T}^{1} \frac{1}{2}$ Limit $=30$ days

Parent Dose Report

OFFSITE Effluent release

File : PADUCAH EFFLUENT RELEASE-NONINVOLVED-WORKER-REV.ROF

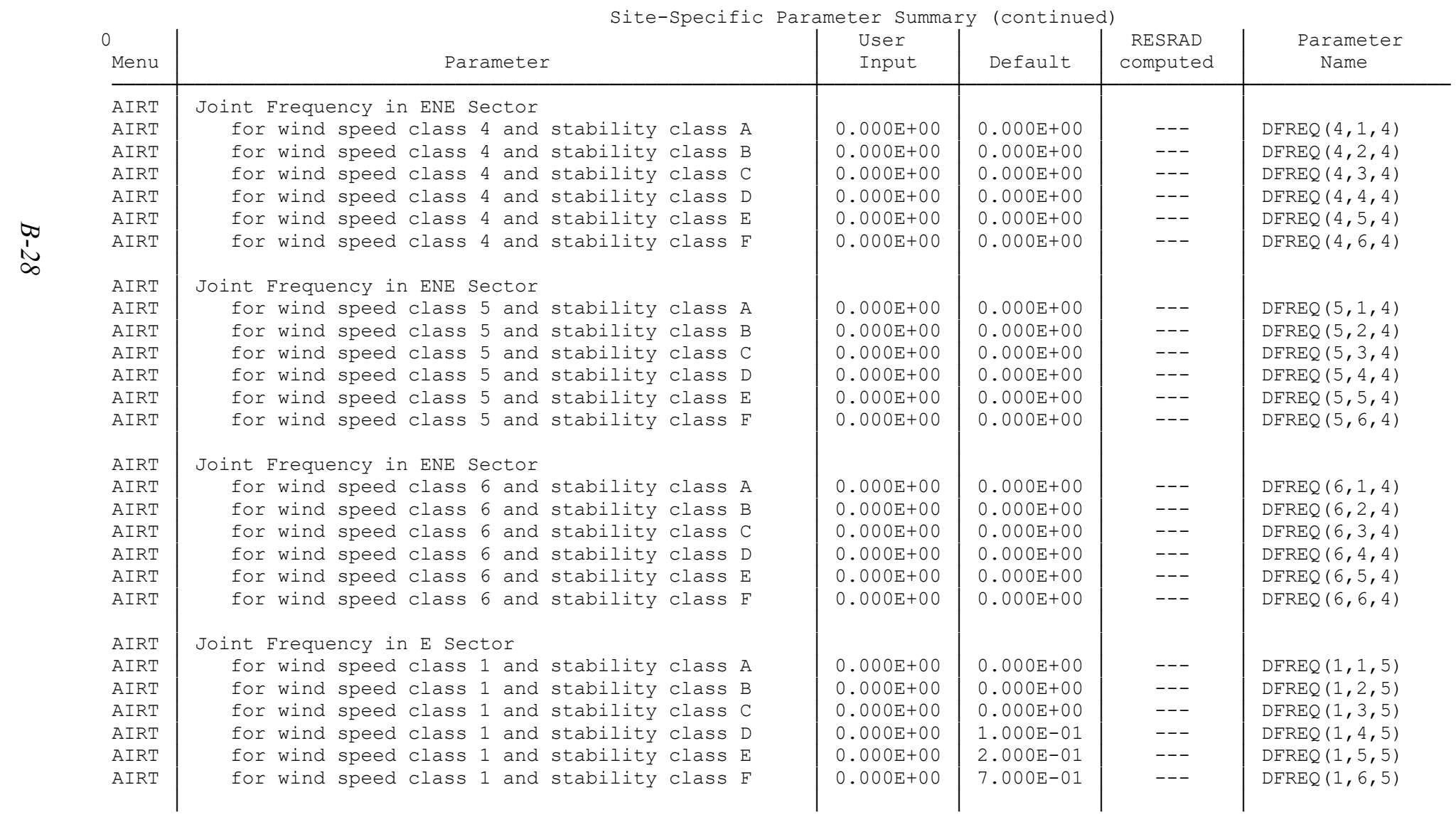

\begin{tabular}{|l|l|l|l}
$0.000 \mathrm{E}+00$ & $0.000 \mathrm{E}+00$ & --- & $\operatorname{DFREQ}(2,5,4)$ \\
$0.000 \mathrm{E}+00$ & $0.000 \mathrm{E}+00$ & --- & $\operatorname{DFREQ}(2,6,4)$ \\
& & & \\
$0.000 \mathrm{E}+00$ & $0.000 \mathrm{E}+00$ & --- & $\operatorname{DFREQ}(3,1,4)$ \\
$0.000 \mathrm{E}+00$ & $0.000 \mathrm{E}+00$ & --- & $\operatorname{DFREQ}(3,2,4)$ \\
$0.000 \mathrm{E}+00$ & $0.000 \mathrm{E}+00$ & -- & $\operatorname{DFREQ}(3,3,4)$ \\
$0.000 \mathrm{E}+00$ & $0.000 \mathrm{E}+00$ & -- & $\operatorname{DFREQ}(3,4,4)$ \\
$0.000 \mathrm{E}+00$ & $0.000 \mathrm{E}+00$ & --- & $\operatorname{DFREQ}(3,5,4)$ \\
$0.000 \mathrm{E}+00$ & $0.000 \mathrm{E}+00$ & --- & $\operatorname{DFREQ}(3,6,4)$
\end{tabular}

$06 / 11 / 2020 \cdot 11 \cdot 39$ Page 27 
\begin{tabular}{l|l} 
AIRT & Joint Frequency in E Sector
\end{tabular}

AIRT for wind speed class 2 and stability class A

AIRT for wind speed class 2 and stability class $B$

AIRT for wind speed class 2 and stability class C

AIRT for wind speed class 2 and stability class D

AIRT for wind speed class 2 and stability class $E$

AIRT for wind speed class 2 and stability class F

AIRT Joint Frequency in E sector

AIRT for wind speed class 3 and stability class A

AIRT for wind speed class 3 and stability class $B$

AIRT for wind speed class 3 and stability class C

AIRT for wind speed class 3 and stability class D

AIRT for wind speed class 3 and stability class $E$ AIRT for wind speed class 3 and stability class F

1RESRAD-OFFSITE, Version 4.0 .3 beta $\mathrm{T}^{\frac{1}{2}} \mathrm{~L}$ Limit $=30$ days Parent Dose Report

Title : RESRAD-OFFSITE Effluent release

File : PADUCAH EFFLUENT RELEASE-NONINVOLVED-WORKER-REV.ROF

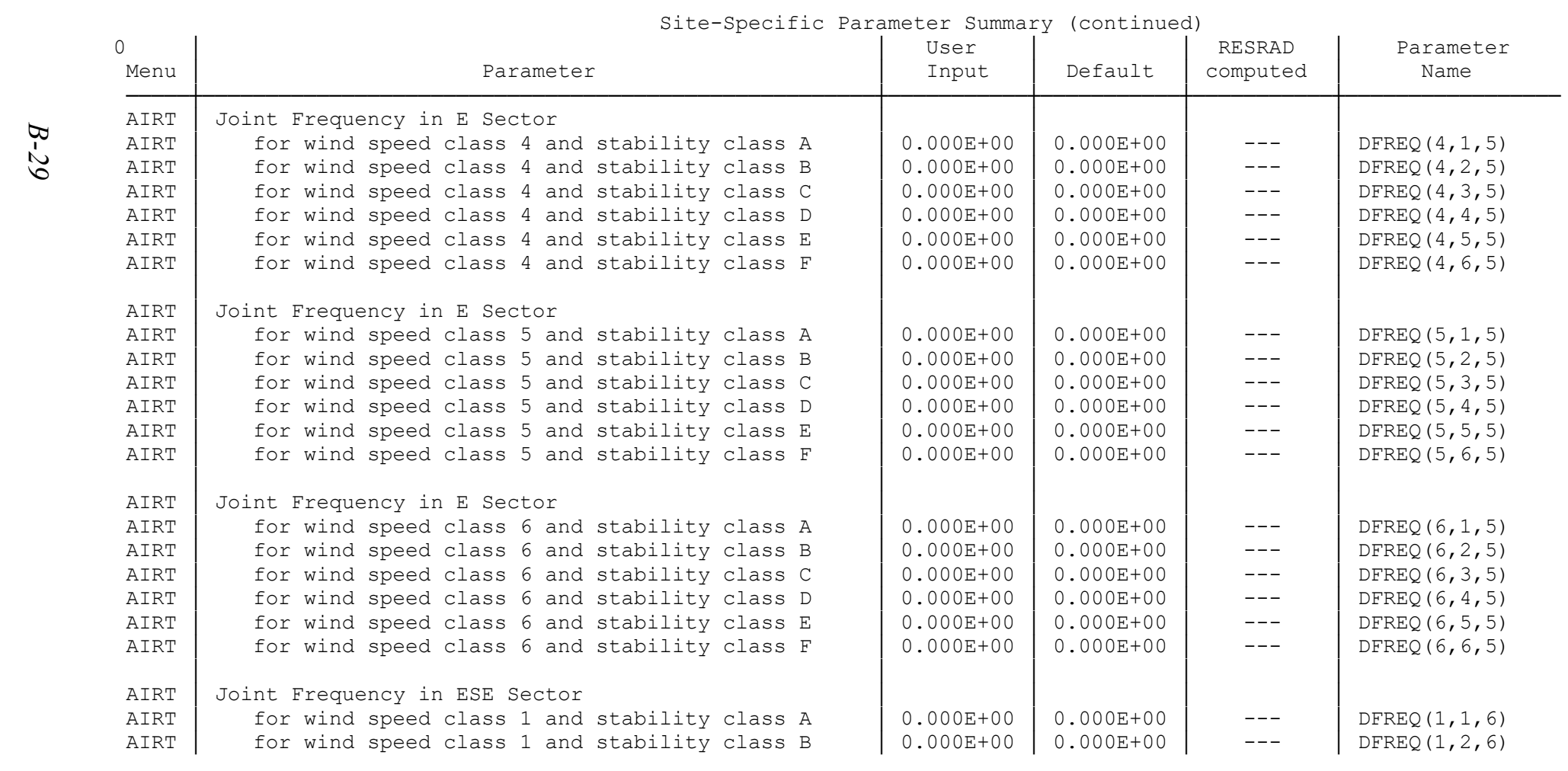

\begin{tabular}{|l|l|l|l}
$0.000 \mathrm{E}+00$ & $0.000 \mathrm{E}+00$ & -- & \\
$0.000 \mathrm{E}+00$ & $0.000 \mathrm{E}+00$ & -- & $\operatorname{DFREQ}(2,1,5)$ \\
$0.000 \mathrm{E}+00$ & $0.000 \mathrm{E}+00$ & -- & $\operatorname{DFREQ}(2,2,5)$ \\
$0.000 \mathrm{E}+00$ & $0.000 \mathrm{E}+00$ & -- & $\operatorname{DFREQ}(2,3,5)$ \\
$0.000 \mathrm{E}+00$ & $0.000 \mathrm{E}+00$ & --- & $\operatorname{DFREQ}(2,5,5)$ \\
$0.000 \mathrm{E}+00$ & $0.000 \mathrm{E}+00$ & -- & $\operatorname{DFREQ}(2,6,5)$ \\
& & & \\
$0.000 \mathrm{E}+00$ & $0.000 \mathrm{E}+00$ & -- & $\operatorname{DFREQ}(3,1,5)$ \\
$0.000 \mathrm{E}+00$ & $0.000 \mathrm{E}+00$ & --- & $\operatorname{DFREQ}(3,2,5)$ \\
$0.000 \mathrm{E}+00$ & $0.000 \mathrm{E}+00$ & --- & $\operatorname{DFREQ}(3,3,5)$ \\
$0.000 \mathrm{E}+00$ & $0.000 \mathrm{E}+00$ & -- & $\operatorname{DFREQ}(3,4,5)$ \\
$0.000 \mathrm{E}+00$ & $0.000 \mathrm{E}+00$ & -- & $\operatorname{DFREQ}(3,5,5)$ \\
$0.000 \mathrm{E}+00$ & $0.000 \mathrm{E}+00$ & --- & $\operatorname{DFREQ}(3,6,5)$
\end{tabular}


AIRT for wind speed class 1 and stability class $C$ AIRT

AIRT

AIRT

AIRT

AIRT

AIRT

AIRT

AIRT

AIRT

AIRT

AIRT

AIRT

AIRT

AIRT

AIRT

IRESRAD-OFFSITE, Version 4.0 .3 beta T1/2 Limit = 30 days

Parent Dose Report

Title : RESRAD-OFFSITE Effluent release
File : PADUCAH EFFLUENT RELEASE-NONINVOLVED-WORKER-REV.ROF

\begin{tabular}{|l|l|l|l}
$0.000 \mathrm{E}+00$ & $0.000 \mathrm{E}+00$ & --- & $\operatorname{DFREQ}(1,3,6)$ \\
$0.000 \mathrm{E}+00$ & $1.000 \mathrm{E}-01$ & -- & $\operatorname{DFREQ}(1,4,6)$ \\
$0.000 \mathrm{E}+00$ & $2.000 \mathrm{E}-01$ & --- & $\operatorname{DFREQ}(1,5,6)$ \\
$0.000 \mathrm{E}+00$ & $7.000 \mathrm{E}-01$ & --- & $\operatorname{DFREQ}(1,6,6)$ \\
& & & \\
$0.000 \mathrm{E}+00$ & $0.000 \mathrm{E}+00$ & -- & $\operatorname{DFREQ}(2,1,6)$ \\
$0.000 \mathrm{E}+00$ & $0.000 \mathrm{E}+00$ & -- & $\operatorname{DFREQ}(2,2,6)$ \\
$0.000 \mathrm{E}+00$ & $0.000 \mathrm{E}+00$ & -- & $\operatorname{DFREQ}(2,3,6)$ \\
$0.000 \mathrm{E}+00$ & $0.000 \mathrm{E}+00$ & --- & $\operatorname{DFREQ}(2,4,6)$ \\
$0.000 \mathrm{E}+00$ & $0.000 \mathrm{E}+00$ & --- & $\operatorname{DFREQ}(2,5,6)$ \\
$0.000 \mathrm{E}+00$ & $0.000 \mathrm{E}+00$ & -- & $\operatorname{DFREQ}(2,6,6)$ \\
& & & \\
$0.000 \mathrm{E}+00$ & $0.000 \mathrm{E}+00$ & --- & $\operatorname{DFREQ}(3,1,6)$ \\
$0.000 \mathrm{E}+00$ & $0.000 \mathrm{E}+00$ & --- & $\operatorname{DFREQ}(3,2,6)$ \\
$0.000 \mathrm{E}+00$ & $0.000 \mathrm{E}+00$ & --- & $\operatorname{DFREQ}(3,3,6)$ \\
$0.000 \mathrm{E}+00$ & $0.000 \mathrm{E}+00$ & -- & $\operatorname{DFREQ}(3,4,6)$ \\
$0.000 \mathrm{E}+00$ & $0.000 \mathrm{E}+00$ & -- & $\operatorname{DFREQ}(3,5,6)$ \\
$0.000 \mathrm{E}+00$ & $0.000 \mathrm{E}+00$ & -- & $\operatorname{DFREQ}(3,6,6)$ \\
$06 / 11 / 2020$ &
\end{tabular}

Site-Specific Parameter Summary (continued)

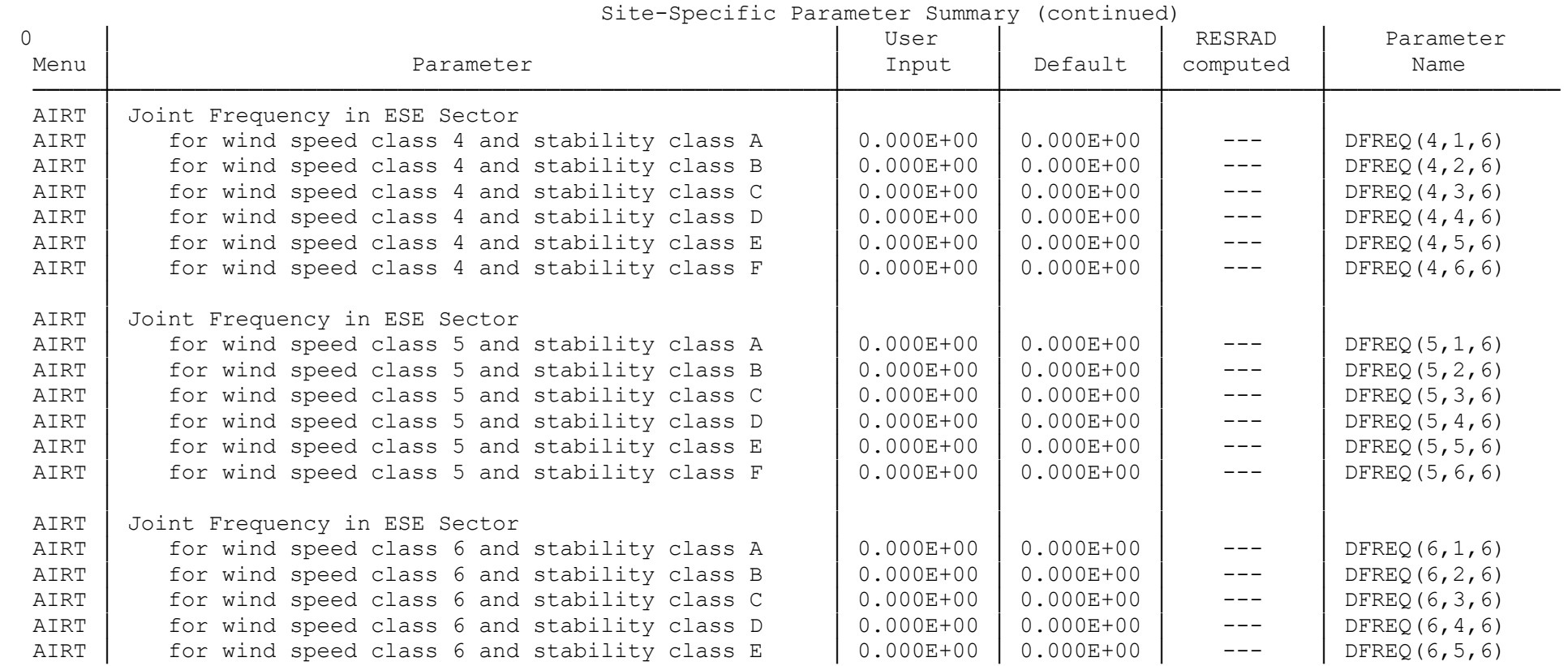


AIRT

AIRT

AIRT

AIRT

AIRT

AIRT

AIRT

AIRT

AIRT

AIRT

AIRT

AIRT

AIRT

AIRT

AIRT

AIRT

AIRT

AIRT

AIRT for wind speed class 6 and stability class F

Joint Frequency in SE Sector

for wind speed class 1 and stability class A for wind speed class 1 and stability class B for wind speed class 1 and stability class C for wind speed class 1 and stability class D for wind speed class 1 and stability class E for wind speed class 1 and stability class F

Joint Frequency in SE Sector

for wind speed class 2 and stability class A for wind speed class 2 and stability class B for wind speed class 2 and stability class C for wind speed class 2 and stability class D for wind speed class 2 and stability class E for wind speed class 2 and stability class F

Joint Frequency in SE Sector

for wind speed class 3 and stability class A for wind speed class 3 and stability class B for wind speed class 3 and stability class C for wind speed class 3 and stability class D for wind speed class 3 and stability class E for wind speed class 3 and stability class $F$ RAD-OFFSITE, Version 4.0 .3 beta $T^{1 / 2}$ Limit $=30$ days Parent Dose Report

Title : RESRAD-OFFSITE Effluent release

File: PADUCAH EFFLUENT RELEASE-NONINVOLVED-WORKER-REV.ROF

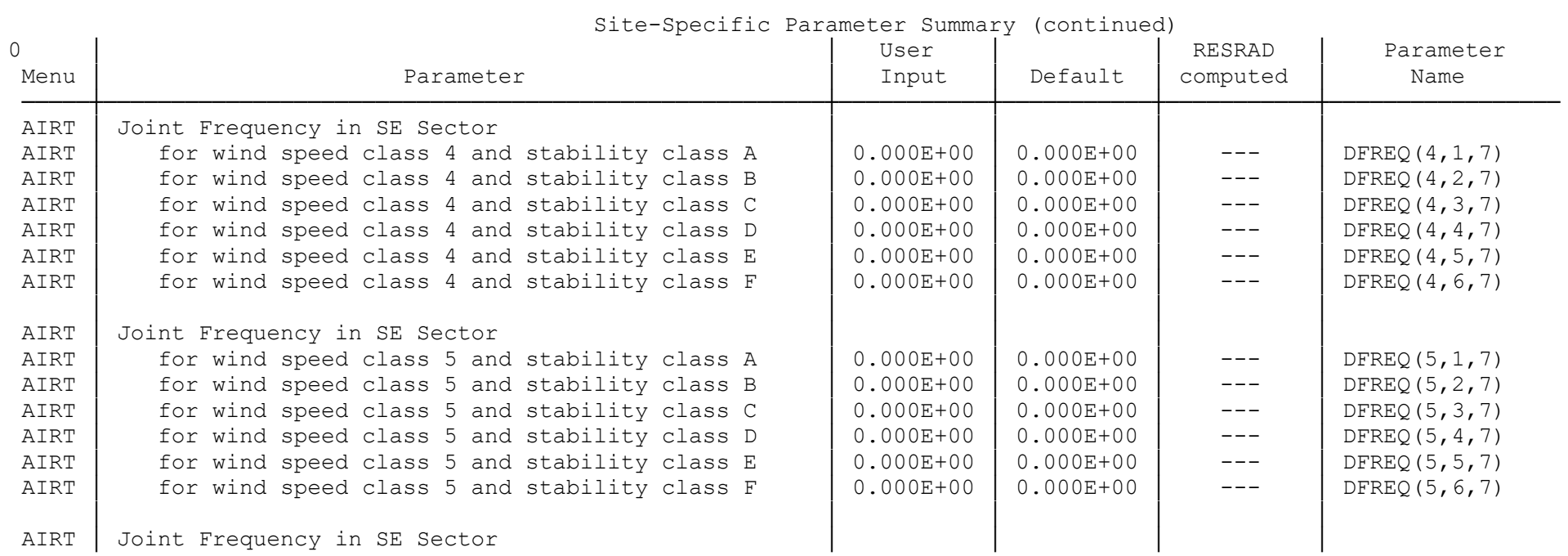

\begin{tabular}{|l|l|l|l}
$0.000 \mathrm{E}+00$ & $0.000 \mathrm{E}+00$ & --- & $\operatorname{DFREQ}(6,6,6)$ \\
$0.000 \mathrm{E}+00$ & $0.000 \mathrm{E}+00$ & --- & $\operatorname{DFREQ}(1,1,7)$ \\
$0.000 \mathrm{E}+00$ & $0.000 \mathrm{E}+00$ & --- & $\operatorname{DFREQ}(1,2,7)$ \\
$0.000 \mathrm{E}+00$ & $0.000 \mathrm{E}+00$ & --- & $\operatorname{DFREQ}(1,3,7)$ \\
$0.000 \mathrm{E}+00$ & $1.000 \mathrm{E}-01$ & --- & $\operatorname{DFREQ}(1,4,7)$ \\
$0.000 \mathrm{E}+00$ & $2.000 \mathrm{E}-01$ & -- & $\operatorname{DFREQ}(1,5,7)$ \\
$0.000 \mathrm{E}+00$ & $7.000 \mathrm{E}-01$ & -- & $\operatorname{DFREQ}(1,6,7)$ \\
& & & \\
$0.000 \mathrm{E}+00$ & $0.000 \mathrm{E}+00$ & --- & $\operatorname{DFREQ}(2,1,7)$ \\
$0.000 \mathrm{E}+00$ & $0.000 \mathrm{E}+00$ & --- & $\operatorname{DFREQ}(2,2,7)$ \\
$0.000 \mathrm{E}+00$ & $0.000 \mathrm{E}+00$ & -- & $\operatorname{DFREQ}(2,3,7)$ \\
$0.000 \mathrm{E}+00$ & $0.000 \mathrm{E}+00$ & -- & $\operatorname{DFREQ}(2,4,7)$ \\
$0.000 \mathrm{E}+00$ & $0.000 \mathrm{E}+00$ & -- & $\operatorname{DFREQ}(2,5,7)$ \\
$0.000 \mathrm{E}+00$ & $0.000 \mathrm{E}+00$ & --- & $\operatorname{DFREQ}(2,6,7)$ \\
& & & \\
$0.000 \mathrm{E}+00$ & $0.000 \mathrm{E}+00$ & -- & $\operatorname{DFREQ}(3,1,7)$ \\
$0.000 \mathrm{E}+00$ & $0.000 \mathrm{E}+00$ & -- & $\operatorname{DFREQ}(3,2,7)$ \\
$0.000 \mathrm{E}+00$ & $0.000 \mathrm{E}+00$ & --- & $\operatorname{DFREQ}(3,3,7)$ \\
$0.000 \mathrm{E}+00$ & $0.000 \mathrm{E}+00$ & --- & $\operatorname{DFREQ}(3,4,7)$ \\
$0.000 \mathrm{E}+00$ & $0.000 \mathrm{E}+00$ & --- & $\operatorname{DFREQ}(3,5,7)$ \\
$0.000 \mathrm{E}+00$ & $0.000 \mathrm{E}+00$ & -- & $\operatorname{DFREQ}(3,6,7)$
\end{tabular}

06/11/2020 11:39 Page 30 


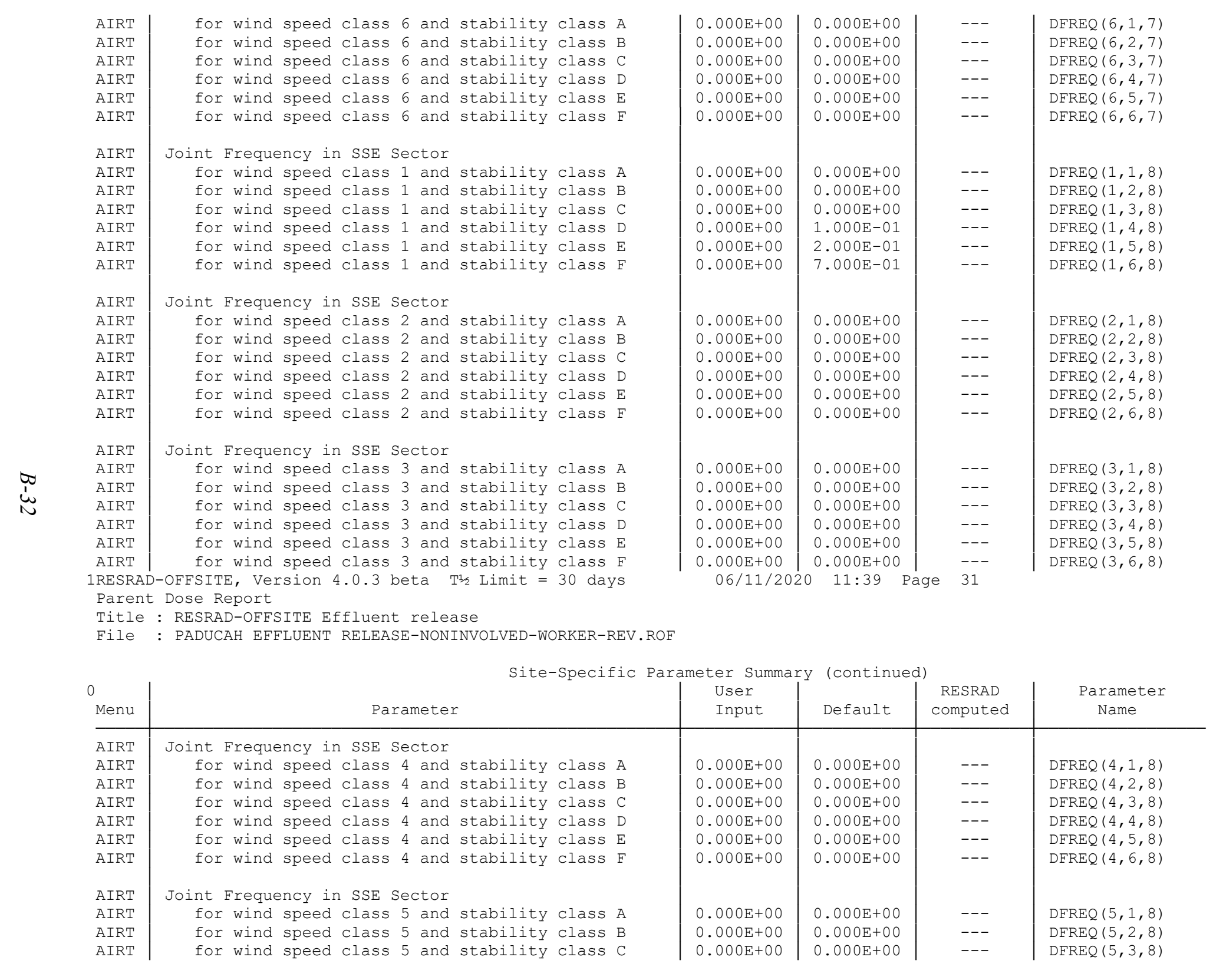



\begin{tabular}{l|ll} 
AIRT & for wind speed class 5 and stability class D \\
AIRT & for wind speed class 5 and stability class $\mathrm{E}$
\end{tabular}

AIRT AIRT AIRT AIRT AIRT

AIRT

AIRT

AIRT

AIRT

AIRT

AIRT

AIRT

AIRT

AIRT

AIRT

AIRT

AIRT

AIRT for wind speed class 5 and stability class F

Joint Frequency in SSE Sector

for wind speed class 6 and stability class A for wind speed class 6 and stability class B for wind speed class 6 and stability class C for wind speed class 6 and stability class D for wind speed class 6 and stability class for wind speed class 6 and stability class F

Joint Frequency in $\mathrm{S}$ Sector

for wind speed class 1 and stability class A for wind speed class 1 and stability class B for wind speed class 1 and stability class C for wind speed class 1 and stability class D for wind speed class 1 and stability Class $E$ for wind speed class 1 and stability class F

Joint Frequency in $S$ Sector

for wind speed class 2 and stability class A for wind speed class 2 and stability class B for wind speed class 2 and stability class C for wind speed class 2 and stability class D for wind speed class 2 and stability class E for wind speed class 2 and stability class F
AIRT Joint Frequency in $S$ sector

AIRT for wind speed class 3 and stability class A

AIRT for wind speed class 3 and stability class B

AIRT for wind speed class 3 and stability class C

AIRT for wind speed class 3 and stability class D

AIRT for wind speed class 3 and stability class E

AIRT for wind speed class 3 and stability Class $F$

1RESRAD-OFFSITE, Version 4.0 .3 beta $\mathrm{T}^{1} \frac{1}{2}$ Limit $=30$ days

Parent Dose Report

Title : RESRAD-OFFSITE Effluent release

File : PADUCAH EFFLUENT RELEASE-NONINVOLVED-WORKER-REV.ROF

\begin{tabular}{|l|l|l|l}
$0.000 \mathrm{E}+00$ & $0.000 \mathrm{E}+00$ & --- & $\operatorname{DFREQ}(5,4,8)$ \\
$0.000 \mathrm{E}+00$ & $0.000 \mathrm{E}+00$ & --- & $\operatorname{DFREQ}(5,5,8)$ \\
$0.000 \mathrm{E}+00$ & $0.000 \mathrm{E}+00$ & --- & $\operatorname{DFREQ}(5,6,8)$ \\
& & & \\
$0.000 \mathrm{E}+00$ & $0.000 \mathrm{E}+00$ & --- & $\operatorname{DFREQ}(6,1,8)$ \\
$0.000 \mathrm{E}+00$ & $0.000 \mathrm{E}+00$ & --- & $\operatorname{DFREQ}(6,2,8)$ \\
$0.000 \mathrm{E}+00$ & $0.000 \mathrm{E}+00$ & --- & $\operatorname{DFREQ}(6,3,8)$ \\
$0.000 \mathrm{E}+00$ & $0.000 \mathrm{E}+00$ & --- & $\operatorname{DFREQ}(6,4,8)$ \\
$0.000 \mathrm{E}+00$ & $0.000 \mathrm{E}+00$ & --- & $\operatorname{DFREQ}(6,5,8)$ \\
$0.000 \mathrm{E}+00$ & $0.000 \mathrm{E}+00$ & --- & $\operatorname{DFREQ}(6,6,8)$ \\
& & & \\
$0.000 \mathrm{E}+00$ & $0.000 \mathrm{E}+00$ & --- & $\operatorname{DFREQ}(1,1,9)$ \\
$0.000 \mathrm{E}+00$ & $0.000 \mathrm{E}+00$ & --- & $\operatorname{DFREQ}(1,2,9)$ \\
$0.000 \mathrm{E}+00$ & $0.000 \mathrm{E}+00$ & --- & $\operatorname{DFREQ}(1,3,9)$ \\
$0.000 \mathrm{E}+00$ & $1.000 \mathrm{E}-01$ & --- & $\operatorname{DFREQ}(1,4,9)$ \\
$0.000 \mathrm{E}+00$ & $2.000 \mathrm{E}-01$ & --- & $\operatorname{DFREQ}(1,5,9)$ \\
$0.000 \mathrm{E}+00$ & $7.000 \mathrm{E}-01$ & --- & $\operatorname{DFREQ}(1,6,9)$ \\
& & & \\
$0.000 \mathrm{E}+00$ & $0.000 \mathrm{E}+00$ & --- & $\operatorname{DFREQ}(2,1,9)$ \\
$0.000 \mathrm{E}+00$ & $0.000 \mathrm{E}+00$ & --- & $\operatorname{DFREQ}(2,2,9)$ \\
$0.000 \mathrm{E}+00$ & $0.000 \mathrm{E}+00$ & --- & $\operatorname{DFREQ}(2,3,9)$ \\
$0.000 \mathrm{E}+00$ & $0.000 \mathrm{E}+00$ & --- & $\operatorname{DFREQ}(2,4,9)$ \\
$0.000 \mathrm{E}+00$ & $0.000 \mathrm{E}+00$ & --- & $\operatorname{DFREQ}(2,5,9)$ \\
$0.000 \mathrm{E}+00$ & $0.000 \mathrm{E}+00$ & --- & $\operatorname{DFREQ}(2,6,9)$ \\
& & & \\
$0.000 \mathrm{E}+00$ & $0.000 \mathrm{E}+00$ & --- & $\operatorname{DFREQ}(3,1,9)$ \\
$0.000 \mathrm{E}+00$ & $0.000 \mathrm{E}+00$ & --- & $\operatorname{DFREQ}(3,2,9)$ \\
$0.000 \mathrm{E}+00$ & $0.000 \mathrm{E}+00$ & --- & $\operatorname{DFREQ}(3,3,9)$ \\
$0.000 \mathrm{E}+00$ & $0.000 \mathrm{E}+00$ & --- & $\operatorname{DFREQ}(3,4,9)$ \\
$0.000 \mathrm{E}+00$ & $0.000 \mathrm{E}+00$ & --- & $\operatorname{DFREQ}(3,5,9)$ \\
$0.000 \mathrm{E}+00$ & $0.000 \mathrm{E}+00$ & --- & $\operatorname{DFREQ}(3,6,9)$
\end{tabular}

$06 / 11 / 202011: 39$ Page 32

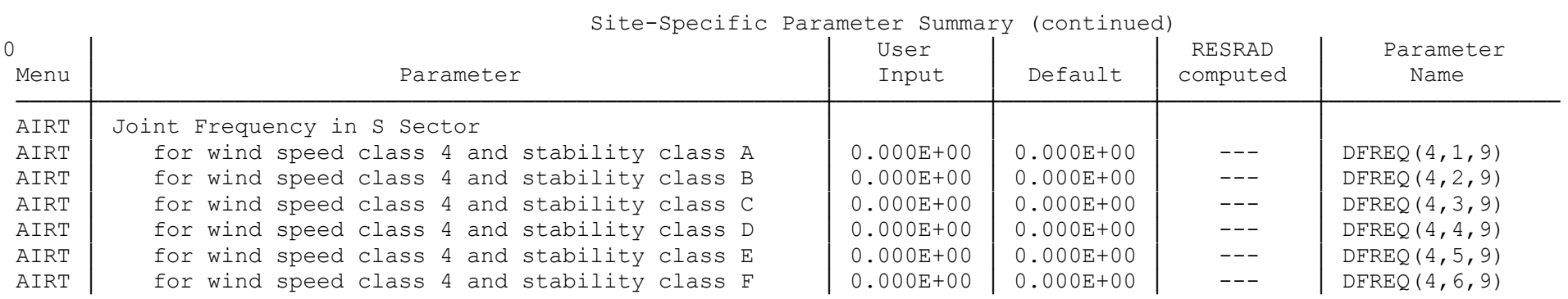




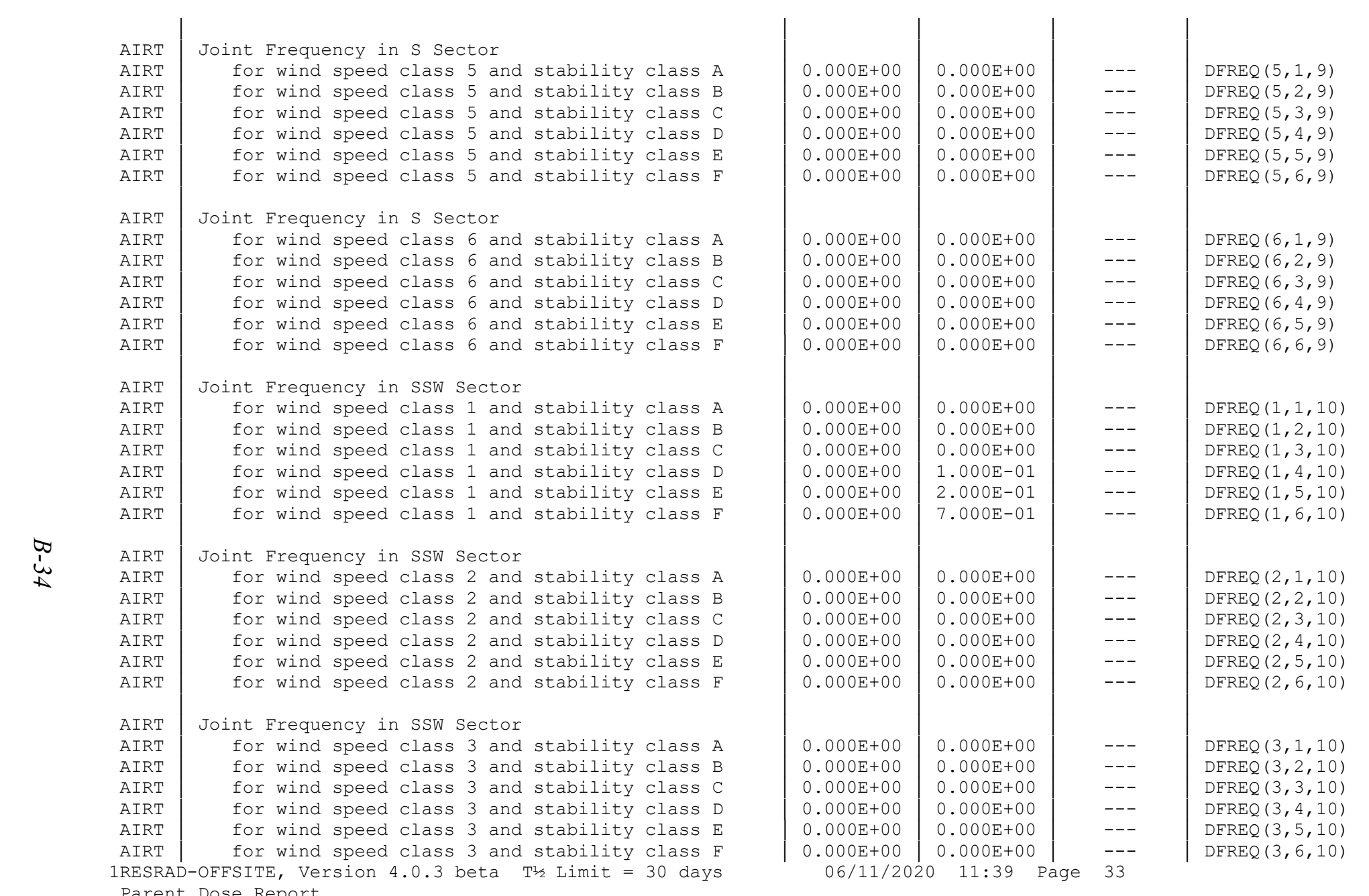

Parent Dose Report

Title: RESRAD-OFFSITE Effluent release

File : PADUCAH EFFLUENT RELEASE-NONINVOLVED-WORKER-REV.ROF

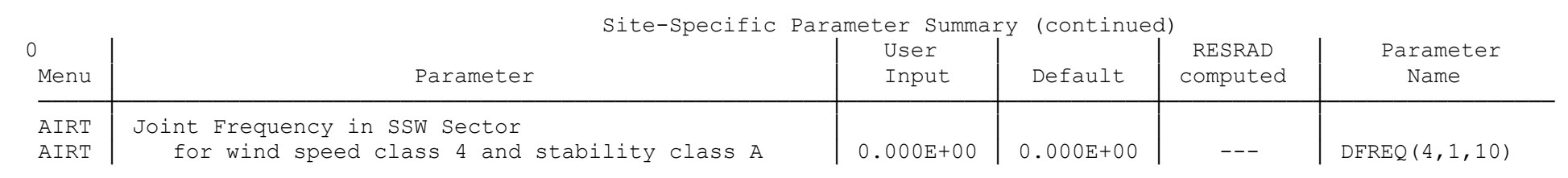


AIRT for wind speed class 4 and stability class B for wind speed class 4 and stability class $C$ for wind speed class 4 and stability class D for wind speed class 4 and stability class E for wind speed class 4 and stability class F

Joint Frequency in SSW Sector

for wind speed class 5 and stability class A for wind speed class 5 and stability class B for wind speed class 5 and stability class C for wind speed class 5 and stability class D for wind speed class 5 and stability class E for wind speed class 5 and stability class F

Joint Frequency in SSW Sector

for wind speed class 6 and stability class A for wind speed class 6 and stability class B for wind speed class 6 and stability class C for wind speed class 6 and stability class D for wind speed class 6 and stability class E for wind speed class 6 and stability class F

Joint Frequency in SW Sector

for wind speed class 1 and stability class A for wind speed class 1 and stability class B for wind speed class 1 and stability class $C$

for wind speed class 1 and stability class D

AIRT AIRT

AIRT

AIRT

AIRT

AIRT

AIRT

AIRT

AIRT

AIRT

AIRT

AIRT

AIRT

AIRT

AIRT for wind speed class 1 and stability class $\mathrm{E}$

Joint Frequency in SW Sector

for wind speed class 2 and stability class A for wind speed class 2 and stability class B for wind speed class 2 and stability class C for wind speed class 2 and stability class D for wind speed class 2 and stability class E for wind speed class 2 and stability class F

Joint Frequency in SW Sector

for wind speed class 3 and stability class A for wind speed class 3 and stability class B for wind speed class 3 and stability class C for wind speed class 3 and stability class D for wind speed class 3 and stability class E for wind speed class 3 and stability class F IRESRAD-OFFSITE, Version 4.0 .3 beta $\mathrm{T}^{1 / 2} \mathrm{Limit}=30$ days Parent Dose Report

Title : RESRAD-OFFSITE Effluent release

File : PADUCAH EFFLUENT RELEASE-NONINVOLVED-WORKER-REV.ROF

\begin{tabular}{|l|l|l|l}
$0.000 \mathrm{E}+00$ & $0.000 \mathrm{E}+00$ & --- & $\operatorname{DFREQ}(4,2,10)$ \\
$0.000 \mathrm{E}+00$ & $0.000 \mathrm{E}+00$ & --- & $\operatorname{DFREQ}(4,3,10)$ \\
$0.000 \mathrm{E}+00$ & $0.000 \mathrm{E}+00$ & --- & $\operatorname{DFREQ}(4,4,10)$ \\
$0.000 \mathrm{E}+00$ & $0.000 \mathrm{E}+00$ & -- & $\operatorname{DFREQ}(4,5,10)$ \\
$0.000 \mathrm{E}+00$ & $0.000 \mathrm{E}+00$ & -- & $\operatorname{DFREQ}(4,6,10)$ \\
& & & \\
$0.000 \mathrm{E}+00$ & $0.000 \mathrm{E}+00$ & --- & $\operatorname{DFREQ}(5,1,10)$ \\
$0.000 \mathrm{E}+00$ & $0.000 \mathrm{E}+00$ & --- & $\operatorname{DFREQ}(5,2,10)$ \\
$0.000 \mathrm{E}+00$ & $0.000 \mathrm{E}+00$ & --- & $\operatorname{DFREQ}(5,3,10)$ \\
$0.000 \mathrm{E}+00$ & $0.000 \mathrm{E}+00$ & --- & $\operatorname{DFREQ}(5,4,10)$ \\
$0.000 \mathrm{E}+00$ & $0.000 \mathrm{E}+00$ & --- & $\operatorname{DFREQ}(5,5,10)$ \\
$0.000 \mathrm{E}+00$ & $0.000 \mathrm{E}+00$ & --- & $\operatorname{DFREQ}(5,6,10)$ \\
& & & \\
$0.000 \mathrm{E}+00$ & $0.000 \mathrm{E}+00$ & --- & $\operatorname{DFREQ}(6,1,10)$ \\
$0.000 \mathrm{E}+00$ & $0.000 \mathrm{E}+00$ & --- & $\operatorname{DFREQ}(6,2,10)$ \\
$0.000 \mathrm{E}+00$ & $0.000 \mathrm{E}+00$ & --- & $\operatorname{DFREQ}(6,3,10)$ \\
$0.000 \mathrm{E}+00$ & $0.000 \mathrm{E}+00$ & --- & $\operatorname{DFREQ}(6,4,10)$ \\
$0.000 \mathrm{E}+00$ & $0.000 \mathrm{E}+00$ & --- & $\operatorname{DFREQ}(6,5,10)$ \\
$0.000 \mathrm{E}+00$ & $0.000 \mathrm{E}+00$ & --- & $\operatorname{DFREQ}(6,6,10)$ \\
& & & \\
$0.000 \mathrm{E}+00$ & $0.000 \mathrm{E}+00$ & --- & $\operatorname{DFREQ}(1,1,11)$ \\
$0.000 \mathrm{E}+00$ & $0.000 \mathrm{E}+00$ & --- & $\operatorname{DFREQ}(1,2,11)$ \\
$0.000 \mathrm{E}+00$ & $0.000 \mathrm{E}+00$ & --- & $\operatorname{DFREQ}(1,3,11)$ \\
$0.000 \mathrm{E}+00$ & $1.000 \mathrm{E}-01$ & --- & $\operatorname{DFREQ}(1,4,11)$ \\
$0.000 \mathrm{E}+00$ & $2.000 \mathrm{E}-01$ & --- & $\operatorname{DFREQ}(1,5,11)$ \\
$0.000 \mathrm{E}+00$ & $7.000 \mathrm{E}-01$ & --- & $\operatorname{DFREQ}(1,6,11)$ \\
$0.000 \mathrm{E}+00$ & $0.000 \mathrm{E}+00$ & --- & $\operatorname{DFREQ}(2,1,11)$ \\
$0.000 \mathrm{E}+00$ & $0.000 \mathrm{E}+00$ & --- & $\operatorname{DFREQ}(2,2,11)$ \\
$0.000 \mathrm{E}+00$ & $0.000 \mathrm{E}+00$ & --- & $\operatorname{DFREQ}(2,3,11)$ \\
$0.000 \mathrm{E}+00$ & $0.000 \mathrm{E}+00$ & --- & $\operatorname{DFREQ}(2,4,11)$ \\
$0.000 \mathrm{E}+00$ & $0.000 \mathrm{E}+00$ & --- & $\operatorname{DFREQ}(2,5,11)$ \\
$0.000 \mathrm{E}+00$ & $0.000 \mathrm{E}+00$ & --- & $\operatorname{DFREQ}(2,6,11)$ \\
$0.000 \mathrm{E}+00$ & $0.000 \mathrm{E}+00$ & --- & $\operatorname{DFREQ}(3,1,11)$ \\
$0.000 \mathrm{E}+00$ & $0.000 \mathrm{E}+00$ & --- & $\operatorname{DFREQ}(3,2,11)$ \\
$0.000 \mathrm{E}+00$ & $0.000 \mathrm{E}+00$ & --- & $\operatorname{DFREQ}(3,3,11)$ \\
$0.000 \mathrm{E}+00$ & $0.000 \mathrm{E}+00$ & --- & $\operatorname{DFREQ}(3,4,11)$ \\
$0.000 \mathrm{E}+00$ & $0.000 \mathrm{E}+00$ & --- & $\operatorname{DFREQ}(3,5,11)$ \\
$0.000 \mathrm{E}+00$ & $0.000 \mathrm{E}+00$ & --- & $\operatorname{DFREQ}(3,6,11)$ \\
$060111 / 2020$ & $111: 39$ & 34 &
\end{tabular}

$\operatorname{DFREQ}(3,5,11)$
$\operatorname{DFREQ}(3,6,11)$ 


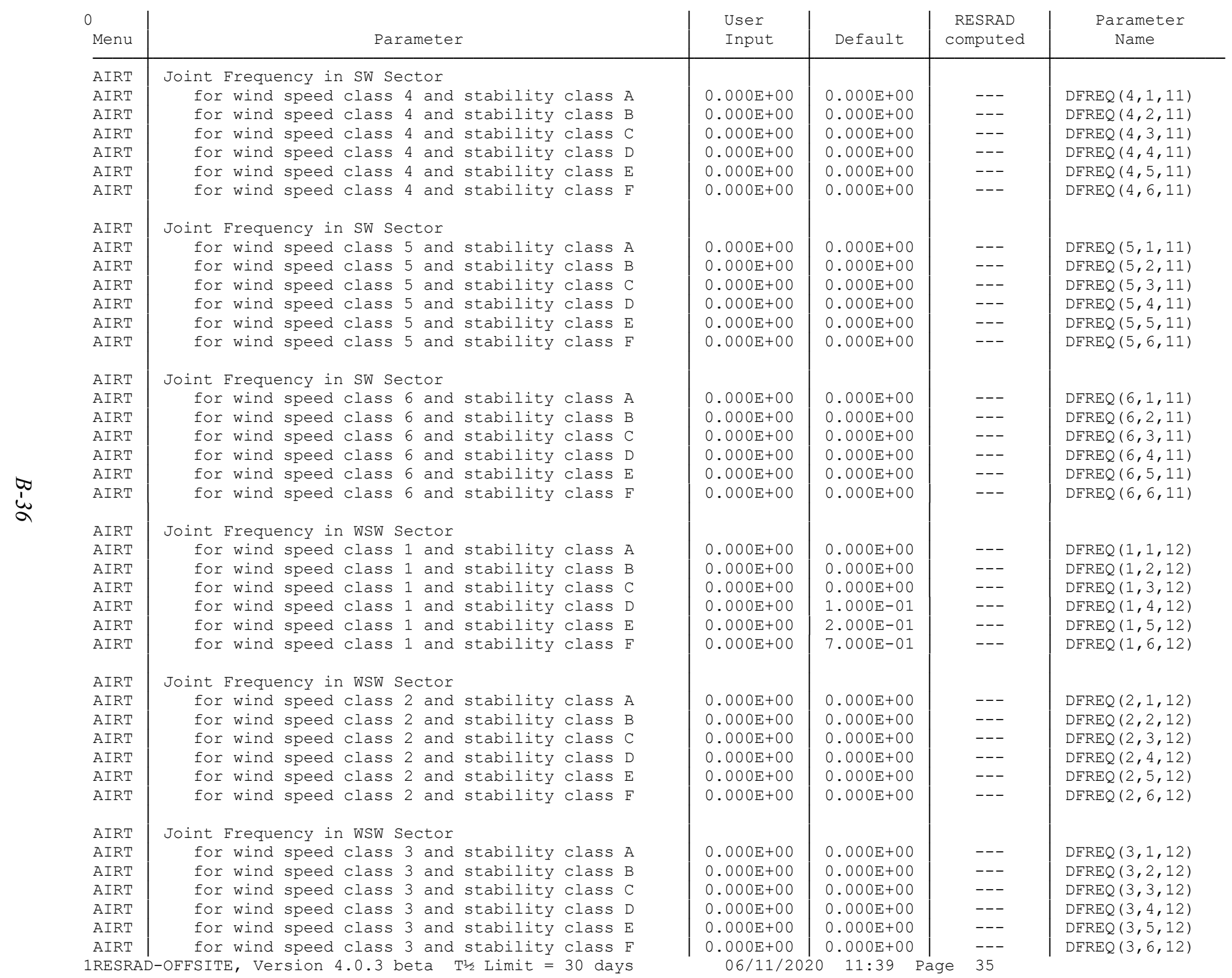


Parent Dose Report

Title : RESRAD-OFFSITE Effluent release

File : PADUCAH EFFLUENT RELEASE-NONINVOLVED-WORKER-REV.ROF

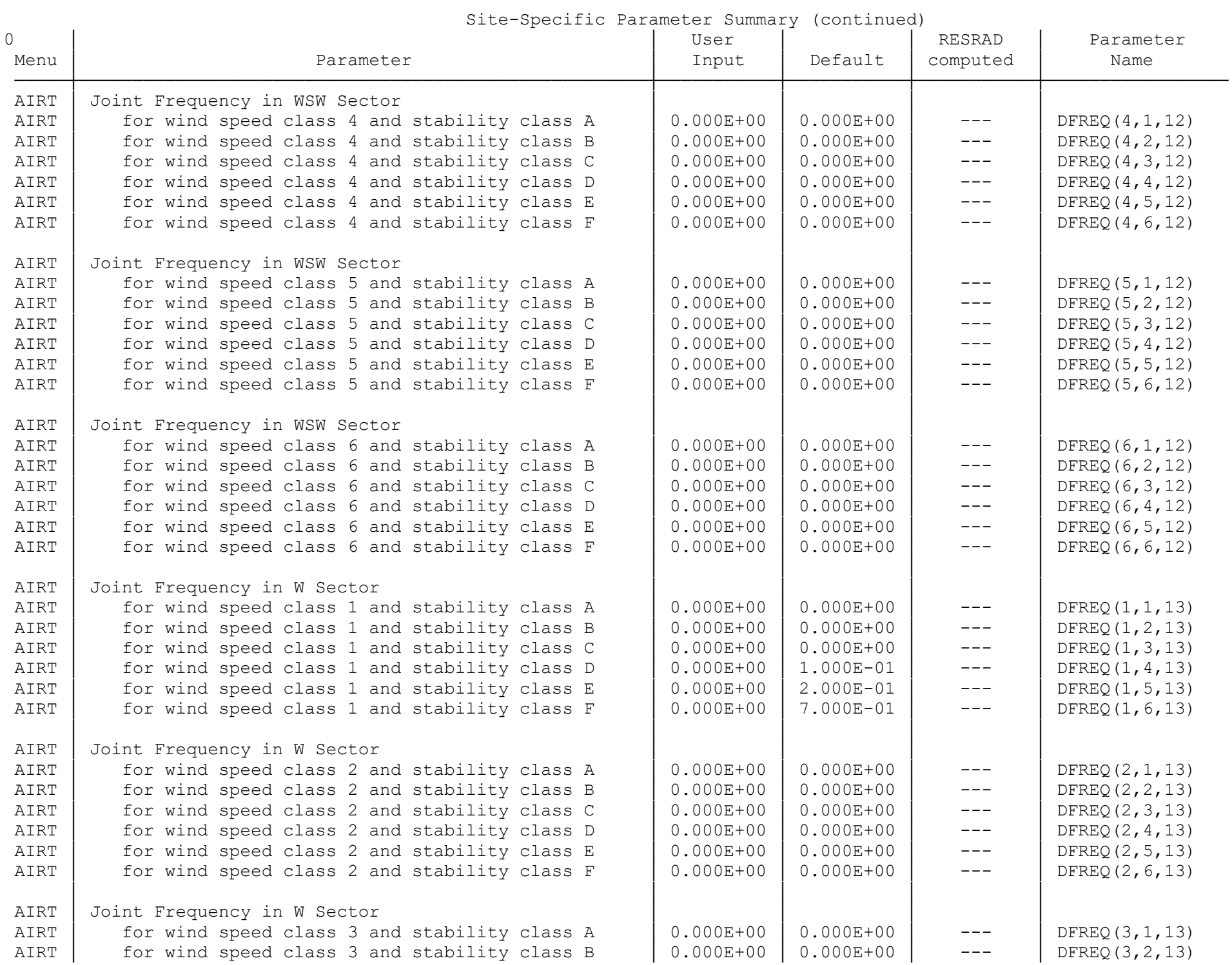


AIRT for wind speed class 3 and stability class C

AIRT for wind speed class 3 and stability class D

AIRT for wind speed class 3 and stability class $E$

AIRT for wind speed class 3 and stability class F

1RESRAD-OFFSITE, Version 4.0 .3 beta T1/2 Limit = 30 days

Parent Dose Report

Title : RESRAD-OFFSITE Effluent release

File : PADUCAH EFFLUENT RELEASE-NONINVOLVED-WORKER-REV.ROF

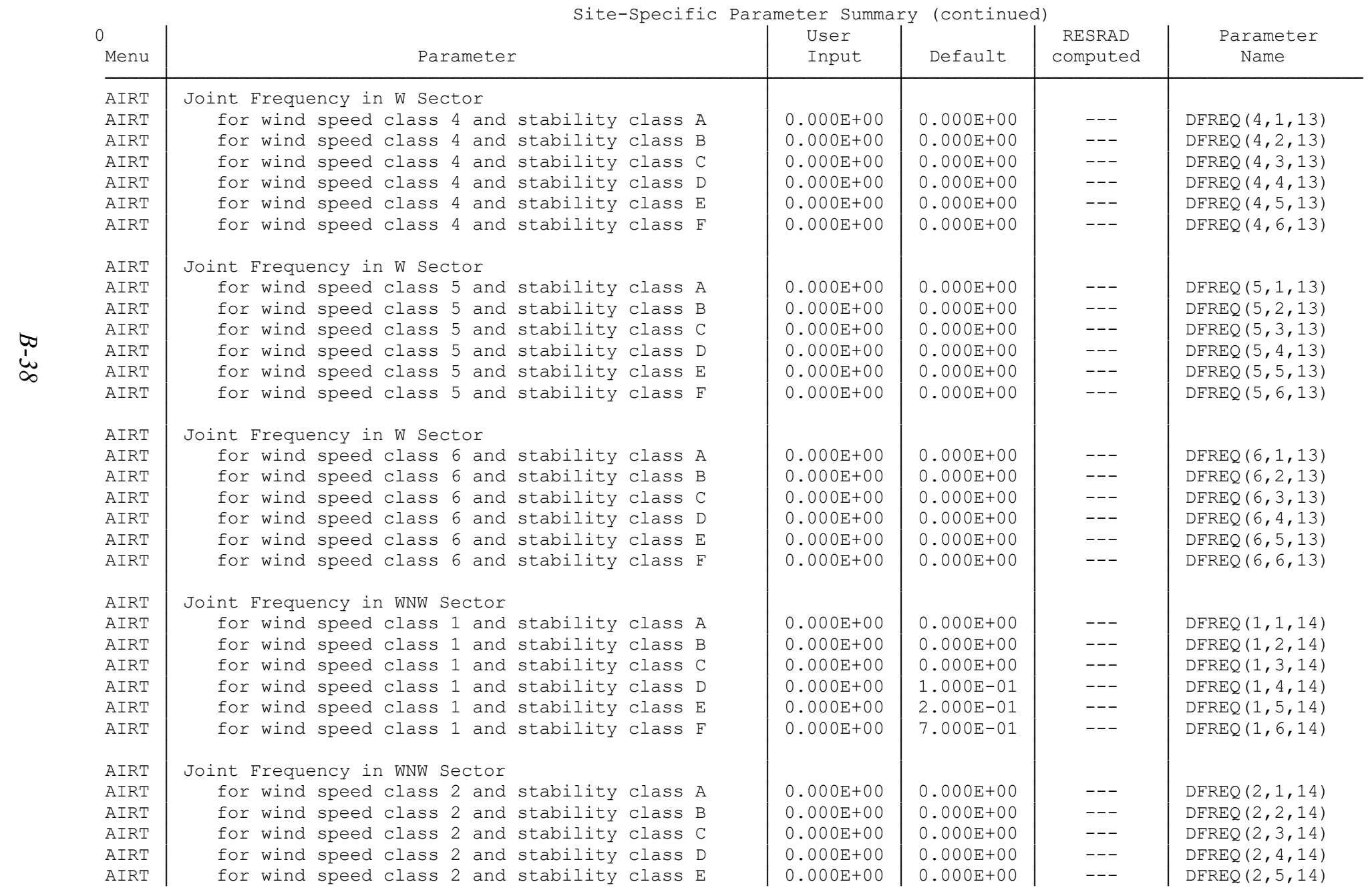

\begin{tabular}{l|l|l|l}
$0.000 \mathrm{E}+00$ & $0.000 \mathrm{E}+00$ & --- & $\operatorname{DFREQ}(3,3,13)$ \\
$0.000 \mathrm{E}+00$ & $0.000 \mathrm{E}+00$ & -- & DFREQ $(3,4,13)$ \\
$0.000 \mathrm{E}+00$ & $0.000 \mathrm{E}+00$ & --- & DFREQ $(3,5,13)$ \\
$0.000 \mathrm{E}+00$ & $0.000 \mathrm{E}+00$ & --- & DFREQ $(3,6,13)$
\end{tabular}

06/11/2020 11:39 Page 36 
AIRT for wind speed class 2 and stability class $F$

AIRT Joint Frequency in WNW Sector

AIRT for wind speed class 3 and stability class A

AIRT for wind speed class 3 and stability class B

AIRT for wind speed class 3 and stability class C

AIRT for wind speed class 3 and stability class D

AIRT for wind speed class 3 and stability class E

AIRT for wind speed class 3 and stability class F

1RESRAD-OFFSITE, Version 4.0 .3 beta T1/2 Limit $=30$ days Parent Dose Report

Title : RESRAD-OFFSITE Effluent release

File: PADUCAH EFFLUENT RELEASE-NONINVOLVED-WORKER-REV.ROF

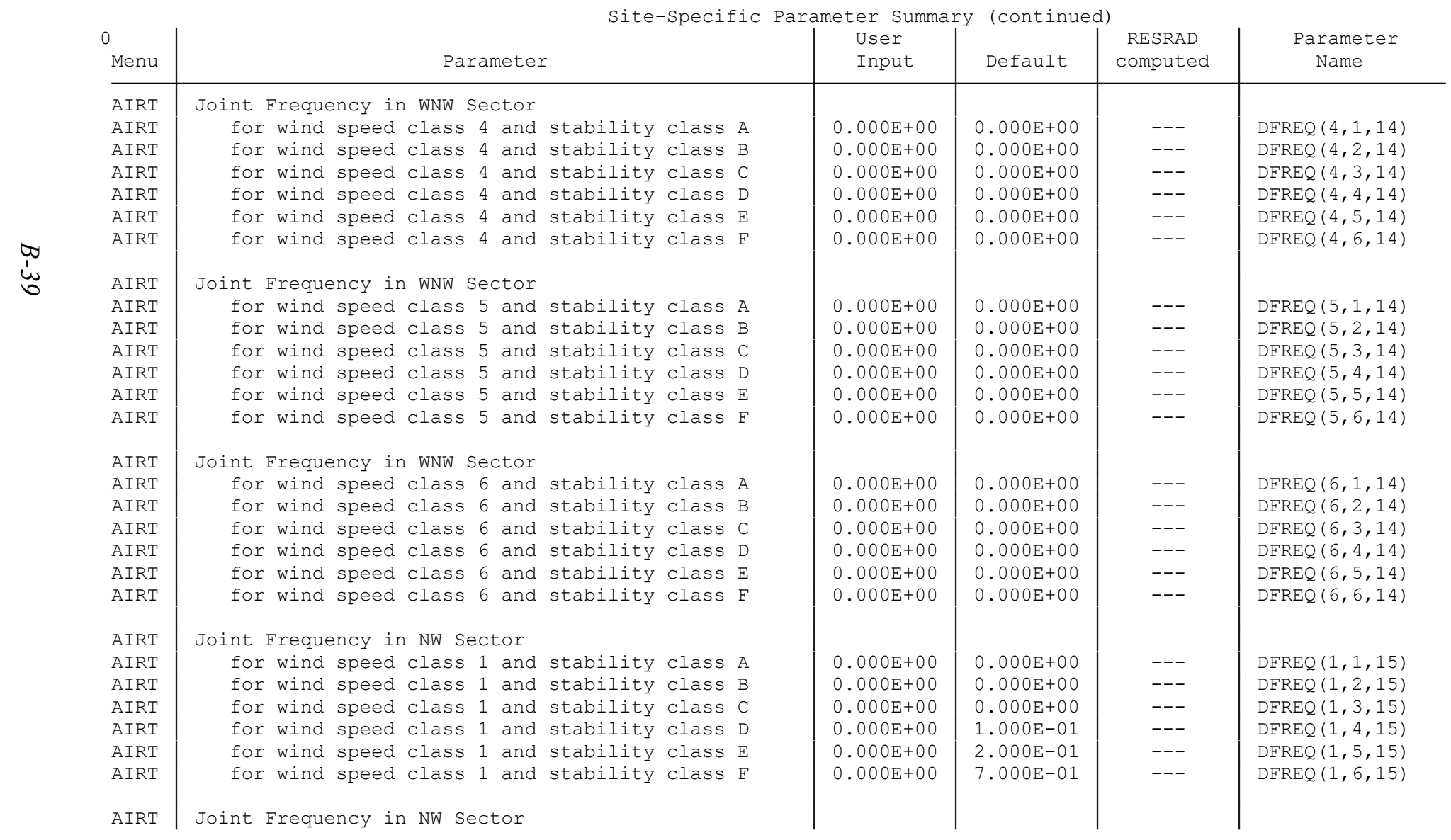

\begin{tabular}{|l|l|l|l|}
$0.000 \mathrm{E}+00$ & $0.000 \mathrm{E}+00$ & --- & $\operatorname{DFREQ}(2,6,14)$ \\
& & & \\
$0.000 \mathrm{E}+00$ & $0.000 \mathrm{E}+00$ & --- & $\operatorname{DFREQ}(3,1,14)$ \\
$0.000 \mathrm{E}+00$ & $0.000 \mathrm{E}+00$ & --- & $\operatorname{DFREQ}(3,2,14)$ \\
$0.000 \mathrm{E}+00$ & $0.000 \mathrm{E}+00$ & --- & $\operatorname{DFREQ}(3,3,14)$ \\
$0.000 \mathrm{E}+00$ & $0.000 \mathrm{E}+00$ & --- & $\operatorname{DFREQ}(3,4,14)$ \\
$0.000 \mathrm{E}+00$ & $0.000 \mathrm{E}+00$ & -- & $\operatorname{DFREQ}(3,5,14)$ \\
$0.000 \mathrm{E}+00$ & $0.000 \mathrm{E}+00$ & -- & $\operatorname{DFREQ}(3,6,14)$
\end{tabular}




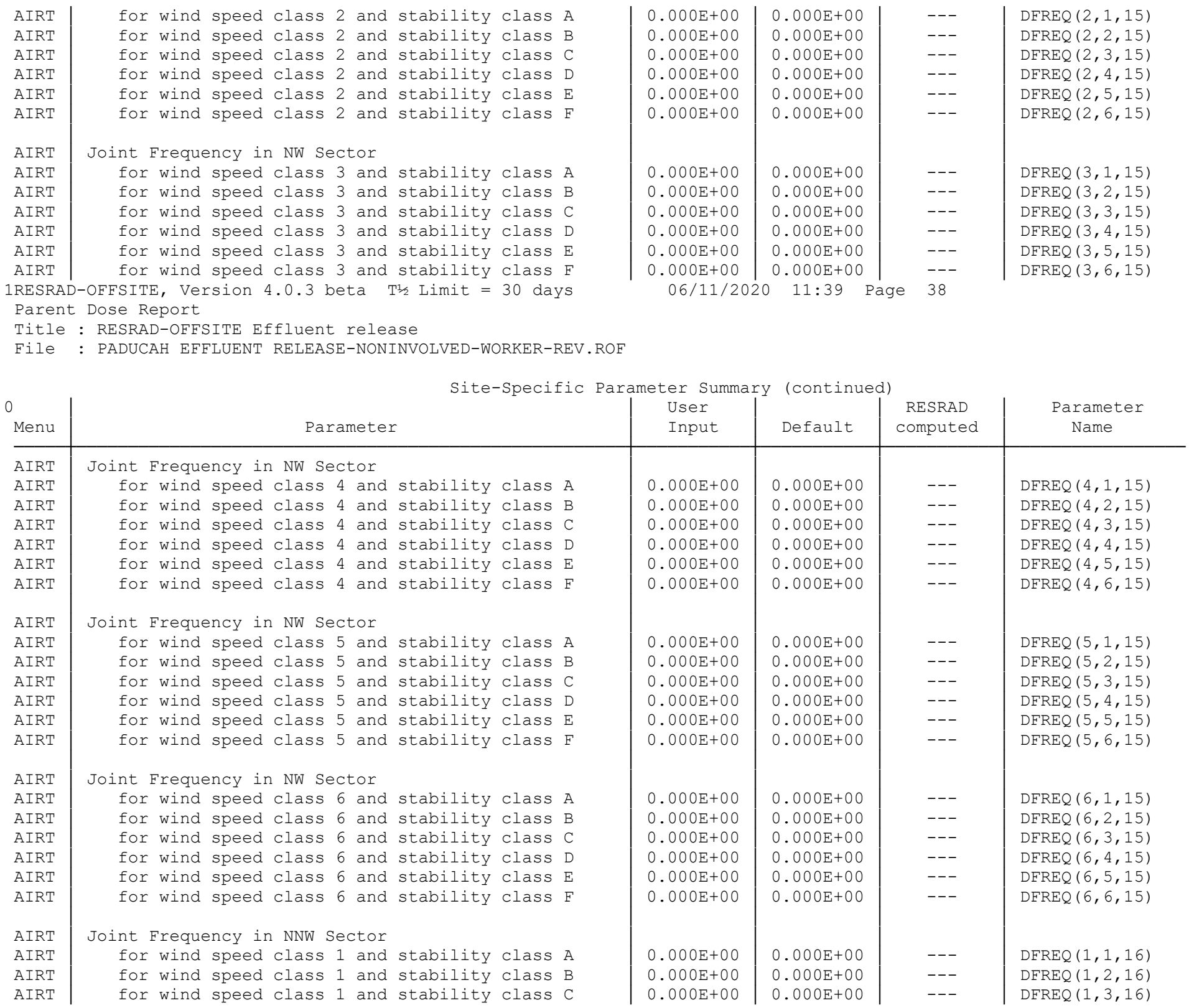




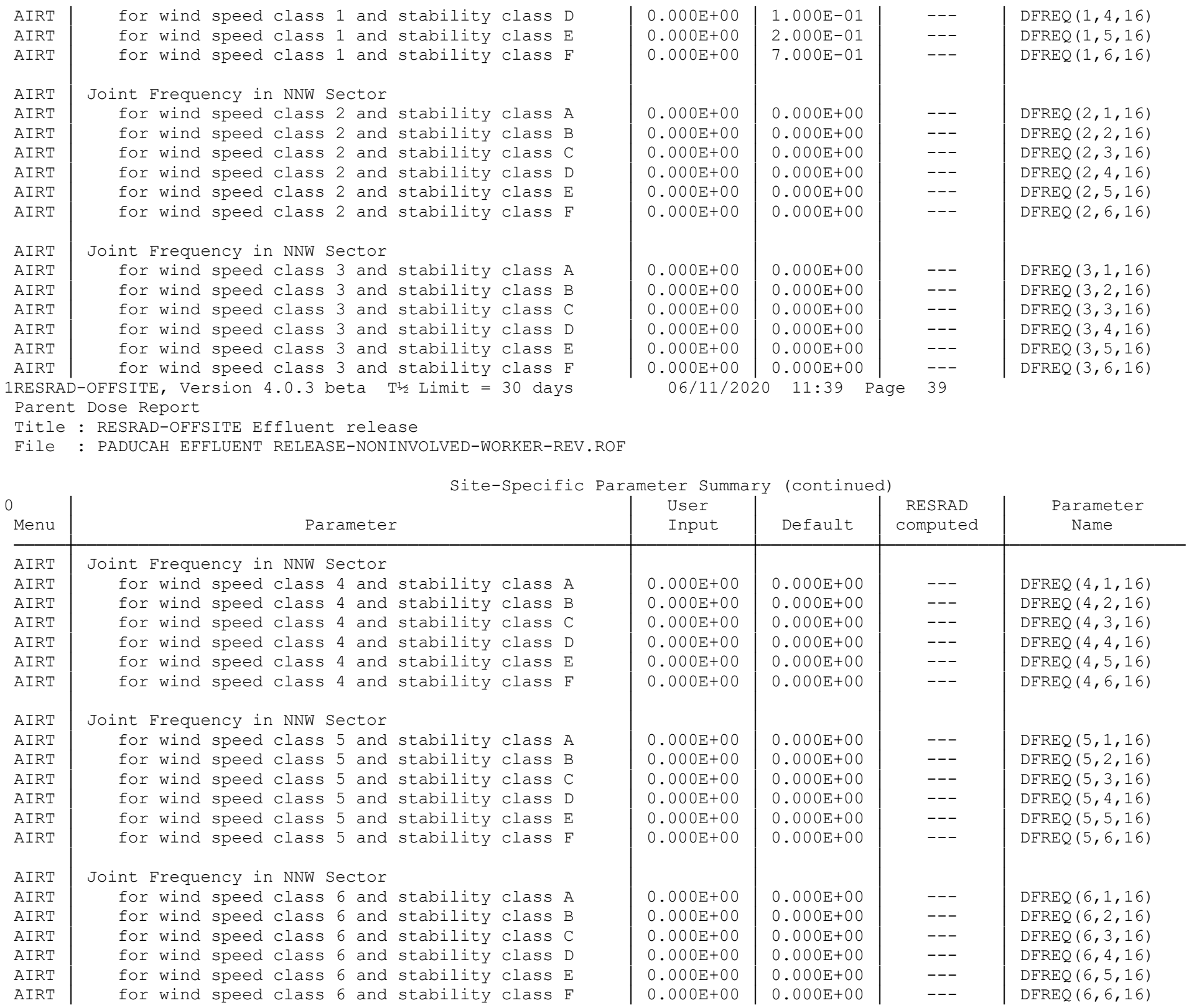


AIRT | Average annual wind speed ( $\mathrm{m} / \mathrm{sec})$

AIRT

GWTR

GWTR

GWTR

GWTR

GWTR

GWTR

GiTTR

Gire

GWTR

GWTR

GWTR

GWTR

GWTR

GWTR

GWTR

GW'R

1 = Nuclide specific distrubution coefficients in

transformation.

$0=$ (total porosity + distribution coefficient*dry bu

1RESRAD-OFFSITE, Version 4.0 .3 beta $\mathrm{T}^{\frac{1}{2}} \mathrm{Limit}=30$ days

Parent Dose Report

Title : RESRAD-OFFSITE Effluent release

File : PADUCAH EFFLUENT RELEASE-NONINVOLVED-WORKER-REV.ROF

\begin{tabular}{|c|c|c|c|c|c|}
\hline Menu & Site-Specific Par & $\begin{array}{c}\text { meter Summ } \\
\text { User } \\
\text { Input }\end{array}$ & $\begin{array}{l}\text { (continu } \\
\text { Default }\end{array}$ & $\begin{array}{l}\text { RESRAD } \\
\text { computed }\end{array}$ & $\begin{array}{l}\text { Parameter } \\
\text { Name }\end{array}$ \\
\hline USZN & Number of unsaturated zone strata & 1 & 1 & --- & NS \\
\hline USZN & Unsat. zone 1 , soil density $(\mathrm{g} / \mathrm{cm} * * 3)$ & $1.500 \mathrm{E}+00$ & $1.500 \mathrm{E}+00$ & --- & DENSUZ (1) \\
\hline USZN & Unsat. zone 1, total porosity & $4.000 \mathrm{E}-01$ & $4.000 \mathrm{E}-01$ & --- & TPUZ (1) \\
\hline USZN & Unsat. zone 1, effective porosity & $2.000 \mathrm{E}-01$ & $2.000 \mathrm{E}-01$ & --- & EPUZ (1) \\
\hline USZN & Unsat. zone 1 , field capacity & $3.000 \mathrm{E}-01$ & $3.000 \mathrm{E}-01$ & --- & FCUZ (1) \\
\hline USZN & Unsat. zone 1 , longitudinal dispersivity (m) & $1.000 \mathrm{E}-01$ & $1.000 \mathrm{E}-01$ & --- & ALPHALU (1) \\
\hline SZNE & Well pump intake depth (m below water table) & $1.000 \mathrm{E}+01$ & $1.000 \mathrm{E}+01$ & --- & DWIBWT \\
\hline SZNE & Depth of aquifer contributing to surface water body & $5.000 \mathrm{E}+00$ & $5.000 \mathrm{E}+00$ & --- & DPTHAQSW \\
\hline SZNE & Thickness of saturated zone (m) & $1.000 \mathrm{E}+02$ & $1.000 \mathrm{E}+02$ & --- & DPTHAQ \\
\hline SZNE & Density of saturated zone $(\mathrm{g} / \mathrm{cm} * * 3)$ & $1.500 \mathrm{E}+00$ & $1.500 \mathrm{E}+00$ & --- & DENSAQ \\
\hline SZNE & Saturated zone total porosity & $4.000 \mathrm{E}-01$ & $4.000 \mathrm{E}-01$ & --- & TPSZ \\
\hline
\end{tabular}

\begin{tabular}{|r|l|l}
$8.900 \mathrm{E}-01$ & --- & WIND \\
$1.000 \mathrm{E}+01$ & --- & ATGRID \\
$1.000 \mathrm{E}-03$ & --- & EPS \\
$1.000 \mathrm{E}+02$ & --- & OFFLPAQW \\
$0.000 \mathrm{E}+00$ & --- & OFFLNAQW \\
$4.500 \mathrm{E}+02$ & --- & OFFLPAQS \\
$-1.500 \mathrm{E}+02$ & --- & OFFLNAQSN \\
$1.500 \mathrm{E}+02$ & --- & OFFLNAQSF \\
1 & --- & NPCM \\
1 & --- & NPCMF \\
1 & --- & NPCZ \\
1 & --- & NPCZF \\
1 & --- & NSPCZ \\
1 & --- & NSPCZF \\
1 & --- & NPSS \\
1 & --- & NPSSF \\
1 & --- & NAQS \\
1 & --- & NAQSF \\
1 & --- &
\end{tabular}

Longitudinal dispersion in all but the subzone of 0 porosity $^{---} \mid$

density) / total porosity 06/11/2020 11:39 Page 40

convergence criterion for groundwater transport calc $1.000 \mathrm{E}-03$ contamination to Well c/c distance normal to flow, m $0.000 \mathrm{E}+00$ $4.500 \mathrm{E}+02$ ation to near edge of swb, c/c normal to flow $-1.500 \mathrm{E}+02$ 列 er of minor sub zones in last main PC sub zone Number of minor sub zones in last main SPC sub zone ( main sub zones in saturated stratum zone sub zone

$8.900 \mathrm{E}-01$
$1.000 \mathrm{E}+01$
$1.000 \mathrm{E}-03$
$-1.000 \mathrm{E}+00$
$0.000 \mathrm{E}+00$
$4.500 \mathrm{E}+02$
$-1.500 \mathrm{E}+02$
$1.500 \mathrm{E}+02$
1
1
1
1
1
1
1
1
1
1
1

HGW 
SZNE | Satur. zone hydraulic gradient to surface water body

SZNE longitudinal dispersivity to well (m)

SZNE longitudinal dispersivity to SWB (m)

SZNE lateral (horizontal) dispersivity to well (m)

SZNE lateral (horizontal) dispersivity to SWB (m)

SZNE lateral (vertical) dispersivity to well (m)

SZNE lateral (vertical) dispersivity to SWB (m)

SZNE Irrigation rate over aquifer to well (m/yr)

SZNE Irrigation rate over aquifer to SWB (m/yr)

SZNE Evapotranspiration coefficient over aquifer to well

SZNE Evapotranspiration coefficient over aquifer to SWB

SZNE Runoff coefficient over aquifer to well

SZNE Runoff coefficient over aquifer to SWB

SZNE Concentration of mobile colloids in the aquifer

SZNE Water - Soil Distribution coefficient of colloids

SZNE Water - Mobile Colloids Distribution coefficient

WTRU Drinking water intake (L/yr)

WTRU Fraction of drinking water from surface water

WTRU Fraction of drinking water from well water

WTRU Fraction of household water from surface water

WTRU Fraction of household water from well wate

WTRU Livestock water intake for meat 1 (L/day)

WTRU Fraction of livestock water 1 from surface water

WTRU Fraction of livestock water 1 from well water

WTRU Livestock water intake for milk (L/day)

WTRU Fraction of dairy cow water from surface water

WTRU Fraction of dairy cow water from well water

WTRU Irrigation rate in Agricultural Area 1 (m/yr)

1RESRAD-OFFSITE, Version 4.0 .3 beta $T^{1 / 2}$ Limit $=30$ days

Parent Dose Report

Title : RESRAD-OFFSITE Effluent release

File : PADUCAH EFFLUENT RELEASE-NONINVOLVED-WORKER-REV.ROF

\begin{tabular}{|c|c|c|c|c|c|c|}
\hline \multirow{2}{*}{$\begin{array}{l}0 \\
\text { Menu } \\
\text { WTRU }\end{array}$} & \multicolumn{2}{|l|}{ Parameter } & $\begin{array}{l}\text { neter Summ } \\
\text { User } \\
\text { Input }\end{array}$ & $\begin{array}{l}\text { y (continu } \\
\text { Default }\end{array}$ & $\begin{array}{l}\text { RESRAD } \\
\text { computed }\end{array}$ & $\begin{array}{c}\text { Parameter } \\
\text { Name }\end{array}$ \\
\hline & Fraction of irrigation water & 1 from surface water & $0.000 \mathrm{E}+00$ & $0.000 \mathrm{E}+00$ & --- & FSWIR (1) \\
\hline WTRU & Fraction of irrigation water & 2 from surface water & $0.000 \mathrm{E}+00$ & $0.000 \mathrm{E}+00$ & --- & FSWIR (2) \\
\hline WTRU & Fraction of irrigation water & 2 from well water & $1.000 \mathrm{E}+00$ & $1.000 \mathrm{E}+00$ & --- & FWWIR (2) \\
\hline WTRU & Irrigation rate in Agricultura & al Area 3 (m/yr) & $2.000 \mathrm{E}-01$ & $2.000 \mathrm{E}-01$ & --- & RIRRIG (3) \\
\hline WTRU & Fraction of irrigation water & 4 from surface water & $0.000 \mathrm{E}+00$ & $0.000 \mathrm{E}+00$ & --- & $\operatorname{FSWIR}(4)$ \\
\hline WTRU & Fraction of irrigation water & 4 from well water & $1.000 \mathrm{E}+00$ & $1.000 \mathrm{E}+00$ & --- & FWWIR (4) \\
\hline WTRU & Irrigation rate in offsite dwe & elling site (m/yr) & $2.000 \mathrm{E}-01$ & $2.000 \mathrm{E}-01$ & --- & RIRRIGDWELL \\
\hline WTRU & Fraction of irrigation water & Erom surface water & $0.000 \mathrm{E}+00$ & $0.000 \mathrm{E}+00$ & --- & FSWIRDWELL \\
\hline
\end{tabular}

\begin{tabular}{|l|l|l|l}
$2.000 \mathrm{E}-02$ & $2.000 \mathrm{E}-02$ & --- & HGSW \\
$3.000 \mathrm{E}+00$ & $3.000 \mathrm{E}+00$ & --- & ALPHALOW \\
$1.000 \mathrm{E}+01$ & $1.000 \mathrm{E}+01$ & --- & ALPHALOSW \\
$4.000 \mathrm{E}-01$ & $4.000 \mathrm{E}-01$ & --- & ALPHATW \\
$1.000 \mathrm{E}+00$ & $1.000 \mathrm{E}+00$ & --- & ALPHATSW \\
$2.000 \mathrm{E}-02$ & $2.000 \mathrm{E}-02$ & --- & ALPHAVW \\
$6.000 \mathrm{E}-02$ & $6.000 \mathrm{E}-02$ & --- & ALPHAVSW \\
not used & $0.000 \mathrm{E}+00$ & --- & RIAQW \\
not used & $0.000 \mathrm{E}+00$ & --- & RIAQSW \\
not used & $1.000 \mathrm{E}+00$ & --- & EVAPTRAQW \\
not used & $1.000 \mathrm{E}+00$ & --- & EVAPTRAQSW \\
not used & $1.000 \mathrm{E}+00$ & --- & RUNOFFAQW \\
not used & $1.000 \mathrm{E}+00$ & --- & RUNOFFAQSW \\
$0.000 \mathrm{E}+00$ & $0.000 \mathrm{E}+00$ & --- & CCOL \\
$0.000 \mathrm{E}+00$ & $0.000 \mathrm{E}+00$ & --- & K1COI \\
$0.000 \mathrm{E}+00$ & $0.000 \mathrm{E}+00$ & --- & K3COI \\
& & & \\
not used & $5.100 \mathrm{E}+02$ & --- & DWI \\
not used & $0.000 \mathrm{E}+00$ & --- & FSWD \\
not used & $1.000 \mathrm{E}+00$ & --- & FWWD \\
$0.000 \mathrm{E}+00$ & $0.000 \mathrm{E}+00$ & --- & FSWHH \\
$1.000 \mathrm{E}+00$ & $1.000 \mathrm{E}+00$ & --- & FWWHH \\
not used & $5.000 \mathrm{E}+01$ & --- & LWI (1) \\
not used & $0.000 \mathrm{E}+00$ & --- & FSWLV(1) \\
not used & $1.000 \mathrm{E}+00$ & --- & FWWLV (1) \\
not used & $1.600 \mathrm{E}+02$ & --- & LWI (2) \\
not used & $0.000 \mathrm{E}+00$ & --- & FSWLV (2) \\
not used & $1.000 \mathrm{E}+00$ & --- & FWWLV (2) \\
$2.000 \mathrm{E}-01$ & $2.000 \mathrm{E}-01$ & --- & RIRRIG (1) \\
$006 / 11 / 202011: 39$ & &
\end{tabular}


WTRU Fraction of irrigation water from well water

WTRU Well pumping rate $(\mathrm{m} * * 3 / \mathrm{yr})$

SWBY Surface area of water in surface water body, $m^{* * 2}$

SWBY Volume of surface water body, $m * * 3$

SWBY Potential evaporation, $\mathrm{m} / \mathrm{y}$

SWBY Stream outflow as a fraction of seepage+stm outflows

SWBY Use inflow ratio for outflow ratio, 1 yes, 0 no

Settling velocity of suspended sediments, $\mathrm{cm} / \mathrm{s}$

SWBY Thickness of bottom sediment absorbing nuclides, $m$

SWBY Number of distinct catchments

SWBY Catchment 1, smaller X coordinate (m)

SWBY Catchment 1, larger X coordinate (m)

SWBY Catchment 1, smaller Y coordinate (m)

SWBY Catchment 1, larger Y coordinate (m)

SWBY Catchment 1, area, $m^{* * 2}$

SWBY Catchment 1, runoff coefficient

SWBY Catchment 1, soil erodibility factor, tons/acre

SWBY Catchment 1, Slope-length-steepness factor

SWBY Catchment 1, Cover and management factor

SWBY Catchment 1, support practice factor

SWBY Catchment 1, sediment delivery ratio

SWBY Catchment 1, use SRD - Area correlation, 1 yes, 0 no

SWBY Catchment 1, deposited radionuclide delivery ratio

SWBY Compute atmospheric deposition on catchment

SWBY Convergence criterion for deposition calculations

INGE Fish consumption ( $\mathrm{kg} / \mathrm{yr}$ )

INGE Fraction of Fish from affected area

INGE Other Aquatic food consumption ( $\mathrm{kg} / \mathrm{yr}$ )

INGE Fraction of Aquatic food from affected area

INGE Non-Leafy vegetables consumption ( $\mathrm{kg} / \mathrm{yr}$ )

INGE Fraction of vegetable 1 from affected area

1RESRAD-OFFSITE, Version 4.0 .3 beta $\mathrm{T}^{1 / 2}$ Limit $=30$ days

Parent Dose Report

Title : RESRAD-OFFSITE Effluent release

File : PADUCAH EFFLUENT RELEASE-NONINVOLVED-WORKER-REV.ROF

\begin{tabular}{|c|c|c|c|}
\hline $1.000 \mathrm{E}+00$ & $1.000 \mathrm{E}+00$ & --- & FWWIRDWELL \\
\hline $5.100 \mathrm{E}+03$ & $5.100 \mathrm{E}+03$ & --- & UW \\
\hline $9.000 \mathrm{E}+04$ & $9.000 \mathrm{E}+04$ & --- & ALAKE \\
\hline $1.500 \mathrm{E}+05$ & $1.500 \mathrm{E}+05$ & --- & VLAKE \\
\hline $1.000 \mathrm{E}+00$ & $1.000 \mathrm{E}+00$ & --- & EVAPOT \\
\hline $9.983 \mathrm{E}-01$ & $9.983 \mathrm{E}-01$ & --- & FSTMFLOW \\
\hline 1 & 1 & --- & FSTMFLOWIN \\
\hline $1.000 \mathrm{E}-01$ & $1.000 \mathrm{E}-01$ & --- & Vsettle \\
\hline $1.500 \mathrm{E}+00$ & $1.500 \mathrm{E}+00$ & --- & Rhobsed \\
\hline $5.000 \mathrm{E}-02$ & $5.000 \mathrm{E}-02$ & --- & Thicksed \\
\hline 1 & 1 & --- & NCATCH \\
\hline$-1.450 \mathrm{E}+03$ & $-1.450 \mathrm{E}+03$ & --- & $\operatorname{CATCHXY}(1,1)$ \\
\hline $1.550 \mathrm{E}+03$ & $1.550 \mathrm{E}+03$ & --- & CATCHXY $(2,1)$ \\
\hline$-2.450 \mathrm{E}+03$ & $-2.450 \mathrm{E}+03$ & --- & $\operatorname{CATCHXY}(3,1)$ \\
\hline $5.500 \mathrm{E}+02$ & $5.500 \mathrm{E}+02$ & --- & CATCHXY $(4,1)$ \\
\hline $9.000 \mathrm{E}+06$ & $9.000 \mathrm{E}+06$ & --- & $\operatorname{AREACA}(1)$ \\
\hline $2.000 \mathrm{E}-01$ & $2.000 \mathrm{E}-01$ & --- & RUNOFFCA (1) \\
\hline $4.000 \mathrm{E}-01$ & $4.000 \mathrm{E}-01$ & --- & ERODIBILITYCA (1) \\
\hline $4.000 \mathrm{E}-01$ & $4.000 \mathrm{E}-01$ & --- & SLPLENSTPCA (1) \\
\hline $3.000 \mathrm{E}-03$ & $3.000 \mathrm{E}-03$ & --- & CRPMANGCA (1) \\
\hline $1.000 \mathrm{E}+00$ & $1.000 \mathrm{E}+00$ & --- & CONVPRACCA (1) \\
\hline $2.121 \mathrm{E}-01$ & $2.121 \mathrm{E}-01$ & --- & SDRCA (1) \\
\hline 1 & 1 & --- & SDRACOR \\
\hline $2.000 \mathrm{E}-02$ & $2.000 E-02$ & --- & DDRCA (1) \\
\hline yes & no & --- & ComputeDep \\
\hline $1.000 \mathrm{E}-03$ & $1.000 \mathrm{E}-03$ & --- & ConvCritAtm \\
\hline not used & $5.400 \mathrm{E}+00$ & --- & $\operatorname{DFI}(1)$ \\
\hline not used & $5.000 \mathrm{E}-01$ & --- & FFISH (1) \\
\hline not used & $9.000 \mathrm{E}-01$ & --- & $\operatorname{DFI}(2)$ \\
\hline not used & $5.000 \mathrm{E}-01$ & --- & FFISH (2) \\
\hline not used & $1.600 \mathrm{E}+02$ & --- & DVI (1) \\
\hline not used & $5.000 \mathrm{E}-01$ & --- & FVEG (1) \\
\hline
\end{tabular}

\begin{tabular}{|c|c|c|c|c|c|}
\hline $\begin{array}{l}0 \\
\text { Menu }\end{array}$ & Site-Spe & $\begin{array}{l}\text { eter Summa } \\
\text { User } \\
\text { Input }\end{array}$ & $\begin{array}{l}\text { (continue } \\
\text { Default }\end{array}$ & $\begin{array}{l}\text { RESRAD } \\
\text { computed }\end{array}$ & $\begin{array}{c}\text { Parameter } \\
\text { Name }\end{array}$ \\
\hline $\begin{array}{l}\text { INGE } \\
\text { INGE } \\
\text { INGE } \\
\text { INGE } \\
\text { INGE } \\
\text { INGE } \\
\text { INGE }\end{array}$ & $\begin{array}{l}\text { Leafy vegetable consumption (kg/yr) } \\
\text { Fraction of vegetable } 2 \text { from affected area } \\
\text { Meat } 1 \text { consumption (kg/yr) } \\
\text { Fraction of meat } 1 \mathrm{from} \text { affected area } \\
\text { Milk consumption (L/yr) } \\
\text { Fraction of milk from affected area } \\
\text { Soil ingestion rate (g/yr) }\end{array}$ & $\begin{array}{l}\text { not used } \\
\text { not used } \\
\text { not used } \\
\text { not used } \\
\text { not used } \\
\text { not used } \\
3.650 \mathrm{E}+01\end{array}$ & $\begin{array}{l}1.400 \mathrm{E}+01 \\
5.000 \mathrm{E}-01 \\
6.300 \mathrm{E}+01 \\
1.000 \mathrm{E}+00 \\
9.200 \mathrm{E}+01 \\
1.000 \mathrm{E}+00 \\
3.650 \mathrm{E}+01\end{array}$ & $\begin{array}{l}--- \\
--- \\
--- \\
--- \\
--- \\
--- \\
---\end{array}$ & $\begin{array}{l}\text { DVI (2) } \\
\text { FVEG (2) } \\
\text { DMI (1) } \\
\text { FMEMI (1) } \\
\text { DMI (2) } \\
\text { FMEMI (2) } \\
\text { SOIL }\end{array}$ \\
\hline
\end{tabular}


VEGE $\mid$ Wet weight crop yield for Non-Leafy ( $\mathrm{kg} / \mathrm{m} * \star 2)$

\begin{tabular}{|c|c|c|c|c|c|}
\hline $\begin{array}{l}0 \\
\text { Menu }\end{array}$ & Site-Specific Para & $\begin{array}{l}\text { neter Summ } \\
\text { User } \\
\text { Input }\end{array}$ & $\begin{array}{l}\text { y (continu } \\
\text { Default }\end{array}$ & $\begin{array}{l}\text { RESRAD } \\
\text { computed }\end{array}$ & $\begin{array}{c}\text { Parameter } \\
\text { Name }\end{array}$ \\
\hline INHE & Inhalation rate $(m * * 3 / y r)$ & $8.400 \mathrm{E}+03$ & $8.400 \mathrm{E}+03$ & --- & INHALR \\
\hline INHE & Offsite mass loading same as onsite mass loading? & $0.000 \mathrm{E}+00$ & & --- & SAMEMLRF \\
\hline INHE & Total mass loading at agricultural area 1 ( $\mathrm{g} / \mathrm{m} * * 3)$ & $1.000 \mathrm{E}-04$ & $1.000 \mathrm{E}-04$ & --- & MLTOTOF (1) \\
\hline
\end{tabular}

YIELD (1)

GROWTIME (1)

FOLI_F (1)

RWEATHER (1)

FINTCEPT $(1,2)$

$\mathrm{DROOT}(1)$

YIELD (2)

FOLI $F(2)$

RWEATTHER (2)

FINTCEPT $(2,1)$

INTCEPT $(2,2)$

DROOT (2)

YIELD (3)

FOLI F (3)

RWEATHER (3)

$\operatorname{FINTCEPT}(3,1)$

FINTCEPT $(3,2)$

$\operatorname{DROOT~(3)~}$

YIELD (4)

FOLI F (4)

RWEATTHER (4)

$\operatorname{FINTCEPT}(4,1)$

$\operatorname{FINTCEPT}(4,2)$

DROOT (4)

$\operatorname{LFI}(1,1)$

$\operatorname{LSI}(1,1)$

$\operatorname{LFI}(2,1)$

$\operatorname{LSI}(2,1)$

$\operatorname{LSI}(1,2)$

$\operatorname{LFI}(2,2)$

$\operatorname{LSI}(2,2)$

not used 
INHE $\mid$ Respirable fraction at agricultural area 1

INHE Total mass loading at agricultural area $2(\mathrm{~g} / \mathrm{m} * * 3)$

INHE Respirable fraction at agricultural area 2

INHE Total mass loading at agricultural area $3(\mathrm{~g} / \mathrm{m} * * 3)$

INHE Respirable fraction at agricultural area 3

INHE Total mass loading at agricultural area $4(\mathrm{~g} / \mathrm{m} * * 3)$

INHE Respirable fraction at agricultural area 4

INHE Total mass loading at offsite dwelling $(\mathrm{g} / \mathrm{m} * * 3)$

INHE Indoor dust filtration factor, inhalation

INHE Shielding factor, external gamma

INHE Shape factor flag, external gamma

SEXT Onsite shape factor array (used if non-circular):

SEXT Radii of shape factor array (used if non-circular):

SEXT Outer annular radius (m), ring 1 :

SEXT Outer annular radius (m), ring 2 :

SEXT Outer annular radius (m), ring 3 :

SEXT Outer annular radius (m), ring 4:

SEXT Outer annular radius $(\mathrm{m})$, ring 5 :

SEXT Outer annular radius $(\mathrm{m})$, ring 6 :

SEXT Outer annular radius (m), ring 7 :

SEXT Outer annular radius ( $\mathrm{m})$, ring 8

SEXT Outer annular radius $(\mathrm{m})$, ring 9:

SEXT Outer annular radius (m), ring 10 :

SEXT Outer annular radius (m), ring 11:

SEXT Outer annular radius $(\mathrm{m})$, ring 12 :

SEXT Fractions of annular areas within AREA:

SEXT Ring 1

SEXT Ring 2

SEXT Ring 3

SEXT Ring 4

$\begin{array}{lll}\text { SEXT } & \text { Ring } 5 \\ \text { SEXT } & \text { Ring }\end{array}$

SEXT Ring 7

SEXT Ring 8

SEXT Ring 9

SEXT Ring 10

SEXT Ring 11

SEXT Ring 12

1RESRAD-OFFSITE, Version 4.0 .3 beta $\mathrm{T}^{1 / 2}$ Limit $=30$ days

Parent Dose Report

Title: RESRAD-OFFSITE Effluent release

File : PADUCAH EFFLUENT RELEASE-NONINVOLVED-WORKER-REV.ROF

\begin{tabular}{|c|c|c|c|}
\hline $1.000 \mathrm{E}+00$ & $1.000 E+00$ & --- & RESPFRACOF (1) \\
\hline $1.000 \mathrm{E}-04$ & $1.000 \mathrm{E}-04$ & --- & MLTOTOF (2) \\
\hline $1.000 \mathrm{E}+00$ & $1.000 \mathrm{E}+00$ & --- & RESPFRACOF (2) \\
\hline $1.000 \mathrm{E}-04$ & $1.000 \mathrm{E}-04$ & --- & MLTOTOF (3) \\
\hline $1.000 \mathrm{E}+00$ & $1.000 \mathrm{E}+00$ & --- & RESPFRACOF (3) \\
\hline $1.000 \mathrm{E}-04$ & $1.000 \mathrm{E}-04$ & --- & MLTOTOF (4) \\
\hline $1.000 \mathrm{E}+00$ & $1.000 \mathrm{E}-04$ & --- & RESPFRACOF (4) \\
\hline $1.000 \mathrm{E}-04$ & $1.000 \mathrm{E}-04$ & --- & MLTOTDWELL \\
\hline $1.000 \mathrm{E}+00$ & $1.000 E+00$ & --- & RESPFRACDWELL \\
\hline $4.000 \mathrm{E}-01$ & $4.000 \mathrm{E}-01$ & --- & SHF 3 \\
\hline $7.000 \mathrm{E}-01$ & $7.000 \mathrm{E}-01$ & --- & SHF1 \\
\hline$-1.000 \mathrm{E}+00$ & $1.000 \mathrm{E}+00$ & noncircular & FS \\
\hline $1.667 \mathrm{E}-01$ & $6.000 \mathrm{E}+00$ & --- & RAD_SHAPE $(1)$ \\
\hline $3.333 \mathrm{E}-01$ & $1.200 \mathrm{E}+01$ & -- & RAD_SHAPE ( 2) \\
\hline $5.000 \mathrm{E}-01$ & $1.800 \mathrm{E}+01$ & --- & RAD_SHAPE ( 3) \\
\hline $6.667 \mathrm{E}-01$ & $2.400 \mathrm{E}+01$ & --- & RAD_SHAPE ( 4) \\
\hline $8.333 \mathrm{E}-01$ & $3.000 \mathrm{E}+01$ & -- & RAD_SHAPE ( 5) \\
\hline $1.000 \mathrm{E}+00$ & $3.600 \mathrm{E}+01$ & --- & RAD_SHAPE ( 6) \\
\hline $1.167 \mathrm{E}+00$ & $4.200 E+01$ & --- & RAD_SHAPE ( 7) \\
\hline $1.333 \mathrm{E}+00$ & $4.800 \mathrm{E}+01$ & --- & RAD_SHAPE ( 8) \\
\hline $1.500 \mathrm{E}+00$ & $5.400 \mathrm{E}+01$ & -- & RAD_SHAPE ( 9) \\
\hline $1.667 \mathrm{E}+00$ & $6.000 \mathrm{E}+01$ & --1 & RAD_SHAPE (10) \\
\hline $1.833 \mathrm{E}+00$ & $6.600 \mathrm{E}+01$ & --- & RAD_SHAPE (11) \\
\hline $2.000 \mathrm{E}+00$ & $7.200 E+01$ & --- & RAD_SHAPE (12) \\
\hline $1.000 \mathrm{E}+00$ & $1.000 \mathrm{E}+00$ & --- & FRACA ( 1) \\
\hline $0.000 \mathrm{E}+00$ & $1.000 \mathrm{E}+00$ & -- & FRACA ( 2) \\
\hline $0.000 \mathrm{E}+00$ & $1.000 \mathrm{E}+00$ & --- & FRACA ( 3) \\
\hline $0.000 \mathrm{E}+00$ & $1.000 \mathrm{E}+00$ & --- & FRACA ( 4) \\
\hline $0.000 \mathrm{E}+00$ & $1.000 \mathrm{E}+00$ & --1 & FRACA ( 5) \\
\hline $0.000 \mathrm{E}+00$ & $1.000 \mathrm{E}+00$ & --- & FRACA ( 6) \\
\hline $0.000 \mathrm{E}+00$ & $1.000 \mathrm{E}+00$ & --- & FRACA ( 7) \\
\hline $0.000 \mathrm{E}+00$ & $1.000 \mathrm{E}+00$ & -- & FRACA ( 8) \\
\hline $0.000 \mathrm{E}+00$ & $7.700 \mathrm{E}-01$ & --- & FRACA ( 9) \\
\hline $0.000 \mathrm{E}+00$ & $3.700 \mathrm{E}-01$ & --- & FRACA (10) \\
\hline $0.000 \mathrm{E}+00$ & $1.700 \mathrm{E}-01$ & --- & FRACA (11) \\
\hline $0.000 \mathrm{E}+00$ & $3.100 \mathrm{E}-02$ & --- & FRACA (12) \\
\hline
\end{tabular}

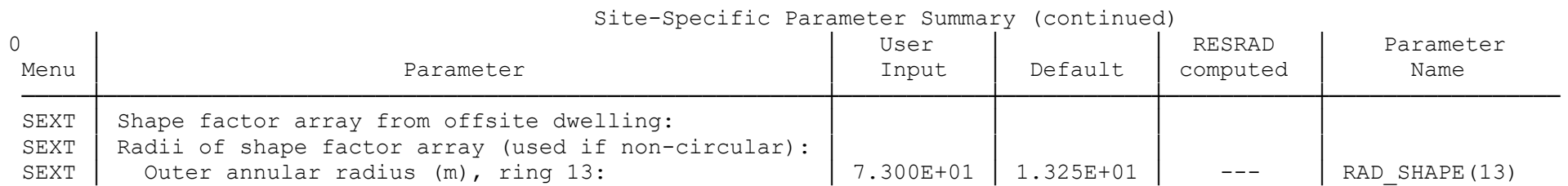


SEXT Outer annular radius (m), ring 14:

SEXT Outer annular radius (m), ring 15:

SEXT Outer annular radius (m), ring 16:

SEXT Outer annular radius (m), ring 17 :

SEXT Outer annular radius $(\mathrm{m})$, ring 18

SEXT Outer annular radius $(m)$, ring 19:

SEXT Outer annular radius (m), ring 20:

SEXT Outer annular radius (m), ring 21:

SEXT Outer annular radius (m), ring 22:

SEXT Outer annular radius (m), ring 23:

SEXT Outer annular radius (m), ring 24 :

SEXT Fractions of annular areas within AREA:

SEXT Ring 13

SEXT Ring 14

SEXT Ring 15

SEXT Ring 17

SEXT Ring 18

SEXT Ring 19

SEXT Ring 20

SEXT Ring 21

SEXT Ring 22

SEXT Ring 23

SEXT Ring 24

SEXT Shape factor array from offsite area 1 :

SEXT Radii of shape factor array (used if non-circular):

SEXT Outer annular radius $(\mathrm{m})$, ring 25 :

SEXT Outer annular radius (m), ring 26:

SEXT Outer annular radius $(\mathrm{m})$, ring 27 :

SEXT Outer annular radius (m), ring 28:

SEXT Outer annular radius (m), ring 29:

SEXT Outer annular radius (m), ring 30:

SEXT Outer annular radius (m), ring 31:

SEXT Outer annular radius $(\mathrm{m})$, ring 32 :

SEXT Outer annular radius $(\mathrm{m})$, ring 33:

SEXT

SEXT Outer annular radius (m), ring $35:$

SEXT Outer annular radius (m), ring 36 :

1RESRAD-OFFSITE, Version 4.0 .3 beta $T^{1 / 2}$ Limit $=30$ days

Parent Dose Report

Title : RESRAD-OFFSITE Effluent release

File : PADUCAH EFFLUENT RELEASE-NONINVOLVED-WORKER-REV.ROF
SEXT Ring 16

\begin{tabular}{|c|c|c|c|}
\hline $1.460 \mathrm{E}+02$ & $2.650 \mathrm{E}+01$ & --- & RAD_SHAPE (14) \\
\hline $2.190 \mathrm{E}+02$ & $3.975 \mathrm{E}+01$ & --- & RAD_SHAPE (15) \\
\hline $2.920 \mathrm{E}+02$ & $5.300 \mathrm{E}+01$ & --- & $\operatorname{RAD}^{-} \mathrm{SHAPE}(16)$ \\
\hline $3.650 \mathrm{E}+02$ & $6.625 \mathrm{E}+01$ & --- & RAD $\mathrm{SHAPE}$ (17) \\
\hline $4.380 \mathrm{E}+02$ & $7.950 \mathrm{E}+01$ & --- & RAD SHAPE (18) \\
\hline $5.110 \mathrm{E}+02$ & $9.275 \mathrm{E}+01$ & --- & RAD_SHAPE (19) \\
\hline $5.840 \mathrm{E}+02$ & $1.060 \mathrm{E}+02$ & --- & RAD $^{-}$SHAPE (20) \\
\hline $6.570 \mathrm{E}+02$ & $1.192 \mathrm{E}+02$ & --- & RAD_SHAPE (21) \\
\hline $7.300 \mathrm{E}+02$ & $1.325 \mathrm{E}+02$ & --- & RAD_SHAPE (22) \\
\hline $8.030 \mathrm{E}+02$ & $1.458 \mathrm{E}+02$ & --- & RAD_SHAPE (23) \\
\hline $8.760 \mathrm{E}+02$ & $1.590 \mathrm{E}+02$ & --- & RAD_SHAPE (24) \\
\hline $0.000 \mathrm{E}+00$ & $0.000 \mathrm{E}+00$ & --- & FRACA (13) \\
\hline $0.000 \mathrm{E}+00$ & $0.000 \mathrm{E}+00$ & --- & FRACA (14) \\
\hline $0.000 \mathrm{E}+00$ & $0.000 \mathrm{E}+00$ & --- & FRACA (15) \\
\hline $0.000 \mathrm{E}+00$ & $2.400 \mathrm{E}-02$ & --- & FRACA (16) \\
\hline $0.000 \mathrm{E}+00$ & $1.900 \mathrm{E}-01$ & --- & FRACA (17) \\
\hline $0.000 \mathrm{E}+00$ & $2.400 \mathrm{E}-01$ & --- & FRACA (18) \\
\hline $0.000 \mathrm{E}+00$ & $2.000 \mathrm{E}-01$ & --- & FRACA (19) \\
\hline $0.000 \mathrm{E}+00$ & $1.700 \mathrm{E}-01$ & --- & FRACA (20) \\
\hline $0.000 \mathrm{E}+00$ & $1.500 \mathrm{E}-01$ & --- & FRACA (21) \\
\hline $0.000 \mathrm{E}+00$ & $1.300 \mathrm{E}-01$ & --- & FRACA (22) \\
\hline $0.000 \mathrm{E}+00$ & $1.200 \mathrm{E}-01$ & --- & FRACA (23) \\
\hline $1.100 \mathrm{E}-06$ & $5.200 \mathrm{E}-02$ & --- & FRACA (24) \\
\hline $1.000 \mathrm{E}+01$ & $1.000 \mathrm{E}+01$ & --- & RAD_SHAPE (25) \\
\hline $1.000 \mathrm{E}+01$ & $1.000 \mathrm{E}+01$ & --- & RAD_SHAPE (26) \\
\hline $1.000 \mathrm{E}+01$ & $1.000 \mathrm{E}+01$ & --- & RAD SHAPE (27) \\
\hline $1.000 \mathrm{E}+01$ & $1.000 \mathrm{E}+01$ & --- & RAD_SHAPE (28) \\
\hline $1.000 \mathrm{E}+01$ & $1.000 \mathrm{E}+01$ & --- & RAD_SHAPE (29) \\
\hline $1.000 \mathrm{E}+01$ & $1.000 \mathrm{E}+01$ & --- & RAD_SHAPE (30) \\
\hline $1.000 \mathrm{E}+01$ & $1.000 \mathrm{E}+01$ & --- & RAD $S H A P E(31)$ \\
\hline $1.000 \mathrm{E}+01$ & $1.000 \mathrm{E}+01$ & --- & RAD_SHAPE (32) \\
\hline $1.000 \mathrm{E}+01$ & $1.000 \mathrm{E}+01$ & --- & RAD_SHAPE (33) \\
\hline $1.000 \mathrm{E}+01$ & $1.000 \mathrm{E}+01$ & --- & $\operatorname{RAD}^{-} \mathrm{SHAPE}(34)$ \\
\hline $1.000 \mathrm{E}+01$ & $1.000 \mathrm{E}+01$ & --- & RAD_SHAPE (35) \\
\hline $1.000 \mathrm{E}+01$ & $1.000 \mathrm{E}+01$ & --- & RAD_SHAPE (36) \\
\hline $06 / 11 / 2$ & $11: 39$ & 45 & \\
\hline
\end{tabular}

Site-Specific Parameter Summary (continued)

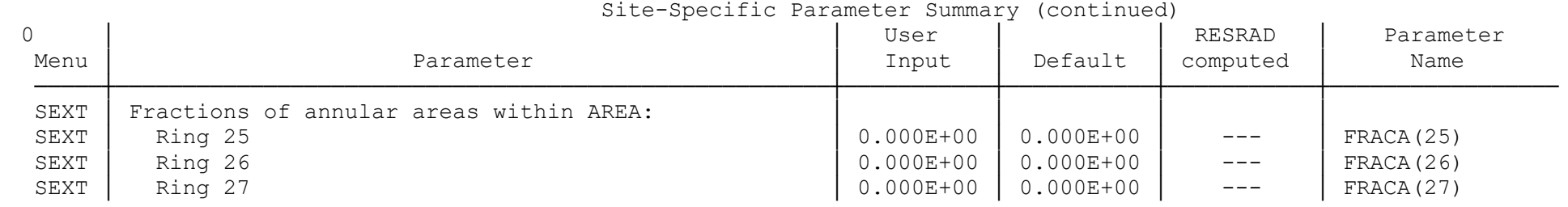




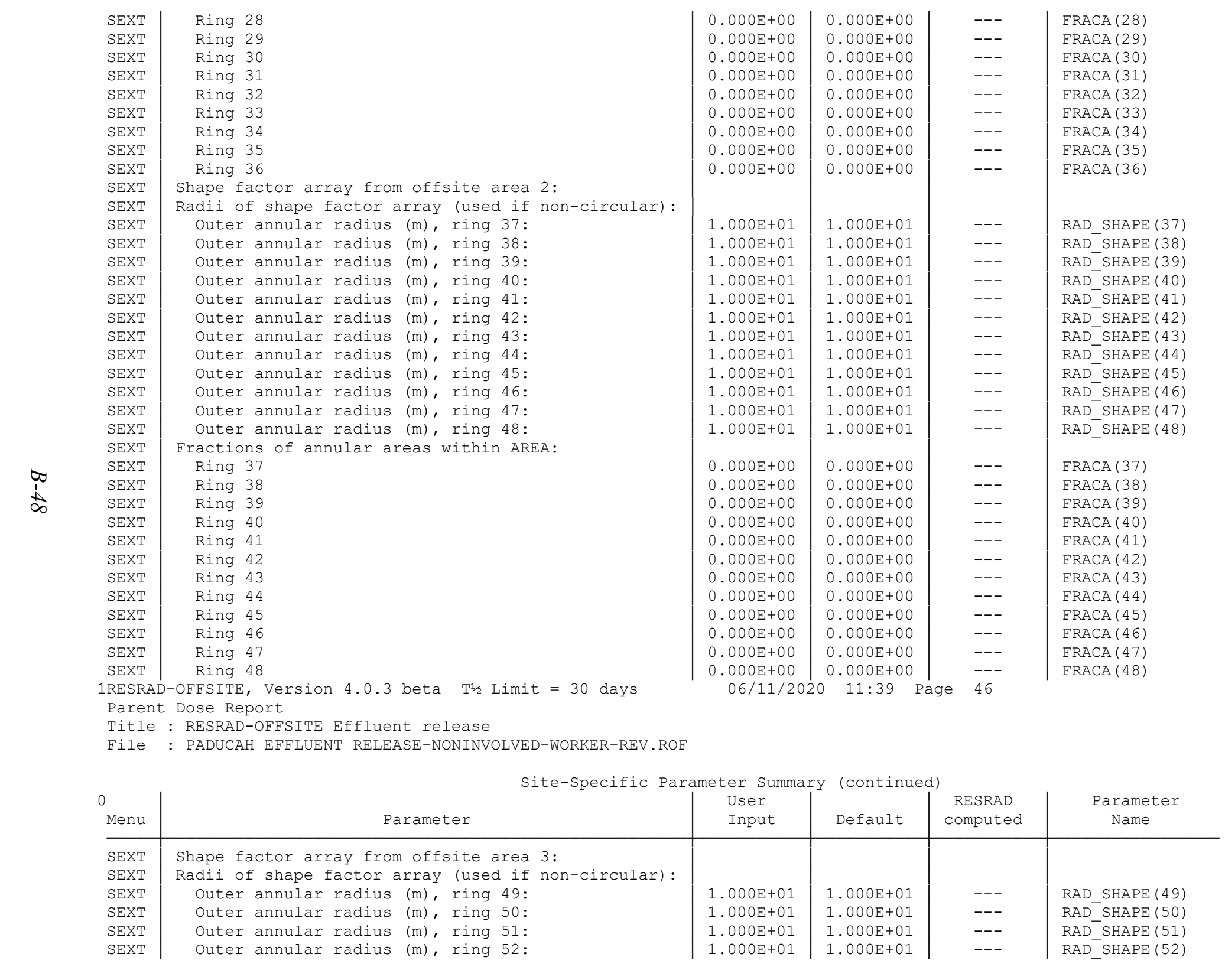


SEXT Outer annular radius (m), ring 53:

SEXT Outer annular radius (m), ring 54:

SEXT Outer annular radius (m), ring 55:

SEXT Outer annular radius (m), ring 56:

SEXT Outer annular radius $(\mathrm{m})$, ring 57:

SEXT Outer annular radius (m), ring 58:

SEXT Outer annular radius ( $\mathrm{m}$ ), ring 59:

SEXT Fractions of annular areas within AREA:

SEXT Ring 49

SEXT Ring 50

SEXT Ring 51

SEXT Ring 52

SEXT Ring 53

SEXT Ring 54

SEXT Ring 55

SEXT Ring 56

SEXT Ring 57

SEXT Ring 58

SEXT Ring 59

SEXT Ring 60

SEXT Shape factor array from offsite area 4:

SEXT Outer annular radius (m), ring 61:

SEXT Outer annular radius (m), ring 63:

SEXT Outer annular radius $(\mathrm{m})$, ring 64:

SEXT Outer annular radius $(\mathrm{m})$, ring 65 :

SEXT

SEXT Outer annular radius (m), ring 67:

SEXT Outer annular radius (m), ring 68:

SEXT Outer annular radius (m), ring 69:

SEXT Outer annular radius (m), ring 70:

SEXT Outer annular radius (m), ring 71:

SEXT Outer annular radius (m), ring 72:

1RESRAD-OFFSITE, Version 4.0 .3 beta $\mathrm{T}^{1 / 2} / \mathrm{Limit}=30$ days

Parent Dose Report

OFFSITE Effluent release

File : PADUCAH EFFLUENT RELEASE-NONINVOLVED-WORKER-REV.ROF
SEXT Outer annular radius (m), ring 60:

SEXT Radii of shape factor array (used if non-circular):

SEXT Outer annular radius (m), ring 62:

\begin{tabular}{|c|c|c|c|}
\hline $1.000 \mathrm{E}+01$ & $1.000 \mathrm{E}+01$ & --- & RAD_SHAPE (53) \\
\hline $1.000 \mathrm{E}+01$ & $1.000 \mathrm{E}+01$ & --- & RAD_SHAPE (54) \\
\hline $1.000 \mathrm{E}+01$ & $1.000 \mathrm{E}+01$ & --- & $\operatorname{RAD}^{-} \mathrm{SHAPE}(55)$ \\
\hline $1.000 \mathrm{E}+01$ & $1.000 \mathrm{E}+01$ & --- & $\operatorname{RAD}^{-} \operatorname{SHAPE}(56)$ \\
\hline $1.000 \mathrm{E}+01$ & $1.000 \mathrm{E}+01$ & --- & $\operatorname{RAD}^{-} \mathrm{SHAPE}(57)$ \\
\hline $1.000 \mathrm{E}+01$ & $1.000 \mathrm{E}+01$ & --- & RAD_SHAPE (58) \\
\hline $1.000 \mathrm{E}+01$ & $1.000 \mathrm{E}+01$ & --- & $\operatorname{RAD}^{-} \operatorname{SHAPE}(59)$ \\
\hline $1.000 \mathrm{E}+01$ & $1.000 \mathrm{E}+01$ & --- & RAD_SHAPE (60) \\
\hline $0.000 \mathrm{E}+00$ & $0.000 \mathrm{E}+00$ & --- & FRACA (49) \\
\hline $0.000 \mathrm{E}+00$ & $0.000 \mathrm{E}+00$ & --- & FRACA $(50)$ \\
\hline $0.000 \mathrm{E}+00$ & $0.000 \mathrm{E}+00$ & --- & FRACA (51) \\
\hline $0.000 \mathrm{E}+00$ & $0.000 \mathrm{E}+00$ & --- & FRACA (52) \\
\hline $0.000 \mathrm{E}+00$ & $0.000 \mathrm{E}+00$ & --- & FRACA (53) \\
\hline $0.000 \mathrm{E}+00$ & $0.000 \mathrm{E}+00$ & --- & FRACA (54) \\
\hline $0.000 \mathrm{E}+00$ & $0.000 \mathrm{E}+00$ & --- & FRACA (55) \\
\hline $0.000 \mathrm{E}+00$ & $0.000 \mathrm{E}+00$ & --- & FRACA (56) \\
\hline $0.000 \mathrm{E}+00$ & $0.000 \mathrm{E}+00$ & --- & FRACA (57) \\
\hline $0.000 \mathrm{E}+00$ & $0.000 \mathrm{E}+00$ & --- & FRACA (58) \\
\hline $0.000 \mathrm{E}+00$ & $0.000 \mathrm{E}+00$ & --- & FRACA (59) \\
\hline $0.000 \mathrm{E}+00$ & $0.000 \mathrm{E}+00$ & --- & FRACA $(60)$ \\
\hline $1.000 \mathrm{E}+01$ & $1.000 \mathrm{E}+01$ & --- & RAD_SHAPE (61) \\
\hline $1.000 \mathrm{E}+01$ & $1.000 \mathrm{E}+01$ & --- & $\operatorname{RAD}^{-} \operatorname{SHAPE}(62)$ \\
\hline $1.000 \mathrm{E}+01$ & $1.000 \mathrm{E}+01$ & --- & $\operatorname{RAD}^{-} \mathrm{SHAPE}(63)$ \\
\hline $1.000 \mathrm{E}+01$ & $1.000 \mathrm{E}+01$ & --- & RAD_SHAPE (64) \\
\hline $1.000 \mathrm{E}+01$ & $1.000 \mathrm{E}+01$ & --- & $\operatorname{RAD}^{-} \operatorname{SHAPE}(65)$ \\
\hline $1.000 \mathrm{E}+01$ & $1.000 \mathrm{E}+01$ & --- & RAD SHAPE (66) \\
\hline $1.000 \mathrm{E}+01$ & $1.000 \mathrm{E}+01$ & --- & RAD_SHAPE (67) \\
\hline $1.000 \mathrm{E}+01$ & $1.000 \mathrm{E}+01$ & --- & RAD_SHAPE (68) \\
\hline $1.000 \mathrm{E}+01$ & $1.000 \mathrm{E}+01$ & --- & $\operatorname{RAD}^{-} \mathrm{SHAPE}(69)$ \\
\hline $1.000 \mathrm{E}+01$ & $1.000 \mathrm{E}+01$ & --- & $\operatorname{RAD}^{-} \operatorname{SHAPE}(70)$ \\
\hline $1.000 \mathrm{E}+01$ & $1.000 \mathrm{E}+01$ & --- & $\operatorname{RAD}^{-} \operatorname{SHAPE}(71)$ \\
\hline $1.000 \mathrm{E}+01$ & $1.000 \mathrm{E}+01$ & --- & $\operatorname{RAD}^{-} \mathrm{SHAPE}(72)$ \\
\hline
\end{tabular}

Site-Specific Parameter Summary (continued)

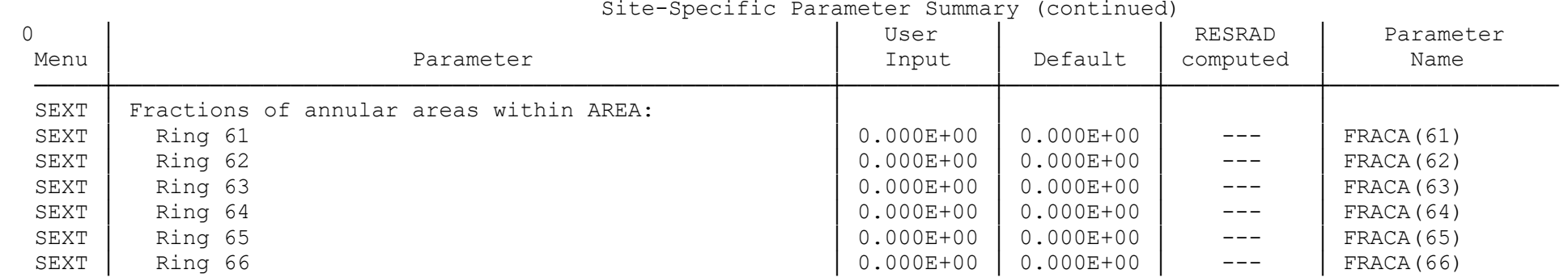




\begin{tabular}{|c|c|c|c|c|c|}
\hline SEXT & Ring 67 & $0.000 \mathrm{E}+00$ & $0.000 \mathrm{E}+00$ & --- & FRACA (67) \\
\hline SEXT & Ring 68 & $0.000 \mathrm{E}+00$ & $0.000 \mathrm{E}+00$ & --- & FRACA (68) \\
\hline SEXT & Ring 69 & $0.000 \mathrm{E}+00$ & $0.000 \mathrm{E}+00$ & --- & FRACA (69) \\
\hline SEXT & Ring 70 & $0.000 \mathrm{E}+00$ & $0.000 \mathrm{E}+00$ & --- & FRACA (70) \\
\hline SEXT & Ring 71 & $0.000 \mathrm{E}+00$ & $0.000 \mathrm{E}+00$ & --- & FRACA (71) \\
\hline SEXT & Ring 72 & $0.000 \mathrm{E}+00$ & $0.000 \mathrm{E}+00$ & --- & FRACA (72) \\
\hline $\mathrm{OCCU}$ & Fraction of time spent indoors on contaminated site & $0.000 \mathrm{E}+00$ & $0.000 \mathrm{E}+00$ & --- & FIND \\
\hline OCCU & Fraction of time spent outdoors on contaminated site & $0.000 \mathrm{E}+00$ & $0.000 \mathrm{E}+00$ & --- & FOTD \\
\hline $\mathrm{OCCU}$ & Fraction of time spent indoors in offsite Dwelling & $0.000 \mathrm{E}+00$ & $5.000 \mathrm{E}-01$ & --- & FINDDWELL \\
\hline $\mathrm{OCCU}$ & Fraction of time spent outdoors in Offsite Dwelling & $2.283 \mathrm{E}-01$ & $1.000 \mathrm{E}-01$ & --- & FOTDDWELL \\
\hline $\mathrm{OCCU}$ & Fraction of time spent outdoors in agri. area 1 & $0.000 \mathrm{E}+00$ & $1.000 \mathrm{E}-01$ & --- & OCCUPANCY (1) \\
\hline OCCU & Fraction of time spent outdoors in agri. area 2 & $0.000 \mathrm{E}+00$ & $1.000 \mathrm{E}-01$ & --- & OCCUPANCY (2) \\
\hline $\mathrm{OCCU}$ & Fraction of time spent outdoors in agri. area 3 & $0.000 \mathrm{E}+00$ & $1.000 \mathrm{E}-01$ & --- & OCCUPANCY (3) \\
\hline $\mathrm{OCCU}$ & Fraction of time spent outdoors in agri. area 4 & $0.000 \mathrm{E}+00$ & $1.000 \mathrm{E}-01$ & --- & OCCUPANCY (4) \\
\hline RADN & Diffusion coefficient for radon gas $(\mathrm{m} / \mathrm{sec})$ : & & & & \\
\hline RADN & in cover material & not used & $2.000 \mathrm{E}-06$ & --- & DIFCV \\
\hline RADN & in contaminated zone soil & not used & $2.000 \mathrm{E}-06$ & --- & DIFCZ \\
\hline RADN & in fruit, grain and non-leafy vegetable field & not used & $2.000 \mathrm{E}-06$ & --- & DIFOS (1) \\
\hline RADN & in leafy vegetable field & not used & $2.000 \mathrm{E}-06$ & --- & DIFOS (2) \\
\hline RADN & in pature & not used & $2.000 \mathrm{E}-06$ & --- & DIFOS (3) \\
\hline RADN & in livestock grain field & not used & $2.000 \mathrm{E}-06$ & --- & DIFOS (4) \\
\hline RADN & in offsite dwelling site & not used & $2.000 \mathrm{E}-06$ & --- & DIFOS (5) \\
\hline RADN & in foundation material & not used & $3.000 \mathrm{E}-07$ & --- & DIFFL \\
\hline RADN & Thickness of building foundation (m) & not used & $1.500 \mathrm{E}-01$ & --- & FLOOR1 \\
\hline RADN & Bulk density of building foundation $(\mathrm{g} / \mathrm{cm} * * 3)$ & not used & $2.400 \mathrm{E}+00$ & --- & DENSFL \\
\hline RADN & Total porosity of the building foundation & not used & $1.000 \mathrm{E}-01$ & --- & TPFL \\
\hline RADN & Volumetric water content of the foundation & not used & $3.000 \mathrm{E}-02$ & --- & PH2OFL \\
\hline RADN & Building depth below ground surface (m) & not used & $-1.000 \mathrm{E}+00$ & --- & DMFL \\
\hline RADN & Radon vertical dimension of mixing (m) & not used & $2.000 \mathrm{E}+00$ & --- & HMIX \\
\hline RADN & Height of the building (room) (m) & not used & $2.500 \mathrm{E}+00$ & --- & HRM \\
\hline RADN & Average building air exchange rate $(1 / \mathrm{hr})$ & not used & $5.000 \mathrm{E}-01$ & --- & REXG \\
\hline RADN & Building interior area factor & not used & $0.000 \mathrm{E}+00$ & --- & FAI \\
\hline RADN & Emanating power of $\mathrm{Rn}-222$ gas & not used & $2.500 \mathrm{E}-01$ & --- & EMANA (1) \\
\hline RADN & Emanating power of $\mathrm{Rn}-220$ gas & not used & $1.500 \mathrm{E}-01$ & --- & EMANA (2) \\
\hline $\mathrm{C} 14$ & $\mathrm{C}-14$ evasion layer thickness in soil (m) & not used & $3.000 \mathrm{E}-01$ & --- & DMC \\
\hline C14 & Vertical dimension of mixing for vegetation (m) & not used & $1.000 \mathrm{E}+00$ & --- & HMIXV \\
\hline $\mathrm{C} 14$ & C-14 evasion flux rate from soil ( $1 / \mathrm{sec})$ & not used & $7.000 \mathrm{E}-07$ & --- & C14EVSN \\
\hline 1RESR & -OFFSITE, Version 4.0 .3 beta $\mathrm{T}^{1} \frac{1}{2}$ Limit $=30$ days & $06 / 11 / 2$ & $11: 39$ & 48 & \\
\hline
\end{tabular}

Parent Dose Report

Title : RESRAD-OFFSITE Effluent release

File : PADUCAH EFFLUENT RELEASE-NONINVOLVED-WORKER-REV.ROF

\begin{tabular}{|c|c|c|c|c|c|}
\hline Menu & Parameter & $\begin{array}{c}\text { neter Sumn } \\
\text { User } \\
\text { Input }\end{array}$ & $\begin{array}{l}\text { y (continue } \\
\text { Default }\end{array}$ & $\begin{array}{l}\text { RESRAD } \\
\text { computed }\end{array}$ & $\begin{array}{l}\text { Parameter } \\
\text { Name }\end{array}$ \\
\hline $\begin{array}{l}\text { C14 } \\
\text { C14 }\end{array}$ & $\begin{array}{l}\text { C-12 evasion flux rate from soil ( } 1 / \mathrm{sec}) \\
\text { Fraction of vegetation carbon from air }\end{array}$ & $\begin{array}{l}\text { not used } \\
\text { not used }\end{array}$ & $\begin{array}{l}1.000 \mathrm{E}-10 \\
9.800 \mathrm{E}-01\end{array}$ & $\begin{array}{l}--- \\
---\end{array}$ & $\begin{array}{l}\text { C12EVSN } \\
\text { CAIR }\end{array}$ \\
\hline
\end{tabular}




\begin{tabular}{|c|c|c|c|c|c|}
\hline $\mathrm{C} 14$ & Fraction of vegetation carbon from soil & not used & $2.000 \mathrm{E}-02$ & --- & CSOIL \\
\hline $\mathrm{C} 12$ & C-12 concentration in contaminated soil ( $\mathrm{g} / \mathrm{g}$ ) & not used & $3.000 \mathrm{E}-02$ & --- & $\mathrm{C} 12 \mathrm{CZ}$ \\
\hline $\mathrm{C} 12$ & $\mathrm{C}-12$ concentration in water $(\mathrm{g} / \mathrm{cm} * * 3)$ & not used & $2.000 \mathrm{E}-05$ & --- & C12WTR \\
\hline $\mathrm{C} 12$ & C-12 concentration in milk ( $\mathrm{g} / \mathrm{g})$ & not used & $7.000 \mathrm{E}-02$ & --- & C12MEAT_MILK (2) \\
\hline $\mathrm{C} 12$ & $\mathrm{C}-12$ concentration in vegetable $1(\mathrm{~g} / \mathrm{g})$ & not used & $4.000 \mathrm{E}-01$ & --- & C12PLANT̄ (1) \\
\hline $\mathrm{C} 12$ & C-12 concentration in vegetable 2 ( $\mathrm{g} / \mathrm{g})$ & not used & $9.000 \mathrm{E}-02$ & --- & C12PLANT (2) \\
\hline H3 & Humidity in air $(\mathrm{g} / \mathrm{cm} * * 3)$ & not used & $8.000 \mathrm{E}+00$ & --- & HUMID \\
\hline H3 & Mass fraction of water in meat 1 ( $\mathrm{g} / \mathrm{g})$ & not used & $6.000 \mathrm{E}-01$ & --- & H2OMEAT MILK (1) \\
\hline Н3 & Mass fraction of water in milk $(\mathrm{g} / \mathrm{g})$ & not used & $8.800 \mathrm{E}-01$ & --- & H2OMEAT_MILK (2) \\
\hline H3 & Mass fraction of water in vegetable $1(\mathrm{~g} / \mathrm{g})$ & not used & $8.000 \mathrm{E}-01$ & --- & H2OPLANT̄ (1) \\
\hline Н3 & Mass fraction of water in vegetable $2(\mathrm{~g} / \mathrm{g})$ & not used & $8.000 \mathrm{E}-01$ & --- & H2OPLANT (2) \\
\hline Н3 & Mass fraction of water in livestock feed 1 ( $\mathrm{g} / \mathrm{g})$ & not used & $8.000 \mathrm{E}-01$ & --- & H2OPLANT (3) \\
\hline Н3 & Mass fraction of water in livestock feed 2 ( $\mathrm{g} / \mathrm{g})$ & not used & $8.000 \mathrm{E}-01$ & --- & H2OPLANT (4) \\
\hline
\end{tabular}

Summary of Pathway Selections

\begin{tabular}{c|c} 
Pathway & User Selection \\
\hline $1--$ external gamma & active \\
$2--$ inhalation (w/o radon) & active \\
3 -- plant ingestion & suppressed \\
$4--$ meat ingestion & suppressed \\
5 -- milk ingestion & suppressed \\
6 -- aquatic foods & suppressed \\
7 -- drinking water & active \\
8 -- soil ingestion & suppressed \\
9 -- radon & P
\end{tabular}

1RESRAD-OFFSITE, Version 4.0 .3 beta $T^{1} \frac{1}{2}$ Limit $=30$ days Parent Dose Report

Title : RESRAD-OFFSITE Effluent release

File : PADUCAH EFFLUENT RELEASE-NONINVOLVED-WORKER-REV.ROF

Contaminated Zone Dimensions

$\begin{aligned} \text { Area: } & 1.00 \text { square meters }\end{aligned}$

Thickness: $\quad 2.00$ meters

Cover Depth: $\quad 0.00$ meters
Initial Soil Concentrations, $\mathrm{pCi} / \mathrm{g}$

$\begin{array}{ll}\mathrm{Am}-241 & 3.000 \mathrm{E}+00 \\ \mathrm{Cs}-137 & 3.000 \mathrm{E}+00 \\ \mathrm{~Np}-237 & 3.000 \mathrm{E}+01 \\ \mathrm{Pu}-238 & 3.000 \mathrm{E}+00 \\ \mathrm{Pu}-239 & 3.000 \mathrm{E}+00 \\ \mathrm{Pu}-240 & 3.000 \mathrm{E}+00 \\ \mathrm{~T}-99 & 3.000 \mathrm{E}+01\end{array}$




$\begin{array}{ll}\text { Th-228 } & 3.000 \mathrm{E}+00 \\ \text { Th-230 } & 3.000 \mathrm{E}+00 \\ \text { Th-232 } & 3.000 \mathrm{E}+00 \\ \mathrm{U}-234 & 3.000 \mathrm{E}+01 \\ \mathrm{U}-235 & 3.000 \mathrm{E}+01 \\ \mathrm{U}-238 & 3.000 \mathrm{E}+01\end{array}$

Total Dose TDOSE(t), mrem/yr

Basic Radiation Dose Limit $=2.500 \mathrm{E}+01 \mathrm{mrem} / \mathrm{yr}$

Total Mixture Sum $M(t)=$ Fraction of Basic Dose Limit Received at Time (t)

$\begin{array}{rlllll}t \text { (years) : } & 0.000 \mathrm{E}+00 & 1.000 \mathrm{E}+00 & 3.000 \mathrm{E}+00 & 6.000 \mathrm{E}+00 & 1.000 \mathrm{E}+01 \\ \text { TDOSE }(t): & 5.718 \mathrm{E}-01 & 5.718 \mathrm{E}-01 & 5.719 \mathrm{E}-01 & 5.721 \mathrm{E}-01 & 5.722 \mathrm{E}-01\end{array}$

$\begin{array}{rlllll}\text { TDOSE }(t): & 5.718 \mathrm{E}-01 & 5.718 \mathrm{E}-01 & 5.719 \mathrm{E}-01 & 5.721 \mathrm{E}-01 & 5.722 \mathrm{E}-01 \\ \mathrm{M}(t): & 2.287 \mathrm{E}-02 & 2.287 \mathrm{E}-02 & 2.288 \mathrm{E}-02 & 2.288 \mathrm{E}-02 & 2.289 \mathrm{E}-02\end{array}$

OMaximum TDOSE $(t): 5.735 \mathrm{E}-01 \mathrm{mrem} / \mathrm{yr}$ at $t=40$ years

RESRAD-OFFSITE, Version 4.0 .3 beta $T^{1 / 2}$ Limit $=30$ days

Parent Dose Report

06/11/2020 11:39 Page 50

Title : RESRAD-OFFSITE Effluent release

File : PADUCAH EFFLUENT RELEASE-NONINVOLVED-WORKER-REV.ROF

Total Dose Contributions TDOSE ( $i, p, t)$ for Individual Radionuclides (i) and Pathways (p) in $\mathrm{mrem} / \mathrm{yr}$ and as a Percentage of Total Dose at $t=0$ years

Radon Plant

Meat

Milk

Soil

Water

Nuclide

Fish

Dose $\%$

Dose \%

Dose $\%$

$\mathrm{Am}-241$

$0.00 \mathrm{E}+00$

$0.00 \mathrm{E}+00$

$0.00 \mathrm{E}+00$

$\overline{0.00 \mathrm{E}+00}$

$\begin{array}{llllllll}\mathrm{Cs}-137 & 0.00 \mathrm{E}+00 & 0 & 0.00 \mathrm{E}+00 & 0 & 0.00 \mathrm{E}+00 & 0 & 0.00 \mathrm{E}+00\end{array}$

$\begin{array}{llll}\mathrm{Np}-237 & 0.00 \mathrm{E}+00 & 0 & 0.00 \mathrm{E}+00\end{array}$

$\begin{array}{llll}\mathrm{Pu}-238 & 0.00 \mathrm{E}+00 & 0 & 0.00 \mathrm{E}+00\end{array}$

$\begin{array}{llll}\mathrm{Pu}-239 & 0.00 \mathrm{E}+00 & 0 & 0.00 \mathrm{E}+00\end{array}$

$\begin{array}{llll}\mathrm{Pu}-240 & 0.00 \mathrm{E}+00 & 0 & 0.00 \mathrm{E}+00 \\ \mathrm{TC}-99 & 0.00 \mathrm{E}+00 & 0 & 0.00 \mathrm{E}+00\end{array}$

$\begin{array}{lll}\mathrm{TC}-92 & .00 \mathrm{E}+00 & 0 \\ \mathrm{Th}-228 & 0.00 \mathrm{E}+00 & 0.00 \mathrm{E}+00\end{array}$

$0.00 \mathrm{E}+00$

$0.00 \mathrm{E}+00$

$0.00 \mathrm{E}+00$

$0.00 \mathrm{E}+00$

$0.00 \mathrm{E}+00$

$\frac{0}{0.00 \mathrm{D}+00} \div$

$0.00 \mathrm{E}+00$

$0.00 \mathrm{E}+00$

Dose

Dose \%

$0.00 \mathrm{E}+00$

$0.00 \mathrm{E}+00$

$\begin{array}{lllll}0.00 \mathrm{E}+00 & 0 & 0.00 \mathrm{E}+00 & 0 & 0.00 \mathrm{E}+00\end{array}$

$\begin{array}{llll}0 & 0.00 \mathrm{E}+00 & 0 & 0.00 \mathrm{E}+00\end{array}$

$0.00 \mathrm{E}+00$

$0 \quad 0.00 \mathrm{E}+00$

$0.00 \mathrm{E}+00$

$\begin{array}{llll}\mathrm{Th}-232 & 0.00 \mathrm{E}+00 & 0 & 0.00 \mathrm{E}+00\end{array}$

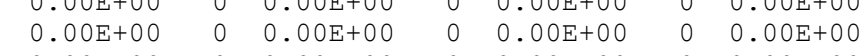

$0.0 .00 \mathrm{E}+00$

$0.00 \mathrm{E}+00$

$\begin{array}{lll}0.00 \mathrm{E}+00 & 0 & 0.00 \mathrm{E}+00\end{array}$

$\mathrm{U}-235$

$0.00 \mathrm{E}+00 \quad 0 \quad 0.00 \mathrm{E}+00$

$0 \quad 0.00 \mathrm{E}+00$

$0.00 \mathrm{E}+00$

$0.00 \mathrm{E}+00$

$0.00 \mathrm{E}+00$

$0.00 \mathrm{E}+00$

$\overline{0.00 \mathrm{E}+00}-\overline{0.00 \mathrm{E}+00} \overline{0}$

$0.00 \mathrm{E}+00 \quad 0 \quad 0.00 \mathrm{E}+00 \quad 0$

$\begin{array}{lllll}0.00 \mathrm{E}+00 & 0 & 0.00 \mathrm{E}+00 & 0 & 0.00 \mathrm{E}+00\end{array}$

$\begin{array}{lllll}0.00 \mathrm{E}+00 & 0 & 0.00 \mathrm{E}+00 & 0 & 0.00 \mathrm{E}+00 \\ 0.00 \mathrm{E}+00 & 0 & 0.00 \mathrm{E}+00 & 0 & 0.00 \mathrm{E}+00\end{array}$

$0.00 \mathrm{E}+00$

$0.00 \mathrm{E}+00$

$0.00 \mathrm{E}+00$

$0.00 \mathrm{E}+00$

$\begin{array}{lllll}0.00 \mathrm{E}+00 & 0 & 0.00 \mathrm{E}+00 & 0 & 0.00 \mathrm{E}+00\end{array}$

$\begin{array}{lllll}0.00 \mathrm{E}+00 & 0 & 0.00 \mathrm{E}+00 & 0 & 0.00 \mathrm{E}+00\end{array}$

$\begin{array}{llllll}0 & 0.00 \mathrm{E}+00 & 0 & 0.00 \mathrm{E}+00 & 0 & 0.00 \mathrm{E}+00 \\ 0 & 0.00 \mathrm{E}+00 & 0 & 0.00 \mathrm{E}+00 & 0 & 0.00 \mathrm{E}+00\end{array}$

$0.00 \mathrm{E}+00$

Total

$\overline{\overline{0.00 \mathrm{E}+00}} \overline{\overline{0}} \overline{\overline{0.00 \mathrm{E}+00}} \overline{\overline{0.00 \mathrm{E}+00}}=$

$0.00 \mathrm{E}+00$

$\overline{0.00 \mathrm{E}+00}$

$0.00 \mathrm{E}+00$

$0.00 \mathrm{E}+00$

Total Dose Contributions TDOSE $(i, p, t)$ for Individual Radionuclides (i) and Pathways (p)

in mrem/yr and as a Percentage of Total Dose at $t=0$ years

0 Directly from primary contamination and from release to atmosphere (Inhalation excludes radon) Ground Inhalation Radon Plant

Meat

$\mathrm{Milk}$ Soil

All Pathways*

\begin{tabular}{|c|c|c|c|c|c|c|c|c|c|c|c|c|c|c|c|c|}
\hline \multirow{2}{*}{$\begin{array}{l}\text { Radio- } \\
\text { Nuclide }\end{array}$} & & & & & & & & & & & & & & & & \\
\hline & Dose & $\%$ & Dose & $\%$ & Dose & \% & Dose & $\%$ & Dose & \% & Dose & $\div$ & Dose & $\%$ & Dose & 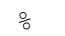 \\
\hline
\end{tabular}




\begin{tabular}{|c|c|c|c|c|c|c|c|c|c|c|c|c|c|c|c|}
\hline Cs-137 & $2.34 \mathrm{E}-06$ & 0 & $1.53 E-05$ & 0 & $0.00 \mathrm{E}+00$ & 0 & $0.00 \mathrm{E}+00$ & 0 & $0.00 \mathrm{E}+00$ & 0 & $0.00 \mathrm{E}+00$ & 0 & $1.53 \mathrm{E}-09$ & 0 & $1.76 \mathrm{E}-05$ \\
\hline $\mathrm{Np}-237$ & $7.96 \mathrm{E}-06$ & 0 & $1.96 \mathrm{E}-01$ & 34 & $0.00 \mathrm{E}+00$ & 0 & $0.00 \mathrm{E}+00$ & 0 & $0.00 \mathrm{E}+00$ & 0 & $0.00 \mathrm{E}+00$ & 0 & $1.32 \mathrm{E}-07$ & 0 & $1.96 \mathrm{E}-01$ \\
\hline $\mathrm{Pu}-238$ & $9.93 \mathrm{E}-11$ & 0 & $4.31 \mathrm{E}-02$ & 8 & $0.00 \mathrm{E}+00$ & 0 & $0.00 \mathrm{E}+00$ & 0 & $0.00 \mathrm{E}+00$ & 0 & $0.00 \mathrm{E}+00$ & 0 & $2.73 E-08$ & 0 & 4. $31 \mathrm{E}-02$ \\
\hline $\mathrm{Pu}-239$ & $2.13 \mathrm{E}-10$ & 0 & $4.70 \mathrm{E}-02$ & 8 & $0.00 \mathrm{E}+00$ & 0 & $0.00 \mathrm{E}+00$ & 0 & $0.00 \mathrm{E}+00$ & 0 & $0.00 \mathrm{E}+00$ & 0 & $2.98 \mathrm{E}-08$ & 0 & $4.70 \mathrm{E}-02$ \\
\hline $\mathrm{Pu}-240$ & $9.68 \mathrm{E}-11$ & 0 & $4.70 \mathrm{E}-02$ & 8 & $0.00 \mathrm{E}+00$ & 0 & $0.00 \mathrm{E}+00$ & 0 & $0.00 \mathrm{E}+00$ & 0 & $0.00 \mathrm{E}+00$ & 0 & $2.98 \mathrm{E}-08$ & 0 & $4.70 \mathrm{E}-02$ \\
\hline TC-99 & $6.54 \mathrm{E}-11$ & 0 & $5.09 E-05$ & 0 & $0.00 \mathrm{E}+00$ & 0 & $0.00 \mathrm{E}+00$ & 0 & $0.00 \mathrm{E}+00$ & 0 & $0.00 \mathrm{E}+00$ & 0 & $5.50 \mathrm{E}-11$ & 0 & $5.09 \mathrm{E}-05$ \\
\hline Th-228 & $5.28 \mathrm{E}-06$ & 0 & $1.71 \mathrm{E}-02$ & 3 & $0.00 \mathrm{E}+00$ & 0 & $0.00 \mathrm{E}+00$ & 0 & $0.00 \mathrm{E}+00$ & 0 & $0.00 \mathrm{E}+00$ & 0 & $1.38 \mathrm{E}-08$ & 0 & $1.71 \mathrm{E}-02$ \\
\hline Th-230 & $2.88 \mathrm{E}-09$ & 0 & $3.92 \mathrm{E}-02$ & 7 & $0.00 \mathrm{E}+00$ & 0 & $0.00 \mathrm{E}+00$ & 0 & $0.00 \mathrm{E}+00$ & 0 & $0.00 \mathrm{E}+00$ & 0 & $2.51 \mathrm{E}-08$ & 0 & $3.92 \mathrm{E}-02$ \\
\hline Th-232 & $3.53 E-07$ & 0 & $4.31 E-02$ & 8 & $0.00 \mathrm{E}+00$ & 0 & $0.00 \mathrm{E}+00$ & 0 & $0.00 \mathrm{E}+00$ & 0 & $0.00 \mathrm{E}+00$ & 0 & $3.35 \mathrm{E}-08$ & 0 & $4.31 \mathrm{E}-02$ \\
\hline$U-234$ & $2.79 E-09$ & 0 & $3.68 \mathrm{E}-02$ & 6 & $0.00 \mathrm{E}+00$ & 0 & $0.00 \mathrm{E}+00$ & 0 & $0.00 \mathrm{E}+00$ & 0 & $0.00 \mathrm{E}+00$ & 0 & $5.69 \mathrm{E}-08$ & 0 & $3.68 \mathrm{E}-02$ \\
\hline U-235 & $5.44 \mathrm{E}-06$ & 0 & $3.33 E-02$ & 6 & $0.00 \mathrm{E}+00$ & 0 & $0.00 \mathrm{E}+00$ & 0 & $0.00 \mathrm{E}+00$ & 0 & $0.00 \mathrm{E}+00$ & 0 & $5.50 \mathrm{E}-08$ & 0 & $3.33 \mathrm{E}-02$ \\
\hline $\mathrm{U}-238$ & $1.14 \mathrm{E}-06$ & 0 & $3.14 \mathrm{E}-02$ & 5 & $0.00 \mathrm{E}+00$ & 0 & $0.00 \mathrm{E}+00$ & 0 & $0.00 \mathrm{E}+00$ & 0 & $0.00 \mathrm{E}+00$ & 0 & $5.62 \mathrm{E}-08$ & 0 & $3.14 \mathrm{E}-02$ \\
\hline & & & & & 00 & & $0.00 \mathrm{E}+00$ & 0 & $0.00 \mathrm{E}+00$ & 0 & 0 & 0 & 7 & 0 & \\
\hline
\end{tabular}

RESRAD-OFFSITE, Version 4.0 .3 beta $T^{\frac{1}{2} / 2}$ Limit $=30$ days

Title: RESRAD-OFFSITE Effluent release

File : PADUCAH EFFLUENT RELEASE-NONINVOLVED-WORKER-REV.ROF

Total Dose Contributions TDOSE (i,p,t) for Individual Radionuclides (i) and Pathways (p) in mrem/yr and as a Percentage of Total Dose at $t=1$ years

\begin{tabular}{|c|c|c|c|c|c|c|c|c|c|c|c|c|c|c|c|c|}
\hline \multirow{2}{*}{$\begin{array}{l}\text { Radio- } \\
\text { Nuclide }\end{array}$} & \multicolumn{2}{|c|}{ Ground } & \multicolumn{2}{|l|}{ Fish } & \multicolumn{2}{|c|}{$\begin{array}{l}\text { From } \\
\text { Radon }\end{array}$} & \multicolumn{2}{|c|}{$\begin{array}{l}\text { es to grour } \\
\text { Plant }\end{array}$} & \multicolumn{2}{|c|}{$\begin{array}{l}\text { water and } \\
\text { Meat }\end{array}$} & \multicolumn{2}{|c|}{$\begin{array}{l}\text { o surface water } \\
\text { Milk }\end{array}$} & \multicolumn{2}{|l|}{ Soil } & \multicolumn{2}{|l|}{ Water } \\
\hline & Dose & $\%$ & Dose & $\%$ & Dose & $\%$ & Dose & $\div$ & Dose & 응 & Dose & 웅 & Dose & $\%$ & Dose & $\%$ \\
\hline$n-241$ & $00 E+00$ & 0 & $.00 E+00$ & 0 & $.00 \mathrm{E}+00$ & 0 & $.00 E+00$ & 0 & $0.00 \mathrm{E}+00$ & 0 & $0.00 \mathrm{E}+00$ & 0 & $0.00 \mathrm{E}+00$ & 0 & $.00 \mathrm{E}+00$ & \\
\hline & $O O E$ & 0 & $00 \mathrm{H}$ & 0 & 001 & 0 & .00 & 0 & 0 & 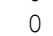 & 0 & 0 & .00 & 0 & 00 & \\
\hline $\mathrm{Np}-237$ & $0.00 \mathrm{E}+00$ & 0 & $0.00 \mathrm{E}+00$ & 0 & $0.00 \mathrm{E}+00$ & 0 & $0.00 \mathrm{E}+00$ & 0 & $0.00 \mathrm{E}+00$ & 0 & $.00 \mathrm{E}+00$ & 0 & $0.00 \mathrm{E}+00$ & 0 & $0.00 \mathrm{E}+00$ & \\
\hline $\mathrm{Pu}-238$ & $00 E+00$ & 0 & $0.00 \mathrm{E}+00$ & 0 & $.00 \mathrm{E}+00$ & 0 & $.00 \mathrm{E}$ & 0 & $.00 \mathrm{E}$ & - & 0 & 0 & $\mathrm{OOH}$ & 0 & $.00 \mathrm{E}+00$ & \\
\hline $\mathrm{Pu}-239$ & $00 E+00$ & 0 & $00 E+00$ & 0 & $00 \mathrm{E}+00$ & 政 & 0 & 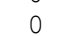 & 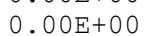 & ( & & - & 0 & 0 & 00 & \\
\hline $\mathrm{Pu}-240$ & $00 E+00$ & 0 & $0.00 \mathrm{E}+00$ & 0 & $0.00 \mathrm{E}+00$ & 0 & $0.00 \mathrm{E}+00$ & 0 & $0.00 \mathrm{E}+$ & 0 & 0 & 0 & $00 \mathrm{E}+$ & 0 & $00 \mathrm{E}+00$ & \\
\hline & (20) & 0 & 0 & 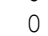 & $00 \mathrm{E}+00$ & 然 & & 0 & & 0 & & & & & & \\
\hline 28 & $0 c$ & 0 & 00 & 0 & 0 & 0 & 0 & 0 & 0 & 0 & 0 & c & 0 & 0 & 0 & \\
\hline Th-230 & $00 E+00$ & 0 & $0.00 \mathrm{E}+00$ & 0 & $00 \mathrm{E}+00$ & 0 & $0.00 \mathrm{E}+00$ & 0 & $0.00 \mathrm{E}+$ & o & 0.001 & 0 & $0.00 \mathrm{~B}$ & 0 & $0.00 \mathrm{E}+00$ & \\
\hline Th-232 & $.00 \mathrm{E}+00$ & 0 & $0.00 \mathrm{E}+00$ & 0 & $0.00 \mathrm{E}+00$ & 0 & $0.00 \mathrm{E}+00$ & 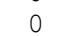 & 0.00 & - & & 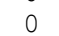 & - & 0 & $0.00 \mathrm{E}+00$ & \\
\hline $\mathrm{U}-234$ & חמת תחת & 0 & مחת תמת ת & 0 & ק & 0 & 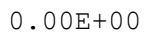 & 0 & 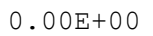 & 0 & & & & & & \\
\hline $\mathrm{U}-23$ & 0 & 0 & 0 & 0 & 0 & U & 0 & 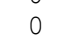 & 0 & 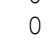 & & 0 & 00 & 0 & $.00 \mathrm{E}+00$ & \\
\hline $\mathrm{U}-238$ & $0.00 \mathrm{E}+00$ & 0 & $0.00 \mathrm{E}+00$ & 0 & $0.00 \mathrm{E}+00$ & 0 & $0.00 \mathrm{E}+00$ & 0 & $0.00 \mathrm{E}+00$ & 0 & $0.00 \mathrm{E}+00$ & 0 & $0.00 \mathrm{E}+00$ & 0 & $0.00 \mathrm{E}+00$ & \\
\hline & & 0 & 00E+00 & 0 & +00 & 0 & Ju & 0 & & 0 & 0 & 0 & 0 & 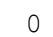 & 10 & \\
\hline
\end{tabular}

0

Total Dose Contributions TDOSE (i, p,t) for Individual Radionuclides (i) and Pathways (p) in $\mathrm{mrem} / \mathrm{yr}$ and as a Percentage of Total Dose at $t=1$ years

0 Directly from primary contamination and from release to atmosphere (Inhalation excludes radon)

\begin{tabular}{|c|c|c|c|c|c|c|c|c|c|c|c|c|c|c|c|c|}
\hline \multirow{2}{*}{$\begin{array}{l}\text { Radio- } \\
\text { Nuclide }\end{array}$} & \multicolumn{2}{|c|}{$\begin{array}{l}\text { Directly } \\
\text { Ground }\end{array}$} & \multicolumn{2}{|c|}{$\begin{array}{c}\text { from primary } \\
\text { Inhalation }\end{array}$} & & \multicolumn{2}{|c|}{$\begin{array}{c}\text { from release } \\
\text { Plant }\end{array}$} & \multicolumn{2}{|c|}{$\begin{array}{c}\text { to atmosphere } \\
\text { Meat }\end{array}$} & \multicolumn{2}{|c|}{$\begin{array}{c}\text { (Inhalation ex } \\
\text { Milk }\end{array}$} & \multicolumn{2}{|c|}{$\begin{array}{c}\text { excludes radon) } \\
\text { Soil }\end{array}$} & \multicolumn{2}{|c|}{ All Pathways* } \\
\hline & Dose & $\%$ & Dose & 응 & Dose & $\frac{\circ}{0}$ & Dose & $\%$ & Dose & 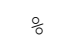 & Dose & $\frac{\circ}{0}$ & Dose & 응 & Dose & $\frac{\circ}{0}$ \\
\hline
\end{tabular}




\begin{tabular}{|c|c|c|c|c|c|c|c|c|c|c|c|c|c|c|c|}
\hline Cs-137 & $6.98 \mathrm{E}-06$ & 0 & $1.53 E-05$ & 0 & $0.00 \mathrm{E}+00$ & 0 & $0.00 \mathrm{E}+00$ & 0 & $0.00 \mathrm{E}+00$ & 0 & $0.00 \mathrm{E}+00$ & 0 & $4.55 \mathrm{E}-09$ & 0 & $2.23 E-05$ \\
\hline $\mathrm{Np}-237$ & $2.38 \mathrm{E}-05$ & 0 & $1.96 \mathrm{E}-01$ & 34 & $0.00 \mathrm{E}+00$ & 0 & $0.00 \mathrm{E}+00$ & 0 & $0.00 \mathrm{E}+00$ & 0 & $0.00 \mathrm{E}+00$ & 0 & $3.94 \mathrm{E}-07$ & 0 & $1.96 \mathrm{E}-01$ \\
\hline $\mathrm{Pu}-238$ & $2.97 \mathrm{E}-10$ & 0 & $4.31 \mathrm{E}-02$ & 8 & $0.00 \mathrm{E}+00$ & 0 & $0.00 \mathrm{E}+00$ & 0 & $0.00 \mathrm{E}+00$ & 0 & $0.00 \mathrm{E}+00$ & 0 & $8.17 \mathrm{E}-08$ & 0 & 4. $31 \mathrm{E}-02$ \\
\hline $\mathrm{Pu}-239$ & $6.38 \mathrm{E}-10$ & 0 & $4.70 \mathrm{E}-02$ & 8 & $0.00 \mathrm{E}+00$ & 0 & $0.00 \mathrm{E}+00$ & 0 & $0.00 \mathrm{E}+00$ & 0 & $0.00 \mathrm{E}+00$ & 0 & $8.95 \mathrm{E}-08$ & 0 & $4.70 \mathrm{E}-02$ \\
\hline $\mathrm{Pu}-240$ & $2.90 \mathrm{E}-10$ & 0 & $4.70 \mathrm{E}-02$ & 8 & $0.00 \mathrm{E}+00$ & 0 & $0.00 \mathrm{E}+00$ & 0 & $0.00 \mathrm{E}+00$ & 0 & $0.00 \mathrm{E}+00$ & 0 & $8.95 \mathrm{E}-08$ & 0 & $4.70 \mathrm{E}-02$ \\
\hline TC-99 & $1.44 \mathrm{E}-10$ & 0 & $5.09 E-05$ & 0 & $0.00 \mathrm{E}+00$ & 0 & $0.00 \mathrm{E}+00$ & 0 & $0.00 \mathrm{E}+00$ & 0 & $0.00 \mathrm{E}+00$ & 0 & $1.21 \mathrm{E}-10$ & 0 & $5.09 \mathrm{E}-05$ \\
\hline Th-228 & $1.43 E-05$ & 0 & $1.71 \mathrm{E}-02$ & 3 & $0.00 \mathrm{E}+00$ & 0 & $0.00 \mathrm{E}+00$ & 0 & $0.00 \mathrm{E}+00$ & 0 & $0.00 \mathrm{E}+00$ & 0 & $3.73 E-08$ & 0 & $1.71 \mathrm{E}-02$ \\
\hline Th-230 & $1.18 \mathrm{E}-08$ & 0 & $3.92 \mathrm{E}-02$ & 7 & $0.00 \mathrm{E}+00$ & 0 & $0.00 \mathrm{E}+00$ & 0 & $0.00 \mathrm{E}+00$ & 0 & $0.00 \mathrm{E}+00$ & 0 & $7.53 \mathrm{E}-08$ & 0 & $3.92 \mathrm{E}-02$ \\
\hline Th-232 & $1.71 \mathrm{E}-06$ & 0 & $4.31 E-02$ & 8 & $0.00 \mathrm{E}+00$ & 0 & $0.00 \mathrm{E}+00$ & 0 & $0.00 \mathrm{E}+00$ & 0 & $0.00 \mathrm{E}+00$ & 0 & $1.09 \mathrm{E}-07$ & 0 & $4.31 E-02$ \\
\hline$U-234$ & $8.25 \mathrm{E}-09$ & 0 & $3.68 \mathrm{E}-02$ & 6 & $0.00 \mathrm{E}+00$ & 0 & $0.00 \mathrm{E}+00$ & 0 & $0.00 \mathrm{E}+00$ & 0 & $0.00 \mathrm{E}+00$ & 0 & $1.68 \mathrm{E}-07$ & 0 & $3.68 \mathrm{E}-02$ \\
\hline U-235 & $1.61 \mathrm{E}-05$ & 0 & $3.33 E-02$ & 6 & $0.00 \mathrm{E}+00$ & 0 & $0.00 \mathrm{E}+00$ & 0 & $0.00 \mathrm{E}+00$ & 0 & $0.00 \mathrm{E}+00$ & 0 & $1.63 \mathrm{E}-07$ & 0 & $3.33 \mathrm{E}-02$ \\
\hline $\mathrm{U}-238$ & $3.38 E-06$ & 0 & $3.14 \mathrm{E}-02$ & 5 & $0.00 \mathrm{E}+00$ & 0 & $0.00 \mathrm{E}+00$ & 0 & $0.00 \mathrm{E}+00$ & 0 & $0.00 \mathrm{E}+00$ & 0 & $1.66 \mathrm{E}-07$ & 0 & $3.14 \mathrm{E}-02$ \\
\hline & & & & & 00 & & $0.00 \mathrm{E}+00$ & 0 & 0 & 0 & 0 & 0 & 6 & 0 & \\
\hline
\end{tabular}

RESRAD-OFFSITE, Version 4.0 .3 beta $T^{\frac{1}{2} / 2}$ Limit $=30$ days

Title: RESRAD-OFFSITE Effluent release

File : PADUCAH EFFLUENT RELEASE-NONINVOLVED-WORKER-REV.ROF

Total Dose Contributions TDOSE (i,p,t) for Individual Radionuclides (i) and Pathways (p) in mrem/yr and as a Percentage of Total Dose at $t=3$ years

\begin{tabular}{|c|c|c|c|c|c|c|c|c|c|c|c|c|c|c|c|c|}
\hline \multirow{2}{*}{$\begin{array}{l}\text { Radio- } \\
\text { Nuclide }\end{array}$} & \multicolumn{2}{|c|}{ Ground } & \multicolumn{2}{|l|}{ Fish } & \multicolumn{2}{|c|}{$\begin{array}{l}\text { From } \\
\text { Radon }\end{array}$} & \multicolumn{2}{|c|}{$\begin{array}{l}\text { es to grour } \\
\text { Plant }\end{array}$} & \multicolumn{2}{|c|}{$\begin{array}{l}\text { water and } \\
\text { Meat }\end{array}$} & \multicolumn{2}{|c|}{$\begin{array}{l}\text { o surface water } \\
\text { Milk }\end{array}$} & \multicolumn{2}{|l|}{ Soil } & \multicolumn{2}{|l|}{ Water } \\
\hline & Dose & $\%$ & Dose & $\%$ & Dose & $\%$ & Dose & $\div$ & Dose & 응 & Dose & 웅 & Dose & $\%$ & Dose & $\%$ \\
\hline$n-241$ & $00 E+00$ & 0 & $.00 E+00$ & 0 & $.00 \mathrm{E}+00$ & 0 & $.00 E+00$ & 0 & $0.00 \mathrm{E}+00$ & 0 & $0.00 \mathrm{E}+00$ & 0 & $0.00 \mathrm{E}+00$ & 0 & $.00 \mathrm{E}+00$ & \\
\hline & $O O E$ & 0 & $00 \mathrm{H}$ & 0 & 001 & 0 & .00 & 0 & 0 & 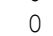 & 0 & 0 & .00 & 0 & 00 & \\
\hline $\mathrm{Np}-237$ & $0.00 \mathrm{E}+00$ & 0 & $0.00 \mathrm{E}+00$ & 0 & $0.00 \mathrm{E}+00$ & 0 & $0.00 \mathrm{E}+00$ & 0 & $0.00 \mathrm{E}+00$ & 0 & $.00 \mathrm{E}+00$ & 0 & $0.00 \mathrm{E}+00$ & 0 & $0.00 \mathrm{E}+00$ & \\
\hline $\mathrm{Pu}-238$ & $00 E+00$ & 0 & $0.00 \mathrm{E}+00$ & 0 & $.00 \mathrm{E}+00$ & 0 & $.00 \mathrm{E}$ & 0 & $.00 \mathrm{E}$ & - & 0 & 0 & $\mathrm{OOH}$ & 0 & $.00 \mathrm{E}+00$ & \\
\hline $\mathrm{Pu}-239$ & $00 E+00$ & 0 & $00 E+00$ & 0 & $00 \mathrm{E}+00$ & 政 & 0 & 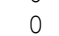 & 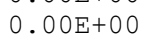 & ( & & - & 0 & 0 & 00 & \\
\hline $\mathrm{Pu}-240$ & $00 E+00$ & 0 & $0.00 \mathrm{E}+00$ & 0 & $0.00 \mathrm{E}+00$ & 0 & $0.00 \mathrm{E}+00$ & 0 & $0.00 \mathrm{E}+$ & 0 & 0 & 0 & $00 \mathrm{E}+$ & 0 & $00 \mathrm{E}+00$ & \\
\hline & (20) & 0 & 0 & 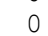 & $00 \mathrm{E}+00$ & 然 & & 0 & & 0 & & & & & & \\
\hline 28 & $0 c$ & 0 & 00 & 0 & 0 & 0 & 0 & 0 & 0 & 0 & 0 & c & 0 & 0 & 0 & \\
\hline Th-230 & $00 E+00$ & 0 & $0.00 \mathrm{E}+00$ & 0 & $00 \mathrm{E}+00$ & 0 & $0.00 \mathrm{E}+00$ & 0 & $0.00 \mathrm{E}+$ & o & 0.001 & 0 & $0.00 \mathrm{~B}$ & 0 & $0.00 \mathrm{E}+00$ & \\
\hline Th-232 & $.00 \mathrm{E}+00$ & 0 & $0.00 \mathrm{E}+00$ & 0 & $0.00 \mathrm{E}+00$ & 0 & $0.00 \mathrm{E}+00$ & 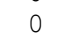 & 0.00 & - & & 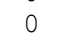 & - & 0 & $0.00 \mathrm{E}+00$ & \\
\hline $\mathrm{U}-234$ & חמת תחת & 0 & مחת תמת ת & 0 & ק & 0 & 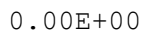 & 0 & 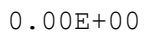 & 0 & & & & & & \\
\hline $\mathrm{U}-23$ & 0 & 0 & 0 & 0 & 0 & U & 0 & 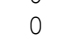 & 0 & 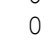 & & 0 & 00 & 0 & $.00 \mathrm{E}+00$ & \\
\hline $\mathrm{U}-238$ & $0.00 \mathrm{E}+00$ & 0 & $0.00 \mathrm{E}+00$ & 0 & $0.00 \mathrm{E}+00$ & 0 & $0.00 \mathrm{E}+00$ & 0 & $0.00 \mathrm{E}+00$ & 0 & $0.00 \mathrm{E}+00$ & 0 & $0.00 \mathrm{E}+00$ & 0 & $0.00 \mathrm{E}+00$ & \\
\hline & & 0 & 00E+00 & 0 & +00 & 0 & Ju & 0 & & 0 & 0 & 0 & 0 & 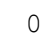 & 10 & \\
\hline
\end{tabular}

0

Total Dose Contributions TDOSE (i,p,t) for Individual Radionuclides (i) and Pathways (p) in $\mathrm{mrem} / \mathrm{yr}$ and as a Percentage of Total Dose at $t=3$ years

0 Directly from primary contamination and from release to atmosphere (Inhalation excludes radon)

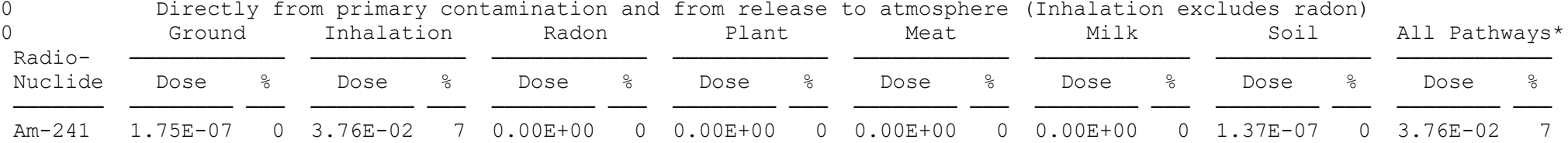




\begin{tabular}{|c|c|c|c|c|c|c|c|c|c|c|c|c|c|c|c|}
\hline $\mathrm{Cs}-137$ & $1.59 \mathrm{E}-05$ & 0 & $1.53 E-05$ & 0 & $0.00 \mathrm{E}+00$ & 0 & $0.00 \mathrm{E}+00$ & 0 & $0.00 \mathrm{E}+00$ & 0 & $0.00 \mathrm{E}+00$ & 0 & $1.04 \mathrm{E}-08$ & 0 & $3.12 \mathrm{E}-05$ \\
\hline Np-237 & $5.51 \mathrm{E}-05$ & 0 & $1.96 \mathrm{E}-01$ & 34 & $0.00 \mathrm{E}+00$ & 0 & $0.00 \mathrm{E}+00$ & 0 & $0.00 \mathrm{E}+00$ & 0 & $0.00 \mathrm{E}+00$ & 0 & $9.12 \mathrm{E}-07$ & 0 & $1.96 \mathrm{E}-01$ \\
\hline $\mathrm{Pu}-238$ & $6.87 \mathrm{E}-10$ & 0 & $4.31 E-02$ & 8 & $0.00 \mathrm{E}+00$ & 0 & $0.00 \mathrm{E}+00$ & 0 & $0.00 \mathrm{E}+00$ & 0 & $0.00 \mathrm{E}+00$ & 0 & $1.89 \mathrm{E}-07$ & 0 & $4.31 \mathrm{E}-02$ \\
\hline $\mathrm{Pu}-239$ & $1.49 \mathrm{E}-09$ & 0 & $4.70 \mathrm{E}-02$ & 8 & $0.00 \mathrm{E}+00$ & 0 & $0.00 \mathrm{E}+00$ & 0 & $0.00 \mathrm{E}+00$ & 0 & $0.00 \mathrm{E}+00$ & 0 & $2.09 \mathrm{E}-07$ & 0 & $4.70 \mathrm{E}-02$ \\
\hline $\mathrm{Pu}-240$ & $6.76 \mathrm{E}-10$ & 0 & $4.70 \mathrm{E}-02$ & 8 & $0.00 \mathrm{E}+00$ & 0 & $0.00 \mathrm{E}+00$ & 0 & $0.00 \mathrm{E}+00$ & 0 & $0.00 \mathrm{E}+00$ & 0 & $2.09 \mathrm{E}-07$ & 0 & $4.70 \mathrm{E}-02$ \\
\hline TC-99 & $1.64 \mathrm{E}-10$ & 0 & $5.09 E-05$ & 0 & $0.00 \mathrm{E}+00$ & 0 & $0.00 \mathrm{E}+00$ & 0 & $0.00 \mathrm{E}+00$ & 0 & $0.00 \mathrm{E}+00$ & 0 & $1.38 \mathrm{E}-10$ & 0 & $5.09 \mathrm{E}-05$ \\
\hline Th-228 & $2.55 \mathrm{E}-05$ & 0 & $1.71 \mathrm{E}-02$ & 3 & $0.00 \mathrm{E}+00$ & 0 & $0.00 \mathrm{E}+00$ & 0 & $0.00 \mathrm{E}+00$ & 0 & $0.00 \mathrm{E}+00$ & 0 & $6.66 \mathrm{E}-08$ & 0 & $1.71 \mathrm{E}-02$ \\
\hline Th-230 & $4.67 \mathrm{E}-08$ & 0 & $3.92 \mathrm{E}-02$ & 7 & $0.00 \mathrm{E}+00$ & 0 & $0.00 \mathrm{E}+00$ & 0 & $0.00 \mathrm{E}+00$ & 0 & $0.00 \mathrm{E}+00$ & 0 & $1.76 \mathrm{E}-07$ & 0 & $3.92 \mathrm{E}-02$ \\
\hline Th-232 & $8.29 \mathrm{E}-06$ & 0 & $4.31 E-02$ & 8 & $0.00 \mathrm{E}+00$ & 0 & $0.00 \mathrm{E}+00$ & 0 & $0.00 \mathrm{E}+00$ & 0 & $0.00 \mathrm{E}+00$ & 0 & $3.07 \mathrm{E}-07$ & 0 & $4.31 \mathrm{E}-02$ \\
\hline $\mathrm{U}-234$ & $1.85 \mathrm{E}-08$ & 0 & $3.68 \mathrm{E}-02$ & 6 & $0.00 \mathrm{E}+00$ & 0 & $0.00 \mathrm{E}+00$ & 0 & $0.00 \mathrm{E}+00$ & 0 & $0.00 \mathrm{E}+00$ & 0 & $3.78 \mathrm{E}-07$ & 0 & $3.68 \mathrm{E}-02$ \\
\hline $\mathrm{U}-235$ & $3.61 E-05$ & 0 & $3.33 E-02$ & 6 & $0.00 \mathrm{E}+00$ & 0 & $0.00 \mathrm{E}+00$ & 0 & $0.00 \mathrm{E}+00$ & 0 & $0.00 \mathrm{E}+00$ & 0 & $3.65 \mathrm{E}-07$ & 0 & $3.34 \mathrm{E}-02$ \\
\hline $\mathrm{U}-238$ & $7.59 \mathrm{E}-06$ & 0 & $3.14 \mathrm{E}-02$ & 5 & $0.00 \mathrm{E}+00$ & 0 & $0.00 \mathrm{E}+00$ & 0 & $0.00 \mathrm{E}+00$ & 0 & $0.00 \mathrm{E}+00$ & 0 & $3.73 \mathrm{E}-07$ & 0 & $3.14 \mathrm{E}-02$ \\
\hline & & & & & & & & 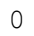 & $0.00 \mathrm{E}+00$ & 0 & $0.00 \mathrm{E}+00$ & 0 & -06 & 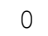 & -01 \\
\hline
\end{tabular}

RESRAD-OFFSITE, Version 4.0 .3 beta $T^{\frac{1}{2} / 2}$ Limit $=30$ days

Title: RESRAD-OFFSITE Effluent release

File : PADUCAH EFFLUENT RELEASE-NONINVOLVED-WORKER-REV.ROF

Total Dose Contributions TDOSE (i,p,t) for Individual Radionuclides (i) and Pathways (p) in mrem/yr and as a Percentage of Total Dose at $t=6$ years

\begin{tabular}{|c|c|c|c|c|c|c|c|c|c|c|c|c|c|c|c|c|}
\hline \multirow{2}{*}{$\begin{array}{l}\text { Radio- } \\
\text { Nuclide }\end{array}$} & \multicolumn{2}{|c|}{ Ground } & \multicolumn{2}{|l|}{ Fish } & \multicolumn{2}{|c|}{$\begin{array}{l}\text { From } \\
\text { Radon }\end{array}$} & \multicolumn{2}{|c|}{$\begin{array}{l}\text { es to groun } \\
\text { Plant }\end{array}$} & \multicolumn{2}{|c|}{$\begin{array}{l}\text { water and } \\
\text { Meat }\end{array}$} & \multicolumn{2}{|c|}{$\begin{array}{l}\text { o surface water } \\
\text { Milk }\end{array}$} & \multicolumn{2}{|l|}{ Soil } & \multicolumn{2}{|l|}{ Water } \\
\hline & Dose & $\%$ & Dose & $\%$ & Dose & $\%$ & Dose & $\div$ & Dose & 응 & Dose & 웅 & Dose & $\%$ & Dose & $\%$ \\
\hline$n-241$ & $00 E+00$ & 0 & $.00 E+00$ & 0 & $.00 \mathrm{E}+00$ & 0 & $.00 E+00$ & 0 & $0.00 \mathrm{E}+00$ & 0 & $0.00 \mathrm{E}+00$ & 0 & $0.00 \mathrm{E}+00$ & 0 & $.00 \mathrm{E}+00$ & \\
\hline & $\mathrm{OOF}$ & 0 & $00 \mathrm{H}$ & 0 & 001 & 0 & .00 & 0 & .00 & 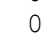 & 0 & 0 & 00 & 0 & 00 & \\
\hline $\mathrm{Np}-237$ & $0.00 \mathrm{E}+00$ & 0 & $0.00 \mathrm{E}+00$ & 0 & $0.00 \mathrm{E}+00$ & 0 & $0.00 \mathrm{E}+00$ & 0 & $0.00 \mathrm{E}+00$ & 0 & $.00 \mathrm{E}+00$ & 0 & $0.00 \mathrm{E}+00$ & 0 & $0.00 \mathrm{E}+00$ & \\
\hline $\mathrm{Pu}-238$ & $00 E+00$ & 0 & $0.00 \mathrm{E}+00$ & 0 & $.00 \mathrm{E}+00$ & 0 & $.00 \mathrm{E}$ & 0 & $.00 \mathrm{E}$ & - & 0 & 0 & $\mathrm{OOH}$ & 0 & 00 & \\
\hline $\mathrm{Pu}-239$ & $00 E+00$ & 0 & $00 E+00$ & 0 & $00 \mathrm{E}+00$ & 政 & 0 & 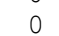 & 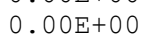 & ( & & - & 0 & 0 & .00 & \\
\hline $\mathrm{Pu}-240$ & $00 E+00$ & 0 & $0.00 \mathrm{E}+00$ & 0 & $0.00 \mathrm{E}+00$ & 0 & $0.00 \mathrm{E}+00$ & 0 & $0.00 \mathrm{E}+$ & 0 & 0 & 0 & $00 \mathrm{E}$ & 0 & $00 \mathrm{E}+00$ & \\
\hline & (20) & 0 & 0 & 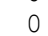 & $00 \mathrm{E}+00$ & 然 & & 0 & & 0 & & & & & & \\
\hline 28 & $0 c$ & 0 & 00 & 0 & 0 & 0 & 0 & 0 & 0 & 0 & 0 & c & 0 & 0 & 0 & \\
\hline Th-230 & $00 E+00$ & 0 & $0.00 \mathrm{E}+00$ & 0 & $00 \mathrm{E}+00$ & 0 & $0.00 \mathrm{E}+00$ & 0 & $0.00 \mathrm{E}+$ & o & 0.001 & 0 & $0.00 \mathrm{~B}$ & 0 & $0.00 \mathrm{E}+00$ & \\
\hline Th-232 & $.00 \mathrm{E}+00$ & 0 & $0.00 \mathrm{E}+00$ & 0 & $0.00 \mathrm{E}+00$ & 0 & $0.00 \mathrm{E}+00$ & 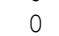 & 0.00 & - & & 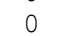 & 0 & 0 & $0.00 \mathrm{E}+00$ & \\
\hline $\mathrm{U}-234$ & חמת תחת & 0 & נותחת ח & 0 & ק & 0 & 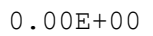 & 0 & 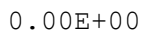 & 0 & & & & & & \\
\hline $\mathrm{U}-23$ & 0 & 0 & 0 & 0 & 0 & U & 0 & 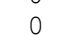 & 0 & 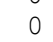 & & 0 & 00 & 0 & $.00 \mathrm{E}+00$ & \\
\hline $\mathrm{U}-238$ & $0.00 \mathrm{E}+00$ & 0 & $0.00 \mathrm{E}+00$ & 0 & $0.00 \mathrm{E}+00$ & 0 & $0.00 \mathrm{E}+00$ & 0 & $0.00 \mathrm{E}+00$ & 0 & $0.00 \mathrm{E}+00$ & 0 & $0.00 \mathrm{E}+00$ & 0 & $0.00 \mathrm{E}+00$ & \\
\hline & & 0 & 00E+00 & 0 & +00 & 0 & Ju & 0 & & 0 & 0 & 0 & 0 & 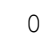 & 10 & \\
\hline
\end{tabular}

0

Total Dose Contributions TDOSE (i, p,t) for Individual Radionuclides (i) and Pathways (p) in mrem/yr and as a Percentage of Total Dose at $t=6$ years

0 Directly from primary contamination and from release to atmosphere (Inhalation excludes radon)

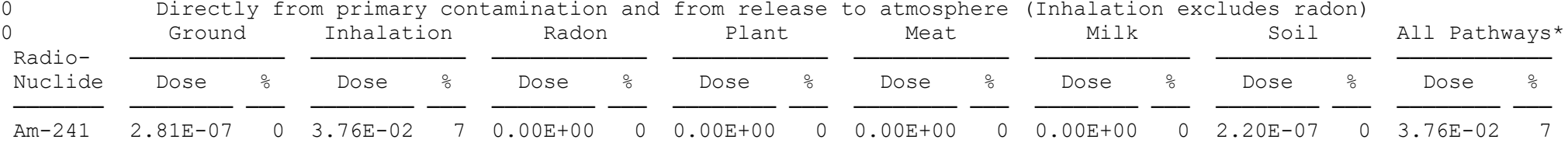




\begin{tabular}{|c|c|c|c|c|c|c|c|c|c|c|c|c|c|c|c|c|}
\hline $\mathrm{Cs}-137$ & $2.86 \mathrm{E}-05$ & 0 & $1.53 E-05$ & 0 & $0.00 \mathrm{E}+00$ & 0 & $0.00 \mathrm{E}+00$ & 0 & $0.00 \mathrm{E}+00$ & 0 & $0.00 \mathrm{E}+00$ & 0 & $1.87 \mathrm{E}-08$ & 0 & $4.39 E-05$ & 0 \\
\hline$N p-237$ & $1.01 \mathrm{E}-04$ & 0 & $1.96 \mathrm{E}-01$ & 34 & $0.00 \mathrm{E}+00$ & 0 & $0.00 \mathrm{E}+00$ & 0 & $0.00 \mathrm{E}+00$ & 0 & $0.00 \mathrm{E}+00$ & 0 & $1.67 \mathrm{E}-06$ & 0 & $1.96 \mathrm{E}-01$ & 34 \\
\hline $\mathrm{Pu}-238$ & $1.26 \mathrm{E}-09$ & 0 & $4.31 \mathrm{E}-02$ & 8 & $0.00 \mathrm{E}+00$ & 0 & $0.00 \mathrm{E}+00$ & 0 & $0.00 \mathrm{E}+00$ & 0 & $0.00 \mathrm{E}+00$ & 0 & $3.47 \mathrm{E}-07$ & 0 & $4.31 E-02$ & 8 \\
\hline $\mathrm{Pu}-239$ & $2.76 \mathrm{E}-09$ & 0 & $4.70 \mathrm{E}-02$ & 8 & $0.00 \mathrm{E}+00$ & 0 & $0.00 \mathrm{E}+00$ & 0 & $0.00 \mathrm{E}+00$ & 0 & $0.00 \mathrm{E}+00$ & 0 & $3.87 \mathrm{E}-07$ & 0 & $4.70 \mathrm{E}-02$ & 8 \\
\hline $\mathrm{Pu}-240$ & $1.25 \mathrm{E}-09$ & 0 & $4.70 \mathrm{E}-02$ & 8 & $0.00 \mathrm{E}+00$ & 0 & $0.00 \mathrm{E}+00$ & 0 & $0.00 \mathrm{E}+00$ & 0 & $0.00 \mathrm{E}+00$ & 0 & $3.87 \mathrm{E}-07$ & 0 & $4.70 \mathrm{E}-02$ & 8 \\
\hline TC-99 & $1.64 \mathrm{E}-10$ & 0 & $5.09 E-05$ & 0 & $0.00 \mathrm{E}+00$ & 0 & $0.00 \mathrm{E}+00$ & 0 & $0.00 \mathrm{E}+00$ & 0 & $0.00 \mathrm{E}+00$ & 0 & $1.38 \mathrm{E}-10$ & 0 & $5.09 \mathrm{E}-05$ & 0 \\
\hline Th-228 & $3.26 \mathrm{E}-05$ & 0 & $1.71 \mathrm{E}-02$ & 3 & $0.00 \mathrm{E}+00$ & 0 & $0.00 \mathrm{E}+00$ & 0 & $0.00 \mathrm{E}+00$ & 0 & $0.00 \mathrm{E}+00$ & 0 & $8.51 \mathrm{E}-08$ & 0 & $1.71 \mathrm{E}-02$ & 3 \\
\hline Th-230 & $1.41 \mathrm{E}-07$ & 0 & $3.92 \mathrm{E}-02$ & 7 & $0.00 \mathrm{E}+00$ & 0 & $0.00 \mathrm{E}+00$ & 0 & $0.00 \mathrm{E}+00$ & 0 & $0.00 \mathrm{E}+00$ & 0 & $3.27 \mathrm{E}-07$ & 0 & $3.92 \mathrm{E}-02$ & 7 \\
\hline Th-232 & $2.81 E-05$ & 0 & $4.31 E-02$ & 8 & $0.00 \mathrm{E}+00$ & 0 & $0.00 \mathrm{E}+00$ & 0 & $0.00 \mathrm{E}+00$ & 0 & $0.00 \mathrm{E}+00$ & 0 & $7.02 \mathrm{E}-07$ & 0 & $4.31 E-02$ & 8 \\
\hline $\mathrm{U}-234$ & $3.24 \mathrm{E}-08$ & 0 & $3.68 \mathrm{E}-02$ & 6 & $0.00 \mathrm{E}+00$ & 0 & $0.00 \mathrm{E}+00$ & 0 & $0.00 \mathrm{E}+00$ & 0 & $0.00 \mathrm{E}+00$ & 0 & $6.60 \mathrm{E}-07$ & 0 & $3.68 \mathrm{E}-02$ & 6 \\
\hline $\mathrm{U}-235$ & $6.31 \mathrm{E}-05$ & 0 & $3.33 E-02$ & 6 & $0.00 \mathrm{E}+00$ & 0 & $0.00 \mathrm{E}+00$ & 0 & $0.00 \mathrm{E}+00$ & 0 & $0.00 \mathrm{E}+00$ & 0 & $6.38 \mathrm{E}-07$ & 0 & $3.34 \mathrm{E}-02$ & 6 \\
\hline $\mathrm{U}-238$ & $1.33 E-05$ & 0 & $3.14 \mathrm{E}-02$ & 5 & $0.00 \mathrm{E}+00$ & 0 & $0.00 \mathrm{E}+00$ & 0 & $0.00 \mathrm{E}+00$ & 0 & $0.00 \mathrm{E}+00$ & 0 & $6.52 \mathrm{E}-07$ & 0 & $3.14 \mathrm{E}-02$ & 5 \\
\hline & . & 0 & & & +00 & 0 & $0 \mathrm{E}+00$ & 0 & 0 & 0 & 0 & 0 & - & 0 & 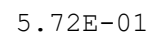 & 0 \\
\hline
\end{tabular}

TRESRAD-OFFSITE, Version 4.0 .3 beta $T^{\frac{1}{2} / 2}$ Limit $=30$ days

Title: RESRAD-OFFSITE Effluent release

File : PADUCAH EFFLUENT RELEASE-NONINVOLVED-WORKER-REV.ROF

Total Dose Contributions TDOSE (i,p,t) for Individual Radionuclides (i) and Pathways (p) in mrem/yr and as a Percentage of Total Dose at $t=10$ years

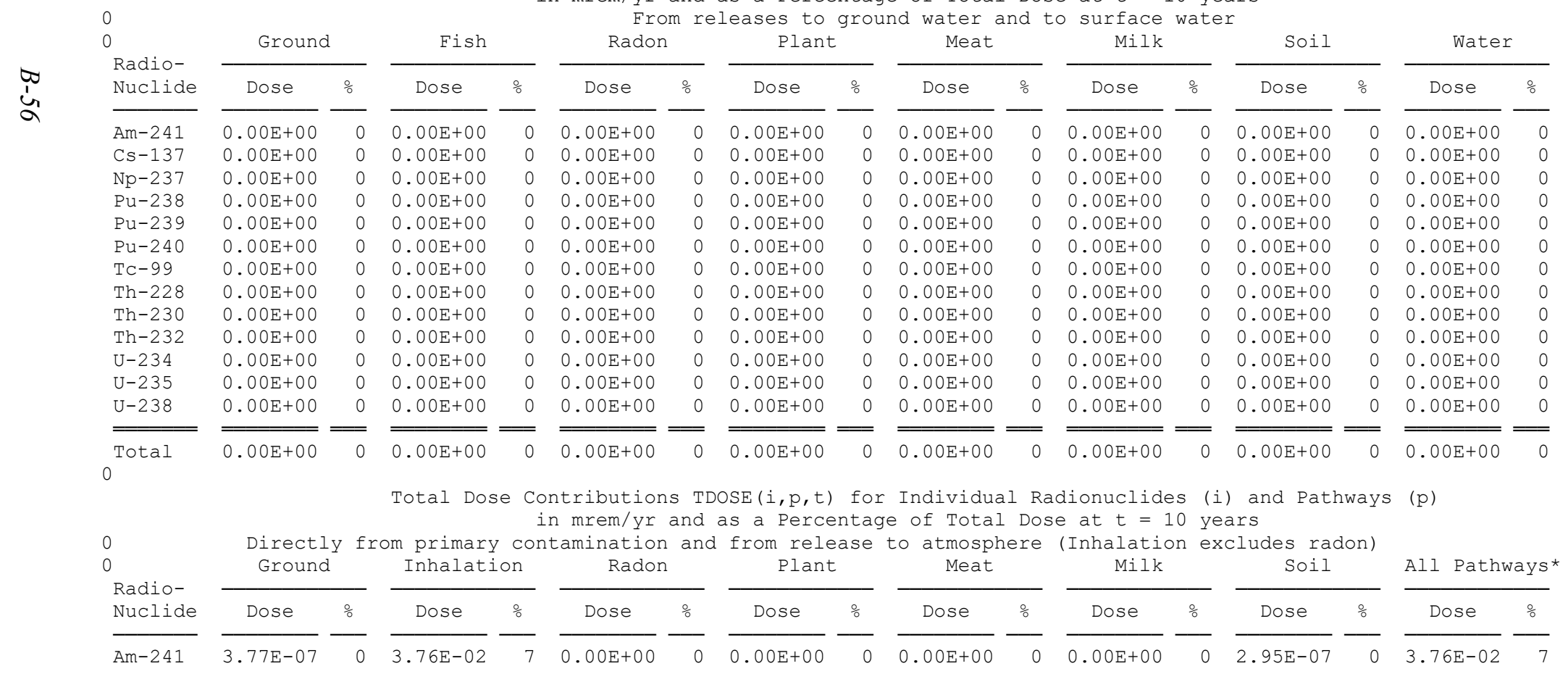




\begin{tabular}{|c|c|c|c|c|c|c|c|c|c|c|c|c|c|c|c|c|}
\hline $\mathrm{Cs}-137$ & $4.42 \mathrm{E}-05$ & 0 & $1.53 \mathrm{E}-05$ & 0 & $0.00 \mathrm{E}+00$ & 0 & $0.00 \mathrm{E}+00$ & 0 & $0.00 \mathrm{E}+00$ & 0 & $0.00 \mathrm{E}+00$ & 0 & $2.89 \mathrm{E}-08$ & 0 & $5.96 \mathrm{E}-05$ & 0 \\
\hline Np-237 & $1.61 \mathrm{E}-04$ & 0 & $1.96 \mathrm{E}-01$ & 34 & $0.00 \mathrm{E}+00$ & 0 & $0.00 \mathrm{E}+00$ & 0 & $0.00 \mathrm{E}+00$ & 0 & $0.00 \mathrm{E}+00$ & 0 & $2.66 \mathrm{E}-06$ & 0 & $1.96 \mathrm{E}-01$ & 34 \\
\hline $\mathrm{Pu}-238$ & $2.00 E-09$ & 0 & $4.31 \mathrm{E}-02$ & 8 & $0.00 \mathrm{E}+00$ & 0 & $0.00 \mathrm{E}+00$ & 0 & $0.00 \mathrm{E}+00$ & 0 & $0.00 \mathrm{E}+00$ & 0 & $5.50 \mathrm{E}-07$ & 0 & $4.31 \mathrm{E}-02$ & 8 \\
\hline Pu-239 & $4.44 \mathrm{E}-09$ & 0 & $4.70 \mathrm{E}-02$ & 8 & $0.00 \mathrm{E}+00$ & 0 & $0.00 \mathrm{E}+00$ & 0 & $0.00 \mathrm{E}+00$ & 0 & $0.00 \mathrm{E}+00$ & 0 & $6.23 \mathrm{E}-07$ & 0 & $4.70 \mathrm{E}-02$ & 8 \\
\hline Pu-240 & $2.02 E-09$ & 0 & $4.70 \mathrm{E}-02$ & 8 & $0.00 \mathrm{E}+00$ & 0 & $0.00 \mathrm{E}+00$ & 0 & $0.00 \mathrm{E}+00$ & 0 & $0.00 \mathrm{E}+00$ & 0 & $6.23 \mathrm{E}-07$ & 0 & $4.70 \mathrm{E}-02$ & 8 \\
\hline TC-99 & $1.64 \mathrm{E}-10$ & 0 & $5.09 \mathrm{E}-05$ & 0 & $0.00 \mathrm{E}+00$ & 0 & $0.00 \mathrm{E}+00$ & 0 & $0.00 \mathrm{E}+00$ & 0 & $0.00 \mathrm{E}+00$ & 0 & $1.38 \mathrm{E}-10$ & 0 & $5.09 \mathrm{E}-05$ & 0 \\
\hline Th-228 & $3.54 \mathrm{E}-05$ & 0 & $1.71 \mathrm{E}-02$ & 3 & $0.00 \mathrm{E}+00$ & 0 & $0.00 \mathrm{E}+00$ & 0 & $0.00 \mathrm{E}+00$ & 0 & $0.00 \mathrm{E}+00$ & 0 & $9.23 \mathrm{E}-08$ & 0 & $1.71 \mathrm{E}-02$ & 3 \\
\hline Th-230 & $3.40 \mathrm{E}-07$ & 0 & $3.92 \mathrm{E}-02$ & 7 & $0.00 \mathrm{E}+00$ & 0 & $0.00 \mathrm{E}+00$ & 0 & $0.00 \mathrm{E}+00$ & 0 & $0.00 \mathrm{E}+00$ & 0 & $5.29 \mathrm{E}-07$ & 0 & $3.92 \mathrm{E}-02$ & 7 \\
\hline Th-232 & $6.96 \mathrm{E}-05$ & 0 & $4.31 \mathrm{E}-02$ & 8 & $0.00 \mathrm{E}+00$ & 0 & $0.00 \mathrm{E}+00$ & 0 & $0.00 \mathrm{E}+00$ & 0 & $0.00 \mathrm{E}+00$ & 0 & $1.36 \mathrm{E}-06$ & 0 & $4.32 \mathrm{E}-02$ & 8 \\
\hline$U-234$ & $4.83 E-08$ & 0 & $3.68 \mathrm{E}-02$ & 6 & $0.00 \mathrm{E}+00$ & 0 & $0.00 \mathrm{E}+00$ & 0 & $0.00 \mathrm{E}+00$ & 0 & $0.00 \mathrm{E}+00$ & 0 & $9.82 \mathrm{E}-07$ & 0 & $3.68 \mathrm{E}-02$ & 6 \\
\hline$U-235$ & $9.39 \mathrm{E}-05$ & 0 & $3.33 \mathrm{E}-02$ & 6 & $0.00 \mathrm{E}+00$ & 0 & $0.00 \mathrm{E}+00$ & 0 & $0.00 \mathrm{E}+00$ & 0 & $0.00 \mathrm{E}+00$ & 0 & $9.51 \mathrm{E}-07$ & 0 & $3.34 \mathrm{E}-02$ & 6 \\
\hline $\mathrm{U}-238$ & $1.97 \mathrm{E}-05$ & 0 & $3.14 \mathrm{E}-02$ & 5 & $0.00 \mathrm{E}+00$ & 0 & $0.00 \mathrm{E}+00$ & 0 & $0.00 \mathrm{E}+00$ & 0 & $0.00 \mathrm{E}+00$ & 0 & $9.70 \mathrm{E}-07$ & 0 & $3.14 \mathrm{E}-02$ & 5 \\
\hline & & 0 & $5.72 E-01$ & & .00 & 0 & 0 & 0 & $0.00 \mathrm{E}+00$ & 0 & $0.00 \mathrm{E}+00$ & 0 & $9.66 \mathrm{E}-06$ & 0 & $5.72 \mathrm{E}-01$ & 00 \\
\hline
\end{tabular}

$0 *$ Sum of dose from all releases and from primary contamination.

1RESRAD-OFFSITE, Version 4.0 .3 beta T1/2 Limit $=30$ days $\quad 06 / 11 / 2020 \quad 11: 39 \quad$ Page 55

Parent Dose Report

Title : RESRAD-OFFSITE Effluent release

File : PADUCAH EFFLUENT RELEASE-NONINVOLVED-WORKER-REV.ROF

Dose/Source Ratios Summed Over All Pathways

Parent and Progeny Principal Radionuclide Contributions Indicated

0 Parent Product Thread DSR $(j, \mathrm{t}) \quad(\mathrm{mrem} / \mathrm{yr}) /(\mathrm{pCi} / \mathrm{g})$

\begin{tabular}{|c|c|c|c|c|c|c|c|}
\hline $\begin{array}{l}\operatorname{aren} \\
\text { (i) }\end{array}$ & $\begin{array}{l}\text { roduct } \\
(j)\end{array}$ & Fraction & $0.000 \mathrm{E}+00$ & $1.000 \mathrm{E}+00$ & $3.000 \mathrm{E}+00$ & $6.000 \mathrm{E}+00$ & $1.000 \mathrm{E}+01$ \\
\hline & 11 & $1.000 \mathrm{E}+00$ & $1.254 \mathrm{E}-02$ & $1.254 \mathrm{E}-02$ & $1.254 \mathrm{E}-02$ & $1.254 \mathrm{E}-02$ & $1.254 \mathrm{E}-02$ \\
\hline 241 & $\mathrm{~Np}-237+\mathrm{D}$ & $1.000 \mathrm{E}+00$ & $6.104 \mathrm{E}-14$ & $2.715 \mathrm{E}-13$ & $1.147 \mathrm{E}-12$ & $3.415 \mathrm{E}-12$ & $7.692 \mathrm{E}-12$ \\
\hline 241 & U-233 & $1.000 \mathrm{E}+00$ & $3.914 \mathrm{E}-21$ & $2.929 \mathrm{E}-20$ & $2.277 \mathrm{E}-19$ & $1.155 \mathrm{E}-18$ & $4.071 E-18$ \\
\hline 241 & $T h-229+D$ & $1.000 \mathrm{E}+00$ & $6.677 \mathrm{E}-24$ & $9.142 \mathrm{E}-23$ & $1.298 \mathrm{E}-21$ & $1.112 \mathrm{E}-20$ & $6.328 \mathrm{E}-20$ \\
\hline 241 & $\Sigma \mathrm{DSR}(j)$ & & $1.254 \mathrm{E}-02$ & $1.254 \mathrm{E}-02$ & $1.254 \mathrm{E}-02$ & $1.254 \mathrm{E}-02$ & $1.254 \mathrm{E}-02$ \\
\hline $37+D$ & $C s-137+D$ & $1.000 \mathrm{E}+00$ & $5.877 \mathrm{E}-06$ & $7.422 \mathrm{E}-06$ & $1.041 E-05$ & $1.465 \mathrm{E}-05$ & $1.985 \mathrm{E}-05$ \\
\hline $237+D$ & $\mathrm{~Np}-237+\mathrm{D}$ & $1.000 \mathrm{E}+00$ & $6.532 \mathrm{E}-03$ & $6.533 \mathrm{E}-03$ & $6.534 \mathrm{E}-03$ & $6.536 \mathrm{E}-03$ & $6.538 \mathrm{E}-03$ \\
\hline$-237+D$ & $U-233$ & $1.000 \mathrm{E}+00$ & $2.986 \mathrm{E}-14$ & $1.353 \mathrm{E}-13$ & $5.929 \mathrm{E}-13$ & $1.865 \mathrm{E}-12$ & $4.506 \mathrm{E}-12$ \\
\hline$-237+D$ & Th $-229+D$ & $1.000 \mathrm{E}+00$ & $6.759 \mathrm{E}-17$ & $5.202 \mathrm{E}-16$ & $4.246 \mathrm{E}-15$ & $2.309 \mathrm{E}-14$ & $8.970 \mathrm{E}-14$ \\
\hline 37 & $\Sigma \mathrm{DSR}(j)$ & & $6.532 \mathrm{E}-03$ & $6.533 \mathrm{E}-03$ & $6.534 \mathrm{E}-03$ & $6.536 \mathrm{E}-03$ & $6.538 \mathrm{E}-03$ \\
\hline 38 & $\mathrm{Pu}-238$ & $1.840 \mathrm{E}-09$ & $0.000 \mathrm{E}+00$ & $0.000 \mathrm{E}+00$ & $0.000 \mathrm{E}+00$ & $0.000 \mathrm{E}+00$ & $0.000 \mathrm{E}+00$ \\
\hline-238 & $\mathrm{Pu}-238$ & $1.000 \mathrm{E}+00$ & $1.437 \mathrm{E}-02$ & $1.437 \mathrm{E}-02$ & $1.437 \mathrm{E}-02$ & $1.437 \mathrm{E}-02$ & $1.437 \mathrm{E}-02$ \\
\hline 238 & U-234 & $1.000 \mathrm{E}+00$ & $1.847 \mathrm{E}-14$ & $8.368 \mathrm{E}-14$ & $3.667 \mathrm{E}-13$ & $1.153 \mathrm{E}-12$ & $2.784 \mathrm{E}-12$ \\
\hline & Th-230 & $1.000 \mathrm{E}+00$ & $6.553 E-19$ & $5.043 \mathrm{E}-18$ & $4.116 \mathrm{E}-17$ & $2.238 \mathrm{E}-16$ & $8.691 \mathrm{E}-16$ \\
\hline & $\mathrm{Ra}-226+\mathrm{D}$ & $1.000 \mathrm{E}+00$ & $2.219 \mathrm{E}-21$ & $3.092 \mathrm{E}-20$ & $4.513 E-19$ & $4.015 \mathrm{E}-18$ & $2.409 \mathrm{E}-17$ \\
\hline 238 & $\mathrm{~Pb}-210+\mathrm{D}$ & $1.000 \mathrm{E}+00$ & $2.362 E-25$ & $6.206 \mathrm{E}-24$ & $1.604 \mathrm{E}-22$ & $2.262 \mathrm{E}-21$ & $2.063 E-20$ \\
\hline & Po-210 & $1.000 \mathrm{E}+00$ & $9.737 \mathrm{E}-26$ & $3.904 \mathrm{E}-24$ & $1.332 \mathrm{E}-22$ & $2.202 \mathrm{E}-21$ & $2.233 E-20$ \\
\hline & $\Sigma \mathrm{DSR}(j)$ & & $1.437 \mathrm{E}-02$ & $1.437 \mathrm{E}-02$ & $1.437 \mathrm{E}-02$ & $1.437 \mathrm{E}-02$ & $1.437 \mathrm{E}-02$ \\
\hline & $\mathrm{Pu}-239$ & $1.000 \mathrm{E}+00$ & $1.568 \mathrm{E}-02$ & $1.568 \mathrm{E}-02$ & $1.568 \mathrm{E}-02$ & $1.568 \mathrm{E}-02$ & $1.568 \mathrm{E}-02$ \\
\hline & $\mathrm{U}-235+\mathrm{D}$ & $1.000 \mathrm{E}+00$ & $1.184 \mathrm{E}-16$ & $5.379 \mathrm{E}-16$ & $2.368 E-15$ & $7.501 \mathrm{E}-15$ & $1.831 \mathrm{E}-14$ \\
\hline & $\mathrm{Pa}-231$ & $1.000 \mathrm{E}+00$ & $1.069 \mathrm{E}-21$ & $8.154 \mathrm{E}-21$ & $6.546 \mathrm{E}-20$ & $3.475 \mathrm{E}-19$ & $1.305 \mathrm{E}-18$ \\
\hline & $A C-227+D$ & $1.000 \mathrm{E}+00$ & $5.387 \mathrm{E}-23$ & $7.268 \mathrm{E}-22$ & $1.007 \mathrm{E}-20$ & $8.350 \mathrm{E}-20$ & $4.537 \mathrm{E}-19$ \\
\hline & $\Sigma \operatorname{DSR}(j)$ & & $1.568 \mathrm{E}-02$ & $1.568 \mathrm{E}-02$ & $1.568 \mathrm{E}-02$ & $1.568 \mathrm{E}-02$ & $1.568 \mathrm{E}-02$ \\
\hline & Pu-240 & $950 E-08$ & $0.000 \mathrm{E}+00$ & $0.000 \mathrm{E}+00$ & $0.000 \mathrm{E}+00$ & $0.000 \mathrm{E}+00$ & $0.000 \mathrm{E}+00$ \\
\hline & $\mathrm{Pu}-240$ & $1.000 \mathrm{E}+00$ & $1.568 \mathrm{E}-02$ & $1.568 \mathrm{E}-02$ & $1.568 \mathrm{E}-02$ & $1.568 \mathrm{E}-02$ & $1.568 \mathrm{E}-02$ \\
\hline
\end{tabular}




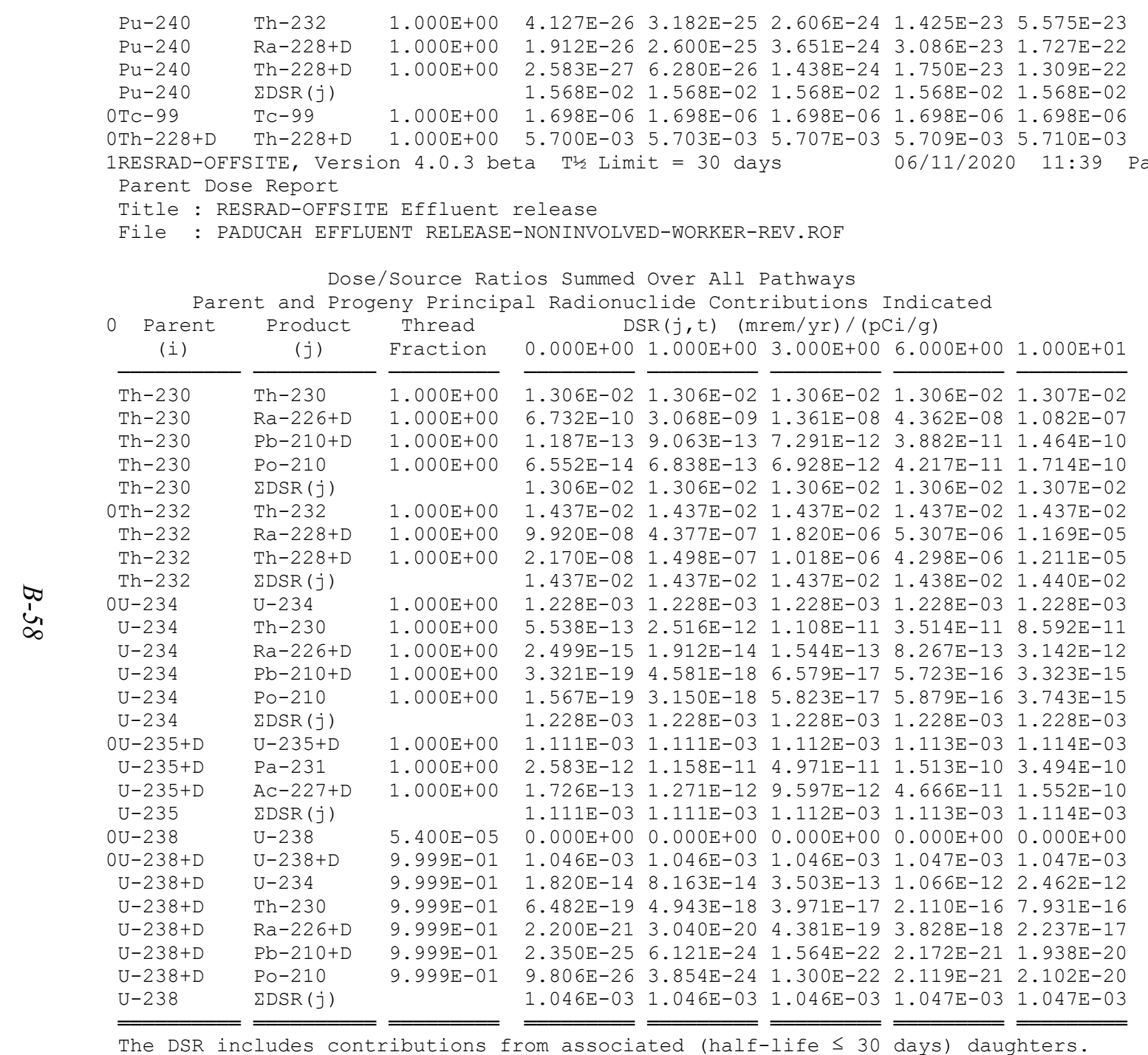

The DSR includes contributions from associated (half-life $\leq 30$ days) daughters.

1RESRAD-OFFSITE, Version 4.0 .3 beta T1/2 Limit $=30$ days $06 / 11 / 2020 \quad 11: 39$ Page

Parent Dose Report

Title : RESRAD-OFFSITE Effluent release

File : PADUCAH EFFLUENT RELEASE-NONINVOLVED-WORKER-REV.ROF

Single Radionuclide Soil Guidelines G(i,t) in pCi/g 
Basic Radiation Dose Limit $=2.500 \mathrm{E}+01 \mathrm{mrem} / \mathrm{yr}$

ONuclide

\begin{tabular}{|c|c|c|c|c|c|c|}
\hline (i) & $t=$ & $0.000 \mathrm{E}+00$ & $1.000 \mathrm{E}+00$ & $3.000 \mathrm{E}+00$ & $6.000 \mathrm{E}+00$ & $1.000 \mathrm{E}+01$ \\
\hline$A m-241$ & & $1.994 \mathrm{E}+03$ & $1.993 \mathrm{E}+03$ & $1.993 \mathrm{E}+03$ & $1.993 \mathrm{E}+03$ & $1.993 \mathrm{E}+03$ \\
\hline Cs -137 & & $4.254 \mathrm{E}+06$ & $3.368 \mathrm{E}+06$ & $2.401 E+06$ & $1.707 \mathrm{E}+06$ & $1.259 \mathrm{E}+06$ \\
\hline Np-237 & & $3.827 \mathrm{E}+03$ & $3.827 \mathrm{E}+03$ & $3.826 \mathrm{E}+03$ & $3.825 E+03$ & $3.824 \mathrm{E}+03$ \\
\hline Pu-238 & & $1.740 \mathrm{E}+03$ & $1.740 \mathrm{E}+03$ & $1.740 \mathrm{E}+03$ & $1.740 \mathrm{E}+03$ & $1.740 \mathrm{E}+03$ \\
\hline $\mathrm{Pu}-239$ & & $1.595 \mathrm{E}+03$ & $1.595 \mathrm{E}+03$ & $1.595 \mathrm{E}+03$ & $1.595 \mathrm{E}+03$ & $1.595 \mathrm{E}+03$ \\
\hline Pu-240 & & $1.595 \mathrm{E}+03$ & $1.595 \mathrm{E}+03$ & $1.595 \mathrm{E}+03$ & $1.595 \mathrm{E}+03$ & $1.595 \mathrm{E}+03$ \\
\hline TC-99 & & $1.472 \mathrm{E}+07$ & $1.472 \mathrm{E}+07$ & $1.472 E+07$ & $1.472 \mathrm{E}+07$ & $1.472 \mathrm{E}+07$ \\
\hline Th-228 & & $4.386 \mathrm{E}+03$ & $4.384 E+03$ & $4.381 E+03$ & $4.379 E+03$ & $4.378 \mathrm{E}+03$ \\
\hline Th-230 & & $1.914 \mathrm{E}+03$ & $1.914 \mathrm{E}+03$ & $1.914 \mathrm{E}+03$ & $1.914 \mathrm{E}+03$ & $1.913 \mathrm{E}+03$ \\
\hline Th-232 & & $1.740 \mathrm{E}+03$ & $1.740 \mathrm{E}+03$ & $1.739 \mathrm{E}+03$ & $1.738 \mathrm{E}+03$ & $1.737 \mathrm{E}+03$ \\
\hline $\mathrm{U}-234$ & & $2.036 \mathrm{E}+04$ & $2.036 \mathrm{E}+04$ & $2.036 \mathrm{E}+04$ & $2.036 \mathrm{E}+04$ & $2.036 \mathrm{E}+04$ \\
\hline $\mathrm{U}-235$ & & $2.251 \mathrm{E}+04$ & $2.250 \mathrm{E}+04$ & $2.249 E+04$ & $2.247 \mathrm{E}+04$ & $2.245 \mathrm{E}+04$ \\
\hline $\mathrm{U}-238$ & & $2.390 \mathrm{E}+04$ & $2.390 \mathrm{E}+04$ & $2.389 \mathrm{E}+04$ & $2.389 \mathrm{E}+04$ & $2.388 \mathrm{E}+04$ \\
\hline
\end{tabular}

Summed Dose/Source Ratios DSR ( $i, t)$ in (mrem/yr)/(pCi/g)

and Single Radionuclide Soil Guidelines $G(i, t)$ in $\mathrm{pCi} / \mathrm{g}$

at tmin = time of minimum single radionuclide soil guideline

and at $\operatorname{tmax}=$ time of maximum total dose $=40$ years

\begin{tabular}{|c|c|c|c|c|c|c|}
\hline $\begin{array}{l}\text { ONuclide } \\
\text { (i) }\end{array}$ & $\begin{array}{l}\text { Initial } \\
\text { (pCi/g) }\end{array}$ & $\begin{array}{c}\operatorname{tmin} \\
\text { (years) }\end{array}$ & $\operatorname{DSR}(i, \operatorname{tmin})$ & $\begin{array}{c}G(i, \operatorname{tmin}) \\
(\mathrm{pCi} / \mathrm{g})\end{array}$ & $\operatorname{DSR}(i, \operatorname{tmax})$ & $\begin{array}{c}\mathrm{G}(\mathrm{i}, \mathrm{tmax}) \\
(\mathrm{pCi} / \mathrm{g})\end{array}$ \\
\hline$A m-241$ & $3.000 \mathrm{E}+00$ & 40 & $1.254 \mathrm{E}-02$ & $1.993 E+03$ & $1.254 \mathrm{E}-02$ & $1.993 \mathrm{E}+03$ \\
\hline Cs -137 & $3.000 \mathrm{E}+00$ & 40 & $4.619 E-05$ & $5.413 E+05$ & $4.619 \mathrm{E}-05$ & $5.413 \mathrm{E}+05$ \\
\hline $\mathrm{Np}-237$ & $3.000 \mathrm{E}+01$ & 40 & $6.554 \mathrm{E}-03$ & $3.815 E+03$ & $6.554 \mathrm{E}-03$ & $3.815 \mathrm{E}+03$ \\
\hline $\mathrm{Pu}-238$ & $3.000 \mathrm{E}+00$ & 40 & $1.438 \mathrm{E}-02$ & $1.739 \mathrm{E}+03$ & $1.438 \mathrm{E}-02$ & $1.739 \mathrm{E}+03$ \\
\hline $\mathrm{Pu}-239$ & $3.000 \mathrm{E}+00$ & 40 & $1.569 \mathrm{E}-02$ & $1.594 \mathrm{E}+03$ & $1.569 \mathrm{E}-02$ & $1.594 \mathrm{E}+03$ \\
\hline $\mathrm{Pu}-240$ & $3.000 \mathrm{E}+00$ & 40 & $1.569 \mathrm{E}-02$ & $1.594 \mathrm{E}+03$ & $1.569 \mathrm{E}-02$ & $1.594 \mathrm{E}+03$ \\
\hline $\mathrm{TC}-99$ & $3.000 \mathrm{E}+01$ & 1.25 & $1.698 \mathrm{E}-06$ & $1.472 \mathrm{E}+07$ & $1.698 \mathrm{E}-06$ & $1.472 \mathrm{E}+07$ \\
\hline Th-228 & $3.000 \mathrm{E}+00$ & 27.5 & $5.711 \mathrm{E}-03$ & $4.378 E+03$ & $5.711 \mathrm{E}-03$ & $4.378 \mathrm{E}+03$ \\
\hline Th-230 & $3.000 \mathrm{E}+00$ & 40 & $1.307 \mathrm{E}-02$ & $1.912 \mathrm{E}+03$ & $1.307 \mathrm{E}-02$ & $1.912 \mathrm{E}+03$ \\
\hline Th-232 & $3.000 \mathrm{E}+00$ & 40 & $1.456 \mathrm{E}-02$ & $1.717 \mathrm{E}+03$ & $1.456 \mathrm{E}-02$ & $1.717 \mathrm{E}+03$ \\
\hline$U-234$ & $3.000 \mathrm{E}+01$ & 40 & $1.228 \mathrm{E}-03$ & $2.035 E+04$ & $1.228 \mathrm{E}-03$ & $2.035 \mathrm{E}+04$ \\
\hline $\mathrm{U}-235$ & $3.000 E+01$ & 40 & $1.118 \mathrm{E}-03$ & $2.237 E+04$ & $1.118 \mathrm{E}-03$ & $2.237 \mathrm{E}+04$ \\
\hline $\mathrm{U}-238$ & $3.000 \mathrm{E}+01$ & 40 & $1.048 \mathrm{E}-03$ & $2.386 \mathrm{E}+04$ & $1.048 \mathrm{E}-03$ & $2.386 \mathrm{E}+04$ \\
\hline
\end{tabular}

1RESRAD-OFFSITE, Version 4.0 .3 beta $T^{1 / 2}$ Limit $=30$ days

Parent Dose Report

Title : RESRAD-OFFSITE Effluent release

File : PADUCAH EFFLUENT RELEASE-NONINVOLVED-WORKER-REV.ROF

Individual Nuclide Dose Summed Over All Pathways

Parent Nuclide and Thread Fraction Indicated

onuclide Parent THF(i)
(j)
$t=0.000 \mathrm{E}+00 \quad 1.000 \mathrm{E}+00 \quad 3.000 \mathrm{E}+00 \quad 6.000 \mathrm{E}+00 \quad 1.000 \mathrm{E}+01$ 


\begin{tabular}{|c|c|c|}
\hline$n-241$ & 241 & $0 \mathrm{E}+$ \\
\hline Npp-237 & Am-241 & $1.000 \mathrm{E}+\mathrm{C}$ \\
\hline Np-237 & $\mathrm{Np}-237$ & $1.000 \mathrm{E}+00$ \\
\hline Np-237 & $\Sigma \operatorname{DOSE}(j)$ & \\
\hline $0 U-233$ & Am-241 & $1.000 \mathrm{E}+00$ \\
\hline $\mathrm{U}-233$ & $\mathrm{~Np}-237$ & $1.000 \mathrm{E}+00$ \\
\hline $\mathrm{U}-233$ & $\Sigma \operatorname{DOSE}(j)$ & \\
\hline $0 T h-229$ & $\mathrm{Am}-241$ & $1.000 \mathrm{E}+0$ \\
\hline Th-229 & Np-237 & $1.000 \mathrm{E}+00$ \\
\hline Th-229 & $\Sigma \operatorname{DOSE}(j)$ & \\
\hline $\mathrm{Cs}-137$ & $\mathrm{Cs}^{-}$ & 1 \\
\hline $0 \mathrm{Pu}-238$ & $\mathrm{Pu}-238$ & $0 E-09$ \\
\hline Pu-238 & $\mathrm{Pu}-238$ & $1.000 \mathrm{E}+00$ \\
\hline $\mathrm{Pu}-238$ & $\Sigma \operatorname{DOSE}(j)$ & \\
\hline bu-234 & $\mathrm{Pu}-238$ & $0 \mathrm{E}+00$ \\
\hline $\mathrm{U}-234$ & $\mathrm{U}-234$ & $0 \mathrm{E}+00$ \\
\hline $\mathrm{U}-234$ & $\mathrm{U}-238$ & $9.999 \mathrm{E}-01$ \\
\hline $\mathrm{U}-234$ & $\Sigma \operatorname{DOSE}(j)$ & : \\
\hline $0 \mathrm{Th}-230$ & $\mathrm{Pu}-238$ & $1.000 \mathrm{E}+00$ \\
\hline Th-230 & Th- & $0 \mathrm{E}+00$ \\
\hline Th-230 & $U-234$ & $1.000 \mathrm{E}+00$ \\
\hline Th-230 & $\mathrm{U}-238$ & $9.999 \mathrm{E}-01$ \\
\hline Th-230 & $\Sigma \operatorname{DOSE}(j)$ & \\
\hline ORa-226 & $\mathrm{Pu}-238$ & $1.000 \mathrm{E}+00$ \\
\hline $\mathrm{Ra}-226$ & Th-230 & $1.000 \mathrm{E}+00$ \\
\hline $\mathrm{Ra}-226$ & $U-234$ & $1.000 \mathrm{E}+00$ \\
\hline $\mathrm{Ra}-226$ & $\mathrm{U}-238$ & $9.999 \mathrm{E}-01$ \\
\hline $\mathrm{Ra}-226$ & $\Sigma \operatorname{DOSE}(j)$ & \\
\hline $0 \mathrm{~Pb}-210$ & $\mathrm{Pu}-238$ & $1.000 \mathrm{E}+00$ \\
\hline $\mathrm{Pb}-210$ & Th-230 & $1.000 \mathrm{E}+00$ \\
\hline $\mathrm{Pb}-210$ & $\mathrm{U}-234$ & $1.000 \mathrm{E}+00$ \\
\hline & $\mathrm{U}-238$ & $9.999 \mathrm{E}-$ \\
\hline 07 & $\Sigma \mathrm{DOSE}$ & \\
\hline
\end{tabular}

$\begin{array}{lllll}3.762 \mathrm{E}-02 & 3.762 \mathrm{E}-02 & 3.762 \mathrm{E}-02 & 3.762 \mathrm{E}-02 & 3.763 \mathrm{E}-02\end{array}$ $\begin{array}{lllll}1.831 \mathrm{E}-13 & 8.145 \mathrm{E}-13 & 3.440 \mathrm{E}-12 & 1.024 \mathrm{E}-11 & 2.307 \mathrm{E}-11\end{array}$ $1.960 \mathrm{E}-01 \quad 1.960 \mathrm{E}-01 \quad 1.960 \mathrm{E}-01 \quad 1.961 \mathrm{E}-01 \quad 1.962 \mathrm{E}-01$ $1.960 \mathrm{E}-01 \quad 1.960 \mathrm{E}-01 \quad 1.960 \mathrm{E}-01 \quad 1.961 \mathrm{E}-01 \quad 1.962 \mathrm{E}-01$ $\begin{array}{lllll}1.960 \mathrm{E}-01 & 1.960 \mathrm{E}-01 & 1.960 \mathrm{E}-01 & 1.961 \mathrm{E}-01 & 1.962 \mathrm{E}-01 \\ 1.174 \mathrm{E}-20 & 8.788 \mathrm{E}-20 & 6.832 \mathrm{E}-19 & 3.464 \mathrm{E}-18 & 1.221 \mathrm{E}-17\end{array}$ $\begin{array}{lllll}1.174 \mathrm{E}-20 & 8.788 \mathrm{E}-20 & 6.832 \mathrm{E}-19 & 3.464 \mathrm{E}-18 & 1.221 \mathrm{E}-17 \\ 8.957 \mathrm{E}-13 & 4.058 \mathrm{E}-12 & 1.779 \mathrm{E}-11 & 5.594 \mathrm{E}-11 & 1.352 \mathrm{E}-10\end{array}$ $\begin{array}{llllll}8.957 \mathrm{E}-13 & 4.058 \mathrm{E}-12 & 1.779 \mathrm{E}-11 & 5.594 \mathrm{E}-11 & 1.352 \mathrm{E}-10\end{array}$ $\begin{array}{llllll}2.003 \mathrm{E}-23 & 2.743 \mathrm{E}-22 & 3.893 \mathrm{E}-21 & 3.335 \mathrm{E}-20 & 1.898 \mathrm{E}-19\end{array}$ $\begin{array}{llllll}2.028 \mathrm{E}-15 & 1.561 \mathrm{E}-14 & 1.274 \mathrm{E}-13 & 6.927 \mathrm{E}-13 & 2.691 \mathrm{E}-12\end{array}$ $\begin{array}{llllll}2.028 \mathrm{E}-15 & 1.561 \mathrm{E}-14 & 1.274 \mathrm{E}-13 & 6.927 \mathrm{E}-13 & 2.691 \mathrm{E}-12\end{array}$ $1.763 \mathrm{E}-05-2.227$ $1.763 \mathrm{E}-052.227 \mathrm{E}-052.124 \mathrm{E}-05.4 .39$ $0.000 \mathrm{E}+0020.000 \mathrm{E}+000.000 \mathrm{E}+0020.000 \mathrm{E}+0020.000 \mathrm{E}+00$ $12 \mathrm{E}-02$

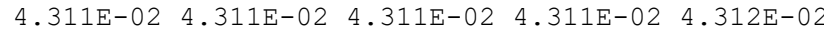
$\begin{array}{lllll}5.541 \mathrm{E}-14 & 2.510 \mathrm{E}-13 & 1.100 \mathrm{E}-12 & 3.458 \mathrm{E}-12 & 8.353 \mathrm{E}-12\end{array}$

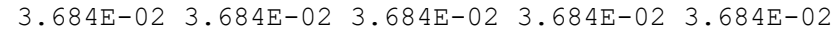
$\begin{array}{llllll}5.460 \mathrm{E}-13 & 2.449 \mathrm{E}-12 & 1.051 \mathrm{E}-11 & 3.199 \mathrm{E}-11 & 7.387 \mathrm{E}-11\end{array}$ $\begin{array}{lllll}3.684 \mathrm{E}-02 & 3.684 \mathrm{E}-02 & 3.684 \mathrm{E}-02 & 3.684 \mathrm{E}-02 & 3.684 \mathrm{E}-02\end{array}$ $\begin{array}{llllll}1.966 \mathrm{E}-18 & 1.513 \mathrm{E}-17 & 1.235 \mathrm{E}-16 & 6.713 \mathrm{E}-16 & 2.607 \mathrm{E}-15\end{array}$ $1.966 \mathrm{E}-1821.513 \mathrm{E}-171.235 \mathrm{E}-162.613 \mathrm{E}-1622.607 \mathrm{E}-15$ $3.919 \mathrm{E}-023.919 \mathrm{E}-023.919 \mathrm{E}-023.919 \mathrm{E}-02 \mathrm{2} 3.920 \mathrm{E}-02$

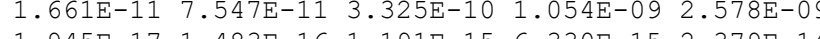
$\begin{array}{llllll}1.945 \mathrm{E}-17 & 1.483 \mathrm{E}-16 & 1.191 \mathrm{E}-15 & 6.330 \mathrm{E}-15 & 2.379 \mathrm{E}-14\end{array}$ $3.919 \mathrm{E}-02 \quad 3.919 \mathrm{E}-02 \quad 3.919 \mathrm{E}-02 \quad 3.919 \mathrm{E}-02 \quad 3.920 \mathrm{E}-02$ $\begin{array}{lllll}6.658 \mathrm{E}-21 & 9.275 \mathrm{E}-20 & 1.354 \mathrm{E}-18 & 1.205 \mathrm{E}-17 & 7.226 \mathrm{E}-17\end{array}$ 2.020E-09 9.205E-09 $4.084 \mathrm{E}-08$ 1.309E-07 $3.245 \mathrm{E}-07$ $\begin{array}{lllll}7.496 \mathrm{E}-14 & 5.735 \mathrm{E}-13 & 4.632 \mathrm{E}-12 & 2.480 \mathrm{E}-11 & 9.425 \mathrm{E}-11\end{array}$ $6.599 \mathrm{E}-20 \quad 9.121 \mathrm{E}-191.314 \mathrm{E}-17 \quad 1.48 \mathrm{E}-16 \quad 6.710 \mathrm{E}-16$ $2.020 \mathrm{E}-099.206 \mathrm{E}-094.085 \mathrm{E}-081.309 \mathrm{E}-073.246 \mathrm{E}-07$ $2.020 \mathrm{E}-099.206 \mathrm{E}-094.085 \mathrm{E}-08 \mathrm{1}-309 \mathrm{E}-07 \mathrm{3} .246 \mathrm{E}-07$ $7.085 \mathrm{E}-251.862 \mathrm{E}-234.813 \mathrm{E}-226.787 \mathrm{E}-216.189 \mathrm{E}-20$ $\begin{array}{lllll}3.562 \mathrm{E}-13 & 2.719 \mathrm{E}-12 & 2.187 \mathrm{E}-11 & 1.165 \mathrm{E}-10 & 4.392 \mathrm{E}-10\end{array}$ $\begin{array}{lllll}9.963 \mathrm{E}-18 & 1.374 \mathrm{E}-16 & 1.974 \mathrm{E}-15 & 1.717 \mathrm{E}-14 & 9.968 \mathrm{E}-14\end{array}$

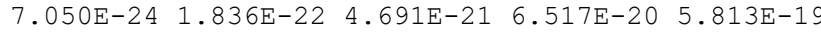
$\begin{array}{lllll}3.562 \mathrm{E}-13 & 2.719 \mathrm{E}-12 & 2.188 \mathrm{E}-11 & 1.165 \mathrm{E}-10 & 4.393 \mathrm{E}-10\end{array}$

1RESRAD-OFFSITE, Version 4.0 .3 beta $T^{1} \frac{1}{2}$ Limit $=30$ days

Parent Dose Report

Title: RESRAD-OFFSITE Effluent release

File : PADUCAH EFFLUENT RELEASE-NONINVOLVED-WORKER-REV.ROF

Individual Nuclide Dose Summed Over All Pathways Parent Nuclide and Thread Fraction Indicated

onuclide Parent THF(i)

\begin{tabular}{|c|c|c|c|c|c|c|c|c|}
\hline (j) & (i) & & $t=$ & $0.000 \mathrm{E}+00$ & $1.000 \mathrm{E}+00$ & $3.000 \mathrm{E}+00$ & $6.000 \mathrm{E}+00$ & $1.000 \mathrm{E}+01$ \\
\hline & -238 & b & & 5 & 23 & 22 & 21 & \\
\hline & & & & & & & 10 & \\
\hline 10 & & & & & & & & \\
\hline Po-210 & $\mathrm{U}-238$ & & & -24 & $E-22$ & $3.899 \mathrm{E}-21$ & 6.35 & $6.307 \mathrm{E}-19$ \\
\hline Po-210 & $\Sigma \operatorname{DOSE}(j)$ & & & $1.966 \mathrm{E}-13$ & 2.05 & $2.079 \mathrm{E}-11$ & -10 & $5.144 \mathrm{E}-10$ \\
\hline & $\mathrm{P}$ & & & & & & & \\
\hline & $\mathrm{Pr}$ & & & 16 & 1 & 5 & 14 & 5 \\
\hline & $\mathrm{U}-235$ & $1.000 \mathrm{E}+00$ & & $3.332 \mathrm{E}-02$ & $3.333 \mathrm{E}-02$ & $3.335 \mathrm{E}-02$ & $3.338 \mathrm{E}-02$ & $3.341 \mathrm{E}-02$ \\
\hline
\end{tabular}




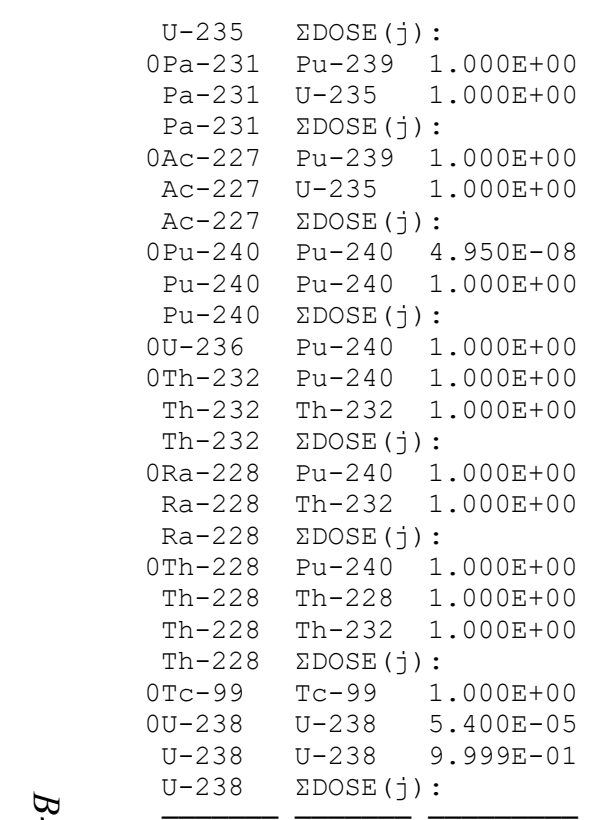

$3.332 \mathrm{E}-02 \quad 3.333 \mathrm{E}-02 \quad 3.335 \mathrm{E}-02 \quad 3.338 \mathrm{E}-02 \quad 3.341 \mathrm{E}-02$

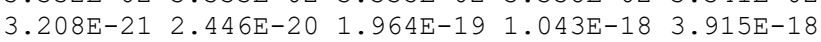
$7.749 \mathrm{E}-113.475 \mathrm{E}-101.964 \mathrm{E}-192.043 \mathrm{E}-183.915 \mathrm{E}-18$

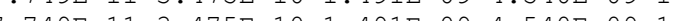
$7.749 \mathrm{E}-11 \mathrm{3} .475 \mathrm{E}-10 \mathrm{1}-4.491 \mathrm{E}-092.540 \mathrm{E}-091.048 \mathrm{E}-08$

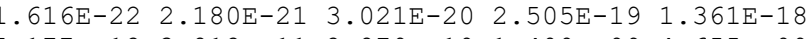
$5.177 \mathrm{E}-12 \quad 3.812 \mathrm{E}-11 \quad 2.879 \mathrm{E}-10 \quad 1.400 \mathrm{E}-09 \quad 4.655 \mathrm{E}-09$ $\begin{array}{lllll}5.177 \mathrm{E}-12 & 3.812 \mathrm{E}-11 & 2.879 \mathrm{E}-10 & 1.400 \mathrm{E}-09 & 4.655 \mathrm{E}-09\end{array}$ $0.000 \mathrm{E}+00 \quad 0.000 \mathrm{E}+00 \quad 0.000 \mathrm{E}+00 \quad 0.000 \mathrm{E}+00 \quad 0.000 \mathrm{E}+00$ $4.703 \mathrm{E}-02 \quad 4.703 \mathrm{E}-02 \quad 4.703 \mathrm{E}-02 \quad 4.703 \mathrm{E}-02 \quad 4.703 \mathrm{E}-02$ 4.703E-02 4.703E-02 4.703E-02 4.703E-02 4.703E-02 $\begin{array}{lllll}5.387 \mathrm{E}-16 & 2.446 \mathrm{E}-15 & 1.077 \mathrm{E}-14 & 3.411 \mathrm{E}-14 & 8.327 \mathrm{E}-14\end{array}$ $\begin{array}{lllll}1.238 \mathrm{E}-25 & 9.546 \mathrm{E}-25 & 7.819 \mathrm{E}-24 & 4.274 \mathrm{E}-23 & 1.673 \mathrm{E}-22\end{array}$

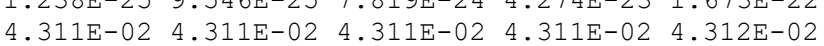
$\begin{array}{lllll}4.311 \mathrm{E}-02 & 4.311 \mathrm{E}-02 & 4.311 \mathrm{E}-02 & 4.311 \mathrm{E}-02 & 4.312 \mathrm{E}-02 \\ 4.311 \mathrm{E}-02 & 4.311 \mathrm{E}-02 & 4.311 \mathrm{E}-02 & 4.311 \mathrm{E}-02 & 4.312 \mathrm{E}-02\end{array}$ $\begin{array}{lllll}5.736 \mathrm{E}-26 & 7.800 \mathrm{E}-25 & 1.095 \mathrm{E}-23 & 9.258 \mathrm{E}-23 & 5.180 \mathrm{E}-22\end{array}$ $2.976 \mathrm{E}-07 \quad 1.313 \mathrm{E}-06 \quad 5.460 \mathrm{E}-06 \quad 1.592 \mathrm{E}-05 \quad 3.506 \mathrm{E}-05$ $2.976 \mathrm{E}-07 \quad 1.313 \mathrm{E}-06 \quad 5.460 \mathrm{E}-06 \quad 1.592 \mathrm{E}-05 \quad 3.506 \mathrm{E}-05$ $\begin{array}{lllll}7.750 \mathrm{E}-27 & 1.884 \mathrm{E}-25 & 4.314 \mathrm{E}-24 & 5.251 \mathrm{E}-23 & 3.928 \mathrm{E}-22\end{array}$ 1.710E-02 $1.711 \mathrm{E}-02 \quad 1.712 \mathrm{E}-02 \quad 1.713 \mathrm{E}-02 \mathrm{1} 1.713 \mathrm{E}-02$ 1.710E-02 1.711E-02 $1.712 \mathrm{E}-021.713 \mathrm{E}-02 \mathrm{c} 1.713 \mathrm{E}-02$ $1.710 \mathrm{E}-02 \mathrm{2} 1.711 \mathrm{E}-02 \quad 1.712 \mathrm{E}-021.714 \mathrm{E}-02 \mathrm{2} 1.717 \mathrm{E}-02$ $1.710 \mathrm{E}-021.711 \mathrm{E}-02 \quad 1.712 \mathrm{E}-021.714 \mathrm{E}-02 \quad 1.717 \mathrm{E}-02$ 5.095E-05 5.095E-05 5.095E-05 5.095E-05 5.095E-05 $\begin{array}{llllll}0.000 \mathrm{E}+00 & 0.000 \mathrm{E}+00 & 0.000 \mathrm{E}+00 & 0.000 \mathrm{E}+00 & 0.000 \mathrm{E}+00\end{array}$ $3.138 \mathrm{E}-02 \quad 3.139 \mathrm{E}-02 \quad 3.139 \mathrm{E}-02 \quad 3.140 \mathrm{E}-02 \quad 3.141 \mathrm{E}-02$ 3.138E-02 3.139E-02 3.139E-02 3.140E-02 3.141E-02

THF(i) is the thread fraction of the parent nuclide.

1RESRAD-OFFSITE, Version 4.0 .3 beta $\mathrm{T}^{1 / 2} \mathrm{Limit}=30$ days

$06 / 11 / 202011: 39$

Parent Dose Report

Title : RESRAD-OFFSITE Effluent release

File : PADUCAH EFFLUENT RELEASE-NONINVOLVED-WORKER-REV.ROF

Individual Nuclide Soil Concentration

Parent Nuclide and Thread Fraction Indicated

ONuclide Parent THF(i) $\mathrm{S}(j, \mathrm{t}), \mathrm{pCi} / \mathrm{g}$

\begin{tabular}{|c|c|c|c|c|c|c|c|c|}
\hline (j) & (i) & & $t=$ & $0.000 \mathrm{E}+00$ & $00 E+00$ & $00 \mathrm{E}+00$ & 00 & $\mathrm{E}+01$ \\
\hline Am-241 & $m-241$ & $1.000 \mathrm{E}+00$ & & $0.000 \mathrm{E}+00$ & $0.000 \mathrm{E}+00$ & $0.000 \mathrm{E}+00$ & $0.000 \mathrm{E}+00$ & $0.000 \mathrm{E}+00$ \\
\hline ONp-237 & $A m-241$ & $1.000 \mathrm{E}+00$ & & $0.000 \mathrm{E}+00$ & $0.000 \mathrm{E}+00$ & $0.000 \mathrm{E}+00$ & $0.000 \mathrm{E}+00$ & $0 E+00$ \\
\hline $\mathrm{Np}-237$ & Np-237 & $1.000 \mathrm{E}+00$ & & $0.000 \mathrm{E}+00$ & $0.000 \mathrm{E}+00$ & $0.000 \mathrm{E}+00$ & $0.000 \mathrm{E}+00$ & $0.000 \mathrm{E}+00$ \\
\hline $\mathrm{Np}-237$ & $\Sigma S(j):$ & & & $0.000 \mathrm{E}+00$ & $0.000 \mathrm{E}+00$ & $0.000 \mathrm{E}+00$ & $0.000 \mathrm{E}+00$ & $0.000 \mathrm{E}+00$ \\
\hline OU-233 & $A m-241$ & +00 & & $0.000 \mathrm{E}+00$ & $0.000 \mathrm{E}+00$ & $0.000 \mathrm{E}+00$ & $0.000 \mathrm{E}+00$ & $0.000 \mathrm{E}+00$ \\
\hline $\mathrm{U}-233$ & Np-237 & $1.000 \mathrm{E}+00$ & & $0.000 \mathrm{E}+00$ & $0.000 \mathrm{E}+00$ & $0.000 \mathrm{E}+00$ & $0.000 \mathrm{E}+00$ & $0.000 \mathrm{E}+00$ \\
\hline $\mathrm{U}-233$ & $\Sigma S(j):$ & & & $0.000 \mathrm{E}+00$ & $0.000 \mathrm{E}+00$ & $0.000 \mathrm{E}+00$ & $0.000 \mathrm{E}+00$ & $0.000 \mathrm{E}+00$ \\
\hline $0 T h-229$ & $A m-241$ & 1.00 & & $0.000 \mathrm{E}+00$ & 0.0 & 0.00 & $E+00$ & $0.000 \mathrm{E}+00$ \\
\hline Th-229 & Np-237 & $1.000 \mathrm{E}+00$ & & $0.000 \mathrm{E}+00$ & $0.000 \mathrm{E}+00$ & $0.000 \mathrm{E}+00$ & $0.000 \mathrm{E}+00$ & $0.000 \mathrm{E}+00$ \\
\hline Th-229 & $\Sigma S(j):$ & & & $0.000 \mathrm{E}+00$ & $0.000 \mathrm{E}+00$ & $0.000 \mathrm{E}+00$ & $0.000 \mathrm{E}+00$ & $0.000 \mathrm{E}+00$ \\
\hline $0 \mathrm{Cs}-137$ & Cs -137 & $1.000 \mathrm{E}+00$ & & 0.00 & 0. & 0.0 & 0.0 & $0.000 \mathrm{E}+00$ \\
\hline $0 \mathrm{Pu}-238$ & $\mathrm{Pu}-238$ & -09 & & 0.00 & 0.00 & 0.00 & 0.00 & $0.000 \mathrm{E}+00$ \\
\hline $\mathrm{Pu}-238$ & $\mathrm{Pu}-238$ & $1.000 \mathrm{E}+00$ & & $0.000 \mathrm{E}+00$ & $0.000 \mathrm{E}+00$ & $0.000 \mathrm{E}+00$ & $0.000 \mathrm{E}+00$ & $0.000 \mathrm{E}+00$ \\
\hline $\mathrm{Pu}-238$ & $\Sigma S(j):$ & & & $0.000 \mathrm{E}+00$ & $0.000 \mathrm{E}+00$ & $0.000 \mathrm{E}+00$ & $0.000 \mathrm{E}+00$ & $0.000 \mathrm{E}+00$ \\
\hline
\end{tabular}




\begin{tabular}{|c|c|c|c|c|c|c|c|}
\hline OU-234 & $\mathrm{Pu}-238$ & $1.000 \mathrm{E}+00$ & $0.000 \mathrm{E}+00$ & $0.000 \mathrm{E}+00$ & $0.000 \mathrm{E}+00$ & $0.000 \mathrm{E}+00$ & $0.000 \mathrm{E}+00$ \\
\hline $\mathrm{U}-234$ & $\mathrm{U}-234$ & $1.000 \mathrm{E}+00$ & $0.000 \mathrm{E}+00$ & $0.000 \mathrm{E}+00$ & $0.000 \mathrm{E}+00$ & $0.000 \mathrm{E}+00$ & $0.000 \mathrm{E}+00$ \\
\hline $\mathrm{U}-234$ & $\mathrm{U}-238$ & $9.999 \mathrm{E}-01$ & $0.000 \mathrm{E}+00$ & $0.000 \mathrm{E}+00$ & $0.000 \mathrm{E}+00$ & $0.000 \mathrm{E}+00$ & $0.000 \mathrm{E}+00$ \\
\hline $\mathrm{U}-234$ & $\Sigma S(j):$ & & $0.000 \mathrm{E}+00$ & $0.000 \mathrm{E}+00$ & $0.000 \mathrm{E}+00$ & $0.000 \mathrm{E}+00$ & $0.000 \mathrm{E}+00$ \\
\hline $0 \mathrm{Th}-230$ & $\mathrm{Pu}-238$ & $1.000 \mathrm{E}+00$ & $0.000 \mathrm{E}+00$ & $0.000 \mathrm{E}+00$ & $0.000 \mathrm{E}+00$ & $0.000 \mathrm{E}+00$ & $0.000 \mathrm{E}+00$ \\
\hline Th-230 & Th-230 & $1.000 \mathrm{E}+00$ & $0.000 \mathrm{E}+00$ & $0.000 \mathrm{E}+00$ & $0.000 \mathrm{E}+00$ & $0.000 \mathrm{E}+00$ & $0.000 \mathrm{E}+00$ \\
\hline Th-230 & $\mathrm{U}-234$ & $1.000 \mathrm{E}+00$ & $0.000 \mathrm{E}+00$ & $0.000 \mathrm{E}+00$ & $0.000 \mathrm{E}+00$ & $0.000 \mathrm{E}+00$ & $0.000 \mathrm{E}+00$ \\
\hline Th-230 & $\mathrm{U}-238$ & $9.999 \mathrm{E}-01$ & $0.000 \mathrm{E}+00$ & $0.000 \mathrm{E}+00$ & $0.000 \mathrm{E}+00$ & $0.000 \mathrm{E}+00$ & $0.000 \mathrm{E}+00$ \\
\hline $\mathrm{Th}-230$ & $\Sigma S(j):$ & & $0.000 \mathrm{E}+00$ & $0.000 \mathrm{E}+00$ & $0.000 \mathrm{E}+00$ & $0.000 \mathrm{E}+00$ & $0.000 \mathrm{E}+00$ \\
\hline $0 \mathrm{Ra}-226$ & $\mathrm{Pu}-238$ & $1.000 \mathrm{E}+00$ & $0.000 \mathrm{E}+00$ & $0.000 \mathrm{E}+00$ & $0.000 \mathrm{E}+00$ & $0.000 \mathrm{E}+00$ & $0.000 \mathrm{E}+00$ \\
\hline $\mathrm{Ra}-226$ & Th-230 & $1.000 \mathrm{E}+00$ & $0.000 \mathrm{E}+00$ & $0.000 \mathrm{E}+00$ & $0.000 \mathrm{E}+00$ & $0.000 \mathrm{E}+00$ & $0.000 \mathrm{E}+00$ \\
\hline $\mathrm{Ra}-226$ & $\mathrm{U}-234$ & $1.000 \mathrm{E}+00$ & $0.000 \mathrm{E}+00$ & $0.000 \mathrm{E}+00$ & $0.000 \mathrm{E}+00$ & $0.000 \mathrm{E}+00$ & $0.000 \mathrm{E}+00$ \\
\hline $\mathrm{Ra}-226$ & $\mathrm{U}-238$ & $9.999 \mathrm{E}-01$ & $0.000 \mathrm{E}+00$ & $0.000 \mathrm{E}+00$ & $0.000 \mathrm{E}+00$ & $0.000 \mathrm{E}+00$ & $0.000 \mathrm{E}+00$ \\
\hline $\mathrm{Ra}-226$ & $\Sigma S(j):$ & & $0.000 \mathrm{E}+00$ & $0.000 \mathrm{E}+00$ & $0.000 \mathrm{E}+00$ & $0.000 \mathrm{E}+00$ & $0.000 \mathrm{E}+00$ \\
\hline $0 \mathrm{~Pb}-210$ & $\mathrm{Pu}-238$ & $1.000 \mathrm{E}+00$ & $0.000 \mathrm{E}+00$ & $0.000 \mathrm{E}+00$ & $0.000 \mathrm{E}+00$ & $0.000 \mathrm{E}+00$ & $0.000 \mathrm{E}+00$ \\
\hline $\mathrm{Pb}-210$ & Th-230 & $1.000 \mathrm{E}+00$ & $0.000 \mathrm{E}+00$ & $0.000 \mathrm{E}+00$ & $0.000 \mathrm{E}+00$ & $0.000 \mathrm{E}+00$ & $0.000 \mathrm{E}+00$ \\
\hline $\mathrm{Pb}-210$ & $\mathrm{U}-234$ & $1.000 \mathrm{E}+00$ & $0.000 \mathrm{E}+00$ & $0.000 \mathrm{E}+00$ & $0.000 \mathrm{E}+00$ & $0.000 \mathrm{E}+00$ & $0.000 \mathrm{E}+00$ \\
\hline $\mathrm{Pb}-210$ & $\mathrm{U}-238$ & $9.999 \mathrm{E}-01$ & $0.000 \mathrm{E}+00$ & $0.000 \mathrm{E}+00$ & $0.000 \mathrm{E}+00$ & $0.000 \mathrm{E}+00$ & $0.000 \mathrm{E}+00$ \\
\hline $\mathrm{Pb}-210$ & $\Sigma S(j):$ & & $0.000 \mathrm{E}+00$ & $0.000 \mathrm{E}+00$ & $0.000 \mathrm{E}+00$ & $0.000 \mathrm{E}+00$ & $0.000 \mathrm{E}+00$ \\
\hline
\end{tabular}

$\mathrm{Pb}-210 \Sigma \mathrm{S}(\mathrm{j}): \quad 0.000 \mathrm{E}+00 \quad 0.000 \mathrm{E}+00 \quad 0.000 \mathrm{E}+00 \quad 0.000 \mathrm{E}+00 \quad 0.000 \mathrm{E}+00$

Version $4 \cdot 0.3$ beta T1/2 Limit $=30$ days $\quad 06 / 11 / 2020 \quad 11: 39$

Parent Dose Repor

Title : RESRAD-OFFSITE Effluent release

File : PADUCAH EFFLUENT RELEASE-NONINVOLVED-WORKER-REV.ROF

Individual Nuclide Soil Concentration

arent Nuclide and Thread Fraction Indicated

\begin{tabular}{|c|c|c|c|c|c|c|c|c|}
\hline $\begin{array}{l}\text { (j) } \\
(j)\end{array}$ & $\begin{array}{c}\text { Parent } \\
\text { (i) }\end{array}$ & & & $0.000 \mathrm{E}+00$ & $1.000 \mathrm{E}+00$ & $3.000 \mathrm{E}+00$ & $6.000 \mathrm{E}+00$ & $1.000 \mathrm{E}+01$ \\
\hline 10 & & $.000 \mathrm{H}$ & & 0 & 0 & $0.000 \mathrm{E}$ & $0 \mathrm{E}+00$ & $0.000 \mathrm{E}$ \\
\hline Po-210 & Th-230 & & & $0.000 \mathrm{E}+00$ & $0.000 \mathrm{E}+00$ & $0.000 \mathrm{E}+00$ & $E+00$ & $00 \mathrm{E}+00$ \\
\hline Po-210 & $\mathrm{HJ}-234$ & & & $000 \mathrm{E}+00$ & $00 E+00$ & $000 \mathrm{E}+00$ & & \\
\hline Po- 210 & & & & & & & & \\
\hline Po-210 & $\Sigma S(j):$ & & & 00 & +00 & +00 & & $E+00$ \\
\hline $\mathrm{Pu}-239$ & $\mathrm{Pu}-239$ & 0 & & & +00 & +00 & & \\
\hline & & & & & & & & \\
\hline & $\mathrm{U}-2$ & 1. & & & & & & \\
\hline & $\Sigma \mathrm{S}($ & & & & & & & \\
\hline $0 \mathrm{~Pa}-231$ & & & & & & & & \\
\hline $\mathrm{Pa}-231$ & $\mathrm{U}-23$ & & & & +00 & 00 & & +00 \\
\hline & $\Sigma S(j):$ & & & & & & & \\
\hline $0 A C-227$ & & & & & 00 & 00 & & -00 \\
\hline Ac-227 & $\mathrm{U}-23$ & 1 & & & +00 & 100 & 00 & 0.0 \\
\hline Ac-227 & $\Sigma S$ & & & & +00 & 0 . & & +00 \\
\hline $0 \mathrm{Pu}-240$ & & & & & & & & \\
\hline $\mathrm{Pu}-240$ & $\mathrm{Pu}-240$ & & & & +00 & 0 & 0. & 0.0 \\
\hline $\mathrm{Pu}-240$ & $\Sigma S$ & & & $0.000 \mathrm{E}+00$ & +00 & 0.0 & 0.0 & $0.000 \mathrm{E}+00$ \\
\hline & & & & & & & & \\
\hline & & & & & & & +00 & +00 \\
\hline Th-232 & Th-232 & $1.000 \mathrm{E}+00$ & & $0.000 \mathrm{E}+00$ & $0.000 \mathrm{E}+00$ & $E+00$ & مอ & \\
\hline
\end{tabular}




\begin{tabular}{rll} 
ORa-228 & Pu-240 & $1.000 \mathrm{E}+00$ \\
$\mathrm{Ra}-228$ & $\mathrm{Th}-232$ & $1.000 \mathrm{E}+00$ \\
$\mathrm{Ra}-228$ & $\Sigma \mathrm{S}(\mathrm{j}):$ & \\
0Th-228 & $\mathrm{Pu}-240$ & $1.000 \mathrm{E}+00$ \\
$\mathrm{Th}-228$ & $\mathrm{Th}-228$ & $1.000 \mathrm{E}+00$ \\
$\mathrm{Th}-228$ & $\mathrm{Th}-232$ & $1.000 \mathrm{E}+00$ \\
$\mathrm{Th}-228$ & $\Sigma \mathrm{S}(\mathrm{j}):$ & \\
OTC-99 & $\mathrm{TC}-99$ & $1.000 \mathrm{E}+00$ \\
$\mathrm{OU}-238$ & $\mathrm{U}-238$ & $5.400 \mathrm{E}-05$ \\
$\mathrm{U}-238$ & $\mathrm{U}-238$ & $9.999 \mathrm{E}-01$ \\
$\mathrm{U}-238$ & $\Sigma \mathrm{S}(\mathrm{j}):$ & \\
\hline
\end{tabular}

$\begin{array}{llllll}0.000 E+00 & 0.000 E+00 & 0.000 E+00 & 0.000 E+00 & 0.000 E+00\end{array}$ $\begin{array}{llllll}0.000 \mathrm{E}+00 & 0.000 \mathrm{E}+00 & 0.000 \mathrm{E}+00 & 0.000 \mathrm{E}+00 & 0.000 \mathrm{E}+00\end{array}$ $\begin{array}{lllll}0.000 \mathrm{E}+00 & 0.000 \mathrm{E}+00 & 0.000 \mathrm{E}+00 & 0.000 \mathrm{E}+00 & 0.000 \mathrm{E}+00\end{array}$

$0.000 \mathrm{E}+00 \quad 0.000 \mathrm{E}+00 \quad 0.000 \mathrm{E}+00 \quad 0.000 \mathrm{E}+00 \quad 0.000 \mathrm{E}+00$

$\begin{array}{lllll}0.000 \mathrm{E}+00 & 0.000 \mathrm{E}+00 & 0.000 \mathrm{E}+00 & 0.000 \mathrm{E}+00 & 0.000 \mathrm{E}+00\end{array}$

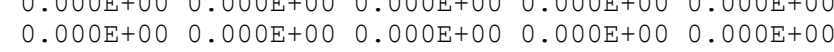

$\begin{array}{lllll}0.000 \mathrm{E}+00 & 0.000 \mathrm{E}+00 & 0.000 \mathrm{E}+00 & 0.000 \mathrm{E}+00 & 0.000 \mathrm{E}+00\end{array}$

$\begin{array}{llllll}0.000 \mathrm{E}+00 & 0.000 \mathrm{E}+00 & 0.000 \mathrm{E}+00 & 0.000 \mathrm{E}+00 & 0.000 \mathrm{E}+00\end{array}$

$\begin{array}{llllll}0.000 \mathrm{E}+00 & 0.000 \mathrm{E}+00 & 0.000 \mathrm{E}+00 & 0.000 \mathrm{E}+00 & 0.000 \mathrm{E}+00\end{array}$

$\begin{array}{lllll}0.000 \mathrm{E}+00 & 0.000 \mathrm{E}+00 & 0.000 \mathrm{E}+00 & 0.000 \mathrm{E}+00 & 0.000 \mathrm{E}+00\end{array}$

$\begin{array}{lllll}0.000 \mathrm{E}+00 & 0.000 \mathrm{E}+00 & 0.000 \mathrm{E}+00 & 0.000 \mathrm{E}+00 & 0.000 \mathrm{E}+00\end{array}$

THF(i) is the thread fraction of the parent nuclide.

IRESRAD-OFFSITE, Version 4.0 .3 beta T1/2 Limit $=30$ days

Parent Dose Report

Title : RESRAD-OFFSITE Effluent release

File : PADUCAH EFFLUENT RELEASE-NONINVOLVED-WORKER-REV.ROF

Run Time Information

ResOCalc.EXE execution began at 11:39 on 06/11/2020

ResoCalc.EXE execution ended at 11:39 on 06/11/2020

Resocalc.EXE execution time .216 seconds

Note: The dose at 6 years is considered from the ground external, inhalation, and soil ingestion pathways (see Table 6 in the main report). Ground concentration will increase from deposition; therefore, the dose during the last year incinerator will be operational is used because this will result in the highest dose.

\section{B.2 RESRAD-OFFSITE RESULTS FOR INCINERATOR NEIGHBOR SCENARIO}

To run the incinerator neighbor scenario, the AIRFLUXIN.DAT file was changed to match the radionuclide's yearly release rate from the El Dorado incinerator's SN-01 stack.

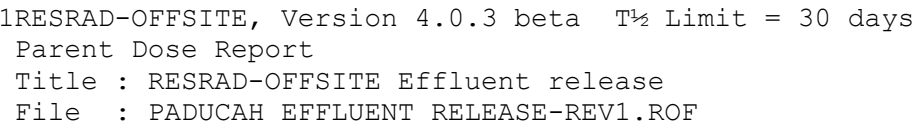

Table of Contents 
Dose Conversion Factor (and Related) Parameter Summary ... 2

Site-Specific Parameter Summary $\ldots \ldots \ldots \ldots \ldots \ldots \ldots \ldots .11$

Summary of Pathway Selections ................... 48

Contaminated Zone and Total Dose Summary .............. 49

Total Dose Components

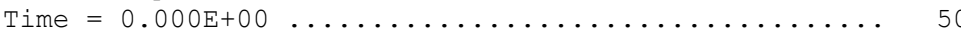

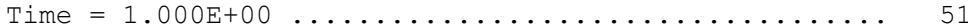

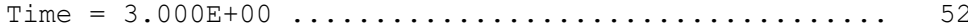

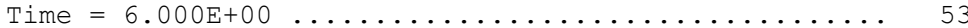

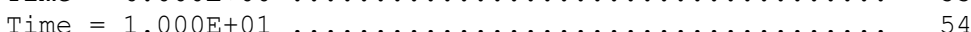

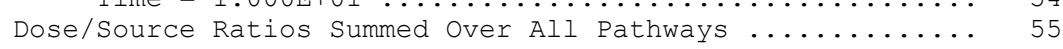

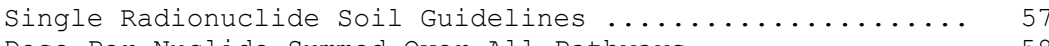

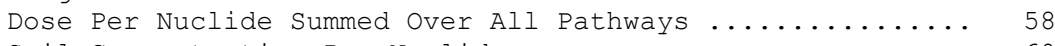

Soil Concentration Per Nuclide $\ldots \ldots \ldots \ldots \ldots \ldots \ldots \ldots \ldots .60$

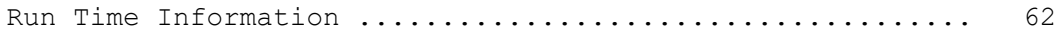

1RESRAD-OFFSITE, Version 4.0 .3 beta $T^{1 / 2}$ Limit $=30$ days

Parent Dose Report

06/11/2020 11:32 Page 2

Title : RESRAD-OFFSITE Effluent release

File : PADUCAH EFFLUENT RELEASE-REV1.ROF

Dose Conversion Factor (and Related) Parameter Summary Current Library: ICRP 60

Default Library: ICRP 60

\begin{tabular}{|c|c|c|c|c|c|c|c|}
\hline $\begin{array}{l}0 \\
\text { Menu }\end{array}$ & \multicolumn{3}{|r|}{ Parameter } & $\begin{array}{c}\text { Current } \\
\text { Value }\end{array}$ & Default & \multicolumn{2}{|c|}{$\begin{array}{l}\text { Parameter } \\
\text { Name }\end{array}$} \\
\hline DCSF & DCF's fo: & E external grour & Ind radiation, (mrem/yr)/(pCi/g) & & & & \\
\hline DCSF & AC -225 & (Source: ICRP & 60) & $5.775 \mathrm{E}-02$ & $5.775 \mathrm{E}-02$ & DCFEXT ( & \\
\hline DCSF & $\mathrm{Ac}-227$ & (Source: ICRP & 60) & $4.485 \mathrm{E}-04$ & $4.485 \mathrm{E}-04$ & DCEEXT ( & 2) \\
\hline DCSF & $A_{C}-228$ & (Source: ICRP & $60)$ & $5.662 \mathrm{E}+00$ & $5.662 \mathrm{E}+00$ & DCEEXT ( & 3) \\
\hline DCSE & $A m-241$ & (Source: ICRP & 60) & $3.719 \mathrm{E}-02$ & $3.719 \mathrm{E}-02$ & DCEEXT ( & 4) \\
\hline DCSF & At -217 & (Source: ICRP & $60)$ & $1.654 \mathrm{E}-03$ & $1.654 \mathrm{E}-03$ & DCEEXT ( & 5) \\
\hline DCSF & At -218 & (Source: ICRP & $60)$ & $4.878 \mathrm{E}-03$ & $4.878 \mathrm{E}-03$ & DCEEXT ( & 6) \\
\hline DCSE & $\mathrm{Ba}-137 \mathrm{~m}$ & (Source: ICRP & 60) & $3.383 \mathrm{E}+00$ & $3.383 E+00$ & DCFEXT ( & 7) \\
\hline DCSE & $\mathrm{Bi}-210$ & (Source: ICRP & 60) & $5.476 \mathrm{E}-03$ & $5.476 \mathrm{E}-03$ & DCEEXT ( & 8) \\
\hline DCSF & $\mathrm{Bi}-211$ & (Source: ICRP & $60)$ & $2.373 \mathrm{E}-01$ & $2.373 \mathrm{E}-01$ & DCEEXT ( & 9) \\
\hline DCSF & $\mathrm{Bi}-212$ & (Source: ICRP & $60)$ & $1.114 \mathrm{E}+00$ & $1.114 \mathrm{E}+00$ & DCEEXT ( & 10) \\
\hline DCSF & $\mathrm{Bi}-213$ & (Source: ICRP & $60)$ & $7.158 \mathrm{E}-01$ & $7.158 \mathrm{E}-01$ & DCEEXT ( & 11) \\
\hline DCSF & $\mathrm{Bi}-214$ & (Source: ICRP & $60)$ & $9.325 \mathrm{E}+00$ & $9.325 E+00$ & DCFEXT ( & 12) \\
\hline DCSF & Cs -137 & (Source: ICRP & $60)$ & $8.372 \mathrm{E}-04$ & $8.372 \mathrm{E}-04$ & DCEEXT ( & 13) \\
\hline DCSF & Fr -221 & (Source: ICRP & $60)$ & $1.413 \mathrm{E}-01$ & $1.413 \mathrm{E}-01$ & DCEEXT ( & 14) \\
\hline DCSF & Fr -223 & (Source: ICRP & $60)$ & $1.813 \mathrm{E}-01$ & $1.813 \mathrm{E}-01$ & DCFEXT ( & 15) \\
\hline DCSF & $\mathrm{Np}-237$ & (Source: ICRP & 60) & $6.971 \mathrm{E}-02$ & $6.971 \mathrm{E}-02$ & DCEEXT ( & 16) \\
\hline DCSE & $\mathrm{Pa}-231$ & (Source: ICRP & 60) & $1.762 \mathrm{E}-01$ & $1.762 \mathrm{E}-01$ & DCEEXT ( & 17) \\
\hline DCSF & $\mathrm{Pa}-233$ & (Source: ICRP & $60)$ & $9.419 \mathrm{E}-01$ & $9.419 \mathrm{E}-01$ & DCEEXT ( & 18) \\
\hline DCSF & $\mathrm{Pa}-234$ & (Source: ICRP & $60)$ & $1.088 \mathrm{E}+01$ & $1.088 \mathrm{E}+01$ & DCEEXT ( & 19) \\
\hline DCSE & $\mathrm{Pa}-234 \mathrm{~m}$ & (Source: ICRP & $60)$ & $9.867 \mathrm{E}-02$ & $9.867 \mathrm{E}-02$ & DCFEXT ( & 20) \\
\hline DCSF & $\mathrm{Pb}-209$ & (Source: ICRP & 60) & $7.550 \mathrm{E}-04$ & $7.550 \mathrm{E}-04$ & DCEEXT ( & 21) \\
\hline DCSE & $\mathrm{Pb}-210$ & (Source: ICRP & $60)$ & $1.981 \mathrm{E}-03$ & $1.981 \mathrm{E}-03$ & DCEEXT ( & 22) \\
\hline DCSE & $\mathrm{Pb}-211$ & (Source: ICRP & $60)$ & $2.915 \mathrm{E}-01$ & $2.915 \mathrm{E}-01$ & DCEEXT ( & 23) \\
\hline
\end{tabular}




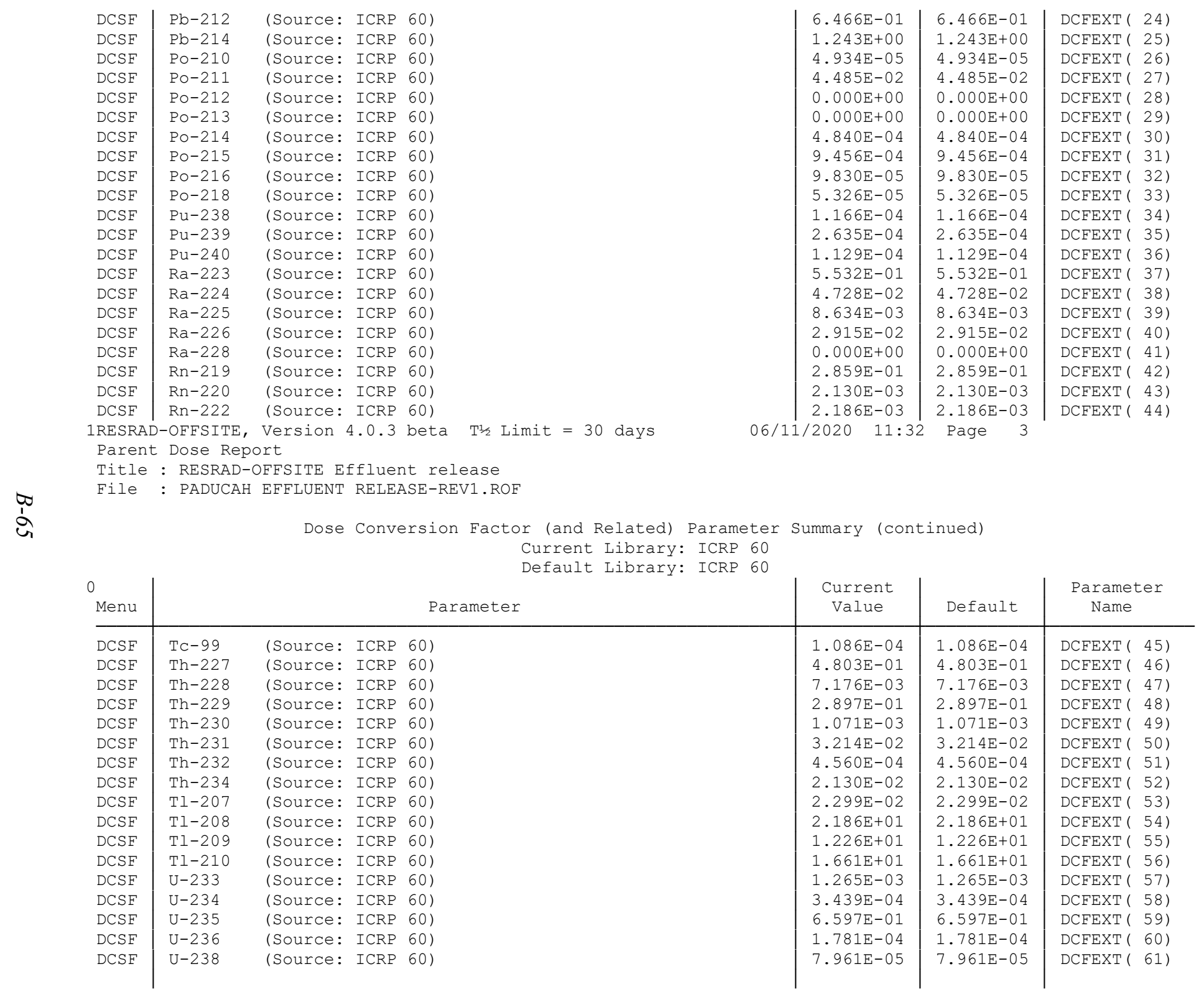


Current Library: ICRP 72 (Adult)

Default Library: ICRP 72 (Adult)

\begin{tabular}{|c|c|c|c|c|}
\hline $\begin{array}{l}0 \\
\text { Menu }\end{array}$ & Parameter & $\begin{array}{l}\text { Current } \\
\text { Value }\end{array}$ & Default & $\begin{array}{l}\text { Parameter } \\
\text { Name }\end{array}$ \\
\hline DCSE & Dose conversion factors for inhalation, mrem/pCi: & & & \\
\hline DCSE & $A C-227+D$ & $2.104 \mathrm{E}+00$ & $2.104 \mathrm{E}+00$ & $\mathrm{DCF} 2(1)$ \\
\hline DCSF & $\mathrm{Am}-241$ & $3.552 \mathrm{E}-01$ & $3.552 \mathrm{E}-01$ & DCF2 (2) \\
\hline DCSF & $\mathrm{Cs}-137+\mathrm{D}$ & $1.443 \mathrm{E}-04$ & $1.443 \mathrm{E}-04$ & DCF2 (3) \\
\hline DCSF & $\mathrm{Np}-237+\mathrm{D}$ & $1.850 \mathrm{E}-01$ & $1.850 \mathrm{E}-01$ & $\mathrm{DCF} 2(4)$ \\
\hline DCSE & $\mathrm{Pa}-231$ & $5.180 \mathrm{E}-01$ & $5.180 \mathrm{E}-01$ & DCF2 (5) \\
\hline DCSE & $\mathrm{Pb}-210+\mathrm{D}$ & $2.106 \mathrm{E}-02$ & $2.106 \mathrm{E}-02$ & DCF2 (6) \\
\hline DCSF & Po-210 & $1.591 \mathrm{E}-02$ & $1.591 \mathrm{E}-02$ & DCF2 (7) \\
\hline DCSF & $\mathrm{Pu}-238$ & $4.070 \mathrm{E}-01$ & $4.070 \mathrm{E}-01$ & DCF2 (8) \\
\hline DCSF & $\mathrm{Pu}-239$ & $4.440 \mathrm{E}-01$ & $4.440 \mathrm{E}-01$ & $\mathrm{DCF} 2(10)$ \\
\hline DCSF & $\mathrm{Pu}-240$ & $4.440 \mathrm{E}-01$ & $4.440 \mathrm{E}-01$ & $\operatorname{DCF} 2(11)$ \\
\hline DCSF & $R a-226+D$ & $3.526 \mathrm{E}-02$ & $3.526 \mathrm{E}-02$ & $\mathrm{DCF} 2(13)$ \\
\hline DCSF & $\mathrm{Ra}-228+\mathrm{D}$ & $5.929 \mathrm{E}-02$ & $5.929 \mathrm{E}-02$ & $\mathrm{DCF} 2(14)$ \\
\hline DCSF & $\mathrm{TC}-99$ & $4.810 \mathrm{E}-05$ & $4.810 \mathrm{E}-05$ & $\mathrm{DCF} 2(15)$ \\
\hline DCSF & Th $-228+D$ & $1.614 \mathrm{E}-01$ & $1.614 \mathrm{E}-01$ & $\operatorname{DCF} 2(16)$ \\
\hline DCSF & Th $-229+D$ & $9.481 \mathrm{E}-01$ & $9.481 \mathrm{E}-01$ & $\operatorname{DCF} 2(17)$ \\
\hline DCSF & Th-230 & $3.700 \mathrm{E}-01$ & $3.700 \mathrm{E}-01$ & $\mathrm{DCF} 2(18)$ \\
\hline DCSF & Th-232 & $4.070 \mathrm{E}-01$ & $4.070 \mathrm{E}-01$ & $\mathrm{DCF} 2$ (19) \\
\hline DCSF & $\mathrm{U}-233$ & $3.552 \mathrm{E}-02$ & $3.552 \mathrm{E}-02$ & $\operatorname{DCF} 2(20)$ \\
\hline DCSF & $\mathrm{U}-234$ & $3.478 \mathrm{E}-02$ & $3.478 \mathrm{E}-02$ & $\operatorname{DCF} 2(21)$ \\
\hline
\end{tabular}

ț

Title : RESRAD-OFFSITE Effluent release

File : PADUCAH EFFLUENT RELEASE-REV1.ROF

Dose Conversion Factor (and Related) Parameter Summary (continued) Current Library: ICRP 72 (Adult)

\begin{tabular}{|c|c|c|c|c|}
\hline $\begin{array}{l}\text { O Menu } \\
\text { Men }\end{array}$ & Parameter & $\begin{array}{l}\text { Current } \\
\text { Value }\end{array}$ & Default & $\begin{array}{l}\text { Parameter } \\
\text { Name }\end{array}$ \\
\hline DCSF & $\mathrm{U}-235+\mathrm{D}$ & $3.145 \mathrm{E}-02$ & $3.145 \mathrm{E}-02$ & DCF2 (22) \\
\hline DCSF & $\mathrm{U}-236$ & $3.219 \mathrm{E}-02$ & $3.219 \mathrm{E}-02$ & DCF2 (23) \\
\hline DCSF & $\mathrm{U}-238$ & $2.960 \mathrm{E}-02$ & $2.960 \mathrm{E}-02$ & DCF2 (24) \\
\hline DCSF & $\mathrm{U}-238+\mathrm{D}$ & $2.963 \mathrm{E}-02$ & $2.963 \mathrm{E}-02$ & $\mathrm{DCF} 2(25)$ \\
\hline DCSF & Dose conversion factors for ingestion, mrem/pCi: & & & \\
\hline DCSF & $A C-227+D$ & $4.473 \mathrm{E}-03$ & $4.473 E-03$ & $\mathrm{DCF} 3(1)$ \\
\hline DCSF & $\mathrm{Am}-241$ & $7.400 \mathrm{E}-04$ & $7.400 \mathrm{E}-04$ & $\mathrm{DCF} 3(2)$ \\
\hline DCSF & $C s-137+D$ & $4.810 \mathrm{E}-05$ & $4.810 \mathrm{E}-05$ & $\mathrm{DCF} 3(3)$ \\
\hline DCSF & $\mathrm{Np}-237+\mathrm{D}$ & $4.102 \mathrm{E}-04$ & $4.102 \mathrm{E}-04$ & $\mathrm{DCF} 3(4)$ \\
\hline DCSF & $\mathrm{Pa}-231$ & $2.627 \mathrm{E}-03$ & $2.627 \mathrm{E}-03$ & $\mathrm{DCF} 3(5)$ \\
\hline DCSF & $\mathrm{Pb}-210+\mathrm{D}$ & $2.558 \mathrm{E}-03$ & $2.558 \mathrm{E}-03$ & $\mathrm{DCF} 3(6)$ \\
\hline DCSF & $\mathrm{Po}-210$ & $4.440 \mathrm{E}-03$ & $4.440 \mathrm{E}-03$ & $\operatorname{DCF} 3(7)$ \\
\hline DCSF & $\mathrm{Pu}-238$ & $8.510 \mathrm{E}-04$ & $8.510 \mathrm{E}-04$ & $\mathrm{DCF} 3(8)$ \\
\hline DCSF & $\mathrm{Pu}-239$ & $9.250 \mathrm{E}-04$ & $9.250 \mathrm{E}-04$ & $\operatorname{DCF} 3(10)$ \\
\hline
\end{tabular}




\begin{tabular}{|c|c|c|c|c|}
\hline DCSF & $\mathrm{Pu}-240$ & $9.250 \mathrm{E}-04$ & $9.250 \mathrm{E}-04$ & $\operatorname{DCF} 3(11)$ \\
\hline DCSF & $\mathrm{Ra}-226+\mathrm{D}$ & $1.037 \mathrm{E}-03$ & $1.037 \mathrm{E}-03$ & $\mathrm{DCF} 3(13)$ \\
\hline DCSF & $\mathrm{Ra}-228+\mathrm{D}$ & $2.555 \mathrm{E}-03$ & $2.555 \mathrm{E}-03$ & $\mathrm{DCF} 3(14)$ \\
\hline DCSF & $T C-99$ & $2.368 \mathrm{E}-06$ & $2.368 \mathrm{E}-06$ & DCF3 (15) \\
\hline DCSF & Th $-228+D$ & $5.301 \mathrm{E}-04$ & $5.301 \mathrm{E}-04$ & DCF3 (16) \\
\hline DCSF & Th-229+D & $2.269 \mathrm{E}-03$ & $2.269 \mathrm{E}-03$ & $\operatorname{DCF} 3(17)$ \\
\hline DCSF & Th -230 & $7.770 \mathrm{E}-04$ & $7.770 \mathrm{E}-04$ & $\operatorname{DCF} 3(18)$ \\
\hline DCSF & Th-232 & $8.510 \mathrm{E}-04$ & $8.510 \mathrm{E}-04$ & DCF3 (19) \\
\hline DCSF & $\mathrm{U}-233$ & $1.887 \mathrm{E}-04$ & $1.887 \mathrm{E}-04$ & $\operatorname{DCF} 3(20)$ \\
\hline DCSF & $\mathrm{U}-234$ & $1.813 \mathrm{E}-04$ & $1.813 \mathrm{E}-04$ & $\operatorname{DCF} 3(21)$ \\
\hline DCSF & $U-235+D$ & $1.752 \mathrm{E}-04$ & $1.752 \mathrm{E}-04$ & $\operatorname{DCF} 3(22)$ \\
\hline DCSF & $\mathrm{U}-236$ & $1.739 \mathrm{E}-04$ & $1.739 \mathrm{E}-04$ & $\operatorname{DCF} 3(23)$ \\
\hline DCSF & $\mathrm{U}-238$ & $1.665 \mathrm{E}-04$ & $1.665 \mathrm{E}-04$ & $\operatorname{DCF} 3(24)$ \\
\hline DCSF & $U-238+D$ & $1.791 \mathrm{E}-04$ & $1.791 E-04$ & $\mathrm{DCF} 3(25)$ \\
\hline
\end{tabular}

Parent Dose Report

Title : RESRAD-OFFSITE Effluent release

File : PADUCAH EFFLUENT RELEASE-REV1.ROF

Dose Conversion Factor (and Related) Parameter Summary (continued)

Current Library: RESRAD Default Transfer factors

Default Library: RESRAD Default Transfer factors

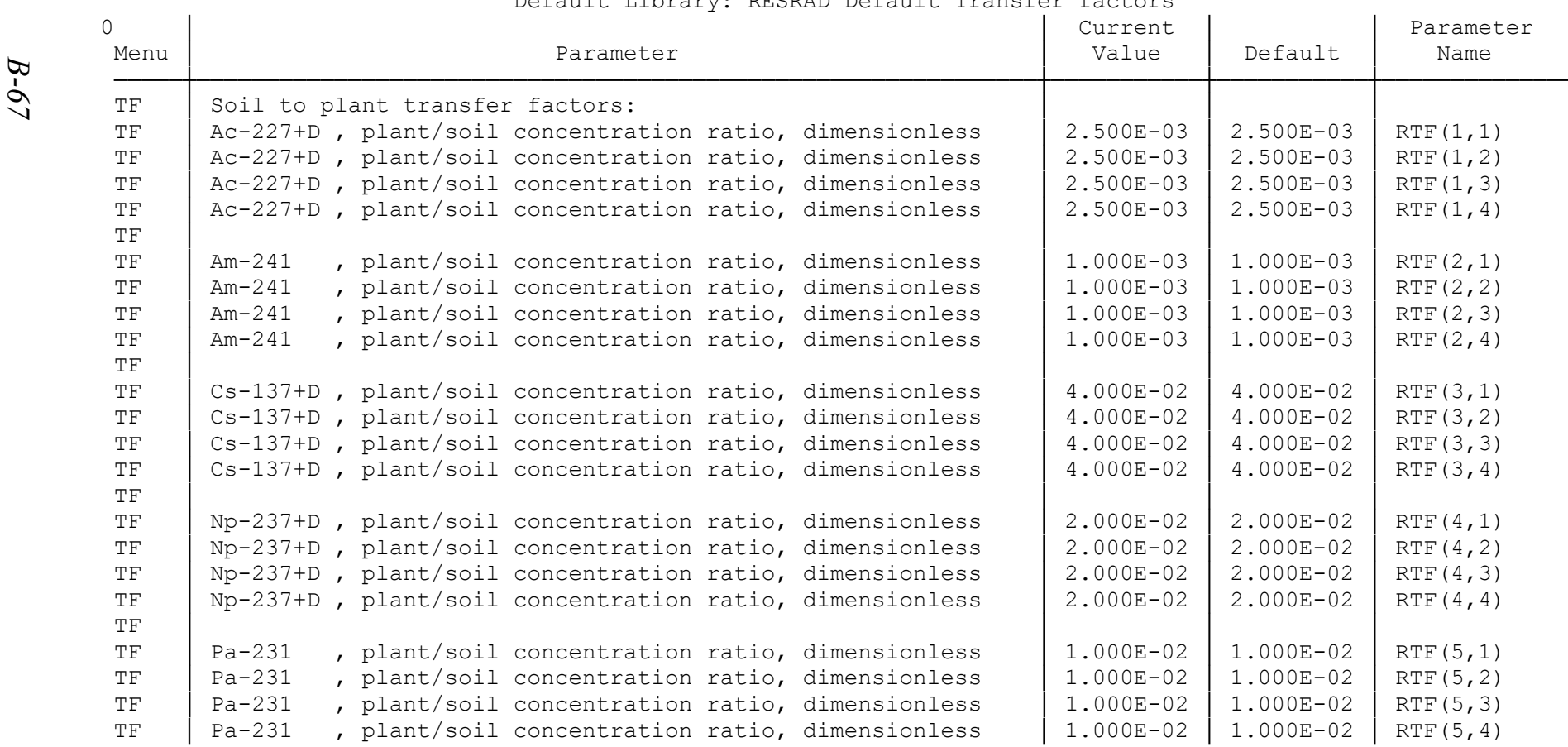




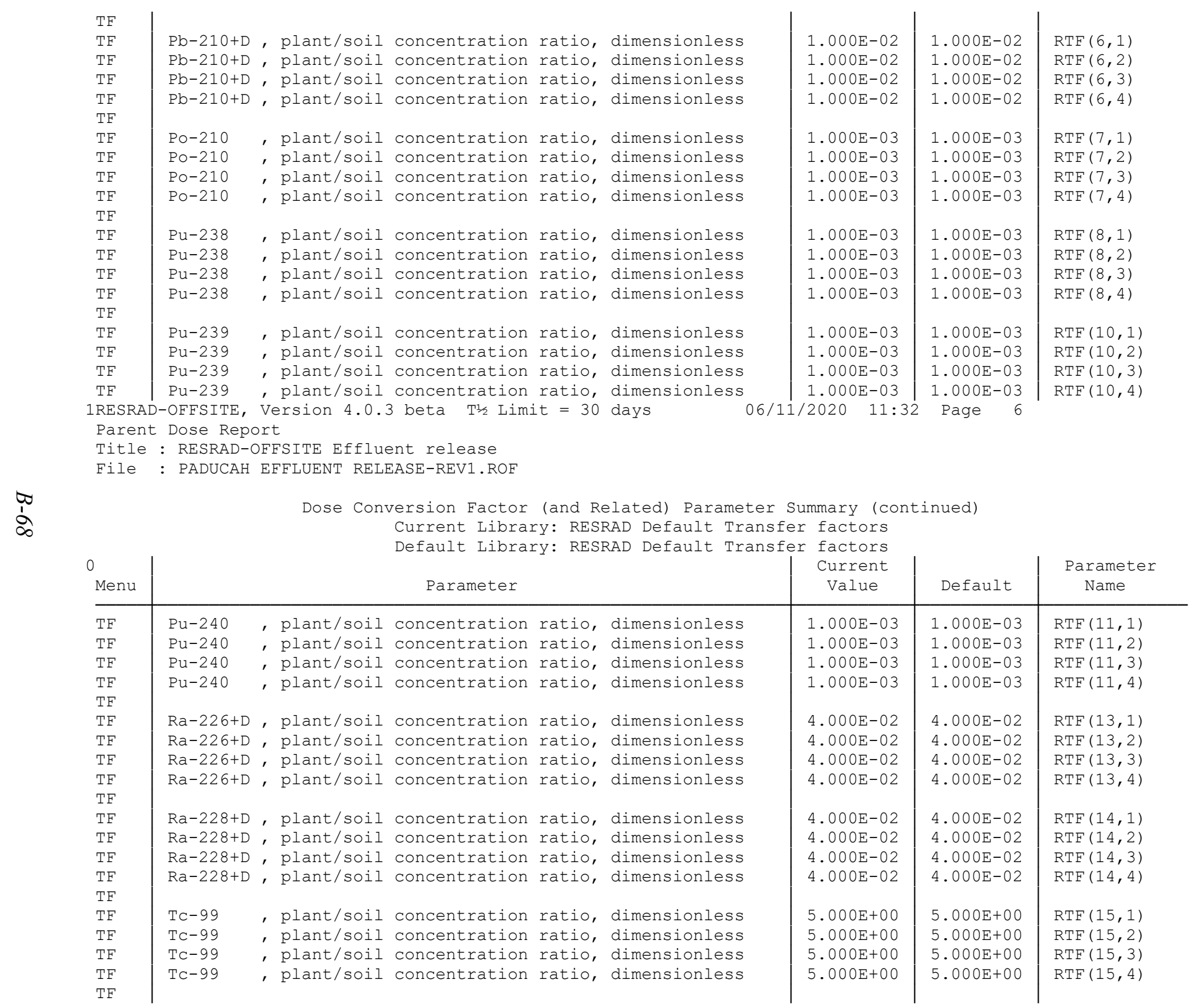




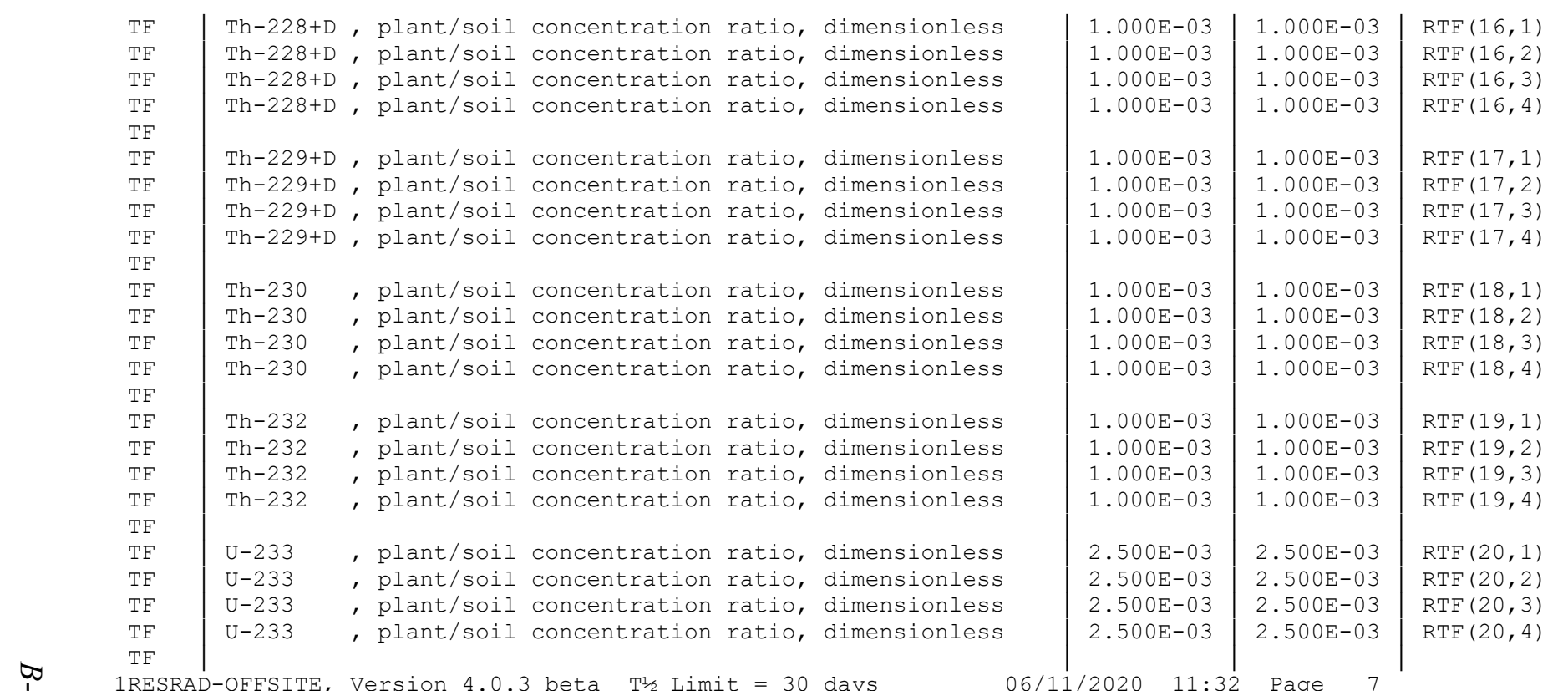

Parent Dose Report

Pitle : RESRAD-OFFSITE Effluent release

File : PADUCAH EFFLUENT RELEASE-REV1.ROF

Dose Conversion Factor (and Related) Parameter Summary (continued) Current Library: RESRAD Default Transfer factors Default Library: RESRAD Default Transfer factors

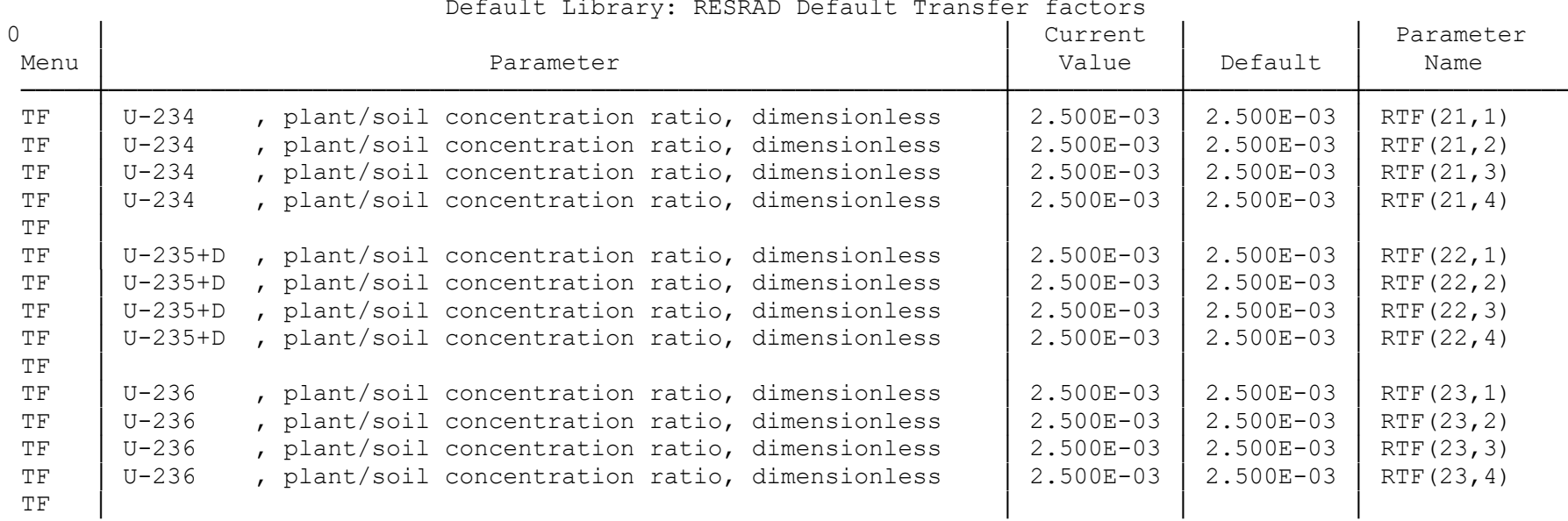




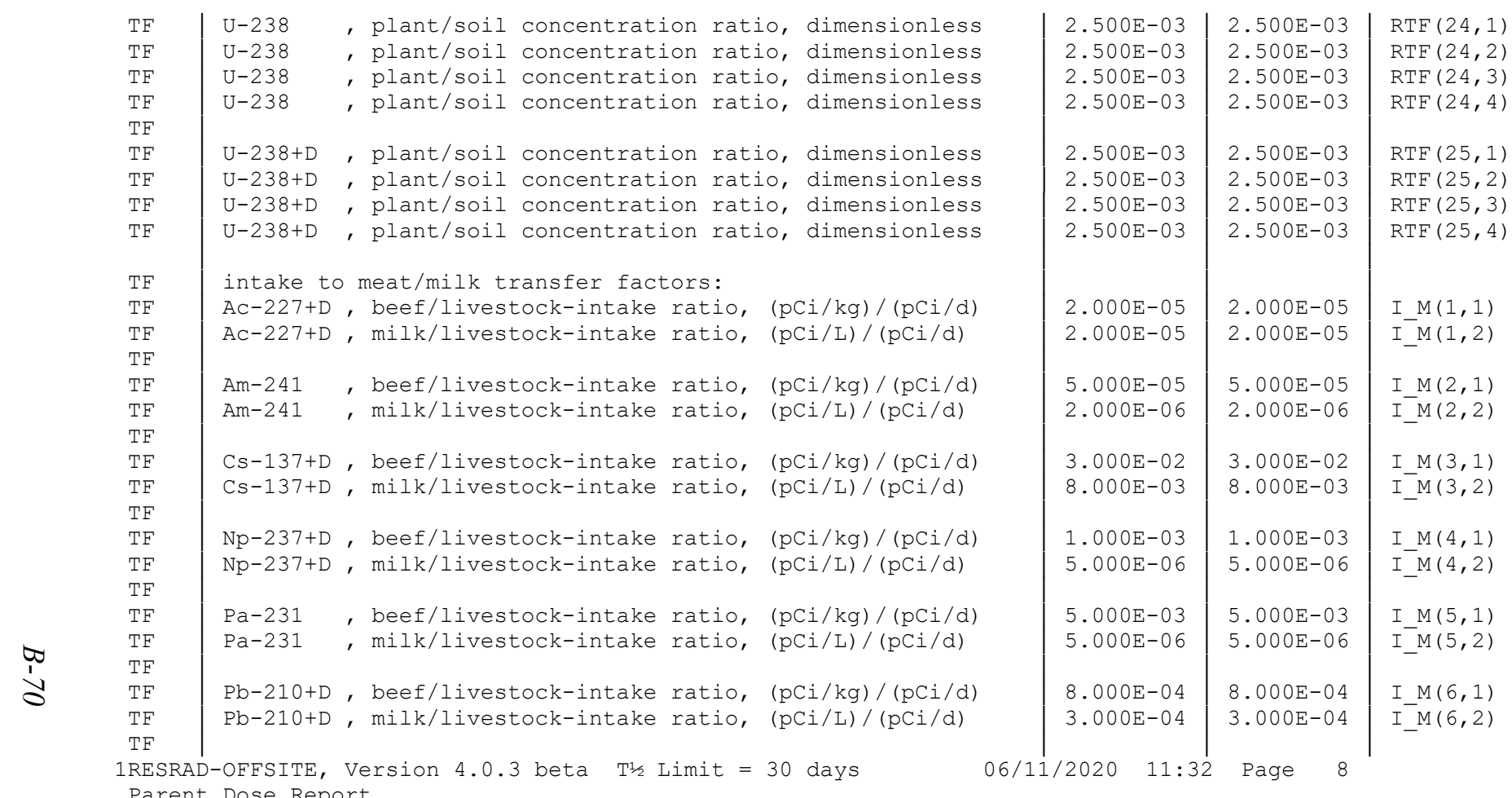

Parent Dose Report

Title : RESRAD-OFFSITE Effluent release

File : PADUCAH EFFLUENT RELEASE-REV1.ROF

Dose Conversion Factor (and Related) Parameter Summary (continued)

Current Library: RESRAD Default Transfer factors

Default Library: RESRAD Default Transfer factors

\begin{tabular}{|c|c|c|c|c|c|c|c|}
\hline \multirow{2}{*}{$\begin{array}{l}0 \\
\text { Menu } \\
\mathrm{TF}\end{array}$} & \multicolumn{4}{|c|}{ Parameter } & \multirow{2}{*}{$\begin{array}{c}\begin{array}{c}\text { Current } \\
\text { Value }\end{array} \\
5.000 \mathrm{E}-03\end{array}$} & \multirow{2}{*}{$\frac{\text { Default }}{5.000 \mathrm{E}-03}$} & \multirow{2}{*}{$\begin{array}{c}\begin{array}{c}\text { Parameter } \\
\text { Name }\end{array} \\
\text { I M }(7,1)\end{array}$} \\
\hline & Po-210 & , beef/livestock-intake & ratio, & $(\mathrm{pCi} / \mathrm{kg}) /(\mathrm{pCi} / \mathrm{d})$ & & & \\
\hline $\begin{array}{l}\mathrm{TF} \\
\mathrm{TF}\end{array}$ & Po-210 & , milk/livestock-intake & ratio, & $(\mathrm{pCi} / \mathrm{L}) /(\mathrm{pCi} / \mathrm{d})$ & $3.400 \mathrm{E}-04$ & $3.400 \mathrm{E}-04$ & $\mathrm{I}_{-}^{-} \mathrm{M}(7,2)$ \\
\hline TF & $\mathrm{Pu}-238$ & , beef/livestock-intake & ratio, & $(\mathrm{pCi} / \mathrm{kg}) /(\mathrm{pCi} / \mathrm{d})$ & $1.000 \mathrm{E}-04$ & $1.000 \mathrm{E}-04$ & $I_{-} M(8,1)$ \\
\hline $\begin{array}{l}\mathrm{TF} \\
\mathrm{TF}\end{array}$ & $\mathrm{Pu}-238$ & , milk/livestock-intake & ratio, & $(\mathrm{pCi} / \mathrm{L}) /(\mathrm{pCi} / \mathrm{d})$ & $1.000 \mathrm{E}-06$ & $1.000 \mathrm{E}-06$ & $I_{-}-M(8,2)$ \\
\hline $\mathrm{TF}$ & $\mathrm{Pu}-239$ & , beef/livestock-intake & ratio, & $(\mathrm{pCi} / \mathrm{kg}) /(\mathrm{pCi} / \mathrm{d})$ & $1.000 \mathrm{E}-04$ & $1.000 \mathrm{E}-04$ & I_M $(10,1)$ \\
\hline $\begin{array}{l}\mathrm{TF} \\
\mathrm{TF}\end{array}$ & $\mathrm{Pu}-239$ & , milk/livestock-intake & ratio, & $(\mathrm{pCi} / \mathrm{L}) /(\mathrm{pCi} / \mathrm{d})$ & $1.000 \mathrm{E}-06$ & $1.000 \mathrm{E}-06$ & $I_{-}^{-} M(10,2)$ \\
\hline TF & $\mathrm{Pu}-240$ & , beef/livestock-intake & ratio, & $(\mathrm{pCi} / \mathrm{kg}) /(\mathrm{pCi} / \mathrm{d})$ & $1.000 \mathrm{E}-04$ & $1.000 \mathrm{E}-04$ & I_M $(11,1)$ \\
\hline TF & $\mathrm{Pu}-240$ & , milk/livestock-intake & ratio, & $(\mathrm{pCi} / \mathrm{L}) /(\mathrm{pCi} / \mathrm{d})$ & $1.000 \mathrm{E}-06$ & $1.000 \mathrm{E}-06$ & $I_{-} M(11,2)$ \\
\hline
\end{tabular}




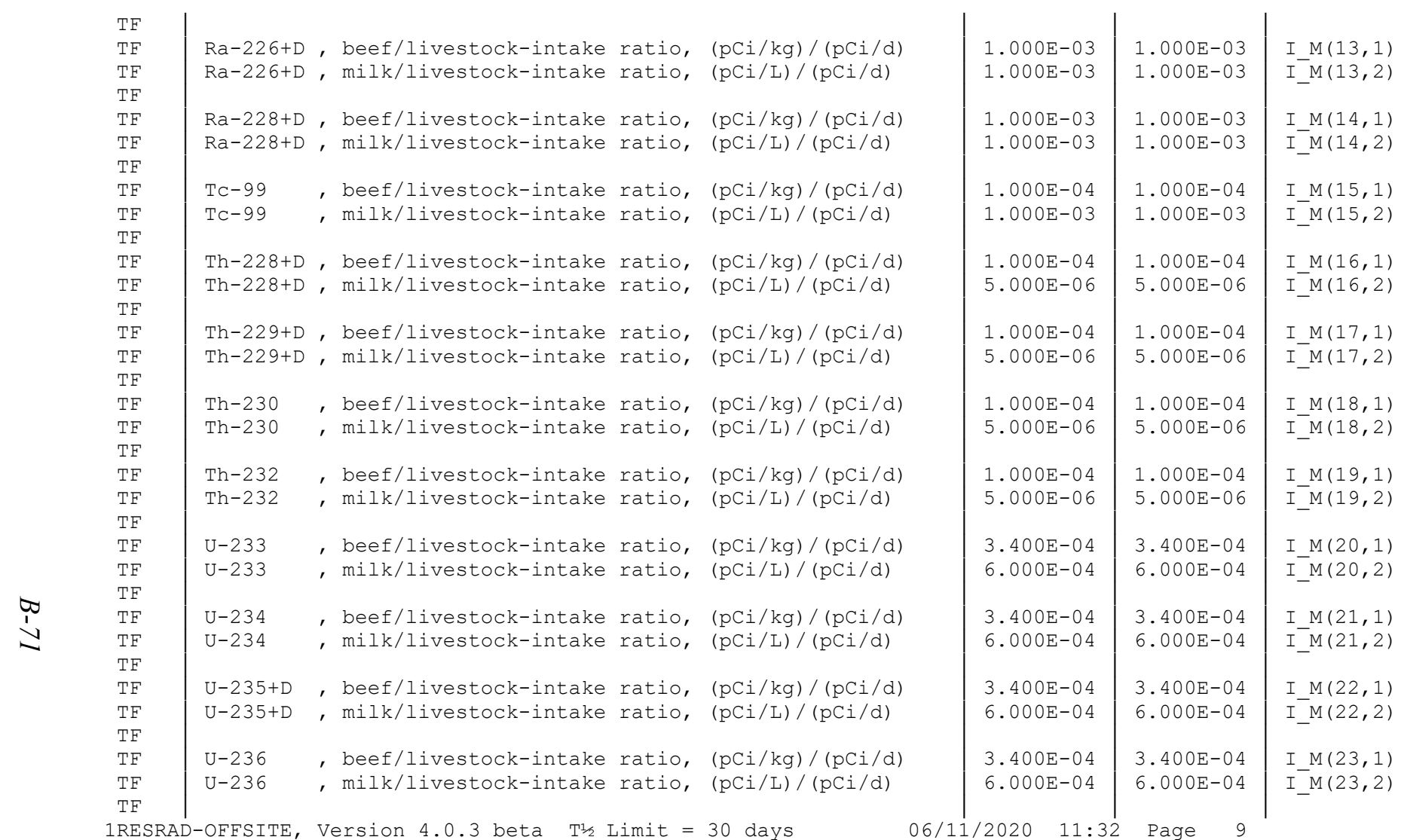

RESRAD-OFFSITE, Version 4.0 .3 beta T1/2 Limit = 30 days

06/11/2020 11:32 Page 9

Parent Dose Report

Title : RESRAD-OFFSITE Effluent release

File : PADUCAH EFFLUENT RELEASE-REV1.ROF

Dose Conversion Factor (and Related) Parameter Summary (continued) Current Library: RESRAD Default Transfer factors

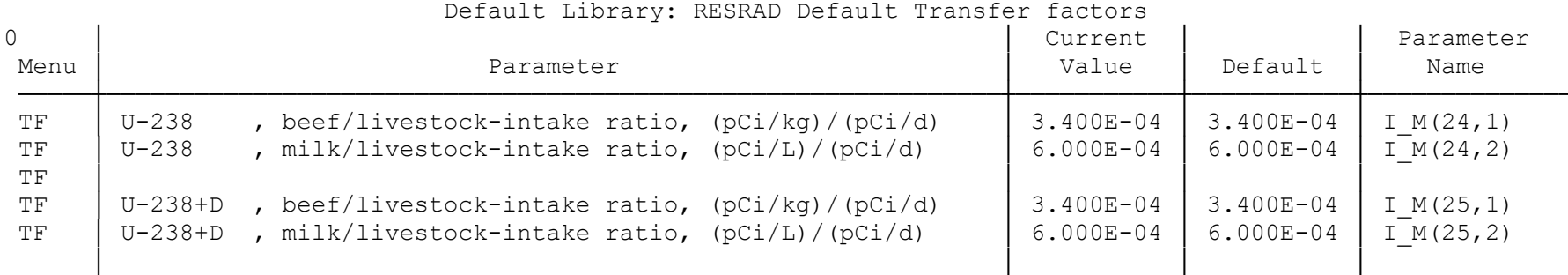




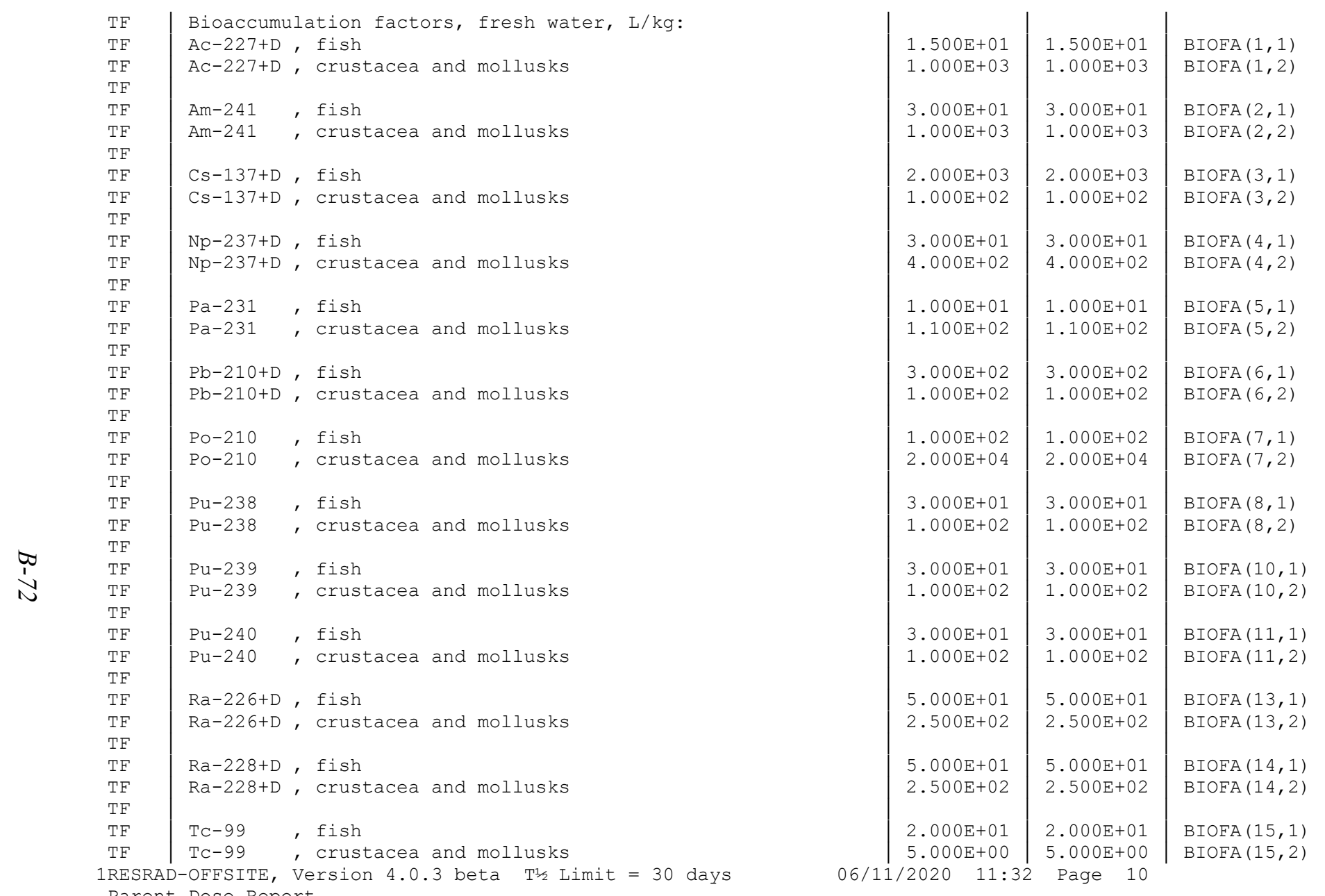

Parent Dose Report
Title : RESRAD-OFFSITE Effluent release

File : PADUCAH EFFLUENT RELEASE-REV1.ROF

Dose Conversion Factor (and Related) Parameter Summary (continued)

Current Library: RESRAD Default Transfer factors

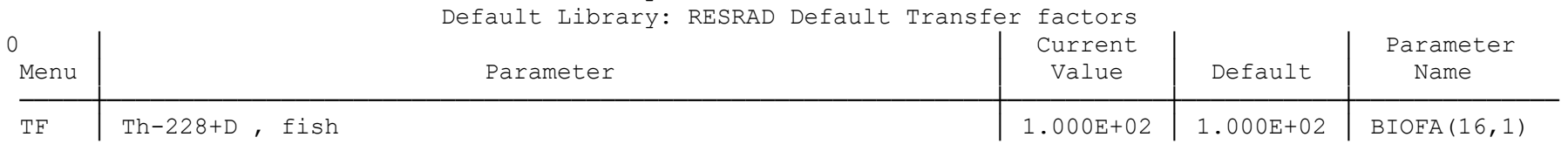




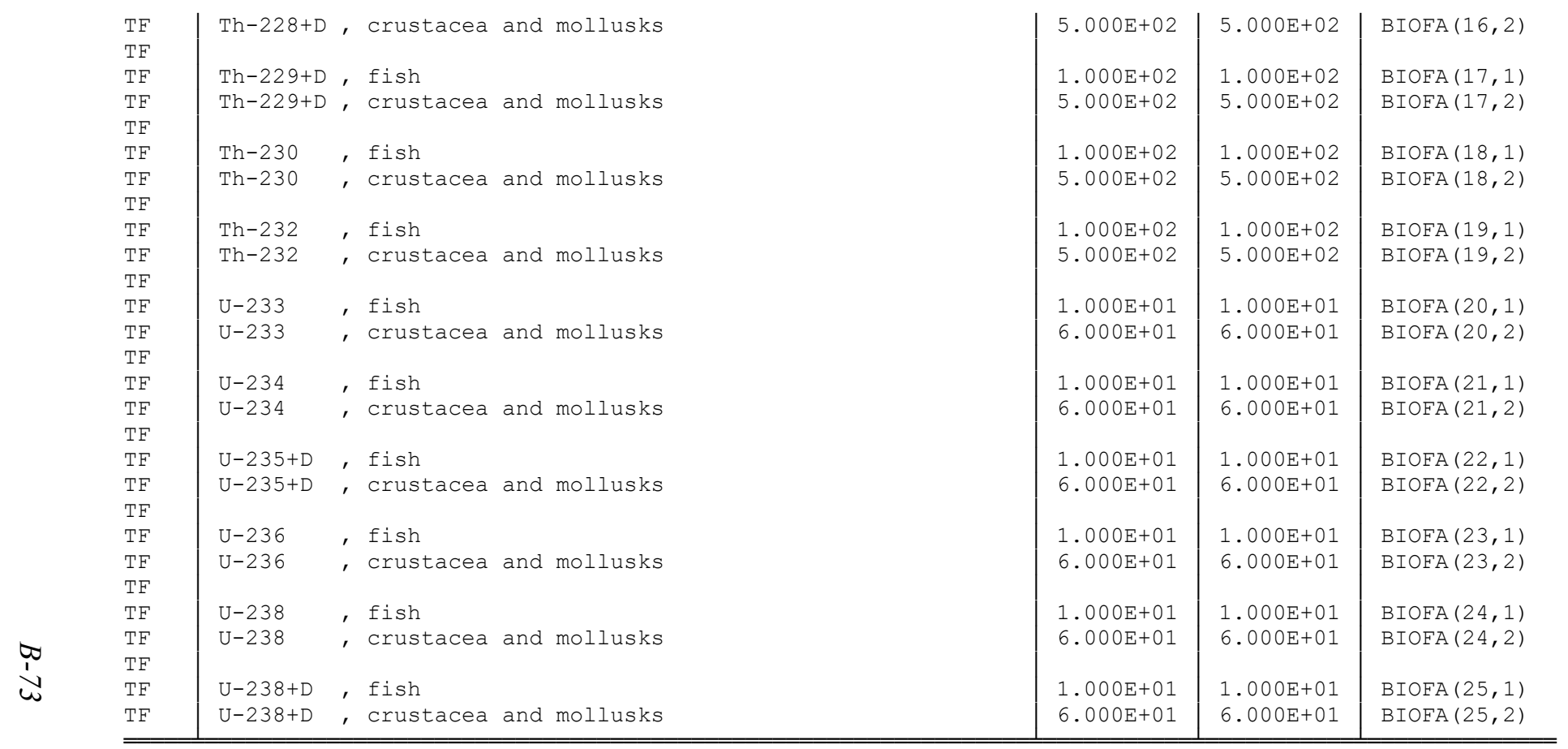

1RESRAD-OFFSITE, Version 4.0.3 beta T1/2 Limit = 30 days $\quad 06 / 11 / 2020 \quad 11: 32 \quad$ Page 11

Parent Dose Report

Title : RESRAD-OFFSITE Effluent release

File : PADUCAH EFFLUENT RELEASE-REV1.ROF

\begin{tabular}{|c|c|c|c|c|c|}
\hline $\begin{array}{l}0 \\
\text { Menu }\end{array}$ & Parameter & $\begin{array}{c}\text { - Parameter } \\
\text { User } \\
\text { Input }\end{array}$ & $\begin{array}{l}\text { Summary } \\
\text { Default }\end{array}$ & $\begin{array}{l}\text { RESRAD } \\
\text { computed }\end{array}$ & $\begin{array}{c}\text { Parameter } \\
\text { Name }\end{array}$ \\
\hline FSTI & Exposure duration for risk & $3.000 \mathrm{E}+01$ & $3.000 \mathrm{E}+01$ & --- & $\mathrm{ED}$ \\
\hline RELT & 1st release time (years) & $0.000 \mathrm{E}+00$ & & --- & RelTime (1) \\
\hline $\mathrm{CONC}$ & Initial concentration of Am-241 (pCi/g) & $3.000 \mathrm{E}+00$ & $0.000 \mathrm{E}+00$ & --- & S1 (2) \\
\hline $\mathrm{CONC}$ & Initial concentration of $\mathrm{Cs}-137$ (pCi/g) & $3.000 \mathrm{E}+00$ & $0.000 \mathrm{E}+00$ & --- & S1 (3) \\
\hline $\mathrm{CONC}$ & Initial concentration of $\mathrm{Pu}-239$ ( $\mathrm{pCi} / \mathrm{g}$ ) & $3.000 \mathrm{E}+00$ & $0.000 \mathrm{E}+00$ & --- & S1(10) \\
\hline $\mathrm{CONC}$ & Initial concentration of $\mathrm{Pu}-240$ (pCi/g) & $3.000 \mathrm{E}+00$ & $0.000 \mathrm{E}+00$ & --- & S1(11) \\
\hline $\mathrm{CONC}$ & Initial concentration of $\mathrm{Tc}-99$ (pCi/g) & $3.000 \mathrm{E}+01$ & $0.000 \mathrm{E}+00$ & --- & S1 (15) \\
\hline $\mathrm{CONC}$ & Initial concentration of $\mathrm{Th}-228$ ( $\mathrm{pCi} / \mathrm{g}$ ) & $3.000 \mathrm{E}+00$ & $0.000 \mathrm{E}+00$ & --- & S1 (16) \\
\hline $\mathrm{CONC}$ & Initial concentration of $\mathrm{Th}-230$ (pCi/g) & $3.000 \mathrm{E}+00$ & $0.000 \mathrm{E}+00$ & --- & S1 (18) \\
\hline
\end{tabular}


CONC Initial concentration of Th-232 (pCi/g)

CONC Initial concentration of $\mathrm{U}-234$ (pCi/g)

CONC Initial concentration of $\mathrm{U}-235 \mathrm{(pCi} / \mathrm{g})$

CONC Initial concentration of $\mathrm{U}-238$ (pCi/g)

VDEP Deposition velocity of Ac-227 on total particulates VDEP Dep. velocity of Ac-227 on respirable particulates

VDEP Deposition velocity of Am-241 on total particulates

VDEP Dep. velocity of Am-241 on respirable particulates

VDEP Deposition velocity of Cs-137 on total particulates

VDEP Dep. velocity of Cs-137 on respirable particulates

VDEP Deposition velocity of Np-237 on total particulates

VDEP Dep. velocity of Np-237 on respirable particulates

VDEP Deposition velocity of $\mathrm{Pa}-231$ on total particulates

VDEP Dep. velocity of $\mathrm{Pa}-231$ on respirable particulates

VDEP Deposition velocity of $\mathrm{Pb}-210$ on total particulates

VDEP Dep. velocity of $\mathrm{Pb}-210$ on respirable particulates

VDEP Deposition velocity of Po-210 on total particulates

VDEP Dep. velocity of Po-210 on respirable particulates

VDEP Deposition velocity of Pu-238 on total particulates

VDEP Dep. velocity of $\mathrm{Pu}-238$ on respirable particulates

VDEP Deposition velocity of Pu-239 on total particulates

VDEP Dep. velocity of Pu-239 on respirable particulates

VDEP Deposition velocity of Pu-240 on total particulates

VDEP Dep. velocity of $\mathrm{Pu}-240$ on respirable particulates

VDEP Deposition velocity of Ra-226 on total particulates

VDEP Dep relocity of Ra-226 on respirable particulates

VDEP Deposition velocity of Ra-228 on total particulates

VDEP Dep. velocity of Ra-228 on respirable particulates

VDEP Deposition velocity of TC-99 on total particulates

VDEP Dep. velocity of Tc-99 on respirable particulates

VDEP Deposition velocity of Th-228 on total particulates

VDEP Dep. velocity of Th-228 on respirable particulates

1RESRAD-OFFSITE, Version 4.0 .3 beta $T^{1} \frac{1}{2}$ Limit $=30$ days

Parent Dose Report

Title: RESRAD-OFFSITE Effluent release

File: PADUCAH EFFLUENT RELEASE-REV1.ROF

\begin{tabular}{|c|c|c|c|c|c|}
\hline Menu & ite-Specific $\mathrm{Pa}$ & $\begin{array}{l}\text { eter Summa } \\
\text { User } \\
\text { Input }\end{array}$ & $\begin{array}{l}\text { (continu } \\
\text { Default }\end{array}$ & $\begin{array}{l}\text { RESRAD } \\
\text { computed }\end{array}$ & $\begin{array}{l}\text { Parameter } \\
\text { Name }\end{array}$ \\
\hline VDEP & Deposition velocity of Th-229 on total particulates & $1.000 \mathrm{E}-03$ & $1.000 \mathrm{E}-03$ & --- & DEPVEL (17) \\
\hline VDEP & Deposition velocity of Th-230 on total particulates & $1.000 \mathrm{E}-03$ & $1.000 \mathrm{E}-03$ & --- & DEPVEL (18) \\
\hline VDEP & Dep. velocity of Th-230 on respirable particulates & $1.000 \mathrm{E}-03$ & $1.000 \mathrm{E}-03$ & --- & DEPVELT (18) \\
\hline VDEP & Deposition velocity of Th-232 on total particulates & $1.000 \mathrm{E}-03$ & $1.000 \mathrm{E}-03$ & --- & DEPVEL (19) \\
\hline VDEP & Dep. velocity of $\mathrm{Th}-232$ on respirable particulates & $1.000 \mathrm{E}-03$ & $1.000 \mathrm{E}-03$ & --- & DEPVELT (19) \\
\hline VDEP & Deposition velocity of $\mathrm{U}-234$ on total particulates & $1.000 \mathrm{E}-03$ & $1.000 \mathrm{E}-03$ & --- & DEPVEL (21) \\
\hline
\end{tabular}

\begin{tabular}{|c|c|c|c|}
\hline $3.000 \mathrm{E}+00$ & $0.000 \mathrm{E}+00$ & --- & S1 (19) \\
\hline $3.000 \mathrm{E}+01$ & $0.000 \mathrm{E}+00$ & --- & S1 (21) \\
\hline $3.000 \mathrm{E}+01$ & $0.000 \mathrm{E}+00$ & --- & S1 (22) \\
\hline $3.000 \mathrm{E}+01$ & $0.000 \mathrm{E}+00$ & --- & S1 (24) \\
\hline $1.000 \mathrm{E}-03$ & $1.000 \mathrm{E}-03$ & --- & DEPVEL (1) \\
\hline $1.000 \mathrm{E}-03$ & $1.000 \mathrm{E}-03$ & --- & DEPVELT (1) \\
\hline $1.000 \mathrm{E}-03$ & $1.000 \mathrm{E}-03$ & --- & DEPVEL (2) \\
\hline $1.000 \mathrm{E}-03$ & $1.000 \mathrm{E}-03$ & --- & DEPVELT (2) \\
\hline $1.000 \mathrm{E}-03$ & $1.000 \mathrm{E}-03$ & --- & DEPVEL (3) \\
\hline $1.000 \mathrm{E}-03$ & $1.000 \mathrm{E}-03$ & --- & DEPVELT (3) \\
\hline $1.000 \mathrm{E}-03$ & $1.000 \mathrm{E}-03$ & --- & DEPVEL (4) \\
\hline $1.000 \mathrm{E}-03$ & $1.000 \mathrm{E}-03$ & --- & DEPVELT (4) \\
\hline $1.000 \mathrm{E}-03$ & $1.000 \mathrm{E}-03$ & --- & DEPVEL (5) \\
\hline $1.000 \mathrm{E}-03$ & $1.000 \mathrm{E}-03$ & --- & DEPVELT (5) \\
\hline $1.000 \mathrm{E}-03$ & $1.000 \mathrm{E}-03$ & --- & DEPVEL (6) \\
\hline $1.000 \mathrm{E}-03$ & $1.000 \mathrm{E}-03$ & --- & DEPVELT (6) \\
\hline $1.000 \mathrm{E}-03$ & $1.000 \mathrm{E}-03$ & --- & DEPVEL (7) \\
\hline $1.000 \mathrm{E}-03$ & $1.000 \mathrm{E}-03$ & --- & DEPVELT (7) \\
\hline $1.000 \mathrm{E}-03$ & $1.000 \mathrm{E}-03$ & --- & DEPVEL ( 8) \\
\hline $1.000 \mathrm{E}-03$ & $1.000 \mathrm{E}-03$ & --- & DEPVELT (8) \\
\hline $1.000 \mathrm{E}-03$ & $1.000 \mathrm{E}-03$ & --- & DEPVEL (10) \\
\hline $1.000 \mathrm{E}-03$ & $1.000 \mathrm{E}-03$ & --- & DEPVELT (10) \\
\hline $1.000 \mathrm{E}-03$ & $1.000 \mathrm{E}-03$ & --- & DEPVEL (11) \\
\hline $1.000 \mathrm{E}-03$ & $1.000 \mathrm{E}-03$ & --- & DEPVELT (11) \\
\hline $1.000 \mathrm{E}-03$ & $1.000 \mathrm{E}-03$ & --- & DEPVEL (13) \\
\hline $1.000 \mathrm{E}-03$ & $1.000 \mathrm{E}-03$ & --- & DEPVELT (13) \\
\hline $1.000 \mathrm{E}-03$ & $1.000 \mathrm{E}-03$ & --- & DEPVEL (14) \\
\hline $1.000 \mathrm{E}-03$ & $1.000 \mathrm{E}-03$ & --- & DEPVELT (14) \\
\hline $1.000 \mathrm{E}-03$ & $1.000 \mathrm{E}-03$ & --- & DEPVEL (15) \\
\hline $1.000 \mathrm{E}-03$ & $1.000 \mathrm{E}-03$ & --- & DEPVELT (15) \\
\hline $1.000 \mathrm{E}-03$ & $1.000 \mathrm{E}-03$ & --- & DEPVEL (16) \\
\hline $1.000 \mathrm{E}-03$ & $1.000 \mathrm{E}-03$ & --- & DEPVELT (16) \\
\hline
\end{tabular}


VDEP $\mid$ Dep. velocity of U-234 on respirable particulates VDEP Deposition velocity of U-235 on total particulate VDEP Dep. velocity of U-235 on respirable particulates

VDEP Deposition velocity of U-236 on total particulates

VDEP Dep. velocity of U-236 on respirable particulates

VDEP Deposition velocity of U-238 on total particulates

VDEP Dep. velocity of U-238 on respirable particulates

DCLR Distribution coefficients for Am-241

DCLR Contaminated zone $(\mathrm{cm} * * 3 / \mathrm{g})$

DCLR Unsaturated zone $1(\mathrm{~cm} * * 3 / \mathrm{g})$

DCLR Saturated zone $(\mathrm{cm} * * 3 / \mathrm{g})$

DCLR Bottom sediment in surface water body $(\mathrm{cm} * * 3 / \mathrm{g})$

DCLR Suspended sediment in surface water body $(\mathrm{cm} * * 3 / \mathrm{g})$

DCLR Agricultural area $1(\mathrm{~cm} * * 3 / \mathrm{g})$

DCLR Agricultural area $2(\mathrm{~cm} * * 3 / \mathrm{g})$

DCLR Agricultural area $3(\mathrm{~cm} * * 3 / \mathrm{g})$

DCLR Agricultural area $4(\mathrm{~cm} * * 3 / \mathrm{g})$

DCLR Offsite Dwelling $(\mathrm{cm} * * 3 / \mathrm{g})$

DCLR Leach rate constant of Am-241 (/yr)

DCLR Distribution coefficients for Cs-137

DCLR Contaminated zone $(\mathrm{cm} * * 3 / \mathrm{g})$

DCLR Unsaturated zone $1(\mathrm{~cm} * * 3 / \mathrm{g})$

DCLR Saturated zone $(\mathrm{cm} * * 3 / \mathrm{g})$

DCLR Bottom sediment in surface water body $(\mathrm{cm} * * 3 / \mathrm{g})$

DCLR Suspended sediment in surface water body $(\mathrm{cm} * * 3 / \mathrm{g})$

DCLR

DCLR

\begin{tabular}{l|l} 
DCLR & Offsite Dwelling $(\mathrm{cm} * * 3 / \mathrm{g})$ \\
DCLR & Leach rate constant of $\mathrm{Cs}-137$ (/yr)
\end{tabular}

1RESRAD-OFFSITE, Version 4.0 .3 beta $T^{1 / 2}$ Limit $=30$ days

Parent Dose Report

Title: RESRAD-OFFSITE Effluent release

File : PADUCAH EFFLUENT RELEASE-REV1.ROF

\begin{tabular}{|c|c|c|c|}
\hline $1.000 \mathrm{E}-03$ & $1.000 \mathrm{E}-03$ & --- & DEPVELT (21) \\
\hline $1.000 \mathrm{E}-03$ & $1.000 \mathrm{E}-03$ & --- & DEPVEL (22) \\
\hline $1.000 \mathrm{E}-03$ & $1.000 \mathrm{E}-03$ & --- & DEPVELT (22) \\
\hline $1.000 \mathrm{E}-03$ & $1.000 \mathrm{E}-03$ & --- & DEPVEL (23) \\
\hline $1.000 \mathrm{E}-03$ & $1.000 \mathrm{E}-03$ & --- & DEPVELT (23) \\
\hline $1.000 \mathrm{E}-03$ & $1.000 \mathrm{E}-03$ & --- & DEPVEL (24) \\
\hline $1.000 \mathrm{E}-03$ & $1.000 \mathrm{E}-03$ & --- & DEPVELT (24) \\
\hline $2.000 \mathrm{E}+01$ & $2.000 \mathrm{E}+01$ & --- & $\operatorname{DCNUCC}(2)$ \\
\hline $2.000 \mathrm{E}+01$ & $2.000 \mathrm{E}+01$ & --- & $\operatorname{DCNUCU}(2,1)$ \\
\hline $2.000 \mathrm{E}+01$ & $2.000 \mathrm{E}+01$ & --- & DCNUCS (2) \\
\hline $2.000 \mathrm{E}+01$ & $2.000 \mathrm{E}+01$ & --- & DCNUCSWB (2) \\
\hline $2.000 \mathrm{E}+01$ & $2.000 \mathrm{E}+01$ & --- & DCNUCSWS (2) \\
\hline $2.000 \mathrm{E}+01$ & $2.000 \mathrm{E}+01$ & --- & $\operatorname{DCNUCOF}(2,1)$ \\
\hline $2.000 \mathrm{E}+01$ & $2.000 \mathrm{E}+01$ & --- & $\operatorname{DCNUCOF}(2,2)$ \\
\hline $2.000 \mathrm{E}+01$ & $2.000 \mathrm{E}+01$ & --- & $\operatorname{DCNUCOF}(2,3)$ \\
\hline $2.000 \mathrm{E}+01$ & $2.000 \mathrm{E}+01$ & --- & $\operatorname{DCNUCOF}(2,4)$ \\
\hline $2.000 \mathrm{E}+01$ & $2.000 \mathrm{E}+01$ & --- & DCNUCDWE (2) \\
\hline $0.000 \mathrm{E}+00$ & $0.000 \mathrm{E}+00$ & $8.245 \mathrm{E}-03$ & Rleach $(1,2)$ \\
\hline $4.600 \mathrm{E}+03$ & $4.600 \mathrm{E}+03$ & --- & DCNUCC (3) \\
\hline $4.600 \mathrm{E}+03$ & $4.600 \mathrm{E}+03$ & --- & $\operatorname{DCNUCU}(3,1)$ \\
\hline $4.600 \mathrm{E}+03$ & $4.600 \mathrm{E}+03$ & --- & DCNUCS (3) \\
\hline $4.600 \mathrm{E}+03$ & $4.600 \mathrm{E}+03$ & --- & DCNUCSWB (3) \\
\hline $4.600 \mathrm{E}+03$ & $4.600 \mathrm{E}+03$ & --- & DCNUCSWS (3) \\
\hline $4.600 \mathrm{E}+03$ & $4.600 \mathrm{E}+03$ & --- & $\operatorname{DCNUCOF}(3,1)$ \\
\hline $4.600 \mathrm{E}+03$ & $4.600 \mathrm{E}+03$ & --- & $\operatorname{DCNUCOF}(3,2)$ \\
\hline $4.600 \mathrm{E}+03$ & $4.600 \mathrm{E}+03$ & --- & $\operatorname{DCNUCOF}(3,3)$ \\
\hline $4.600 \mathrm{E}+03$ & $4.600 \mathrm{E}+03$ & --- & $\operatorname{DCNUCOF}(3,4)$ \\
\hline $4.600 \mathrm{E}+03$ & $4.600 \mathrm{E}+03$ & --- & DCNUCDWE (3) \\
\hline $0.000 \mathrm{E}+00$ & $0.000 \mathrm{E}+00$ & $3.623 \mathrm{E}-05$ & $\operatorname{Rleach}(1,3)$ \\
\hline
\end{tabular}

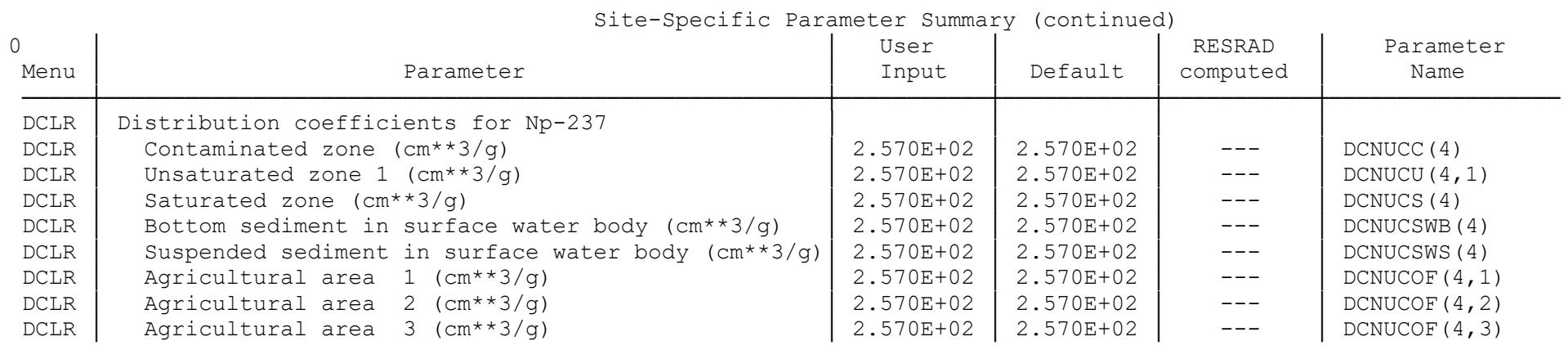




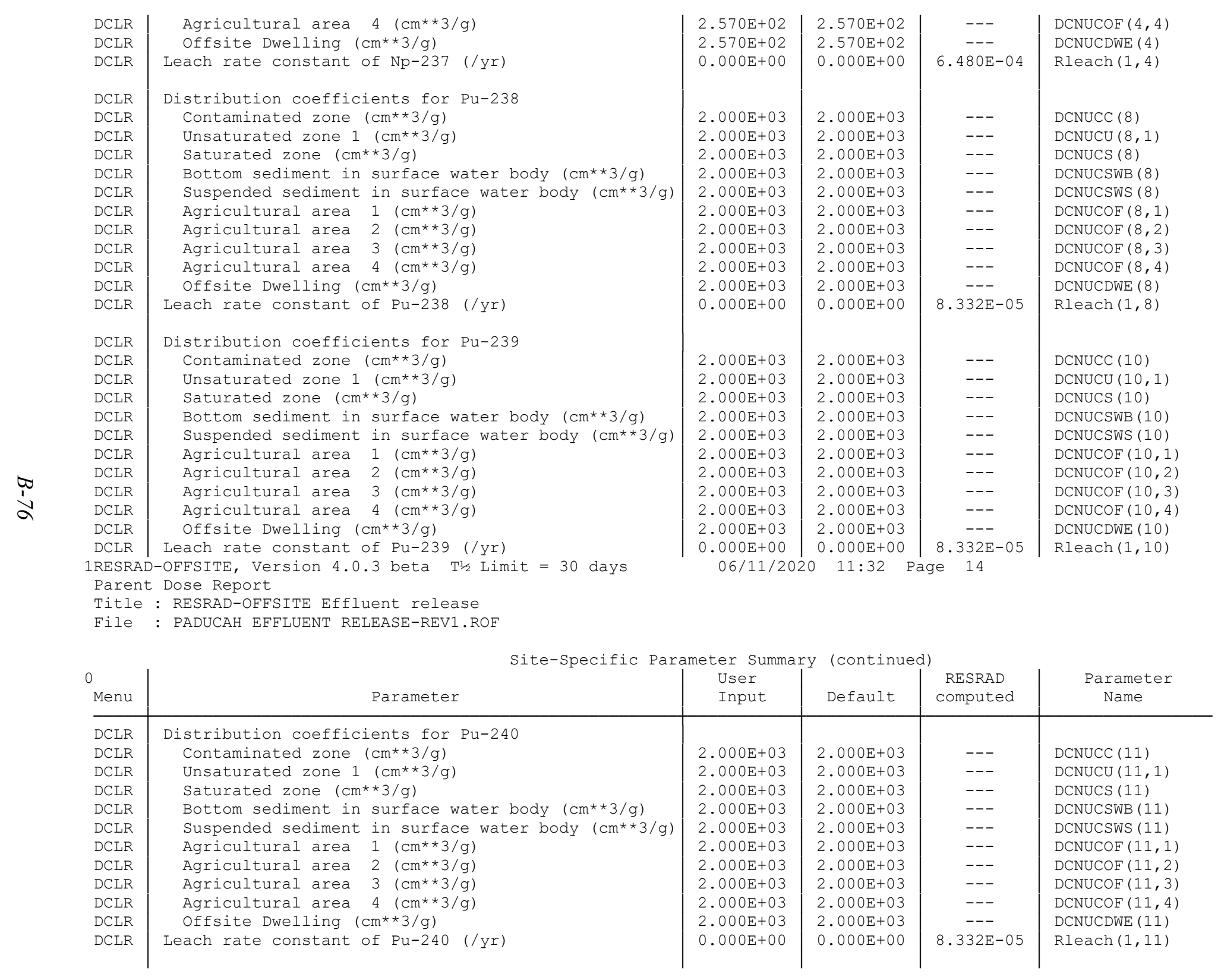




\begin{tabular}{|c|c|c|c|c|c|}
\hline CLR & Distribution coefficients for TC-99 & & & & \\
\hline DCLR & Contaminated zone $(\mathrm{cm} * * 3 / \mathrm{g})$ & $0.000 \mathrm{E}+00$ & $0.000 \mathrm{E}+00$ & --- & DCNUCC (15) \\
\hline DCLR & Unsaturated zone $1(\mathrm{~cm} * * 3 / \mathrm{g})$ & $0.000 \mathrm{E}+00$ & $0.000 \mathrm{E}+00$ & --- & $\operatorname{DCNUCU}(15,1)$ \\
\hline DCLR & Saturated zone $(\mathrm{cm} * * 3 / \mathrm{g})$ & $0.000 \mathrm{E}+00$ & $0.000 \mathrm{E}+00$ & --- & DCNUCS (15) \\
\hline DCLR & Bottom sediment in surface water body $(\mathrm{cm} * * 3 / \mathrm{g})$ & $0.000 \mathrm{E}+00$ & $0.000 \mathrm{E}+00$ & --- & DCNUCSWB (15) \\
\hline DCLR & Suspended sediment in surface water body $(\mathrm{cm} * * 3 / \mathrm{g})$ & $0.000 \mathrm{E}+00$ & $0.000 \mathrm{E}+00$ & --- & DCNUCSWS (15) \\
\hline DCLR & Agricultural area $1(\mathrm{~cm} * * 3 / \mathrm{g})$ & $0.000 \mathrm{E}+00$ & $0.000 \mathrm{E}+00$ & --- & $\operatorname{DCNUCOF}(15,1)$ \\
\hline DCLR & Agricultural area $2(\mathrm{~cm} * * 3 / \mathrm{g})$ & $0.000 \mathrm{E}+00$ & $0.000 \mathrm{E}+00$ & --- & $\operatorname{DCNUCOF}(15,2)$ \\
\hline DCLR & Agricultural area $3(\mathrm{~cm} * * 3 / \mathrm{g})$ & $0.000 \mathrm{E}+00$ & $0.000 \mathrm{E}+00$ & --- & $\operatorname{DCNUCOF}(15,3)$ \\
\hline DCLR & Agricultural area $4(\mathrm{~cm} * \star 3 / \mathrm{g})$ & $0.000 \mathrm{E}+00$ & $0.000 \mathrm{E}+00$ & --- & $\operatorname{DCNUCOF}(15,4)$ \\
\hline DCLR & Offsite Dwelling $(\mathrm{cm} * \star 3 / \mathrm{g})$ & $00 \mathrm{E}+00$ & $0.000 \mathrm{E}+00$ & --- & DCNUCDWE (15) \\
\hline DCLR & Leach rate constant of $\mathrm{Tc}-99$ (/yr) & $0.000 \mathrm{E}+00$ & $0.000 \mathrm{E}+00$ & $7.790 \mathrm{E}-01$ & Rleach $(1,15)$ \\
\hline DCLR & Distribution coefficients fo & & & & \\
\hline DCLR & Contaminated zone $(\mathrm{cm} * * 3 / \mathrm{g})$ & $6.000 \mathrm{E}+04$ & $6.000 \mathrm{E}+04$ & --- & DCNUCC (16) \\
\hline DCLR & Unsaturated zone $1(\mathrm{~cm} * \star 3 / \mathrm{g})$ & $6.000 \mathrm{E}+04$ & $6.000 \mathrm{E}+04$ & --- & $\operatorname{DCNUCU}(16,1)$ \\
\hline DCLR & Saturated zone $(\mathrm{cm} * * 3 / \mathrm{g})$ & $0 \mathrm{E}+04$ & $0 \mathrm{E}+04$ & --- & DCNUCS (16) \\
\hline DCLR & Bottom sediment in surface water body $(\mathrm{cm} * * 3 / \mathrm{g})$ & $6.000 \mathrm{E}+04$ & $6.000 \mathrm{E}+04$ & --- & $\operatorname{DCNUCSWB}(16)$ \\
\hline DCLR & Suspended sediment in surface water body $(\mathrm{cm} * * 3 / \mathrm{g})$ & $6.000 \mathrm{E}+04$ & $6.000 \mathrm{E}+04$ & --- & DCNUCSWS (16) \\
\hline DCLR & Agricultural area $1(\mathrm{~cm} * * 3 / \mathrm{g})$ & $6.000 \mathrm{E}+04$ & $6.000 \mathrm{E}+04$ & --- & $\operatorname{DCNUCOF}(16,1)$ \\
\hline DCLR & Agricultural area $2(\mathrm{~cm} * * 3 / \mathrm{g})$ & $6.000 \mathrm{E}+04$ & $\mathrm{DE}+04$ & --- & $\operatorname{DCNUCOF}(16,2)$ \\
\hline DCLR & Agricultural area $3(\mathrm{~cm} * * 3 / \mathrm{g})$ & $6.000 \mathrm{E}+04$ & $6.000 \mathrm{E}+04$ & --- & $\operatorname{DCNUCOF}(16,3)$ \\
\hline DCLR & Agricultural area $4(\mathrm{~cm} * * 3 / \mathrm{g})$ & $6.000 \mathrm{E}+04$ & $6.000 \mathrm{E}+04$ & --- & $\operatorname{DCNUCOF}(16,4)$ \\
\hline DCLR & Offsite Dwelling $\left(\mathrm{cm}^{* * 3 / \mathrm{g})}\right.$ & $6.000 \mathrm{E}+04$ & $6.000 \mathrm{E}+04$ & --- & DCNUCDWE (16) \\
\hline DCLR & Leach rate constant of Th-228 (/yr) & $0.000 \mathrm{E}+00$ & $0.000 \mathrm{E}+00$ & $2.778 \mathrm{E}-06$ & Rleach $(1,16)$ \\
\hline
\end{tabular}

1RESRAD-OFFSITE, Version 4.0 .3 beta T1/2 Limit = 30 days

Parent Dose Report

Title : RESRAD-OFFSITE Effluent release

File : PADUCAH EFFLUENT RELEASE-REVI.ROF

\begin{tabular}{|c|c|c|c|c|c|}
\hline $\begin{array}{l}0 \\
\text { Menu }\end{array}$ & Site-Specific Par & $\begin{array}{l}\text { ter Summ } \\
\text { User } \\
\text { Input }\end{array}$ & $\begin{array}{l}\text { (continu } \\
\text { Default }\end{array}$ & $\begin{array}{l}\text { RESRAD } \\
\text { computed }\end{array}$ & $\begin{array}{l}\text { Parameter } \\
\text { Name }\end{array}$ \\
\hline DCLR & Distribution coefficients for Th-230 & & & & \\
\hline DCLR & Contaminated zone $(\mathrm{cm} * * 3 / \mathrm{g})$ & $6.000 \mathrm{E}+04$ & $6.000 \mathrm{E}+04$ & --- & DCNUCC (18) \\
\hline DCLR & Unsaturated zone $1(\mathrm{~cm} * * 3 / \mathrm{g})$ & $6.000 \mathrm{E}+04$ & $6.000 \mathrm{E}+04$ & --- & $\operatorname{DCNUCU}(18,1)$ \\
\hline DCLR & Saturated zone $(\mathrm{cm} * * 3 / \mathrm{g})$ & $6.000 \mathrm{E}+04$ & $6.000 \mathrm{E}+04$ & --- & DCNUCS (18) \\
\hline DCLR & Bottom sediment in surface water body $(\mathrm{cm} * * 3 / \mathrm{g})$ & $6.000 \mathrm{E}+04$ & $6.000 \mathrm{E}+04$ & --- & DCNUCSWB (18) \\
\hline DCLR & Suspended sediment in surface water body $(\mathrm{cm} * * 3 / \mathrm{g})$ & $6.000 \mathrm{E}+04$ & $6.000 \mathrm{E}+04$ & --- & DCNUCSWS (18) \\
\hline DCLR & Agricultural area $1(\mathrm{~cm} * * 3 / \mathrm{g})$ & $6.000 \mathrm{E}+04$ & $6.000 \mathrm{E}+04$ & --- & $\operatorname{DCNUCOF}(18,1)$ \\
\hline DCLR & Agricultural area $2(\mathrm{~cm} * * 3 / \mathrm{g})$ & $6.000 \mathrm{E}+04$ & $6.000 \mathrm{E}+04$ & --- & $\operatorname{DCNUCOF}(18,2)$ \\
\hline DCLR & Agricultural area $3(\mathrm{~cm} * * 3 / \mathrm{g})$ & $6.000 \mathrm{E}+04$ & $6.000 \mathrm{E}+04$ & --- & $\operatorname{DCNUCOF}(18,3)$ \\
\hline DCLR & Agricultural area $4(\mathrm{~cm} * * 3 / \mathrm{g})$ & $6.000 \mathrm{E}+04$ & $6.000 \mathrm{E}+04$ & --- & $\operatorname{DCNUCOF}(18,4)$ \\
\hline DCLR & Offsite Dwelling $(\mathrm{cm} * * 3 / \mathrm{g})$ & $6.000 \mathrm{E}+04$ & $6.000 \mathrm{E}+04$ & --- & DCNUCDWE (18) \\
\hline DCLR & Leach rate constant of $\mathrm{Th}-230$ (/yr) & $0.000 \mathrm{E}+00$ & $0.000 \mathrm{E}+00$ & $2.778 \mathrm{E}-06$ & Rleach $(1,18)$ \\
\hline DCLR & Distribution coefficients for Th-232 & & & & \\
\hline DCLR & Contaminated zone $(\mathrm{cm} * * 3 / \mathrm{g})$ & $6.000 \mathrm{E}+04$ & $6.000 \mathrm{E}+04$ & --- & $\operatorname{DCNUCC}(19)$ \\
\hline DCLR & Unsaturated zone $1(\mathrm{~cm} * * 3 / \mathrm{g})$ & $6.000 \mathrm{E}+04$ & $6.000 \mathrm{E}+04$ & --- & $\operatorname{DCNUCU}(19,1)$ \\
\hline DCLR & Saturated zone $(\mathrm{cm} * * 3 / \mathrm{g})$ & $6.000 \mathrm{E}+04$ & $6.000 \mathrm{E}+04$ & --- & DCNUCS (19) \\
\hline
\end{tabular}


\begin{tabular}{l|l} 
DCLR & Bottom sediment in surface water body $(\mathrm{cm} * * 3 / \mathrm{g})$ \\
DCLR & Suspended sediment in surface water body $(\mathrm{cm} * * 3 / \mathrm{g})$
\end{tabular} Agricultural area $1(\mathrm{~cm} * * 3 / \mathrm{g})$ Agricultural area $2(\mathrm{~cm} * * 3 / \mathrm{g})$

Agricultural area $3(\mathrm{~cm} * * 3 / \mathrm{g})$

Agricultural area $4(\mathrm{~cm} * \star 3 / \mathrm{g})$

$\begin{array}{lll}\text { DCLR } & \text { Offsite Dwelling }\left(\mathrm{cm}^{*} * 3 / \mathrm{g}\right) & \\ \text { DCLR } & \text { Leach rate constant of } \mathrm{Th}-232 \text { (/yr) }\end{array}$

DCLR Distribution coefficients for U-234

DCLR Contaminated zone $(\mathrm{cm} * * 3 / \mathrm{g})$

DCLR Unsaturated zone $1(\mathrm{~cm} * * 3 / \mathrm{g})$

DCLR Saturated zone $(\mathrm{cm} * * 3 / \mathrm{g})$

DCLR Bottom sediment in surface water body $(\mathrm{cm} * * 3 / \mathrm{g})$

DCLR Suspended sediment in surface water body $(\mathrm{cm} * * 3 / \mathrm{g})$

DCLR Agricultural area $1(\mathrm{~cm} * * 3 / \mathrm{g})$

DCLR Agricultural area $2(\mathrm{~cm} * * 3 / \mathrm{g})$

DCLR Agricultural area $3(\mathrm{~cm} * * 3 / \mathrm{g})$

DCLR Agricultural area $4(\mathrm{~cm} * * 3 / \mathrm{g})$

DCLR Offsite Dwelling $(\mathrm{cm} * * 3 / \mathrm{g})$

DCLR Leach rate constant of U-234 (/yr)

1RESRAD-OFFSITE, Version 4.0 .3 beta T1/2 Limit $=30$ days

Parent Dose Report

Title : RESRAD-OFFSITE Effluent release

File : PADUCAH EFFLUENT RELEASE-REV1.ROF

\begin{tabular}{|c|c|c|c|c|c|}
\hline $\begin{array}{l}0 \\
\text { Menu }\end{array}$ & Site-Specific $\mathrm{Pa}$ & $\begin{array}{l}\text { eter Summa } \\
\text { User } \\
\text { Input }\end{array}$ & $\begin{array}{l}\text { (continu } \\
\text { Default }\end{array}$ & $\begin{array}{l}\text { RESRAD } \\
\text { computed }\end{array}$ & $\begin{array}{l}\text { Parameter } \\
\text { Name }\end{array}$ \\
\hline DCLR & Distribution coefficients for U-235 & & & & \\
\hline DCLR & Saturated zone $(\mathrm{cm} * * 3 / \mathrm{g})$ & $5.000 \mathrm{E}+01$ & $5.000 \mathrm{E}+01$ & --- & DCNUCS (22) \\
\hline DCLR & Bottom sediment in surface water body $(\mathrm{cm} * * 3 / \mathrm{g})$ & $5.000 \mathrm{E}+01$ & $5.000 \mathrm{E}+01$ & --- & DCNUCSWB (22) \\
\hline DCLR & Suspended sediment in surface water body $(\mathrm{cm} * * 3 / \mathrm{g})$ & $5.000 \mathrm{E}+01$ & $5.000 \mathrm{E}+01$ & --- & DCNUCSWS (22) \\
\hline DCLR & Agricultural area $4(\mathrm{~cm} * * 3 / \mathrm{g})$ & $5.000 E+01$ & $5.000 \mathrm{E}+01$ & --- & $\operatorname{DCNUCOF}(22,4)$ \\
\hline DCLR & Offsite Dwelling $(\mathrm{cm} * * 3 / \mathrm{g})$ & $5.000 \mathrm{E}+01$ & $5.000 \mathrm{E}+01$ & --- & DCNUCDWE (22) \\
\hline DCLR & Leach rate constant of $\mathrm{U}-235$ (/yr) & $0.000 \mathrm{E}+00$ & $0.000 \mathrm{E}+00$ & $3.319 E-03$ & Rleach $(1,22)$ \\
\hline DCLR & Distribution coefficients for U-238 & & & & \\
\hline DCLR & Contaminated zone $(\mathrm{cm} * * 3 / \mathrm{g})$ & $5.000 \mathrm{E}+01$ & $5.000 \mathrm{E}+01$ & --- & DCNUCC (24) \\
\hline DCLR & Unsaturated zone $1(\mathrm{~cm} * * 3 / \mathrm{g})$ & $5.000 \mathrm{E}+01$ & $5.000 \mathrm{E}+01$ & --- & $\operatorname{DCNUCU}(24,1)$ \\
\hline DCLR & Agricultural area $2(\mathrm{~cm} * * 3 / \mathrm{g})$ & $5.000 \mathrm{E}+01$ & $5.000 \mathrm{E}+01$ & --- & $\operatorname{DCNUCOF}(24,2)$ \\
\hline
\end{tabular}

\begin{tabular}{|c|c|c|c|}
\hline $6.000 \mathrm{E}+04$ & $6.000 \mathrm{E}+04$ & --- & DCNUCSWB (19) \\
\hline $6.000 \mathrm{E}+04$ & $6.000 \mathrm{E}+04$ & --- & DCNUCSWS (19) \\
\hline $6.000 \mathrm{E}+04$ & $6.000 \mathrm{E}+04$ & --- & $\operatorname{DCNUCOF}(19,1)$ \\
\hline $6.000 \mathrm{E}+04$ & $6.000 \mathrm{E}+04$ & --- & $\operatorname{DCNUCOF}(19,2)$ \\
\hline $6.000 \mathrm{E}+04$ & $6.000 \mathrm{E}+04$ & --- & $\operatorname{DCNUCOF}(19,3)$ \\
\hline $6.000 \mathrm{E}+04$ & $6.000 \mathrm{E}+04$ & --- & $\operatorname{DCNUCOF}(19,4)$ \\
\hline $6.000 \mathrm{E}+04$ & $6.000 \mathrm{E}+04$ & --- & DCNUCDWE (19) \\
\hline $0.000 \mathrm{E}+00$ & $0.000 \mathrm{E}+00$ & $2.778 \mathrm{E}-06$ & Rleach $(1,19)$ \\
\hline $5.000 \mathrm{E}+01$ & $5.000 \mathrm{E}+01$ & --- & $\operatorname{DCNUCC}(21)$ \\
\hline $5.000 \mathrm{E}+01$ & $5.000 \mathrm{E}+01$ & --- & $\operatorname{DCNUCU}(21,1)$ \\
\hline $5.000 \mathrm{E}+01$ & $5.000 \mathrm{E}+01$ & --- & DCNUCS (21) \\
\hline $5.000 \mathrm{E}+01$ & $5.000 \mathrm{E}+01$ & --- & DCNUCSWB (21) \\
\hline $5.000 \mathrm{E}+01$ & $5.000 \mathrm{E}+01$ & --- & DCNUCSWS (21) \\
\hline $5.000 \mathrm{E}+01$ & $5.000 \mathrm{E}+01$ & --- & $\operatorname{DCNUCOF}(21,1)$ \\
\hline $5.000 \mathrm{E}+01$ & $5.000 \mathrm{E}+01$ & --- & $\operatorname{DCNUCOF}(21,2)$ \\
\hline $5.000 \mathrm{E}+01$ & $5.000 \mathrm{E}+01$ & --- & $\operatorname{DCNUCOF}(21,3)$ \\
\hline $5.000 \mathrm{E}+01$ & $5.000 \mathrm{E}+01$ & --- & $\operatorname{DCNUCOF}(21,4)$ \\
\hline $5.000 \mathrm{E}+01$ & $5.000 \mathrm{E}+01$ & --- & DCNUCDWE (21) \\
\hline $0.000 \mathrm{E}+00$ & $0.000 \mathrm{E}+00$ & $3.319 \mathrm{E}-03$ & Rleach $(1,21)$ \\
\hline
\end{tabular}

\begin{tabular}{l|l|l|l}
$0.000 \mathrm{E}+00$ & $0.000 \mathrm{E}+00$ & $3.319 \mathrm{E}-03$ & Rleach $(1,21)$
\end{tabular} $06 / 11 / 2020$ 11:32 Page 16 


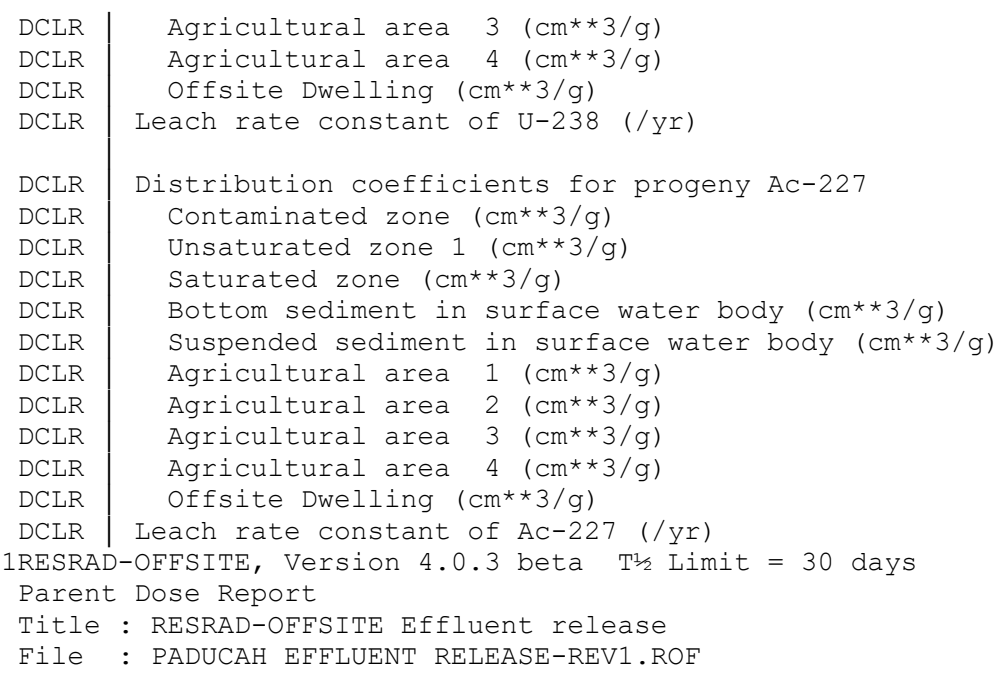

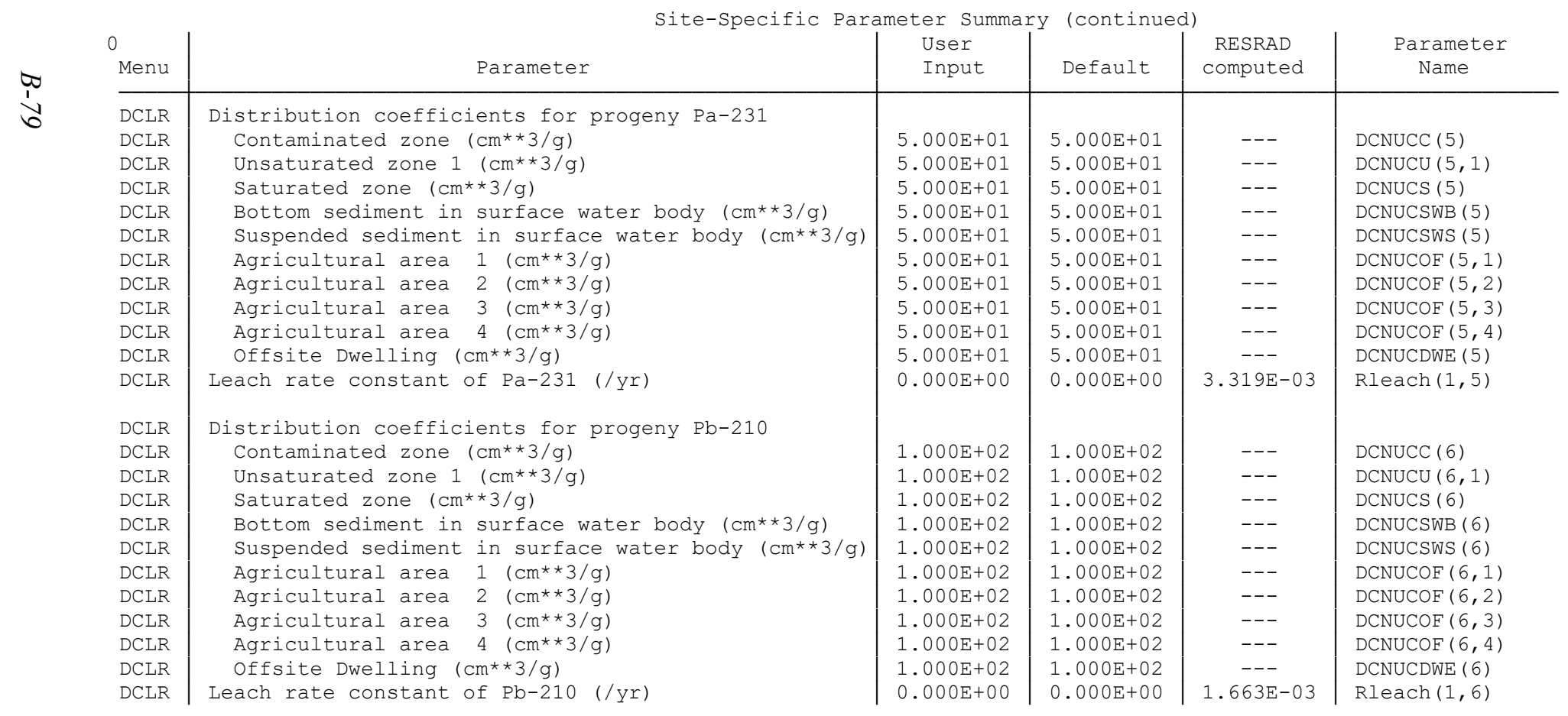

\begin{tabular}{|l|c|c|l}
$5.000 \mathrm{E}+01$ & $5.000 \mathrm{E}+01$ & --- & $\operatorname{DCNUCOF}(24,3)$ \\
$5.000 \mathrm{E}+01$ & $5.000 \mathrm{E}+01$ & --- & $\operatorname{DCNUCOF}(24,4)$ \\
$5.000 \mathrm{E}+01$ & $5.000 \mathrm{E}+01$ & --- & $\operatorname{DCNUCDWE}(24)$ \\
$0.000 \mathrm{E}+00$ & $0.000 \mathrm{E}+00$ & $3.319 \mathrm{E}-03$ & $\operatorname{RleaCh}(1,24)$ \\
& & & \\
$2.000 \mathrm{E}+01$ & $2.000 \mathrm{E}+01$ & --- & $\operatorname{DCNUCC}(1)$ \\
$2.000 \mathrm{E}+01$ & $2.000 \mathrm{E}+01$ & --- & $\operatorname{DCNUCU}(1,1)$ \\
$2.000 \mathrm{E}+01$ & $2.000 \mathrm{E}+01$ & --- & $\operatorname{DCNUCS}(1)$ \\
$2.000 \mathrm{E}+01$ & $2.000 \mathrm{E}+01$ & --- & $\operatorname{DCNUCSWB}(1)$ \\
$2.000 \mathrm{E}+01$ & $2.000 \mathrm{E}+01$ & --- & $\operatorname{DCNUCSWS}(1)$ \\
$2.000 \mathrm{E}+01$ & $2.000 \mathrm{E}+01$ & --- & $\operatorname{DCNUCOF}(1,1)$ \\
$2.000 \mathrm{E}+01$ & $2.000 \mathrm{E}+01$ & --- & $\operatorname{DCNUCOF}(1,2)$ \\
$2.000 \mathrm{E}+01$ & $2.000 \mathrm{E}+01$ & --- & $\operatorname{DCNUCOF}(1,3)$ \\
$2.000 \mathrm{E}+01$ & $2.000 \mathrm{E}+01$ & --- & $\operatorname{DCNUCOF}(1,4)$ \\
$2.000 \mathrm{E}+01$ & $2.000 \mathrm{E}+01$ & --- & $\operatorname{DCNUCDWE}(1)$ \\
$0.000 \mathrm{E}+00$ & $0.000 \mathrm{E}+00$ & $8.245 \mathrm{E}-03$ & $\operatorname{Rl} \operatorname{RaCh}(1,1)$
\end{tabular}

06/11/2020 11:32 Page 17 


\begin{tabular}{|c|c|c|c|c|c|c|}
\hline & DCLR & Distribution coefficients for progeny Po-210 & & & & \\
\hline & DCLR & Contaminated zone $(\mathrm{cm} * \star 3 / \mathrm{g})$ & $1.000 \mathrm{E}+01$ & $1.000 \mathrm{E}+01$ & --- & DCNUCC (7) \\
\hline & DCLR & Unsaturated zone $1(\mathrm{~cm} * * 3 / \mathrm{g})$ & $1.000 \mathrm{E}+01$ & $1.000 \mathrm{E}+01$ & --- & $\operatorname{DCNUCU}(7,1)$ \\
\hline & DCLR & Saturated zone $(\mathrm{cm} * * 3 / \mathrm{g})$ & $1.000 \mathrm{E}+01$ & $1.000 \mathrm{E}+01$ & --- & DCNUCS (7) \\
\hline & DCLR & Bottom sediment in surface water body $(\mathrm{cm} * * 3 / \mathrm{g})$ & $1.000 \mathrm{E}+01$ & $1.000 \mathrm{E}+01$ & --- & DCNUCSWB (7) \\
\hline & DCLR & Suspended sediment in surface water body $(\mathrm{cm} * * 3 / \mathrm{g})$ & $1.000 \mathrm{E}+01$ & $1.000 \mathrm{E}+01$ & --- & DCNUCSWS (7) \\
\hline & DCLR & Agricultural area $1(\mathrm{~cm} * * 3 / \mathrm{g})$ & $1.000 \mathrm{E}+01$ & $1.000 \mathrm{E}+01$ & --- & $\operatorname{DCNUCOF}(7,1)$ \\
\hline & DCLR & Agricultural area $2(\mathrm{~cm} * * 3 / \mathrm{g})$ & $1.000 \mathrm{E}+01$ & $1.000 \mathrm{E}+01$ & --- & $\operatorname{DCNUCOF}(7,2)$ \\
\hline & DCLR & Agricultural area $3(\mathrm{~cm} * * 3 / \mathrm{g})$ & $1.000 \mathrm{E}+01$ & $1.000 \mathrm{E}+01$ & --- & $\operatorname{DCNUCOF}(7,3)$ \\
\hline & DCLR & Agricultural area $4(\mathrm{~cm} * * 3 / \mathrm{g})$ & $1.000 \mathrm{E}+01$ & $1.000 \mathrm{E}+01$ & --- & $\operatorname{DCNUCOF}(7,4)$ \\
\hline & DCLR & Offsite Dwelling $(\mathrm{cm} * \star 3 / \mathrm{g})$ & $1.000 \mathrm{E}+01$ & $1.000 \mathrm{E}+01$ & --- & DCNUCDWE ( 7 ) \\
\hline & DCLR & Leach rate constant of Po-210 (/yr) & $0.000 \mathrm{E}+00$ & $0.000 \mathrm{E}+00$ & $1.632 \mathrm{E}-02$ & Rleach $(1,7)$ \\
\hline & 1RESRA & -OFFSITE, Version 4.0 .3 beta $T^{1 / 2}$ Limit $=30$ days & $06 / 11 / 20$ & $11: 32$ & 18 & \\
\hline & Paren & Dose Report & & & & \\
\hline & Title & : RESRAD-OFFSITE Effluent release & & & & \\
\hline & File & : PADUCAH EFFLUENT RELEASE-REV1.ROF & & & & \\
\hline & & Site-Specific Pa & eter summa & y (continue & & \\
\hline & 0 & & User & & RESRAD & Parameter \\
\hline & Menu & Parameter & Input & Default & computed & Name \\
\hline & DCLR & Distribution coefficients for progeny Ra-226 & & & & \\
\hline & DCLR & Contaminated zone $\left(\mathrm{cm}^{* * 3 / \mathrm{g})}\right.$ & $7.000 \mathrm{E}+01$ & $7.000 \mathrm{E}+01$ & --- & DCNUCC (13) \\
\hline & DCLR & Unsaturated zone $1(\mathrm{~cm} * * 3 / \mathrm{g})$ & $7.000 \mathrm{E}+01$ & $7.000 \mathrm{E}+01$ & --- & $\operatorname{DCNUCU}(13,1)$ \\
\hline & DCLR & Saturated zone $(\mathrm{cm} * * 3 / \mathrm{g})$ & $7.000 \mathrm{E}+01$ & $7.000 \mathrm{E}+01$ & --- & DCNUCS (13) \\
\hline & DCLR & Bottom sediment in surface water body $(\mathrm{cm} * * 3 / \mathrm{g})$ & $7.000 \mathrm{E}+01$ & $7.000 \mathrm{E}+01$ & --- & DCNUCSWB (13) \\
\hline & DCLR & Suspended sediment in surface water body $(\mathrm{cm} * * 3 / \mathrm{g})$ & $7.000 \mathrm{E}+01$ & $7.000 \mathrm{E}+01$ & --- & DCNUCSWS (13) \\
\hline & DCLR & Agricultural area $1(\mathrm{~cm} * * 3 / \mathrm{g})$ & $7.000 \mathrm{E}+01$ & $7.000 \mathrm{E}+01$ & --- & $\operatorname{DCNUCOF}(13,1)$ \\
\hline & DCLR & Agricultural area $2(\mathrm{~cm} * * 3 / \mathrm{g})$ & $7.000 \mathrm{E}+01$ & $7.000 \mathrm{E}+01$ & --- & $\operatorname{DCNUCOF}(13,2)$ \\
\hline & DCLR & Agricultural area $3(\mathrm{~cm} * * 3 / \mathrm{g})$ & $7.000 \mathrm{E}+01$ & $7.000 \mathrm{E}+01$ & --- & $\operatorname{DCNUCOF}(13,3)$ \\
\hline & DCLR & Agricultural area $4(\mathrm{~cm} * * 3 / \mathrm{g})$ & $7.000 \mathrm{E}+01$ & $7.000 \mathrm{E}+01$ & --- & $\operatorname{DCNUCOF}(13,4)$ \\
\hline & DCLR & Offsite Dwelling $(\mathrm{cm} * * 3 / \mathrm{g})$ & $7.000 \mathrm{E}+01$ & $7.000 \mathrm{E}+01$ & --- & DCNUCDWE (13) \\
\hline & DCLR & Leach rate constant of $\mathrm{Ra}-226$ (/yr) & $0.000 \mathrm{E}+00$ & $0.000 \mathrm{E}+00$ & $2.374 \mathrm{E}-03$ & Rleach $(1,13)$ \\
\hline & DCLR & Distribution coefficients for progeny Ra-228 & & & & \\
\hline & DCLR & Contaminated zone $(\mathrm{cm} * * 3 / \mathrm{g})$ & $7.000 \mathrm{E}+01$ & $7.000 \mathrm{E}+01$ & --- & DCNUCC (14) \\
\hline & DCLR & Unsaturated zone $1(\mathrm{~cm} * * 3 / \mathrm{g})$ & $7.000 \mathrm{E}+01$ & $7.000 \mathrm{E}+01$ & --- & $\operatorname{DCNUCU}(14,1)$ \\
\hline & DCLR & Saturated zone $(\mathrm{cm} * * 3 / \mathrm{g})$ & $7.000 \mathrm{E}+01$ & $7.000 \mathrm{E}+01$ & --- & DCNUCS (14) \\
\hline & DCLR & Bottom sediment in surface water body $(\mathrm{cm} * * 3 / \mathrm{g})$ & $7.000 \mathrm{E}+01$ & $7.000 \mathrm{E}+01$ & --- & DCNUCSWB (14) \\
\hline & DCLR & Suspended sediment in surface water body $\left(\mathrm{cm}^{* *} 3 / \mathrm{g}\right)$ & $7.000 \mathrm{E}+01$ & $7.000 \mathrm{E}+01$ & --- & DCNUCSWS (14) \\
\hline & DCLR & Agricultural area $1(\mathrm{~cm} * * 3 / \mathrm{g})$ & $7.000 \mathrm{E}+01$ & $7.000 \mathrm{E}+01$ & --- & $\operatorname{DCNUCOF}(14,1)$ \\
\hline & DCLR & Agricultural area $2(\mathrm{~cm} * * 3 / \mathrm{g})$ & $7.000 \mathrm{E}+01$ & $7.000 \mathrm{E}+01$ & --- & $\operatorname{DCNUCOF}(14,2)$ \\
\hline & DCLR & Agricultural area $3(\mathrm{~cm} * * 3 / \mathrm{g})$ & $7.000 \mathrm{E}+01$ & $7.000 \mathrm{E}+01$ & --- & $\operatorname{DCNUCOF}(14,3)$ \\
\hline & DCLR & Agricultural area $4(\mathrm{~cm} * * 3 / \mathrm{g})$ & $7.000 \mathrm{E}+01$ & $7.000 \mathrm{E}+01$ & --- & $\operatorname{DCNUCOF}(14,4)$ \\
\hline & DCLR & Offsite Dwelling $(\mathrm{cm} * * 3 / \mathrm{g})$ & $7.000 \mathrm{E}+01$ & $7.000 \mathrm{E}+01$ & --- & DCNUCDWE (14) \\
\hline & DCLR & Leach rate constant of $\mathrm{Ra}-228$ (/yr) & $0.000 \mathrm{E}+00$ & $0.000 \mathrm{E}+00$ & $2.374 E-03$ & Rleach $(1,14)$ \\
\hline & DCLR & Distribution coefficients for progeny Th-229 & & & & \\
\hline & DCLR & Contaminated zone $(\mathrm{cm} * * 3 / \mathrm{g})$ & $6.000 \mathrm{E}+04$ & $6.000 \mathrm{E}+04$ & --- & DCNUCC (17) \\
\hline & DCLR & Unsaturated zone $1(\mathrm{~cm} * * 3 / \mathrm{g})$ & $6.000 \mathrm{E}+04$ & $6.000 \mathrm{E}+04$ & --- & $\operatorname{DCNUCU}(17,1)$ \\
\hline
\end{tabular}


DCLR Saturated zone $(\mathrm{cm} * * 3 / \mathrm{g})$

DCLR Bottom sediment in surface water body $(\mathrm{cm} * * 3 / \mathrm{g})$

DCLR Suspended sediment in surface water body $(\mathrm{cm} \star \star 3 / \mathrm{g})$

DCLR Agricultural area $1(\mathrm{~cm} * * 3 / \mathrm{g})$

DCLR Agricultural area $2(\mathrm{~cm} * * 3 / \mathrm{g})$

DCLR Agricultural area $3(\mathrm{~cm} * * 3 / \mathrm{g})$

DCLR Agricultural area $4(\mathrm{~cm} * * 3 / \mathrm{g})$

DCLR Offsite Dwelling $(\mathrm{cm} * * 3 / \mathrm{g})$

DCLR Leach rate constant of Th-229 (/yr)

1RESRAD-OFFSITE, Version 4.0 .3 beta $\mathrm{T}^{1} \frac{1}{2}$ Limit $=30$ days

Parent Dose Report

Title : RESRAD-OFFSITE Effluent release

File: PADUCAH EFFLUENT RELEASE-REV1.ROF

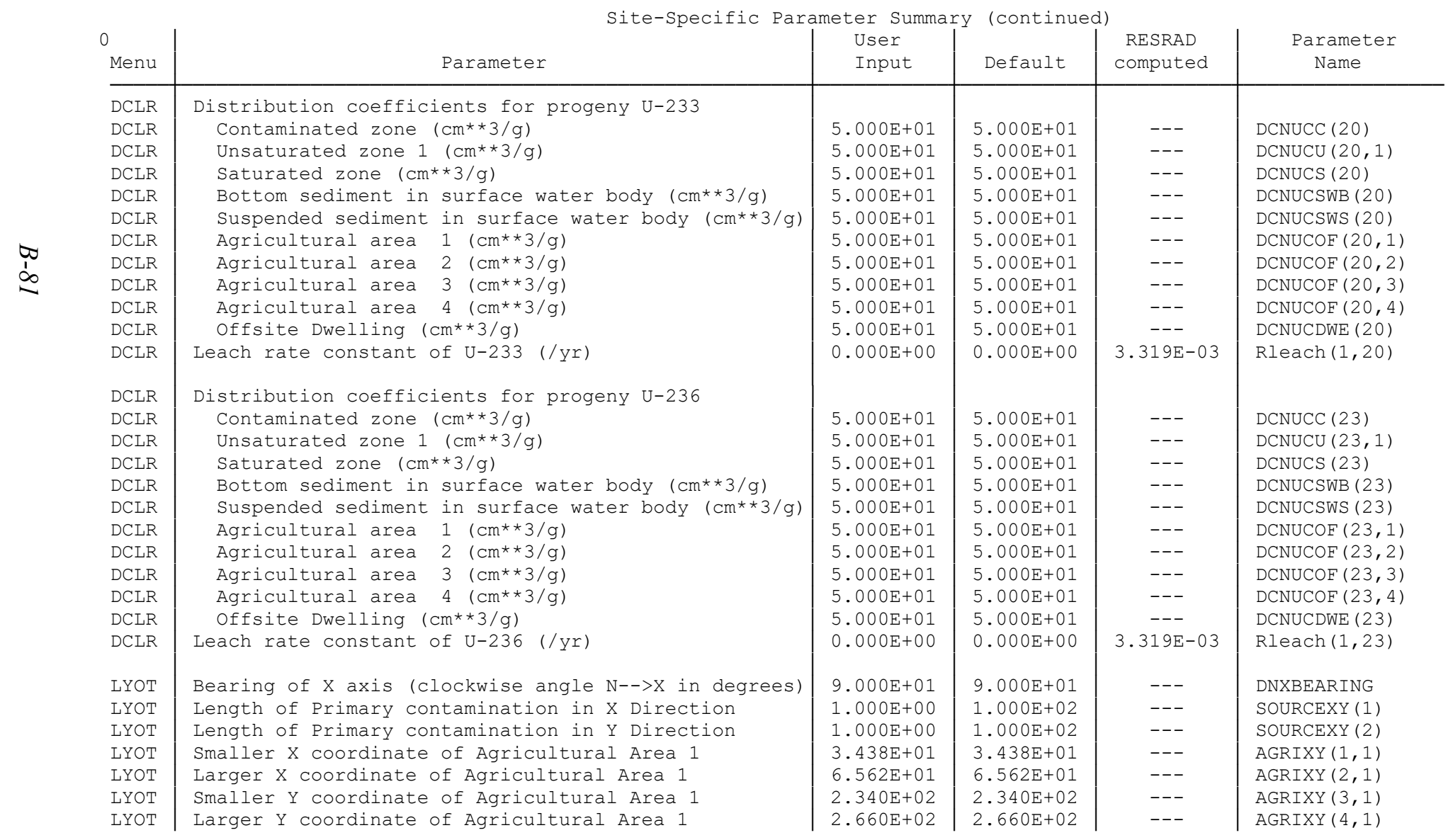

\begin{tabular}{l|c|c|l}
$6.000 \mathrm{E}+04$ & $6.000 \mathrm{E}+04$ & --- & $\operatorname{DCNUCS}(17)$ \\
$6.000 \mathrm{E}+04$ & $6.000 \mathrm{E}+04$ & --- & $\operatorname{DCNUCSWB}(17)$ \\
$6.000 \mathrm{E}+04$ & $6.000 \mathrm{E}+04$ & --- & $\operatorname{DCNUCSWS}(17)$ \\
$6.000 \mathrm{E}+04$ & $6.000 \mathrm{E}+04$ & --- & $\operatorname{DCNUCOF}(17,1)$ \\
$6.000 \mathrm{E}+04$ & $6.000 \mathrm{E}+04$ & --- & $\operatorname{DCNUCOF}(17,2)$ \\
$6.000 \mathrm{E}+04$ & $6.000 \mathrm{E}+04$ & --- & $\operatorname{DCNUCOF}(17,3)$ \\
$6.000 \mathrm{E}+04$ & $6.000 \mathrm{E}+04$ & --- & $\operatorname{DCNUCOF}(17,4)$ \\
$6.000 \mathrm{E}+04$ & $6.000 \mathrm{E}+04$ & --- & $\operatorname{DCNUCDWE}(17)$ \\
$0.000 \mathrm{E}+00$ & $0.000 \mathrm{E}+00$ & $2.778 \mathrm{E}-06$ & $\operatorname{Rleach}(1,17)$
\end{tabular}

$06 / 11 / 2020 \quad 11 \cdot 32$ Page 19

Rleach $(1,17)$

$.660 \mathrm{E}+02-12.660 \mathrm{E}+02$

$\operatorname{AGRIXY}(4,1)$ 
LYOT Smaller X coordinate of Agricultural Area 2

LYOT Larger X coordinate of Agricultural Area 2

LYOT Smaller Y coordinate of Agricultural Area 2

LYOT Larger Y coordinate of Agricultural Area 2

LYOT Smaller X coordinate of Agricultural Area 3

LYOT Larger X coordinate of Agricultural Area 3

LYOT Smaller $Y$ coordinate of Agricultural Area 3

LYOT Larger Y coordinate of Agricultural Area 3

LYOT Smaller X coordinate of Agricultural Area 4

LYOT Larger X coordinate of Agricultural Area 4

LYOT Smaller Y coordinate of Agricultural Area 4

LYOT Larger Y coordinate of Agricultural Area 4

LYOT Smaller X coordinate of Dwelling Area

LYOT Larger $X$ coordinate of Dwelling Area

1RESRAD-OFFSITE, Version 4.0 .3 beta $\mathrm{T}^{1 / 2} \mathrm{Limit}=30$ days

Parent Dose Report

Title : RESRAD-OFFSITE Effluent release

File : PADUCAH EFFLUENT RELEASE-REV1 ROE

\begin{tabular}{|c|c|c|c|}
\hline $3.438 \mathrm{E}+01$ & $3.438 \mathrm{E}+01$ & --- & $\operatorname{AGRIXY}(1,2)$ \\
\hline $6.562 \mathrm{E}+01$ & $6.562 \mathrm{E}+01$ & --- & $\operatorname{AGRIXY}(2,2)$ \\
\hline $2.680 \mathrm{E}+02$ & $2.680 \mathrm{E}+02$ & --- & $\operatorname{AGRIXY}(3,2)$ \\
\hline $3.000 \mathrm{E}+02$ & $3.000 \mathrm{E}+02$ & --- & $\operatorname{AGRIXY}(4,2)$ \\
\hline $0.000 \mathrm{E}+00$ & $0.000 \mathrm{E}+00$ & --- & $\operatorname{AGRIXY}(1,3)$ \\
\hline $1.000 \mathrm{E}+02$ & $1.000 \mathrm{E}+02$ & --- & $\operatorname{AGRIXY}(2,3)$ \\
\hline $4.500 \mathrm{E}+02$ & $4.500 \mathrm{E}+02$ & --- & $\operatorname{AGRIXY}(3,3)$ \\
\hline $5.500 \mathrm{E}+02$ & $5.500 \mathrm{E}+02$ & --- & $\operatorname{AGRIXY}(4,3)$ \\
\hline $0.000 \mathrm{E}+00$ & $0.000 \mathrm{E}+00$ & --- & $\operatorname{AGRIXY}(1,4)$ \\
\hline $1.000 \mathrm{E}+02$ & $1.000 \mathrm{E}+02$ & --- & $\operatorname{AGRIXY}(2,4)$ \\
\hline $3.000 \mathrm{E}+02$ & $3.000 \mathrm{E}+02$ & --- & $\operatorname{AGRIXY}(3,4)$ \\
\hline $4.000 \mathrm{E}+02$ & $4.000 \mathrm{E}+02$ & --- & $\operatorname{AGRIXY}(4,4)$ \\
\hline$-1.512 \mathrm{E}+01$ & $3.438 \mathrm{E}+01$ & --- & DWELLXY (1) \\
\hline $1.612 \mathrm{E}+01$ & $6.562 \mathrm{E}+01$ & --- & DWELLXY (2) \\
\hline
\end{tabular}

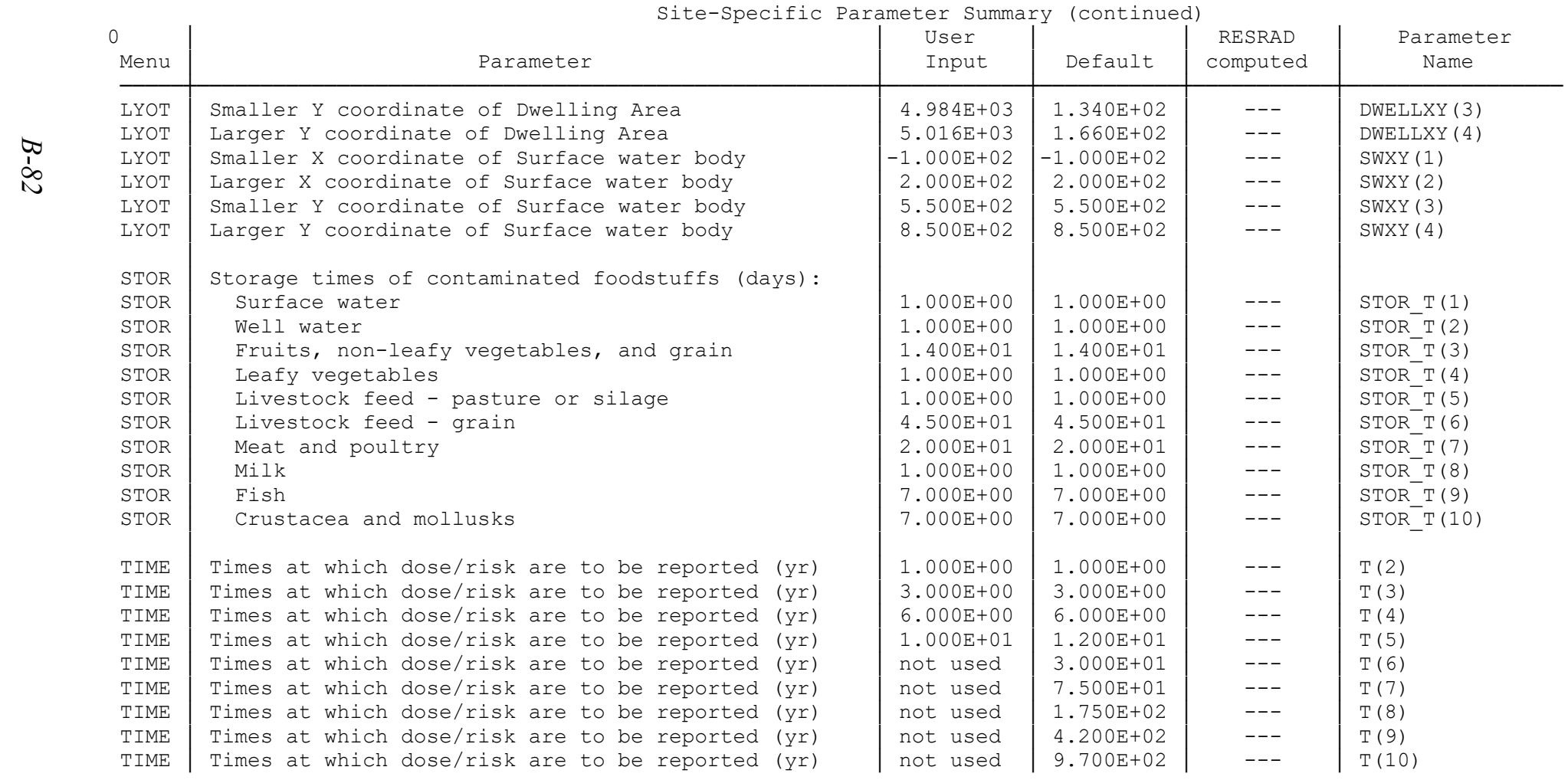


SITE Precipitation (m/yr)

SITE Rainfall Erosion Index

PRCZ Area of primary contamination ( $\left.m^{* * 2}\right)$

PRCZ Length parallel to aquifer flow (m)

PRCZ Depth of soil mixing layer (m)

PRCZ Mass loading of all particulates for release $(\mathrm{g} / \mathrm{m} * * 3)$

PRCZ DepositionVelocityofAllParticulates for release $(\mathrm{m} / \mathrm{s}$ )

PRCZ Respirable particulates as a fraction of total

PRCZ DepositionVelocityofRespirableParticulatesForRe $(\mathrm{m} / \mathrm{s})$

PRCZ Irrigation (m/yr)

PRCZ Evapotranspiration coefficient

PRCZ Runoff coefficient

PRCZ Slope-length-steepness factor of prim. contamination PRCZ Cropping-management factor of primary contamination PRCZ Conservation practice factor of prim. contamination PRCZ Fraction of primary contamination that is sumberged PRCZ Thickness of primary contamination (m)

1RESRAD-OFFSITE, Version 4.0 .3 beta T1/2 Limit $=30$ days Parent Dose Report

Title : RESRAD-OFFSITE Effluent release
File : PADUCAH EFFLUENT RELEASE-REV1.ROF

\begin{tabular}{|l|l|l|l}
$1.000 \mathrm{E}+00$ & $1.000 \mathrm{E}+00$ & --- & PRECIP \\
$1.600 \mathrm{E}+02$ & $1.600 \mathrm{E}+02$ & --- & RAINEROS \\
& & & \\
$1.000 \mathrm{E}+00$ & $1.000 \mathrm{E}+04$ & --- & AREA \\
$1.000 \mathrm{E}+02$ & $1.000 \mathrm{E}+02$ & --- & LCZPAQ \\
$1.500 \mathrm{E}-01$ & $1.500 \mathrm{E}-01$ & --- & DM \\
$1.000 \mathrm{E}-04$ & $1.000 \mathrm{E}-04$ & -- & MLFD \\
$1.000 \mathrm{E}-03$ & $1.000 \mathrm{E}-03$ & -- & DEPVEL_DUSTT \\
$1.000 \mathrm{E}+00$ & $1.000 \mathrm{E}+00$ & --- & RESPFRACPC \\
$1.000 \mathrm{E}-03$ & $1.000 \mathrm{E}-03$ & --- & DEPVEL_DUST \\
$2.000 \mathrm{E}-01$ & $2.000 \mathrm{E}-01$ & --- & RI \\
$5.000 \mathrm{E}-01$ & $5.000 \mathrm{E}-01$ & --- & EVAPTR \\
$2.000 \mathrm{E}-01$ & $2.000 \mathrm{E}-01$ & --- & RUNOFF \\
$4.000 \mathrm{E}-01$ & $4.000 \mathrm{E}-01$ & --- & SLPLENSTPPC \\
$3.000 \mathrm{E}-03$ & $3.000 \mathrm{E}-03$ & --- & CRPMANGPC \\
$1.000 \mathrm{E}+00$ & $1.000 \mathrm{E}+00$ & --- & CONVPRACPC \\
$0.000 \mathrm{E}+00$ & $0.000 \mathrm{E}+00$ & --- & SUBMERGEDF \\
$2.000 \mathrm{E}+00$ & $2.000 \mathrm{E}+00$ & --- & THICKO
\end{tabular}

Site-Specific Parameter Summary (continued)

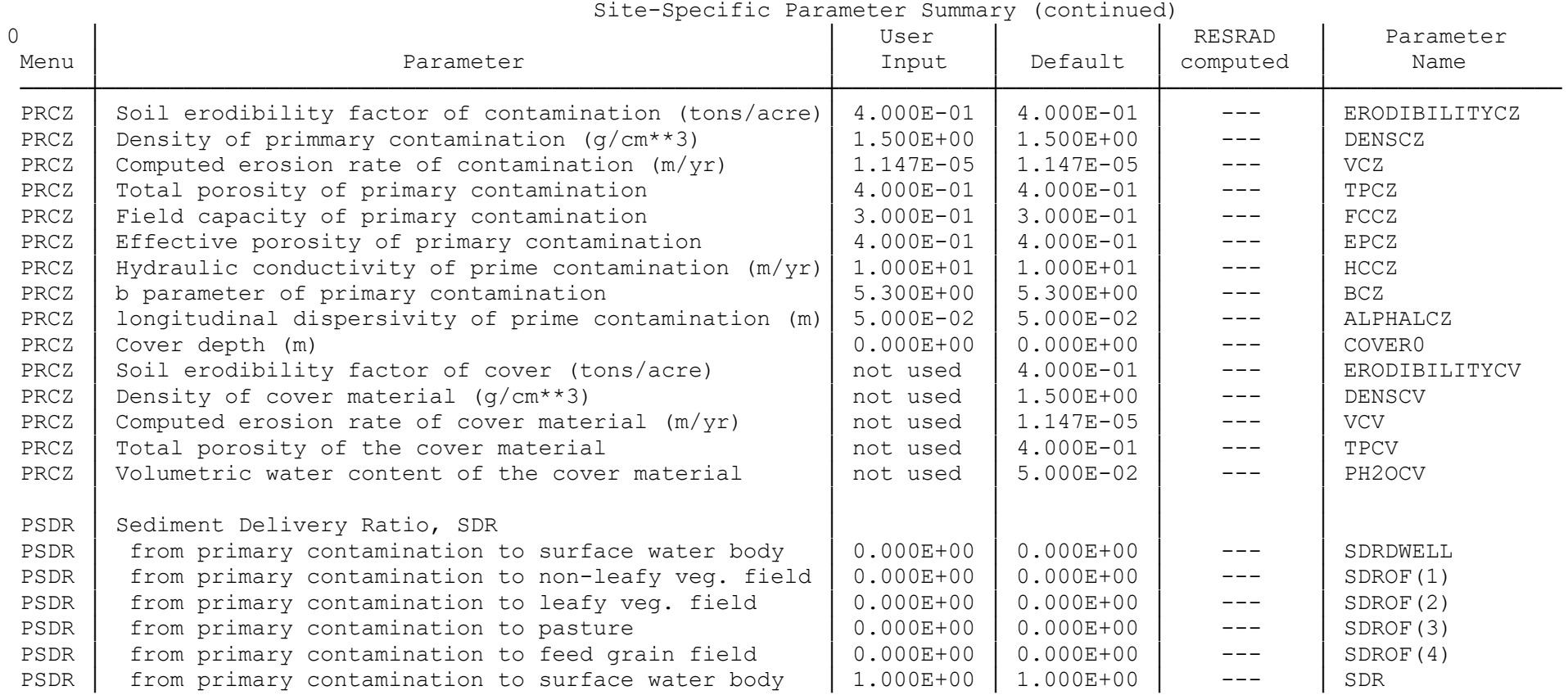




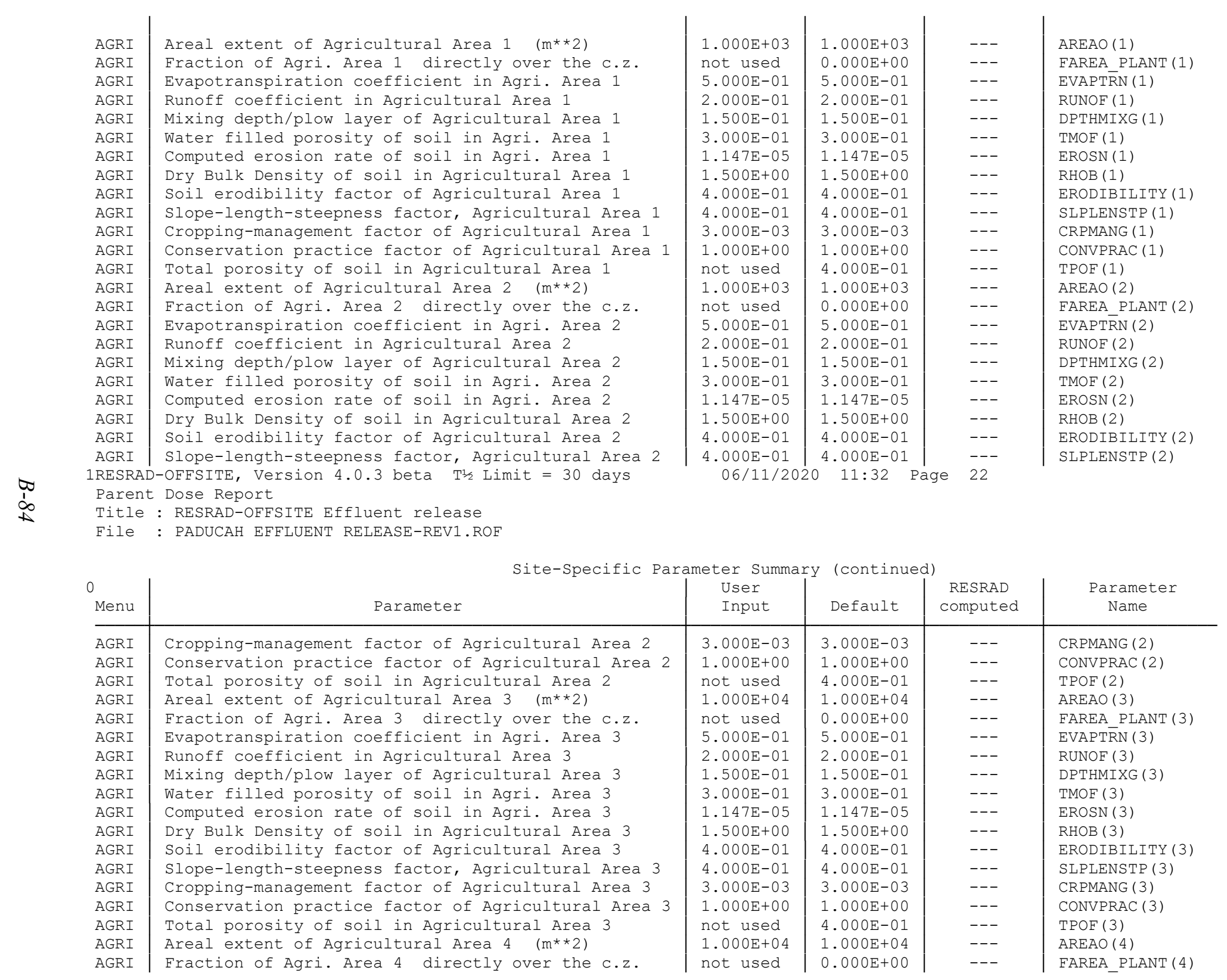


AGRI Evapotranspiration coefficient in Agri. Area 4

AGRI Runoff coefficient in Agricultural Area 4

AGRI Mixing depth/plow layer of Agricultural Area 4

AGRI Water filled porosity of soil in Agri. Area 4

AGRI Computed erosion rate of soil in Agri. Area 4

AGRI Dry Bulk Density of soil in Agricultural Area 4

AGRI Soil erodibility factor of Agricultural Area 4

AGRI Slope-length-steepness factor, Agricultural Area 4

AGRI Cropping-management factor of Agricultural Area 4

AGRI Conservation practice factor of Agricultural Area 4

AGRI Total porosity of soil in Agricultural Area 4

DWEL Areal extent of offsite dwelling site ( $m * * 2)$

DWEL Evapotranspiration coefficient in dwelling (off) site

DWEL Runoff coefficient in offsite dwelling site

DWEL Mixing depth of Offsite dwelling site

DWEL Water filled porosity of soil in offsite Dwelling

DWEL Computed erosion rate of soil in offsite Dwelling

DWEL Dry Bulk Density of soil in Offsite dwelling site

DWET Soil erodibility factor of soil in Dwelling site

DWEL Slope-length-steepness factor of Dwelling site

DWEL Cropping-management factor of Dwelling site

DWEL Conservation practice factor of offsite Dwelling sit

DWEL Total porosity of soil in Offsite Dwelling

AIRT Dispersion Coefficients; 1 = Pasquill-Gifford

AIRT Population zone; 1 = Rural

AIRT Release height, (m)

AIRT Heat flux for buoyant plume (cal/s),

1RESRAD-OFFSITE, Version 4.0 .3 beta T1/2 Limit = 30 days Parent Dose Report

Title : RESRAD-OFFSITE Effluent release

File : PADUCAH EFFLUENT RELEASE-REV1.ROF

\begin{tabular}{|c|c|c|c|c|c|}
\hline Menu & Site-Specific Pa & $\begin{array}{l}\text { ter Summ } \\
\text { User } \\
\text { Input }\end{array}$ & $\begin{array}{l}\text { (contin } \\
\text { Default }\end{array}$ & $\begin{array}{l}\text { RESRAD } \\
\text { computed }\end{array}$ & $\begin{array}{l}\text { Parameter } \\
\text { Name }\end{array}$ \\
\hline AIRT & Anemometer height, (m) & $1.000 \mathrm{E}+01$ & $1.000 \mathrm{E}+01$ & --- & ANH \\
\hline AIRT & AM atmospheric mixing height (m) & $4.000 \mathrm{E}+02$ & $4.000 \mathrm{E}+02$ & --- & AMIX \\
\hline AIRT & PM atmospheric mixing height (m) & $1.600 \mathrm{E}+03$ & $1.600 \mathrm{E}+03$ & --- & PMIX \\
\hline AIRT & Elevation of Agricultural Area 1 above primary cont. & $0.000 \mathrm{E}+00$ & $0.000 \mathrm{E}+00$ & --- & AGRIELEV (1) \\
\hline AIRT & Elevation of Agricultural Area 2 above primary cont. & $0.000 \mathrm{E}+00$ & $0.000 \mathrm{E}+00$ & --- & AGRIELEV (2) \\
\hline AIRT & Elevation of Dwelling Site relative to primary cont. & $0.000 \mathrm{E}+00$ & $0.000 \mathrm{E}+00$ & --- & DWELLELEV \\
\hline AIRT & Elevation of Surf.Wtr body relative to primary cont. & $0.000 \mathrm{E}+00$ & $0.000 \mathrm{E}+00$ & --- & SWELEV \\
\hline AIRT & Joint frequency Meteorological data & & & & \\
\hline AIRT & Upper limit for windspeed class $1(\mathrm{~m} / \mathrm{s})$ & $8.900 \mathrm{E}-01$ & $8.900 \mathrm{E}-01$ & --- & WINDSPEED (1) \\
\hline
\end{tabular}

\begin{tabular}{|c|c|c|c|}
\hline $5.000 \mathrm{E}-01$ & $5.000 \mathrm{E}-01$ & --- & EVAPTRN (4) \\
\hline $2.000 \mathrm{E}-01$ & $2.000 \mathrm{E}-01$ & --- & RUNOF (4) \\
\hline $1.500 \mathrm{E}-01$ & $1.500 \mathrm{E}-01$ & --- & DPTHMIXG (4) \\
\hline $3.000 \mathrm{E}-01$ & $3.000 \mathrm{E}-01$ & --- & TMOF (4) \\
\hline $1.147 \mathrm{E}-05$ & $1.147 \mathrm{E}-05$ & --- & $\operatorname{EROSN}(4)$ \\
\hline $1.500 \mathrm{E}+00$ & $1.500 \mathrm{E}+00$ & --- & RHOB ( 4) \\
\hline $4.000 \mathrm{E}-01$ & $4.000 \mathrm{E}-01$ & --- & ERODIBILITY (4) \\
\hline $4.000 \mathrm{E}-01$ & $4.000 \mathrm{E}-01$ & --- & SLPLENSTP (4) \\
\hline $3.000 \mathrm{E}-03$ & $3.000 \mathrm{E}-03$ & --- & CRPMANG ( 4 ) \\
\hline $1.000 \mathrm{E}+00$ & $1.000 \mathrm{E}+00$ & --- & CONVPRAC (4) \\
\hline not used & $4.000 \mathrm{E}-01$ & --- & TPOF ( 4 ) \\
\hline $1.000 \mathrm{E}+03$ & $1.000 \mathrm{E}+03$ & --- & AREAODWELL \\
\hline $5.000 \mathrm{E}-01$ & $5.000 \mathrm{E}-01$ & --- & EVAPTRNDWELL \\
\hline $2.000 \mathrm{E}-01$ & $2.000 \mathrm{E}-01$ & --- & RUNOFDWELL \\
\hline $1.500 \mathrm{E}-01$ & $1.500 \mathrm{E}-01$ & --- & DPTHMIXGDWELL \\
\hline $3.000 \mathrm{E}-01$ & $3.000 \mathrm{E}-01$ & --- & TMOFDWELL \\
\hline $0.000 \mathrm{E}+00$ & $0.000 \mathrm{E}+00$ & --- & EROSNDWELL \\
\hline $1.500 \mathrm{E}+00$ & $1.500 \mathrm{E}+00$ & --- & RHOBDWELL \\
\hline $0.000 \mathrm{E}+00$ & $0.000 \mathrm{E}+00$ & --- & ERODIBILITYDWELL \\
\hline $4.000 \mathrm{E}-01$ & $4.000 \mathrm{E}-01$ & --- & SLPLENSTPDWELL \\
\hline $3.000 \mathrm{E}-03$ & $3.000 \mathrm{E}-03$ & --- & CRPMANGDWELL \\
\hline $1.000 \mathrm{E}+00$ & $1.000 \mathrm{E}+00$ & --- & CONVPRACDWELL \\
\hline not used & $4.000 \mathrm{E}-01$ & --- & TPOFDWELL \\
\hline 1 & 1 & --- & IDISPMOD \\
\hline 1 & 1 & --- & IZONE \\
\hline $5.944 \mathrm{E}+01$ & $1.000 \mathrm{E}+00$ & --- & AIRRELHT \\
\hline $0.000 \mathrm{E}+00$ & $0.000 \mathrm{E}+00$ & --- & HEATFLX \\
\hline
\end{tabular}

$06 / 11 / 2020 \quad 11: 32$ Page 23

AIRRELHT
HEATFLX 
AIRT Upper limit for windspeed class 2 (m/s)

AIRT Upper limit for windspeed class $3(\mathrm{~m} / \mathrm{s})$

AIRT Upper limit for windspeed class $4(\mathrm{~m} / \mathrm{s})$

AIRT Upper limit for windspeed class $5(\mathrm{~m} / \mathrm{s})$

AIRT Upper limit for windspeed class $6(\mathrm{~m} / \mathrm{s})$

AIRT Joint Frequency in $\mathrm{N}$ Sector

AIRT for wind speed class 1 and stability class A

AIRT for wind speed class 1 and stability class B

AIRT for wind speed class 1 and stability class $C$

AIRT - for wind speed class 1 and stability class $D$

ATRT for wind speed class 1 and stability class

AIRT for wind speed class 1 and stability class F

AIRT Joint Frequency in $\mathrm{N}$ Sector

AIRT for wind speed class 2 and stability class A

AIRT for wind speed class 2 and stability class B

AIRT for wind speed class 2 and stability class $C$

AIRT

$\operatorname{TrT}$

for wind speed class 2 and stability class D

for wind speed class 2 and stability class E

for wind speed class 2 and stability class F

AIRT Joint Frequency in $\mathrm{N}$ Sector

AIRT for wind speed class 3 and stability class A

AIRT for wind speed class 3 and stability class B

AIRT

AIRT

AIRT for wind speed class 3 and stability class $C$ for wind speed Class 3 and stability Class D for wind speed class 3 and stability class E for wind speed class 3 and stability class $\mathrm{F}$ RESRAD-OFFSITE, Version 4.0 .3 beta T1/2 Limit $=30$ days Parent Dose Report

Title : RESRAD-OFFSITE Effluent release

File : PADUCAH EFFLUENT RELEASE-REV1.ROF

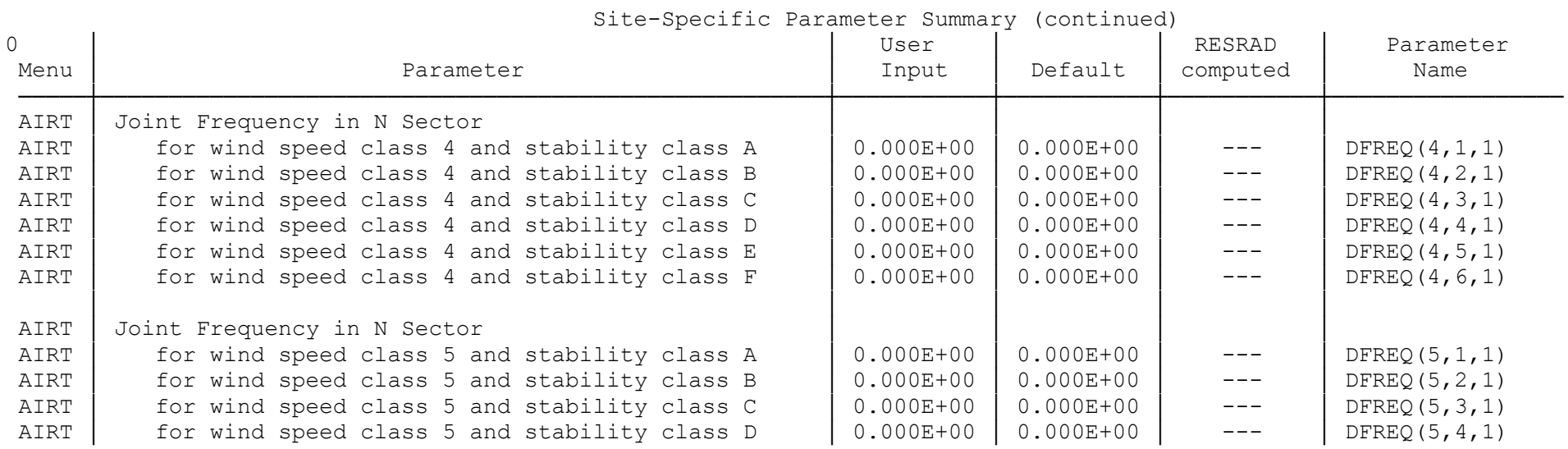

\begin{tabular}{|c|c|c|c|}
\hline $2.460 \mathrm{E}+00$ & $2.460 \mathrm{E}+00$ & --- & WINDSPEED (2) \\
\hline $4.470 \mathrm{E}+00$ & $4.470 \mathrm{E}+00$ & --- & WINDSPEED (3) \\
\hline $6.930 \mathrm{E}+00$ & $6.930 \mathrm{E}+00$ & --- & WINDSPEED (4) \\
\hline $9.610 \mathrm{E}+00$ & $9.610 \mathrm{E}+00$ & --- & WINDSPEED (5) \\
\hline $1.252 \mathrm{E}+01$ & $1.252 \mathrm{E}+01$ & --- & WINDSPEED (6) \\
\hline $0.000 \mathrm{E}+00$ & $0.000 \mathrm{E}+00$ & --- & $\operatorname{DFREQ}(1,1,1)$ \\
\hline $0.000 \mathrm{E}+00$ & $0.000 \mathrm{E}+00$ & --- & $\operatorname{DFREQ}(1,2,1)$ \\
\hline $0.000 \mathrm{E}+00$ & $0.000 \mathrm{E}+00$ & --- & $\operatorname{DFREQ}(1,3,1)$ \\
\hline $1.000 \mathrm{E}+00$ & $1.000 \mathrm{E}-01$ & --- & $\operatorname{DFREQ}(1,4,1)$ \\
\hline $0.000 \mathrm{E}+00$ & $2.000 \mathrm{E}-01$ & --- & $\operatorname{DFREQ}(1,5,1)$ \\
\hline $0.000 \mathrm{E}+00$ & $7.000 \mathrm{E}-01$ & --- & $\operatorname{DFREQ}(1,6,1)$ \\
\hline $0.000 \mathrm{E}+00$ & $0.000 \mathrm{E}+00$ & --- & $\operatorname{DFREQ}(2,1,1)$ \\
\hline $0.000 \mathrm{E}+00$ & $0.000 \mathrm{E}+00$ & --- & $\operatorname{DFREQ}(2,2,1)$ \\
\hline $0.000 \mathrm{E}+00$ & $0.000 \mathrm{E}+00$ & --- & $\operatorname{DFREQ}(2,3,1)$ \\
\hline $0.000 \mathrm{E}+00$ & $0.000 \mathrm{E}+00$ & --- & $\operatorname{DFREQ}(2,4,1)$ \\
\hline $0.000 \mathrm{E}+00$ & $0.000 \mathrm{E}+00$ & --- & $\operatorname{DFREQ}(2,5,1)$ \\
\hline $0.000 \mathrm{E}+00$ & $0.000 \mathrm{E}+00$ & --- & $\operatorname{DFREQ}(2,6,1)$ \\
\hline $0.000 \mathrm{E}+00$ & $0.000 \mathrm{E}+00$ & --- & $\operatorname{DFREQ}(3,1,1)$ \\
\hline $0.000 \mathrm{E}+00$ & $0.000 \mathrm{E}+00$ & -- & $\operatorname{DFREQ}(3,2,1)$ \\
\hline $0.000 \mathrm{E}+00$ & $0.000 \mathrm{E}+00$ & --- & $\operatorname{DFREQ}(3,3,1)$ \\
\hline $0.000 \mathrm{E}+00$ & $0.000 \mathrm{E}+00$ & --- & $\operatorname{DFREQ}(3,4,1)$ \\
\hline $0.000 \mathrm{E}+00$ & $0.000 \mathrm{E}+00$ & --- & $\operatorname{DFREQ}(3,5,1)$ \\
\hline $0.000 \mathrm{E}+00$ & $0.000 \mathrm{E}+00$ & --- & $\operatorname{DFREQ}(3,6,1)$ \\
\hline $06 / 11 / 20$ & $11: 32$ & 24 & \\
\hline
\end{tabular}

$\operatorname{DFREQ}(3,5,1)$
$\operatorname{DFREQ}(3,6,1)$
$0.000 \mathrm{E}+0$
DEREQ $(5,4$ 
\begin{tabular}{l|l} 
AIRT & for wind speed class 5 and stability class $E$
\end{tabular} for wind speed class 5 and stability class F

ATRT

AIRT

AIRT

AIRT

AIRT

AIRT

AIRT

AIRT

AIRT

AIRT

AIRT

AIRT

AIRT

AIRT

AIRT

AIRT

AIRT

AIRT

Joint Frequency in $\mathrm{N}$ Sector

for wind speed class 6 and stability class A for wind speed class 6 and stability class B for wind speed class 6 and stability class C for wind speed class 6 and stability class D for wind speed class 6 and stability class $\mathrm{E}$ for wind speed class 6 and stability class F

Joint Frequency in NNE Sector

for wind speed class 1 and stability class A for wind speed class 1 and stability class B for wind speed class 1 and stability class C for wind speed class 1 and stability class D for wind speed class 1 and stability class E for wind speed class 1 and stability class F

Joint Frequency in NNE Sector

for wind speed class 2 and stability class A for wind speed class 2 and stability class B for wind speed class 2 and stability class C for wind speed Class 2 and stability Class D for wind speed class 2 and stability class $\mathrm{E}$ for wind speed class 2 and stability class F
ATRT

AIRT

AIRT

AIRT

AIRT

AIRT

AIRT

Joint Frequency in NNE Sector

for wind speed class 3 and stability class A for wind speed class 3 and stability class B for wind speed class 3 and stability class C for wind speed class 3 and stability class D for wind speed class 3 and stability class E for wind speed class 3 and stability class F RESRAD-OFFSITE, Version 4.0 .3 beta T1/2 Limit $=30$ days Parent Dose Report

Title : RESRAD-OFFSITE Effluent release

File: PADUCAH EFFLUENT RELEASE-REVI.ROF

\begin{tabular}{|c|c|c|c|}
\hline $0.000 \mathrm{E}+00$ & $0.000 \mathrm{E}+00$ & --- & $\operatorname{DFREQ}(5,5,1)$ \\
\hline $0.000 \mathrm{E}+00$ & $0.000 \mathrm{E}+00$ & --- & $\operatorname{DFREQ}(5,6,1)$ \\
\hline $0.000 \mathrm{E}+00$ & $0.000 \mathrm{E}+00$ & --- & $\operatorname{DFREQ}(6,1,1)$ \\
\hline $0.000 \mathrm{E}+00$ & $0.000 \mathrm{E}+00$ & --- & $\operatorname{DFREQ}(6,2,1)$ \\
\hline $0.000 \mathrm{E}+00$ & $0.000 \mathrm{E}+00$ & --- & $\operatorname{DFREQ}(6,3,1)$ \\
\hline $0.000 \mathrm{E}+00$ & $0.000 \mathrm{E}+00$ & --- & $\operatorname{DFREQ}(6,4,1)$ \\
\hline $0.000 \mathrm{E}+00$ & $0.000 \mathrm{E}+00$ & --- & $\operatorname{DFREQ}(6,5,1)$ \\
\hline $0.000 \mathrm{E}+00$ & $0.000 \mathrm{E}+00$ & --- & $\operatorname{DFREQ}(6,6,1)$ \\
\hline $0.000 \mathrm{E}+00$ & $0.000 \mathrm{E}+00$ & --- & $\operatorname{DFREQ}(1,1,2)$ \\
\hline $0.000 \mathrm{E}+00$ & $0.000 \mathrm{E}+00$ & --- & DFREQ $(1,2,2)$ \\
\hline $0.000 \mathrm{E}+00$ & $0.000 \mathrm{E}+00$ & --- & $\operatorname{DFREQ}(1,3,2)$ \\
\hline $0.000 \mathrm{E}+00$ & $1.000 \mathrm{E}-01$ & --- & DFREQ $(1,4,2)$ \\
\hline $0.000 \mathrm{E}+00$ & $2.000 \mathrm{E}-01$ & --- & $\operatorname{DFREQ}(1,5,2)$ \\
\hline $0.000 \mathrm{E}+00$ & $7.000 \mathrm{E}-01$ & --- & $\operatorname{DFREQ}(1,6,2)$ \\
\hline $0.000 \mathrm{E}+00$ & $0.000 \mathrm{E}+00$ & --- & $\operatorname{DFREQ}(2,1,2)$ \\
\hline $0.000 \mathrm{E}+00$ & $0.000 \mathrm{E}+00$ & --- & DFREQ $(2,2,2)$ \\
\hline $0.000 \mathrm{E}+00$ & $0.000 \mathrm{E}+00$ & --- & $\operatorname{DFREQ}(2,3,2)$ \\
\hline $0.000 \mathrm{E}+00$ & $0.000 \mathrm{E}+00$ & --- & DFREQ $(2,4,2)$ \\
\hline $0.000 \mathrm{E}+00$ & $0.000 \mathrm{E}+00$ & --- & $\operatorname{DFREQ}(2,5,2)$ \\
\hline $0.000 \mathrm{E}+00$ & $0.000 \mathrm{E}+00$ & --- & $\operatorname{DFREQ}(2,6,2)$ \\
\hline $0.000 \mathrm{E}+00$ & $0.000 \mathrm{E}+00$ & --- & $\operatorname{DFREQ}(3,1,2)$ \\
\hline $0.000 \mathrm{E}+00$ & $0.000 \mathrm{E}+00$ & --- & $\operatorname{DFREQ}(3,2,2)$ \\
\hline $0.000 \mathrm{E}+00$ & $0.000 \mathrm{E}+00$ & --- & $\operatorname{DFREQ}(3,3,2)$ \\
\hline $0.000 \mathrm{E}+00$ & $0.000 \mathrm{E}+00$ & --- & DFREQ $(3,4,2)$ \\
\hline $0.000 \mathrm{E}+00$ & $0.000 \mathrm{E}+00$ & --- & $\operatorname{DFREQ}(3,5,2)$ \\
\hline $0.000 \mathrm{E}+00$ & $0.000 \mathrm{E}+00$ & --- & $\operatorname{DFREQ}(3,6,2)$ \\
\hline
\end{tabular}

Site-Specific Parameter Summary (continued)

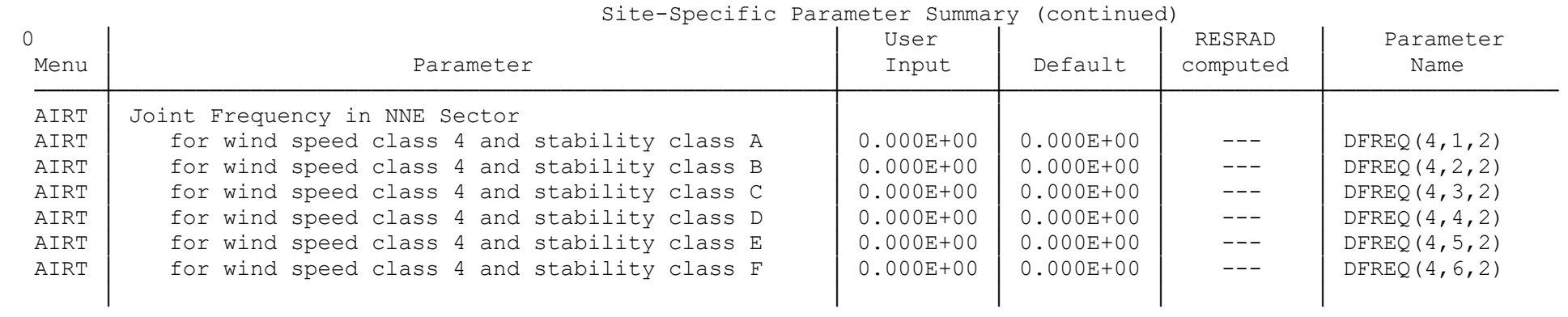


AIRT | Joint Frequency in NNE Sector

AIRT for wind speed class 5 and stability class A

AIRT for wind speed class 5 and stability class B

AIRT for wind speed class 5 and stability class $C$

AIRT for wind speed class 5 and stability class D

AIRT for wind speed class 5 and stability class E

AIRT for wind speed class 5 and stability class $F$

AIRT Joint Frequency in NNE Sector

AIRT for wind speed class 6 and stability class A

AIRT for wind speed class 6 and stability class B

AIRT for wind speed class 6 and stability class C

AIRT for wind speed class 6 and stability class D

AIRT for wind speed class 6 and stability class E

AIRT for wind speed class 6 and stability class F

AIRT Joint Frequency in NE Sector

AIRT for wind speed class 1 and stability class A

AIRT for wind speed class 1 and stability class $B$

AIRT for wind speed class 1 and stability class $C$

AIRT for wind speed class 1 and stability class D

AIRT for wind speed class 1 and stability class $E$

AIRT for wind speed class 1 and stability class F

क AIRT Joint Frequency in NE Sector

AIRT for wind speed class 2 and stability class A

AIRT for wind speed class 2 and stability class $B$

AIRT for wind speed class 2 and stability class $C$

AIRT for wind speed class 2 and stability class D

AIRT for wind speed class 2 and stability class E

AIRT for wind speed class 2 and stability class F

AIRT Joint Frequency in NE Sector

AIRT for wind speed class 3 and stability class A

AIRT for wind speed class 3 and stability class B

AIRT for wind speed class 3 and stability class $C$

AIRT for wind speed class 3 and stability class D

AIRT for wind speed class 3 and stability class $E$

AIRT for wind speed class 3 and stability class F

1RESRAD-OFFSITE, Version 4.0 .3 beta $T^{\frac{1}{2}}$ Limit $=30$ days

Parent Dose Report

Title : RESRAD-OFFSITE Effluent release

File : PADUCAH EFFLUENT RELEASE-REV1.ROF

\begin{tabular}{|c|c|c|c|}
\hline $0.000 \mathrm{E}+00$ & $0.000 \mathrm{E}+00$ & --- & $\operatorname{DFREQ}(5,1,2)$ \\
\hline $0.000 \mathrm{E}+00$ & $0.000 \mathrm{E}+00$ & --- & $\operatorname{DFREQ}(5,2,2)$ \\
\hline $0.000 \mathrm{E}+00$ & $0.000 \mathrm{E}+00$ & --- & $\operatorname{DFREQ}(5,3,2)$ \\
\hline $0.000 \mathrm{E}+00$ & $0.000 \mathrm{E}+00$ & --- & $\operatorname{DFREQ}(5,4,2)$ \\
\hline $0.000 \mathrm{E}+00$ & $0.000 \mathrm{E}+00$ & --- & $\operatorname{DFREQ}(5,5,2)$ \\
\hline $0.000 \mathrm{E}+00$ & $0.000 \mathrm{E}+00$ & --- & $\operatorname{DFREQ}(5,6,2)$ \\
\hline $0.000 \mathrm{E}+00$ & $0.000 \mathrm{E}+00$ & --- & $\operatorname{DFREQ}(6,1,2)$ \\
\hline $0.000 \mathrm{E}+00$ & $0.000 \mathrm{E}+00$ & --- & $\operatorname{DFREQ}(6,2,2)$ \\
\hline $0.000 \mathrm{E}+00$ & $0.000 \mathrm{E}+00$ & --- & $\operatorname{DFREQ}(6,3,2)$ \\
\hline $0.000 \mathrm{E}+00$ & $0.000 \mathrm{E}+00$ & --- & $\operatorname{DFREQ}(6,4,2)$ \\
\hline $0.000 \mathrm{E}+00$ & $0.000 \mathrm{E}+00$ & --- & $\operatorname{DFREQ}(6,5,2)$ \\
\hline $0.000 \mathrm{E}+00$ & $0.000 \mathrm{E}+00$ & --- & $\operatorname{DFREQ}(6,6,2)$ \\
\hline $0.000 \mathrm{E}+00$ & $0.000 \mathrm{E}+00$ & --- & $\operatorname{DFREQ}(1,1,3)$ \\
\hline $0.000 \mathrm{E}+00$ & $0.000 \mathrm{E}+00$ & --- & $\operatorname{DFREQ}(1,2,3)$ \\
\hline $0.000 \mathrm{E}+00$ & $0.000 \mathrm{E}+00$ & --- & $\operatorname{DFREQ}(1,3,3)$ \\
\hline $0.000 \mathrm{E}+00$ & $1.000 \mathrm{E}-01$ & --- & $\operatorname{DFREQ}(1,4,3)$ \\
\hline $0.000 \mathrm{E}+00$ & $2.000 \mathrm{E}-01$ & --- & $\operatorname{DFREQ}(1,5,3)$ \\
\hline $0.000 \mathrm{E}+00$ & $7.000 \mathrm{E}-01$ & --- & $\operatorname{DFREQ}(1,6,3)$ \\
\hline $0.000 \mathrm{E}+00$ & $0.000 \mathrm{E}+00$ & --- & $\operatorname{DFREQ}(2,1,3)$ \\
\hline $0.000 \mathrm{E}+00$ & $0.000 \mathrm{E}+00$ & --- & $\operatorname{DFREQ}(2,2,3)$ \\
\hline $0.000 \mathrm{E}+00$ & $0.000 \mathrm{E}+00$ & --- & $\operatorname{DFREQ}(2,3,3)$ \\
\hline $0.000 \mathrm{E}+00$ & $0.000 \mathrm{E}+00$ & --- & $\operatorname{DFREQ}(2,4,3)$ \\
\hline $0.000 \mathrm{E}+00$ & $0.000 \mathrm{E}+00$ & --- & $\operatorname{DFREQ}(2,5,3)$ \\
\hline $0.000 \mathrm{E}+00$ & $0.000 \mathrm{E}+00$ & --- & $\operatorname{DFREQ}(2,6,3)$ \\
\hline $0.000 \mathrm{E}+00$ & $0.000 \mathrm{E}+00$ & --- & $\operatorname{DFREQ}(3,1,3)$ \\
\hline $0.000 \mathrm{E}+00$ & $0.000 \mathrm{E}+00$ & --- & $\operatorname{DFREQ}(3,2,3)$ \\
\hline $0.000 \mathrm{E}+00$ & $0.000 \mathrm{E}+00$ & --- & $\operatorname{DFREQ}(3,3,3)$ \\
\hline $0.000 \mathrm{E}+00$ & $0.000 \mathrm{E}+00$ & --- & $\operatorname{DFREQ}(3,4,3)$ \\
\hline $0.000 \mathrm{E}+00$ & $0.000 \mathrm{E}+00$ & --- & $\operatorname{DFREQ}(3,5,3)$ \\
\hline $0.000 \mathrm{E}+00$ & $0.000 \mathrm{E}+00$ & --- & $\operatorname{DFREQ}(3,6,3)$ \\
\hline
\end{tabular}

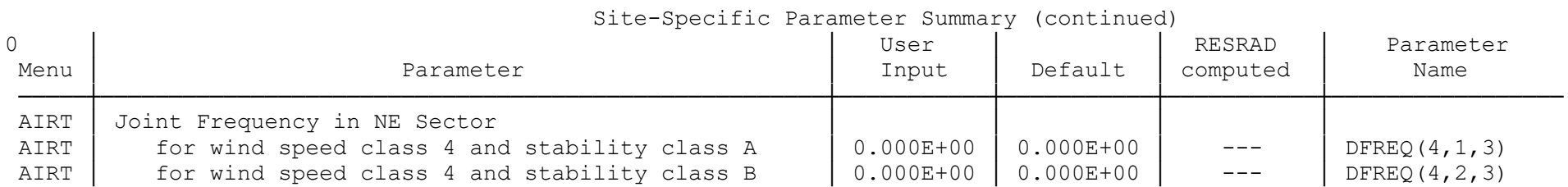




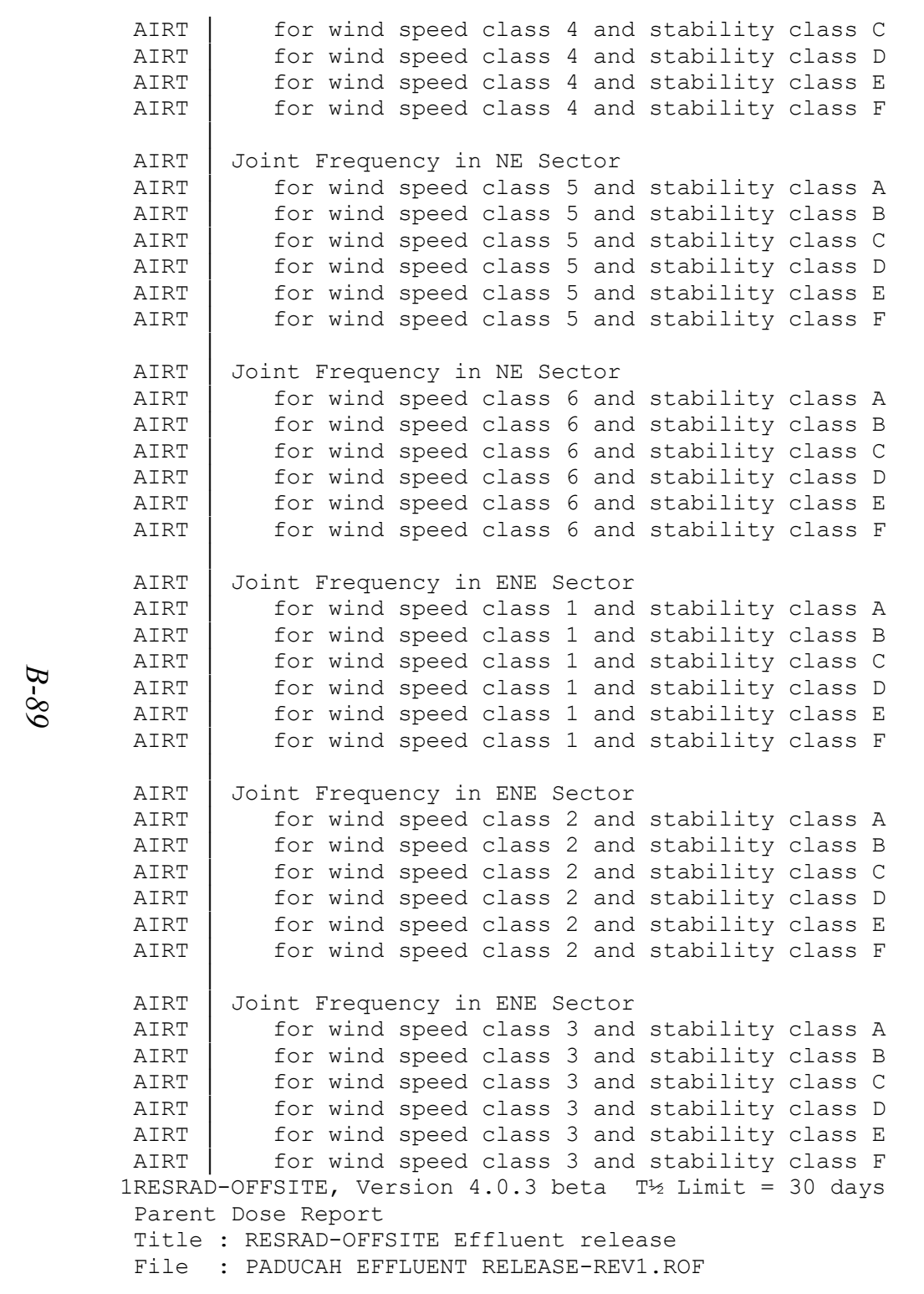

\begin{tabular}{|c|c|c|c|}
\hline $0.000 \mathrm{E}+00$ & $0.000 \mathrm{E}+00$ & --- & $\operatorname{DFREQ}(4,3,3)$ \\
\hline $0.000 \mathrm{E}+00$ & $0.000 \mathrm{E}+00$ & --- & $\operatorname{DFREQ}(4,4,3)$ \\
\hline $0.000 \mathrm{E}+00$ & $0.000 \mathrm{E}+00$ & --- & $\operatorname{DFREQ}(4,5,3)$ \\
\hline $0.000 \mathrm{E}+00$ & $0.000 \mathrm{E}+00$ & --- & $\operatorname{DFREQ}(4,6,3)$ \\
\hline $0.000 \mathrm{E}+00$ & $0.000 \mathrm{E}+00$ & --- & $\operatorname{DFREQ}(5,1,3)$ \\
\hline $0.000 \mathrm{E}+00$ & $0.000 \mathrm{E}+00$ & & $\operatorname{DFREQ}(5,2,3)$ \\
\hline $0.000 \mathrm{E}+00$ & $0.000 \mathrm{E}+00$ & --- & $\operatorname{DFREQ}(5,3,3)$ \\
\hline $0.000 \mathrm{E}+00$ & $0.000 \mathrm{E}+00$ & & $\operatorname{DFREQ}(5,4,3)$ \\
\hline $0.000 \mathrm{E}+00$ & $0.000 \mathrm{E}+00$ & & $\operatorname{DFREQ}(5,5,3)$ \\
\hline $0.000 \mathrm{E}+00$ & $0.000 \mathrm{E}+00$ & - & $\operatorname{DFREQ}(5,6,3)$ \\
\hline $0.000 \mathrm{E}+00$ & $0.000 \mathrm{E}+00$ & - & $\operatorname{DFREQ}(6,1,3)$ \\
\hline $0.000 \mathrm{E}+00$ & $0.000 \mathrm{E}+00$ & & $\operatorname{DFREQ}(6,2,3)$ \\
\hline $0.000 \mathrm{E}+00$ & $0.000 \mathrm{E}+00$ & & $\operatorname{DFREQ}(6,3,3)$ \\
\hline $0.000 \mathrm{E}+00$ & $0.000 \mathrm{E}+00$ & & $\operatorname{DFREQ}(6,4,3)$ \\
\hline $0.000 \mathrm{E}+00$ & $0.000 \mathrm{E}+00$ & - & $\operatorname{DFREQ}(6,5,3)$ \\
\hline $0.000 \mathrm{E}+00$ & $0.000 \mathrm{E}+00$ & & $\operatorname{DFREQ}(6,6,3)$ \\
\hline $0.000 \mathrm{E}+00$ & $0.000 \mathrm{E}+00$ & 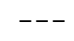 & $\operatorname{DFREQ}(1,1,4)$ \\
\hline $0.000 \mathrm{E}+00$ & $0.000 \mathrm{E}+00$ & & $\operatorname{DFREQ}(1,2,4)$ \\
\hline $0.000 \mathrm{E}+00$ & $0.000 \mathrm{E}+00$ & & $\operatorname{DFREQ}(1,3,4)$ \\
\hline $0.000 \mathrm{E}+00$ & $1.000 \mathrm{E}-01$ & & $\operatorname{DFREQ}(1,4,4)$ \\
\hline $0.000 \mathrm{E}+00$ & $2.000 \mathrm{E}-01$ & & $\operatorname{DFREQ}(1,5,4)$ \\
\hline $0.000 \mathrm{E}+00$ & $7.000 \mathrm{E}-01$ & & $\operatorname{DFREQ}(1,6,4)$ \\
\hline $0.000 \mathrm{E}+00$ & $0.000 \mathrm{E}+00$ & --- & $\operatorname{DFREQ}(2,1,4)$ \\
\hline $0.000 \mathrm{E}+00$ & $0.000 \mathrm{E}+00$ & & $\operatorname{DFREQ}(2,2,4)$ \\
\hline $0.000 \mathrm{E}+00$ & $0.000 \mathrm{E}+00$ & & $\operatorname{DFREQ}(2,3,4)$ \\
\hline $0.000 \mathrm{E}+00$ & $0.000 \mathrm{E}+00$ & & $\operatorname{DFREQ}(2,4,4)$ \\
\hline $0.000 \mathrm{E}+00$ & $0.000 \mathrm{E}+00$ & & $\operatorname{DFREQ}(2,5,4)$ \\
\hline $0.000 \mathrm{E}+00$ & $0.000 \mathrm{E}+00$ & & $\operatorname{DFREQ}(2,6,4)$ \\
\hline & $0.000 \mathrm{E}+00$ & & \\
\hline $0.000 \mathrm{E}+00$ & $0.000 \mathrm{E}+00$ & & $\operatorname{DFREQ}(3,2,4)$ \\
\hline $0.000 \mathrm{E}+$ & $0.000 \mathrm{E}+00$ & & $\operatorname{DFREQ}(3,3,4)$ \\
\hline $0.000 \mathrm{E}+00$ & $0.000 \mathrm{E}+00$ & & $\operatorname{DFREQ}(3,4,4)$ \\
\hline $0.000 \mathrm{E}+00$ & $0.000 \mathrm{E}+00$ & & $\operatorname{DFREQ}(3,5,4)$ \\
\hline $0.000 \mathrm{E}+00$ & $0.000 \mathrm{E}+00$ & & $\operatorname{DFREQ}(3,6,4)$ \\
\hline
\end{tabular}

Site-Specific Parameter Summary (continued) 


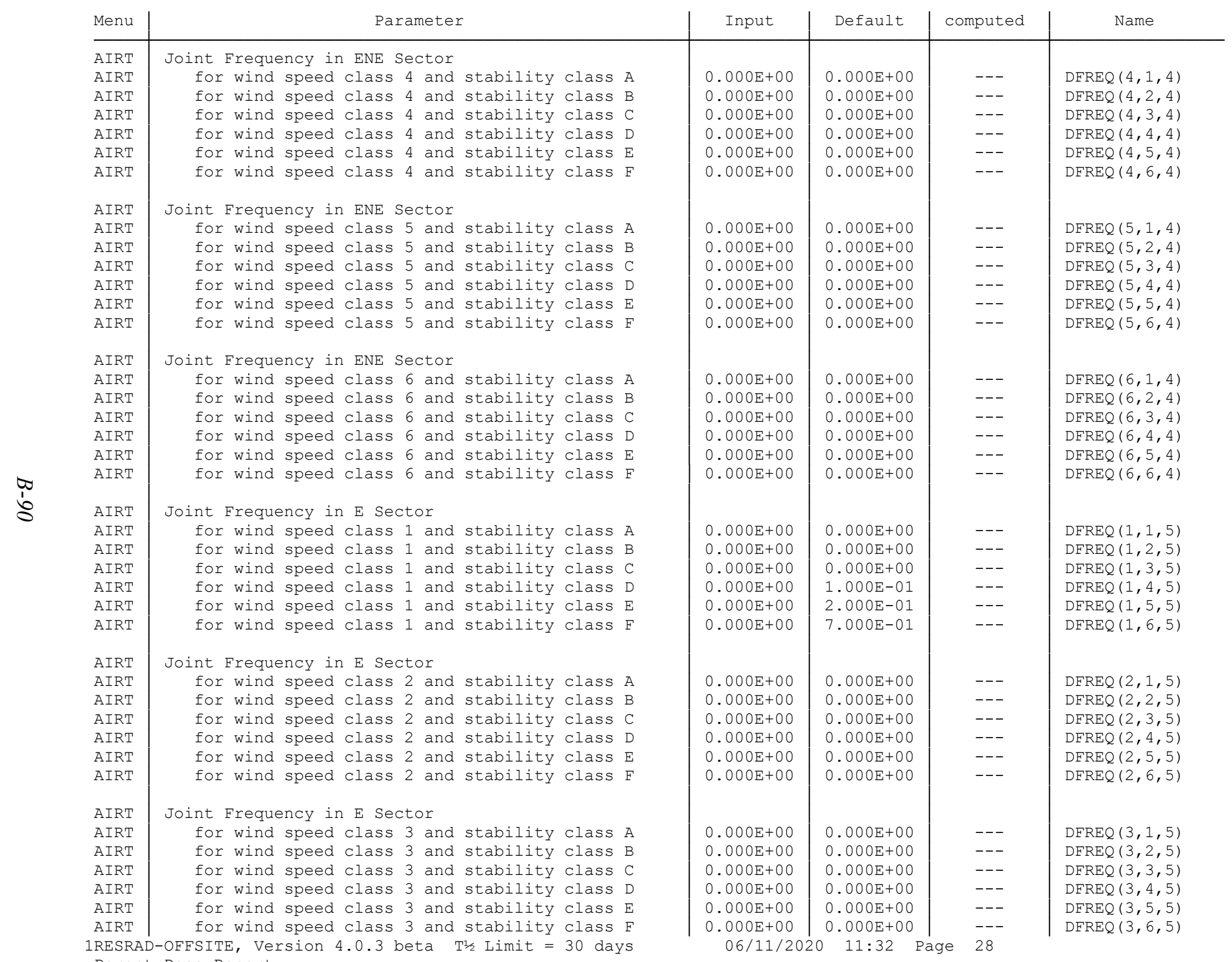


Title : RESRAD-OFFSITE Effluent release

File : PADUCAH FFFLUENT RELFASE-REV1 ROF

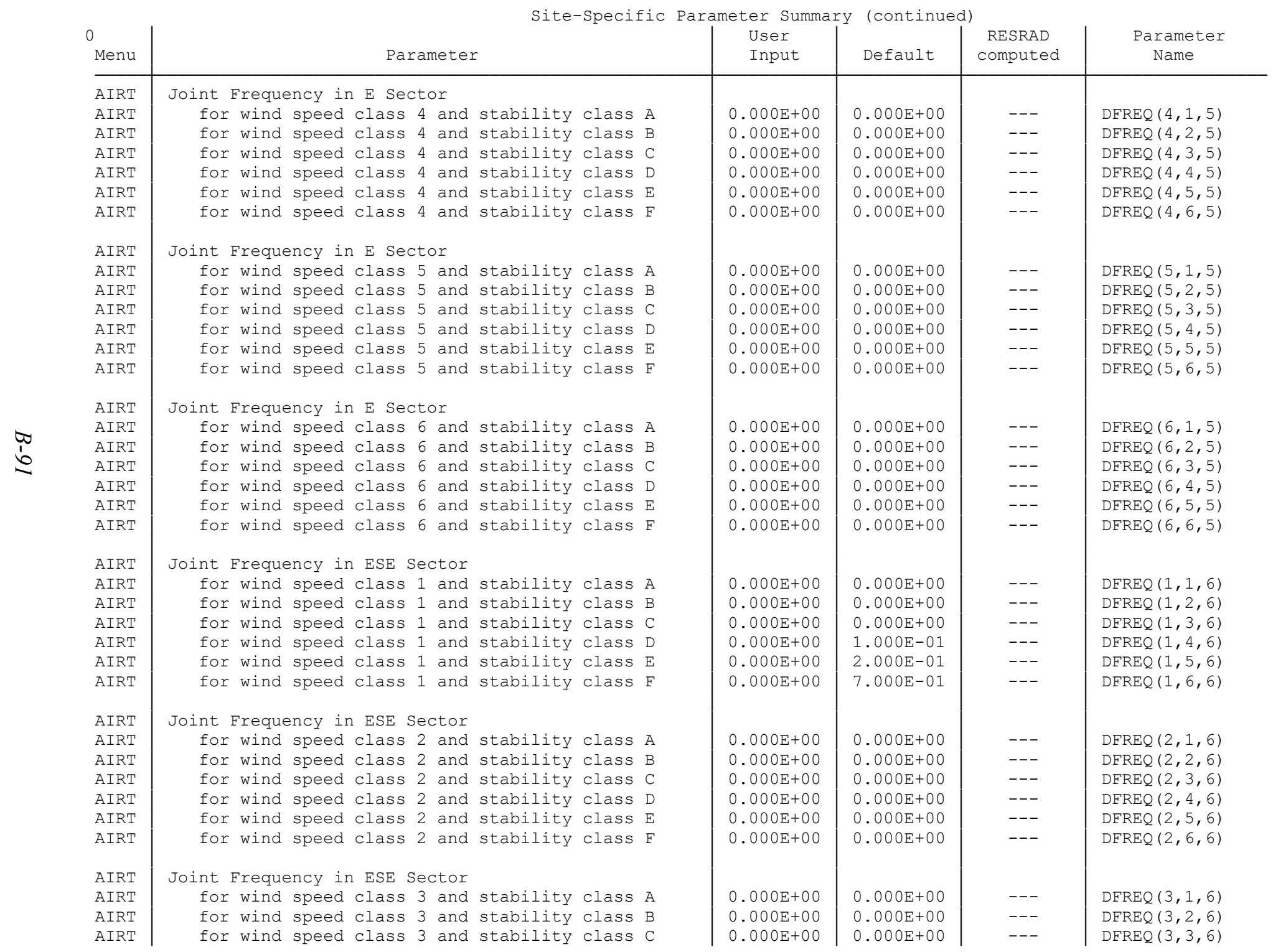


AIRT for wind speed class 3 and stability class D AIRT for wind speed class 3 and stability class E AIRT for wind speed class 3 and stability class $F$ 1RESRAD-OFFSITE, Version 4.0 .3 beta T1/2 Limit $=30$ days Parent Dose Report

Title : RESRAD-OFFSITE Effluent release

File : PADUCAH EFFLUENT RELEASE-REVI.ROF

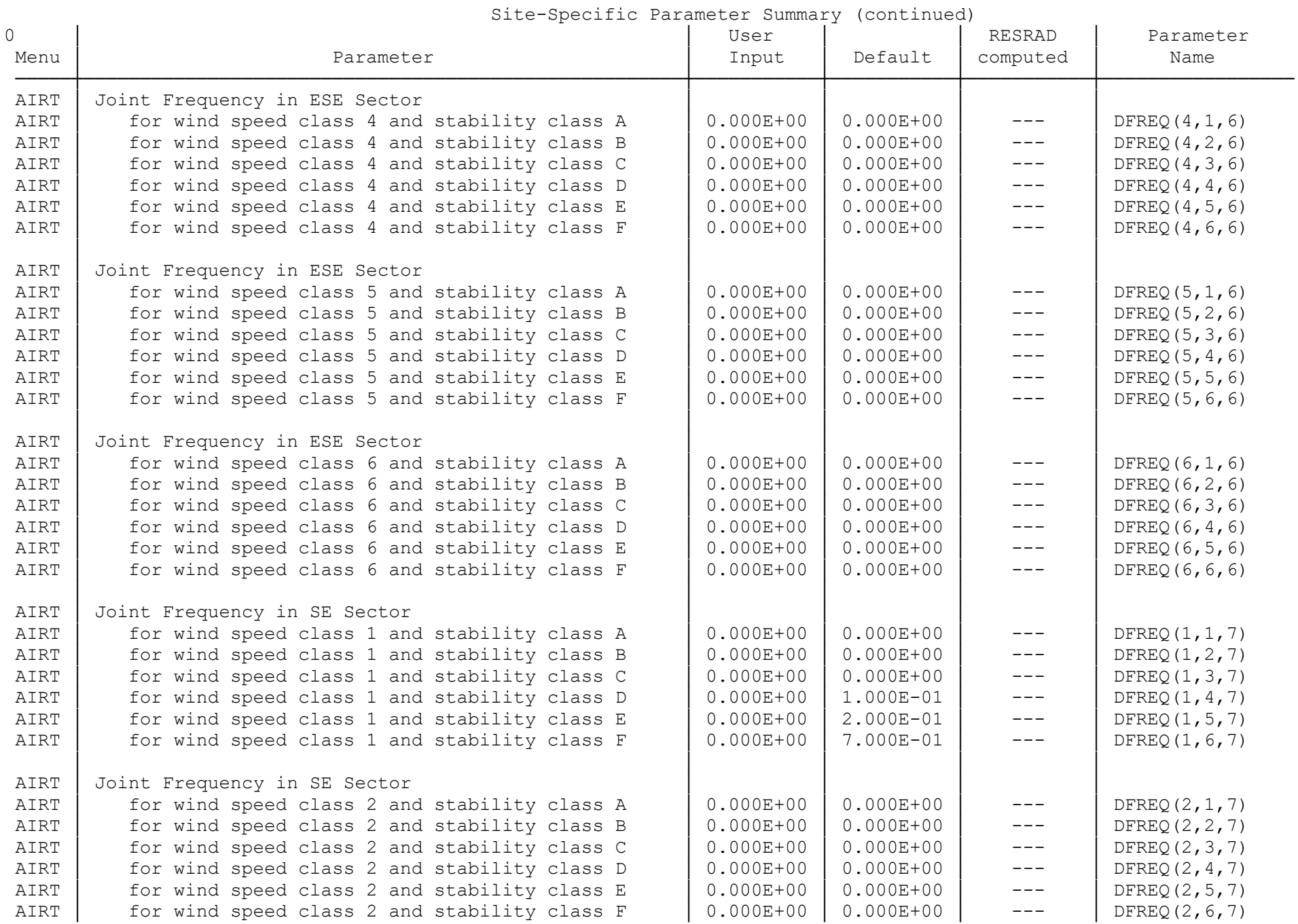


AIRT Joint Frequency in SE Sector

AIRT for wind speed class 3 and stability class A

AIRT for wind speed class 3 and stability class B

AIRT for wind speed class 3 and stability class $C$

AIRT for wind speed class 3 and stability class D

AIRT for wind speed class 3 and stability class E

AIRT for wind speed class 3 and stability class F

IRESRAD-OFFSITE, Version 4.0 .3 beta $\mathrm{T}^{1} \frac{1}{2}$ Limit $=30$ days

Parent Dose Report

Title: RESRAD-OFFSITE Effluent release

File : PADUCAH EFFLUENT RELEASE-REV1.ROF

\begin{tabular}{|l|l|l|l} 
& & & \\
$0.000 \mathrm{E}+00$ & $0.000 \mathrm{E}+00$ & -- & $\operatorname{DFREQ}(3,1,7)$ \\
$0.000 \mathrm{E}+00$ & $0.000 \mathrm{E}+00$ & -- & $\operatorname{DFREQ}(3,2,7)$ \\
$0.000 \mathrm{E}+00$ & $0.000 \mathrm{E}+00$ & -- & $\operatorname{DFREQ}(3,3,7)$ \\
$0.000 \mathrm{E}+00$ & $0.000 \mathrm{E}+00$ & --- & $\operatorname{DFREQ}(3,4,7)$ \\
$0.000 \mathrm{E}+00$ & $0.000 \mathrm{E}+00$ & --- & $\operatorname{DFREQ}(3,5,7)$ \\
$0.000 \mathrm{E}+00$ & $0.000 \mathrm{E}+00$ & --- & $\operatorname{DFREQ}(3,6,7)$
\end{tabular}

$\operatorname{DFREQ}(3,5,7)$

06/11/2020 11:32 Page 30

Site-Specific Parameter Summary (continued)

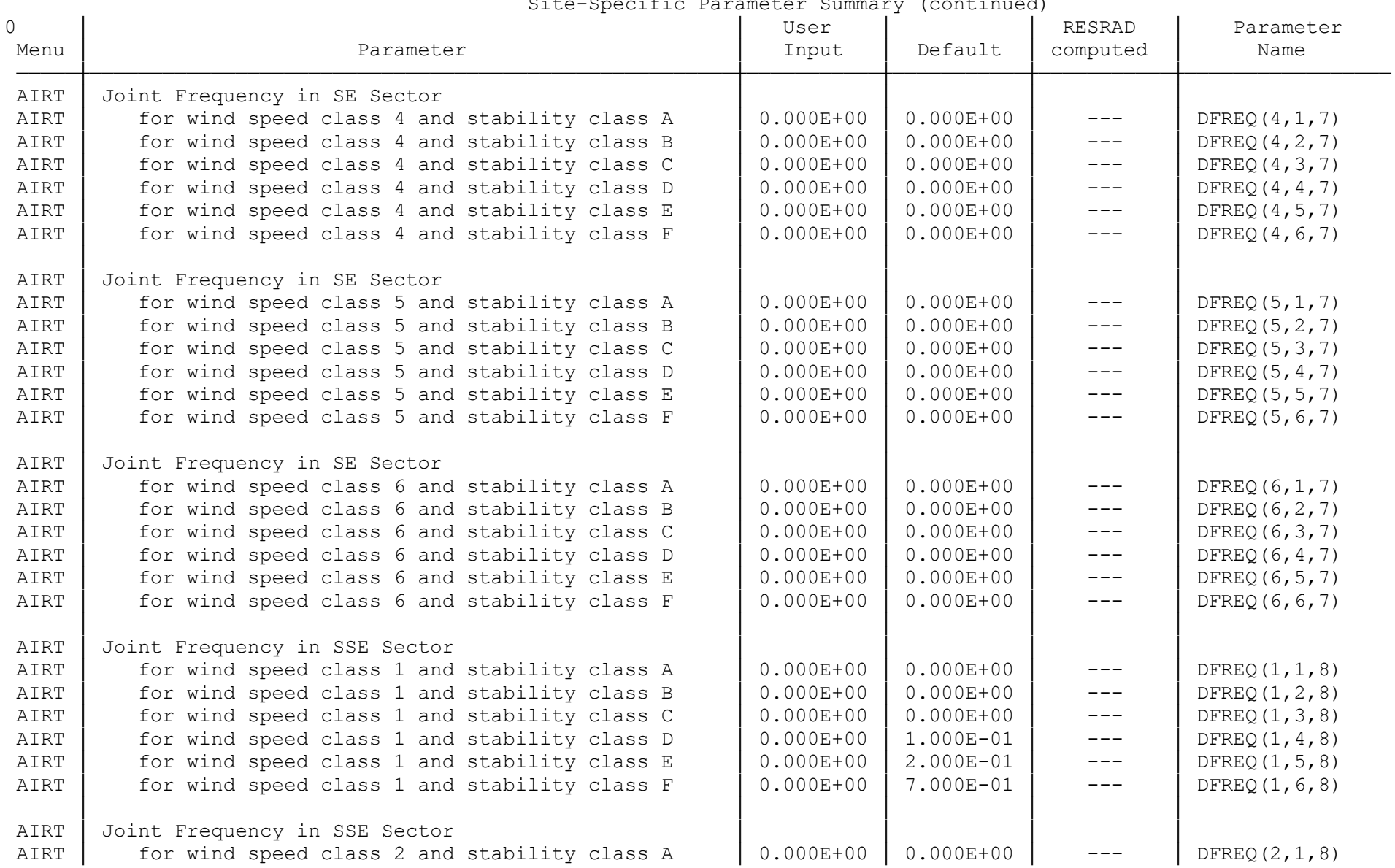




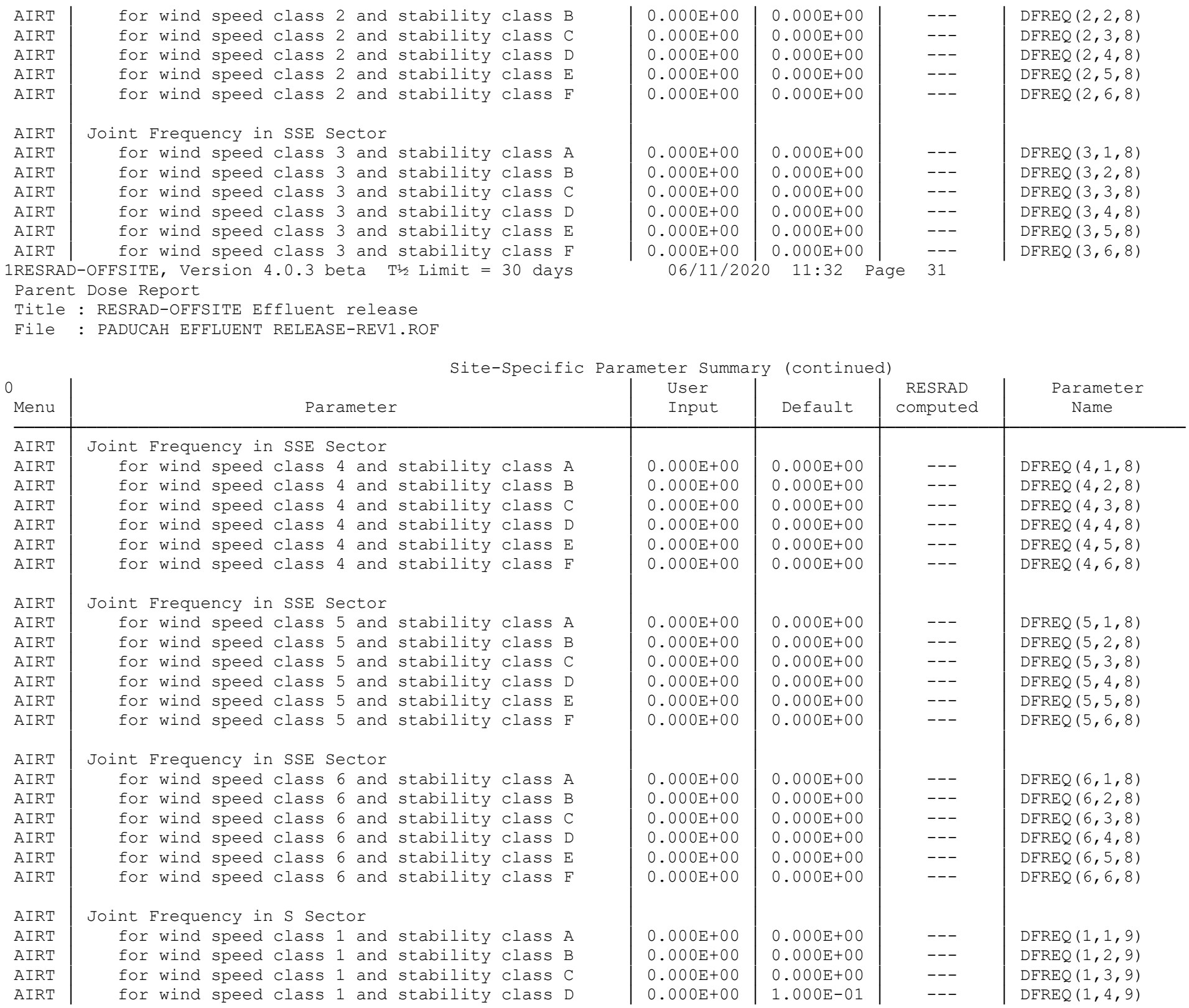




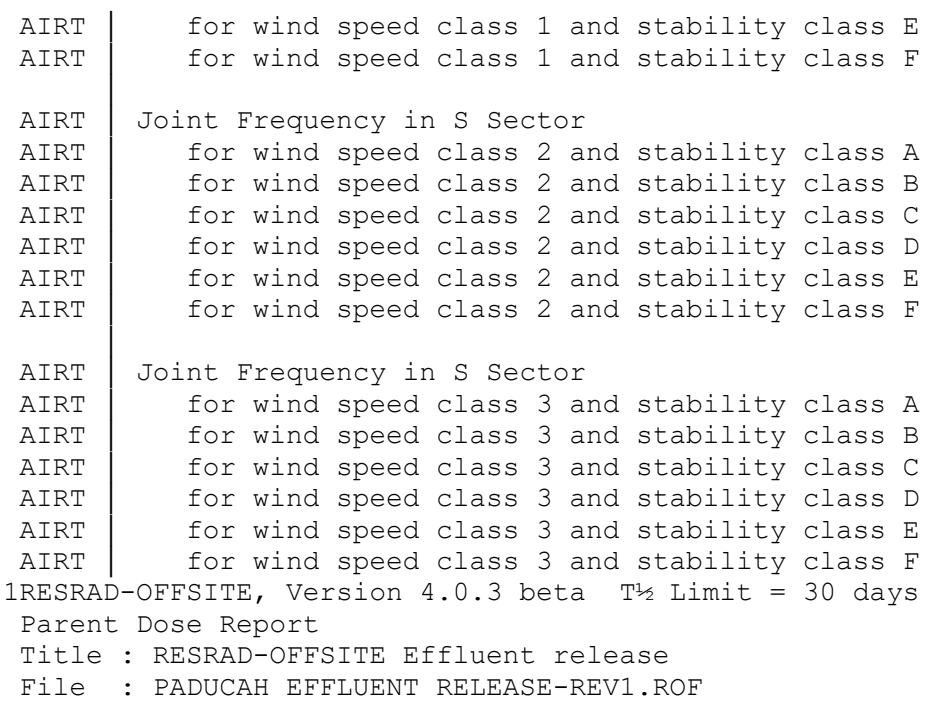

\begin{tabular}{|l|l|l|l}
$0.000 \mathrm{E}+00$ & $2.000 \mathrm{E}-01$ & --- & $\operatorname{DFREQ}(1,5,9)$ \\
$0.000 \mathrm{E}+00$ & $7.000 \mathrm{E}-01$ & -- & $\operatorname{DFREQ}(1,6,9)$ \\
& & & \\
$0.000 \mathrm{E}+00$ & $0.000 \mathrm{E}+00$ & -- & $\operatorname{DFREQ}(2,1,9)$ \\
$0.000 \mathrm{E}+00$ & $0.000 \mathrm{E}+00$ & --- & $\operatorname{DFREQ}(2,2,9)$ \\
$0.000 \mathrm{E}+00$ & $0.000 \mathrm{E}+00$ & --- & $\operatorname{DFREQ}(2,3,9)$ \\
$0.000 \mathrm{E}+00$ & $0.000 \mathrm{E}+00$ & --- & $\operatorname{DFREQ}(2,4,9)$ \\
$0.000 \mathrm{E}+00$ & $0.000 \mathrm{E}+00$ & --- & $\operatorname{DFREQ}(2,5,9)$ \\
$0.000 \mathrm{E}+00$ & $0.000 \mathrm{E}+00$ & -- & $\operatorname{DFREQ}(2,6,9)$ \\
& & & \\
$0.000 \mathrm{E}+00$ & $0.000 \mathrm{E}+00$ & --- & $\operatorname{DFREQ}(3,1,9)$ \\
$0.000 \mathrm{E}+00$ & $0.000 \mathrm{E}+00$ & --- & $\operatorname{DFREQ}(3,2,9)$ \\
$0.000 \mathrm{E}+00$ & $0.000 \mathrm{E}+00$ & --- & $\operatorname{DFREQ}(3,3,9)$ \\
$0.000 \mathrm{E}+00$ & $0.000 \mathrm{E}+00$ & --- & $\operatorname{DFREQ}(3,4,9)$ \\
$0.000 \mathrm{E}+00$ & $0.000 \mathrm{E}+00$ & --- & $\operatorname{DFREQ}(3,5,9)$ \\
$0.000 \mathrm{E}+00$ & $0.000 \mathrm{E}+00$ & --- & $\operatorname{DFREQ}(3,6,9)$
\end{tabular}

Site-Specific Parameter Summary (continued)

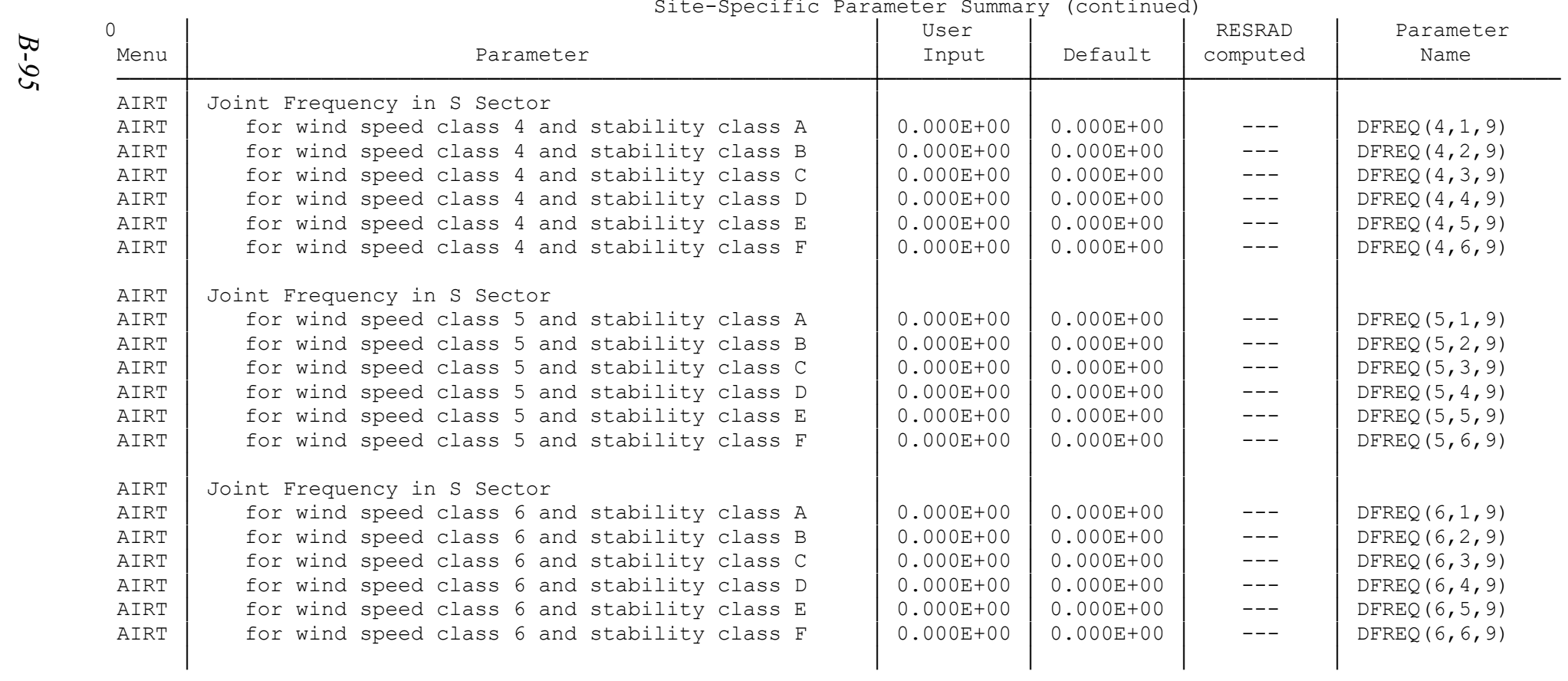


AIRT | Joint Frequency in SSW Sector

AIRT for wind speed class 1 and stability class A

AIRT for wind speed class 1 and stability class $B$

AIRT for wind speed class 1 and stability class $C$

AIRT for wind speed class 1 and stability class D

AIRT for wind speed class 1 and stability class E

AIRT for wind speed class 1 and stability class $F$

AIRT Joint Frequency in SSW Sector

AIRT for wind speed class 2 and stability class A

AIRT for wind speed class 2 and stability class B

AIRT for wind speed class 2 and stability class $C$

AIRT for wind speed class 2 and stability class D

AIRT for wind speed class 2 and stability class E

AIRT for wind speed class 2 and stability class $F$

AIRT Joint Frequency in SSW Sector

AIRT for wind speed class 3 and stability class A

AIRT for wind speed class 3 and stability class $B$

AIRT for wind speed class 3 and stability class $C$

AIRT for wind speed class 3 and stability class D

AIRT for wind speed class 3 and stability class $E$

AIRT for wind speed class 3 and stability class F

1RESRAD-OFFSITE, Version 4.0 .3 beta $T^{\frac{1}{2} / 2}$ Limit $=30$ days

Torent Dose Report

File : PADUCAH FFFLUENT REIFASE-REV1 ROF

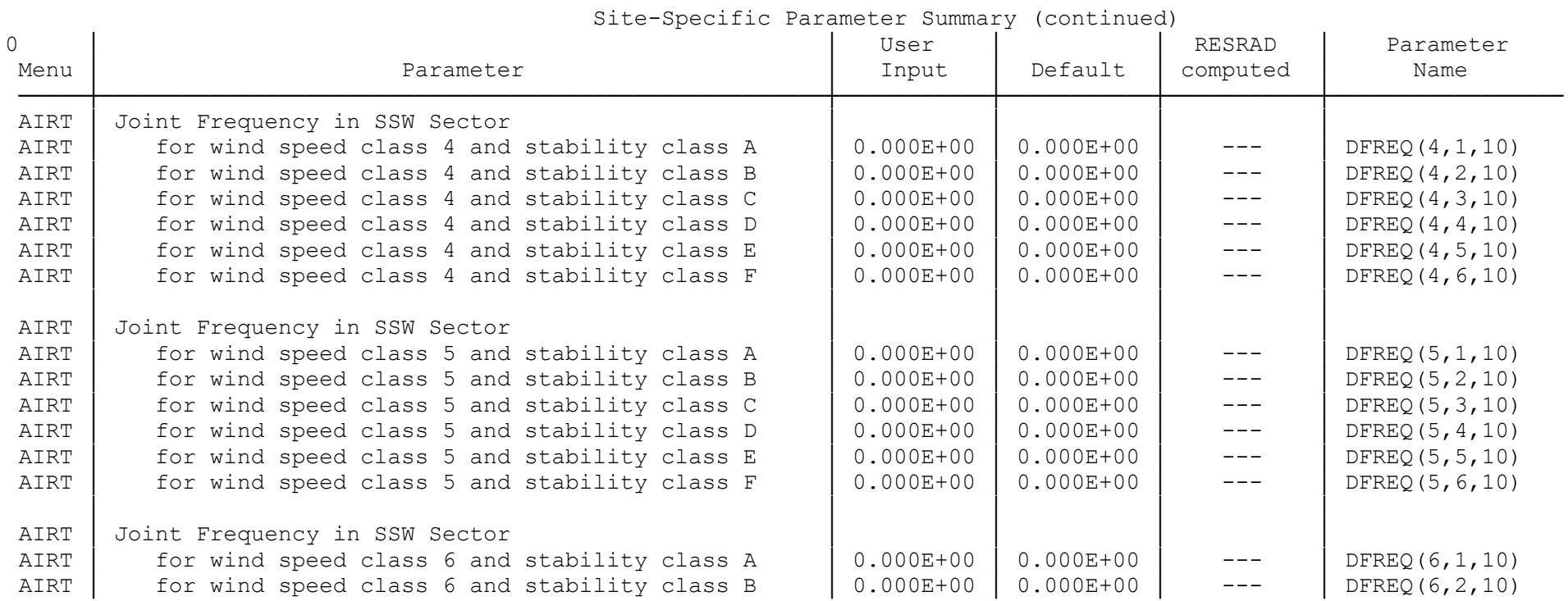

\begin{tabular}{|c|c|c|c|}
\hline $0.000 \mathrm{E}+00$ & $0.000 \mathrm{E}+00$ & --- & $\operatorname{DFREQ}(1,1,10)$ \\
\hline $0.000 \mathrm{E}+00$ & $0.000 \mathrm{E}+00$ & --- & $\operatorname{DFREQ}(1,2,10)$ \\
\hline $0.000 \mathrm{E}+00$ & $0.000 \mathrm{E}+00$ & --- & $\operatorname{DFREQ}(1,3,10)$ \\
\hline $0.000 \mathrm{E}+00$ & $1.000 \mathrm{E}-01$ & --- & $\operatorname{DFREQ}(1,4,10)$ \\
\hline $0.000 \mathrm{E}+00$ & $2.000 \mathrm{E}-01$ & --- & $\operatorname{DFREQ}(1,5,10)$ \\
\hline $0.000 \mathrm{E}+00$ & $7.000 \mathrm{E}-01$ & --- & $\operatorname{DFREQ}(1,6,10)$ \\
\hline $0.000 \mathrm{E}+00$ & $0.000 \mathrm{E}+00$ & --- & $\operatorname{DFREQ}(2,1,10)$ \\
\hline $0.000 \mathrm{E}+00$ & $0.000 \mathrm{E}+00$ & --- & $\operatorname{DFREQ}(2,2,10)$ \\
\hline $0.000 \mathrm{E}+00$ & $0.000 \mathrm{E}+00$ & --- & $\operatorname{DFREQ}(2,3,10)$ \\
\hline $0.000 \mathrm{E}+00$ & $0.000 \mathrm{E}+00$ & --- & $\operatorname{DFREQ}(2,4,10)$ \\
\hline $0.000 \mathrm{E}+00$ & $0.000 \mathrm{E}+00$ & --- & $\operatorname{DFREQ}(2,5,10)$ \\
\hline $0.000 \mathrm{E}+00$ & $0.000 \mathrm{E}+00$ & --- & $\operatorname{DFREQ}(2,6,10)$ \\
\hline $0.000 \mathrm{E}+00$ & $0.000 \mathrm{E}+00$ & --- & $\operatorname{DFREQ}(3,1,10)$ \\
\hline $0.000 \mathrm{E}+00$ & $0.000 \mathrm{E}+00$ & --- & $\operatorname{DFREQ}(3,2,10)$ \\
\hline $0.000 \mathrm{E}+00$ & $0.000 \mathrm{E}+00$ & --- & $\operatorname{DFREQ}(3,3,10)$ \\
\hline $0.000 \mathrm{E}+00$ & $0.000 \mathrm{E}+00$ & --- & $\operatorname{DFREQ}(3,4,10)$ \\
\hline $0.000 \mathrm{E}+00$ & $0.000 \mathrm{E}+00$ & --- & $\operatorname{DFREQ}(3,5,10)$ \\
\hline $0.000 \mathrm{E}+00$ & $0.000 \mathrm{E}+00$ & --- & $\operatorname{DFREQ}(3,6,10)$ \\
\hline
\end{tabular}

06/11/2020 11:32 Page 33

$\operatorname{DFREQ}(3,5,10)$
$\operatorname{DFREQ}(3,6,10)$

$0.000 \mathrm{E}+00-0.000 \mathrm{E}+00$

$\operatorname{DFREQ}(6,2,10)$ 
\begin{tabular}{l|l} 
AIRT & for wind speed class 6 and stability class C
\end{tabular} AIRT AIRT

AIRT

AIRT

AIRT

AIRT

AIRT

AIRT

AIRT

AIRT

AIRT

AIRT

AIRT

AIRT

AIRT

AIRT

AIRT

AIRT

AIRT

AIRT

AIRT

AIRT

AIRT for wind speed class 3 and stability class $F$
IRESRAD-OFFSITE, Version 4.0 .3 beta T1/2 Limit $=30$ days

Parent Dose Report

Title : RESRAD-OFFSITE Effluent release

File : PADUCAH EFFLUENT RELEASE-REV1.ROF

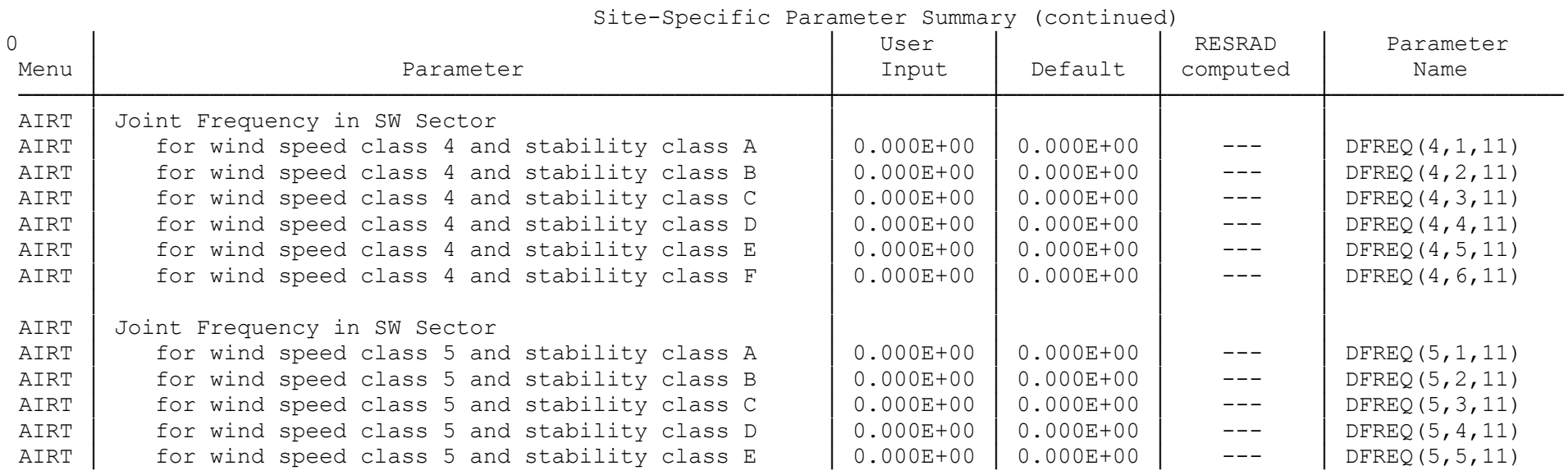

\begin{tabular}{|l|l|l|l}
$0.000 \mathrm{E}+00$ & $0.000 \mathrm{E}+00$ & --- & $\operatorname{DFREQ}(6,3,10)$ \\
$0.000 \mathrm{E}+00$ & $0.000 \mathrm{E}+00$ & --- & $\operatorname{DFREQ}(6,4,10)$ \\
$0.000 \mathrm{E}+00$ & $0.000 \mathrm{E}+00$ & --- & $\operatorname{DFREQ}(6,5,10)$ \\
$0.000 \mathrm{E}+00$ & $0.000 \mathrm{E}+00$ & --- & $\operatorname{DFREQ}(6,6,10)$ \\
& & & \\
$0.000 \mathrm{E}+00$ & $0.000 \mathrm{E}+00$ & --- & $\operatorname{DFREQ}(1,1,11)$ \\
$0.000 \mathrm{E}+00$ & $0.000 \mathrm{E}+00$ & --- & $\operatorname{DFREQ}(1,2,11)$ \\
$0.000 \mathrm{E}+00$ & $0.000 \mathrm{E}+00$ & --- & $\operatorname{DFREQ}(1,3,11)$ \\
$0.000 \mathrm{E}+00$ & $1.000 \mathrm{E}-01$ & --- & $\operatorname{DFREQ}(1,4,11)$ \\
$0.000 \mathrm{E}+00$ & $2.000 \mathrm{E}-01$ & --- & $\operatorname{DFREQ}(1,5,11)$ \\
$0.000 \mathrm{E}+00$ & $7.000 \mathrm{E}-01$ & --- & $\operatorname{DFREQ}(1,6,11)$ \\
& & & \\
$0.000 \mathrm{E}+00$ & $0.000 \mathrm{E}+00$ & --- & $\operatorname{DFREQ}(2,1,11)$ \\
$0.000 \mathrm{E}+00$ & $0.000 \mathrm{E}+00$ & --- & $\operatorname{DFREQ}(2,2,11)$ \\
$0.000 \mathrm{E}+00$ & $0.000 \mathrm{E}+00$ & --- & $\operatorname{DFREQ}(2,3,11)$ \\
$0.000 \mathrm{E}+00$ & $0.000 \mathrm{E}+00$ & --- & $\operatorname{DFREQ}(2,4,11)$ \\
$0.000 \mathrm{E}+00$ & $0.000 \mathrm{E}+00$ & --- & $\operatorname{DFREQ}(2,5,11)$ \\
$0.000 \mathrm{E}+00$ & $0.000 \mathrm{E}+00$ & --- & $\operatorname{DFREQ}(2,6,11)$ \\
& & & \\
$0.000 \mathrm{E}+00$ & $0.000 \mathrm{E}+00$ & --- & $\operatorname{DFREQ}(3,1,11)$ \\
$0.000 \mathrm{E}+00$ & $0.000 \mathrm{E}+00$ & --- & $\operatorname{DFREQ}(3,2,11)$ \\
$0.000 \mathrm{E}+00$ & $0.000 \mathrm{E}+00$ & --- & $\operatorname{DFREQ}(3,3,11)$ \\
$0.000 \mathrm{E}+00$ & $0.000 \mathrm{E}+00$ & --- & $\operatorname{DFREQ}(3,4,11)$ \\
$0.000 \mathrm{E}+00$ & $0.000 \mathrm{E}+00$ & --- & $\operatorname{DFREQ}(3,5,11)$ \\
$0.000 \mathrm{E}+00$ & $0.000 \mathrm{E}+00$ & --- & $\operatorname{DFREQ}(3,6,11)$ \\
$06 / 11 / 2020$ & 111.32 &
\end{tabular}

$06 / 11 / 2020 \cdot 11: 32$ Page 34
$\operatorname{DFREQ}(3,5,11)$
AIRT for wind speed class 5 and stability class E

\begin{tabular}{l|l|l|l}
$0.000 \mathrm{E}+00$ & $0.000 \mathrm{E}+00$ & --- & $\operatorname{DEREQ}(5,5,11)$
\end{tabular} 
AIRT

AIRT

AIRT

AIRT

AIRT

AIRT

AIRT

AIRT

AIRT

AIRT

AIRT

AIRT

AIRT

AIRT

AIRT

AIRT

AIRT

AIRT

AIRT

AIRT

AIRT

AIRT

AIRT

AIRT

AIRT

AIRT

AIRT

AIRT

AIRT for wind speed class 3 and stability class $\mathrm{F}$

Title: RESRAD-OFFSITE Effluent release

File : PADUCAH EFFLUENT RELEASE-REV1.ROF

int Frequency in SW Sector for wind speed class 6 and stability class B for wind speed class 6 and stability class D for wind speed class 6 and stability class E

oint Frequency in WSW Sector for wind speed class 1 and stability class B for wind speed class 1 and stability class D for wind speed class 1 and stability class $E$

oint Frequency in WSW Sector for wind speed class 2 and stability class $B$ for wind speed class 2 and stability class D for wind speed Class 2 and stability Class E

int Frequency in WSW Sector

for wind speed class 3 and stability class A for wind speed class 3 and stability class C for wind speed class 3 and stability class D for wind speed Class 3 and stability class E
Parent Dose Report

\begin{tabular}{|c|c|c|c|}
\hline $0.000 \mathrm{E}+00$ & $0.000 \mathrm{E}+00$ & --- & $\operatorname{DFREQ}(5,6,11)$ \\
\hline $0.000 \mathrm{E}+00$ & $0.000 \mathrm{E}+00$ & --- & $\operatorname{DFREQ}(6,1,11)$ \\
\hline $0.000 \mathrm{E}+00$ & $0.000 \mathrm{E}+00$ & --- & $\operatorname{DFREQ}(6,2,11)$ \\
\hline $0.000 \mathrm{E}+00$ & $0.000 \mathrm{E}+00$ & --- & $\operatorname{DFREQ}(6,3,11)$ \\
\hline $0.000 \mathrm{E}+00$ & $0.000 \mathrm{E}+00$ & --- & $\operatorname{DFREQ}(6,4,11)$ \\
\hline $0.000 \mathrm{E}+00$ & $0.000 \mathrm{E}+00$ & --- & $\operatorname{DFREQ}(6,5,11)$ \\
\hline $0.000 \mathrm{E}+00$ & $0.000 \mathrm{E}+00$ & --- & $\operatorname{DFREQ}(6,6,11)$ \\
\hline $0.000 \mathrm{E}+00$ & $0.000 \mathrm{E}+00$ & --- & $\operatorname{DFREQ}(1,1,12)$ \\
\hline $0.000 \mathrm{E}+00$ & $0.000 \mathrm{E}+00$ & --- & $\operatorname{DFREQ}(1,2,12)$ \\
\hline $0.000 \mathrm{E}+00$ & $0.000 \mathrm{E}+00$ & --- & $\operatorname{DFREQ}(1,3,12)$ \\
\hline $0.000 \mathrm{E}+00$ & $1.000 \mathrm{E}-01$ & --- & $\operatorname{DFREQ}(1,4,12)$ \\
\hline $0.000 \mathrm{E}+00$ & $2.000 \mathrm{E}-01$ & --- & $\operatorname{DFREQ}(1,5,12)$ \\
\hline $0.000 \mathrm{E}+00$ & $7.000 \mathrm{E}-01$ & --- & $\operatorname{DFREQ}(1,6,12)$ \\
\hline $0.000 \mathrm{E}+00$ & $0.000 \mathrm{E}+00$ & --- & DFREQ $(2,1,12)$ \\
\hline $0.000 \mathrm{E}+00$ & $0.000 \mathrm{E}+00$ & --- & $\operatorname{DFREQ}(2,2,12)$ \\
\hline $0.000 \mathrm{E}+00$ & $0.000 \mathrm{E}+00$ & --- & $\operatorname{DFREQ}(2,3,12)$ \\
\hline $0.000 \mathrm{E}+00$ & $0.000 \mathrm{E}+00$ & --- & $\operatorname{DFREQ}(2,4,12)$ \\
\hline $0.000 \mathrm{E}+00$ & $0.000 \mathrm{E}+00$ & --- & DFREQ $(2,5,12)$ \\
\hline $0.000 \mathrm{E}+00$ & $0.000 \mathrm{E}+00$ & --- & $\operatorname{DFREQ}(2,6,12)$ \\
\hline $0.000 \mathrm{E}+00$ & $0.000 \mathrm{E}+00$ & --- & $\operatorname{DFREQ}(3,1,12)$ \\
\hline $0.000 \mathrm{E}+00$ & $0.000 \mathrm{E}+00$ & --- & $\operatorname{DFREQ}(3,2,12)$ \\
\hline $0.000 \mathrm{E}+00$ & $0.000 \mathrm{E}+00$ & --- & $\operatorname{DFREQ}(3,3,12)$ \\
\hline $0.000 \mathrm{E}+00$ & $0.000 \mathrm{E}+00$ & --- & $\operatorname{DFREQ}(3,4,12)$ \\
\hline $0.000 \mathrm{E}+00$ & $0.000 \mathrm{E}+00$ & --- & $\operatorname{DFREQ}(3,5,12)$ \\
\hline $0.000 \mathrm{E}+00$ & $0.000 \mathrm{E}+00$ & --- & $\operatorname{DFREQ}(3,6,12)$ \\
\hline
\end{tabular}

Site-Specific Parameter Summary (continued)

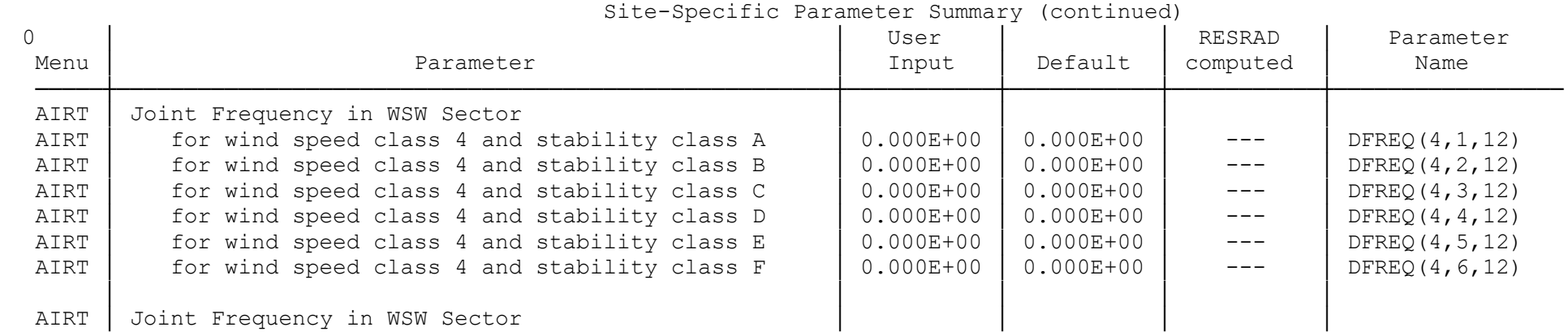
for wind speed class 3 and stability class B

\begin{tabular}{l|l}
$0.000 \mathrm{E}+00$ & $0.000 \mathrm{E}+00$
\end{tabular}

06/11/2020 11:32 Page 35
$\operatorname{DFREQ}(3,6,12)$ 


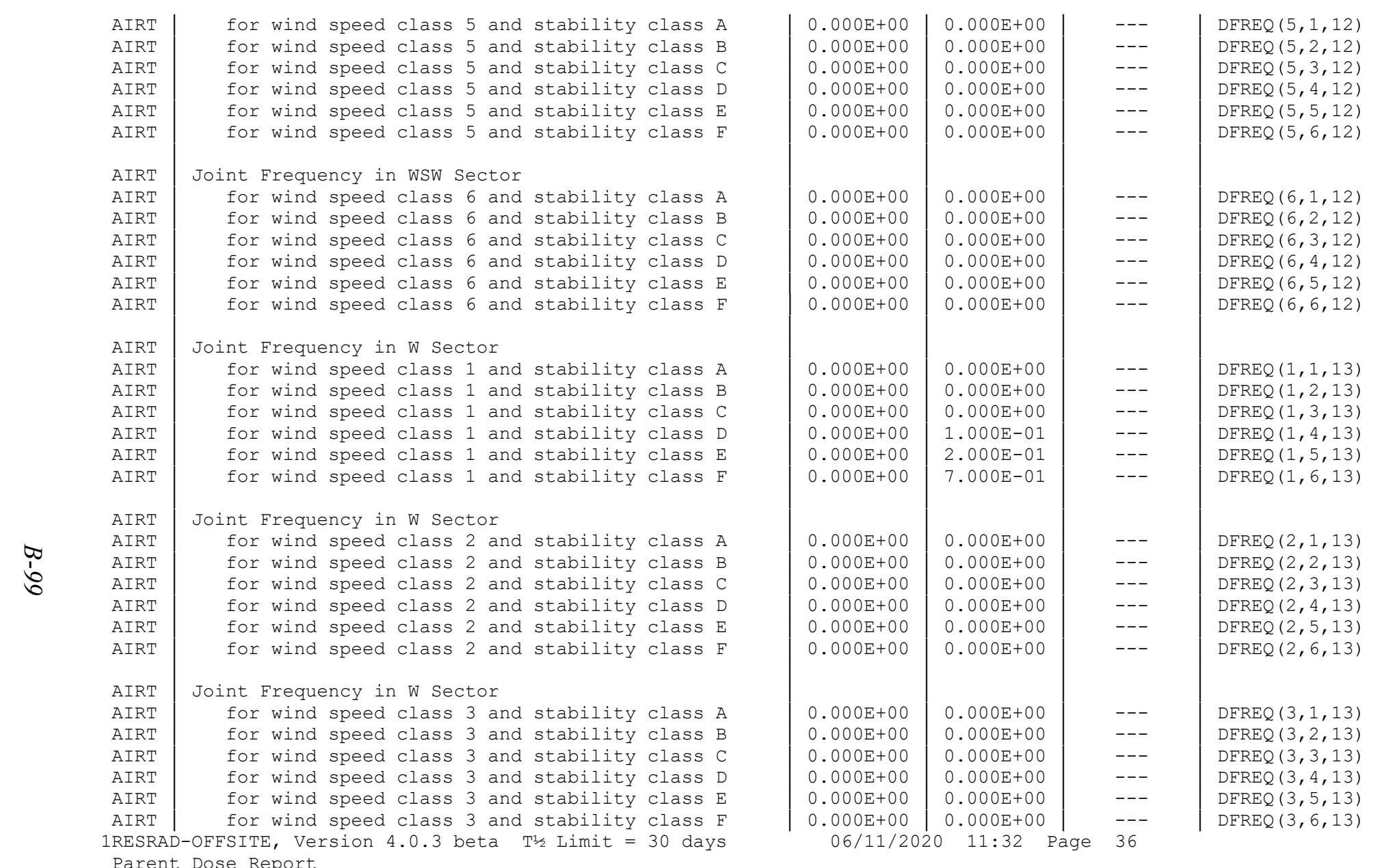

Parent Dose Report

File : PADUCAH EFFLUENT RELEASE-REV1.ROF

\begin{tabular}{|c|c|c|c|c|c|c|}
\hline $\begin{array}{l}\text { O } \\
\text { Menu }\end{array}$ & & Site-Specifi & $\begin{array}{l}\text { heter Summ } \\
\text { User } \\
\text { Input }\end{array}$ & $\begin{array}{l}\text { (continue } \\
\text { Default }\end{array}$ & $\begin{array}{l}\text { RESRAD } \\
\text { computed }\end{array}$ & $\begin{array}{l}\text { Parameter } \\
\text { Name }\end{array}$ \\
\hline
\end{tabular}




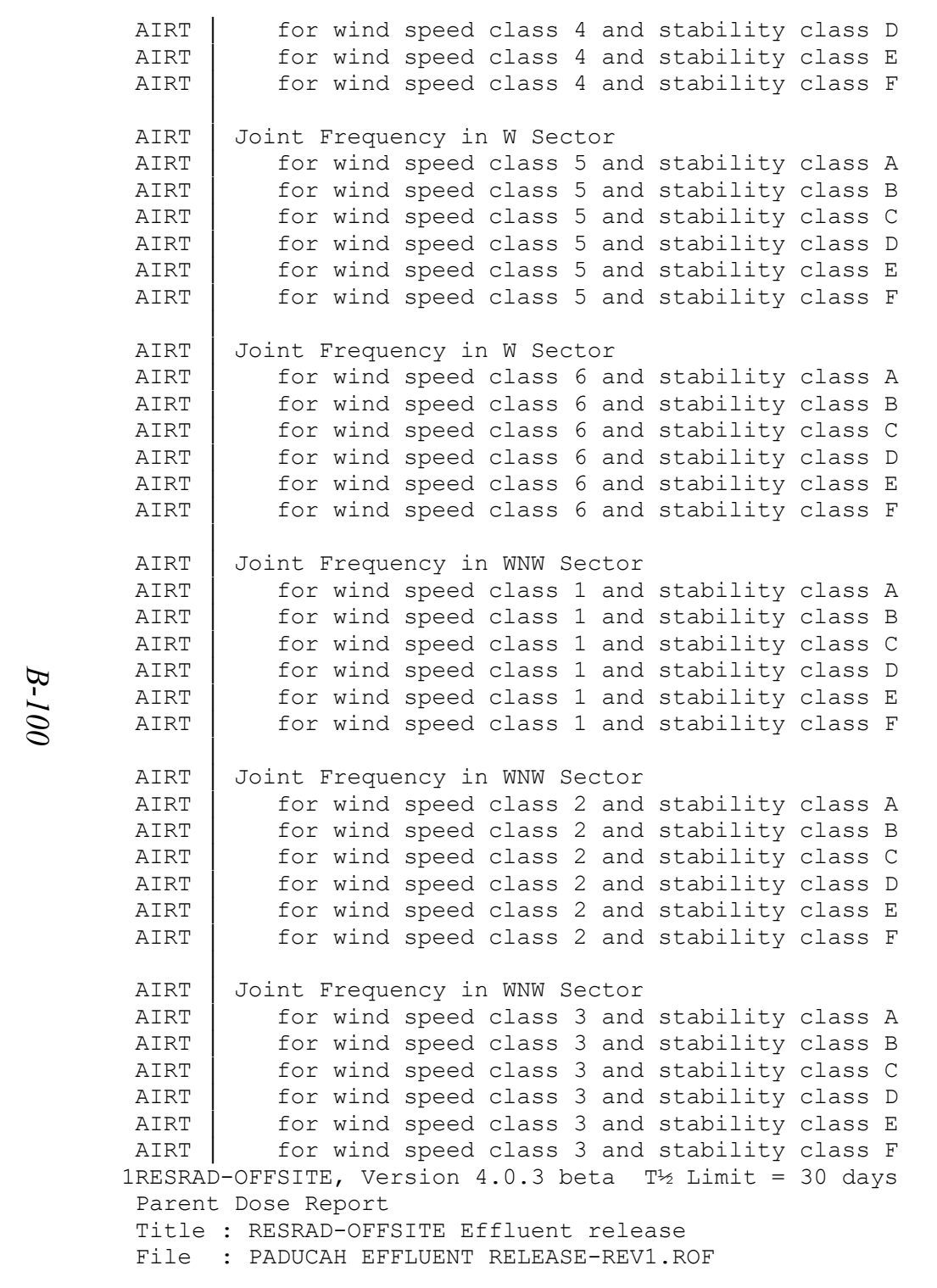

\begin{tabular}{|c|c|c|c|}
\hline $0.000 \mathrm{E}+00$ & $0.000 \mathrm{E}+00$ & --- & $\operatorname{DFREQ}(4,4,13)$ \\
\hline $0.000 \mathrm{E}+00$ & $0.000 \mathrm{E}+00$ & --- & $\operatorname{DFREQ}(4,5,13)$ \\
\hline $0.000 \mathrm{E}+00$ & $0.000 \mathrm{E}+00$ & --- & $\operatorname{DFREQ}(4,6,13)$ \\
\hline $0.000 \mathrm{E}+00$ & $0.000 \mathrm{E}+00$ & --- & $\operatorname{DFREQ}(5,1,13)$ \\
\hline $0.000 \mathrm{E}+00$ & $0.000 \mathrm{E}+00$ & --- & $\operatorname{DFREQ}(5,2,13)$ \\
\hline $0.000 \mathrm{E}+00$ & $0.000 \mathrm{E}+00$ & --- & $\operatorname{DFREQ}(5,3,13)$ \\
\hline $0.000 \mathrm{E}+00$ & $0.000 \mathrm{E}+00$ & --- & $\operatorname{DFREQ}(5,4,13)$ \\
\hline $0.000 \mathrm{E}+00$ & $0.000 \mathrm{E}+00$ & --- & $\operatorname{DFREQ}(5,5,13)$ \\
\hline $0.000 \mathrm{E}+00$ & $0.000 \mathrm{E}+00$ & --- & $\operatorname{DFREQ}(5,6,13)$ \\
\hline $0.000 \mathrm{E}+00$ & $0.000 \mathrm{E}+00$ & --- & $\operatorname{DFREQ}(6,1,13)$ \\
\hline $0.000 \mathrm{E}+00$ & $0.000 \mathrm{E}+00$ & --- & $\operatorname{DFREQ}(6,2,13)$ \\
\hline $0.000 \mathrm{E}+00$ & $0.000 \mathrm{E}+00$ & --- & $\operatorname{DFREQ}(6,3,13)$ \\
\hline $0.000 \mathrm{E}+00$ & $0.000 \mathrm{E}+00$ & --- & $\operatorname{DFREQ}(6,4,13)$ \\
\hline $0.000 \mathrm{E}+00$ & $0.000 \mathrm{E}+00$ & --- & $\operatorname{DFREQ}(6,5,13)$ \\
\hline $0.000 \mathrm{E}+00$ & $0.000 \mathrm{E}+00$ & --- & $\operatorname{DFREQ}(6,6,13)$ \\
\hline $0.000 \mathrm{E}+00$ & $0.000 \mathrm{E}+00$ & --- & $\operatorname{DFREQ}(1,1,14)$ \\
\hline $0.000 \mathrm{E}+00$ & $0.000 \mathrm{E}+00$ & --- & $\operatorname{DFREQ}(1,2,14)$ \\
\hline $0.000 \mathrm{E}+00$ & $0.000 \mathrm{E}+00$ & --- & $\operatorname{DFREQ}(1,3,14)$ \\
\hline $0.000 \mathrm{E}+00$ & $1.000 \mathrm{E}-01$ & --- & $\operatorname{DFREQ}(1,4,14)$ \\
\hline $0.000 \mathrm{E}+00$ & $2.000 \mathrm{E}-01$ & --- & $\operatorname{DFREQ}(1,5,14)$ \\
\hline $0.000 \mathrm{E}+00$ & $7.000 \mathrm{E}-01$ & --- & $\operatorname{DFREQ}(1,6,14)$ \\
\hline $0.000 \mathrm{E}+00$ & $0.000 \mathrm{E}+00$ & --- & $\operatorname{DFREQ}(2,1,14)$ \\
\hline $0.000 \mathrm{E}+00$ & $0.000 \mathrm{E}+00$ & --- & $\operatorname{DFREQ}(2,2,14)$ \\
\hline $0.000 \mathrm{E}+00$ & $0.000 \mathrm{E}+00$ & --- & $\operatorname{DFREQ}(2,3,14)$ \\
\hline $0.000 \mathrm{E}+00$ & $0.000 \mathrm{E}+00$ & --- & $\operatorname{DFREQ}(2,4,14)$ \\
\hline $0.000 \mathrm{E}+00$ & $0.000 \mathrm{E}+00$ & --- & $\operatorname{DFREQ}(2,5,14)$ \\
\hline $0.000 \mathrm{E}+00$ & $0.000 \mathrm{E}+00$ & --- & $\operatorname{DFREQ}(2,6,14)$ \\
\hline $0.000 \mathrm{E}+00$ & $0.000 \mathrm{E}+00$ & --- & $\operatorname{DFREQ}(3,1,14)$ \\
\hline $0.000 \mathrm{E}+00$ & $0.000 \mathrm{E}+00$ & --- & $\operatorname{DFREQ}(3,2,14)$ \\
\hline $0.000 \mathrm{E}+00$ & $0.000 \mathrm{E}+00$ & --- & $\operatorname{DFREQ}(3,3,14)$ \\
\hline $0.000 \mathrm{E}+00$ & $0.000 \mathrm{E}+00$ & --- & $\operatorname{DFREQ}(3,4,14)$ \\
\hline $0.000 \mathrm{E}+00$ & $0.000 \mathrm{E}+00$ & --- & $\operatorname{DFREQ}(3,5,14)$ \\
\hline $0.000 \mathrm{E}+00$ & $0.000 \mathrm{E}+00$ & --- & $\operatorname{DFREQ}(3,6,14)$ \\
\hline
\end{tabular}

\begin{tabular}{c|c} 
Menu & Parameter
\end{tabular}

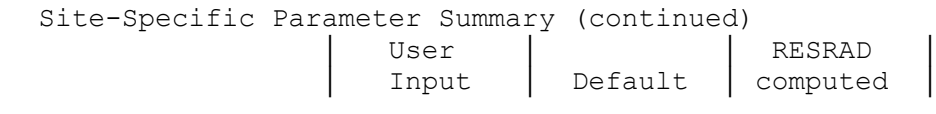

Parameter 


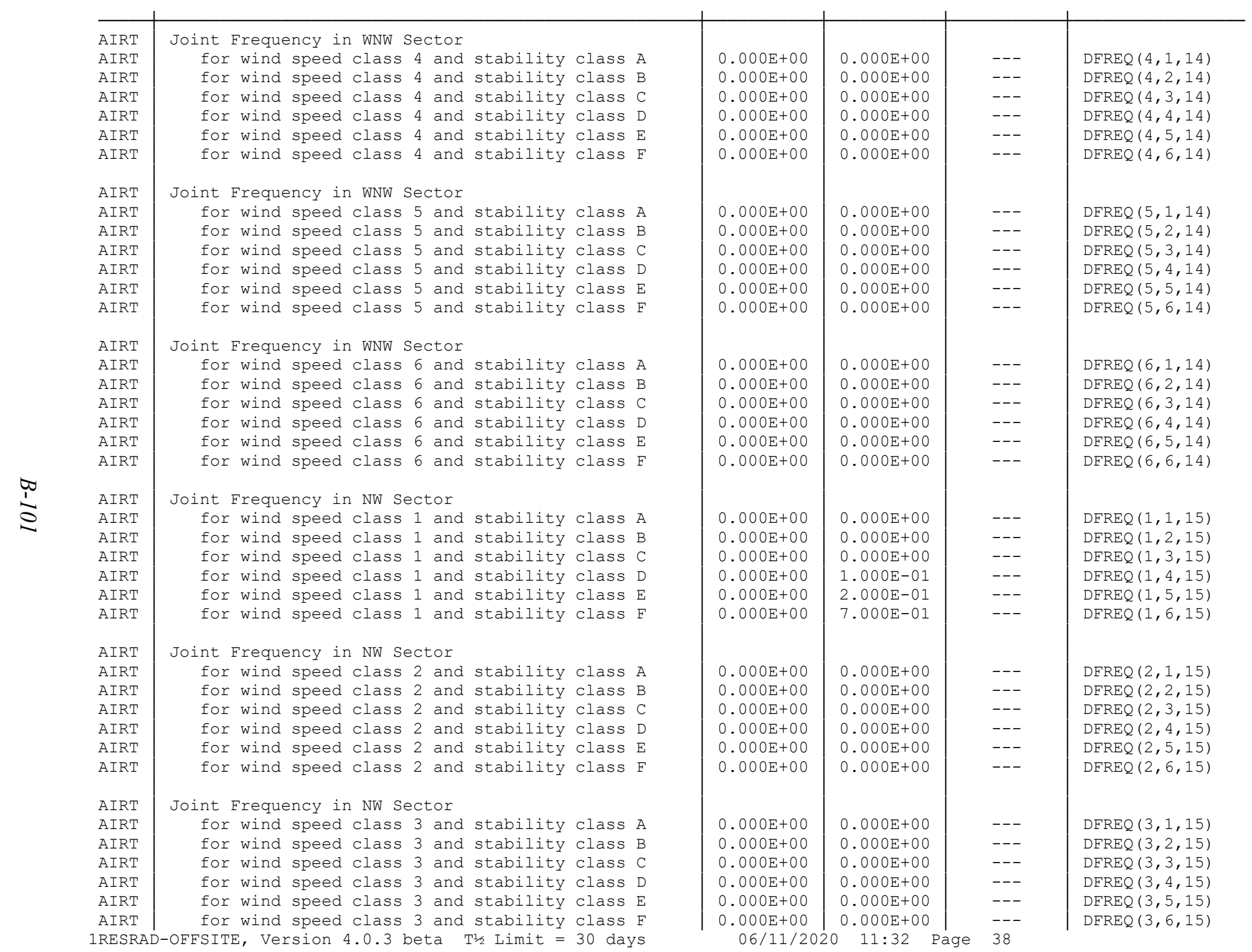




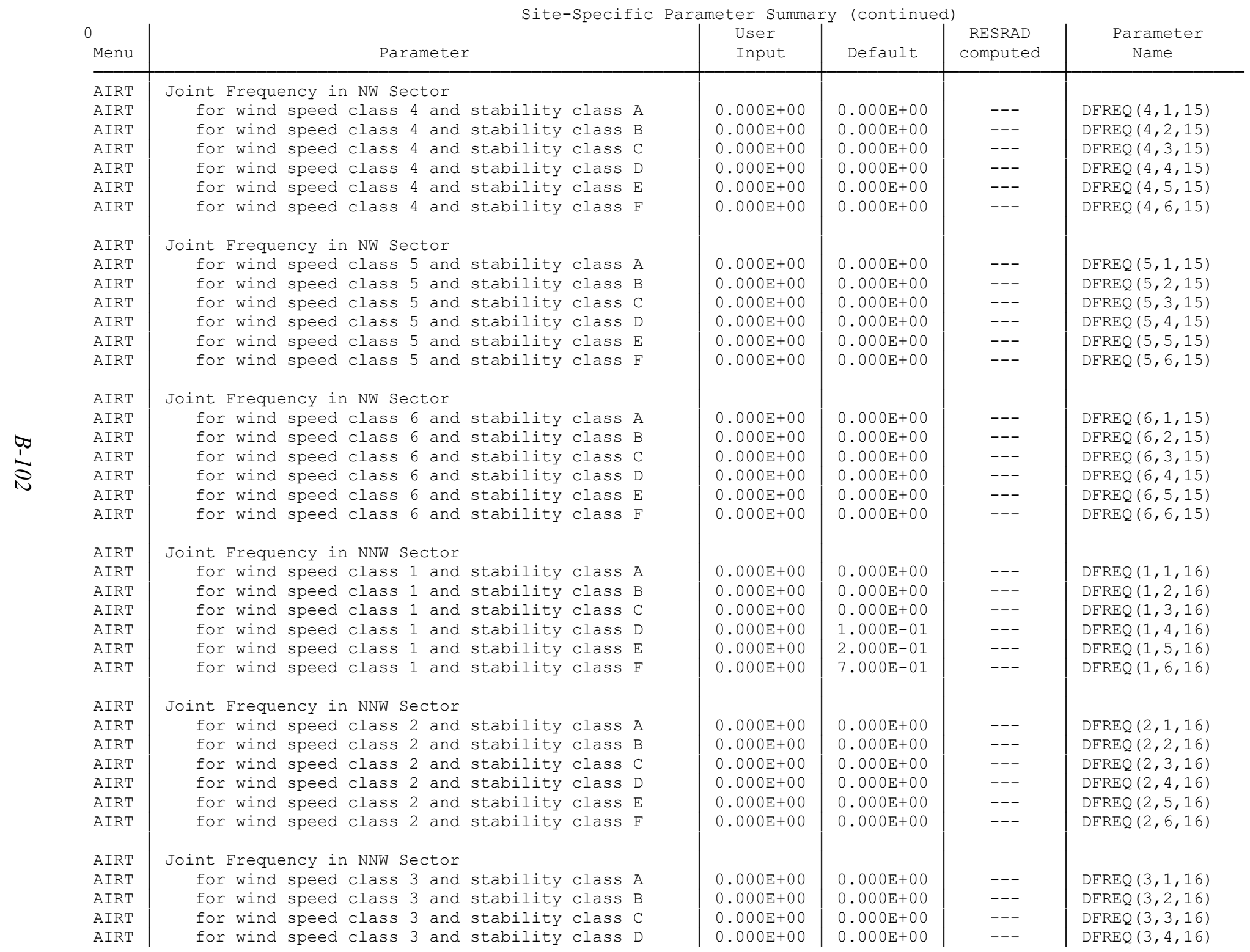


AIRT for wind speed class 3 and stability class E AIRT for wind speed class 3 and stability class $F$ 1RESRAD-OFFSITE, Version 4.0 .3 beta $\mathrm{T}^{1} \frac{1}{2}$ Limit $=30$ days Parent Dose Report

D-OFFSITE Effluent release

File : PADUCAH EFFLUENT RELEASE-REV1.ROF

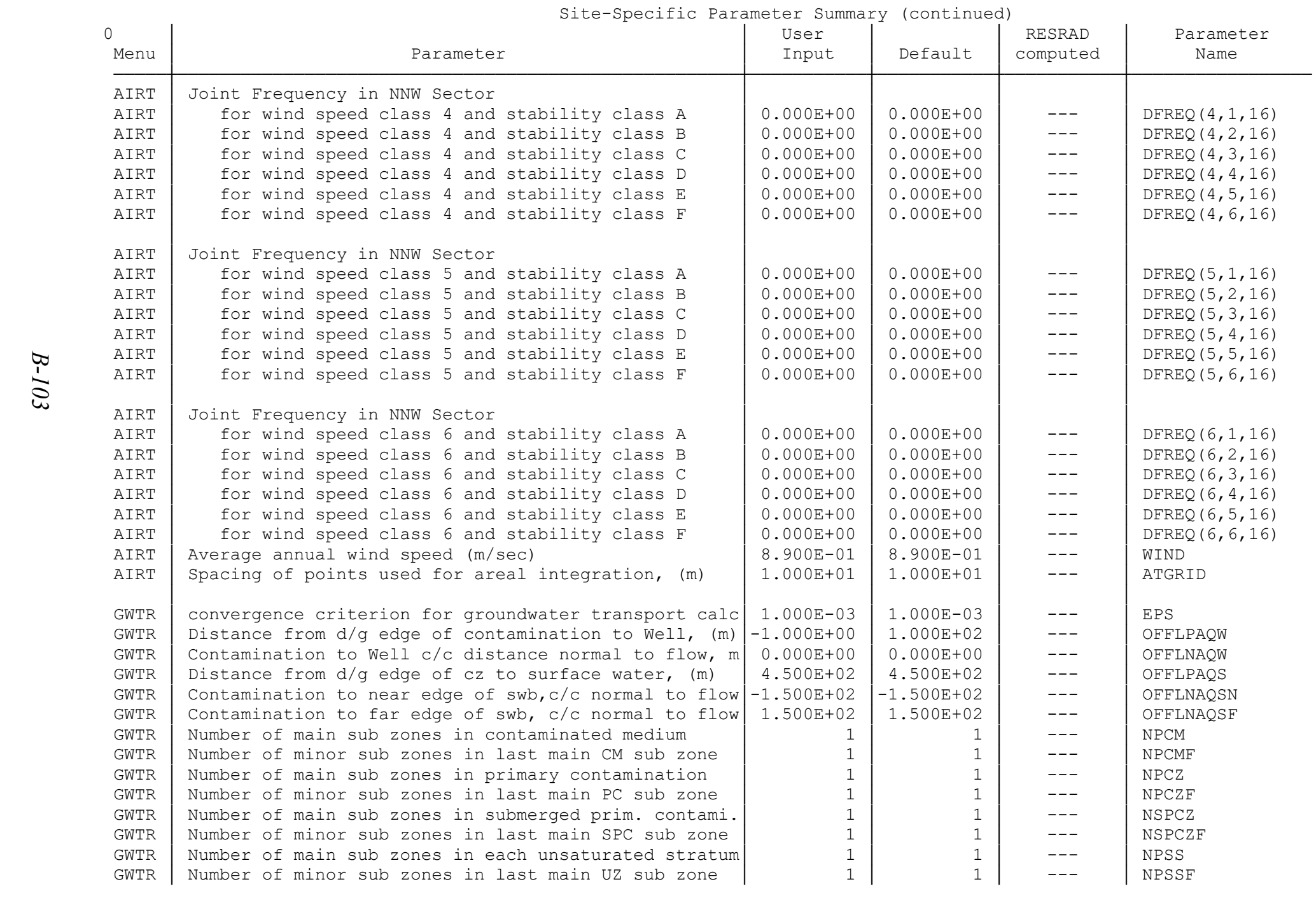

\begin{tabular}{c|c|c|c}
$0.000 \mathrm{E}+00$ & $0.000 \mathrm{E}+00$ & --- & DFREQ $(3,5,16)$ \\
$0.000 \mathrm{E}+00$ & $0.000 \mathrm{E}+00$ & -- & $\mathrm{DFREQ}(3,6,16)$
\end{tabular} 06/11/2020 11:32 Page 39 
GWTR $\mid$ Number of main sub zones in saturated stratum

GWTR Number of minor sub zones in last main Sz sub zone

GWTR Distribution coefficient and longitudinal dispersion

1 = Nuclide specific distrubution coefficients in al subzones. transformation.

GWTR | Retardation factor flag for groundwater transport $0=$ (total porosity + distribution coefficient*dr

1RESRAD-OFFSITE, Version 4.0 .3 beta T1/2 Limit $=30$ days

Parent Dose Report

Title : RESRAD-OFFSITE Effluent release

File : PADUCAH EFFLUENT RELEASE-REV1.ROF

Site-Specific Parameter summary (continued)

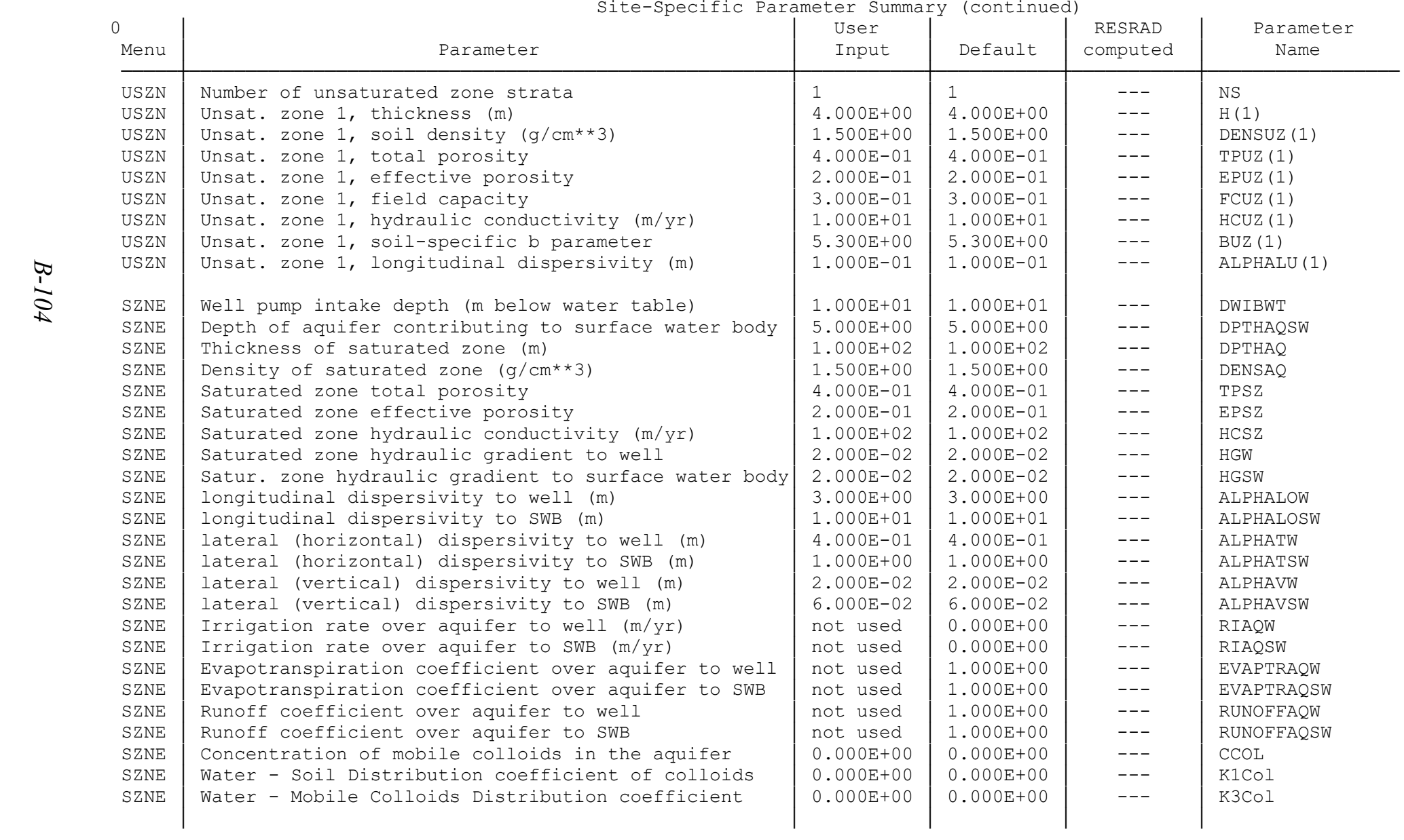

Longitudinal dispersion in all but the subzone of
0 | $--\quad \mid$

density) / total porosity

$11: 32$ Page 40 
WTRU Drinking water intake (L/yr)

WTRU Fraction of drinking water from surface water

WTRU Fraction of drinking water from well water

WTRU Fraction of household water from surface water

WTRU Fraction of household water from well water

WTRU Livestock water intake for meat 1 (L/day)

WTRU Fraction of livestock water 1 from surface water

WTRU Fraction of livestock water 1 from well water

WTRU Livestock water intake for milk (L/day)

WTRU Fraction of dairy cow water from surface water

WTRU Fraction of dairy cow water from well water

WTRU Irrigation rate in Agricultural Area 1 (m/yr)

1RESRAD-OFFSITE, Version 4.0 .3 beta T1/2 Limit $=30$ days Parent Dose Report

Title : RESRAD-OFFSITE Effluent release

File : PADUCAH EFFLUENT RELEASE-REV1.ROF

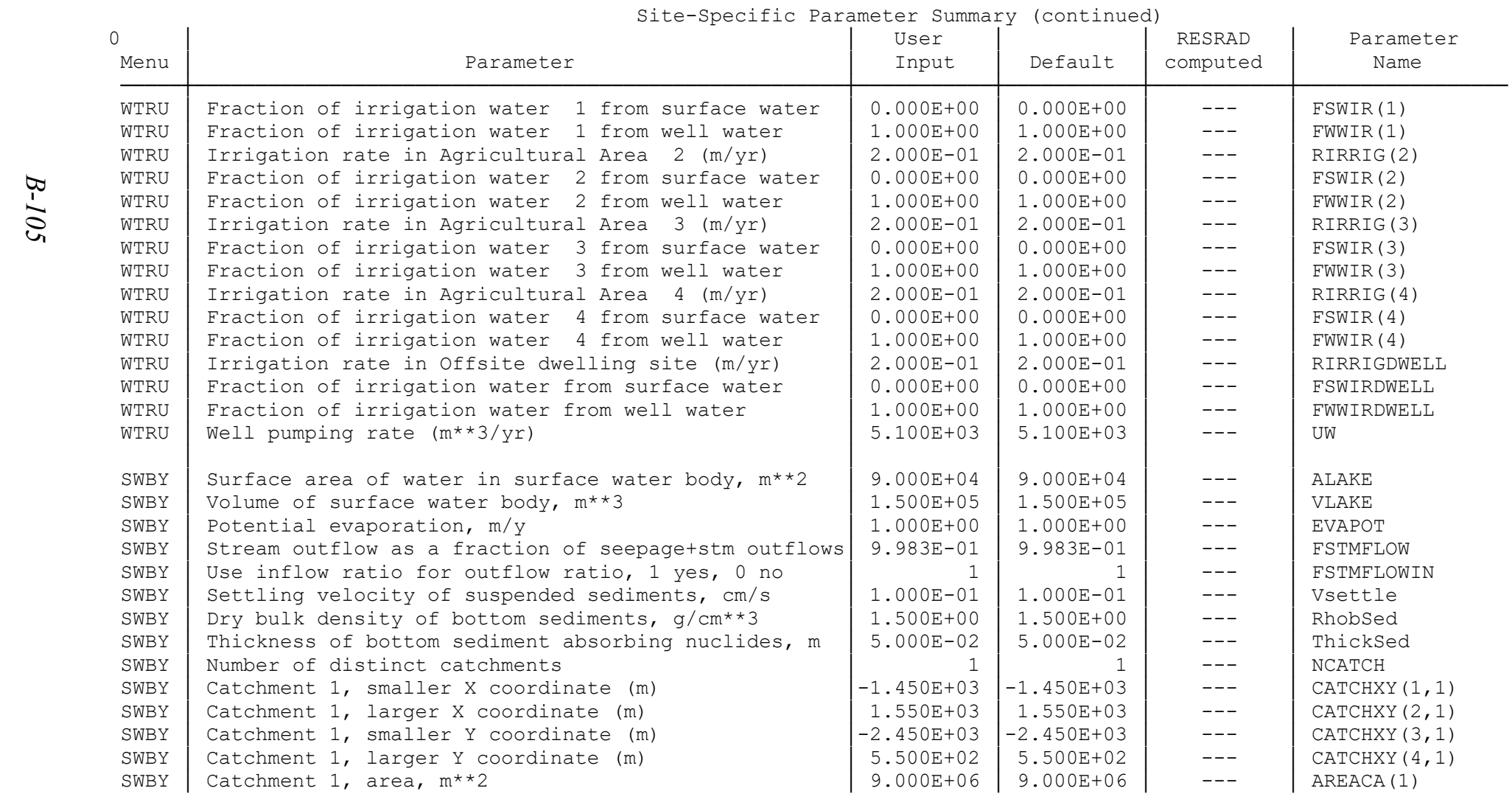

$06 / 11 / 202011: 32$ Page 41

RIRRIG (1)

\begin{tabular}{|l|l|l|l} 
not used & $5.100 \mathrm{E}+02$ & --- & DWI \\
not used & $0.000 \mathrm{E}+00$ & --- & FSWD \\
not used & $1.000 \mathrm{E}+00$ & --- & FWWD \\
$0.000 \mathrm{E}+00$ & $0.000 \mathrm{E}+00$ & --- & FSWHH \\
$1.000 \mathrm{E}+00$ & $1.000 \mathrm{E}+00$ & --- & FWWHH \\
not used & $5.000 \mathrm{E}+01$ & --- & LWI $(1)$ \\
not used & $0.000 \mathrm{E}+00$ & --- & FSWLV(1) \\
not used & $1.000 \mathrm{E}+00$ & --- & FWWLV(1) \\
not used & $1.600 \mathrm{E}+02$ & --- & LWI (2) \\
not used & $0.000 \mathrm{E}+00$ & --- & FSWLV (2) \\
not used & $1.000 \mathrm{E}+00$ & --- & FWWLV (2) \\
$2.000 \mathrm{E}-01$ & $2.000 \mathrm{E}-01$ & --- & RIRRIG (1)
\end{tabular}


SWBY Catchment 1, runoff coefficient

SWBY Catchment 1, soil erodibility factor, tons/acre

SWBY Catchment 1, Slope-length-steepness factor

SWBY Catchment 1, Cover and management factor

SWBY Catchment 1, support practice factor

SWBY Catchment 1, sediment delivery ratio

SWBY Catchment 1, use SRD - Area correlation, 1 yes, 0 no

SWBY Compute atmospheric deposition on catchment

SWBY Convergence criterion for deposition calculations

INGE Fish consumption ( $\mathrm{kg} / \mathrm{yr}$ )

INGE Fraction of Fish from affected area

INGE Other Aquatic food consumption ( $\mathrm{kg} / \mathrm{yr}$ )

INGE Fraction of Aquatic food from affected area

INGE Non-Leafy vegetables consumption ( $\mathrm{kg} / \mathrm{yr}$ )

INGE Fraction of vegetable 1 from affected area

1RESRAD-OFFSITE, Version 4.0 .3 beta $\mathrm{T}^{1 / 2}$ Limit $=30$ days

Parent Dose Report

Title : RESRAD-OFFSITE Effluent release

File : PADUCAH EFFLUENT RELEASE-REV1.ROF

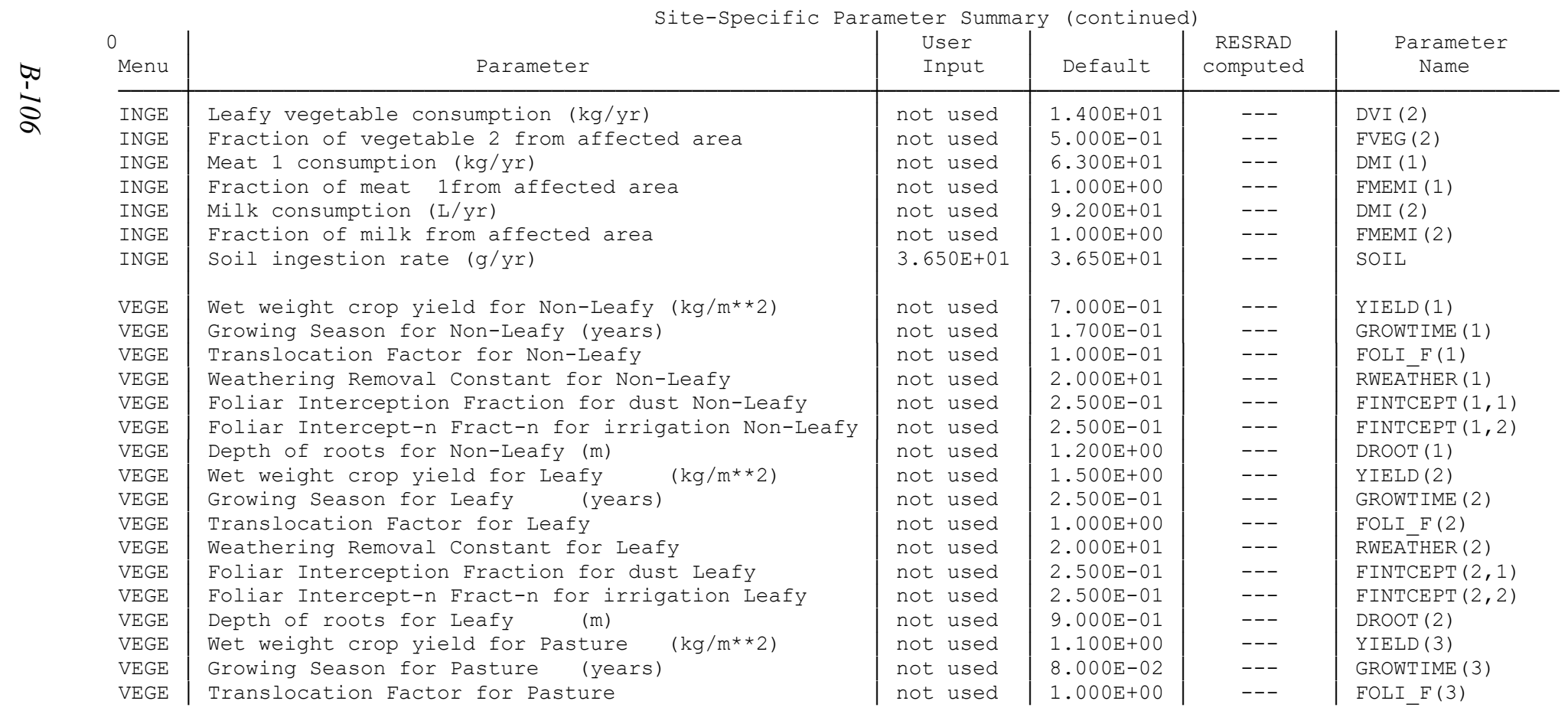

RUNOFFCA (1)

ERODIBILITYCA ( 1

SLPLENSTPCA ( 1 )

CRPMANGCA ( 1

DDRCA (1)

ComputeDep

ConvCritAtm

$\operatorname{DFI}(1)$

FFISH (1)

$\operatorname{DFI}(2)$ used $5.000 \mathrm{E}-01 \mid$ - 11 FVEG(1)

\begin{tabular}{|c|c|c|c|}
\hline $2.000 \mathrm{E}-01$ & $2.000 \mathrm{E}-01$ & --- & RUNOFFCA (1) \\
\hline $4.000 \mathrm{E}-01$ & $4.000 \mathrm{E}-01$ & --- & ERODIBILITYCA (1) \\
\hline $4.000 \mathrm{E}-01$ & $4.000 \mathrm{E}-01$ & --- & SLPLENSTPCA (1) \\
\hline $3.000 \mathrm{E}-03$ & $3.000 \mathrm{E}-03$ & --- & CRPMANGCA (1) \\
\hline $1.000 \mathrm{E}+00$ & $1.000 \mathrm{E}+00$ & --- & CONVPRACCA (1) \\
\hline $2.121 \mathrm{E}-01$ & $2.121 \mathrm{E}-01$ & --- & SDRCA (1) \\
\hline & 1 & --- & SDRACOR \\
\hline $2.000 \mathrm{E}-02$ & $2.000 \mathrm{E}-02$ & --- & DDRCA (1) \\
\hline yes & no & --- & ComputeDep \\
\hline $1.000 \mathrm{E}-03$ & $1.000 \mathrm{E}-03$ & --- & ConvCritAtm \\
\hline not used & $5.400 \mathrm{E}+00$ & --- & $\operatorname{DFI}(1)$ \\
\hline not used & $5.000 \mathrm{E}-01$ & --- & FFISH (1) \\
\hline not used & $9.000 \mathrm{E}-01$ & --- & DFI (2) \\
\hline not used & $5.000 \mathrm{E}-01$ & --- & FFISH (2) \\
\hline not used & $1.600 \mathrm{E}+02$ & --- & DVI (1) \\
\hline not used & $5.000 \mathrm{E}-01$ & --- & FVEG (1) \\
\hline $06 / 11 / 2$ & $11: 32$ & 42 & \\
\hline
\end{tabular}


VEGE Weathering Removal Constant for Pasture

VEGE Foliar Interception Fraction for dust Pasture

VEGE Depth of roots for Pasture (m)

VEGE Wet weight crop yield for Grain ( $\mathrm{kg} / \mathrm{m} * * 2)$

\begin{tabular}{l|l} 
VEGE & Growing Season for Grain (y \\
VEGE & Translocation Factor for Grain
\end{tabular}

VEGE Weathering Removal Constant for Grain

VEGE Foliar Interception Fraction for dust Grain

VEGE Foliar Intercept-n Fract-n for irrigation Grain

VEGE

$\begin{array}{ll}\text { Depth of roots for Grain } & \text { (m) }\end{array}$

Feed 1 intake by livestock 1 ( $\mathrm{kg} /$ day)

LINT Soil intake with feed 1 by livestock 1 ( $\mathrm{kg} /$ day)

LINT Feed 1 intake by dairy cow (kg/day)

LINT Soil intake with feed 1 by dairy cow (kg/day)

LINT Feed 2 intake by livestock 1 (kg/day)

LINT Soil intake with feed 2 by livestock 1 ( $\mathrm{kg} / \mathrm{day}$ )

LINT Feed 2 intake by dairy cow (kg/day)

LINT Soil intake with feed 2 by dairy cow (kg/day)

IRESRAD-OFFSITE, Version 4.0 .3 beta $T^{1 / 2}$ Limit $=30$ days Parent Dose Report

Title : RESRAD-OFFSITE Effluent release

File : PADUCAH EFFLUENT RELEASE-REV1.ROF

\begin{tabular}{|c|c|c|c|c|c|}
\hline $\begin{array}{l}0 \\
\text { Menu }\end{array}$ & Site-Specific Para & $\begin{array}{l}\text { eter Summ } \\
\text { User } \\
\text { Input }\end{array}$ & $\begin{array}{l}\text { (contin } \\
\text { Default }\end{array}$ & $\begin{array}{l}\text { RESRAD } \\
\text { computed }\end{array}$ & $\begin{array}{l}\text { Parameter } \\
\text { Name }\end{array}$ \\
\hline INHE & Inhalation rate $(m * * 3 / y r)$ & $8.400 \mathrm{E}+03$ & $8.400 \mathrm{E}+03$ & --- & INHALR \\
\hline INHE & Mass loading of all particulates from Primary contam & $1.000 \mathrm{E}-04$ & $1.000 \mathrm{E}-04$ & --- & MLFD \\
\hline INHE & Respirable particulates as a fraction of total & $1.000 \mathrm{E}+00$ & $1.000 \mathrm{E}+00$ & --- & RESPFRACPC \\
\hline INHE & Offsite mass loading same as onsite mass loading? & $0.000 \mathrm{E}+00$ & & --- & SAMEMLRF \\
\hline INHE & Total mass loading at agricultural area 1 ( $g / \mathrm{m} * * 3)$ & $1.000 \mathrm{E}-04$ & $1.000 \mathrm{E}-04$ & --- & MLTOTOF (1) \\
\hline INHE & Respirable fraction at agricultural area 1 & $1.000 \mathrm{E}+00$ & $1.000 \mathrm{E}+00$ & --- & RESPFRACOF (1) \\
\hline INHE & Total mass loading at agricultural area $2(\mathrm{~g} / \mathrm{m} * * 3)$ & $1.000 \mathrm{E}-04$ & $1.000 \mathrm{E}-04$ & --- & MLTOTOF (2) \\
\hline INHE & Respirable fraction at agricultural area 2 & $1.000 \mathrm{E}+00$ & $1.000 \mathrm{E}+00$ & --- & RESPFRACOF (2) \\
\hline INHE & Total mass loading at agricultural area $3(\mathrm{~g} / \mathrm{m} * * 3)$ & $1.000 \mathrm{E}-04$ & $1.000 \mathrm{E}-04$ & --- & MLTOTOF (3) \\
\hline INHE & Respirable fraction at agricultural area 3 & $1.000 \mathrm{E}+00$ & $1.000 \mathrm{E}+00$ & --- & RESPFRACOF (3) \\
\hline INHE & Total mass loading at agricultural area 4 ( $\mathrm{g} / \mathrm{n}$ & $1.000 \mathrm{E}-04$ & $1.000 \mathrm{E}-04$ & --- & MLTOTOF (4) \\
\hline INHE & Respirable fraction at agricultural area 4 & $1.000 \mathrm{E}+00$ & $1.000 \mathrm{E}-04$ & --- & RESPFRACOF (4) \\
\hline INHE & Total mass loading at offsite dwelling $(\mathrm{g} / \mathrm{m} * * 3)$ & $1.000 \mathrm{E}-04$ & $1.000 \mathrm{E}-04$ & --- & MLTOTDWELL \\
\hline INHE & Respirable fraction at offsite dwelling $(\mathrm{g} / \mathrm{m} * * 3)$ & $1.000 \mathrm{E}+00$ & $1.000 \mathrm{E}+00$ & --- & RESPFRACDWELL \\
\hline INHE & Indoor dust filtration factor, inhalation & $4.000 \mathrm{E}-01$ & $4.000 \mathrm{E}-01$ & --- & SHF3 \\
\hline INHE & Shielding factor, external gamma & $7.000 \mathrm{E}-01$ & $7.000 \mathrm{E}-01$ & --- & SHF1 \\
\hline INHE & Shape factor flag, external gamma & $-1.000 \mathrm{E}+00$ & $1.000 \mathrm{E}+00$ & noncircular & FS \\
\hline SEXT & Onsite shape factor array (used if non-circular): & & & & \\
\hline SEXT & Radii of shape factor array (used if non-circular): & & & & \\
\hline SEXT & Outer annular radius (m), ring 1 : & $1.667 \mathrm{E}-01$ & $6.000 \mathrm{E}+00$ & --- & RAD_SHAPE ( 1) \\
\hline SEXT & Outer annular radius (m), ring 2 : & $3.333 \mathrm{E}-01$ & $1.200 \mathrm{E}+01$ & --- & RAD $\operatorname{SHAPE}(2)$ \\
\hline SEXT & Outer annular radius ( $\mathrm{m}$ ), ring 3 : & $5.000 \mathrm{E}-01$ & $1.800 \mathrm{E}+01$ & --- & RAD_SHAPE ( 3) \\
\hline
\end{tabular}

\begin{tabular}{|l|l|l|l} 
not used & $2.000 \mathrm{E}+01$ & --- & $\operatorname{RWEATHER}(3)$ \\
not used & $2.500 \mathrm{E}-01$ & --- & $\operatorname{FINTCEPT}(3,1)$ \\
not used & $2.500 \mathrm{E}-01$ & --- & $\operatorname{FINTCEPT}(3,2)$ \\
not used & $9.000 \mathrm{E}-01$ & --- & $\operatorname{DROOT}(3)$ \\
not used & $7.000 \mathrm{E}-01$ & --- & $\operatorname{YIELD}(4)$ \\
not used & $1.700 \mathrm{E}-01$ & --- & $\operatorname{GROWTIME}(4)$ \\
not used & $1.000 \mathrm{E}-01$ & --- & $\operatorname{FOLI} \mathrm{F}(4)$ \\
not used & $2.000 \mathrm{E}+01$ & --- & $\operatorname{RWEATHER}(4)$ \\
not used & $2.500 \mathrm{E}-01$ & --- & $\operatorname{FINTCEPT}(4,1)$ \\
not used & $2.500 \mathrm{E}-01$ & --- & $\operatorname{FINTCEPT}(4,2)$ \\
not used & $1.200 \mathrm{E}+00$ & -- & $\operatorname{DROOT}(4)$ \\
& & & \\
not used & $1.400 \mathrm{E}+01$ & --- & $\operatorname{LFI}(1,1)$ \\
not used & $1.000 \mathrm{E}-01$ & --- & $\operatorname{LSI}(1,1)$ \\
not used & $4.400 \mathrm{E}+01$ & --- & $\operatorname{LFI}(2,1)$ \\
not used & $4.000 \mathrm{E}-01$ & --- & $\operatorname{LSI}(2,1)$ \\
not used & $5.400 \mathrm{E}+01$ & --- & $\operatorname{LFI}(1,2)$ \\
not used & $4.000 \mathrm{E}-01$ & --- & $\operatorname{LSI}(1,2)$ \\
not used & $1.100 \mathrm{E}+01$ & --- & $\operatorname{LFI}(2,2)$ \\
not used & $1.000 \mathrm{E}-01$ & --- & $\operatorname{LSI}(2,2)$ \\
&
\end{tabular}

06/11/2020 11:32 Page 43

$\operatorname{LFI}(2,2)$
$\operatorname{LSI}(2,2)$

RAD_SHAPE ( 3) 
SEXT Outer annular radius (m), ring 4:

SEXT Outer annular radius $(\mathrm{m})$, ring 5:

SEXT Outer annular radius (m), ring 6:

SEXT Outer annular radius (m), ring 7:

SEXT Outer annular radius (m), ring 8 :

SEXT Outer annular radius (m), ring 9:

SEXT Outer annular radius (m), ring 10:

SEXT Outer annular radius (m), ring 11

SEXT Fractions of annular areas within AREA:

SEXT Ring 1

SEXT Ring 2

SEXT Ring 3

SEXT Ring 4

SEXT Ring 5

SEXT Ring 6

SEXT Ring 7

SEXT Ring 8

SEXT

SEXT RI Ring 10

SEXT Ring 11

SEXT Ring 12

RESRAD-OFFSITE, Version 4.0 .3 beta $T^{1 / 2}$ Limit $=30$ days

Parent Dose Report

File : RESRAD-OFFSITE Effluent release

\begin{tabular}{|c|c|c|c|}
\hline $6.667 \mathrm{E}-01$ & $2.400 \mathrm{E}+01$ & --- & RAD_SHAPE ( 4) \\
\hline $8.333 \mathrm{E}-01$ & $3.000 \mathrm{E}+01$ & --- & RAD_SHAPE ( 5) \\
\hline $1.000 \mathrm{E}+00$ & $3.600 \mathrm{E}+01$ & --- & RAD_SHAPE ( 6) \\
\hline $1.167 \mathrm{E}+00$ & $4.200 \mathrm{E}+01$ & --- & RAD_SHAPE ( 7) \\
\hline $1.333 \mathrm{E}+00$ & $4.800 \mathrm{E}+01$ & --- & RAD_SHAPE ( 8) \\
\hline $1.500 \mathrm{E}+00$ & $5.400 \mathrm{E}+01$ & --- & RAD_SHAPE ( 9) \\
\hline $1.667 \mathrm{E}+00$ & $6.000 \mathrm{E}+01$ & --- & RAD_SHAPE (10) \\
\hline $1.833 \mathrm{E}+00$ & $6.600 \mathrm{E}+01$ & --- & RAD_SHAPE (11) \\
\hline $2.000 \mathrm{E}+00$ & $7.200 \mathrm{E}+01$ & --- & RAD_SHAPE (12) \\
\hline $1.000 \mathrm{E}+00$ & $1.000 \mathrm{E}+00$ & --- & FRACA ( 1) \\
\hline $0.000 \mathrm{E}+00$ & $1.000 \mathrm{E}+00$ & --- & FRACA ( 2) \\
\hline $0.000 \mathrm{E}+00$ & $1.000 \mathrm{E}+00$ & --- & FRACA ( 3) \\
\hline $0.000 \mathrm{E}+00$ & $1.000 \mathrm{E}+00$ & --- & FRACA ( 4) \\
\hline $0.000 \mathrm{E}+00$ & $1.000 \mathrm{E}+00$ & --- & FRACA ( 5) \\
\hline $0.000 \mathrm{E}+00$ & $1.000 \mathrm{E}+00$ & --- & FRACA ( 6) \\
\hline $0.000 \mathrm{E}+00$ & $1.000 \mathrm{E}+00$ & --- & FRACA ( 7) \\
\hline $0.000 \mathrm{E}+00$ & $1.000 \mathrm{E}+00$ & --- & FRACA ( 8) \\
\hline $0.000 \mathrm{E}+00$ & $7.700 \mathrm{E}-01$ & --- & FRACA ( 9) \\
\hline $0.000 \mathrm{E}+00$ & $3.700 \mathrm{E}-01$ & --- & FRACA (10) \\
\hline $0.000 \mathrm{E}+00$ & $1.700 \mathrm{E}-01$ & --- & FRACA (11) \\
\hline $000 \mathrm{E}+00$ & $3.100 \mathrm{E}-02$ & --- & FRACA (12) \\
\hline
\end{tabular}

\begin{tabular}{|c|c|c|c|c|c|}
\hline $\begin{array}{l}0 \\
\text { Menu }\end{array}$ & Site-Specific Pa & $\begin{array}{l}\text { eter Summa } \\
\text { User } \\
\text { Input }\end{array}$ & $\begin{array}{l}\text { (continu } \\
\text { Default }\end{array}$ & $\begin{array}{l}\text { RESRAD } \\
\text { computed }\end{array}$ & $\begin{array}{l}\text { Parameter } \\
\text { Name }\end{array}$ \\
\hline SEXT & Shape factor array from offsite dwelling: & & & & \\
\hline SEXT & Outer annular radius (m), ring 14: & $8.335 \mathrm{E}+02$ & $2.650 \mathrm{E}+01$ & --- & RAD_SHAPE (14) \\
\hline SEXT & Outer annular radius (m), ring 15: & $1.250 \mathrm{E}+03$ & $3.975 \mathrm{E}+01$ & --- & RAD ${ }^{-}$SHAPE (15) \\
\hline SEXT & Outer annular radius (m), ring 16: & $1.667 \mathrm{E}+03$ & $5.300 \mathrm{E}+01$ & --- & $\mathrm{RAD}^{-} \mathrm{SHAPE}(16)$ \\
\hline SEXT & Outer annular radius (m), ring 20: & $3.334 \mathrm{E}+03$ & $1.060 \mathrm{E}+02$ & --- & RAD_SHAPE (20) \\
\hline SEXT & Outer annular radius (m), ring 21: & $3.751 \mathrm{E}+03$ & $1.192 \mathrm{E}+02$ & --- & $\mathrm{RAD}^{-} \mathrm{SHAPE}(21)$ \\
\hline SEXT & Outer annular radius (m), ring 22: & $4.168 \mathrm{E}+03$ & $1.325 \mathrm{E}+02$ & --- & RAD SHAPE (22) \\
\hline SEXT & Outer annular radius (m), ring 23: & $4.584 \mathrm{E}+03$ & $1.458 \mathrm{E}+02$ & --- & RAD_SHAPE (23) \\
\hline SEXT & Outer annular radius (m), ring 24: & $5.001 \mathrm{E}+03$ & $1.590 \mathrm{E}+02$ & --- & $\mathrm{RAD}^{-} \mathrm{SHAPE}(24)$ \\
\hline SEXT & Fractions of annular areas within AREA: & & & & \\
\hline SEXT & Ring 13 & $0.000 \mathrm{E}+00$ & $0.000 \mathrm{E}+00$ & --- & FRACA (13) \\
\hline
\end{tabular}




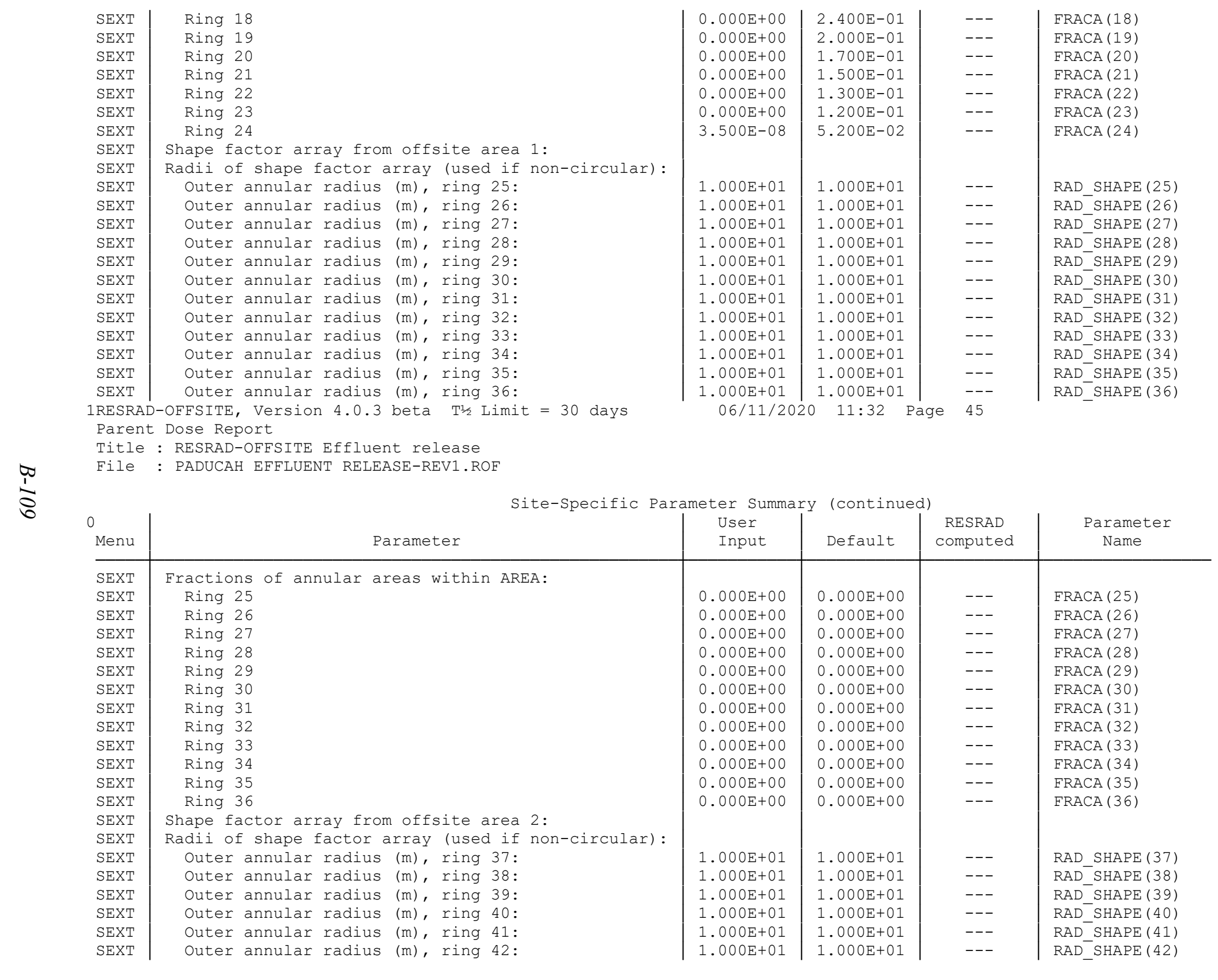


SEXT Outer annular radius (m), ring 43:

SEXT Outer annular radius (m), ring 44:

SEXT Outer annular radius (m), ring 45:

Outer annular radius (m), ring 46

SEXT Outer annular radius (m), ring 47

SEXT Outer annular radius (m), ring 48 :

SEXT Fractions of annular areas within AREA:

SEXT Ring 37

Ring 38

SEXT

SEXT Ring 41

SEXT Ring 42

SEXT Ring 43

SEXT Ring 44

SEXT Ring 45

SEXT Ring 46

SEXT Ring 47

SEXT Ring 48

IRESRAD-OFFSITE, Version 4.0 .3 beta $T^{1 / 1} / 2$ Limit $=30$ days

Parent Dose Report

Title : RESRAD-OFFSITE Effluent release

File : PADUCAH EFFLUENT RELEASE-REV1.ROF

\begin{tabular}{|c|c|c|c|}
\hline $1.000 \mathrm{E}+01$ & $1.000 \mathrm{E}+01$ & --- & RAD_SHAPE (43) \\
\hline $1.000 \mathrm{E}+01$ & $1.000 \mathrm{E}+01$ & --- & RAD_SHAPE (44) \\
\hline $1.000 \mathrm{E}+01$ & $1.000 \mathrm{E}+01$ & --- & RAD $^{-} \operatorname{SHAPE}(45)$ \\
\hline $1.000 \mathrm{E}+01$ & $1.000 \mathrm{E}+01$ & --- & $\mathrm{RAD}^{-} \operatorname{SHAPE}(46)$ \\
\hline $1.000 \mathrm{E}+01$ & $1.000 \mathrm{E}+01$ & --- & RAD $^{-} \operatorname{SHAPE}(47)$ \\
\hline $1.000 \mathrm{E}+01$ & $1.000 \mathrm{E}+01$ & --- & RAD_SHAPE (48) \\
\hline $0.000 \mathrm{E}+00$ & $0.000 \mathrm{E}+00$ & --- & FRACA (37) \\
\hline $0.000 \mathrm{E}+00$ & $0.000 \mathrm{E}+00$ & --- & FRACA (38) \\
\hline $0.000 \mathrm{E}+00$ & $0.000 \mathrm{E}+00$ & --- & FRACA (39) \\
\hline $0.000 \mathrm{E}+00$ & $0.000 \mathrm{E}+00$ & --- & FRACA (40) \\
\hline $0.000 \mathrm{E}+00$ & $0.000 \mathrm{E}+00$ & --- & FRACA (41) \\
\hline $0.000 \mathrm{E}+00$ & $0.000 \mathrm{E}+00$ & --- & FRACA (42) \\
\hline $0.000 \mathrm{E}+00$ & $0.000 \mathrm{E}+00$ & --- & FRACA (43) \\
\hline $0.000 \mathrm{E}+00$ & $0.000 \mathrm{E}+00$ & --- & FRACA (44) \\
\hline $0.000 \mathrm{E}+00$ & $0.000 \mathrm{E}+00$ & --- & FRACA (45) \\
\hline $0.000 \mathrm{E}+00$ & $0.000 \mathrm{E}+00$ & --- & FRACA (46) \\
\hline $0.000 \mathrm{E}+00$ & $0.000 \mathrm{E}+00$ & --- & FRACA (47) \\
\hline $0.000 \mathrm{E}+00$ & $0.000 \mathrm{E}+00$ & --- & FRACA (48) \\
\hline
\end{tabular}

Site-Specific Parameter Summary (continued)

\section{$\stackrel{\infty}{5}$}

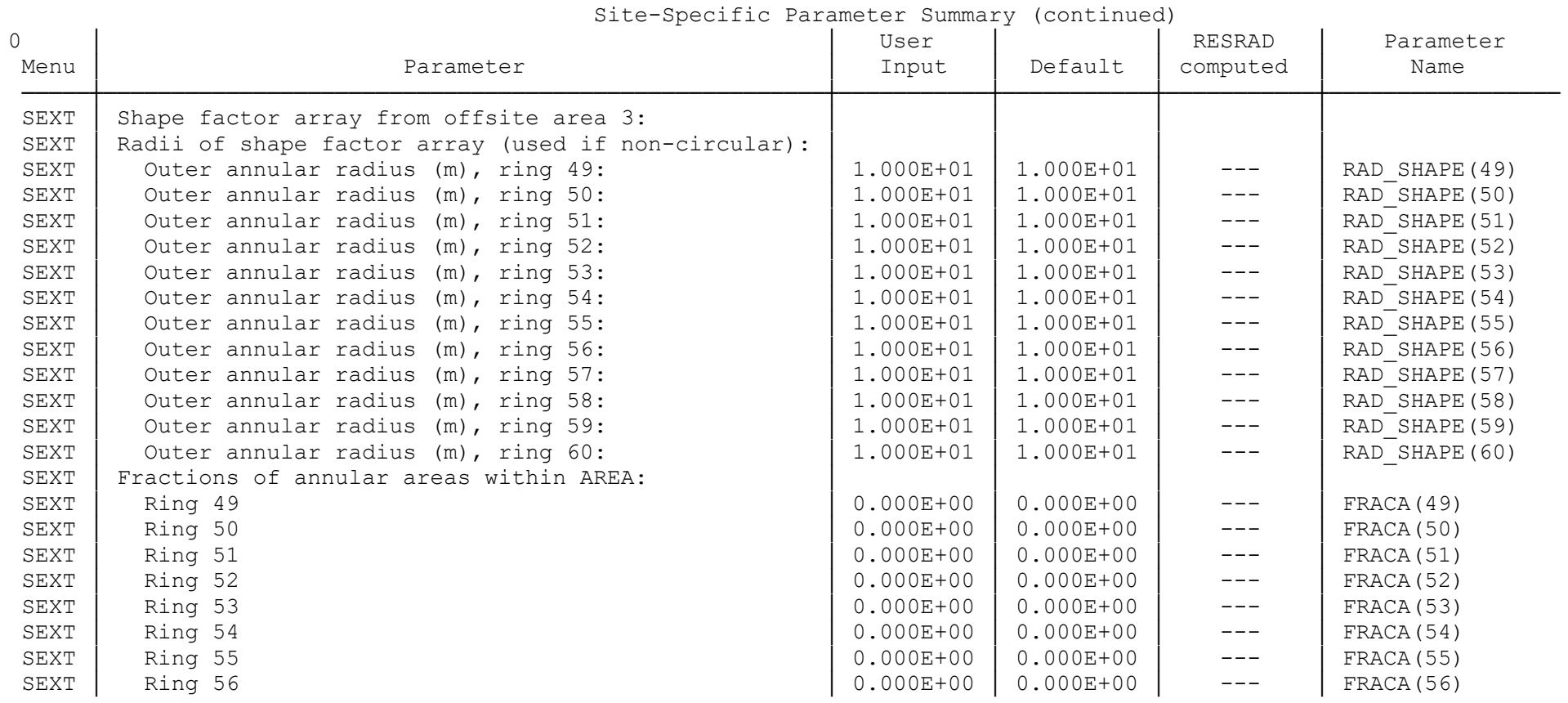




\begin{tabular}{l|ll} 
SEXT & Ring 57 & \\
SEXT & Ring 58 & \\
SEXT & Ring 59 & \\
SEXT & Ring 60 & \\
SEXT & Shape factor array from offsite area $4:$ \\
SEXT & Radii of shape factor array (used if non-circular): \\
SEXT & Outer annular radius (m), ring $61:$ \\
SEXT & Outer annular radius (m), ring $62:$ \\
SEXT & Outer annular radius (m), ring $63:$ \\
SEXT & Outer annular radius (m), ring $64:$ \\
SEXT & Outer annular radius (m), ring $65:$ \\
SEXT & Outer annular radius (m), ring $66:$ \\
SEXT & Outer annular radius (m), ring $67:$ \\
SEXT & Outer annular radius (m), ring $68:$ \\
SEXT & Outer annular radius (m), ring $69:$ \\
SEXT & Outer annular radius (m), ring $70:$ \\
SEXT & Outer annular radius (m), ring $71:$ \\
SEXT & Outer annular radius (m), ring $72:$ \\
REERAD-OFFSITE, Version 4.0 .3 beta T1/2 Limit $=30$ days \\
Parent Dose Report \\
Title : RESRAD-OFFSITE Effluent release \\
File : PADUCAH EFFUENT RELEASE-REV1.ROF
\end{tabular}

\begin{tabular}{|l|l|l|l}
$0.000 \mathrm{E}+00$ & $0.000 \mathrm{E}+00$ & --- & FRACA (57) \\
$0.000 \mathrm{E}+00$ & $0.000 \mathrm{E}+00$ & -- & FRACA (58) \\
$0.000 \mathrm{E}+00$ & $0.000 \mathrm{E}+00$ & --- & FRACA (59) \\
$0.000 \mathrm{E}+00$ & $0.000 \mathrm{E}+00$ & --- & FRACA (60) \\
& & & \\
& & & \\
$1.000 \mathrm{E}+01$ & $1.000 \mathrm{E}+01$ & --- & RAD_SHAPE (61) \\
$1.000 \mathrm{E}+01$ & $1.000 \mathrm{E}+01$ & --- & RAD_SHAPE (62) \\
$1.000 \mathrm{E}+01$ & $1.000 \mathrm{E}+01$ & --- & RAD_SHAPE (63) \\
$1.000 \mathrm{E}+01$ & $1.000 \mathrm{E}+01$ & --- & RAD_SHAPE (64) \\
$1.000 \mathrm{E}+01$ & $1.000 \mathrm{E}+01$ & --- & RAD_SHAPE (65) \\
$1.000 \mathrm{E}+01$ & $1.000 \mathrm{E}+01$ & -- & RAD_SHAPE (66) \\
$1.000 \mathrm{E}+01$ & $1.000 \mathrm{E}+01$ & --- & RAD_SHAPE (67) \\
$1.000 \mathrm{E}+01$ & $1.000 \mathrm{E}+01$ & --- & RAD_SHAPE (68) \\
$1.000 \mathrm{E}+01$ & $1.000 \mathrm{E}+01$ & --- & RAD_SHAPE (69) \\
$1.000 \mathrm{E}+01$ & $1.000 \mathrm{E}+01$ & --- & RAD_SHAPE (70) \\
$1.000 \mathrm{E}+01$ & $1.000 \mathrm{E}+01$ & -- & RAD_SHAPE (71) \\
$1.000 \mathrm{E}+01$ & $1.000 \mathrm{E}+01$ & --- & RAD_SHAPE (72)
\end{tabular}

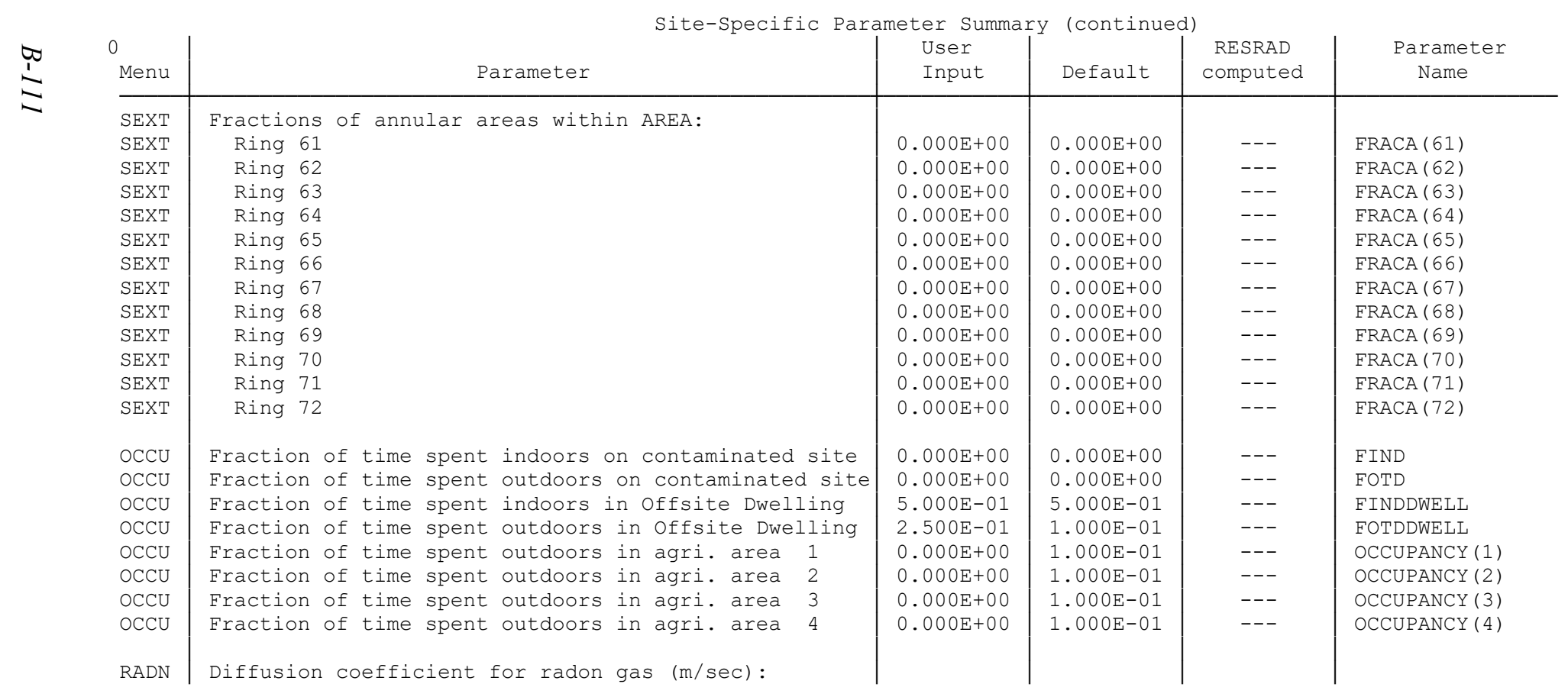




\begin{tabular}{|c|c|c|c|c|c|}
\hline & & & \\
\hline RADN & in cover material & not used & $2.000 \mathrm{E}-06$ & --- & DIFCV \\
\hline RADN & in contaminated zone soil & not used & $2.000 \mathrm{E}-06$ & --- & DIFCZ \\
\hline RADN & in fruit, grain and non-leafy vegetable field & not used & $2.000 \mathrm{E}-06$ & --- & DIFOS (1) \\
\hline RADN & in leafy vegetable field & not used & $2.000 \mathrm{E}-06$ & --- & $\operatorname{DIFOS}(2)$ \\
\hline RADN & in pature & not used & $2.000 \mathrm{E}-06$ & --- & DIFOS (3) \\
\hline RADN & in livestock grain field & not used & $2.000 \mathrm{E}-06$ & --- & DIFOS (4) \\
\hline RADN & in offsite dwelling site & not used & $2.000 \mathrm{E}-06$ & --- & DIFOS (5) \\
\hline RADN & in foundation material & not used & $3.000 \mathrm{E}-07$ & --- & DIFFL \\
\hline RADN & Thickness of building foundation (m) & not used & $1.500 \mathrm{E}-01$ & --- & FLOOR1 \\
\hline RADN & Bulk density of building foundation $(\mathrm{g} / \mathrm{cm} * * 3)$ & not used & $2.400 \mathrm{E}+00$ & --- & DENSFL \\
\hline RADN & Total porosity of the building foundation & not used & $1.000 \mathrm{E}-01$ & --- & TPFL \\
\hline RADN & Volumetric water content of the foundation & not used & $3.000 \mathrm{E}-02$ & --- & PH2OFL \\
\hline RADN & Building depth below ground surface (m) & not used & $-1.000 \mathrm{E}+00$ & --- & DMFL \\
\hline RADN & Radon vertical dimension of mixing (m) & not used & $2.000 \mathrm{E}+00$ & --- & HMIX \\
\hline RADN & Height of the building (room) (m) & not used & $2.500 \mathrm{E}+00$ & --- & HRM \\
\hline RADN & Average building air exchange rate $(1 / h r)$ & not used & $5.000 \mathrm{E}-01$ & --- & REXG \\
\hline RADN & Building interior area factor & not used & $0.000 \mathrm{E}+00$ & --- & FAI \\
\hline RADN & Emanating power of $\mathrm{Rn}-222$ gas & not used & $2.500 \mathrm{E}-01$ & --- & EMANA (1) \\
\hline RADN & Emanating power of $\mathrm{Rn}-220$ gas & not used & $1.500 \mathrm{E}-01$ & --- & EMANA (2) \\
\hline C14 & $\mathrm{C}-14$ evasion layer thickness in soil (m) & not used & $3.000 \mathrm{E}-01$ & --- & DMC \\
\hline $\mathrm{C} 14$ & Vertical dimension of mixing for vegetation (m) & not used & $1.000 \mathrm{E}+00$ & --- & HMIXV \\
\hline C14 & C-14 evasion flux rate from soil ( $1 / \mathrm{sec})$ & not used & $7.000 \mathrm{E}-07$ & --- & C14EVSN \\
\hline $1 \mathrm{RESR}$ & OFFSITE, Version 4.0 .3 beta $T^{1} \frac{1}{2}$ Limit $=30$ days & $06 / 11 / 2$ & $20 \quad 11: 32$ & 48 & \\
\hline
\end{tabular}

i $\quad$ Parent Dose Report

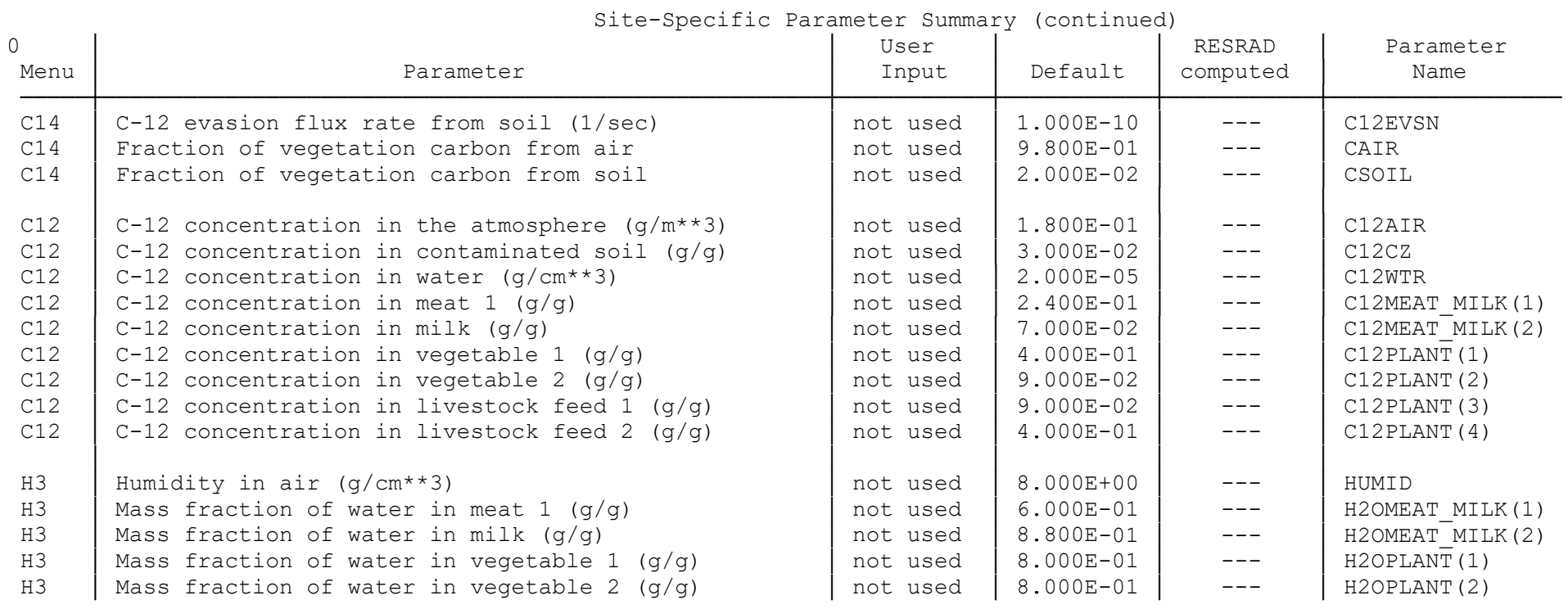


Summary of Pathway Selections

\begin{tabular}{c|c} 
Pathway & User Selection \\
\hline $1--$ external gamma & active \\
$2--$ inhalation (w/o radon) & active \\
$3--$ plant ingestion & suppressed \\
$4--$ meat ingestion & suppressed \\
$5--$ milk ingestion & suppressed \\
$6--$ aquatic foods & suppressed \\
$7--$ drinking water & active \\
$8--$ soil ingestion & suppressed \\
\hline
\end{tabular}

1RESRAD-OFFSITE, Version 4.0 .3 beta $T^{1 \frac{1}{2}}$ Limit $=30$ days

Parent Dose Report

Title : RESRAD-OFFSITE Effluent release

File : PADUCAH EFFLUENT RELEASE-REV1.ROF

Contaminated Zone Dimensions

Initial Soil Concentrations, pCi/g

Area: $\quad 1.00$ square meters

Thickness: $\quad 2.00$ meters

Cover Depth: $\quad 0.00$ meters

\begin{tabular}{ll}
\hline $\mathrm{Am}-241$ & $3.000 \mathrm{E}+00$ \\
$\mathrm{Cs}-137$ & $3.000 \mathrm{E}+00$ \\
$\mathrm{~Np}-237$ & $3.000 \mathrm{E}+01$ \\
$\mathrm{Pu}-238$ & $3.000 \mathrm{E}+00$ \\
$\mathrm{Pu}-239$ & $3.000 \mathrm{E}+00$ \\
$\mathrm{Pu}-240$ & $3.000 \mathrm{E}+00$ \\
$\mathrm{Tc}-99$ & $3.000 \mathrm{E}+01$ \\
$\mathrm{Th}-228$ & $3.000 \mathrm{E}+00$ \\
$\mathrm{Th}-230$ & $3.000 \mathrm{E}+00$ \\
$\mathrm{Th}-232$ & $3.000 \mathrm{E}+00$ \\
$\mathrm{U}-234$ & $3.000 \mathrm{E}+01$ \\
$\mathrm{U}-235$ & $3.000 \mathrm{E}+01$ \\
$\mathrm{U}-238$ & $3.000 \mathrm{E}+01$
\end{tabular}

Total Dose TDOSE(t), mrem/yr

Basic Radiation Dose Iimit $=2.500 \mathrm{~F}+01 \mathrm{mrem} / \mathrm{yr}$

Total Mixture Sum M(t) = Fraction of Basic Dose Limit Received at Time (t)

$t$ (years): $\begin{array}{llllll}0.000 \mathrm{E}+00 & 1.000 \mathrm{E}+00 & 3.000 \mathrm{E}+00 & 6.000 \mathrm{E}+00 & 1.000 \mathrm{E}+01\end{array}$

TDOSE $(t): \begin{array}{lllll}1.350 \mathrm{E}-01 & 1.350 \mathrm{E}-01 & 1.350 \mathrm{E}-01 & 1.351 \mathrm{E}-01 & 1.352 \mathrm{E}-01\end{array}$

$\mathrm{M}(\mathrm{t}): \begin{array}{lllll}5.400 \mathrm{E}-03 & 5.400 \mathrm{E}-03 & 5.402 \mathrm{E}-03 & 5.404 \mathrm{E}-03 & 5.407 \mathrm{E}-03\end{array}$

OMaximum TDOSE(t): $1.356 \mathrm{E}-01 \mathrm{mrem} / \mathrm{yr}$ at $t=40$ years

1RESRAD-OFFSITE, Version 4.0 .3 beta T1/2 Limit $=30$ days

Parent Dose Report 
Title : RESRAD-OFFSITE Effluent release

File : PADUCAH EFFLUENT RELEASE-REV1.ROF

Total Dose Contributions TDOSE (i,p,t) for Individual Radionuclides (i) and Pathways (p) in mrem/yr and as a Percentage of Total Dose at $t=0$ years

0

Radio

Nuclide

Am-241

$\begin{array}{llll}\mathrm{Cs}-137 & 0.00 \mathrm{E}+00 & 0 & 0.00 \mathrm{E}+00\end{array}$

$\begin{array}{llll}\mathrm{Pu}-238 & 0.00 \mathrm{E}+00 & 0 & 0.00 \mathrm{E}+00\end{array}$

$\begin{array}{llll}\mathrm{Pu}-239 & 0.00 \mathrm{E}+00 & 0 & 0.00 \mathrm{E}+00\end{array}$

$\begin{array}{llll}\mathrm{Pu}-240 & 0.00 \mathrm{E}+00 & 0 & 0.00 \mathrm{E}+00\end{array}$

$\begin{array}{llll}\mathrm{TC}-99 & 0.00 \mathrm{E}+00 & 0 & 0.00 \mathrm{E}+00\end{array}$

Th-228 $0.00 \mathrm{E}+00 \quad 0 \quad 0.00 \mathrm{E}+00$

Th-230 $-0.00 \mathrm{E}+00$

$\mathrm{U}-234=0.00 \mathrm{E}+00-0.0 .00 \mathrm{E}+00$

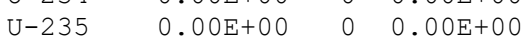

$\mathrm{U}-238$

Radon
Plant Meat
Milk Soi Water

Dose $\%$ Dose

$0.00 \mathrm{E}+00$

Dose $\frac{\circ}{0}$

$0.00 \mathrm{E}+00 \quad 0 \quad 0.00 \mathrm{E}+00$

$\overline{0.00 E+00}-$

$0.00 \mathrm{E}+00$

$0.00 \mathrm{E}+00$

$0.00 \mathrm{E}+00$

$0.00 \mathrm{E}+00$

Dose $\frac{\circ}{0}$
Dose Dose \%

$0.00 \mathrm{E}+00$

$0.00 \mathrm{E}+00$

$0.00 \mathrm{E}+00$

$0.0 .00 \mathrm{E}+00$

$0.00 \mathrm{E}+00$

$0.00 \mathrm{E}+00$

$0.00 \mathrm{E}+00$

$0.00 \mathrm{E}+00$

$0.00 \mathrm{E}+00$

$0 \quad 0.00 \mathrm{E}+00$

$\begin{array}{ll}0 & 0.00 \mathrm{E}+00 \\ 0 & 0.00 \mathrm{E}+00\end{array}$

$0.00 \mathrm{E}+00$

$0.00 \mathrm{E}+00$

$0 \quad 0.00 \mathrm{E}+00$

$0.00 \mathrm{E}+00 \quad 0.000 \mathrm{E}+00$

$0.00 \mathrm{E}+00$

$0.00 \mathrm{E}+00$

$0.00 \mathrm{E}+00$

$0.00 \mathrm{E}+00$
$0.00 \mathrm{E}+00$

$0.00 \mathrm{E}+00$

$0.00 \mathrm{E}+00$

$0.00 \mathrm{E}+00$

Total Dose Con

$0.00 \mathrm{E}+00$

$0.00 \mathrm{E}+00$

$0.00 \mathrm{E}+00$

$0.00 \mathrm{E}+00$

$0.00 \mathrm{E}+00$

$0.00 \mathrm{E}+00$

$0.00 \mathrm{E}+00$

$0.00 \mathrm{E}+00$

$0.00 \mathrm{E}+00$
$0.00 \mathrm{E}+00$

$0.00 \mathrm{E}+00$

$0.00 \mathrm{E}+00$

$0.00 \mathrm{E}+00$

$\overline{0.00 \mathrm{E}+00}$

$\overline{0.00 \mathrm{E}+00}=\overline{0.00 \mathrm{E}+00}$

$\begin{array}{llll}0.00 \mathrm{E}+00 & 0 & 0.00 \mathrm{E}+00\end{array}$

$\begin{array}{lll}0.00 \mathrm{E}+00 & 0 & 0.00 \mathrm{E}+00\end{array}$

$\begin{array}{llll}0 & 0.00 \mathrm{E}+00 & 0 & 0.00 \mathrm{E}+00\end{array}$

$0.00 \mathrm{E}+00 \quad 0 \quad 0.00 \mathrm{E}+00$

$0.00 \mathrm{E}+00 \quad 0 \quad 0.00 \mathrm{E}+00$

$\begin{array}{llll}0 & 0.00 \mathrm{E}+00 & 0 & 0.00 \mathrm{E}+00\end{array}$

$\begin{array}{llll}0 & 0.00 \mathrm{E}+00 & 0 & 0.00 \mathrm{E}+00\end{array}$

$\begin{array}{llll}0 & 0.00 \mathrm{E}+00 & 0 & 0.00 \mathrm{E}+00\end{array}$

$0 \quad 0.00 \mathrm{E}+00 \quad 0 \quad 0.00 \mathrm{E}+00$

$0 \quad 0.00 \mathrm{E}+00 \quad 0 \quad 0.00 \mathrm{E}+00 \quad 0$

$\overline{0.00 \mathrm{E}+00}=\overline{\overline{0.00 \mathrm{E}+00}}=$

$0 \quad 0.00 E+00$

\begin{tabular}{lll}
$0.00 \mathrm{E}+00$ & 0 & $0.00 \mathrm{E}+00$ \\
\hline
\end{tabular}

$=\frac{0.00 \mathrm{E}+00}{\overline{0.00 \mathrm{E}+00}}=\frac{0.00 \mathrm{E}+00}{\overline{0.00 \mathrm{E}+00}}=$

Dose Contributions TDOSE(i,p,t) for Individual Radionuclides (i) and Pathways (p)
in mrem/yr and as a Percentage of Total Dose at $t=0$ years

Directly from primary contamination and from release to atmosphere (Inhalation excludes radon)

\begin{tabular}{|c|c|c|c|c|c|c|c|c|c|c|c|c|c|c|c|c|}
\hline \multirow{2}{*}{$\begin{array}{l}\text { Radio- } \\
\text { Nuclide }\end{array}$} & \multicolumn{2}{|c|}{ Ground } & \multicolumn{2}{|c|}{ Inhalation } & \multicolumn{2}{|c|}{ Radon } & \multicolumn{2}{|c|}{ Plant } & \multicolumn{2}{|c|}{ Meat } & \multicolumn{2}{|c|}{ Milk } & \multicolumn{2}{|l|}{ Soil } & \multicolumn{2}{|c|}{ All Pathways } \\
\hline & Dose & $\%$ & Dose & $\%$ & Dose & $\%$ & Dose & $\%$ & Dose & $\%$ & Dose & $\%$ & Dose & $\%$ & Dose & $\%$ \\
\hline$A m-241$ & $8.88 \mathrm{E}-09$ & 0 & $8.88 \mathrm{E}-03$ & 7 & $0.00 \mathrm{E}+00$ & 0 & $0.00 \mathrm{E}+00$ & 0 & $0.00 \mathrm{E}+00$ & 0 & $0.00 \mathrm{E}+00$ & 0 & $8.77 \mathrm{E}-09$ & 0 & $8.88 \mathrm{E}-03$ & 7 \\
\hline Cs -137 & $9.16 \mathrm{E}-07$ & 0 & $3.61 \mathrm{E}-06$ & 0 & $0.00 \mathrm{E}+00$ & 0 & $0.00 \mathrm{E}+00$ & 0 & $0.00 \mathrm{E}+00$ & 0 & $0.00 \mathrm{E}+00$ & 0 & $6.02 \mathrm{E}-10$ & 0 & $4.53 \mathrm{E}-06$ & 0 \\
\hline $\mathrm{Np}-237$ & $3.07 \mathrm{E}-06$ & 0 & $4.63 \mathrm{E}-02$ & 34 & $0.00 \mathrm{E}+00$ & 0 & $0.00 \mathrm{E}+00$ & 0 & $0.00 \mathrm{E}+00$ & 0 & $0.00 \mathrm{E}+00$ & 0 & $5.18 \mathrm{E}-08$ & 0 & $4.63 \mathrm{E}-02$ & 34 \\
\hline $\mathrm{Pu}-238$ & $3.12 \mathrm{E}-11$ & 0 & $1.02 \mathrm{E}-02$ & 8 & $0.00 \mathrm{E}+00$ & 0 & $0.00 \mathrm{E}+00$ & 0 & $0.00 \mathrm{E}+00$ & 0 & $0.00 \mathrm{E}+00$ & 0 & $1.07 \mathrm{E}-08$ & 0 & $1.02 \mathrm{E}-02$ & 8 \\
\hline $\mathrm{Pu}-239$ & $6.67 \mathrm{E}-11$ & 0 & $1.11 \mathrm{E}-02$ & 8 & $0.00 \mathrm{E}+00$ & 0 & $0.00 \mathrm{E}+00$ & 0 & $0.00 \mathrm{E}+00$ & 0 & $0.00 \mathrm{E}+00$ & 0 & $1.17 \mathrm{E}-08$ & 0 & $1.11 \mathrm{E}-02$ & 8 \\
\hline Pu-240 & $3.04 \mathrm{E}-11$ & 0 & $1.11 \mathrm{E}-02$ & 8 & $0.00 \mathrm{E}+00$ & 0 & $0.00 \mathrm{E}+00$ & 0 & $0.00 \mathrm{E}+00$ & 0 & $0.00 \mathrm{E}+00$ & 0 & $1.17 \mathrm{E}-08$ & 0 & $1.11 \mathrm{E}-02$ & 8 \\
\hline TC-99 & $2.05 \mathrm{E}-11$ & 0 & $1.20 \mathrm{E}-05$ & 0 & $0.00 \mathrm{E}+00$ & 0 & $0.00 \mathrm{E}+00$ & 0 & $0.00 \mathrm{E}+00$ & 0 & $0.00 \mathrm{E}+00$ & 0 & $2.17 \mathrm{E}-11$ & 0 & $1.20 \mathrm{E}-05$ & 0 \\
\hline Th-228 & $2.06 \mathrm{E}-06$ & 0 & $4.04 \mathrm{E}-03$ & 3 & $0.00 \mathrm{E}+00$ & 0 & $0.00 \mathrm{E}+00$ & 0 & $0.00 \mathrm{E}+00$ & 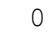 & $0.00 \mathrm{E}+00$ & 0 & $5.41 \mathrm{E}-09$ & 0 & $4.04 \mathrm{E}-03$ & 3 \\
\hline Th-230 & $1.06 \mathrm{E}-09$ & 0 & $9.25 \mathrm{E}-03$ & 7 & $0.00 \mathrm{E}+00$ & 0 & $0.00 \mathrm{E}+00$ & 0 & $0.00 \mathrm{E}+00$ & 0 & $0.00 \mathrm{E}+00$ & 0 & $9.87 \mathrm{E}-09$ & 0 & $9.25 \mathrm{E}-03$ & 7 \\
\hline Th-232 & $1.38 \mathrm{E}-07$ & 0 & $1.02 \mathrm{E}-02$ & 8 & $0.00 \mathrm{E}+00$ & 0 & $0.00 \mathrm{E}+00$ & 0 & $0.00 \mathrm{E}+00$ & 0 & $0.00 \mathrm{E}+00$ & 0 & $1.32 \mathrm{E}-08$ & 0 & $1.02 \mathrm{E}-02$ & 8 \\
\hline $\mathrm{U}-234$ & $8.75 \mathrm{E}-10$ & 0 & $8.70 \mathrm{E}-03$ & 6 & $0.00 \mathrm{E}+00$ & 0 & $0.00 \mathrm{E}+00$ & 0 & $0.00 \mathrm{E}+00$ & 0 & $0.00 \mathrm{E}+00$ & 0 & $2.24 \mathrm{E}-08$ & 0 & $8.70 \mathrm{E}-03$ & 6 \\
\hline $\mathrm{U}-235$ & $1.73 \mathrm{E}-06$ & 0 & $7.86 \mathrm{E}-03$ & 6 & $0.00 \mathrm{E}+00$ & 0 & $0.00 \mathrm{E}+00$ & 0 & $0.00 \mathrm{E}+00$ & 0 & $0.00 \mathrm{E}+00$ & 0 & & 0 & $7.87 \mathrm{E}-03$ & 6 \\
\hline $\mathrm{U}-238$ & $4.47 \mathrm{E}-07$ & 0 & $7.41 \mathrm{E}-03$ & 5 & $0.00 \mathrm{E}+00$ & 0 & $0.00 \mathrm{E}+00$ & 0 & $0.00 \mathrm{E}+00$ & 0 & $0.00 \mathrm{E}+00$ & 0 & $2.21 \mathrm{E}-08$ & 0 & $7.41 \mathrm{E}-03$ & 5 \\
\hline
\end{tabular}

$8.37 \mathrm{E}-06$

$\begin{array}{lll}1.35 \mathrm{E}-01 & 100 & 0.00 \mathrm{E}+00\end{array}$

$0.00 \mathrm{E}+00$

IRESRAD-OFFSITE, Version 4.0 .3 beta T1/2 Limit $=30$ days

06/11/2020 11:32 Page 51

Parent Dose Report 
Title : RESRAD-OFFSITE Effluent release

File : PADUCAH EFFLUENT RELEASE-REV1.ROF

Total Dose Contributions TDOSE (i,p,t) for Individual Radionuclides (i) and Pathways (p) in mrem/yr and as a Percentage of Total Dose at $t=1$ years

0

Radio

Nuclide

Am-241

$\begin{array}{llll}\mathrm{Cs}-137 & 0.00 \mathrm{E}+00 & 0 & 0.00 \mathrm{E}+00\end{array}$

$\begin{array}{llll}\mathrm{Pu}-238 & 0.00 \mathrm{E}+00 & 0 & 0.00 \mathrm{E}+00\end{array}$

$\begin{array}{llll}\mathrm{Pu}-239 & 0.00 \mathrm{E}+00 & 0 & 0.00 \mathrm{E}+00\end{array}$

$\begin{array}{llll}\mathrm{Pu}-240 & 0.00 \mathrm{E}+00 & 0 & 0.00 \mathrm{E}+00\end{array}$

$\begin{array}{llll}\mathrm{TC}-99 & 0.00 \mathrm{E}+00 & 0 & 0.00 \mathrm{E}+00\end{array}$

Th-228 $0.00 \mathrm{E}+00 \quad 0 \quad 0.00 \mathrm{E}+00$

$\mathrm{U}-234=0.00 \mathrm{E}+00=0.0 .00 \mathrm{E}+00$

$\mathrm{U}-2350.00 \mathrm{E}+00 \quad 0 \quad 0.00 \mathrm{E}+00$

$\mathrm{U}-238$

Radon Plant Meat
Meat to surface water
Milk Soil Water

\begin{tabular}{|c|c|c|c|c|c|c|c|c|c|c|c|}
\hline Dose & \% & Dose & $\%$ & Dose & $\%$ & Dose & $\frac{\circ}{0}$ & Dose & $\%$ & Dose & 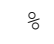 \\
\hline
\end{tabular}

$\begin{array}{lll}0 & 0.00 \mathrm{E}+00 & 0 \\ 0 & 0.00 \mathrm{E}+000 & 0\end{array}$

$0.00 \mathrm{E}+000.00 \mathrm{E}+00$

$\begin{array}{lll}0.00 \mathrm{E}+00 & 0 & 0.00 \mathrm{E}+00\end{array}$

$0.00 \mathrm{E}+00$

$0.00 \mathrm{E}+00$

$0.00 \mathrm{E}+00$

$0.00 \mathrm{E}+00$

$0.00 \mathrm{E}+00$

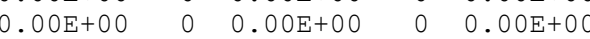

$\begin{array}{lllll}0.00 \mathrm{E}+00 & 0 & 0.00 \mathrm{E}+00 & 0 & 0.00 \mathrm{E}+00\end{array}$

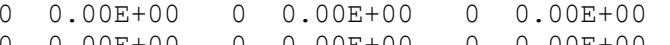

$\begin{array}{llllll}0.00 \mathrm{E}+00 & 0 & 0.00 \mathrm{E}+00 & 0 & 0.00 \mathrm{E}+00\end{array}$

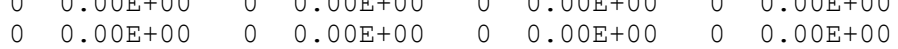

$0.00 \mathrm{E}+00 \quad 0 \quad 0.00 \mathrm{E}+00$

$0 \quad 0.00 \mathrm{E}+00$

$0.00 \mathrm{E}+00$

$0.00 \mathrm{E}+00$

$0.00 \mathrm{E}+00$

$0.00 \mathrm{E}+00 \quad 0 \quad 0.00 \mathrm{E}+00$

$0.0 .00 \mathrm{E}+00.00 .00 \mathrm{E}+00$

$\begin{array}{lll}0.00 \mathrm{E}+00 & 0 & 0.00 \mathrm{E}+00 \\ 0.00 \mathrm{E}+00 & 0 & 0.00 \mathrm{E}+00\end{array}$

$0 \quad 0.00 \mathrm{E}+00$

$0.00 \mathrm{E}+00$

$0.00 \mathrm{E}+00 \quad 0 \quad 0.00 \mathrm{E}+00$

$\begin{array}{lllllll}0.00 \mathrm{E}+00 & 0 & 0.00 \mathrm{E}+00 & 0 & 0.00 \mathrm{E}+00 & 0 & 0.00 \mathrm{E}+00\end{array}$

$\begin{array}{lllllll}0.00 \mathrm{E}+00 & 0 & 0.00 \mathrm{E}+00 & 0 & 0.00 \mathrm{E}+00\end{array}$

$\begin{array}{llllllllllll}0 & 0.00 \mathrm{E}+00 & 0 & 0.00 \mathrm{E}+00 & 0 & 0.00 \mathrm{E}+00 & 0 & 0.00 \mathrm{E}+00 & 0 & 0.00 \mathrm{E}+00 & 0 & 0.00 \mathrm{E}+00 \\ 0 & 0.00 \mathrm{E}+00 & 0 & 0.00 \mathrm{E}+00 & 0 & 0.00 \mathrm{E}+00 & 0 & 0.00 \mathrm{E}+00 & 0 & 0.00 \mathrm{E}+00 & 0 & 0.00 \mathrm{E}+00\end{array}$

$0.00 \mathrm{E}+00 \quad 0-0.00 \mathrm{E}+00$

$0.00 \mathrm{E}+00$

$0.00 \mathrm{E}+00$

$0.00 \mathrm{E}+00$

$\overline{0.00 \mathrm{E}+00}=$

$0.00 \mathrm{E}+00 \quad 0$

$\begin{array}{ll}0.00 \mathrm{E}+00 & 0\end{array}$

$0.00 \mathrm{E}+00=0$

$0.00 \mathrm{E}+00$

Total Dose Contributions TDOSE ( $i, p, t)$ for Individual Radionuclides ( $i$ ) and Pathways ( $p$ ) in mrem/yr and as a Percentage of Total Dose at $t=1$ years

0 Directly from primary contamination and from release to atmosphere (Inhalation excludes radon) Ground Inhalation Radon Plant

Radio

Nuclide

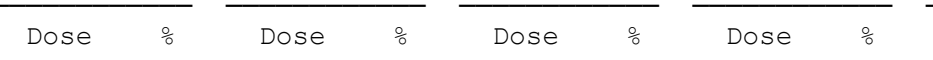

Meat

Milk Soil

All Pathways*

$\overline{A m-241} \overline{2.57 \mathrm{E}-08} \overline{0} \overline{8.88 \mathrm{E}-03}-\overline{7} \overline{0.00 \mathrm{E}+00}-\overline{0} \overline{0.00 \mathrm{E}+00}-{ }_{0}$

$2.57 \mathrm{E}-08$
$2.73 \mathrm{E}-06$

$8.88 \mathrm{E}-03$

$0.00 \mathrm{E}+00$

$0.00 \mathrm{E}+00$

Np-237 9.18E-06

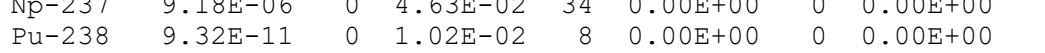

$\begin{array}{llllllll}\mathrm{Pu}-239 & 2.00 \mathrm{E}-10 & 0 & 1.11 \mathrm{E}-02 & 8 & 0.00 \mathrm{E}+00 & 0 & 0.00 \mathrm{E}+00\end{array}$

$\begin{array}{llllllll}\mathrm{Pu}-240 & 9.11 \mathrm{E}-11 & 0 & 1.11 \mathrm{E}-02 & 8 & 0.00 \mathrm{E}+00 & 0 & 0.00 \mathrm{E}+00\end{array}$

TC-99 $4.51 \mathrm{E}-11$

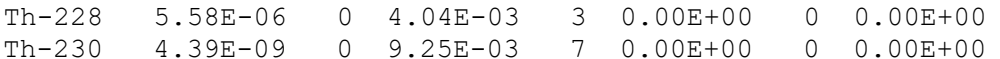

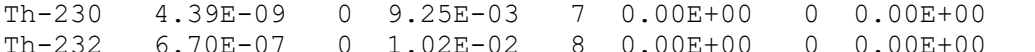

$\begin{array}{lllllll}T h-232 & 1.02 \mathrm{E}-02 & 8 & 0.00 \mathrm{E}+00 & 0 & 0.00 \mathrm{E}+00\end{array}$

$\mathrm{U}-234-2.59 \mathrm{E}-090.00 \mathrm{E}+00$

$\mathrm{U}-235$
$\mathrm{U}-238$

Total

$0.00 \mathrm{E}+00$

1. $32 \mathrm{E}-06$

$7.41 \mathrm{E}-03$

$2.46 \mathrm{E}-05$

1.35E-01 100

$0.00 \mathrm{E}+00$

$00.00 \mathrm{E}+00$

$\frac{0.00 \mathrm{E}+00}{0.00 \mathrm{E}+00}$

$\overline{0.00 \mathrm{E}+00} \frac{\frac{\circ}{0}}{0}$

$0.00 \mathrm{E}+00$

$0.00 \mathrm{E}+00$

$0.00 \mathrm{E}+00$

$0.00 \mathrm{E}+00$

$0.00 \mathrm{E}+00$

$0.00 \mathrm{E}+00$
$0.00 \mathrm{E}+00$

$0.00 \mathrm{E}+00$

$0.00 \mathrm{E}+00$

$0.00 \mathrm{E}+00$

$0.00 \mathrm{E}+00$

$0.00 \mathrm{E}+00$

Sum of dose from all releases and from primary contamina

Parent Dose Report

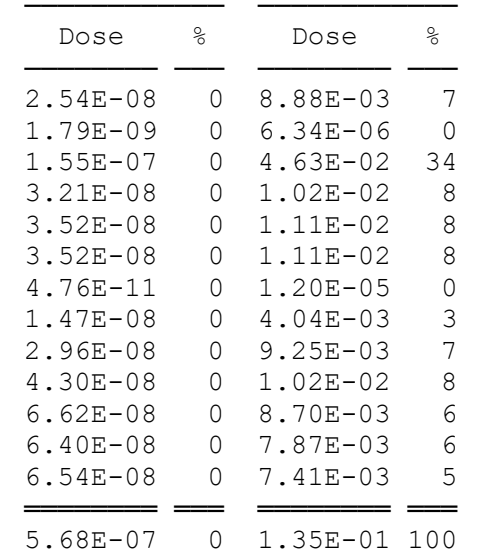


Title : RESRAD-OFFSITE Effluent release

File : PADUCAH EFFLUENT RELEASE-REV1.ROF

Total Dose Contributions TDOSE (i,p,t) for Individual Radionuclides (i) and Pathways (p) in mrem/yr and as a Percentage of Total Dose at $t=3$ years

0

Radio

Nuclide

Am-241

$\begin{array}{llll}\mathrm{Cs}-137 & 0.00 \mathrm{E}+00 & 0 & 0.00 \mathrm{E}+00\end{array}$

$\begin{array}{llll}\mathrm{Pu}-238 & 0.00 \mathrm{E}+00 & 0 & 0.00 \mathrm{E}+00\end{array}$

$\begin{array}{llll}\mathrm{Pu}-239 & 0.00 \mathrm{E}+00 & 0 & 0.00 \mathrm{E}+00\end{array}$

$\begin{array}{llll}\mathrm{Pu}-240 & 0.00 \mathrm{E}+00 & 0 & 0.00 \mathrm{E}+00\end{array}$

$\begin{array}{llll}\mathrm{TC}-99 & 0.00 \mathrm{E}+00 & 0 & 0.00 \mathrm{E}+00\end{array}$

Th-228 $0.00 \mathrm{E}+00 \quad 0 \quad 0.00 \mathrm{E}+00$

Th-230 $-0.00 \mathrm{E}+00$

$\mathrm{U}-234-0.00 \mathrm{E}+00-0.0 .00 \mathrm{E}+00$

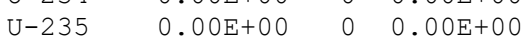

$\mathrm{U}-238$

Radon
Plant und water and to surface wate
Milk Soi Water

Dose $\%$ Dose

$0.00 \mathrm{E}+00$

$0.00 \mathrm{E}+00-0$

Dose $\%$

$0.00 \mathrm{E}+00$

$\begin{array}{lll}0.00 \mathrm{E}+00 \quad 0 & 0.00 \mathrm{E}+00\end{array}$

$\overline{0.00 \mathrm{E}+00}-0$

Dose $\frac{\circ}{0.00 E+00}$
Dose Dose \%

$0.00 \mathrm{E}+00$

$0.00 \mathrm{E}+00$

$0.00 \mathrm{E}+00$

$0.00 \mathrm{E}+00$

$\begin{array}{lllll}0.00 \mathrm{E}+00 & 0 & 0.00 \mathrm{E}+00 & 0 & 0.00 \mathrm{E}+00\end{array}$

$\begin{array}{lll}0.00 \mathrm{E}+00 & 0 & 0.00 \mathrm{E}+00\end{array}$

$0 \quad 0.00 \mathrm{E}+00$

$0.00 \mathrm{E}+00$

$0 \quad 0.00 \mathrm{E}+00$

$0.00 \mathrm{E}+00$

$0.00 \mathrm{E}+00$

$0.0 .00 \mathrm{E}+00$

$0.00 \mathrm{E}+00$

$0.0 .00 \mathrm{E}+00$ O $0.00 \mathrm{E}+00$

$0.00 \mathrm{E}+00$

$0.00 \mathrm{E}+00$

$0.00 \mathrm{E}+00$

$0.00 \mathrm{E}+00$

$0.00 \mathrm{E}+00$

$0.0 .00 \mathrm{E}+00$

$0.00 \mathrm{E}+00$

$0.00 \mathrm{E}+00$

$0.00 \mathrm{E}+00$
$0.00 \mathrm{E}+00$

$0.00 \mathrm{E}+00$

$0.00 \mathrm{E}+00$

$0.00 \mathrm{E}+00$

$0.00 \mathrm{E}+00$

$0.00 \mathrm{E}+00$

$0.00 \mathrm{E}+00$

$0.00 \mathrm{E}+00$

$\overline{0.00 \mathrm{E}+00}=$

\begin{tabular}{l}
$0.00 \mathrm{E}+00$ \\
$0.00 \mathrm{E}+00$ \\
\hline
\end{tabular}

$\overline{0.00 \mathrm{E}+00}=$

Total Dose Contributions TDOSE ( $i, p, t)$ for Individual Radionuclides (i) and Pathways (p) in mrem/yr and as a Percentage of Total Dose at $t=3$ years

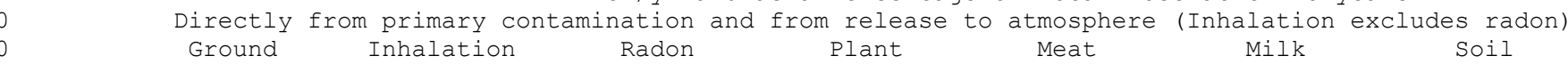

Radio

Nuclide

Dose $\frac{\%}{5.46 \mathrm{E}-08} \frac{\text { Dose }}{8.88 \mathrm{E}-03} \frac{\%}{7} \frac{\text { Dose }}{0.00 \mathrm{E}+00} \frac{\frac{\circ}{0}}{0.00 \mathrm{E}+00} \frac{\text { Dose }}{0}$

$6.23 \mathrm{E}-06$

$8.88 \mathrm{E}-03$

$0.00 \mathrm{E}+00$

$0.00 \mathrm{E}+00$

$\mathrm{Np}-237 \quad 2.13 \mathrm{E}-05$

$\begin{array}{llllll}0 & 4.63 \mathrm{E}-02 & 34 & 0.00 \mathrm{E}+00 & 0 & 0.00 \mathrm{E}+00\end{array}$

$\begin{array}{llllllll}\mathrm{Pu}-238 & 2.16 \mathrm{E}-10 & 0 & 1.02 \mathrm{E}-02 & 8 & 0.00 \mathrm{E}+00 & 0 & 0.00 \mathrm{E}+00 \\ \mathrm{Pu}-239 & 4.66 \mathrm{E}-10 & 0 & 1.11 \mathrm{E}-02 & 8 & 0.00 \mathrm{E}+00 & 0 & 0.00 \mathrm{E}+00\end{array}$

$\mathrm{Pu}-240 \quad 2.12 \mathrm{E}-10$

$1.11 \mathrm{E}-02$

$0.00 \mathrm{E}+00$

$0.00 \mathrm{E}+00$

$\begin{array}{llllllll}\text { TC-99 } & 5.13 \mathrm{E}-11 & 0 & 1.20 \mathrm{E}-05 & 0 & 0.00 \mathrm{E}+00 & 0 & 0.00 \mathrm{E}+00 \\ \mathrm{Th}-228 & 9.97 \mathrm{E}-06 & 0 & 4.04 \mathrm{E}-03 & 3 & 0.00 \mathrm{E}+00 & 0 & 0.00 \mathrm{E}+00\end{array}$

$1.20 \mathrm{E}-05$

$0.00 \mathrm{E}+00$

Meat

$\mathrm{Milk}$

Soil

All Pathways*

$\begin{array}{llllllll}\text { Th-230 } & 1.77 \mathrm{E}-08 & 0 & 9.25 \mathrm{E}-03 & 7 & 0.00 \mathrm{E}+00 & 0 & 0.00 \mathrm{E}+00\end{array}$

$\begin{array}{llllllll}\text { Th-232 } & 3.24 \mathrm{E}-06 & 0 & 1.02 \mathrm{E}-02 & 8 & 0.00 \mathrm{E}+00 & 0 & 0.00 \mathrm{E}+00\end{array}$

$\mathrm{U}-234$

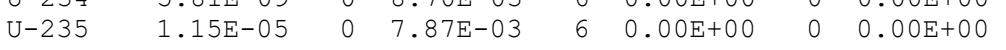

$\mathrm{U}-238$

Total

$\frac{2.96 \mathrm{E}-06}{\overline{5.52 \mathrm{E}-05}}=\frac{0}{0} \frac{7.41 \mathrm{E}-03}{\overline{1.35 \mathrm{E}-01}} \overline{\overline{100}}$ $0.00 \mathrm{E}+00$

\begin{tabular}{ll}
0 & $0.00 \mathrm{E}+00$ \\
0 & $0.00 \mathrm{E}+00$ \\
\hline
\end{tabular}

$\frac{\text { Dose }}{0.00 \mathrm{E}+00} \frac{\frac{\circ}{0}}{0.00 \mathrm{E}+00}$

$0.00 \mathrm{E}+00$

$0.00 \mathrm{E}+00$

$0.00 \mathrm{E}+00$

$0.00 \mathrm{E}+00$

$0.00 \mathrm{E}+00$

$0.00 \mathrm{E}+00$

$0.00 \mathrm{E}+00$

$0.00 \mathrm{E}+00$

$0.00 \mathrm{E}+00$

$0.00 \mathrm{E}+00$

$0.00 \mathrm{E}+00$

$0.00 \mathrm{E}+00$

Sum primary contamin

$06 / 11 / 2020 \quad 11: 32$ Page 53

Parent Dose Report 
Title : RESRAD-OFFSITE Effluent release

File : PADUCAH EFFLUENT RELEASE-REV1.ROF

Total Dose Contributions TDOSE (i,p,t) for Individual Radionuclides (i) and Pathways (p) in mrem/yr and as a Percentage of Total Dose at $t=6$ years

0

Radio

Nuclide

Am-241

$\begin{array}{llll}\mathrm{Cs}-137 & 0.00 \mathrm{E}+00 & 0 & 0.00 \mathrm{E}+00\end{array}$

$\begin{array}{llll}\mathrm{Pu}-238 & 0.00 \mathrm{E}+00 & 0 & 0.00 \mathrm{E}+00\end{array}$

$\begin{array}{llll}\mathrm{Pu}-239 & 0.00 \mathrm{E}+00 & 0 & 0.00 \mathrm{E}+00\end{array}$

$\begin{array}{llll}\mathrm{Pu}-240 & 0.00 \mathrm{E}+00 & 0 & 0.00 \mathrm{E}+00\end{array}$

$\begin{array}{llll}\mathrm{TC}-99 & 0.00 \mathrm{E}+00 & 0 & 0.00 \mathrm{E}+00\end{array}$

Th-228 $0.00 \mathrm{E}+00 \quad 0 \quad 0.00 \mathrm{E}+00$

Th-230 $-0.00 \mathrm{E}+00$

$\mathrm{Th}-232=0.00 \mathrm{E}+00-0=0.00 \mathrm{E}+00$

$\mathrm{U}-234-0.00 \mathrm{E}+00-0=0.00 \mathrm{E}+00$

$\mathrm{U}-235 \quad 0.00 \mathrm{E}+00 \quad 0 \quad 0.00 \mathrm{E}+00$

$\mathrm{U}-238$

Radon

From releases to ground water and to surface water

$\overline{0.00 \mathrm{E}+00}=$

$\overline{0.00 \mathrm{E}+00}=$

Dose $\div$ Dose

Meat

$\frac{\text { Milk }}{\text { Dose }} \frac{\frac{\circ}{0}}{0.00 \mathrm{E}+00} \frac{0}{0.00 \mathrm{E}+00}$

Soil

Water

$\begin{array}{llll}0.00 \mathrm{E}+00 & 0 & 0.00 \mathrm{E}+00 & 0 \\ 0.00 \mathrm{E}+00 & 0 & 0.00 \mathrm{E}+00 & 0\end{array}$

$\overline{0.00 \mathrm{E}+00}-$

$0.00 \mathrm{E}+00$

$\begin{array}{lll}0.00 \mathrm{E}+00 & 0 & 0.00 \mathrm{E}+00\end{array}$

$0.00 \mathrm{E}+00$

$0.00 \mathrm{E}+00$

$0.00 \mathrm{E}+00$

$0.00 \mathrm{E}+00$

$\begin{array}{lllll}0.00 \mathrm{E}+00 & 0 & 0.00 \mathrm{E}+00 & 0 & 0.00 \mathrm{E}+00 \\ 0.00 \mathrm{E}+00 & 0 & 0.00 \mathrm{E}+00 & 0 & 0.00 \mathrm{E}+00\end{array}$

$0.00 \mathrm{E}+00$

$0.00 \mathrm{E}+00$

$0.00 \mathrm{E}+00$

$0.00 \mathrm{E}+00 \quad 0 \quad 0.00 \mathrm{E}+00$

$0.00 \mathrm{E}+00$

$0.00 \mathrm{E}+00$

$0.00 \mathrm{E}+00$

$0.00 \mathrm{E}+00$

$0.00 \mathrm{E}+00$

$0.00 \mathrm{E}+00$

$0.00 \mathrm{E}+00$

$0.00 \mathrm{E}+00$

$\begin{array}{lll}0.00 \mathrm{E}+00 & 0 & 0.00 \mathrm{E}+00\end{array}$

$0.00 \mathrm{E}+00$

$0.00 \mathrm{E}+00$

$0.00 \mathrm{E}+00 \quad 0 \quad 0.00 \mathrm{E}+00$

$0.00 \mathrm{E}+00$

$0.00 \mathrm{E}+00$

$0.00 \mathrm{E}+00$

$0.00 \mathrm{E}+00$

$0.00 \mathrm{E}+00$

$0.00 \mathrm{E}+00$

$\overline{0.00 \mathrm{E}+00}=0$

$\overline{0.00 \mathrm{E}+00}$

Total Dose Contributions TDOSE ( $i, p, t)$ for Individual Radionuclides (i) and Pathways (p)

in mrem/yr and as a Percentage of Total Dose at $t=6$ years

0 Directly from primary contamination and from release to atmosphere (Inhalation excludes radon)

Ground Inhalation Radon Plant

Radio-

Nuclide

Dose $\%$ Dose $\frac{\circ}{\text { Dose }}$

Meat

Milk

Soil

All Pathways*

$\begin{array}{llllllllll}\overline{\mathrm{Am}-241} & \overline{8.77 \mathrm{E}-08} & 0 & \overline{8.88 \mathrm{E}-03} & 7 & \overline{0.00 \mathrm{E}+00} & 0 & \overline{0.00 \mathrm{E}+00} & 0 \\ \mathrm{Cs}-137 & 1.12 \mathrm{E}-05 & 0 & 3.61 \mathrm{E}-06 & 0 & 0.00 \mathrm{E}+00 & 0 & 0.00 \mathrm{E}+00 & 0\end{array}$

$0.00 \mathrm{E}+00$

$0.00 \mathrm{E}+00$

$\overline{\frac{\text { Dose }}{0.00 \mathrm{E}+00}-\frac{\mathrm{O}}{0}}$

$\overline{\text { Dose }} \frac{\%}{0.00 E+00}$

$0.00 \mathrm{E}+00$

$0.00 \mathrm{E}+00$

Dose $\frac{\%}{8.66 \mathrm{E}-08} \frac{\text { Dose }}{8.88 \mathrm{E}-03} \frac{\%}{7}$

$\mathrm{Np}-237 \quad 3.90 \mathrm{E}-05$

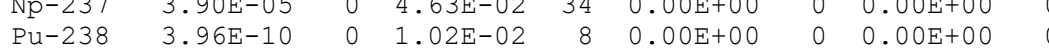

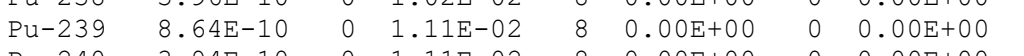

$\mathrm{Pu}-240 \quad 3.94 \mathrm{E}-10$

$0.00 \mathrm{E}+00$

TC-99 $5.13 \mathrm{E}-11$

$\begin{array}{lllllllll}10 & 0 & 1.11 \mathrm{E}-02 & 8 & 0.00 \mathrm{E}+00 & 0 & 0.00 \mathrm{E}+00 & 0 & 0.00 \mathrm{E}+00 \\ 11 & 0 & 1.20 \mathrm{E}-05 & 0 & 0.00 \mathrm{E}+00 & 0 & 0.00 \mathrm{E}+00 & 0 & 0.00 \mathrm{E}+00\end{array}$

$\begin{array}{lllllllll}10 & 0 & 1.11 \mathrm{E}-02 & 8 & 0.00 \mathrm{E}+00 & 0 & 0.00 \mathrm{E}+00 & 0 & 0.00 \mathrm{E}+00 \\ 11 & 0 & 1.20 \mathrm{E}-05 & 0 & 0.00 \mathrm{E}+00 & 0 & 0.00 \mathrm{E}+00 & 0 & 0.00 \mathrm{E}+00\end{array}$

$0.00 \mathrm{E}+00$

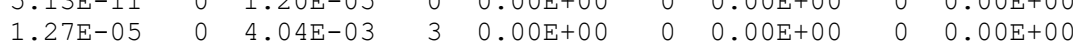

$\begin{array}{llllllll}\mathrm{Th}-230 & 5.43 \mathrm{E}-08 & 0 & 9.25 \mathrm{E}-03 & 7 & 0.00 \mathrm{E}+00 & 0 & 0.00 \mathrm{E}+00\end{array}$

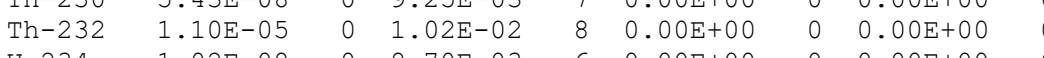

$\begin{array}{lll}1.02 \mathrm{E}-08 & 0 & 8.70 \mathrm{E}-03 \\ 2.00 \mathrm{E}-05 & 0 & 7.87 \mathrm{E}-03\end{array}$

$\mathrm{U}-238$

Total

$.00 \mathrm{E}-05$

$0.00 \mathrm{E}+00$

$0.00 \mathrm{E}+00$

$6 \quad 0.00 \mathrm{E}+00$

$0.00 \mathrm{E}+00$

$\overline{\overline{9.92 \mathrm{E}-05}} \overline{\overline{0}} \overline{\overline{1.35 \mathrm{E}-01}} \overline{\overline{100}}$

$\overline{0.00 \mathrm{E}+00}$

$0 \quad 0.00 \mathrm{E}+00$

$0.00 \mathrm{E}+00$

$0.00 \mathrm{E}+00$

$0.00 \mathrm{E}+00$

$0.00 \mathrm{E}+00$

$0.00 \mathrm{E}+00$
$0.00 \mathrm{E}+00$

$7.35 \mathrm{E}-09 \quad 0 \quad 1.48 \mathrm{E}-05-0$

$0-6.58 \mathrm{E}-07 \quad 0 \quad 4.63 \mathrm{E}-0234$

$\begin{array}{llll}0 & 1.52 \mathrm{E}-07 & 0 & 1.11 \mathrm{E}-02\end{array}$

$\begin{array}{lllll}0.00 \mathrm{E}+00 & 0 & 1.52 \mathrm{E}-07 & 0 & 1.11 \mathrm{E}-02\end{array}$

$\begin{array}{lllll}0.00 \mathrm{E}+00 & 0 & 5.41 \mathrm{E}-11 & 0 & 1.20 \mathrm{E}-05\end{array}$

$0.00 \mathrm{E}+00 \quad 0 \quad 3.35 \mathrm{E}-08 \quad 0 \quad 4.05 \mathrm{E}-03$

$0 \quad 0.00 \mathrm{E}+00$

$0.00 \mathrm{E}+00$

$0.00 \mathrm{E}+00$

$\underline{0.00 \mathrm{E}+00}$

$0 \quad 1.29 \mathrm{E}-07$

$\begin{array}{lll}0.60 \mathrm{E}-07 & 0 & 1.02 \mathrm{E}-02 \\ 0 & 0 & 8.70 \mathrm{E}-03\end{array}$

$$
0
$$

$0.00 \mathrm{E}+00$

\begin{tabular}{llll}
$2.60 \mathrm{E}-07$ & 0 & $8.70 \mathrm{E}-03$ & 6 \\
$2.51 \mathrm{E}-07$ & 0 & $7.89 \mathrm{E}-03$ & 6 \\
\hline
\end{tabular}

orom primary contamination.

Parent Dose Report 
Title : RESRAD-OFFSITE Effluent release

File : PADUCAH EFFLUENT RELEASE-REV1.ROF

Total Dose Contributions TDOSE (i,p,t) for Individual Radionuclides (i) and Pathways (p) in mrem/yr and as a Percentage of Total Dose at $t=10$ years

\begin{tabular}{|c|c|c|c|c|c|c|c|c|c|c|c|c|c|c|c|c|}
\hline \multirow{2}{*}{$\begin{array}{l}0 \\
0 \\
\text { Radio- } \\
\text { Nuclide }\end{array}$} & \multicolumn{2}{|c|}{ Ground } & \multicolumn{2}{|l|}{ Fish } & \multicolumn{2}{|c|}{$\begin{array}{l}\text { From } \\
\text { Radon }\end{array}$} & \multicolumn{2}{|c|}{ Plant } & \multicolumn{2}{|l|}{$\begin{array}{l}\text { water a } \\
\text { Meat }\end{array}$} & \multicolumn{2}{|c|}{$\begin{array}{c}\text { o surface wate } \\
\text { Milk }\end{array}$} & \multicolumn{2}{|l|}{ Soil } & \multicolumn{2}{|c|}{ Water } \\
\hline & Dose & $\%$ & Dose & $\div$ & Dose & $\%$ & Dose & $\%$ & Dose & $\%$ & Dose & $\%$ & Dose & $\%$ & Dose & $\frac{\%}{0}$ \\
\hline Am-241 & $0.00 \mathrm{E}+00$ & 0 & $0.00 \mathrm{E}+00$ & 0 & $0.00 \mathrm{E}+00$ & 0 & $0.00 \mathrm{E}+00$ & 0 & $0.00 \mathrm{E}+00$ & 0 & $0.00 \mathrm{E}+00$ & 0 & $0.00 \mathrm{E}+00$ & 0 & $0.00 \mathrm{E}+00$ & 0 \\
\hline $\mathrm{Cs}-137$ & $0.00 \mathrm{E}+00$ & 0 & $0.00 \mathrm{E}+00$ & 0 & $0.00 \mathrm{E}+00$ & 0 & $0.00 \mathrm{E}+00$ & 0 & $0.00 \mathrm{E}+00$ & 0 & $0.00 \mathrm{E}+00$ & 0 & $0.00 \mathrm{E}+00$ & 0 & $0.00 \mathrm{E}+00$ & 0 \\
\hline $\mathrm{Np}-237$ & $0.00 \mathrm{E}+00$ & 0 & $0.00 \mathrm{E}+00$ & 0 & $0.00 \mathrm{E}+00$ & 0 & $0.00 \mathrm{E}+00$ & 0 & $0.00 \mathrm{E}+00$ & 0 & $0.00 \mathrm{E}+00$ & 0 & $0.00 \mathrm{E}+00$ & 0 & $0.00 \mathrm{E}+00$ & 0 \\
\hline $\mathrm{Pu}-238$ & $0.00 \mathrm{E}+00$ & 0 & $0.00 \mathrm{E}+00$ & 0 & $0.00 \mathrm{E}+00$ & 0 & $0.00 \mathrm{E}+00$ & 0 & $0.00 \mathrm{E}+00$ & 0 & $0.00 \mathrm{E}+00$ & 0 & $0.00 \mathrm{E}+00$ & 0 & $0.00 \mathrm{E}+00$ & 0 \\
\hline $\mathrm{Pu}-239$ & $0.00 \mathrm{E}+00$ & 0 & $0.00 \mathrm{E}+00$ & 0 & $0.00 \mathrm{E}+00$ & 0 & $0.00 \mathrm{E}+00$ & 0 & $0.00 \mathrm{E}+00$ & 0 & $0.00 \mathrm{E}+00$ & 0 & $0.00 \mathrm{E}+00$ & 0 & $0.00 \mathrm{E}+00$ & 0 \\
\hline $\mathrm{Pu}-240$ & $0.00 \mathrm{E}+00$ & 0 & $0.00 \mathrm{E}+00$ & 0 & $0.00 \mathrm{E}+00$ & 0 & $0.00 \mathrm{E}+00$ & 0 & $0.00 \mathrm{E}+00$ & 0 & $0.00 \mathrm{E}+00$ & 0 & $0.00 \mathrm{E}+00$ & 0 & $0.00 \mathrm{E}+00$ & 0 \\
\hline TC-99 & $0.00 \mathrm{E}+00$ & 0 & $0.00 \mathrm{E}+00$ & 0 & $0.00 \mathrm{E}+00$ & 0 & $0.00 \mathrm{E}+00$ & 0 & $0.00 \mathrm{E}+00$ & 0 & $0.00 \mathrm{E}+00$ & 0 & $0.00 \mathrm{E}+00$ & 0 & $0.00 \mathrm{E}+00$ & 0 \\
\hline Th-228 & $0.00 \mathrm{E}+00$ & 0 & $0.00 \mathrm{E}+00$ & 0 & $0.00 \mathrm{E}+00$ & 0 & $0.00 \mathrm{E}+00$ & 0 & $0.00 \mathrm{E}+00$ & 0 & $0.00 \mathrm{E}+00$ & 0 & $0.00 \mathrm{E}+00$ & 0 & $0.00 \mathrm{E}+00$ & 0 \\
\hline Th-230 & $0.00 \mathrm{E}+00$ & 0 & $0.00 \mathrm{E}+00$ & 0 & $0.00 \mathrm{E}+00$ & 0 & $0.00 \mathrm{E}+00$ & 0 & 0.00 & 0 & 0.001 & 0 & $0.00 \mathrm{E}+00$ & 0 & $0.00 \mathrm{E}+00$ & 0 \\
\hline Th-232 & $0.00 \mathrm{E}+00$ & 0 & $0.00 \mathrm{E}+00$ & 0 & $0.00 \mathrm{E}+00$ & 0 & $0.00 \mathrm{E}+00$ & 0 & $0.00 \mathrm{E}+00$ & 0 & $0.00 \mathrm{E}+00$ & 0 & $0.00 \mathrm{E}+00$ & 0 & $0.00 \mathrm{E}+00$ & 0 \\
\hline $\mathrm{U}-234$ & $0.00 \mathrm{E}+00$ & 0 & $0.00 \mathrm{E}+00$ & 0 & $0.00 \mathrm{E}+00$ & 0 & $0.00 \mathrm{E}+00$ & 0 & $0.00 \mathrm{E}+00$ & 0 & $0.00 \mathrm{E}+00$ & 0 & $0.00 \mathrm{E}+00$ & 0 & $0.00 \mathrm{E}+00$ & 0 \\
\hline$U-235$ & $0.00 \mathrm{E}+00$ & 0 & $0.00 \mathrm{E}+00$ & 0 & $0.00 \mathrm{E}+00$ & 0 & $0.00 \mathrm{E}+00$ & 0 & $0.00 \mathrm{E}+00$ & 0 & $0.00 \mathrm{E}+00$ & 0 & $0.00 \mathrm{E}+00$ & 0 & $0.00 \mathrm{E}+00$ & 0 \\
\hline $\mathrm{U}-238$ & $0.00 \mathrm{E}+00$ & 0 & $0.00 \mathrm{E}+00$ & 0 & $0.00 \mathrm{E}+00$ & 0 & $0.00 \mathrm{E}+00$ & 0 & $0.00 \mathrm{E}+00$ & 0 & $0.00 \mathrm{E}+00$ & 0 & $0.00 \mathrm{E}+00$ & 0 & $0.00 \mathrm{E}+00$ & 0 \\
\hline & $0 U E+00$ & 0 & $00 \mathrm{E}+00$ & 0 & $.00 \mathrm{E}+00$ & 0 & $.00 \mathrm{E}+00$ & 0 & $0.00 \mathrm{E}+00$ & 0 & $0.00 \mathrm{E}+00$ & 0 & $0.00 \mathrm{E}+00$ & 0 & $0.00 \mathrm{E}+00$ & J \\
\hline
\end{tabular}

Total Dose Contributions TDOSE $(i, p, t)$ for Individual Radionuclides (i) and Pathways (p) in mrem/yr and as a Percentage of Total Dose at $t=10$ years

0 Directly from primary contamination and from release to atmosphere (Inhalation excludes radon)

\begin{tabular}{|c|c|c|c|c|c|c|c|c|c|c|c|c|c|c|c|c|}
\hline \multirow{2}{*}{$\begin{array}{l}\text { Radio- } \\
\text { Nuclide }\end{array}$} & \multicolumn{2}{|c|}{ Ground } & \multicolumn{2}{|c|}{ Inhalation } & \multicolumn{2}{|c|}{ Radon } & \multicolumn{2}{|l|}{ Plant } & \multicolumn{2}{|l|}{ Meat } & \multicolumn{2}{|c|}{ Milk } & \multicolumn{2}{|c|}{ Soil } & \multicolumn{2}{|c|}{ All Pathways } \\
\hline & Dose & $\%$ & Dose & $\%$ & Dose & \% & Dose & $\%$ & Dose & \% & Dose & $\%$ & Dose & $\%$ & Dose & $\div$ \\
\hline$A m-241$ & $.18 \mathrm{E}-07$ & 0 & $8.88 \mathrm{E}-03$ & 7 & $0.00 \mathrm{E}+00$ & 0 & $0.00 \mathrm{E}+00$ & 0 & $0.00 \mathrm{E}+00$ & 0 & $0.00 \mathrm{E}+00$ & 0 & $1.16 \mathrm{E}-07$ & 0 & $8.88 \mathrm{E}-03$ & 7 \\
\hline $\mathrm{Cs}-137$ & $1.73 \mathrm{E}-05$ & 0 & $3.61 \mathrm{E}-06$ & 0 & $0.00 \mathrm{E}+00$ & 0 & $0.00 \mathrm{E}+00$ & 0 & $0.00 \mathrm{E}+00$ & 0 & $0.00 \mathrm{E}+00$ & 0 & $1.14 \mathrm{E}-08$ & 0 & $2.09 \mathrm{E}-05$ & 0 \\
\hline $\mathrm{Np}-237$ & $6.19 \mathrm{E}-05$ & 0 & $4.63 \mathrm{E}-02$ & 34 & $0.00 \mathrm{E}+00$ & 0 & $0.00 \mathrm{E}+00$ & 0 & $0.00 \mathrm{E}+00$ & 0 & $0.00 \mathrm{E}+00$ & 0 & $1.05 \mathrm{E}-06$ & 0 & $4.63 \mathrm{E}-02$ & 34 \\
\hline $\mathrm{Pu}-238$ & $6.28 \mathrm{E}-10$ & 0 & $1.02 \mathrm{E}-02$ & 8 & $0.00 \mathrm{E}+00$ & 0 & $0.00 \mathrm{E}+00$ & 0 & $0.00 \mathrm{E}+00$ & 0 & $0.00 \mathrm{E}+00$ & 0 & $2.17 \mathrm{E}-07$ & 0 & $1.02 \mathrm{E}-02$ & 8 \\
\hline $\mathrm{Pu}-239$ & $1.39 \mathrm{E}-09$ & 0 & $1.11 \mathrm{E}-02$ & 8 & $0.00 \mathrm{E}+00$ & 0 & $0.00 \mathrm{E}+00$ & 0 & $0.00 \mathrm{E}+00$ & 0 & $0.00 \mathrm{E}+00$ & 0 & $2.45 \mathrm{E}-07$ & 0 & $1.11 \mathrm{E}-02$ & 8 \\
\hline $\mathrm{Pu}-240$ & $6.34 \mathrm{E}-10$ & 0 & $1.11 \mathrm{E}-02$ & 8 & $0.00 \mathrm{E}+00$ & 0 & $0.00 \mathrm{E}+00$ & 0 & $0.00 \mathrm{E}+00$ & 0 & $0.00 \mathrm{E}+00$ & 0 & $2.45 \mathrm{E}-07$ & 0 & $1.11 \mathrm{E}-02$ & 8 \\
\hline $\mathrm{TC}-99$ & $5.13 \mathrm{E}-11$ & 0 & $1.20 \mathrm{E}-05$ & 0 & $0.00 \mathrm{E}+00$ & 0 & $0.00 \mathrm{E}+00$ & 0 & $0.00 \mathrm{E}+00$ & 0 & $0.00 \mathrm{E}+00$ & 0 & $5.41 \mathrm{E}-11$ & 0 & $1.20 \mathrm{E}-05$ & 0 \\
\hline Th-228 & $1.38 \mathrm{E}-05$ & 0 & $4.04 \mathrm{E}-03$ & 3 & $0.00 \mathrm{E}+00$ & 0 & $0.00 \mathrm{E}+00$ & 0 & $0.00 \mathrm{E}+00$ & 0 & $0.00 \mathrm{E}+00$ & 0 & $3.63 \mathrm{E}-08$ & 0 & $4.05 \mathrm{E}-03$ & 3 \\
\hline Th-230 & $1.31 \mathrm{E}-07$ & 0 & $9.25 \mathrm{E}-03$ & 7 & $0.00 \mathrm{E}+00$ & 0 & $0.00 \mathrm{E}+00$ & 0 & $0.00 \mathrm{E}+00$ & 0 & $0.00 \mathrm{E}+00$ & 0 & $2.08 \mathrm{E}-07$ & 0 & $9.25 \mathrm{E}-03$ & 7 \\
\hline Th-232 & $2.72 \mathrm{E}-05$ & 0 & $1.02 \mathrm{E}-02$ & 8 & $0.00 \mathrm{E}+00$ & 0 & $0.00 \mathrm{E}+00$ & 0 & $0.00 \mathrm{E}+00$ & 0 & $0.00 \mathrm{E}+00$ & 0 & $5.34 \mathrm{E}-07$ & 0 & $1.02 \mathrm{E}-02$ & 8 \\
\hline $\mathrm{U}-234$ & $1.51 \mathrm{E}-08$ & 0 & $8.70 \mathrm{E}-03$ & 6 & $0.00 \mathrm{E}+00$ & 0 & $0.00 \mathrm{E}+00$ & 0 & $0.00 \mathrm{E}+00$ & 0 & $0.00 \mathrm{E}+00$ & 0 & $3.87 \mathrm{E}-07$ & 0 & $8.70 \mathrm{E}-03$ & 6 \\
\hline $\mathrm{U}-235$ & $2.98 \mathrm{E}-05$ & 0 & $7.87 \mathrm{E}-03$ & 6 & $0.00 \mathrm{E}+00$ & 0 & $0.00 \mathrm{E}+00$ & 0 & $0.00 \mathrm{E}+00$ & 0 & $0.00 \mathrm{E}+00$ & 0 & $3.74 \mathrm{E}-07$ & 0 & $7.90 \mathrm{E}-03$ & 6 \\
\hline $\mathrm{U}-238$ & $7.71 \mathrm{E}-06$ & 0 & $7.41 \mathrm{E}-03$ & 5 & $0.00 \mathrm{E}+00$ & 0 & $0.00 \mathrm{E}+00$ & 0 & $0.00 \mathrm{E}+00$ & 0 & $0.00 \mathrm{E}+00$ & 0 & $3.82 \mathrm{E}-07$ & 0 & $7.42 \mathrm{E}-03$ & 5 \\
\hline & $.58 E-04$ & 0 & $.35 E-01$ & 100 & $0.00 \mathrm{E}+00$ & 0 & $0.00 \mathrm{E}+00$ & 0 & $0.00 \mathrm{E}+00$ & 0 & $0.00 \mathrm{E}+00$ & 0 & $3.80 E-06$ & 0 & $1.35 \mathrm{E}-01$ & 100 \\
\hline
\end{tabular}

Total

$1.58 \mathrm{E}-04$

$0.00 \mathrm{E}+00$

$0.00 \mathrm{E}+00$

RESRAD-OFFSITE, Version 4.0.3 beta T1/2 Limit $=30$ days 06/11/2020 11:32 Page 55

Parent Dose Report 
Title : RESRAD-OFFSITE Effluent release

File : PADUCAH EFFLUENT RELEASE-REV1.ROF

Dose/Source Ratios Summed Over All Pathways

Parent and Progeny Principal Radionuclide Contributions Indicated

0 Parent Product Thread DSR $(j, t) \quad(\mathrm{mrem} / \mathrm{yr}) /(\mathrm{pCi} / \mathrm{g})$

\begin{tabular}{|c|c|c|c|c|c|c|c|}
\hline (i) & $(j)$ & ion & 0 & 0 & 3 & 0 & 1 \\
\hline & $A m-241$ & $1.000 \mathrm{E}+00$ & $2.961 E-03$ & $2.961 \mathrm{E}-03$ & $2.961 E-03$ & $2.961 \mathrm{E}-03$ & $2.961 E-03$ \\
\hline 241 & $N p-237+D$ & $1.000 \mathrm{E}+00$ & $2.223 E-14$ & $9.889 \mathrm{E}-14$ & $4.176 \mathrm{E}-13$ & $1.244 \mathrm{E}-12$ & $2.801 E-12$ \\
\hline & $\mathrm{U}-233$ & $1.000 \mathrm{E}+00$ & $1.045 \mathrm{E}-21$ & $7.823 \mathrm{E}-21$ & $6.082 \mathrm{E}-20$ & $3.084 \mathrm{E}-19$ & $1.087 \mathrm{E}-18$ \\
\hline & Th $-229+\mathrm{D}$ & $1.000 \mathrm{E}+00$ & $2.167 \mathrm{E}-24$ & $2.968 \mathrm{E}-23$ & $4.212 \mathrm{E}-22$ & $3.609 \mathrm{E}-21$ & $2.054 \mathrm{E}-20$ \\
\hline & $\Sigma \mathrm{DSR}(j)$ & & $2.961 E-03$ & $2.961 \mathrm{E}-03$ & $2.961 E-03$ & $2.961 \mathrm{E}-03$ & $2.961 E-03$ \\
\hline $37+1$ & $\mathrm{Cs}-137+\mathrm{D}$ & $1.000 \mathrm{E}+00$ & $1.508 \mathrm{E}-06$ & $2.112 \mathrm{E}-06$ & $3.281 \mathrm{E}-06$ & $4.936 \mathrm{E}-06$ & $6.969 \mathrm{E}-06$ \\
\hline $237+D$ & $\mathrm{~Np}-237+\mathrm{D}$ & $1.000 \mathrm{E}+00$ & $1.542 \mathrm{E}-03$ & $1.543 \mathrm{E}-03$ & $1.543 \mathrm{E}-03$ & 1.544 & $E-03$ \\
\hline $237+D$ & $\mathrm{U}-233$ & $1.000 \mathrm{E}+00$ & $7.974 \mathrm{E}-15$ & $3.613 \mathrm{E}-14$ & $1.584 \mathrm{E}-13$ & $4.980 \mathrm{E}-13$ & $1.203 \mathrm{E}-12$ \\
\hline$-237+D$ & Th $-229+\mathrm{D}$ & $1.000 \mathrm{E}+00$ & $2.194 \mathrm{E}-17$ & $1.689 \mathrm{E}-16$ & $1.378 \mathrm{E}-15$ & $7.495 \mathrm{E}-15$ & $2.912 E-14$ \\
\hline-237 & $\Sigma \mathrm{DSR}(j)$ & & $1.542 \mathrm{E}-03$ & $1.543 \mathrm{E}-03$ & $1.543 \mathrm{E}-03$ & $1.544 \mathrm{E}-03$ & $1.544 \mathrm{E}-03$ \\
\hline & $\mathrm{Pu}-238$ & $1.840 \mathrm{E}-09$ & $0.000 \mathrm{E}+00$ & $0.000 \mathrm{E}+00$ & $0.000 \mathrm{E}+00$ & $0.000 \mathrm{E}+00$ & $0.000 \mathrm{E}+00$ \\
\hline 38 & $\mathrm{Pu}-238$ & $1.000 \mathrm{E}+00$ & $3.393 \mathrm{E}-03$ & $3.393 \mathrm{E}-03$ & $3.393 \mathrm{E}-03$ & $3.393 \mathrm{E}-03$ & $3.393 E-03$ \\
\hline 238 & $\mathrm{U}-234$ & $1.000 \mathrm{E}+00$ & $4.905 E-15$ & $2.222 \mathrm{E}-14$ & $9.739 \mathrm{E}-14$ & $3.062 \mathrm{E}-13$ & $7.395 \mathrm{E}-13$ \\
\hline 80 & Th -230 & $1.000 \mathrm{E}+00$ & $1.635 \mathrm{E}-19$ & $1.258 \mathrm{E}-18$ & $1.027 \mathrm{E}-17$ & $5.582 \mathrm{E}-17$ & $2.168 E-16$ \\
\hline & $\mathrm{Ra}-226+\mathrm{D}$ & $1.000 \mathrm{E}+00$ & $8.651 E-22$ & $1.205 \mathrm{E}-20$ & $1.759 \mathrm{E}-19$ & $1.565 \mathrm{E}-18$ & $9.390 \mathrm{E}-18$ \\
\hline & $\mathrm{Pb}-210+\mathrm{D}$ & $1.000 \mathrm{E}+00$ & $8.704 \mathrm{E}-26$ & $2.287 \mathrm{E}-24$ & $5.912 \mathrm{E}-23$ & $8.337 \mathrm{E}-22$ & $7.602 \mathrm{E}-21$ \\
\hline 38 & Po-210 & $1.000 \mathrm{E}+00$ & $3.716 \mathrm{E}-26$ & $1.489 \mathrm{E}-24$ & $5.080 \mathrm{E}-23$ & $8.401 \mathrm{E}-22$ & $8.519 \mathrm{E}-21$ \\
\hline 238 & $\Sigma \mathrm{DSR}(j)$ & & $3.393 E-03$ & $3.393 \mathrm{E}-03$ & $3.393 E-03$ & $3.393 E-03$ & $3.393 E-03$ \\
\hline & $\mathrm{Pu}-239$ & $1.000 \mathrm{E}+00$ & $3.701 \mathrm{E}-03$ & $3.701 \mathrm{E}-03$ & $3.701 \mathrm{E}-03$ & $3.701 \mathrm{E}-03$ & $3.702 \mathrm{E}-03$ \\
\hline & $U-235+D$ & $1.000 \mathrm{E}+00$ & 3.72 & 1.693 & 7.4 & 2.36 & E-15 \\
\hline & $\mathrm{Pa}-231$ & $1.000 \mathrm{E}+00$ & $2.947 E-22$ & $2.247 \mathrm{E}-21$ & $1.804 \mathrm{E}-20$ & $9.577 \mathrm{E}-20$ & $3.596 E-19$ \\
\hline 239 & $A C-227+D$ & $1.000 \mathrm{E}+00$ & $1.696 \mathrm{E}-23$ & $2.289 \mathrm{E}-22$ & $3.171 E-21$ & $2.629 \mathrm{E}-20$ & $1.429 \mathrm{E}-19$ \\
\hline 330 & $\Sigma \mathrm{DSR}(j)$ & & $3.701 E-03$ & $3.701 \mathrm{E}-03$ & $3.701 \mathrm{E}-03$ & $3.701 \mathrm{E}-03$ & $3.702 E-03$ \\
\hline & $\mathrm{Pu}-240$ & $E-08$ & $0.000 \mathrm{E}+00$ & $0.000 \mathrm{E}+00$ & $0.000 \mathrm{E}+00$ & $0.000 \mathrm{E}+00$ & $0.000 \mathrm{E}+00$ \\
\hline 240 & $\mathrm{Pu}-240$ & $1.000 \mathrm{E}+00$ & $3.701 \mathrm{E}-03$ & $3.701 \mathrm{E}-03$ & $3.701 \mathrm{E}-03$ & $3.701 \mathrm{E}-03$ & $3.702 \mathrm{E}-03$ \\
\hline & $\mathrm{U}-236$ & $1.000 \mathrm{E}+00$ & $4.781 E-17$ & $2.171 \mathrm{E}-16$ & $9.559 \mathrm{E}-16$ & $3.028 \mathrm{E}-15$ & $7.390 E-15$ \\
\hline & Th -232 & $1.000 \mathrm{E}+00$ & $1.029 \mathrm{E}-26$ & $7.932 \mathrm{E}-26$ & $6.497 \mathrm{E}-25$ & $3.551 \mathrm{E}-24$ & $1.390 \mathrm{E}-23$ \\
\hline-240 & $\mathrm{Ra}-228+\mathrm{D}$ & $1.000 \mathrm{E}+00$ & $7.441 E-27$ & $1.012 \mathrm{E}-25$ & $1.421 \mathrm{E}-24$ & $1.201 \mathrm{E}-23$ & $6.720 E-23$ \\
\hline & Th $-228+D$ & $1.000 \mathrm{E}+00$ & $1.002 \mathrm{E}-27$ & $2.435 \mathrm{E}-26$ & $5.576 \mathrm{E}-25$ & $6.787 \mathrm{E}-24$ & $5.077 \mathrm{E}-23$ \\
\hline & $\Sigma \operatorname{DSR}(j)$ & & $3.701 \mathrm{E}-03$ & $3.701 \mathrm{E}-03$ & $3.701 \mathrm{E}-03$ & $3.701 \mathrm{E}-03$ & $3.702 \mathrm{E}-03$ \\
\hline & $\mathrm{TC}-99$ & $1.000 \mathrm{E}+00$ & $4.009 \mathrm{E}-07$ & $4.009 \mathrm{E}-07$ & $4.009 \mathrm{E}-07$ & $4.009 \mathrm{E}-07$ & $4.009 \mathrm{E}-07$ \\
\hline $28+D$ & $\mathrm{Th}-228+\mathrm{D}$ & $1.000 \mathrm{E}+00$ & $1.346 \mathrm{E}-03$ & $1.347 \mathrm{E}-03$ & $1.349 \mathrm{E}-03$ & $1.350 \mathrm{E}-03$ & $1.350 \mathrm{E}-03$ \\
\hline
\end{tabular}

Th-228+D Th-228+D $\quad 1.000 \mathrm{E}+00 \quad 1.346 \mathrm{E}-03 \quad 1.347 \mathrm{E}-03 \quad 1.349 \mathrm{E}-03 \quad 1.350 \mathrm{E}-03 \quad 1.350 \mathrm{E}-03$

1RESRAD-OFFSITE, Version 4.0.3 beta T1/2 Limit = 30 days 06/11/2020 11:32 Page Parent Dose Report

Title : RESRAD-OFFSITE Effluent release

File : PADUCAH EFFLUENT RELEASE-REV1.ROF

Dose/Source Ratios Summed Over All Pathways

Parent and Progeny Principal Radionuclide Contributions Indicated

0 Parent Product Thread DSR $(j, t)$ (mrem/yr)/(pCi/g)

(i) $\frac{(j)}{\text { Th }-230} \frac{\text { Fraction }}{1.000 \mathrm{E}+00} \frac{0.000 \mathrm{E}+00}{3.084 \mathrm{E}-03} \frac{1.000 \mathrm{E}+00}{3.084 \mathrm{E}-03} \frac{3.000 \mathrm{E}+00}{3.084 \mathrm{E}-03} \frac{6.000 \mathrm{E}+00}{3.084 \mathrm{E}-03} \frac{1.000 \mathrm{E}+01}{3.085 \mathrm{E}-03}$

$\overline{\text { Th-230 }} \overline{\text { Th-230 }} \overline{1.000 \mathrm{E}+00} \overline{3.084 \mathrm{E}-03} \overline{3.084 \mathrm{E}-03} \overline{3.084 \mathrm{E}-03} \overline{3.084 \mathrm{E}-03} \overline{3.085 \mathrm{E}-03}$ 


\begin{tabular}{|c|c|c|}
\hline Th-230 & $\mathrm{Ra}-226+\mathrm{D}$ & $1.000 \mathrm{E}+00$ \\
\hline Th-230 & $\mathrm{Pb}-210+\mathrm{D}$ & $1.000 \mathrm{E}+00$ \\
\hline Th-230 & Po-210 & $1.000 \mathrm{E}+00$ \\
\hline Th-230 & $\Sigma \mathrm{DSR}(j)$ & \\
\hline $0 \mathrm{Th}-232$ & Th-232 & $1.000 \mathrm{E}+00$ \\
\hline Th-232 & $\mathrm{Ra}-228+\mathrm{D}$ & $1.000 \mathrm{E}+00$ \\
\hline Th-232 & $T h-228+D$ & $1.000 \mathrm{E}+00$ \\
\hline Th-232 & $\operatorname{\Sigma DSR}(j)$ & \\
\hline OU-234 & $\mathrm{U}-234$ & $1.000 \mathrm{E}+00$ \\
\hline$U-234$ & Th-230 & $1.000 \mathrm{E}+00$ \\
\hline $\mathrm{U}-234$ & $\mathrm{Ra}-226+\mathrm{D}$ & $1.000 \mathrm{E}+00$ \\
\hline $\mathrm{U}-234$ & $\mathrm{~Pb}-210+\mathrm{D}$ & $1.000 \mathrm{E}+00$ \\
\hline $\mathrm{U}-234$ & Po-210 & $1.000 \mathrm{E}+00$ \\
\hline $\mathrm{U}-234$ & $\Sigma \operatorname{DSR}(j)$ & \\
\hline$O U-235+D$ & $\mathrm{U}-235+\mathrm{D}$ & $1.000 \mathrm{E}+00$ \\
\hline $\mathrm{U}-235+\mathrm{D}$ & $\mathrm{Pa}-231$ & $1.000 \mathrm{E}+00$ \\
\hline $\mathrm{U}-235+\mathrm{D}$ & $A C-227+D$ & $1.000 \mathrm{E}+00$ \\
\hline $\mathrm{U}-235$ & $\operatorname{\Sigma DSR}(j)$ & \\
\hline OU-238 & $\mathrm{U}-238$ & $5.400 \mathrm{E}-05$ \\
\hline$O U-238+D$ & $U-238+D$ & $9.999 \mathrm{E}-01$ \\
\hline $\mathrm{U}-238+\mathrm{D}$ & $\mathrm{U}-234$ & $9.999 \mathrm{E}-01$ \\
\hline$U-238+D$ & Th-230 & $9.999 \mathrm{E}-01$ \\
\hline$U-238+D$ & $\mathrm{Ra}-226+\mathrm{D}$ & $9.999 \mathrm{E}-01$ \\
\hline$U-238+D$ & $\mathrm{~Pb}-210+\mathrm{D}$ & $9.999 \mathrm{E}-01$ \\
\hline$U-238+D$ & Po-210 & $9.999 \mathrm{E}-01$ \\
\hline $\mathrm{U}-238$ & $\Sigma \mathrm{DSR}(j)$ & \\
\hline
\end{tabular}

$\begin{array}{lllll}2.624 \mathrm{E}-10 & 1.196 \mathrm{E}-09 & 5.307 \mathrm{E}-09 & 1.701 \mathrm{E}-08 & 4.217 \mathrm{E}-08\end{array}$ $\begin{array}{lllll}4.376 \mathrm{E}-14 & 3.340 \mathrm{E}-13 & 2.687 \mathrm{E}-12 & 1.431 \mathrm{E}-11 & 5.395 \mathrm{E}-11\end{array}$ $\begin{array}{lllll}2.499 \mathrm{E}-14 & 2.609 \mathrm{E}-13 & 2.643 \mathrm{E}-12 & 1.609 \mathrm{E}-11 & 6.540 \mathrm{E}-11\end{array}$ $3.084 \mathrm{E}-03 \quad 3.084 \mathrm{E}-03 \quad 3.084 \mathrm{E}-03 \quad 3.084 \mathrm{E}-03 \quad 3.085 \mathrm{E}-03$ $3.393 \mathrm{E}-03 \quad 3.393 \mathrm{E}-03 \quad 3.393 \mathrm{E}-03 \quad 3.393 \mathrm{E}-03 \quad 3.393 \mathrm{E}-03$ $3.860 \mathrm{E}-08 \quad 1.703 \mathrm{E}-07 \quad 7.082 \mathrm{E}-07 \quad 2.065 \mathrm{E}-06 \quad 4.548 \mathrm{E}-06$ $\begin{array}{lllll}8.413 \mathrm{E}-09 & 5.809 \mathrm{E}-08 & 3.946 \mathrm{E}-07 & 1.667 \mathrm{E}-06 & 4.697 \mathrm{E}-06\end{array}$

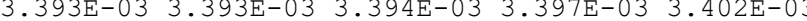
$\begin{array}{lllll}2.899 \mathrm{E}-04 & 2.899 \mathrm{E}-04 & 2.899 \mathrm{E}-04 & 2.899 \mathrm{E}-04 & 2.900 \mathrm{E}-04\end{array}$ $\begin{array}{llllll}1.381 \mathrm{E}-13 & 6.275 \mathrm{E}-13 & 2.765 \mathrm{E}-12 & 8 & .766 \mathrm{E}-12 & 2.143 \mathrm{E}-11\end{array}$ $9.741 \mathrm{E}-16 \quad 7.452 \mathrm{E}-156.019 \mathrm{E}-14 \quad 3.223 \mathrm{E}-12 \mathrm{2} 2.143 \mathrm{E}-11$ $5.978 \mathrm{E}-201.08 \mathrm{E}-182.424 \mathrm{E}-172.109 \mathrm{E}-161.224 \mathrm{E}-15$ $5.999 \mathrm{E}-042.202 \mathrm{E}-182.221 \mathrm{E}-172.243 \mathrm{E}-161.428 \mathrm{E}-15$ $\begin{array}{lllll}2.899 \mathrm{E}-04 & 2.899 \mathrm{E}-04 & 2.899 \mathrm{E}-04 & 2.899 \mathrm{E}-04 & 2.900 \mathrm{E}-0\end{array}$ $\begin{array}{lllll}.622 \mathrm{E}-04 & 2.623 \mathrm{E}-04 & 2.626 \mathrm{E}-04 & 2.629 \mathrm{E}-04 & 2.632 \mathrm{E}-04\end{array}$ $\begin{array}{lllll}.118 \mathrm{E}-13 & 3.192 \mathrm{E}-12 & 1.370 \mathrm{E}-11 & 4.170 \mathrm{E}-11 & 9.628 \mathrm{E}-11\end{array}$ $\begin{array}{llllll}5.435 \mathrm{E}-14 & 4.002 \mathrm{E}-13 & 3.022 \mathrm{E}-12 & 1.469 \mathrm{E}-11 & 4.886 \mathrm{E}-11\end{array}$ $2.622 \mathrm{E}-04 \quad 2.623 \mathrm{E}-04 \quad 2.626 \mathrm{E}-04 \quad 2.629 \mathrm{E}-04 \quad 2.632 \mathrm{E}-04$ $0.000 \mathrm{E}+000.000 \mathrm{E}+00 \quad 0.000 \mathrm{E}+00 \quad 0.000 \mathrm{E}+00-0.000 \mathrm{E}+00$ . $4.834 \mathrm{E}-152.168 \mathrm{E}-142.304 \mathrm{E}-142.2032 \mathrm{E}-133.6 .539 \mathrm{E}-13$

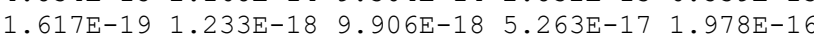
$8.575 \mathrm{E}-22 \quad 1.185 \mathrm{E}-20 \quad 1.708 \mathrm{E}-19 \quad 1.492 \mathrm{E}-18 \quad 8.719 \mathrm{E}-18$ $8.728 \mathrm{E}-26 \quad 2.257 \mathrm{E}-24 \quad 5.762 \mathrm{E}-23 \quad 8.005 \mathrm{E}-22 \quad 7.140 \mathrm{E}-21$ $3.765 \mathrm{E}-26 \quad 1.472 \mathrm{E}-24 \quad 4.959 \mathrm{E}-23 \quad 8.083 \mathrm{E}-22 \quad 8.020 \mathrm{E}-21$

The DSR includes contributions from associated (half-life $\leq 30$ days) daughters.

1RESRAD-OFFSITE, Version 4.0 .3 beta T1/2 Limit $=30$ days $06 / 11 / 2020 \quad 11: 32$ Page

Parent Dose Report

Title : RESRAD-OFFSITE Effluent release

File : PADUCAH EFFLUENT RELEASE-REV1.ROF

Single Radionuclide Soil Guidelines $\mathrm{G}(i, t)$ in $\mathrm{pCi} / \mathrm{g}$ Basic Radiation Dose Limit $=2.500 \mathrm{E}+01 \mathrm{mrem} / \mathrm{yr}$

ONuclide

\begin{tabular}{|c|c|c|c|c|c|c|}
\hline (i) & $t=$ & $0.000 \mathrm{E}+00$ & $1.000 \mathrm{E}+00$ & $3.000 \mathrm{E}+00$ & $6.000 \mathrm{E}+00$ & $1.000 \mathrm{E}+01$ \\
\hline$A m-241$ & & $8.444 \mathrm{E}+03$ & $8.444 \mathrm{E}+03$ & $8.443 E+03$ & $8.443 \mathrm{E}+03$ & $8.443 \mathrm{E}+03$ \\
\hline $\mathrm{Cs}-137$ & & $1.657 \mathrm{E}+07$ & $1.183 \mathrm{E}+07$ & $7.619 \mathrm{E}+06$ & $5.065 \mathrm{E}+06$ & $3.587 \mathrm{E}+06$ \\
\hline $\mathrm{Np}-237$ & & $1.621 \mathrm{E}+04$ & $1.621 \mathrm{E}+04$ & $1.620 \mathrm{E}+04$ & $1.620 \mathrm{E}+04$ & $1.619 \mathrm{E}+04$ \\
\hline $\mathrm{Pu}-238$ & & $7.369 \mathrm{E}+03$ & $7.369 \mathrm{E}+03$ & $7.369 \mathrm{E}+03$ & $7.368 \mathrm{E}+03$ & $7.368 \mathrm{E}+03$ \\
\hline $\mathrm{Pu}-239$ & & $6.755 \mathrm{E}+03$ & $6.755 \mathrm{E}+03$ & $6.755 \mathrm{E}+03$ & $6.754 \mathrm{E}+03$ & $6.754 \mathrm{E}+03$ \\
\hline $\mathrm{Pu}-240$ & & $6.755 \mathrm{E}+03$ & $6.755 \mathrm{E}+03$ & $6.755 \mathrm{E}+03$ & $6.754 \mathrm{E}+03$ & $6.754 \mathrm{E}+03$ \\
\hline$T_{C}-99$ & & $6.235 \mathrm{E}+07$ & $6.235 \mathrm{E}+07$ & $6.235 \mathrm{E}+07$ & $6.235 \mathrm{E}+07$ & $6.235 \mathrm{E}+07$ \\
\hline Th-228 & & $1.857 \mathrm{E}+04$ & $1.856 \mathrm{E}+04$ & $1.854 \mathrm{E}+04$ & $1.852 \mathrm{E}+04$ & $1.852 \mathrm{E}+04$ \\
\hline Th-230 & & $8.106 \mathrm{E}+03$ & $8.106 \mathrm{E}+03$ & $8.106 \mathrm{E}+03$ & $8.105 \mathrm{E}+03$ & $8.105 \mathrm{E}+03$ \\
\hline Th-232 & & $7.369 \mathrm{E}+03$ & $7.368 \mathrm{E}+03$ & $7.366 \mathrm{E}+03$ & $7.360 \mathrm{E}+03$ & $7.348 \mathrm{E}+03$ \\
\hline $\mathrm{U}-234$ & & $8.623 \mathrm{E}+04$ & $8.623 E+04$ & $8.623 E+04$ & $8.623 \mathrm{E}+04$ & $8.622 \mathrm{E}+04$ \\
\hline $\mathrm{U}-235$ & & $9.534 \mathrm{E}+04$ & $9.530 \mathrm{E}+04$ & $9.522 \mathrm{E}+04$ & $9.511 \mathrm{E}+04$ & $9.499 \mathrm{E}+04$ \\
\hline $\mathrm{U}-238$ & & $1.012 \mathrm{E}+05$ & $1.012 \mathrm{E}+05$ & $1.012 \mathrm{E}+05$ & $1.011 \mathrm{E}+05$ & $1.011 \mathrm{E}+05$ \\
\hline
\end{tabular}




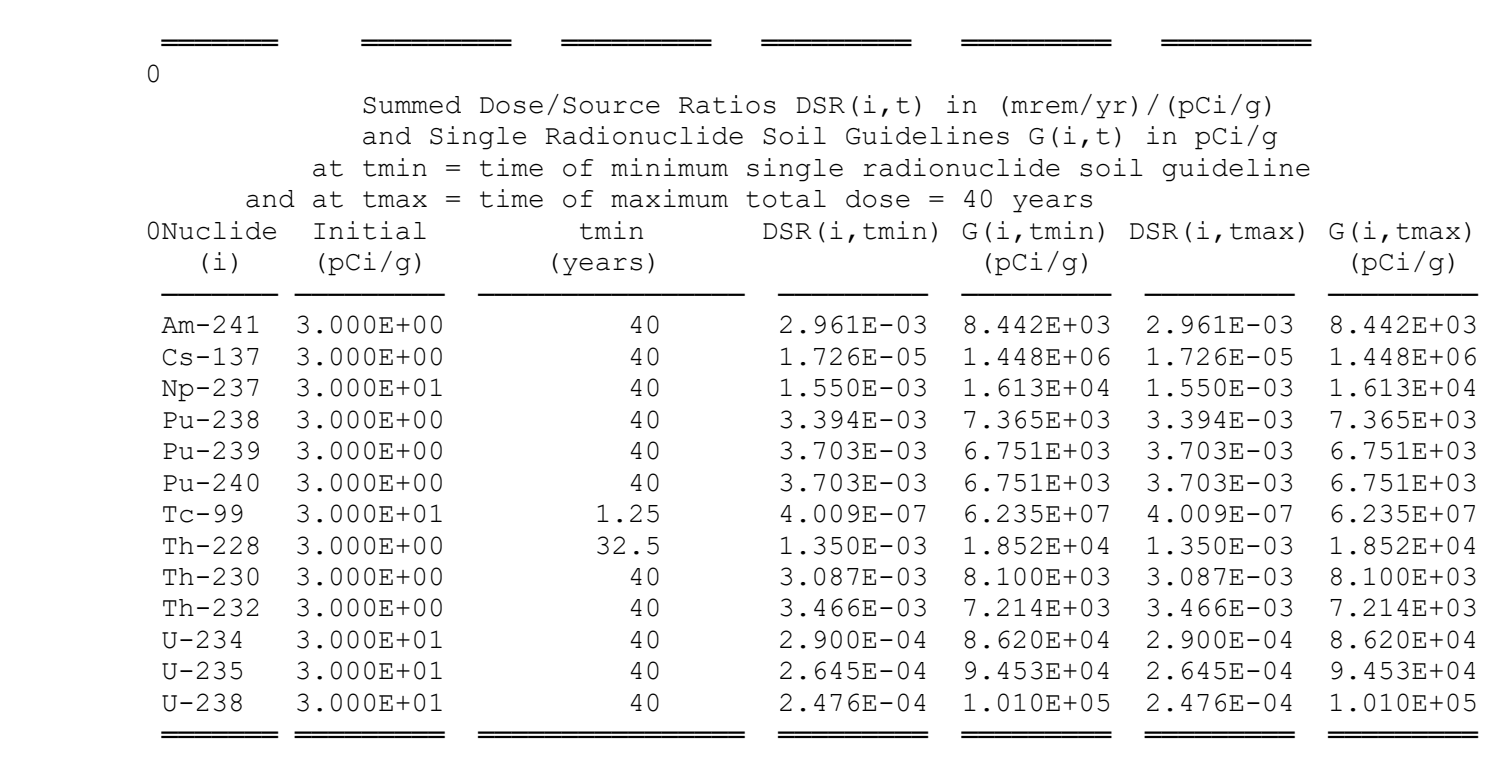

1RESRAD-OFFSITE, Version 4.0 .3 beta $T^{\frac{1}{2} / 2}$ Limit $=30$ days

Parent Dose Report

Title : RESRAD-OFFSITE Effluent release

File : PADUCAH EFFLUENT RELEASE-REV1.ROF

Individual Nuclide Dose Summed Over All Pathways Parent Nuclide and Thread Fraction Indicated

ONuclide Parent THF(i) DOSE $(j, t), \mathrm{mrem} / \mathrm{yr}$

\begin{tabular}{|c|c|c|c|c|c|c|c|}
\hline (j) & (i) & & $.000 \mathrm{E}+00$ & 00 & & 0 & 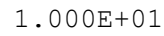 \\
\hline$A m-241$ & $n-241$ & $000 \mathrm{E}+00$ & $8.882 \mathrm{E}-03$ & $8.882 \mathrm{E}-03$ & $8.883 \mathrm{E}-03$ & $8.883 \mathrm{E}-03$ & $8.883 \mathrm{E}-03$ \\
\hline$n=237$ & $A m-241$ & & $6.670 \mathrm{E}-14$ & $2.967 \mathrm{E}-13$ & & & \\
\hline $\mathrm{Np}-237$ & $\mathrm{~Np}-237$ & 1. & $.627 \mathrm{E}-02$ & $4.628 \mathrm{E}-02$ & $4.629 \mathrm{E}-02$ & -02 & 4.6 \\
\hline $\mathrm{Np}-237$ & $\Sigma \operatorname{DOSE}(j)$ & & $.627 \mathrm{E}-02$ & $4.628 \mathrm{E}-02$ & $4.629 \mathrm{E}-02$ & $4.631 \mathrm{E}-02$ & $4.633 \mathrm{E}-02$ \\
\hline OU-233 & $A m-241$ & 1 & -21 & -20 & -19 & -19 & \\
\hline $\mathrm{U}-2$ & $\mathrm{~Np}-237$ & & & & & & \\
\hline $\mathrm{U}-233$ & $\Sigma \operatorname{DOSE}(j)$ & & -13 & 1.0 & -12 & 1.4 & 3.61 \\
\hline Th-229 & $A m-241$ & 1. & & -23 & -21 & -20 & \\
\hline Th-229 & $\mathrm{Np}-237$ & 1.0 & & 5. & 4. & -13 & \\
\hline Th-229 & $\Sigma \mathrm{DOSE}(j)$ & & -16 & $5.066 \mathrm{E}-15$ & $4.135 \mathrm{E}-14$ & $2.248 \mathrm{E}-13$ & $8.735 \mathrm{E}-13$ \\
\hline $0 \mathrm{Cs}-137$ & $\mathrm{Cs}-137$ & & & & & 05 & \\
\hline $0 \mathrm{Pu}-238$ & $\mathrm{Pu}-238$ & & & 0. & 00 & 00 & \\
\hline $\mathrm{Pu}-238$ & $\mathrm{Pu}-238$ & $1.000 \mathrm{E}+00$ & $1.018 \mathrm{E}-02$ & $1.018 \mathrm{E}-02$ & $1.018 \mathrm{E}-02$ & $1.018 \mathrm{E}-02$ & $1.018 \mathrm{E}-02$ \\
\hline $\mathrm{Pu}-238$ & $\Sigma \operatorname{DOSE}(j)$ & & $1.018 \mathrm{E}-02$ & $1.018 \mathrm{E}-02$ & $1.018 \mathrm{E}-02$ & $1.018 \mathrm{E}-02$ & $1.018 \mathrm{E}-02$ \\
\hline & $\mathrm{Pu}-2$ & & & & & & \\
\hline $\mathrm{U}-2$ & $\mathrm{U}-234$ & & $8.697 \mathrm{E}-03$ & $8.697 \mathrm{E}-03$ & $8.698 \mathrm{E}-03$ & $8.698 \mathrm{E}-03$ & $8.699 \mathrm{E}-$ \\
\hline $\mathrm{U}-234$ & $\mathrm{U}-238$ & $9.999 \mathrm{E}-01$ & $1.450 \mathrm{E}-13$ & $6.504 \mathrm{E}-13$ & $2.791 \mathrm{E}-12$ & $8.496 \mathrm{E}-12$ & $1.962 \mathrm{E}-11$ \\
\hline
\end{tabular}




$\begin{array}{rll}\mathrm{U}-234 & \mathrm{IDOSE}(j): \\ 0 \mathrm{Th}-230 & \mathrm{Pu}-238 & 1.000 \mathrm{E}+00 \\ \mathrm{Th}-230 & \mathrm{Th}-230 & 1.000 \mathrm{E}+00 \\ \mathrm{Th}-230 & \mathrm{U}-234 & 1.000 \mathrm{E}+00 \\ \mathrm{Th}-230 & \mathrm{U}-238 & 9.999 \mathrm{E}-01 \\ \mathrm{Th}-230 & \sum \mathrm{DOSE}(j): \\ 0 \mathrm{Ra}-226 & \mathrm{Pu}-238 & 1.000 \mathrm{E}+00 \\ \mathrm{Ra}-226 & \mathrm{Th}-230 & 1.000 \mathrm{E}+00 \\ \mathrm{Ra}-226 & \mathrm{U}-234 & 1.000 \mathrm{E}+00 \\ \mathrm{Ra}-226 & \mathrm{U}-238 & 9.999 \mathrm{E}-01 \\ \mathrm{Ra}-226 & \mathrm{DDOSE}(j): \\ 0 \mathrm{~Pb}-210 & \mathrm{Pu}-238 & 1.000 \mathrm{E}+00 \\ \mathrm{~Pb}-210 & \mathrm{Th}-230 & 1.000 \mathrm{E}+00 \\ \mathrm{~Pb}-210 & \mathrm{U}-234 & 1.000 \mathrm{E}+00 \\ \mathrm{~Pb}-210 & \mathrm{U}-238 & 9.999 \mathrm{E}-01 \\ \mathrm{~Pb}-210 & \mathrm{EDOSE}(j):\end{array}$

$\begin{array}{lllll}8.697 \mathrm{E}-03 & 8.697 \mathrm{E}-03 & 8.698 \mathrm{E}-03 & 8.698 \mathrm{E}-03 & 8.699 \mathrm{E}-03\end{array}$ $\begin{array}{llllll}4.904 \mathrm{E}-19 & 3.774 \mathrm{E}-18 & 3.080 \mathrm{E}-17 & 1.675 \mathrm{E}-16 & 6.504 \mathrm{E}-16\end{array}$ $\begin{array}{lllll}9.252 \mathrm{E}-03 & 9.253 \mathrm{E}-03 & 9.253 \mathrm{E}-03 & 9.253 \mathrm{E}-03 & 9.254 \mathrm{E}-03\end{array}$

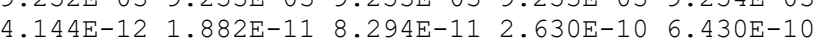
$\begin{array}{lllll}4.850 \mathrm{E}-18 & 3.699 \mathrm{E}-17 & 2.972 \mathrm{E}-16 & 1.579 \mathrm{E}-15 & 5.935 \mathrm{E}-15\end{array}$ $\begin{array}{lllll}4.850 \mathrm{E}-18 & 3.699 \mathrm{E}-17 & 2.972 \mathrm{E}-16 & 1.579 \mathrm{E}-15 & 5.935 \mathrm{E}-15 \\ 9.252 \mathrm{E}-03 & 9.253 \mathrm{E}-03 & 9.253 \mathrm{E}-03 & 9.253 \mathrm{E}-03 & 9.254 \mathrm{E}-03\end{array}$ $\begin{array}{lllll}2.595 \mathrm{E}-21 & 3.616 \mathrm{E}-20 & 5.278 \mathrm{E}-19 & 4.696 \mathrm{E}-18 & 2.817 \mathrm{E}-17\end{array}$ $7.873 \mathrm{E}-10 \quad 3.588 \mathrm{E}-09 \quad 1.592 \mathrm{E}-08 \quad 5.102 \mathrm{E}-08$ 1.265E-07 $2.922 \mathrm{E}-14 \quad 2.236 \mathrm{E}-13 \quad 1.806 \mathrm{E}-12 \quad 9.668 \mathrm{E}-12 \quad 3.674 \mathrm{E}-11$ $2.573 \mathrm{E}-20 \quad 3.556 \mathrm{E}-19 \quad 5.123 \mathrm{E}-18 \quad 4.476 \mathrm{E}-17 \quad 2.616 \mathrm{E}-16$

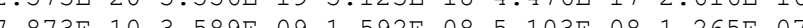
$2.611 \mathrm{E}-256.861 \mathrm{E}-241.774 \mathrm{E}-22.2 .501 \mathrm{E}-212.281 \mathrm{E}-20$

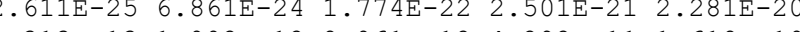
$1.313 \mathrm{E}-13 \quad 1.002 \mathrm{E}-12 \quad 8.061 \mathrm{E}-12 \quad 4.292 \mathrm{E}-11 \quad 1.618 \mathrm{E}-10$ $\begin{array}{lllll}3.672 \mathrm{E}-18 & 5.065 \mathrm{E}-17 & 7.273 \mathrm{E}-16 & 6.327 \mathrm{E}-15 & 3.673 \mathrm{E}-14\end{array}$ $\begin{array}{llllll}2.618 \mathrm{E}-24 & 6.771 \mathrm{E}-23 & 1.729 \mathrm{E}-21 & 2.402 \mathrm{E}-20 & 2.142 \mathrm{E}-19\end{array}$ $\begin{array}{lllll}.313 \mathrm{E}-13 & 1.002 \mathrm{E}-12 & 8.062 \mathrm{E}-12 & 4.293 \mathrm{E}-11 & 1.619 \mathrm{E}-10\end{array}$

IRESRAD-OFFSITE, Version 4.0 .3 beta $T^{1} \frac{1}{2}$ Limit $=30$ days

Parent Dose Report

Title : RESRAD-OFFSITE Effluent release

File : PADUCAH EFFLUENT RELEASE-REV1.ROF

Individual Nuclide Dose Summed Over All Pathways

Parent Nuclide and Thread Fraction Indicated

ONuclide Parent THF(i) $\operatorname{DOSE}(j, t), \mathrm{mrem} / \mathrm{y}$

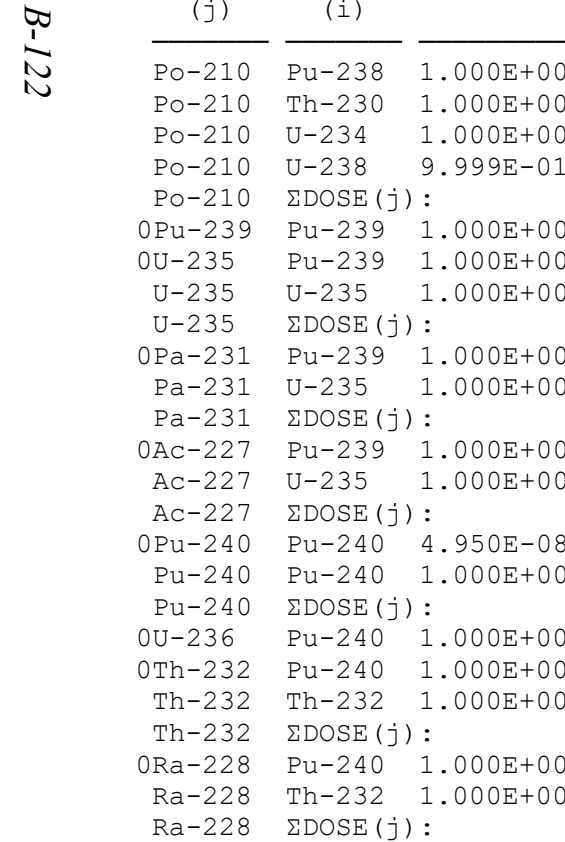

$t=0.000 \mathrm{E}+00 \quad 1.000 \mathrm{E}+00 \quad 3.000 \mathrm{E}+00 \quad 6.000 \mathrm{E}+00 \quad 1.000 \mathrm{E}+01$

$\begin{array}{lllll}1.115 \mathrm{E}-25 & 4.468 \mathrm{E}-24 & 1.524 \mathrm{E}-22 & 2.520 \mathrm{E}-21 & 2.556 \mathrm{E}-20\end{array}$ $\begin{array}{lllll}7.498 \mathrm{E}-14 & 7.826 \mathrm{E}-13 & 7.929 \mathrm{E}-12 & 4.826 \mathrm{E}-11 & 1.962 \mathrm{E}-10\end{array}$ $1.793 \mathrm{E}-18 \quad 3.605 \mathrm{E}-17 \quad 6.664 \mathrm{E}-16 \quad 6.728 \mathrm{E}-15 \quad 4.284 \mathrm{E}-14$ $\begin{array}{lllll}1.130 \mathrm{E}-24 & 4.417 \mathrm{E}-23 & 1.488 \mathrm{E}-21 & 2.425 \mathrm{E}-20 & 2.406 \mathrm{E}-19\end{array}$ $7.499 \mathrm{E}-14 \quad 7.826 \mathrm{E}-13 \quad 7.929 \mathrm{E}-12 \quad 4.826 \mathrm{E}-11 \quad 1.962 \mathrm{E}-10$ $1.110 \mathrm{E}-02 \quad 1.110 \mathrm{E}-02 \quad 1.110 \mathrm{E}-02 \quad 1.110 \mathrm{E}-02 \quad 1.110 \mathrm{E}-02$ $\begin{array}{lllll}1.118 \mathrm{E}-16 & 5.078 \mathrm{E}-16 & 2.236 \mathrm{E}-15 & 7.082 \mathrm{E}-15 & 1.729 \mathrm{E}-14\end{array}$ $7.867 \mathrm{E}-03 \quad 7.870 \mathrm{E}-03 \quad 7.877 \mathrm{E}-03 \quad 7.886 \mathrm{E}-03 \quad 7.896 \mathrm{E}-03$ $7.867 \mathrm{E}-03 \quad 7.870 \mathrm{E}-03 \quad 7.877 \mathrm{E}-03 \quad 7.886 \mathrm{E}-03 \quad 7.896 \mathrm{E}-03$ $8.841 \mathrm{E}-22 \quad 6.741 \mathrm{E}-215.412 \mathrm{E}-202.873 \mathrm{E}-19 \mathrm{~g}-1.079 \mathrm{E}-18$

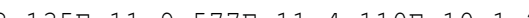
$2.135 \mathrm{E}-1129.577 \mathrm{E}-114.110 \mathrm{E}-101.251 \mathrm{E}-092.889 \mathrm{E}-09$ $2.135 \mathrm{E}-11 \quad 9.577 \mathrm{E}-11 \quad 4.110 \mathrm{E}-10 \quad 1.251 \mathrm{E}-092.889 \mathrm{E}-09$ $\begin{array}{lllll}5.089 \mathrm{E}-23 & 6.866 \mathrm{E}-22 & 9.513 \mathrm{E}-21 & 7.888 \mathrm{E}-20 & 4.286 \mathrm{E}-19\end{array}$ $\begin{array}{lllll}1.630 \mathrm{E}-12 & 1.200 \mathrm{E}-11 & 9.067 \mathrm{E}-11 & 4.408 \mathrm{E}-10 & 1.466 \mathrm{E}-09\end{array}$ $\begin{array}{lllll}1.630 \mathrm{E}-12 & 1.200 \mathrm{E}-11 & 9.067 \mathrm{E}-11 & 4.408 \mathrm{E}-10 & 1.466 \mathrm{E}-09\end{array}$ $\begin{array}{lllll}0.000 \mathrm{E}+00 & 0.000 \mathrm{E}+00 & 0.000 \mathrm{E}+00 & 0.000 \mathrm{E}+00 & 0.000 \mathrm{E}+00\end{array}$ $1.110 \mathrm{E}-02 \quad 1.110 \mathrm{E}-02 \quad 1.110 \mathrm{E}-02 \quad 1.110 \mathrm{E}-02 \quad 1.110 \mathrm{E}-02$ $110 \mathrm{E}-021.11 \mathrm{C}$ $1.110 \mathrm{E}-02$ 1.110E-02 $1.110 \mathrm{E}-02$ 1.110E-02 $1.110 \mathrm{E}-02$

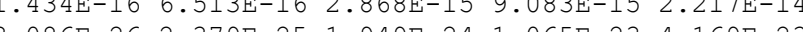
$3.086 \mathrm{E}-26 \quad 2.379 \mathrm{E}-25 \quad 1.949 \mathrm{E}-24 \quad 1.065 \mathrm{E}-23 \quad 4.169 \mathrm{E}-23$ $1.018 \mathrm{E}-02 \quad 1.018 \mathrm{E}-02 \quad 1.018 \mathrm{E}-02 \quad 1.018 \mathrm{E}-02 \quad 1.018 \mathrm{E}-02$ $1.018 \mathrm{E}-02 \quad 1.018 \mathrm{E}-02 \quad 1.018 \mathrm{E}-02 \quad 1.018 \mathrm{E}-02 \quad 1.018 \mathrm{E}-02$ $2.232 \mathrm{E}-26 \quad 3.035 \mathrm{E}-25 \quad 4.262 \mathrm{E}-24 \quad 3.603 \mathrm{E}-23 \quad 2.016 \mathrm{E}-22$ $1.158 \mathrm{E}-07 \quad 5.110 \mathrm{E}-07 \quad 2.125 \mathrm{E}-06 \quad 6.196 \mathrm{E}-06 \quad 1.364 \mathrm{E}-05$ $1.158 \mathrm{E}-07 \quad 5.110 \mathrm{E}-07 \quad 2.125 \mathrm{E}-06 \quad 6.196 \mathrm{E}-06 \quad 1.364 \mathrm{E}-05$ 


\begin{tabular}{cllllllll} 
OTh-228 & Pu-240 & $1.000 \mathrm{E}+00$ & $3.005 \mathrm{E}-27$ & $7.305 \mathrm{E}-26$ & $1.673 \mathrm{E}-24$ & $2.036 \mathrm{E}-23$ & $1.523 \mathrm{E}-22$ \\
Th-228 & Th-228 & $1.000 \mathrm{E}+00$ & $4.038 \mathrm{E}-03$ & $4.042 \mathrm{E}-03$ & $4.046 \mathrm{E}-03$ & $4.049 \mathrm{E}-03$ & $4.050 \mathrm{E}-03$ \\
Th-228 & Th-232 & $1.000 \mathrm{E}+00$ & $2.524 \mathrm{E}-08$ & $1.743 \mathrm{E}-07$ & $1.184 \mathrm{E}-06$ & $5.000 \mathrm{E}-06$ & $1.409 \mathrm{E}-05$ \\
Th-228 & EDOSE $(j):$ & $4.038 \mathrm{E}-03$ & $4.042 \mathrm{E}-03$ & $4.047 \mathrm{E}-03$ & $4.054 \mathrm{E}-03$ & $4.064 \mathrm{E}-03$ \\
0TC-99 & TC-99 & $1.000 \mathrm{E}+00$ & $1.203 \mathrm{E}-05$ & $1.203 \mathrm{E}-05$ & $1.203 \mathrm{E}-05$ & $1.203 \mathrm{E}-05$ & $1.203 \mathrm{E}-05$ \\
OU-238 & $\mathrm{U}-238$ & $5.400 \mathrm{E}-05$ & $0.000 \mathrm{E}+00$ & $0.000 \mathrm{E}+00$ & $0.000 \mathrm{E}+00$ & $0.000 \mathrm{E}+00$ & $0.000 \mathrm{E}+00$ \\
$\mathrm{U}-238$ & $\mathrm{U}-238$ & $9.999 \mathrm{E}-01$ & $7.409 \mathrm{E}-03$ & $7.411 \mathrm{E}-03$ & $7.412 \mathrm{E}-03$ & $7.415 \mathrm{E}-03$ & $7.418 \mathrm{E}-03$ \\
$\mathrm{U}-238$ & EDOSE $(j):$ & $7.409 \mathrm{E}-03$ & $7.411 \mathrm{E}-03$ & $7.412 \mathrm{E}-03$ & $7.415 \mathrm{E}-03$ & $7.418 \mathrm{E}-03$ \\
\hline \hline
\end{tabular}

THF(i) is the thread fraction of the parent nuclide.

1RESRAD-OFFSITE, Version 4.0 .3 beta T1/2 Limit $=30$ days 06/11/2020 11:32 Page

Parent Dose Report

Title : RESRAD-OFFSITE Effluent release

File : PADUCAH EFFLUENT RELEASE-REV1.ROF

Individual Nuclide Soil Concentration

Parent Nuclide and Thread Fraction Indicated

onuclide Parent THF(i) $\quad S(j, t), p C i / g$

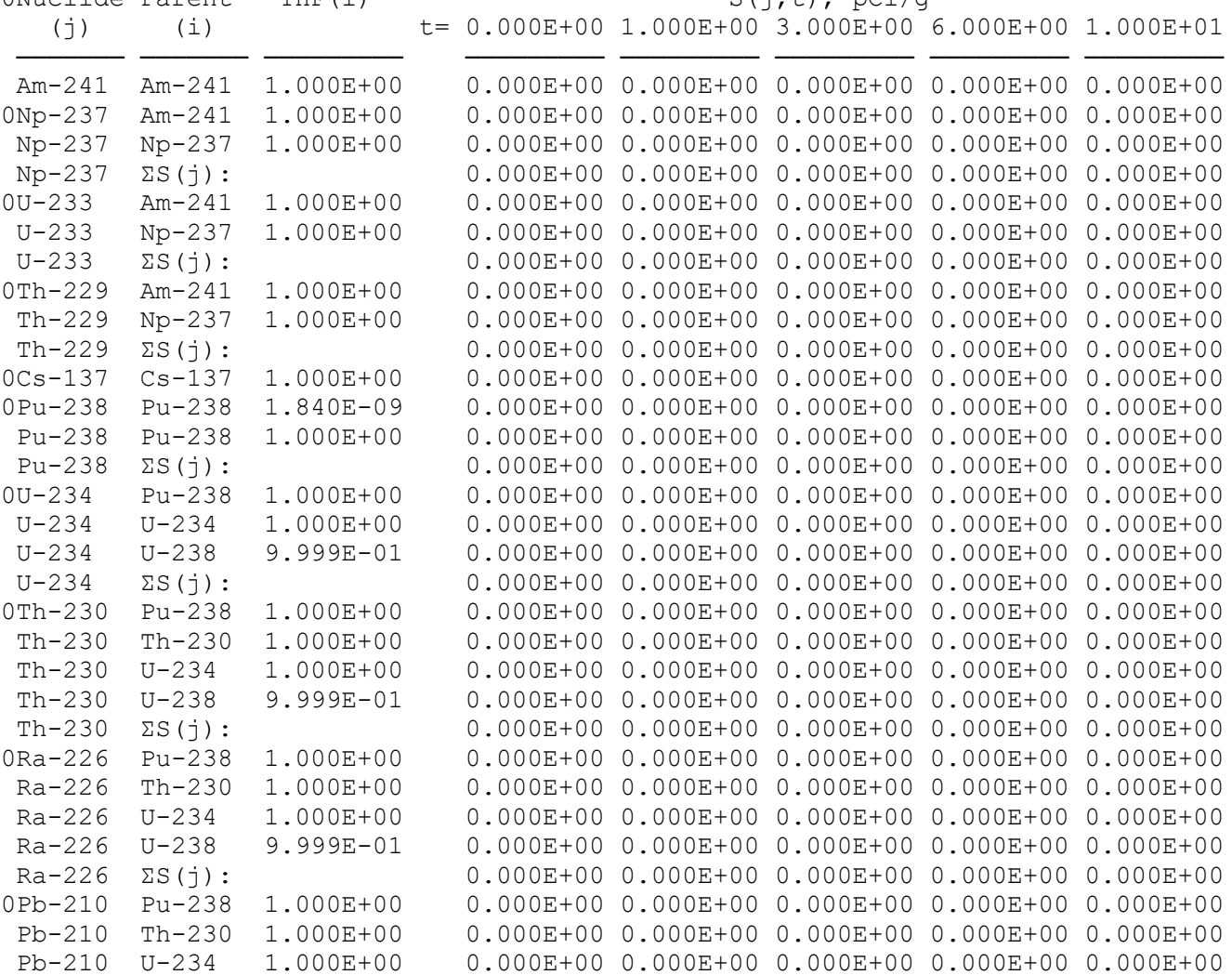


$\begin{array}{llllllll}\mathrm{Pb}-210 & \mathrm{U}-238 & 9.999 \mathrm{E}-01 & 0.000 \mathrm{E}+00 & 0.000 \mathrm{E}+00 & 0.000 \mathrm{E}+00 & 0.000 \mathrm{E}+00 & 0.000 \mathrm{E}+00\end{array}$ $\mathrm{Pb}-210 \Sigma \mathrm{S}(\mathrm{j}): \quad 0.000 \mathrm{E}+00 \quad 0.000 \mathrm{E}+00 \quad 0.000 \mathrm{E}+00 \quad 0.000 \mathrm{E}+00 \quad 0.000 \mathrm{E}+00$ Parent Dose Report

Title: RESRAD-OFFSITE Effluent release

File : PADUCAH EFFLUENT RELEASE-REV1.ROF

Individual Nuclide Soil Concentration Parent Nuclide and Thread Fraction Indicated

ONuclide Parent THF(i) $S(j, t)$, pCi/g

\begin{tabular}{|c|c|c|c|c|c|c|c|}
\hline $\begin{array}{l}C \perp 1 d e \\
(j)\end{array}$ & $\begin{array}{l}\text { arent } \\
\text { (i) }\end{array}$ & & $t=0.000 \mathrm{E}+00$ & $1.000 \mathrm{E}+00$ & $\begin{array}{l}l, t), \quad p C 1 / 9 \\
3.000 \mathrm{E}+00\end{array}$ & $6.000 \mathrm{E}+00$ & $1.000 \mathrm{E}+01$ \\
\hline Po-210 & $\mathrm{Pu}-238$ & $1.000 \mathrm{E}+00$ & $0.000 \mathrm{E}+00$ & $0.000 \mathrm{E}+00$ & $0.000 \mathrm{E}+00$ & $0.000 \mathrm{E}+00$ & $0.000 \mathrm{E}+00$ \\
\hline Po-210 & Th-230 & $1.000 \mathrm{E}+00$ & $0.000 \mathrm{E}+00$ & $0.000 \mathrm{E}+00$ & $0.000 \mathrm{E}+00$ & $0.000 \mathrm{E}+00$ & $0.000 \mathrm{E}+00$ \\
\hline Po-210 & $\mathrm{U}-234$ & $1.000 \mathrm{E}+00$ & $0.000 \mathrm{E}+00$ & $0.000 \mathrm{E}+00$ & $0.000 \mathrm{E}+00$ & $0.000 \mathrm{E}+00$ & $0.000 \mathrm{E}+00$ \\
\hline Po-210 & $\mathrm{U}-238$ & $9.999 \mathrm{E}-01$ & $0.000 \mathrm{E}+00$ & $0.000 \mathrm{E}+00$ & $0.000 \mathrm{E}+00$ & $0.000 \mathrm{E}+00$ & $0.000 \mathrm{E}+00$ \\
\hline Po-210 & $\Sigma S(j):$ & & $0.000 \mathrm{E}+00$ & $0.000 \mathrm{E}+00$ & $0.000 \mathrm{E}+00$ & $0.000 \mathrm{E}+00$ & $0.000 \mathrm{E}+00$ \\
\hline oPu-239 & $\mathrm{Pu}-239$ & $1.000 \mathrm{E}+00$ & $0.000 \mathrm{E}+00$ & $0.000 \mathrm{E}+00$ & $0.000 \mathrm{E}+00$ & $0.000 \mathrm{E}+00$ & $0.000 \mathrm{E}+00$ \\
\hline OU-235 & $\mathrm{Pu}-239$ & $1.000 \mathrm{E}+00$ & $0.000 \mathrm{E}+00$ & $0.000 \mathrm{E}+00$ & $0.000 \mathrm{E}+00$ & $0.000 \mathrm{E}+00$ & $0.000 \mathrm{E}+00$ \\
\hline $\mathrm{U}-235$ & $\mathrm{U}-235$ & $1.000 \mathrm{E}+00$ & $0.000 \mathrm{E}+00$ & $0.000 \mathrm{E}+00$ & $0.000 \mathrm{E}+00$ & $0.000 \mathrm{E}+00$ & $0.000 \mathrm{E}+00$ \\
\hline $\mathrm{U}-235$ & $\Sigma S(j):$ & & $0.000 \mathrm{E}+00$ & $0.000 \mathrm{E}+00$ & $0.000 \mathrm{E}+00$ & $0.000 \mathrm{E}+00$ & $0.000 \mathrm{E}+00$ \\
\hline $0 \mathrm{~Pa}-231$ & $\mathrm{Pu}-239$ & $1.000 \mathrm{E}+00$ & $0.000 \mathrm{E}+00$ & $0.000 \mathrm{E}+00$ & $0.000 \mathrm{E}+00$ & $0.000 \mathrm{E}+00$ & $0.000 \mathrm{E}+00$ \\
\hline $\mathrm{Pa}-231$ & $\mathrm{U}-235$ & $1.000 \mathrm{E}+00$ & $0.000 \mathrm{E}+00$ & $0.000 \mathrm{E}+00$ & $0.000 \mathrm{E}+00$ & $0.000 \mathrm{E}+00$ & $0.000 \mathrm{E}+00$ \\
\hline $\mathrm{Pa}-231$ & $\Sigma S(j):$ & & $0.000 \mathrm{E}+00$ & $0.000 \mathrm{E}+00$ & $0.000 \mathrm{E}+00$ & $0.000 \mathrm{E}+00$ & $0.000 \mathrm{E}+00$ \\
\hline $0 A C-227$ & $\mathrm{Pu}-239$ & $1.000 \mathrm{E}+00$ & $0.000 \mathrm{E}+00$ & $0.000 \mathrm{E}+00$ & $0.000 \mathrm{E}+00$ & $0.000 \mathrm{E}+00$ & $0.000 \mathrm{E}+00$ \\
\hline$A c-227$ & $\mathrm{U}-235$ & & $0.000 \mathrm{E}+00$ & $0.000 \mathrm{E}+00$ & $0.000 \mathrm{E}+00$ & 0.00 & $0.000 \mathrm{E}+00$ \\
\hline$A C-227$ & $\Sigma S(j):$ & & $0.000 \mathrm{E}+00$ & $0.000 \mathrm{E}+00$ & $0.000 \mathrm{E}+00$ & $0.000 \mathrm{E}+00$ & $0.000 \mathrm{E}+00$ \\
\hline $0 \mathrm{Pu}-240$ & $\mathrm{Pu}-240$ & 4.95 & $0.000 \mathrm{E}+00$ & $0.000 \mathrm{E}+00$ & $0.000 \mathrm{E}+00$ & $0.000 \mathrm{E}+00$ & $0.000 \mathrm{E}+00$ \\
\hline $\mathrm{Pu}-240$ & $\mathrm{Pu}-240$ & & $0.000 \mathrm{E}+00$ & $0.000 \mathrm{E}+00$ & $0.000 \mathrm{E}+00$ & $0.000 \mathrm{E}+00$ & $0.000 \mathrm{E}+00$ \\
\hline $\mathrm{Pu}-240$ & $\Sigma S(j):$ & & $0.000 \mathrm{E}+00$ & $0.000 \mathrm{E}+00$ & $0.000 \mathrm{E}+00$ & $0.000 \mathrm{E}+00$ & $0.000 \mathrm{E}+00$ \\
\hline OU-236 & $\mathrm{Pu}-240$ & $1.000 \mathrm{E}+00$ & $0.000 \mathrm{E}+00$ & $0.000 \mathrm{E}+00$ & $0.000 \mathrm{E}+00$ & $0.000 \mathrm{E}+00$ & $0.000 \mathrm{E}+00$ \\
\hline 0 Th-232 & $\mathrm{Pu}-240$ & 1.00 & $0.000 \mathrm{E}+00$ & $0.000 \mathrm{E}+00$ & $0.000 \mathrm{E}+00$ & $0.000 \mathrm{E}+00$ & $0.000 \mathrm{E}+00$ \\
\hline Th-232 & Th-232 & $1.000 \mathrm{E}+00$ & $0.000 \mathrm{E}+00$ & $0.000 \mathrm{E}+00$ & $0.000 \mathrm{E}+00$ & $0.000 \mathrm{E}+00$ & $0.000 \mathrm{E}+00$ \\
\hline Th-232 & $\Sigma S(j):$ & & $0.000 \mathrm{E}+00$ & $0.000 \mathrm{E}+00$ & $0.000 \mathrm{E}+00$ & $0.000 \mathrm{E}+00$ & $0.000 \mathrm{E}+00$ \\
\hline $0 R a-228$ & $\mathrm{Pu}-240$ & $1.000 \mathrm{E}+00$ & $0.000 \mathrm{E}+00$ & $0.000 \mathrm{E}+00$ & $0.000 \mathrm{E}+00$ & $0.000 \mathrm{E}+00$ & $0.000 \mathrm{E}+00$ \\
\hline $\mathrm{Ra}-228$ & Th-232 & $1.000 \mathrm{E}+00$ & $0.000 \mathrm{E}+00$ & $0.000 \mathrm{E}+00$ & $0.000 \mathrm{E}+00$ & $0.000 \mathrm{E}+00$ & $0.000 \mathrm{E}+00$ \\
\hline $\mathrm{Ra}-228$ & $\Sigma S(j):$ & & $0.000 \mathrm{E}+00$ & $0.000 \mathrm{E}+00$ & $0.000 \mathrm{E}+00$ & $0.000 \mathrm{E}+00$ & $0.000 \mathrm{E}+00$ \\
\hline $0 T h-228$ & $\mathrm{Pu}-240$ & $1.000 \mathrm{E}+00$ & $0.000 \mathrm{E}+00$ & $0.000 \mathrm{E}+00$ & $0.000 \mathrm{E}+00$ & $0.000 \mathrm{E}+00$ & $0.000 \mathrm{E}+00$ \\
\hline Th-228 & Th-228 & $1.000 \mathrm{E}+00$ & $0.000 \mathrm{E}+00$ & $0.000 \mathrm{E}+00$ & $0.000 \mathrm{E}+00$ & $0.000 \mathrm{E}+00$ & $0.000 \mathrm{E}+00$ \\
\hline Th-228 & Th-232 & $1.000 \mathrm{E}+00$ & $0.000 \mathrm{E}+00$ & $0.000 \mathrm{E}+00$ & $0.000 \mathrm{E}+00$ & $0.000 \mathrm{E}+00$ & $0.000 \mathrm{E}+00$ \\
\hline Th-228 & $\Sigma S(j):$ & & $0.000 \mathrm{E}+00$ & $0.000 \mathrm{E}+00$ & $0.000 \mathrm{E}+00$ & $0.000 \mathrm{E}+00$ & $0.000 \mathrm{E}+00$ \\
\hline $0 T C-99$ & TC-99 & $1.000 \mathrm{E}+00$ & $0.000 \mathrm{E}+00$ & $0.000 \mathrm{E}+00$ & $0.000 \mathrm{E}+00$ & 0.00 & $0.000 \mathrm{E}+00$ \\
\hline OU-238 & $\mathrm{U}-238$ & $5.400 \mathrm{E}-05$ & $0.000 \mathrm{E}+00$ & $0.000 \mathrm{E}+00$ & $0.000 \mathrm{E}+00$ & $0.000 \mathrm{E}+00$ & $0.000 \mathrm{E}+00$ \\
\hline $\mathrm{U}-238$ & $\mathrm{U}-238$ & $9.999 \mathrm{E}-01$ & $0.000 \mathrm{E}+00$ & $0.000 \mathrm{E}+00$ & $0.000 \mathrm{E}+00$ & $0.000 \mathrm{E}+00$ & $0.000 \mathrm{E}+00$ \\
\hline $\mathrm{U}-238$ & $\Sigma S(j):$ & & $0.000 \mathrm{E}+00$ & $0.000 \mathrm{E}+00$ & $0.000 \mathrm{E}+00$ & $0.000 \mathrm{E}+00$ & $0.000 \mathrm{E}+00$ \\
\hline
\end{tabular}

is the thread fraction of the parent nuclide.

1RESRAD-OFFSITE, Version 4.0 .3 beta $T^{1 / 1} / 2$ Limit $=30$ days

06/11/2020 11:32 Page 62

Parent Dose Report

DARA-OFFSITE Effluent release

File : PADUCAH EFFLUENT RELEASE-REV1.ROF 
Run Time Information

ResoCalc.EXE execution began at 11:32 on 06/11/2020

ResOCalc.EXE execution ended at 11:32 on 06/11/2020

ResOCalc.EXE execution time .216 seconds

Note: The dose at 6 years is considered for the ground external, inhalation, and soil ingestion pathways (see Table 7 in the main report). Ground concentration will increase from deposition; therefore, the dose during the last year incinerator will be operational is used because this will result in the highest dose.

\section{B.3 RESRAD-OFFSITE RESULTS FOR LANDFILL NEIGHBOR SCENARIO}

1RESRAD-OFFSITE, Version 4.0 .3 beta $\mathrm{T}^{\frac{1}{2}}$ Limit $=30$ days Parent Dose Report

Title : Landfill Scenario

File : LANDFILL-PADUCAH-AUTHORIZED-RELEASE-REV.ROF

Table of Contents

Part I: Mixture Sums and Single Radionuclide Guidelines

Dose Conversion Factor (and Related) Parameter Summary ...

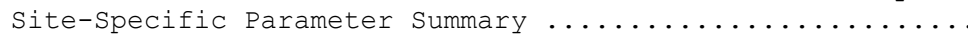

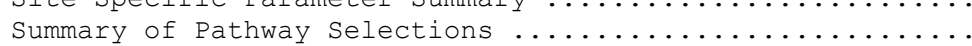
Contaminated Zone and Total Dose Summary $\ldots \ldots \ldots \ldots \ldots \ldots \ldots \ldots$

Total Dose Components

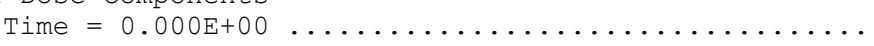

Time $=1.000 \mathrm{E}+00$

$=2.000 \mathrm{E}+00$

Time $=1.400 \mathrm{E}+01$

Time $=1.700 \mathrm{E}+01$

Time $=4.200 \mathrm{E}+02$

Time $=7.780 \mathrm{E}+02$

Time $=8.000 \mathrm{E}+02$

Time $=1.000 \mathrm{E}+03$

06/11/2020 11:17 Page

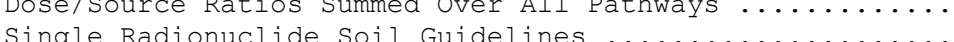

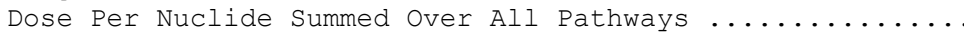

Soil Concentration Per Nuclide $\ldots \ldots \ldots \ldots \ldots \ldots \ldots \ldots$

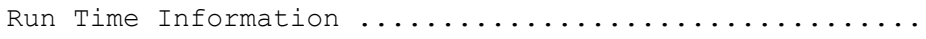

1RESRAD-OFFSITE, Version 4.0 .3 beta $T^{1 / 2}$ Limit $=30$ days

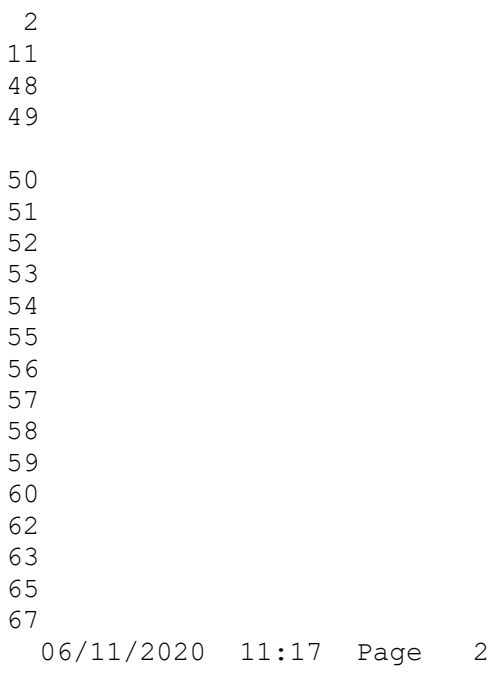


Parent Dose Report

Title : Landfill Scenario

File : LANDFILL-PADUCAH-AUTHORIZED-RELEASE-REV.ROF

Dose Conversion Factor (and Related) Parameter Summary Current Library: ICRP 60

Default Library: ICRP 60

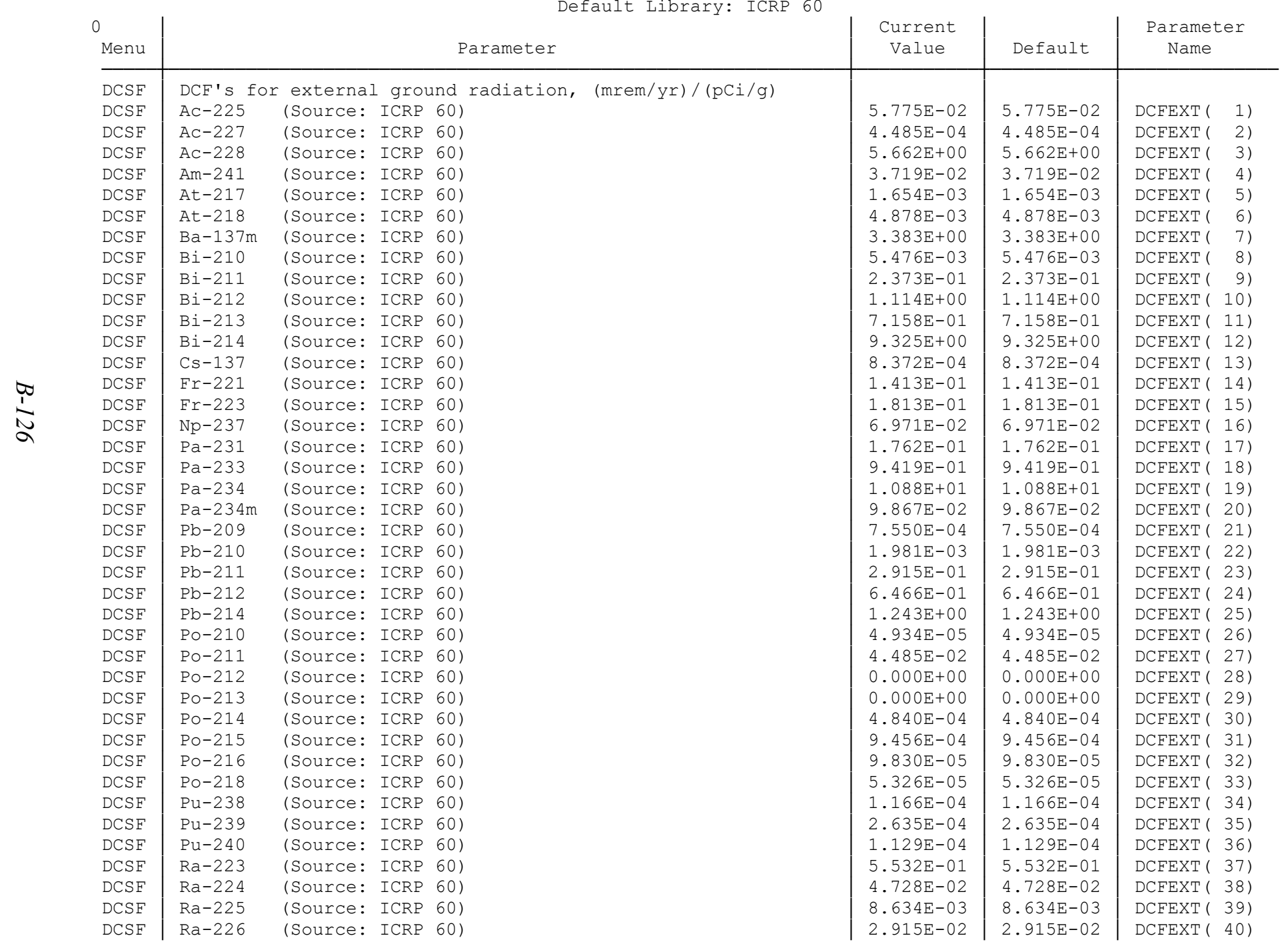


\begin{tabular}{l|ll} 
DCSF & Ra-228 (Source: ICRP 60)
\end{tabular}

DCSF Rn-219 (Source: ICRP 60)

DCSF $\mathrm{Rn}-220$ (Source: ICRP 60)

$\begin{array}{lll}\text { DCSF Rn-222 (Source: ICRP } 60 \text { ) } & \\ \text { RESRAD-OFFSITE, Version } 4.0 .3 \text { beta T1/2 Limit }=30 \text { days }\end{array}$

Parent Dose Report

Title : Landfill Scenario

File : LANDFILL-PADUCAH-AUTHORIZED-RELEASE-REV.ROF

Dose Conversion Factor (and Related) Parameter Summary (continued)

Current Library: ICRP 60

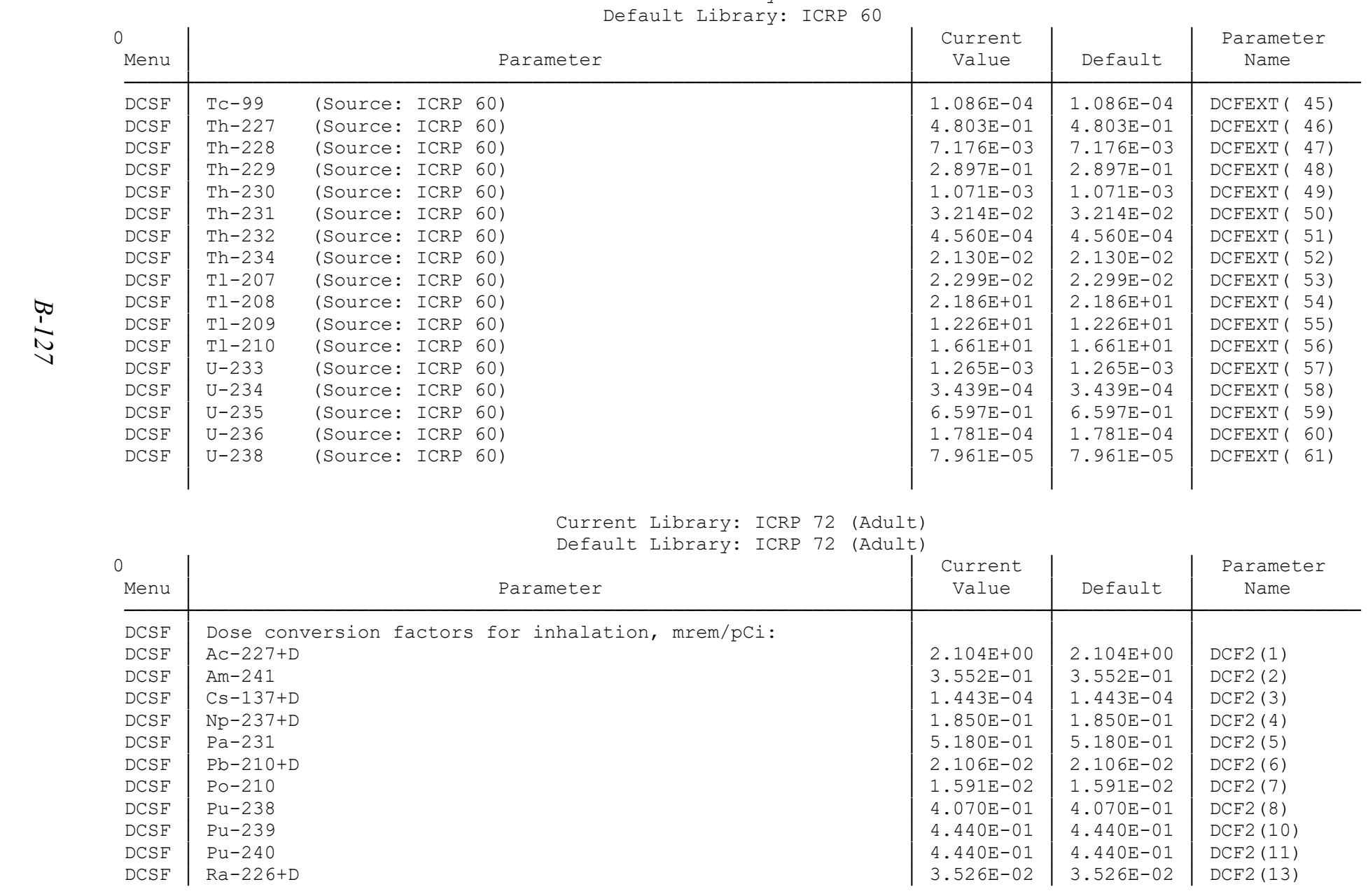

Current Library: ICRP 72 (Adult)

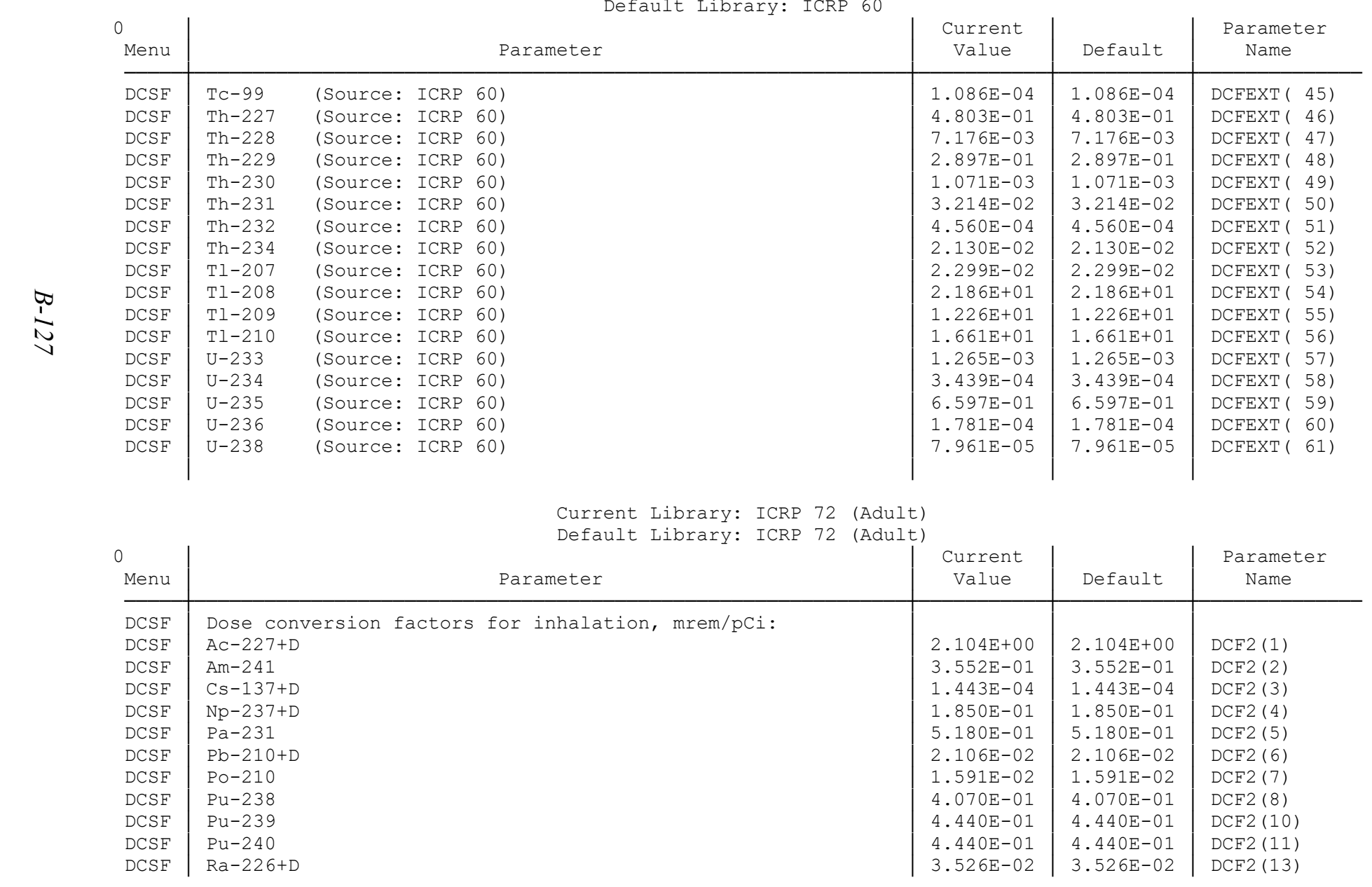




\begin{tabular}{l|l} 
DCSF & Ra $-228+\mathrm{D}$ \\
DCSF & TC -99 \\
DCSF & Th-228+D \\
DCSF & Th $-229+D$ \\
DCSF & $T h-230$ \\
DCSF & Th-232 \\
DCSF & $\mathrm{U}-233$ \\
DCSF & $\mathrm{U}-234$
\end{tabular}

1RESRAD-OFFSITE, Version 4.0 .3 beta $T^{\frac{1}{2}}$ Limit $=30$ days Parent Dose Report

Title : Landfill Scenario

File : LANDFILL-PADUCAH-AUTHORIZED-RELEASE-REV.ROF

Dose Conversion Factor (and Related) Parameter Summary (continued) Current Library: ICRP 72 (Adult)

\begin{tabular}{|c|c|c|c|c|}
\hline $\begin{array}{l}0 \\
\text { Menu }\end{array}$ & Parameter & $\begin{array}{c}\text { Current } \\
\text { Value }\end{array}$ & Default & $\begin{array}{l}\text { Parameter } \\
\text { Name }\end{array}$ \\
\hline DCSF & $\mathrm{U}-235+\mathrm{D}$ & $3.145 \mathrm{E}-02$ & $3.145 \mathrm{E}-02$ & $\mathrm{DCF} 2(22)$ \\
\hline DCSF & $\mathrm{U}-236$ & $3.219 \mathrm{E}-02$ & $3.219 \mathrm{E}-02$ & $\mathrm{DCF} 2(23)$ \\
\hline DCSF & $\mathrm{U}-238$ & $2.960 \mathrm{E}-02$ & $2.960 \mathrm{E}-02$ & $\mathrm{DCF} 2(24)$ \\
\hline DCSF & $\mathrm{U}-238+\mathrm{D}$ & $2.963 \mathrm{E}-02$ & $2.963 \mathrm{E}-02$ & $\mathrm{DCF} 2(25)$ \\
\hline DCSF & Dose conversion factors for ingestion, mrem/pCi: & & & \\
\hline DCSF & $A C-227+D$ & $4.473 \mathrm{E}-03$ & $4.473 \mathrm{E}-03$ & $\mathrm{DCF} 3(1)$ \\
\hline $\mathrm{DCSF}$ & $A m-241$ & $7.400 \mathrm{E}-04$ & $7.400 \mathrm{E}-04$ & DCF3 (2) \\
\hline DCSF & $\mathrm{Cs}-137+\mathrm{D}$ & $4.810 \mathrm{E}-05$ & $4.810 \mathrm{E}-05$ & DCF3 (3) \\
\hline DCSF & $\mathrm{Np}-237+\mathrm{D}$ & $4.102 \mathrm{E}-04$ & $4.102 \mathrm{E}-04$ & $\mathrm{DCF} 3(4)$ \\
\hline $\mathrm{DCSF}$ & $\mathrm{Pa}-231$ & $2.627 \mathrm{E}-03$ & $2.627 \mathrm{E}-03$ & DCF $3(5)$ \\
\hline DCSF & $\mathrm{Pb}-210+\mathrm{D}$ & $2.558 \mathrm{E}-03$ & $2.558 \mathrm{E}-03$ & DCF3 (6) \\
\hline DCSF & $\mathrm{Po}-210$ & $4.440 \mathrm{E}-03$ & $4.440 \mathrm{E}-03$ & DCF3 (7) \\
\hline DCSF & $\mathrm{Pu}-238$ & $8.510 \mathrm{E}-04$ & $8.510 \mathrm{E}-04$ & DCF3 (8) \\
\hline DCSF & $\mathrm{Pu}-239$ & $9.250 \mathrm{E}-04$ & $9.250 \mathrm{E}-04$ & $\mathrm{DCF} 3(10)$ \\
\hline $\mathrm{DCSF}$ & $\mathrm{Pu}-240$ & $9.250 \mathrm{E}-04$ & $9.250 \mathrm{E}-04$ & DCF3 (11) \\
\hline DCSF & $R a-226+D$ & $1.037 \mathrm{E}-03$ & $1.037 \mathrm{E}-03$ & $\mathrm{DCF} 3(13)$ \\
\hline DCSF & $\mathrm{Ra}-228+\mathrm{D}$ & $2.555 \mathrm{E}-03$ & $2.555 \mathrm{E}-03$ & $\mathrm{DCF} 3(14)$ \\
\hline DCSF & $\mathrm{TC}-99$ & $2.368 \mathrm{E}-06$ & $2.368 \mathrm{E}-06$ & $\mathrm{DCF} 3(15)$ \\
\hline DCSF & Th $-228+D$ & $5.301 \mathrm{E}-04$ & $5.301 \mathrm{E}-04$ & $\mathrm{DCF} 3(16)$ \\
\hline $\mathrm{DCSF}$ & $T h-229+D$ & $2.269 \mathrm{E}-03$ & $2.269 \mathrm{E}-03$ & $\mathrm{DCF} 3(17)$ \\
\hline DCSF & Th-230 & $7.770 \mathrm{E}-04$ & $7.770 \mathrm{E}-04$ & $\mathrm{DCF} 3(18)$ \\
\hline DCSF & Th-232 & $8.510 \mathrm{E}-04$ & $8.510 \mathrm{E}-04$ & $\mathrm{DCF} 3(19)$ \\
\hline $\mathrm{DCSF}$ & $\mathrm{U}-233$ & $1.887 \mathrm{E}-04$ & $1.887 \mathrm{E}-04$ & $\mathrm{DCF} 3(20)$ \\
\hline DCSF & $\mathrm{U}-234$ & $1.813 \mathrm{E}-04$ & $1.813 \mathrm{E}-04$ & $\mathrm{DCF} 3(21)$ \\
\hline DCSF & $\mathrm{U}-235+\mathrm{D}$ & $1.752 \mathrm{E}-04$ & $1.752 \mathrm{E}-04$ & $\mathrm{DCF} 3(22)$ \\
\hline DCSF & $\mathrm{U}-236$ & $1.739 \mathrm{E}-04$ & $1.739 \mathrm{E}-04$ & $\mathrm{DCF} 3(23)$ \\
\hline DCSF & $\mathrm{U}-238$ & $1.665 \mathrm{E}-04$ & $1.665 \mathrm{E}-04$ & $\operatorname{DCF} 3(24)$ \\
\hline DCSF & $\mathrm{U}-238+\mathrm{D}$ & $1.791 \mathrm{E}-04$ & $1.791 \mathrm{E}-04$ & $\mathrm{DCF} 3(25)$ \\
\hline $1 \mathrm{~K}$ & $\mathrm{~T}^{1 / 2}$ Limit $=30$ days & /2020 & Page & \\
\hline
\end{tabular}

\begin{tabular}{l|l|l}
$5.929 \mathrm{E}-02$ & $5.929 \mathrm{E}-02$ & DCF2 (14) \\
$4.810 \mathrm{E}-05$ & $4.810 \mathrm{E}-05$ & DCF2 (15) \\
$1.614 \mathrm{E}-01$ & $1.614 \mathrm{E}-01$ & DCF2 (16) \\
$9.481 \mathrm{E}-01$ & $9.481 \mathrm{E}-01$ & DCF2 $(17)$ \\
$3.700 \mathrm{E}-01$ & $3.700 \mathrm{E}-01$ & DCF2 $(18)$ \\
$4.070 \mathrm{E}-01$ & $4.070 \mathrm{E}-01$ & DCF2 (19) \\
$3.552 \mathrm{E}-02$ & $3.552 \mathrm{E}-02$ & DCF2 (20) \\
$3.478 \mathrm{E}-02$ & $3.478 \mathrm{E}-02$ & DCF2 (21)
\end{tabular}

$06 / 11 / 2020 \quad 11: 17$ Page 4
Parent DOSE RePOr 
Title : Landfill Scenario

File : LANDFILL-PADUCAH-AUTHORIZED-RELEASE-REV.ROF

Dose Conversion Factor (and Related) Parameter Summary (continued)

Current Library: RESRAD Default Transfer factors

Default Library: RESRAD Default Transfer factors

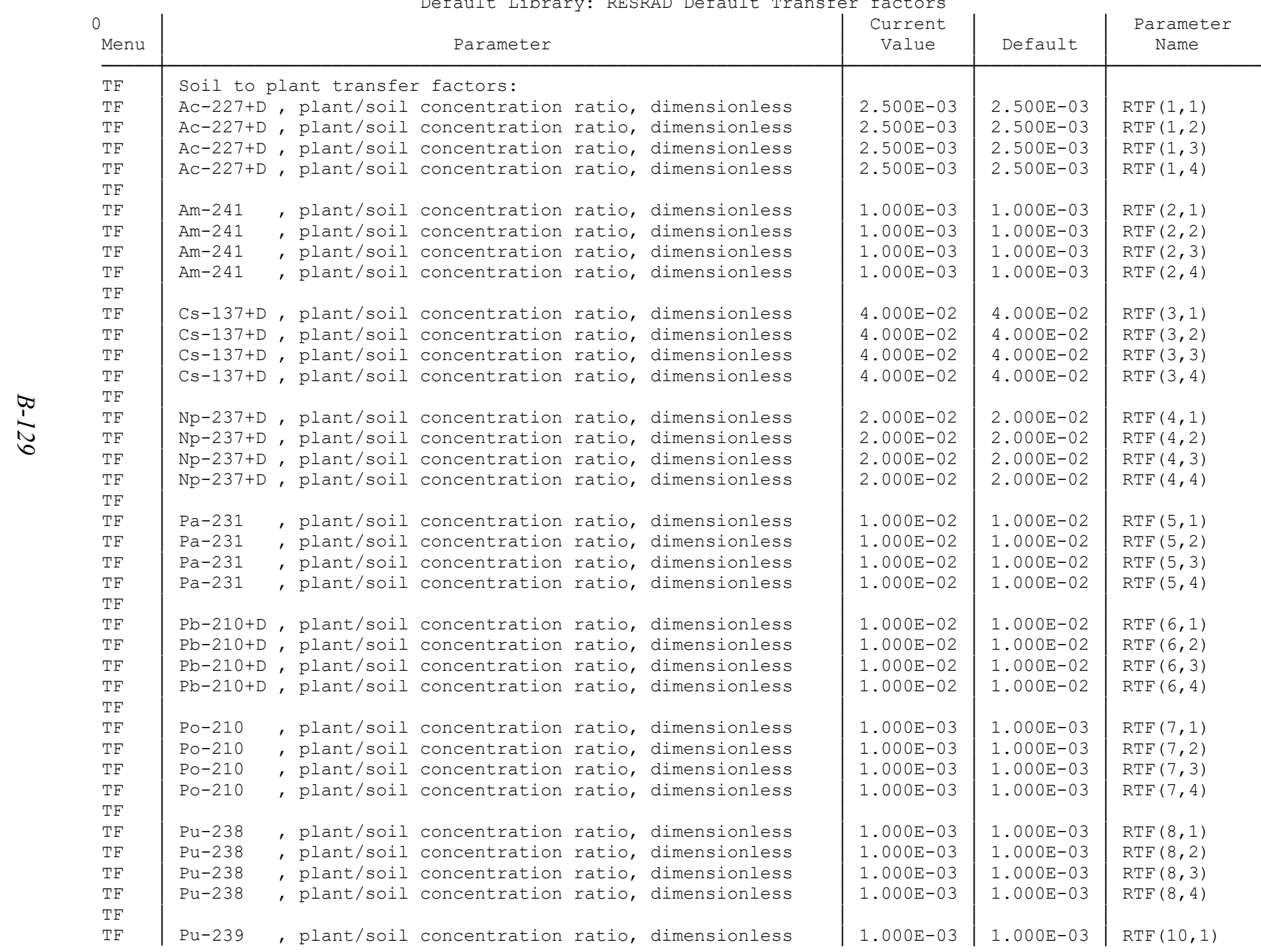




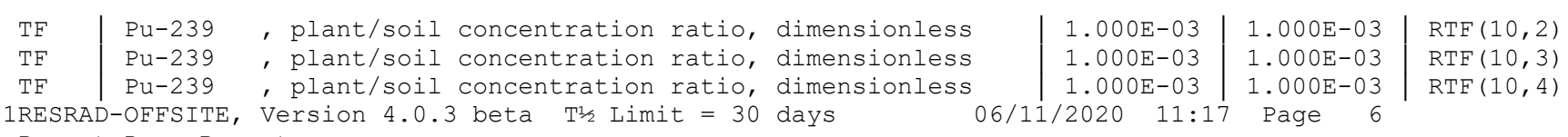
IRESRAD-OFFSITE, Version 4.0.3 beta T1/2 Limit $=30$ days $\quad 06 / 11 / 2020 \quad 11: 17$ Page 6 Parent Dose Report

Title : Landfill Scenario

File : LANDFILL-PADUCAH-AUTHORIZED-RELEASE-REV.ROF

Dose Conversion Factor (and Related) Parameter Summary (continued)

Current Library: RESRAD Default Transfer factors

Default Library: RESRAD Default Transfer factors

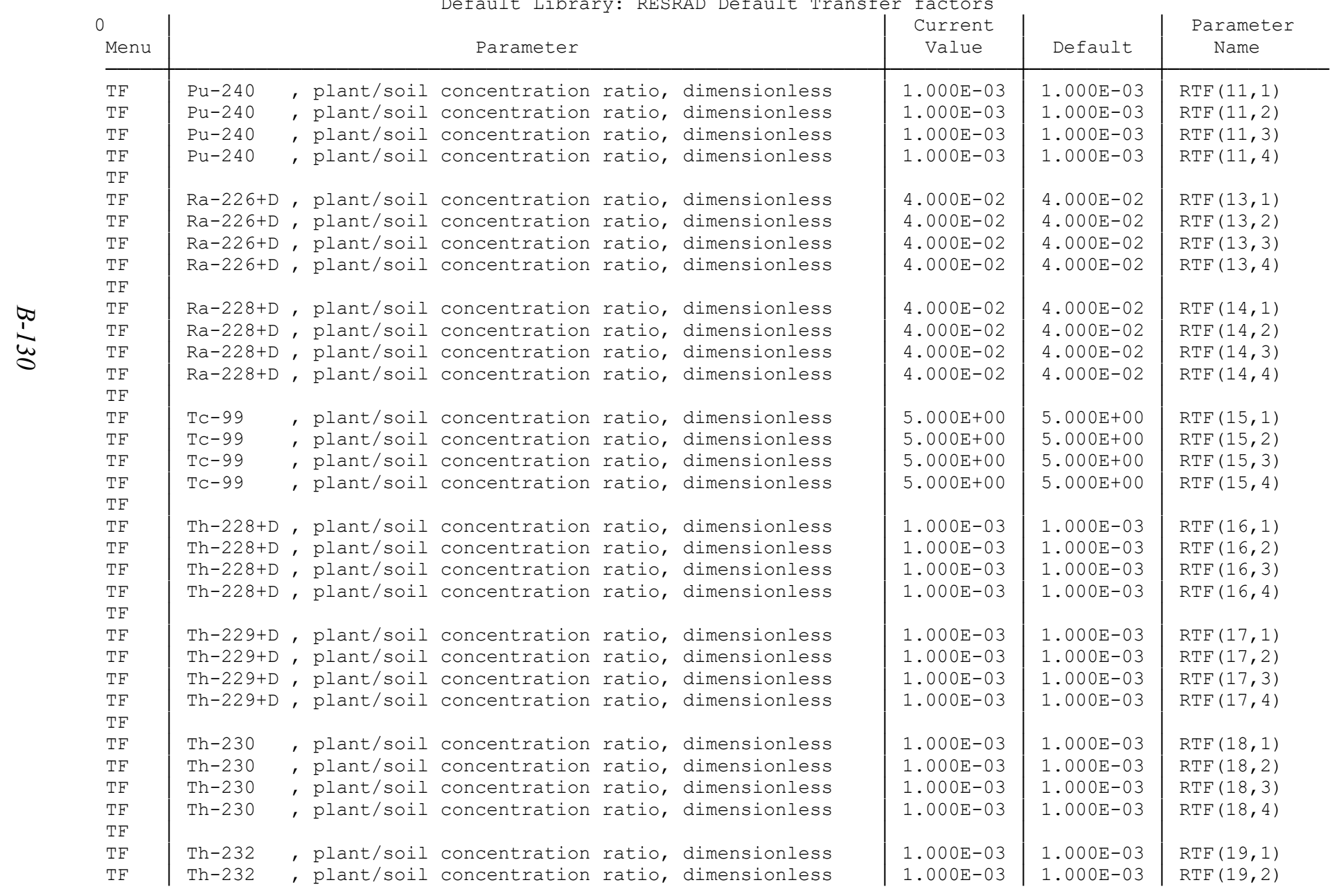




\begin{tabular}{l|lll|l|l|l} 
TF & Th-232 & , plant/soil concentration ratio, dimensionless & $1.000 \mathrm{E}-03$ & $1.000 \mathrm{E}-03$ & $\mathrm{RTF}(19,3)$ \\
$\mathrm{TF}$ & $\mathrm{Th}-232$ &, plant/soil concentration ratio, dimensionless & $1.000 \mathrm{E}-03$ & $1.000 \mathrm{E}-03$ & $\mathrm{RTF}(19,4)$ \\
$\mathrm{TF}$ & & & & & \\
$\mathrm{TF}$ & $\mathrm{U}-233$ &, plant/soil concentration ratio, dimensionless & $2.500 \mathrm{E}-03$ & $2.500 \mathrm{E}-03$ & $\mathrm{RTF}(20,1)$ \\
$\mathrm{TF}$ & $\mathrm{U}-233$ &, plant/soil concentration ratio, dimensionless & $2.500 \mathrm{E}-03$ & $2.500 \mathrm{E}-03$ & $\mathrm{RTF}(20,2)$ \\
$\mathrm{TF}$ & $\mathrm{U}-233$ &, plant/soil concentration ratio, dimensionless & $2.500 \mathrm{E}-03$ & $2.500 \mathrm{E}-03$ & $\mathrm{RTF}(20,3)$ \\
$\mathrm{TF}$ & $\mathrm{U}-233$ &, plant/soil concentration ratio, dimensionless & $2.500 \mathrm{E}-03$ & $2.500 \mathrm{E}-03$ & $\mathrm{RTF}(20,4)$ \\
$\mathrm{TF}$ & & &
\end{tabular}

RESRAD-OFFSITE, Version 4.0 .3 beta $\mathrm{T}^{1 / 2} \mathrm{Limit}=30$ days

Parent Dose Report

Title : Landfill Scenario

File : LANDFILL-PADUCAH-AUTHORIZED-RELEASE-REV.ROF

Dose Conversion Factor (and Related) Parameter Summary (continued) Current Library: RESRAD Default Transfer factors Default Library: RESRAD Default Transfer factors

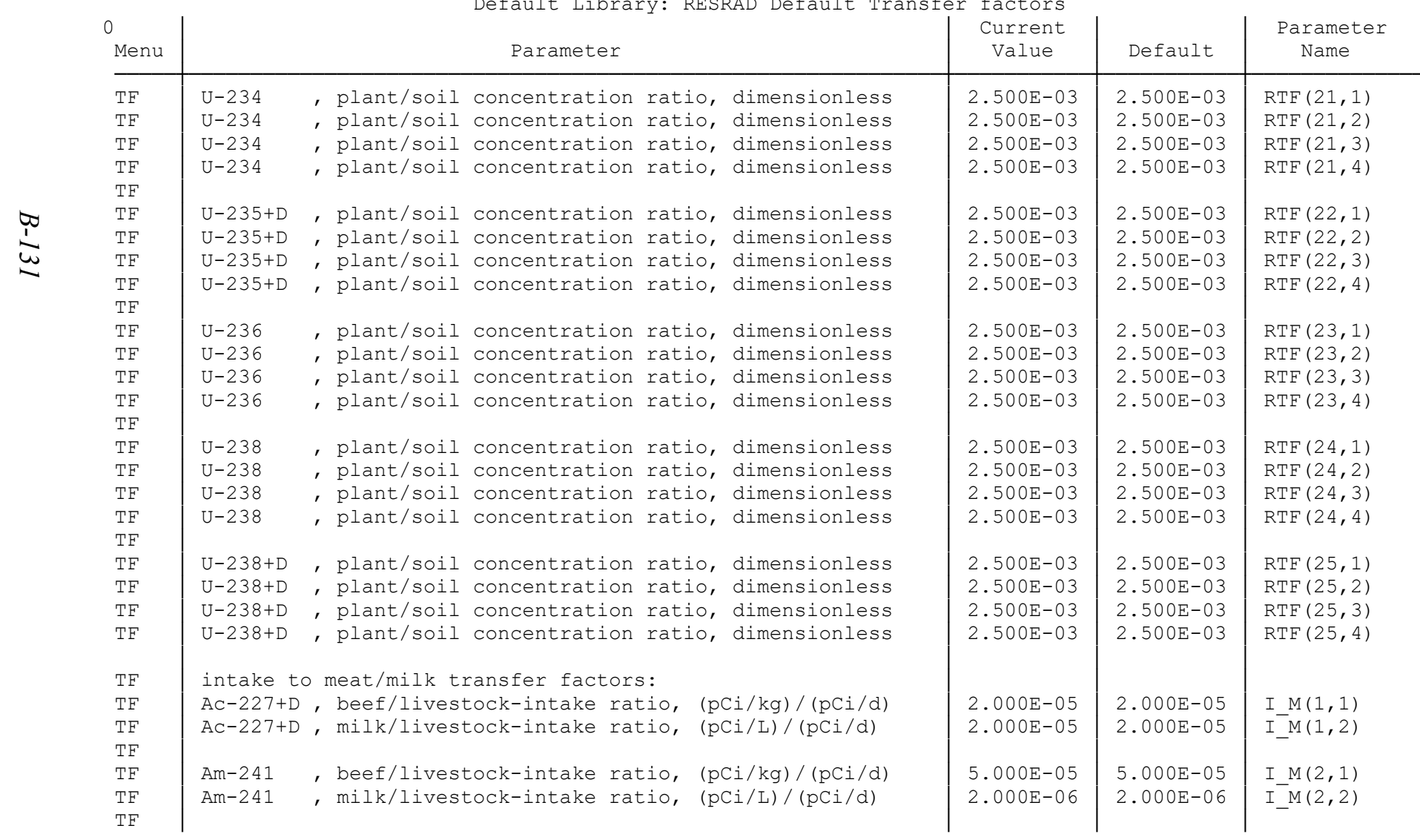




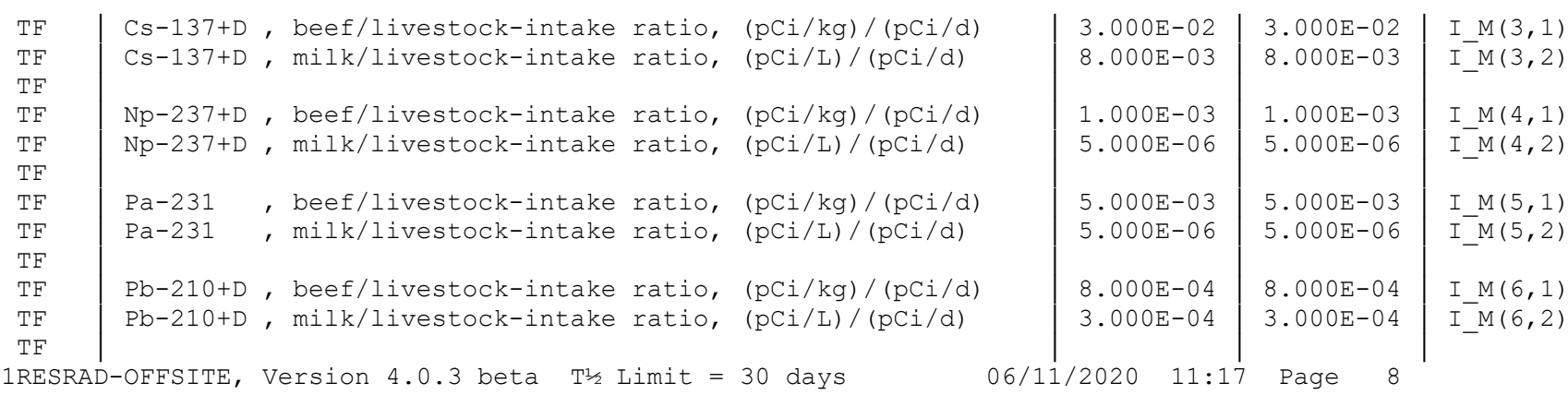

1RESRAD-OFFSITE, Version 4.0 .3 beta T1/2 Limit $=30$ days

06/11/2020 11:17 Page 8

Title : Landfill Scenario

File : LANDFILL-PADUCAH-AUTHORIZED-RELEASE-REV.ROF

Dose Conversion Factor (and Related) Parameter Summary (continued)

Current Library: RESRAD Default Transfer factors

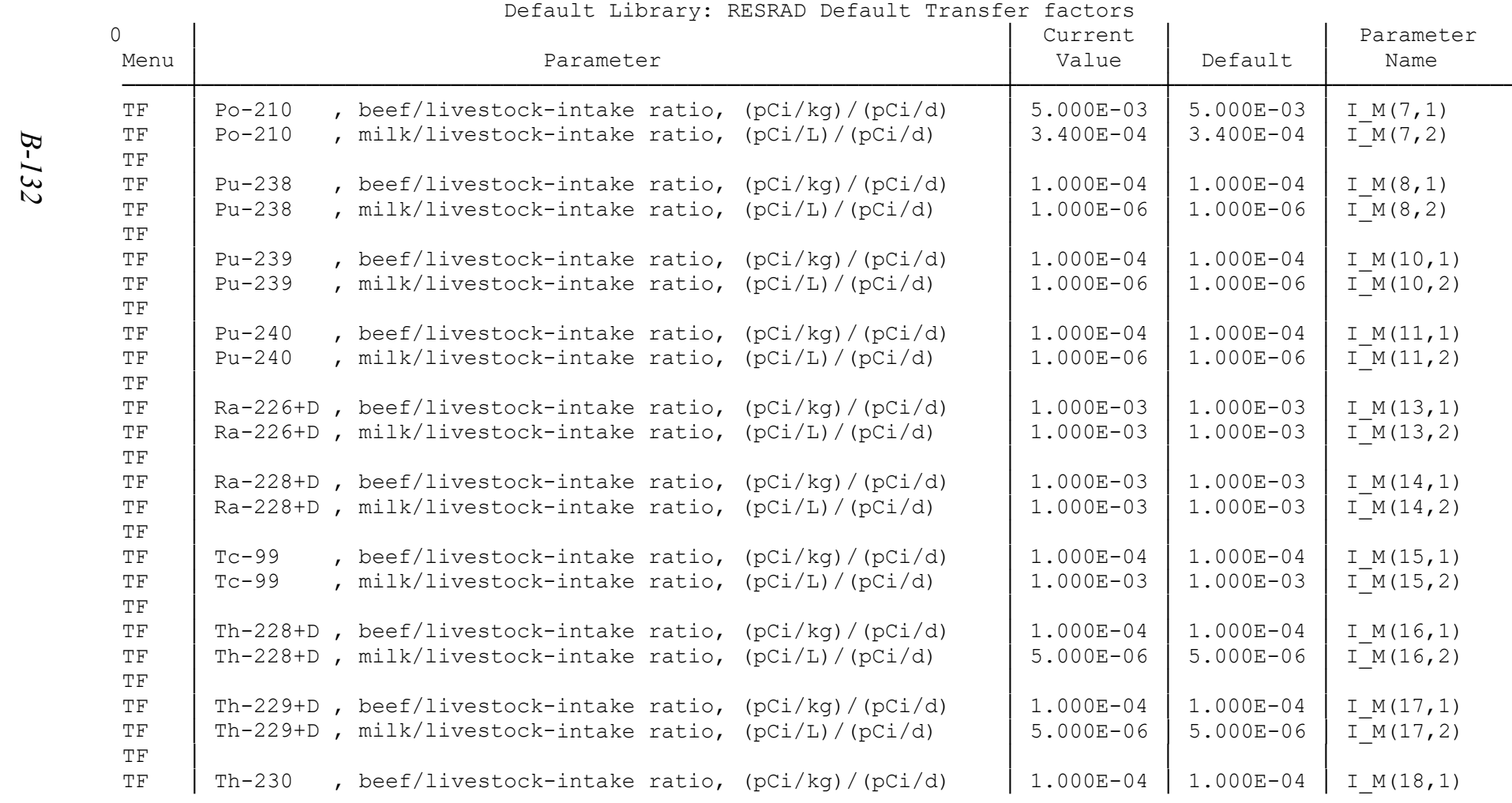




\begin{tabular}{|c|c|c|c|c|c|c|c|}
\hline $\begin{array}{l}\mathrm{TF} \\
\mathrm{TF}\end{array}$ & Th -230 & , milk/livestock-intake & ratio, & $(\mathrm{pCi} / \mathrm{L}) /(\mathrm{pCi} / \mathrm{d})$ & $5.000 \mathrm{E}-06$ & $5.000 \mathrm{E}-06$ & I_M $(18,2)$ \\
\hline $\mathrm{TF}$ & Th -232 & , beef/livestock-intake & ratio, & $(\mathrm{pCi} / \mathrm{kg}) /(\mathrm{pCi} / \mathrm{d})$ & $1.000 \mathrm{E}-04$ & $1.000 \mathrm{E}-04$ & I M $(19,1)$ \\
\hline $\begin{array}{l}\mathrm{TF} \\
\mathrm{TF}\end{array}$ & Th -232 & , milk/livestock-intake & ratio, & $(\mathrm{pCi} / \mathrm{L}) /(\mathrm{pCi} / \mathrm{d})$ & $5.000 \mathrm{E}-06$ & $5.000 \mathrm{E}-06$ & $I_{-}^{-} M(19,2)$ \\
\hline $\mathrm{TF}$ & $\mathrm{U}-233$ & , beef/livestock-intake & ratio, & $(\mathrm{pCi} / \mathrm{kg}) /(\mathrm{pCi} / \mathrm{d})$ & $3.400 \mathrm{E}-04$ & $3.400 \mathrm{E}-04$ & I_M $(20,1)$ \\
\hline $\begin{array}{l}\mathrm{TF} \\
\mathrm{TF}\end{array}$ & $\mathrm{U}-233$ & , milk/livestock-intake & ratio, & $(\mathrm{pCi} / \mathrm{L}) /(\mathrm{pCi} / \mathrm{d})$ & $6.000 \mathrm{E}-04$ & $6.000 \mathrm{E}-04$ & $I_{-} \mathrm{M}(20,2)$ \\
\hline $\mathrm{TF}$ & $\mathrm{U}-234$ & , beef/livestock-intake & ratio, & $(\mathrm{pCi} / \mathrm{kg}) /(\mathrm{pCi} / \mathrm{d})$ & $3.400 \mathrm{E}-04$ & $3.400 \mathrm{E}-04$ & I_M $(21,1)$ \\
\hline $\begin{array}{l}\mathrm{TF} \\
\mathrm{TF}\end{array}$ & $\mathrm{U}-234$ & , milk/livestock-intake & ratio, & $(\mathrm{pCi} / \mathrm{L}) /(\mathrm{pCi} / \mathrm{d})$ & $6.000 \mathrm{E}-04$ & $6.000 \mathrm{E}-04$ & I_M $(21,2)$ \\
\hline $\mathrm{TF}$ & $U-235+D$ & , beef/livestock-intake & ratio, & $(\mathrm{pCi} / \mathrm{kg}) /(\mathrm{pCi} / \mathrm{d})$ & $3.400 \mathrm{E}-04$ & $3.400 \mathrm{E}-04$ & I_M $(22,1)$ \\
\hline $\begin{array}{l}\mathrm{TF} \\
\mathrm{TF}\end{array}$ & $\mathrm{U}-235+\mathrm{D}$ & , milk/livestock-intake & ratio, & $(\mathrm{pCi} / \mathrm{L}) /(\mathrm{pCi} / \mathrm{d})$ & $6.000 \mathrm{E}-04$ & $6.000 \mathrm{E}-04$ & I_M $(22,2)$ \\
\hline $\mathrm{TF}$ & $\mathrm{U}-236$ & , beef/livestock-intake & ratio, & $(\mathrm{pCi} / \mathrm{kg}) /(\mathrm{pCi} / \mathrm{d})$ & $3.400 \mathrm{E}-04$ & $3.400 \mathrm{E}-04$ & I_M $(23,1)$ \\
\hline $\mathrm{TF}$ & $\mathrm{U}-236$ & , milk/livestock-intake & ratio, & $(\mathrm{pCi} / \mathrm{L}) /(\mathrm{pCi} / \mathrm{d})$ & $6.000 \mathrm{E}-04$ & $6.000 \mathrm{E}-04$ & I_M $(23,2)$ \\
\hline
\end{tabular}

Parent Dose Report

06/11/2020 11:17 Page 9

Parent Dose Report

Title : Landfill Scenario

File : LANDFILL-PADUCAH-AUTHORIZED-RELEASE-REV.ROF

Dose Conversion Factor (and Related) Parameter Summary (continued) Current Library: RESRAD Default Transfer factors

\begin{tabular}{|c|c|c|c|c|c|}
\hline $\begin{array}{l}0 \\
\text { Menu }\end{array}$ & & Parameter & $\begin{array}{c}\text { Current } \\
\text { Value }\end{array}$ & Default & $\begin{array}{l}\text { Parameter } \\
\text { Name }\end{array}$ \\
\hline TF & $\mathrm{U}-238$ & , beef/livestock-intake ratio, $(\mathrm{pCi} / \mathrm{kg}) /(\mathrm{pCi} / \mathrm{d})$ & $3.400 \mathrm{E}-04$ & $3.400 \mathrm{E}-04$ & I_M $(24,1)$ \\
\hline TF & $\mathrm{U}-238+\mathrm{D}$ & , beef/livestock-intake ratio, $(\mathrm{pCi} / \mathrm{kg}) /(\mathrm{pCi} / \mathrm{d})$ & $3.400 \mathrm{E}-04$ & $3.400 \mathrm{E}-04$ & I M $(25,1)$ \\
\hline TF & $\mathrm{U}-238+\mathrm{D}$ & , milk/livestock-intake ratio, $(\mathrm{pCi} / \mathrm{L}) /(\mathrm{pCi} / \mathrm{d})$ & $6.000 \mathrm{E}-04$ & $6.000 \mathrm{E}-04$ & $I_{-} M(25,2)$ \\
\hline $\begin{array}{l}\mathrm{TF} \\
\mathrm{TF}\end{array}$ & $A C-227+D$ & , crustacea and mollusks & $1.000 \mathrm{E}+03$ & $1.000 \mathrm{E}+03$ & $\operatorname{BIOFA}(1,2)$ \\
\hline $\mathrm{TF}$ & Am-241 & , fish & $3.000 \mathrm{E}+01$ & $3.000 \mathrm{E}+01$ & $\operatorname{BIOFA}(2,1)$ \\
\hline $\begin{array}{l}\mathrm{TF} \\
\mathrm{TF}\end{array}$ & $A m-241$ & , crustacea and mollusks & $1.000 \mathrm{E}+03$ & $1.000 \mathrm{E}+03$ & $\operatorname{BIOFA}(2,2)$ \\
\hline $\mathrm{TF}$ & $C s-137+D$ & , fish & $2.000 \mathrm{E}+03$ & $2.000 \mathrm{E}+03$ & $\operatorname{BIOFA}(3,1)$ \\
\hline $\begin{array}{l}\text { TF } \\
\text { TF }\end{array}$ & $C s-137+D$ & , crustacea and mollusks & $1.000 \mathrm{E}+02$ & $1.000 \mathrm{E}+02$ & $\operatorname{BIOFA}(3,2)$ \\
\hline $\mathrm{TF}$ & $\mathrm{Np}-237+\mathrm{D}$ & , fish & $3.000 \mathrm{E}+01$ & $3.000 \mathrm{E}+01$ & $\operatorname{BIOFA}(4,1)$ \\
\hline $\begin{array}{l}\mathrm{TF} \\
\mathrm{TF}\end{array}$ & $\mathrm{Np}-237+\mathrm{D}$ & , crustacea and mollusks & $4.000 \mathrm{E}+02$ & $4.000 \mathrm{E}+02$ & $\operatorname{BIOFA}(4,2)$ \\
\hline TF & $\mathrm{Pa}-231$ & , fish & $1.000 \mathrm{E}+01$ & $1.000 \mathrm{E}+01$ & $\operatorname{BIOFA}(5,1)$ \\
\hline $\begin{array}{l}\mathrm{TF} \\
\mathrm{TF}\end{array}$ & $\mathrm{Pa}-231$ & , crustacea and mollusks & $1.100 \mathrm{E}+02$ & $1.100 \mathrm{E}+02$ & $\operatorname{BIOFA}(5,2)$ \\
\hline $\mathrm{TF}$ & $\mathrm{Pb}-210+\mathrm{D}$ & , fish & $3.000 \mathrm{E}+02$ & $3.000 \mathrm{E}+02$ & $\operatorname{BIOFA}(6,1)$ \\
\hline
\end{tabular}




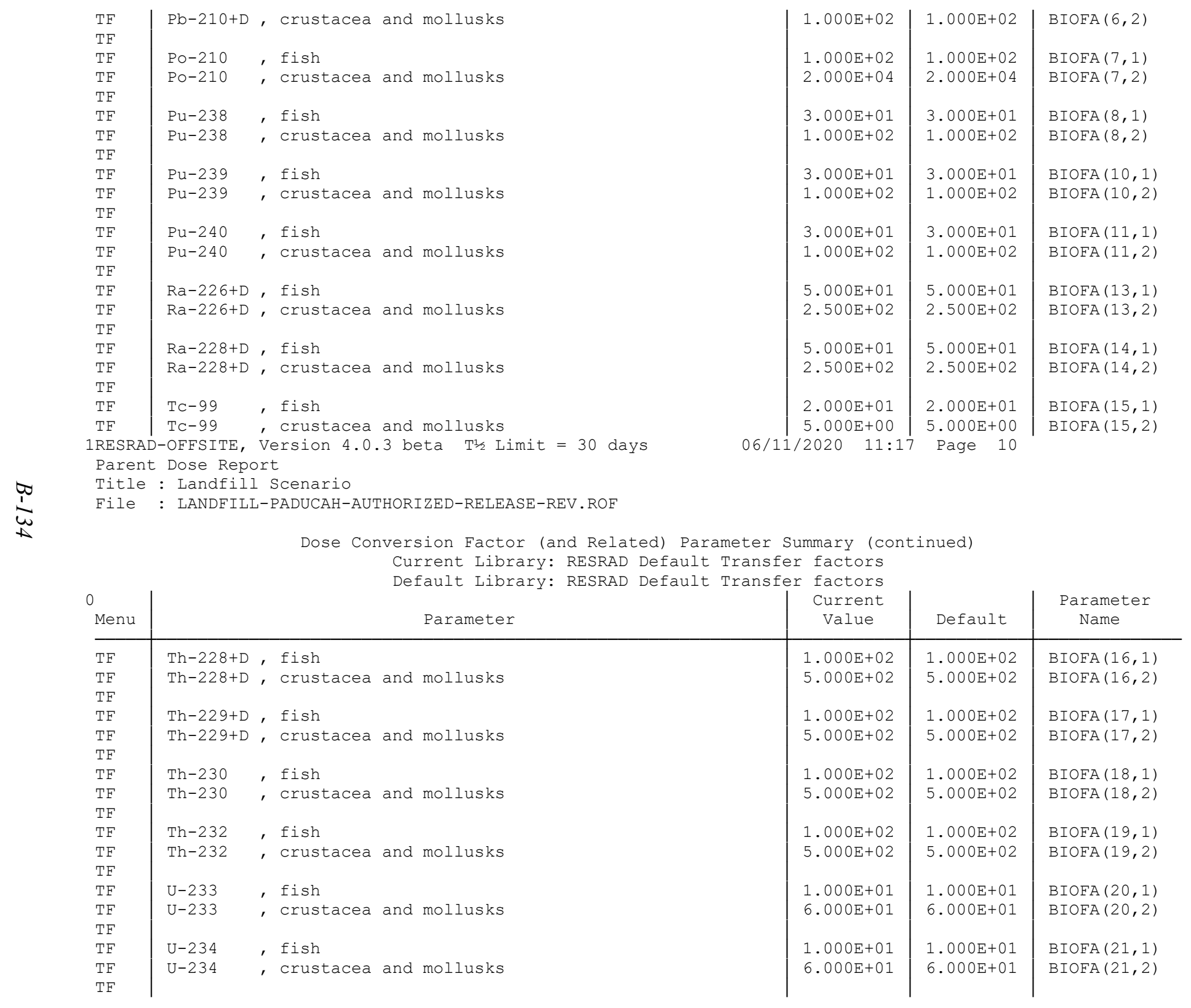




\begin{tabular}{|c|c|c|c|c|c|}
\hline $\mathrm{TF}$ & $U-235+D$ & , fish & $1.000 \mathrm{E}+01$ & $1.000 \mathrm{E}+01$ & $\operatorname{BIOFA}(22,1)$ \\
\hline $\begin{array}{l}\mathrm{TF} \\
\mathrm{TF}\end{array}$ & $\mathrm{U}-235+\mathrm{D}$ & , crustacea and mollusks & $6.000 \mathrm{E}+01$ & $6.000 \mathrm{E}+01$ & $\operatorname{BIOFA}(22,2)$ \\
\hline $\mathrm{TF}$ & $\mathrm{U}-236$ & fish & $1.000 \mathrm{E}+01$ & $1.000 \mathrm{E}+01$ & $\operatorname{BIOFA}(23,1)$ \\
\hline $\begin{array}{l}\mathrm{TF} \\
\mathrm{TF}\end{array}$ & $\mathrm{U}-236$ & , crustacea and mollusks & $6.000 \mathrm{E}+01$ & $6.000 \mathrm{E}+01$ & $\operatorname{BIOFA}(23,2)$ \\
\hline$T F$ & $\mathrm{U}-238$ & , fish & $1.000 \mathrm{E}+01$ & $1.000 \mathrm{E}+01$ & $\operatorname{BIOFA}(24,1)$ \\
\hline $\begin{array}{l}\mathrm{TF} \\
\mathrm{TF}\end{array}$ & $\mathrm{U}-238$ & , crustacea and mollusks & $6.000 \mathrm{E}+01$ & $6.000 \mathrm{E}+01$ & $\operatorname{BIOFA}(24,2)$ \\
\hline $\mathrm{TF}$ & $\mathrm{U}-238+\mathrm{D}$ & , fish & $1.000 \mathrm{E}+01$ & $1.000 \mathrm{E}+01$ & $\operatorname{BIOFA}(25,1)$ \\
\hline $\mathrm{TF}$ & $\mathrm{U}-238+\mathrm{D}$ & , crustacea and mollusks & $6.000 \mathrm{E}+01$ & $6.000 \mathrm{E}+01$ & $\operatorname{BIOFA}(25,2)$ \\
\hline
\end{tabular}

1RESRAD-OFFSITE, Version 4.0 .3 beta $T^{1 / 2}$ Limit $=30$ days

06/11/2020 11:17 Page 11

Parent Dose Report

Title : Landfill Scenario

File : LANDFILL-PADUCAH-AUTHORIZED-RELEASE-REV.ROF

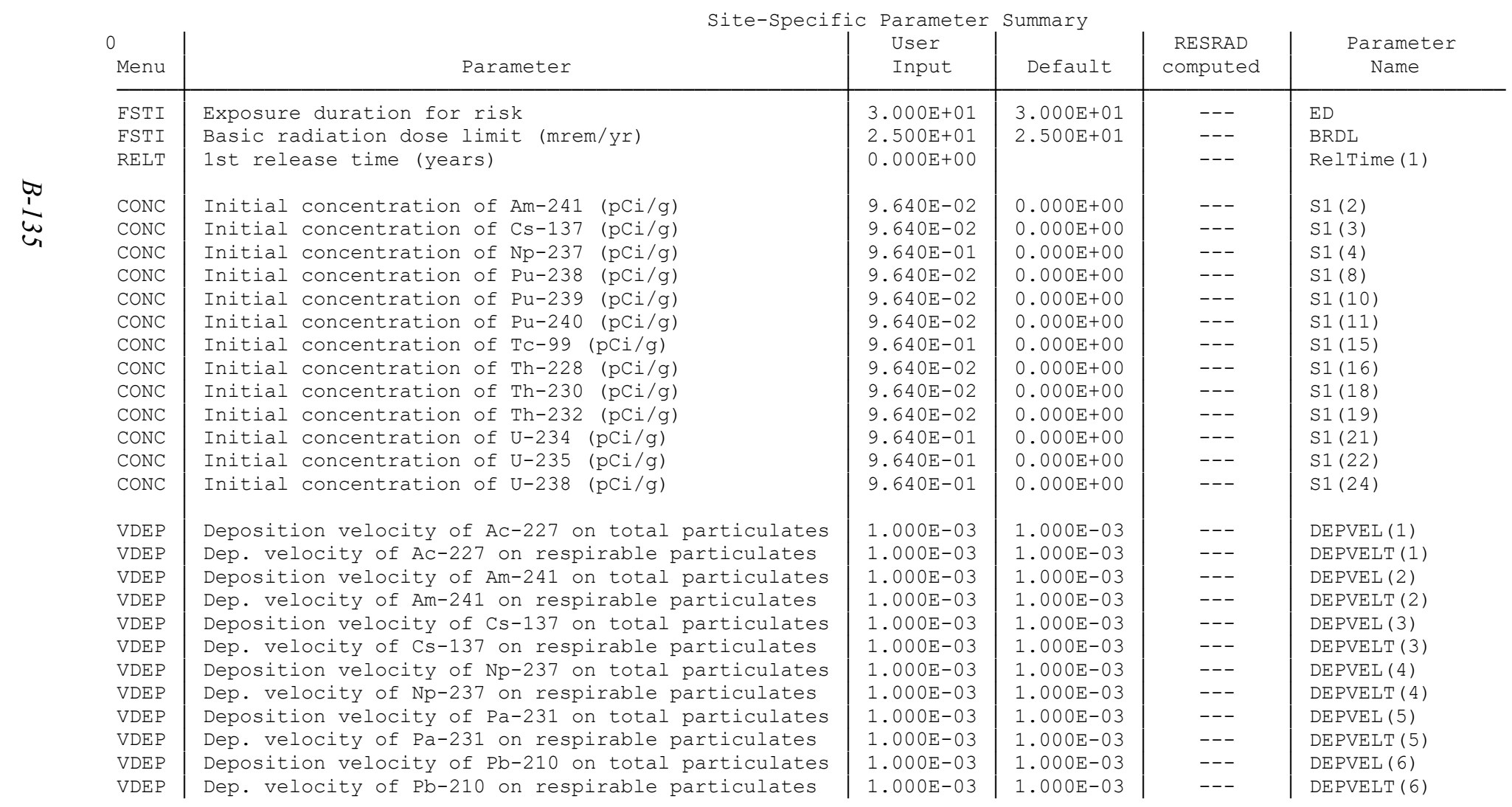


VDEP $\mid$ Deposition velocity of Po-210 on total particulates VDEP Dep. velocity of Po-210 on respirable particulates VDEP Deposition velocity of $\mathrm{Pu}-238$ on total particulates VDEP Dep. velocity of $\mathrm{Pu}-238$ on respirable particulates VDEP Deposition velocity of $\mathrm{Pu}-239$ on total particulates VDEP Dep. velocity of Pu-239 on respirable particulates VDEP Deposition velocity of Pu-240 on total particulates VDEP Dep. velocity of Pu-240 on respirable particulates VDEP Deposition velocity of Ra-226 on total particulates VDEP Dep velocity of Ra-226 on respirable particulates VDEP VDEP Dep. velocity of Ra-228 on respirable particulates Sosition velocity of Dep. velocity of Tc-99 on respirable particulates Deposition velocity of Th-228 on total particulates VDEP Dep. velocity of Th-228 on respirable particulates RESRAD-OFFSITE, Version 4.0 .3 beta $\mathrm{T}^{1} \frac{1}{2}$ Limit $=30$ days Parent Dose Report

Title : Landfill Scenario

File : LANDFILL-PADUCAH-AUTHORIZED-RELEASE-REV.ROF

\begin{tabular}{|l|l|l|l}
$1.000 \mathrm{E}-03$ & $1.000 \mathrm{E}-03$ & --- & DEPVEL (7) \\
$1.000 \mathrm{E}-03$ & $1.000 \mathrm{E}-03$ & --- & DEPVELT (7) \\
$1.000 \mathrm{E}-03$ & $1.000 \mathrm{E}-03$ & --- & DEPVEL (8) \\
$1.000 \mathrm{E}-03$ & $1.000 \mathrm{E}-03$ & --- & DEPVELT (8) \\
$1.000 \mathrm{E}-03$ & $1.000 \mathrm{E}-03$ & --- & DEPVEL (10) \\
$1.000 \mathrm{E}-03$ & $1.000 \mathrm{E}-03$ & --- & DEPVELT (10) \\
$1.000 \mathrm{E}-03$ & $1.000 \mathrm{E}-03$ & --- & DEPVEL (11) \\
$1.000 \mathrm{E}-03$ & $1.000 \mathrm{E}-03$ & --- & DEPVELT (11) \\
$1.000 \mathrm{E}-03$ & $1.000 \mathrm{E}-03$ & --- & DEPVEL (13) \\
$1.000 \mathrm{E}-03$ & $1.000 \mathrm{E}-03$ & --- & DEPVELT (13) \\
$1.000 \mathrm{E}-03$ & $1.000 \mathrm{E}-03$ & --- & DEPVEL $(14)$ \\
$1.000 \mathrm{E}-03$ & $1.000 \mathrm{E}-03$ & --- & DEPVELT (14) \\
$1.000 \mathrm{E}-03$ & $1.000 \mathrm{E}-03$ & --- & DEPVEL (15) \\
$1.000 \mathrm{E}-03$ & $1.000 \mathrm{E}-03$ & --- & DEPVELT (15) \\
$1.000 \mathrm{E}-03$ & $1.000 \mathrm{E}-03$ & --- & DEPVEL (16) \\
$1.000 \mathrm{E}-03$ & $1.000 \mathrm{E}-03$ & --- & DEPVELT (16)
\end{tabular}

\begin{tabular}{|c|c|c|c|c|c|}
\hline $\begin{array}{l}0 \\
\text { Menu }\end{array}$ & ite-Specific Pa & $\begin{array}{l}\text { eter Sum } \\
\text { User } \\
\text { Input }\end{array}$ & Default & $\begin{array}{l}\text { RESRAD } \\
\text { computed }\end{array}$ & $\begin{array}{c}\text { Parameter } \\
\text { Name }\end{array}$ \\
\hline VDEP & Deposition velocity of Th-229 on total particulates & $1.000 \mathrm{E}-03$ & $1.000 \mathrm{E}-03$ & --- & DEPVEL (17) \\
\hline VDEP & Dep. velocity of Th-229 on respirable particulates & $1.000 \mathrm{E}-03$ & $1.000 \mathrm{E}-03$ & --- & DEPVELT (17) \\
\hline VDEP & Deposition velocity of Th-230 on total particulates & $1.000 \mathrm{E}-03$ & $1.000 \mathrm{E}-03$ & --- & DEPVEL (18) \\
\hline VDEP & Dep. velocity of Th-230 on respirable particulates & $1.000 \mathrm{E}-03$ & $1.000 \mathrm{E}-03$ & --- & DEPVELT (18) \\
\hline VDEP & Deposition velocity of Th-232 on total particulates & $1.000 \mathrm{E}-03$ & $1.000 \mathrm{E}-03$ & --- & DEPVEL (19) \\
\hline VDEP & Dep. velocity of Th-232 on respirable particulates & $1.000 \mathrm{E}-03$ & $1.000 \mathrm{E}-03$ & --- & DEPVELT (19) \\
\hline VDEP & Deposition velocity of U-233 on total particulates & $1.000 \mathrm{E}-03$ & $1.000 \mathrm{E}-03$ & --- & DEPVEL (20) \\
\hline VDEP & Dep. velocity of U-233 on respirable particulates & $1.000 \mathrm{E}-03$ & $1.000 \mathrm{E}-03$ & --- & DEPVELT (20) \\
\hline VDEP & Deposition velocity of U-234 on total particulates & $1.000 \mathrm{E}-03$ & $1.000 \mathrm{E}-03$ & --- & DEPVEL (21) \\
\hline VDEP & Dep. velocity of U-234 on respirable particulates & $1.000 \mathrm{E}-03$ & $1.000 \mathrm{E}-03$ & --- & DEPVELT (21) \\
\hline VDEP & Deposition velocity of U-235 on total particulates & $1.000 \mathrm{E}-03$ & $1.000 \mathrm{E}-03$ & --- & DEPVEL (22) \\
\hline VDEP & Dep. velocity of U-235 on respirable particulates & $1.000 \mathrm{E}-03$ & $1.000 \mathrm{E}-03$ & --- & DEPVELT (22) \\
\hline VDEP & Deposition velocity of $\mathrm{U}-236$ on total particulates & $1.000 \mathrm{E}-03$ & $1.000 \mathrm{E}-03$ & --- & DEPVEL (23) \\
\hline VDEP & Dep. velocity of U-236 on respirable particulates & $1.000 \mathrm{E}-03$ & $1.000 \mathrm{E}-03$ & --- & DEPVELT (23) \\
\hline VDEP & Deposition velocity of $\mathrm{U}-238$ on total particulates & $1.000 \mathrm{E}-03$ & $1.000 \mathrm{E}-03$ & --- & DEPVEL (24) \\
\hline VDEP & Dep. velocity of U-238 on respirable particulates & $1.000 \mathrm{E}-03$ & $1.000 \mathrm{E}-03$ & --- & DEPVELT (24) \\
\hline DCLR & Distribution coefficients for Am-241 & & & & \\
\hline DCLR & Contaminated zone $(\mathrm{cm} * * 3 / \mathrm{g})$ & $2.000 \mathrm{E}+01$ & $2.000 \mathrm{E}+01$ & --- & DCNUCC (2) \\
\hline DCLR & Unsaturated zone $1(\mathrm{~cm} * * 3 / \mathrm{g})$ & $2.000 \mathrm{E}+01$ & $2.000 \mathrm{E}+01$ & --- & $\operatorname{DCNUCU}(2,1)$ \\
\hline DCLR & Saturated zone $(\mathrm{cm} * * 3 / \mathrm{g})$ & $2.000 \mathrm{E}+01$ & $2.000 \mathrm{E}+01$ & --- & DCNUCS (2) \\
\hline DCLR & Bottom sediment in surface water body $(\mathrm{cm} * * 3 / \mathrm{g})$ & $2.000 \mathrm{E}+01$ & $2.000 \mathrm{E}+01$ & --- & DCNUCSWB (2) \\
\hline DCLR & Suspended sediment in surface water body $\left(\mathrm{cm}^{* *} 3 / \mathrm{g}\right)$ & $2.000 \mathrm{E}+01$ & $2.000 \mathrm{E}+01$ & --- & DCNUCSWS (2) \\
\hline DCLR & Agricultural area $1(\mathrm{~cm} * * 3 / \mathrm{g})$ & $2.000 \mathrm{E}+01$ & $2.000 \mathrm{E}+01$ & --- & $\operatorname{DCNUCOF}(2,1)$ \\
\hline DCLR & Agricultural area $2(\mathrm{~cm} * * 3 / \mathrm{g})$ & $2.000 \mathrm{E}+01$ & $2.000 \mathrm{E}+01$ & --- & $\operatorname{DCNUCOF}(2,2)$ \\
\hline DCLR & Agricultural area $3(\mathrm{~cm} * * 3 / \mathrm{g})$ & $2.000 \mathrm{E}+01$ & $2.000 \mathrm{E}+01$ & --- & $\operatorname{DCNUCOF}(2,3)$ \\
\hline
\end{tabular}


DCLR | Distribution coefficients for Pu-239

DCLR Contaminated zone $(\mathrm{cm} * * 3 / \mathrm{g})$

DCLR Unsaturated zone $1(\mathrm{~cm} * * 3 / \mathrm{g})$

DCLR Saturated zone $(\mathrm{cm} * * 3 / \mathrm{g})$

DCLR Bottom sediment in surface water body $(\mathrm{cm} * * 3 / \mathrm{g})$

DCLR Suspended sediment in surface water body $(\mathrm{cm} * * 3 / \mathrm{g})$

DCLR Agricultural area $1\left(\mathrm{~cm}^{* * 3 / \mathrm{g})}\right.$

DCLR Agricultural area $2(\mathrm{~cm} * * 3 / \mathrm{g})$

DCLR Agricultural area $3(\mathrm{~cm} * * 3 / \mathrm{g})$

DCLR Agricultural area $4(\mathrm{~cm} * * 3 / \mathrm{g})$

DCLR Offsite Dwelling $(\mathrm{cm} * * 3 / \mathrm{g})$

DCLR Leach rate constant of Pu-239 (/yr)

IRESRAD-OFFSITE, Version 4.0 .3 beta $T^{1 / 2}$ Limit $=30$ days

Parent Dose Report

Title : Landfill Scenario

File : LANDFILL-PADUCAH-AUTHORIZED-RELEASE-REV.ROF

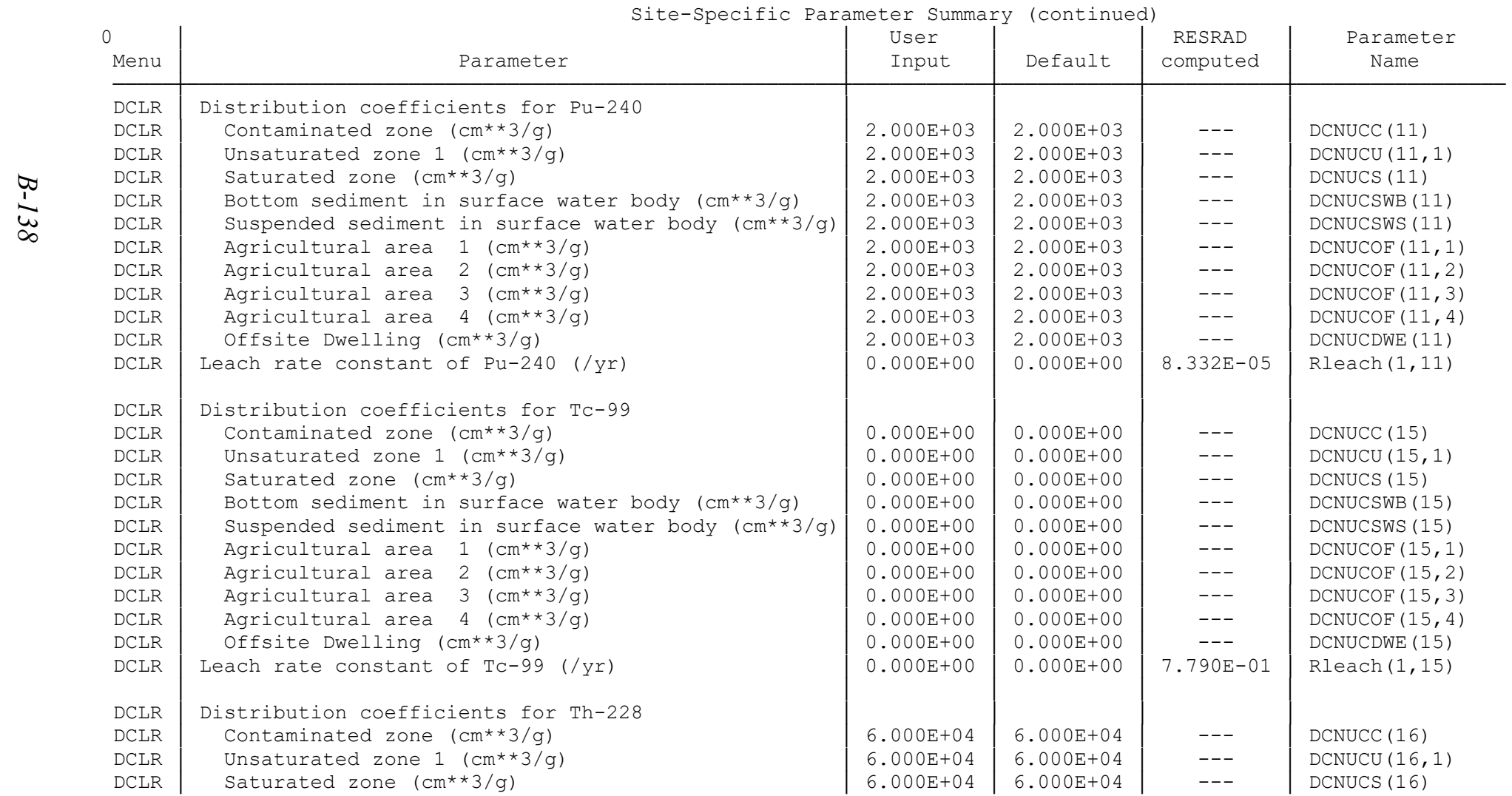

\begin{tabular}{l|l|l|l}
$2.000 \mathrm{E}+03$ & $2.000 \mathrm{E}+03$ & --- & $\operatorname{DCNUCC}(10)$ \\
$2.000 \mathrm{E}+03$ & $2.000 \mathrm{E}+03$ & --- & $\operatorname{DCNUCU}(10,1)$ \\
$2.000 \mathrm{E}+03$ & $2.000 \mathrm{E}+03$ & -- & $\operatorname{DCNUCS}(10)$ \\
$2.000 \mathrm{E}+03$ & $2.000 \mathrm{E}+03$ & --- & $\operatorname{DCNUCSWB}(10)$ \\
$2.000 \mathrm{E}+03$ & $2.000 \mathrm{E}+03$ & --- & $\operatorname{DCNUCSWS}(10)$ \\
$2.000 \mathrm{E}+03$ & $2.000 \mathrm{E}+03$ & --- & $\operatorname{DCNUCOF}(10,1)$ \\
$2.000 \mathrm{E}+03$ & $2.000 \mathrm{E}+03$ & --- & $\operatorname{DCNUCOF}(10,2)$ \\
$2.000 \mathrm{E}+03$ & $2.000 \mathrm{E}+03$ & --- & $\operatorname{DCNUCOF}(10,3)$ \\
$2.000 \mathrm{E}+03$ & $2.000 \mathrm{E}+03$ & --- & $\operatorname{DCNUCOF}(10,4)$ \\
$2.000 \mathrm{E}+03$ & $2.000 \mathrm{E}+03$ & --- & $\operatorname{DCNUCDWE}(10)$ \\
$0.000 \mathrm{E}+00$ & $0.000 \mathrm{E}+00$ & $8.332 \mathrm{E}-05$ & $\operatorname{Rleach}(1,10)$
\end{tabular}

$06 / 11 / 202011: 17$ Page 14 
DCLR Bottom sediment in surface water body $(\mathrm{cm} * * 3 / \mathrm{g})$

DCLR Suspended sediment in surface water body $(\mathrm{cm} * * 3 / \mathrm{g})$

DCIR

DCLR

DCLR

DCLR

Afte Dwelling $(\mathrm{cm} * * 3 / \mathrm{g})$

Leach rate constant of Th-228 (/yr)

RESRAD-OFFSITE, Version 4.0 .3 beta $\mathrm{T}^{1} \frac{1}{2}$ Limit $=30$ days

Parent Dose Report

Title : Landfill Scenario

File : LANDFILL-PADUCAH-AUTHORIZED-RELEASE-REV.ROF

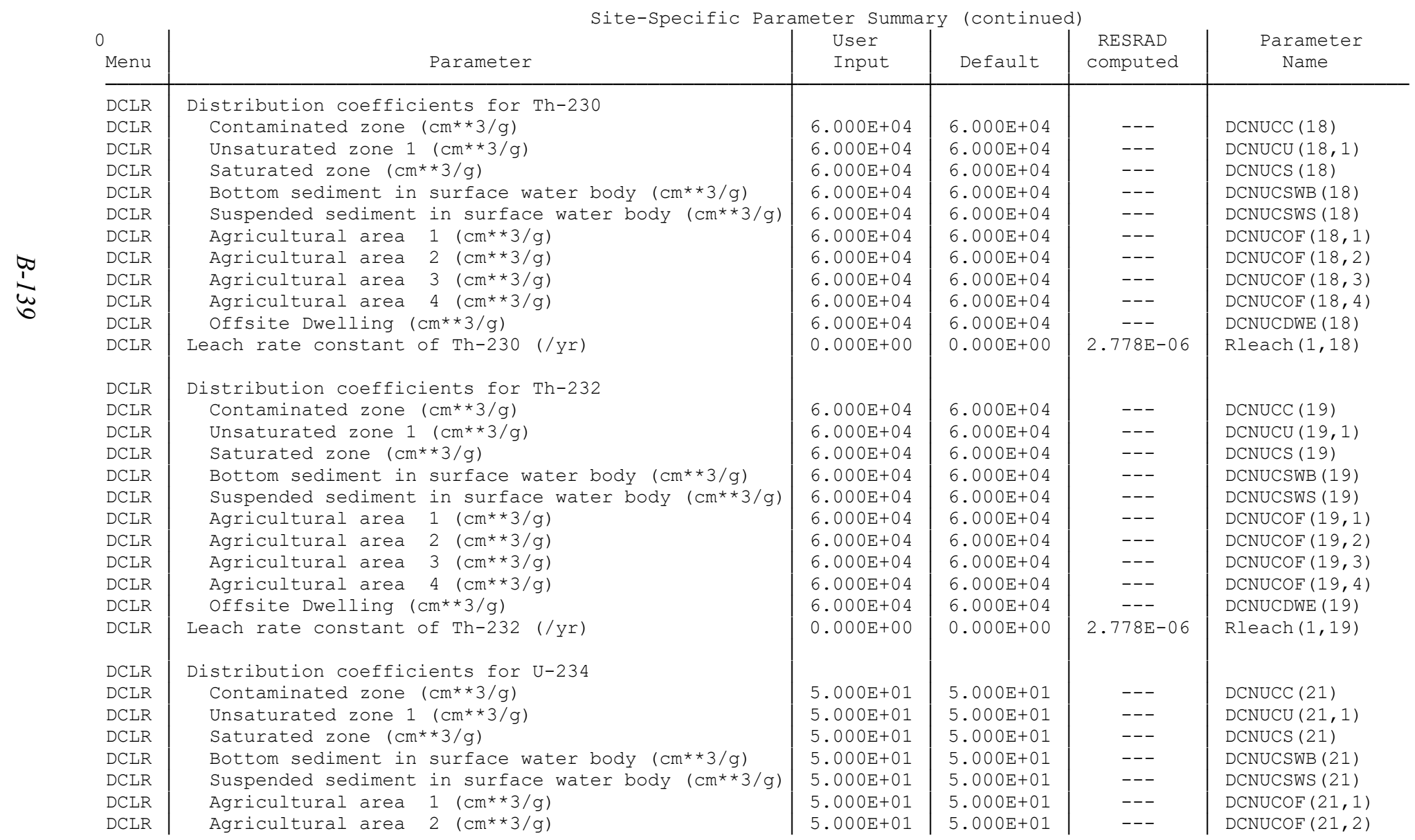


DCLR Agricultural area $3(\mathrm{~cm} * * 3 / \mathrm{g})$

DCLR Agricultural area $4(\mathrm{~cm} * * 3 / \mathrm{g})$

DCLR Offsite Dwelling $(\mathrm{cm} * * 3 / \mathrm{g})$

DCLR Leach rate constant of U-234 (/yr)

1RESRAD-OFFSITE, Version 4.0 .3 beta $T^{\frac{1}{2}}$ Limit $=30$ days

Parent Dose Report

Title : Landfill Scenario

File : LANDFILL-PADUCAH-AUTHORIZED-RELEASE-REV.ROF

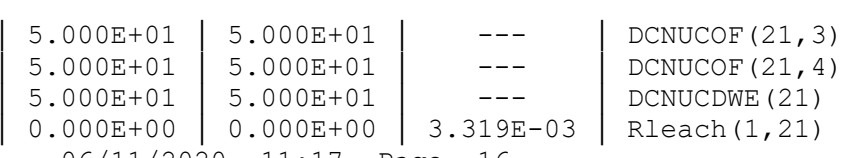

$06 / 11 / 2020$ 11:17 Page 16

\begin{tabular}{|c|c|c|c|c|c|}
\hline $\begin{array}{l}0 \\
\text { Menu }\end{array}$ & Site-Specific Para & $\begin{array}{l}\text { ter Summa } \\
\text { User } \\
\text { Input }\end{array}$ & $\begin{array}{l}\text { (continu } \\
\text { Default }\end{array}$ & $\begin{array}{l}\text { RESRAD } \\
\text { computed }\end{array}$ & $\begin{array}{l}\text { Parameter } \\
\text { Name }\end{array}$ \\
\hline DCLR & Distribution coefficients for U-235 & & & & \\
\hline DCLR & Contaminated zone $(\mathrm{cm} * * 3 / \mathrm{g})$ & $5.000 \mathrm{E}+01$ & $5.000 \mathrm{E}+01$ & --- & DCNUCC (22) \\
\hline DCLR & Unsaturated zone $1 \quad(\mathrm{~cm} * \star 3 / \mathrm{g})$ & $5.000 \mathrm{E}+01$ & $5.000 \mathrm{E}+01$ & --- & $\operatorname{DCNUCU}(22,1)$ \\
\hline DCLR & Saturated zone $(\mathrm{cm} * * 3 / \mathrm{g})$ & $5.000 \mathrm{E}+01$ & $5.000 \mathrm{E}+01$ & --- & DCNUCS (22) \\
\hline DCLR & Bottom sediment in surface water body $(\mathrm{cm} * * 3 / \mathrm{g})$ & $5.000 \mathrm{E}+01$ & $5.000 \mathrm{E}+01$ & --- & DCNUCSWB (22) \\
\hline DCLR & Suspended sediment in surface water body $(\mathrm{cm} * * 3 / \mathrm{g})$ & $5.000 \mathrm{E}+01$ & $5.000 \mathrm{E}+01$ & --- & DCNUCSWS (22) \\
\hline DCLR & Agricultural area $1(\mathrm{~cm} * * 3 / \mathrm{g})$ & $5.000 \mathrm{E}+01$ & $5.000 \mathrm{E}+01$ & --- & $\operatorname{DCNUCOF}(22,1)$ \\
\hline DCLR & Agricultural area $2(\mathrm{~cm} * * 3 / \mathrm{g})$ & $5.000 \mathrm{E}+01$ & $5.000 \mathrm{E}+01$ & --- & $\operatorname{DCNUCOF}(22,2)$ \\
\hline DCLR & Agricultural area $3(\mathrm{~cm} * * 3 / \mathrm{g})$ & $5.000 \mathrm{E}+01$ & $5.000 \mathrm{E}+01$ & --- & $\operatorname{DCNUCOF}(22,3)$ \\
\hline DCLR & Agricultural area $4(\mathrm{~cm} * * 3 / \mathrm{g})$ & $5.000 \mathrm{E}+01$ & $5.000 \mathrm{E}+01$ & --- & $\operatorname{DCNUCOF}(22,4)$ \\
\hline DCLR & Offsite Dwelling $(\mathrm{cm} * * 3 / \mathrm{g})$ & $5.000 \mathrm{E}+01$ & $5.000 \mathrm{E}+01$ & --- & DCNUCDWE (22) \\
\hline DCLR & Leach rate constant of $\mathrm{U}-235$ (/yr) & $0.000 \mathrm{E}+00$ & $0.000 \mathrm{E}+00$ & $3.319 \mathrm{E}-03$ & Rleach $(1,22)$ \\
\hline DCLR & Distribution coefficients for U-238 & & & & \\
\hline DCLR & Contaminated zone $(\mathrm{cm} * * 3 / \mathrm{g})$ & $5.000 \mathrm{E}+01$ & $5.000 \mathrm{E}+01$ & --- & DCNUCC (24) \\
\hline DCLR & Unsaturated zone $1(\mathrm{~cm} * * 3 / \mathrm{g})$ & $5.000 \mathrm{E}+01$ & $5.000 \mathrm{E}+01$ & --- & $\operatorname{DCNUCU}(24,1)$ \\
\hline DCLR & Saturated zone $(\mathrm{cm} * * 3 / \mathrm{g})$ & $5.000 \mathrm{E}+01$ & $5.000 \mathrm{E}+01$ & --- & DCNUCS (24) \\
\hline DCLR & Bottom sediment in surface water body $(\mathrm{cm} * * 3 / \mathrm{g})$ & $5.000 \mathrm{E}+01$ & $5.000 \mathrm{E}+01$ & --- & DCNUCSWB (24) \\
\hline DCLR & Suspended sediment in surface water body $(\mathrm{cm} * * 3 / \mathrm{g})$ & $5.000 \mathrm{E}+01$ & $5.000 \mathrm{E}+01$ & --- & DCNUCSWS $(24)$ \\
\hline DCLR & Agricultural area $1(\mathrm{~cm} * * 3 / \mathrm{g})$ & $5.000 \mathrm{E}+01$ & $5.000 \mathrm{E}+01$ & --- & $\operatorname{DCNUCOF}(24,1)$ \\
\hline DCLR & Agricultural area $2(\mathrm{~cm} * * 3 / \mathrm{g})$ & $5.000 \mathrm{E}+01$ & $5.000 \mathrm{E}+01$ & --- & $\operatorname{DCNUCOF}(24,2)$ \\
\hline DCLR & Agricultural area $3(\mathrm{~cm} * * 3 / \mathrm{g})$ & $5.000 \mathrm{E}+01$ & $5.000 \mathrm{E}+01$ & --- & $\operatorname{DCNUCOF}(24,3)$ \\
\hline DCLR & Agricultural area $4(\mathrm{~cm} * * 3 / \mathrm{g})$ & $5.000 \mathrm{E}+01$ & $5.000 \mathrm{E}+01$ & --- & $\operatorname{DCNUCOF}(24,4)$ \\
\hline DCLR & Offsite Dwelling $(\mathrm{cm} * * 3 / \mathrm{g})$ & $5.000 \mathrm{E}+01$ & $5.000 \mathrm{E}+01$ & --- & DCNUCDWE $(24)$ \\
\hline DCLR & Leach rate constant of $\mathrm{U}-238$ (/yr) & $0.000 \mathrm{E}+00$ & $0.000 \mathrm{E}+00$ & $3.319 \mathrm{E}-03$ & Rleach $(1,24)$ \\
\hline DCLR & Distribution coefficients for progeny Ac-227 & & & & \\
\hline DCLR & Contaminated zone $(\mathrm{cm} * * 3 / \mathrm{g})$ & $2.000 \mathrm{E}+01$ & $2.000 \mathrm{E}+01$ & --- & DCNUCC (1) \\
\hline DCLR & Unsaturated zone $1(\mathrm{~cm} * * 3 / \mathrm{g})$ & $2.000 E+01$ & $2.000 E+01$ & --- & $\operatorname{DCNUCU}(1,1)$ \\
\hline DCLR & Saturated zone $(\mathrm{cm} * * 3 / \mathrm{g})$ & $2.000 \mathrm{E}+01$ & $2.000 \mathrm{E}+01$ & --- & $\operatorname{DCNUCS}(1)$ \\
\hline DCLR & Bottom sediment in surface water body $(\mathrm{cm} * * 3 / \mathrm{g})$ & $2.000 \mathrm{E}+01$ & $2.000 \mathrm{E}+01$ & --- & DCNUCSWB (1) \\
\hline DCLR & Suspended sediment in surface water body $(\mathrm{cm} * * 3 / \mathrm{g})$ & $2.000 \mathrm{E}+01$ & $2.000 \mathrm{E}+01$ & --- & DCNUCSWS (1) \\
\hline DCLR & Agricultural area $1(\mathrm{~cm} * * 3 / \mathrm{g})$ & $2.000 \mathrm{E}+01$ & $2.000 \mathrm{E}+01$ & --- & $\operatorname{DCNUCOF}(1,1)$ \\
\hline DCLR & Agricultural area $2(\mathrm{~cm} * * 3 / \mathrm{g})$ & $2.000 \mathrm{E}+01$ & $2.000 \mathrm{E}+01$ & --- & $\operatorname{DCNUCOF}(1,2)$ \\
\hline DCLR & Agricultural area $3(\mathrm{~cm} * * 3 / \mathrm{g})$ & $2.000 \mathrm{E}+01$ & $2.000 \mathrm{E}+01$ & --- & $\operatorname{DCNUCOF}(1,3)$ \\
\hline DCLR & Agricultural area $4(\mathrm{~cm} * * 3 / \mathrm{g})$ & $2.000 \mathrm{E}+01$ & $2.000 \mathrm{E}+01$ & --- & $\operatorname{DCNUCOF}(1,4)$ \\
\hline DCLR & Offsite Dwelling $(\mathrm{cm} * * 3 / \mathrm{g})$ & $2.000 \mathrm{E}+01$ & $2.000 \mathrm{E}+01$ & --- & DCNUCDWE (1) \\
\hline DCLR & Leach rate constant of $\mathrm{Ac}-227$ (/yr) & $0.000 \mathrm{E}+00$ & $0.000 \mathrm{E}+00$ & $8.245 E-03$ & Rleach $(1,1)$ \\
\hline
\end{tabular}


Parent Dose Report

Title : Landfill Scenario

File : LANDFILL-PADUCAH-AUTHORIZED-RELEASE-REV.ROF

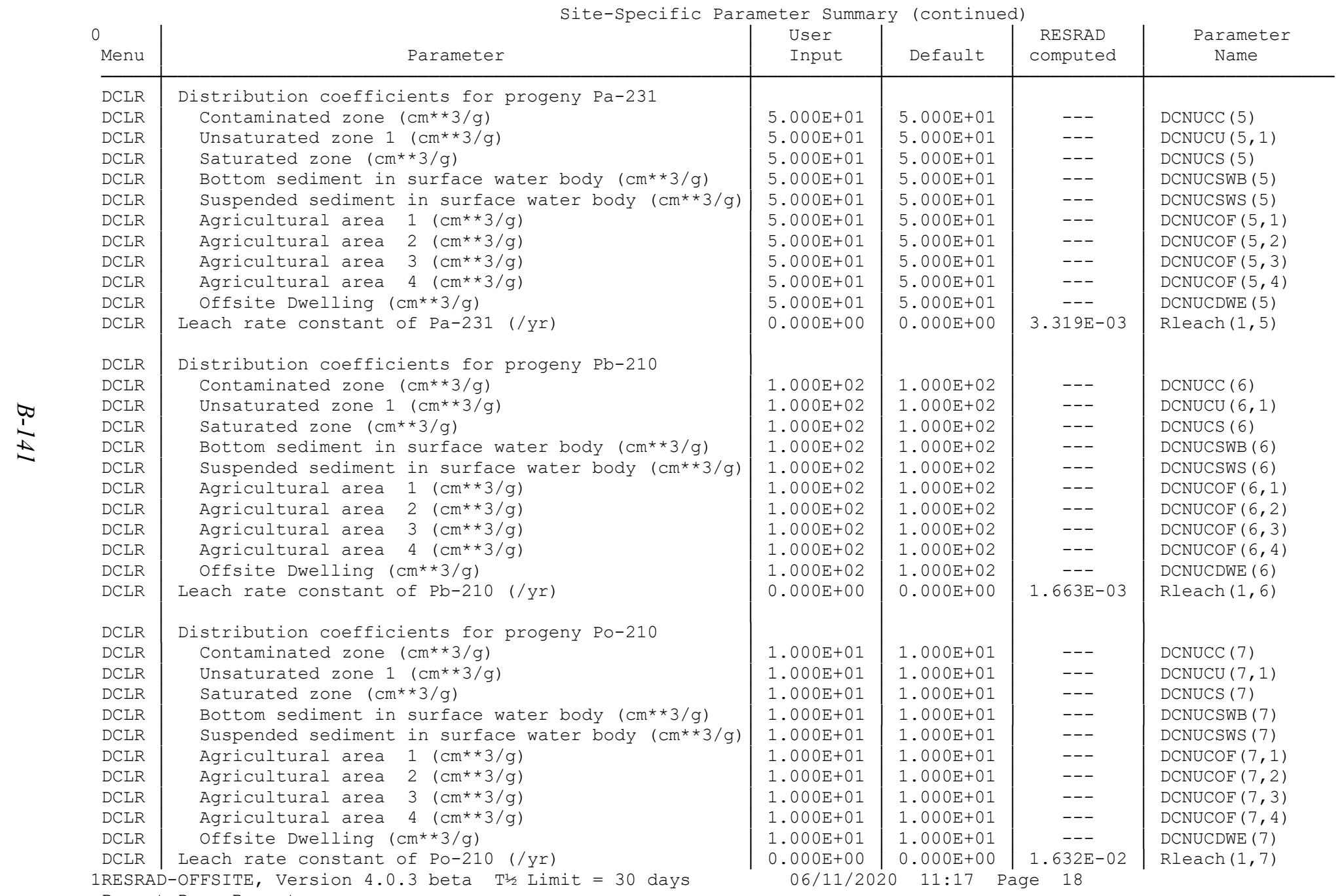

Parent Dose Report

Title : Landfill Scenario

File : LANDFILL-PADUCAH-AUTHORIZED-RELEASE-REV.ROF 


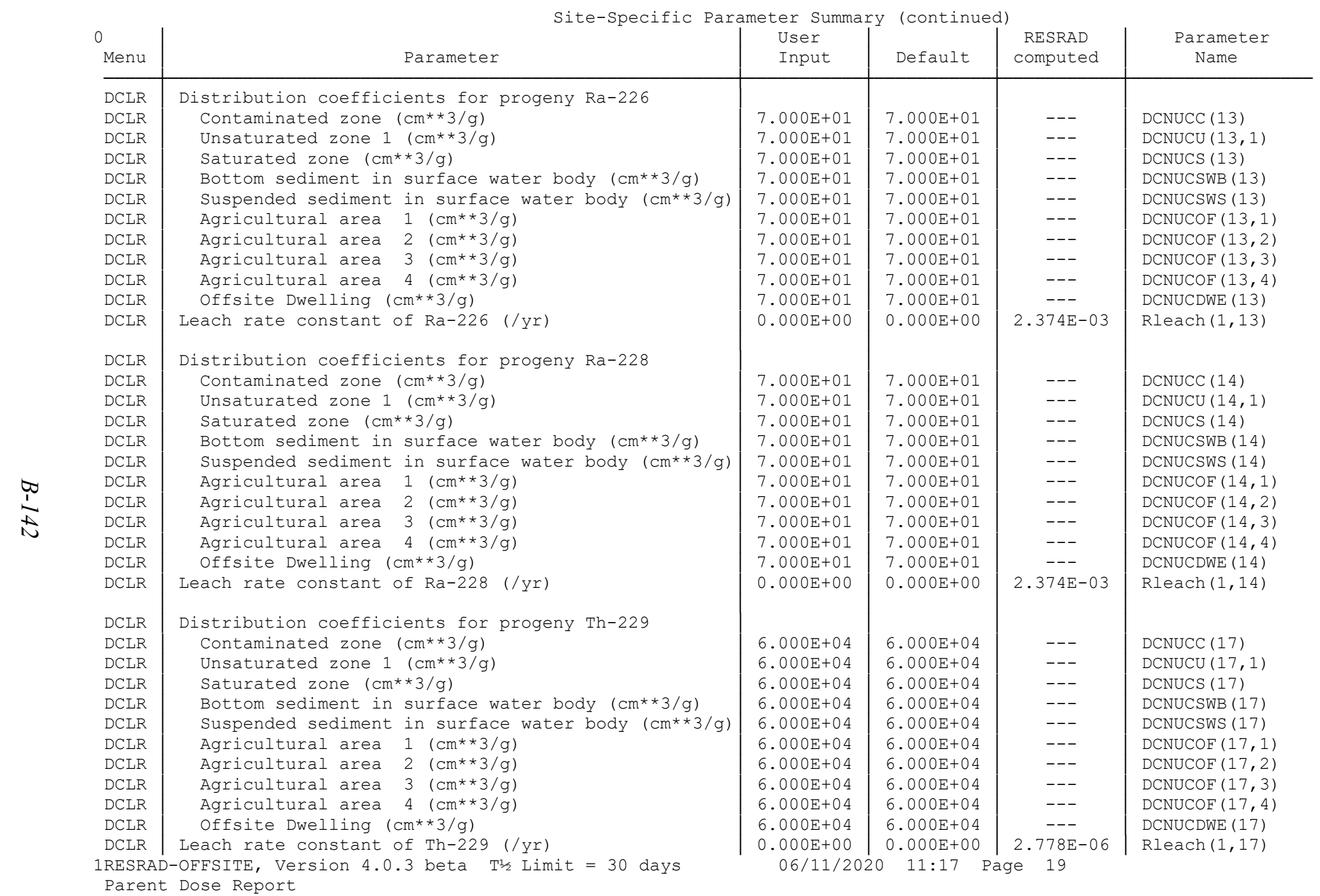

Parent Dose Report
Title : Landfill Scenario

File: LANDFILL-PADUCAH-AUTHORIZED-RELEASE-REV.ROF

0 Menu
Site-Specific Parameter Summary (continued)

Parameter

$$
\begin{array}{l|l|c}
\text { User } & \text { RESRAD } \\
\text { Input } & \text { Default } & \text { computed }
\end{array}
$$




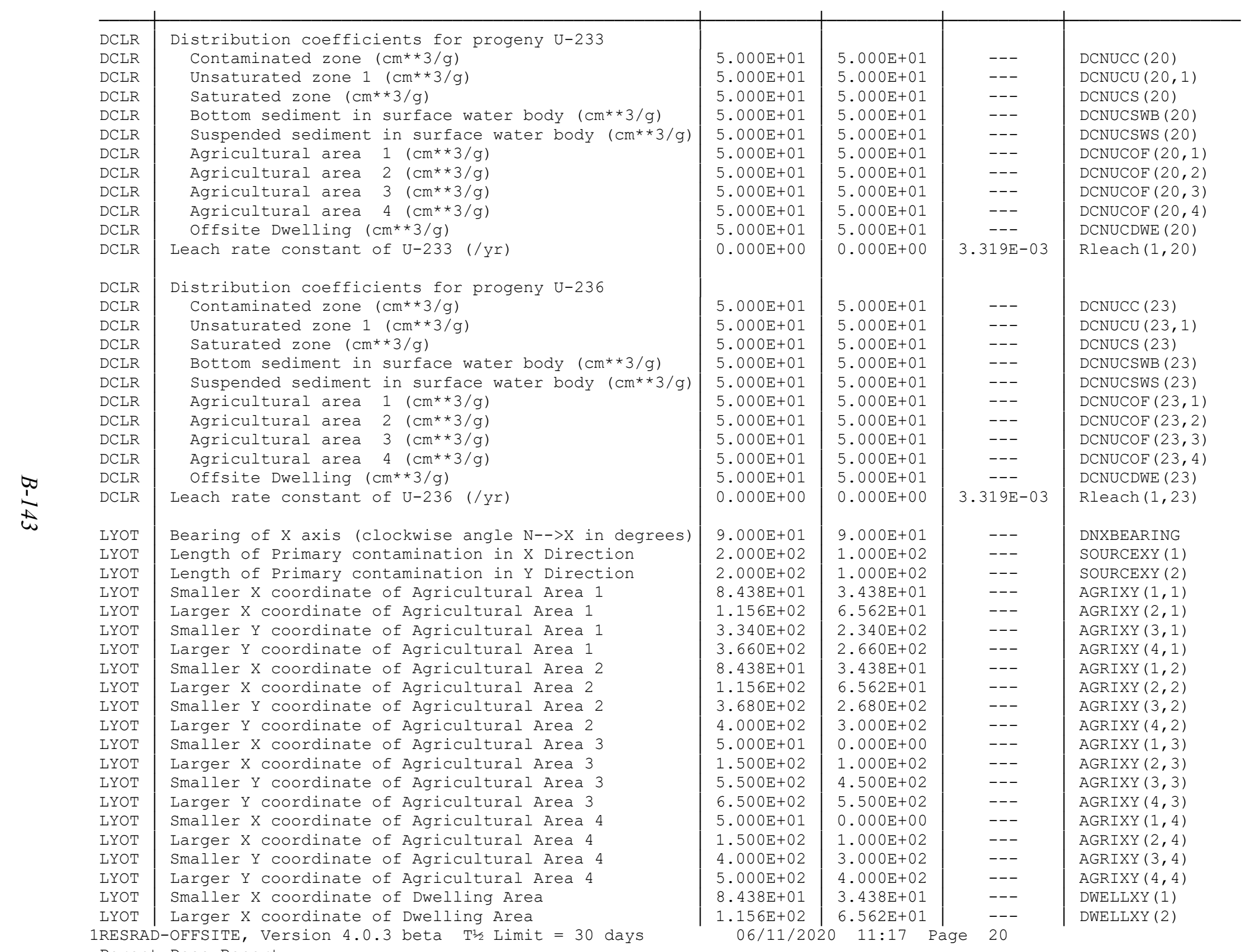


File : LANDFILL-PADUCAH-AUTHORIZED-RELEASE-REV.ROF

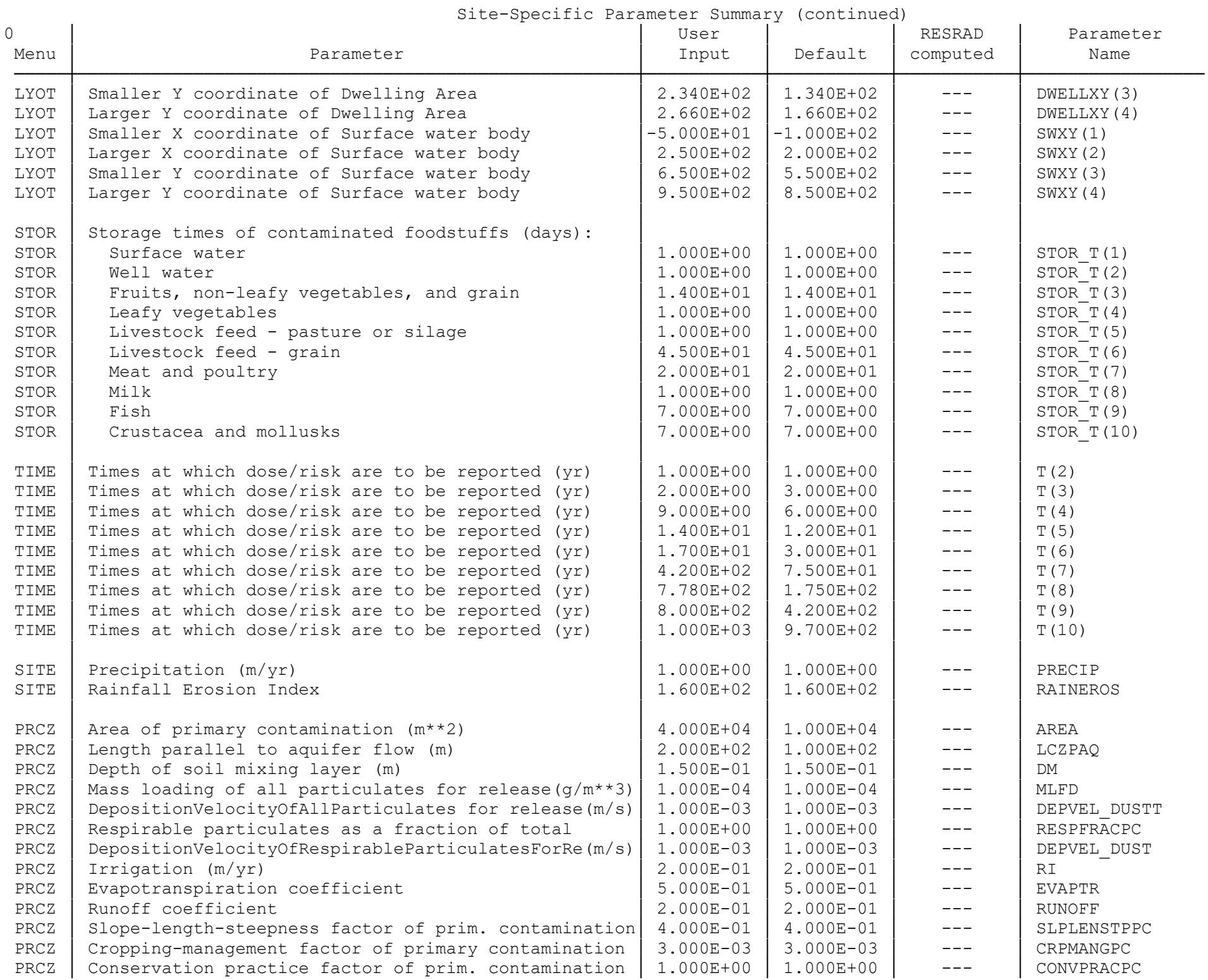


\begin{tabular}{l|l|l|l|l|l} 
PRCZ & Fraction of primary contamination that is sumberged & $0.000 \mathrm{E}+00$ & $0.000 \mathrm{E}+00$ & --- & SUBMERGEDF \\
PRCZ & Thickness of primary contamination $(\mathrm{m})$ & $2.000 \mathrm{E}+00$ & $2.000 \mathrm{E}+00$ & --- & THICKO
\end{tabular}

PRCZ Thickness of primary contamination (m)

1RESRAD-OFFSITE, Version 4.0 .3 beta $T^{1 / 1} / 2$ Limit $=30$ days

$06 / 11 / 2020-11: 17$ Page 21

THICKO

Parent Dose Report

Title : Landfill Scenario

File : LANDFILL-PADUCAH-AUTHORIZED-RELEASE-REV.ROF

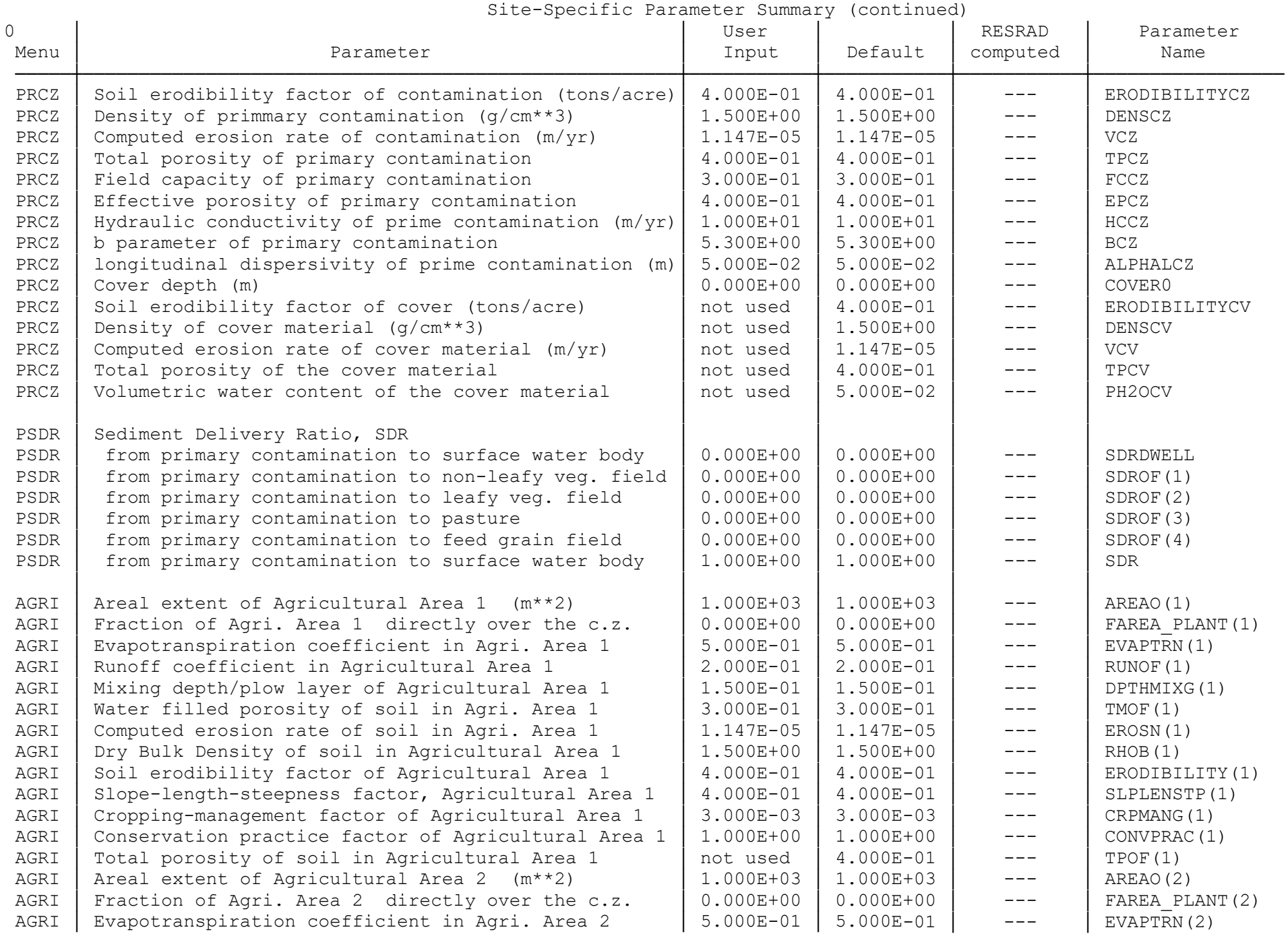


AGRI $\mid$ Runoff coefficient in Agricultural Area 2

AGRI Mixing depth/plow layer of Agricultural Area 2

AGRI Water filled porosity of soil in Agri. Area 2

AGRI Computed erosion rate of soil in Agri. Area 2

AGRI Dry Bulk Density of soil in Agricultural Area 2

AGRI Soil erodibility factor of Agricultural Area 2

AGRI Slope-length-steepness factor, Agricultural Area 2

IRESRAD-OFFSITE, Version 4.0 .3 beta $\mathrm{T}^{1} \frac{1}{2}$ Limit $=30$ days

Parent Dose Report

Title : Landfill Scenario

File : LANDFILL-PADUCAH-AUTHORIZED-RELEASE-REV.ROF

\begin{tabular}{|l|l|l|l}
$2.000 \mathrm{E}-01$ & $2.000 \mathrm{E}-01$ & --- & $\operatorname{RUNOF}(2)$ \\
$1.500 \mathrm{E}-01$ & $1.500 \mathrm{E}-01$ & --- & $\operatorname{DPTHMIXG}(2)$ \\
$3.000 \mathrm{E}-01$ & $3.000 \mathrm{E}-01$ & --- & $\operatorname{TMOF}(2)$ \\
$1.147 \mathrm{E}-05$ & $1.147 \mathrm{E}-05$ & -- & $\operatorname{EROSN}(2)$ \\
$1.500 \mathrm{E}+00$ & $1.500 \mathrm{E}+00$ & -- & $\operatorname{RHOB}(2)$ \\
$4.000 \mathrm{E}-01$ & $4.000 \mathrm{E}-01$ & --- & $\operatorname{ERODIBILITY}(2)$ \\
$4.000 \mathrm{E}-01$ & $4.000 \mathrm{E}-01$ & --- & $\operatorname{SLPLENSTP}(2)$
\end{tabular}

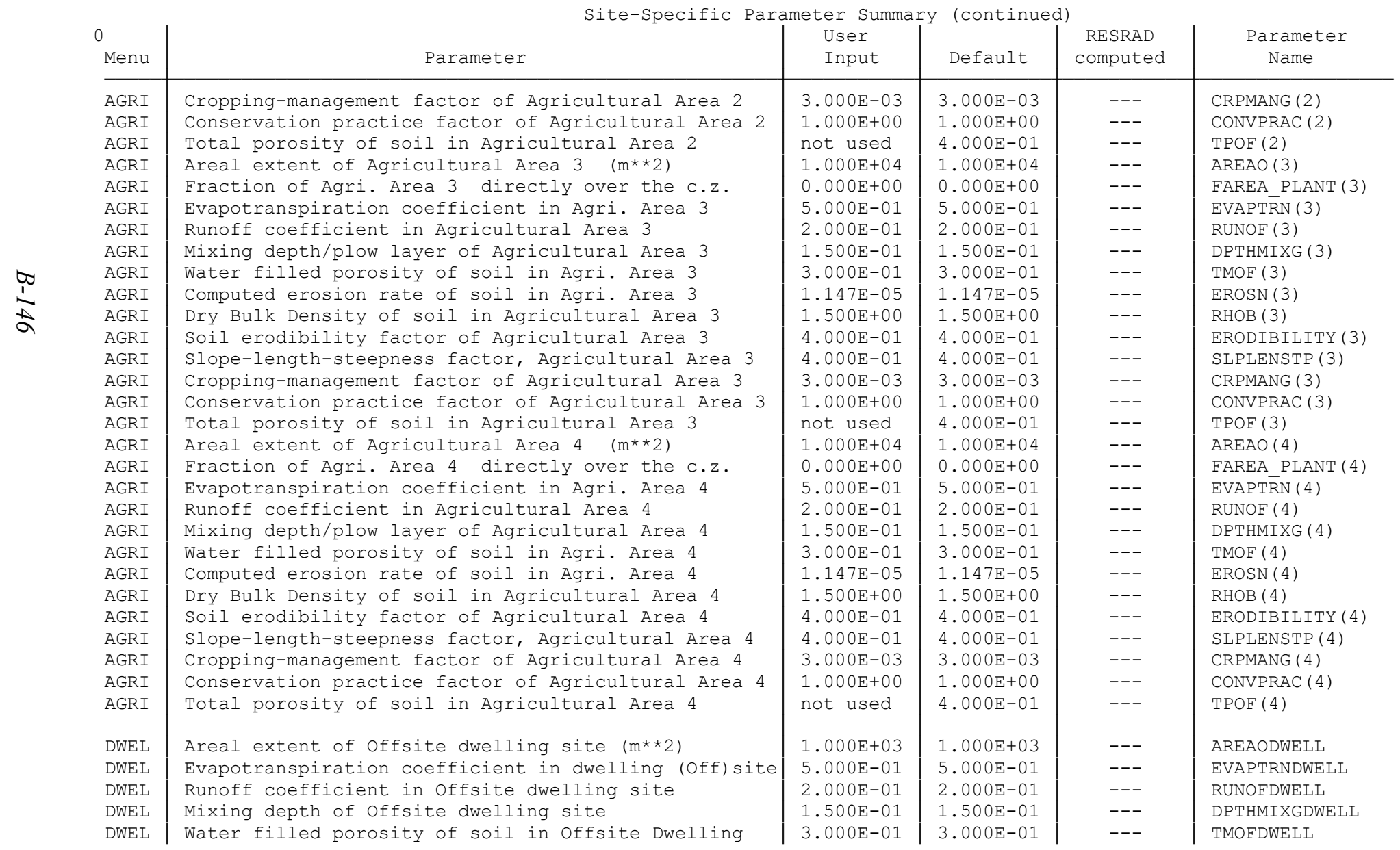


DWEL $\mid$ Computed erosion rate of soil in Offsite Dwelling DWEL Dry Bulk Density of soil in offsite dwelling site DWEL Soil erodibility factor of soil in Dwelling site

DWEL Slope-length-steepness factor of Dwelling site

DWEL Cropping-management factor of Dwelling site

DWEL Conservation practice factor of offsite Dwelling sit

DWEL Total porosity of soil in Offsite Dwelling

AIRT Dispersion Coefficients; 1 = Pasquill-Gifford

AIRT Population zone; 1 = Rural

AIRT Release height, (m)

AIRT Heat flux for buoyant plume (cal/s)

1RESRAD-OFFSITE, Version 4.0 .3 beta T1/2 Limit = 30 days Parent Dose Report

Title : Landfill Scenario

File : LANDFILL-PADUCAH-AUTHORIZED-RELEASE-REV.ROF

\begin{tabular}{|r|r|l|l}
$0.000 \mathrm{E}+00$ & $0.000 \mathrm{E}+00$ & --- & EROSNDWELL \\
$1.500 \mathrm{E}+00$ & $1.500 \mathrm{E}+00$ & -- & RHOBDWELL \\
$0.000 \mathrm{E}+00$ & $0.000 \mathrm{E}+00$ & -- & ERODIBILITYDWELL \\
$4.000 \mathrm{E}-01$ & $4.000 \mathrm{E}-01$ & -- & SLPLENSTPDWELL \\
$3.000 \mathrm{E}-03$ & $3.000 \mathrm{E}-03$ & --- & CRPMANGDWELL \\
$1.000 \mathrm{E}+00$ & $1.000 \mathrm{E}+00$ & --- & CONVPRACDWELL \\
not used & $4.000 \mathrm{E}-01$ & --- & TPOFDWELL \\
& & & \\
1 & 1 & --- & IDISPMOD \\
1 & 1 & --- & IZONE \\
$1.000 \mathrm{E}+00$ & $1.000 \mathrm{E}+00$ & --- & AIRRELHT \\
$0.000 \mathrm{E}+00$ & $0.000 \mathrm{E}+00$ & --- & HEATFLX
\end{tabular}

06/11/2020 11:17 Page 23

Site-Specific Parameter Summary (continued)

\begin{tabular}{|c|c|c|c|c|c|}
\hline $\begin{array}{l}\text { O } \\
\text { Menu }\end{array}$ & Parameter & $\begin{array}{l}\text { eter Summa } \\
\text { User } \\
\text { Input }\end{array}$ & $\begin{array}{l}\text { y lcontinu } \\
\text { Default }\end{array}$ & $\begin{array}{l}\text { RESRAD } \\
\text { computed }\end{array}$ & $\begin{array}{c}\text { Parameter } \\
\text { Name }\end{array}$ \\
\hline AIRT & Anemometer height, (m) & $1.000 \mathrm{E}+01$ & $1.000 \mathrm{E}+01$ & --- & ANH \\
\hline AIRT & Absolute temperature (Kelvin) & $2.850 \mathrm{E}+02$ & $2.850 \mathrm{E}+02$ & --- & TABK \\
\hline AIRT & PM atmospheric mixing height (m) & $1.600 \mathrm{E}+03$ & $1.600 \mathrm{E}+03$ & --- & PMIX \\
\hline AIRT & Elevation of Agricultural Area 1 above primary cont. & $0.000 \mathrm{E}+00$ & $0.000 \mathrm{E}+00$ & --- & AGRIELEV (1) \\
\hline AIRT & Elevation of Agricultural Area 2 above primary cont. & $0.000 \mathrm{E}+00$ & $0.000 \mathrm{E}+00$ & --- & AGRIELEV (2) \\
\hline AIRT & Elevation of Agricultural Area 3 above primary cont. & $0.000 \mathrm{E}+00$ & $0.000 \mathrm{E}+00$ & --- & AGRIELEV (3) \\
\hline AIRT & Elevation of Surf.Wtr body relative to primary cont. & $0.000 \mathrm{E}+00$ & $0.000 \mathrm{E}+00$ & --- & SWELEV \\
\hline AIRT & Joint frequency Meteorological data: & & & & \\
\hline AIRT & Upper limit for windspeed class 1 ( $/ \mathrm{s})$ & $8.900 \mathrm{E}-01$ & $8.900 \mathrm{E}-01$ & --- & WINDSPEED (1) \\
\hline AIRT & Upper limit for windspeed class 2 ( $\mathrm{m} / \mathrm{s})$ & $2.460 \mathrm{E}+00$ & $2.460 \mathrm{E}+00$ & --- & WINDSPEED (2) \\
\hline AIRT & Upper limit for windspeed class 3 ( $\mathrm{m} / \mathrm{s})$ & $4.470 \mathrm{E}+00$ & $4.470 \mathrm{E}+00$ & --- & WINDSPEED (3) \\
\hline AIRT & Upper limit for windspeed class 4 ( $\mathrm{m} / \mathrm{s})$ & $6.930 \mathrm{E}+00$ & $6.930 \mathrm{E}+00$ & --- & WINDSPEED ( 4 ) \\
\hline AIRT & for wind speed class 1 and stability class B & $0.000 \mathrm{E}+00$ & $0.000 \mathrm{E}+00$ & --- & $\operatorname{DFREQ}(1,2,1)$ \\
\hline AIRT & for wind speed class 1 and stability class $C$ & $0.000 \mathrm{E}+00$ & $0.000 \mathrm{E}+00$ & --- & $\operatorname{DFREQ}(1,3,1)$ \\
\hline AIRT & for wind speed class 1 and stability class D & $1.000 \mathrm{E}-01$ & $1.000 \mathrm{E}-01$ & --- & $\operatorname{DFREQ}(1,4,1)$ \\
\hline AIRT & for wind speed class 1 and stability class E & $2.000 \mathrm{E}-01$ & $2.000 \mathrm{E}-01$ & --- & $\operatorname{DFREQ}(1,5,1)$ \\
\hline AIRT & for wind speed class 1 and stability class F & $7.000 \mathrm{E}-01$ & $7.000 \mathrm{E}-01$ & --- & $\operatorname{DFREQ}(1,6,1)$ \\
\hline AIRT & Joint Frequency in $\mathrm{N}$ Sector & & & & \\
\hline AIRT & for wind speed class 2 and stability class A & $0.000 \mathrm{E}+00$ & $0.000 \mathrm{E}+00$ & --- & $\operatorname{DFREQ}(2,1,1)$ \\
\hline AIRT & for wind speed class 2 and stability class $B$ & $0.000 \mathrm{E}+00$ & $0.000 \mathrm{E}+00$ & --- & $\operatorname{DFREQ}(2,2,1)$ \\
\hline
\end{tabular}


AIRT for wind speed class 2 and stability class $C$ AIRT

AIRT

AIRT

AIRT

AIRT

AIRT

AIRT

AIRT

IRESRAD-OFFSITE, Version 4.0 .3 beta T1/2 Limit = 30 days Parent Dose Report

Title : Landfill Scenario

File : LANDFILL-PADUCAH-AUTHORIZED-RELEASE-REV.ROF

\begin{tabular}{|l|l|l|l}
$0.000 \mathrm{E}+00$ & $0.000 \mathrm{E}+00$ & --- & $\operatorname{DFREQ}(2,3,1)$ \\
$0.000 \mathrm{E}+00$ & $0.000 \mathrm{E}+00$ & -- & $\operatorname{DFREQ}(2,4,1)$ \\
$0.000 \mathrm{E}+00$ & $0.000 \mathrm{E}+00$ & -- & $\operatorname{DFREQ}(2,5,1)$ \\
$0.000 \mathrm{E}+00$ & $0.000 \mathrm{E}+00$ & -- & $\operatorname{DFREQ}(2,6,1)$ \\
& & & \\
$0.000 \mathrm{E}+00$ & $0.000 \mathrm{E}+00$ & -- & $\operatorname{DFREQ}(3,1,1)$ \\
$0.000 \mathrm{E}+00$ & $0.000 \mathrm{E}+00$ & -- & $\operatorname{DFREQ}(3,2,1)$ \\
$0.000 \mathrm{E}+00$ & $0.000 \mathrm{E}+00$ & -- & $\operatorname{DFREQ}(3,3,1)$ \\
$0.000 \mathrm{E}+00$ & $0.000 \mathrm{E}+00$ & -- & $\operatorname{DFREQ}(3,4,1)$ \\
$0.000 \mathrm{E}+00$ & $0.000 \mathrm{E}+00$ & --- & $\operatorname{DFREQ}(3,5,1)$ \\
$0.000 \mathrm{E}+00$ & $0.000 \mathrm{E}+00$ & --- & $\operatorname{DFREQ}(3,6,1)$
\end{tabular}

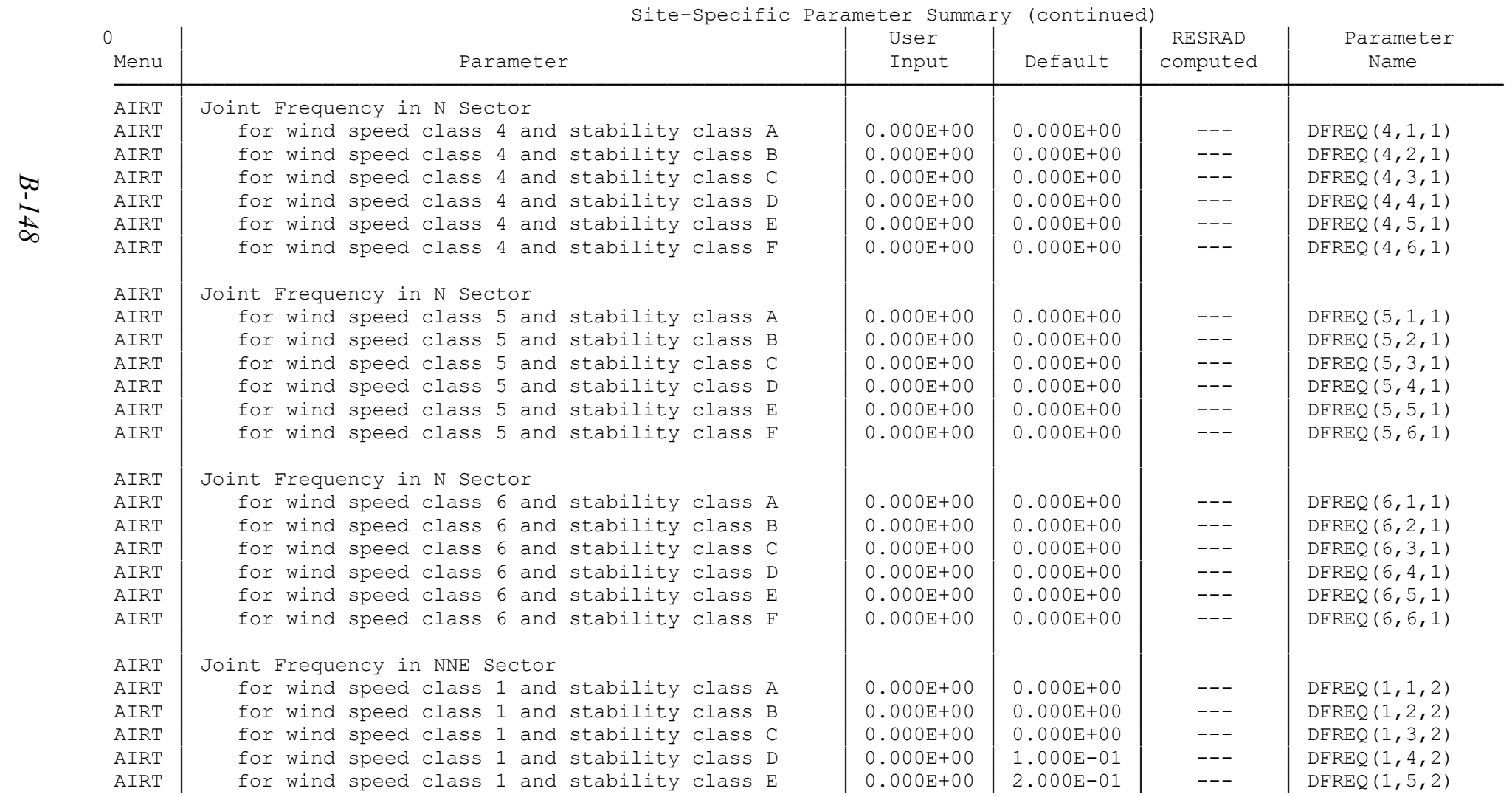


AIRT

AIRT

AIRT

AIRT

AIRT

AIRT

AIRT

AIRT

AIRT

AIRT

for wind speed class 3 and stability class $\mathrm{F}$

Parent Dose

Title : Landfill Scenario

File : LANDFILL-PADUCAH-AUTHORIZED-RELEASE-REV.ROF

\begin{tabular}{|l|l|l|l}
$0.000 \mathrm{E}+00$ & $7.000 \mathrm{E}-01$ & --- & $\operatorname{DFREQ}(1,6,2)$ \\
$0.000 \mathrm{E}+00$ & $0.000 \mathrm{E}+00$ & --- & $\operatorname{DFREQ}(2,1,2)$ \\
$0.000 \mathrm{E}+00$ & $0.000 \mathrm{E}+00$ & -- & $\operatorname{DFREQ}(2,2,2)$ \\
$0.000 \mathrm{E}+00$ & $0.000 \mathrm{E}+00$ & --- & $\operatorname{DFREQ}(2,3,2)$ \\
$0.000 \mathrm{E}+00$ & $0.000 \mathrm{E}+00$ & --- & $\operatorname{DFREQ}(2,4,2)$ \\
$0.000 \mathrm{E}+00$ & $0.000 \mathrm{E}+00$ & --- & $\operatorname{DFREQ}(2,5,2)$ \\
$0.000 \mathrm{E}+00$ & $0.000 \mathrm{E}+00$ & -- & $\operatorname{DFREQ}(2,6,2)$ \\
& & & \\
$0.000 \mathrm{E}+00$ & $0.000 \mathrm{E}+00$ & -- & $\operatorname{DFREQ}(3,1,2)$ \\
$0.000 \mathrm{E}+00$ & $0.000 \mathrm{E}+00$ & --- & $\operatorname{DFREQ}(3,2,2)$ \\
$0.000 \mathrm{E}+00$ & $0.000 \mathrm{E}+00$ & --- & $\operatorname{DFREQ}(3,3,2)$ \\
$0.000 \mathrm{E}+00$ & $0.000 \mathrm{E}+00$ & --- & $\operatorname{DFREQ}(3,4,2)$ \\
$0.000 \mathrm{E}+00$ & $0.000 \mathrm{E}+00$ & --- & $\operatorname{DFREQ}(3,5,2)$ \\
$0.000 \mathrm{E}+00$ & $0.000 \mathrm{E}+00$ & --- & $\operatorname{DFREQ}(3,6,2)$
\end{tabular}

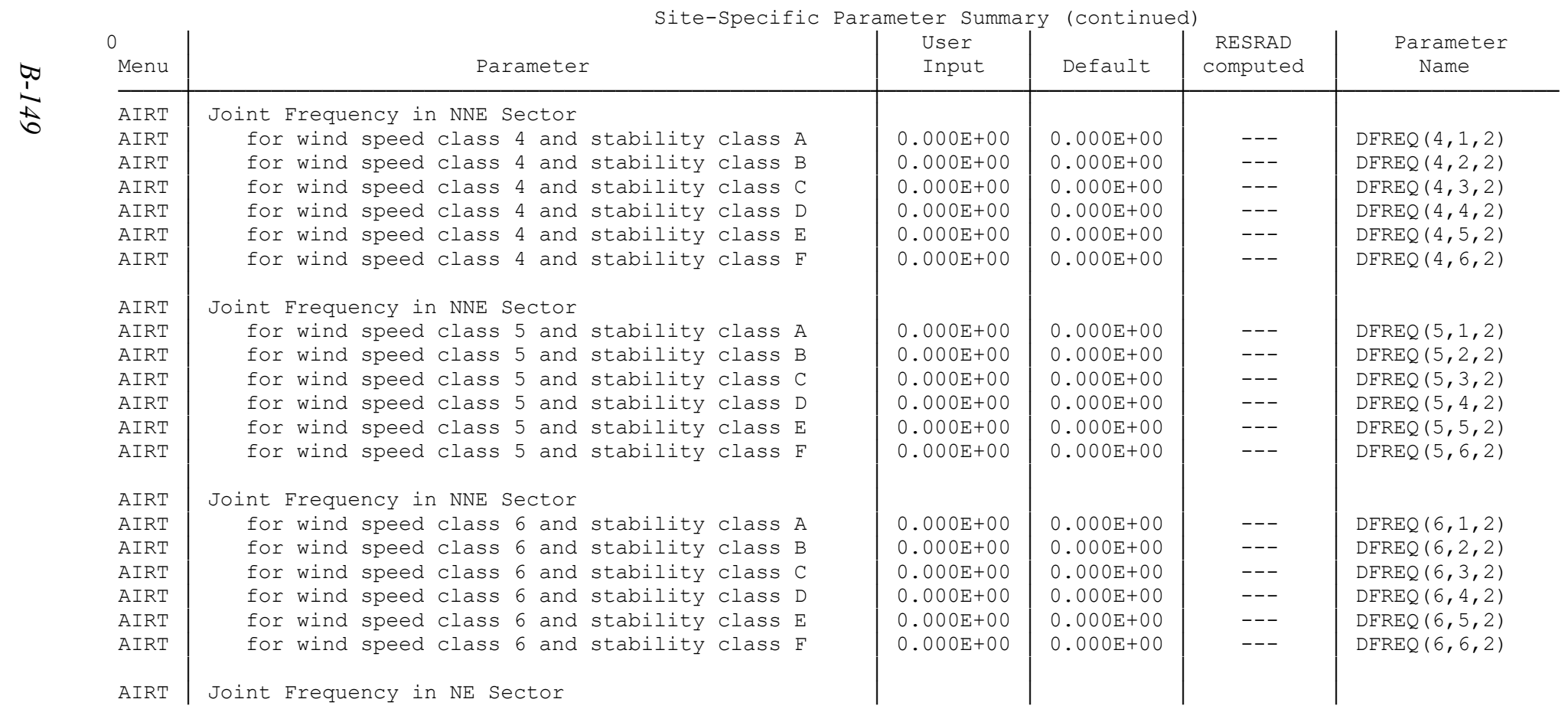




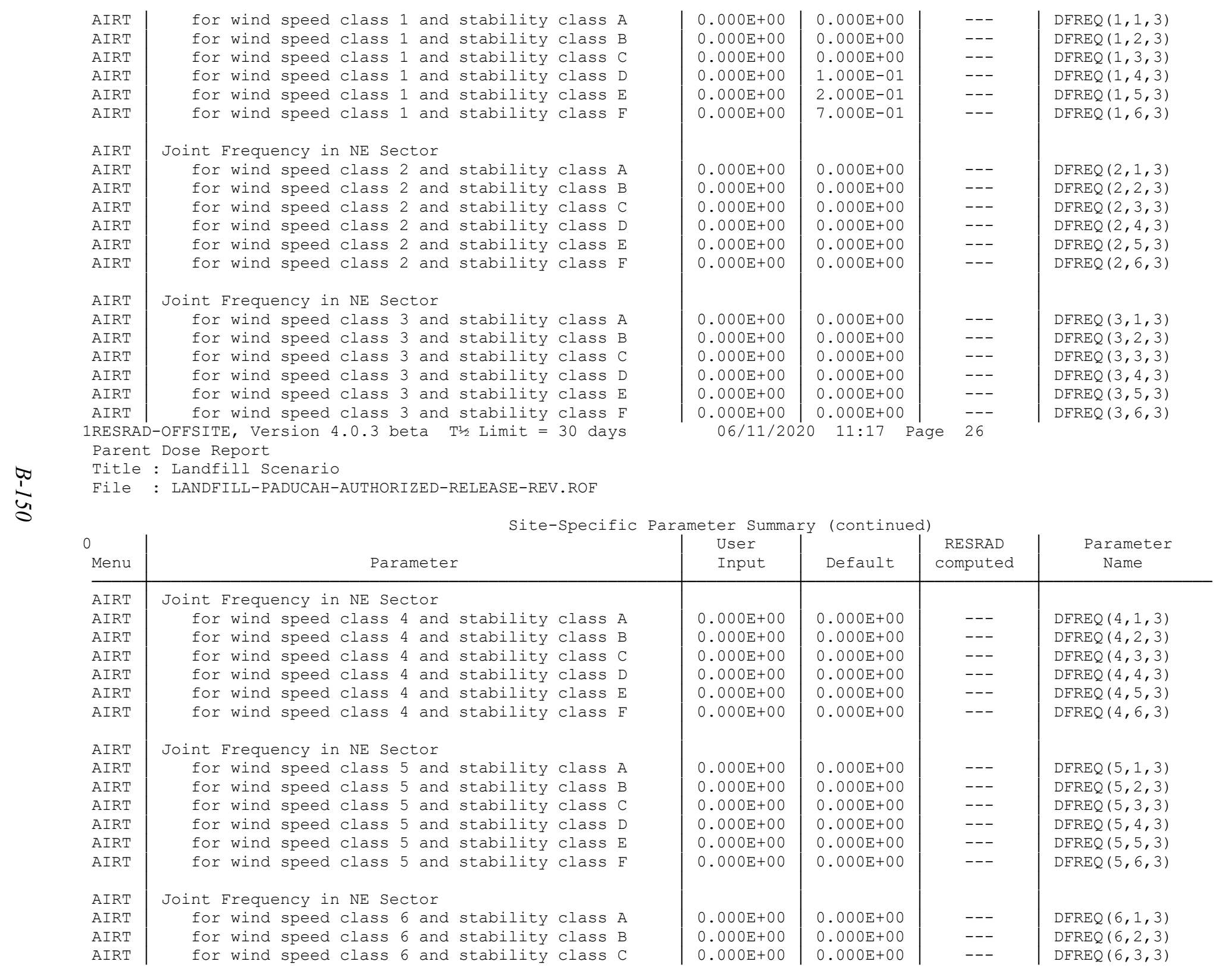




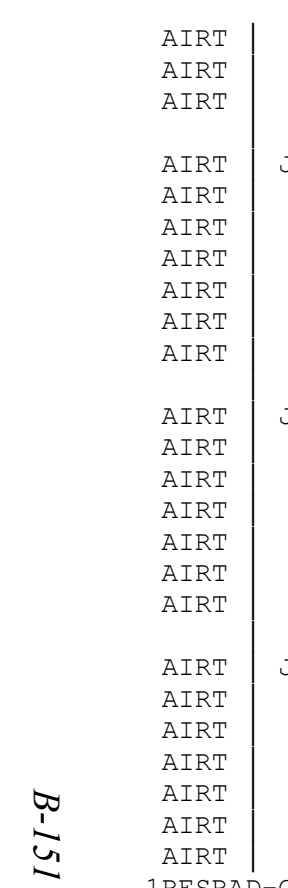

for wind speed class 6 and stability class D for wind speed class 6 and stability class E for wind speed class 6 and stability class F

Joint Frequency in ENE Sector

for wind speed class 1 and stability class A for wind speed class 1 and stability class B for wind speed class 1 and stability class C for wind speed class 1 and stability class D for wind speed class 1 and stability class E for wind speed class 1 and stability class F

Joint Frequency in ENE Sector

for wind speed class 2 and stability class A for wind speed class 2 and stability class B for wind speed class 2 and stability class C for wind speed class 2 and stability class D for wind speed class 2 and stability class E for wind speed class 2 and stability class F

Joint Frequency in ENE Sector

for wind speed class 3 and stability class A for wind speed class 3 and stability class B for wind speed class 3 and stability class C for wind speed class 3 and stability class D for wind speed class 3 and stability class E for wind speed class 3 and stability class $F$ 1RESRAD-OFFSITE, Version 4.0 .3 beta T1/2 Limit $=30$ days Parent Dose Report

Title : Landfill Scenari

File : LANDFILL-PADUCAH-AUTHORIZED-RELEASE-REV.ROF

\begin{tabular}{|l|l|l|l}
$0.000 \mathrm{E}+00$ & $0.000 \mathrm{E}+00$ & --- & $\operatorname{DFREQ}(6,4,3)$ \\
$0.000 \mathrm{E}+00$ & $0.000 \mathrm{E}+00$ & --- & $\operatorname{DFREQ}(6,5,3)$ \\
$0.000 \mathrm{E}+00$ & $0.000 \mathrm{E}+00$ & --- & $\operatorname{DFREQ}(6,6,3)$ \\
& & & \\
$0.000 \mathrm{E}+00$ & $0.000 \mathrm{E}+00$ & --- & $\operatorname{DFREQ}(1,1,4)$ \\
$0.000 \mathrm{E}+00$ & $0.000 \mathrm{E}+00$ & --- & $\operatorname{DFREQ}(1,2,4)$ \\
$0.000 \mathrm{E}+00$ & $0.000 \mathrm{E}+00$ & --- & $\operatorname{DFREQ}(1,3,4)$ \\
$0.000 \mathrm{E}+00$ & $1.000 \mathrm{E}-01$ & --- & $\operatorname{DFREQ}(1,4,4)$ \\
$0.000 \mathrm{E}+00$ & $2.000 \mathrm{E}-01$ & --- & $\operatorname{DFREQ}(1,5,4)$ \\
$0.000 \mathrm{E}+00$ & $7.000 \mathrm{E}-01$ & -- & $\operatorname{DFREQ}(1,6,4)$ \\
& & & \\
$0.000 \mathrm{E}+00$ & $0.000 \mathrm{E}+00$ & --- & $\operatorname{DFREQ}(2,1,4)$ \\
$0.000 \mathrm{E}+00$ & $0.000 \mathrm{E}+00$ & --- & $\operatorname{DFREQ}(2,2,4)$ \\
$0.000 \mathrm{E}+00$ & $0.000 \mathrm{E}+00$ & --- & $\operatorname{DFREQ}(2,3,4)$ \\
$0.000 \mathrm{E}+00$ & $0.000 \mathrm{E}+00$ & --- & $\operatorname{DFREQ}(2,4,4)$ \\
$0.000 \mathrm{E}+00$ & $0.000 \mathrm{E}+00$ & -- & $\operatorname{DFREQ}(2,5,4)$ \\
$0.000 \mathrm{E}+00$ & $0.000 \mathrm{E}+00$ & --- & $\operatorname{DFREQ}(2,6,4)$ \\
& & & \\
$0.000 \mathrm{E}+00$ & $0.000 \mathrm{E}+00$ & --- & $\operatorname{DFREQ}(3,1,4)$ \\
$0.000 \mathrm{E}+00$ & $0.000 \mathrm{E}+00$ & --- & $\operatorname{DFREQ}(3,2,4)$ \\
$0.000 \mathrm{E}+00$ & $0.000 \mathrm{E}+00$ & --- & $\operatorname{DFREQ}(3,3,4)$ \\
$0.000 \mathrm{E}+00$ & $0.000 \mathrm{E}+00$ & --- & $\operatorname{DFREQ}(3,4,4)$ \\
$0.000 \mathrm{E}+00$ & $0.000 \mathrm{E}+00$ & --- & $\operatorname{DFREQ}(3,5,4)$ \\
$0.000 \mathrm{E}+00$ & $0.000 \mathrm{E}+00$ & --- & $\operatorname{DFREQ}(3,6,4)$ \\
$06 / 11 / 2020$ & $11: 17$ & &
\end{tabular}

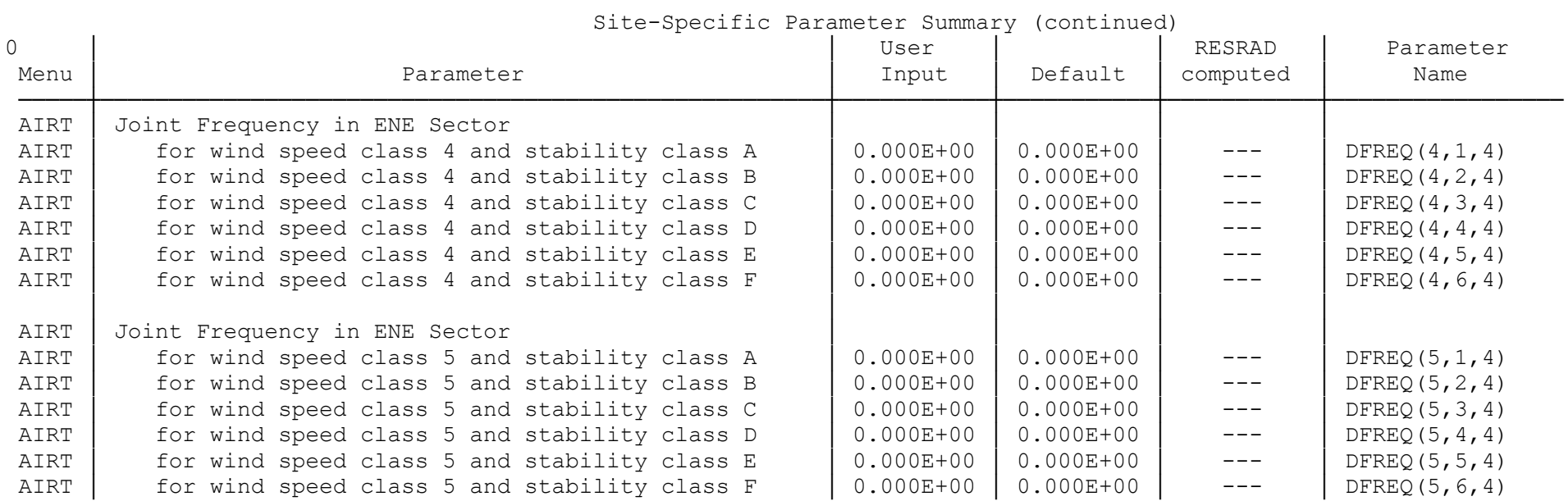




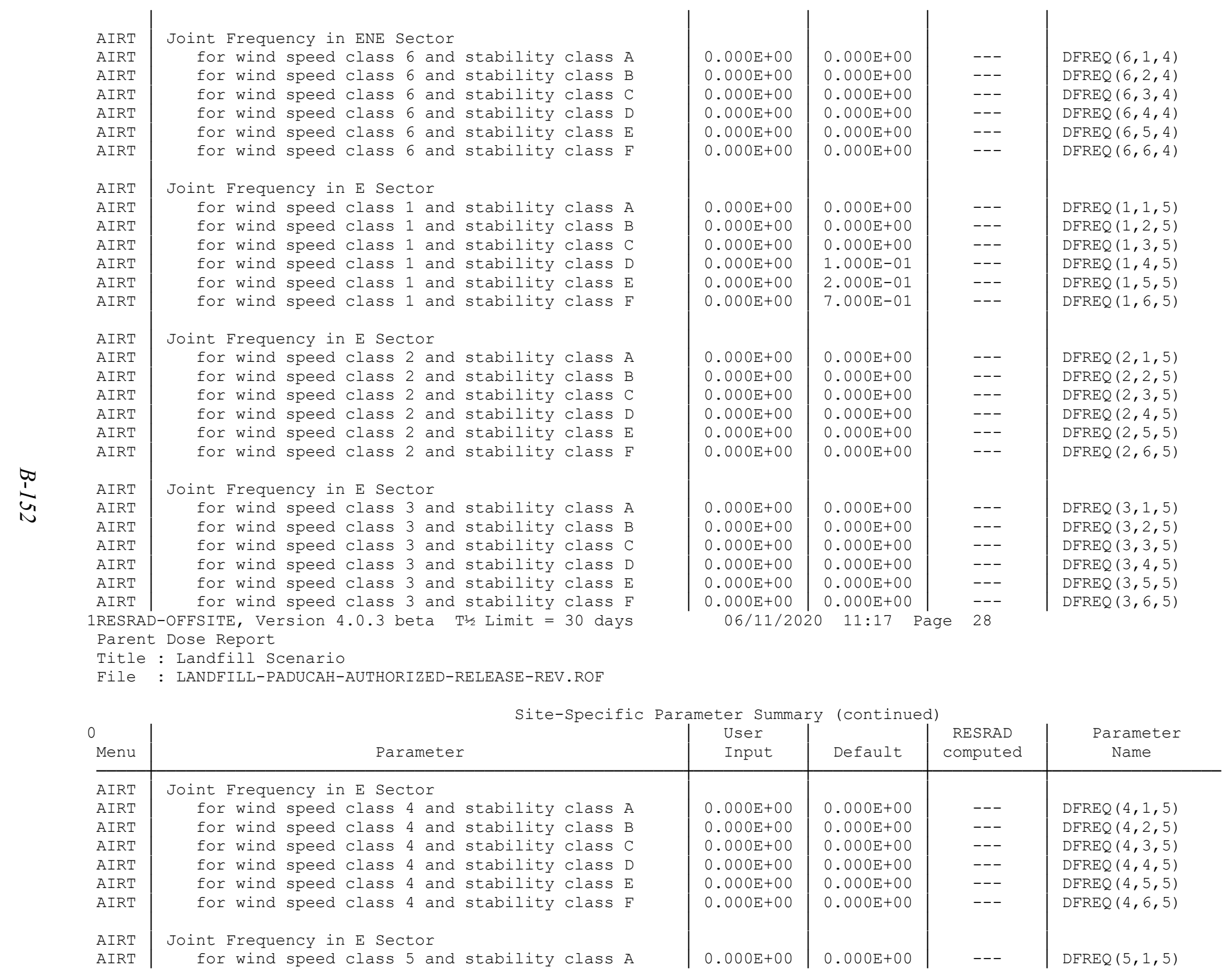




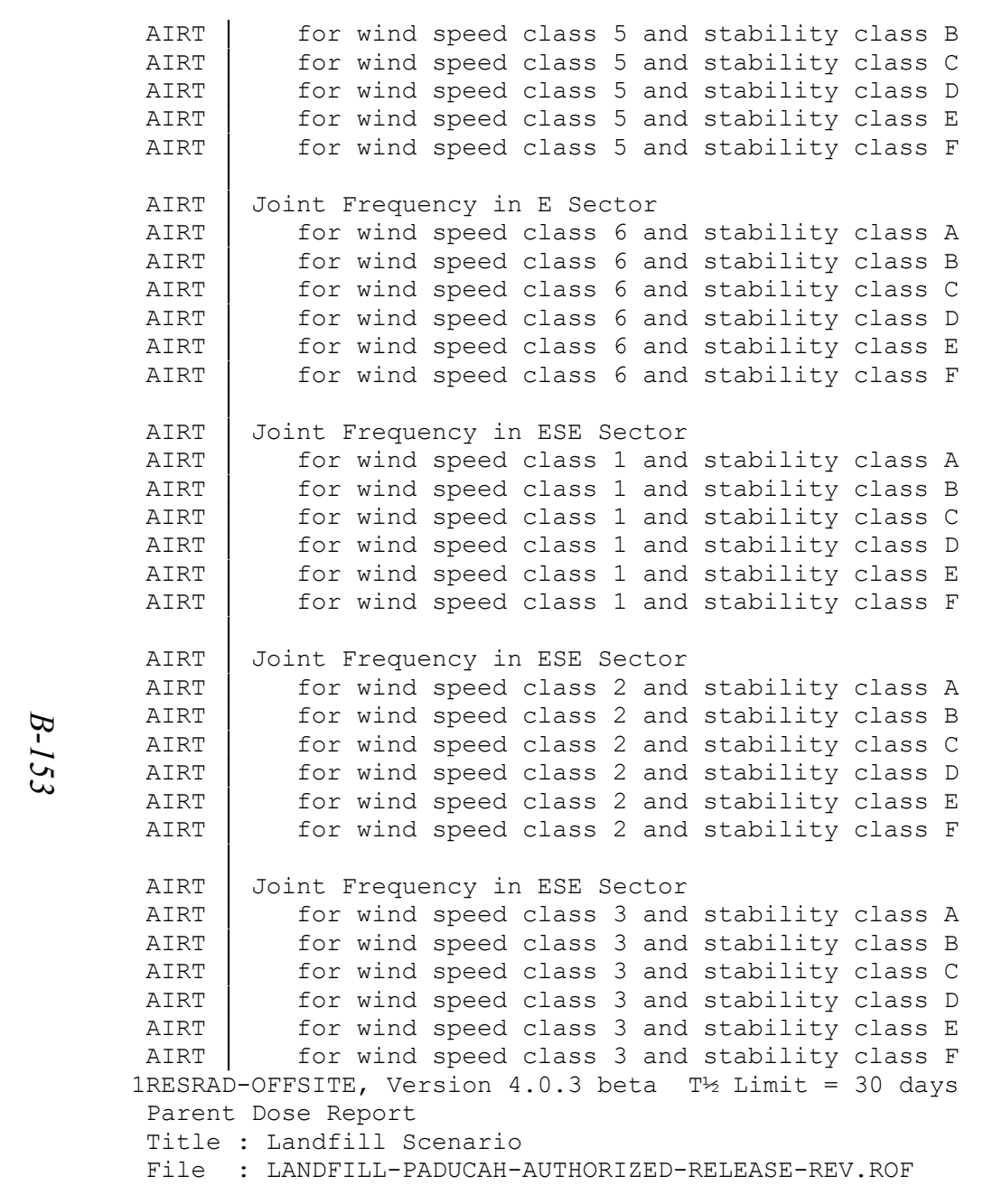

\begin{tabular}{|c|c|c|c|}
\hline $0.000 \mathrm{E}+00$ & $0.000 \mathrm{E}+00$ & --- & $\operatorname{DFREQ}(5,2,5)$ \\
\hline $0.000 \mathrm{E}+00$ & $0.000 \mathrm{E}+00$ & --- & $\operatorname{DFREQ}(5,3,5)$ \\
\hline $0.000 \mathrm{E}+00$ & $0.000 \mathrm{E}+00$ & --- & $\operatorname{DFREQ}(5,4,5)$ \\
\hline $0.000 \mathrm{E}+00$ & $0.000 \mathrm{E}+00$ & --- & $\operatorname{DFREQ}(5,5,5)$ \\
\hline $0.000 \mathrm{E}+00$ & $0.000 \mathrm{E}+00$ & --- & $\operatorname{DFREQ}(5,6,5)$ \\
\hline $0.000 \mathrm{E}+00$ & $0.000 \mathrm{E}+00$ & --- & $\operatorname{DFREQ}(6,1,5)$ \\
\hline $0.000 \mathrm{E}+00$ & $0.000 \mathrm{E}+00$ & --- & $\operatorname{DFREQ}(6,2,5)$ \\
\hline $0.000 \mathrm{E}+00$ & $0.000 \mathrm{E}+00$ & --- & $\operatorname{DFREQ}(6,3,5)$ \\
\hline $0.000 \mathrm{E}+00$ & $0.000 \mathrm{E}+00$ & --- & $\operatorname{DFREQ}(6,4,5)$ \\
\hline $0.000 \mathrm{E}+00$ & $0.000 \mathrm{E}+00$ & --- & $\operatorname{DFREQ}(6,5,5)$ \\
\hline $0.000 \mathrm{E}+00$ & $0.000 \mathrm{E}+00$ & --- & $\operatorname{DFREQ}(6,6,5)$ \\
\hline $0.000 \mathrm{E}+00$ & $0.000 \mathrm{E}+00$ & --- & $\operatorname{DFREQ}(1,1,6)$ \\
\hline $0.000 \mathrm{E}+00$ & $0.000 \mathrm{E}+00$ & --- & $\operatorname{DFREQ}(1,2,6)$ \\
\hline $0.000 \mathrm{E}+00$ & $0.000 \mathrm{E}+00$ & --- & $\operatorname{DFREQ}(1,3,6)$ \\
\hline $0.000 \mathrm{E}+00$ & $1.000 \mathrm{E}-01$ & --- & $\operatorname{DFREQ}(1,4,6)$ \\
\hline $0.000 \mathrm{E}+00$ & $2.000 \mathrm{E}-01$ & --- & $\operatorname{DFREQ}(1,5,6)$ \\
\hline $0.000 \mathrm{E}+00$ & $7.000 \mathrm{E}-01$ & --- & $\operatorname{DFREQ}(1,6,6)$ \\
\hline $0.000 \mathrm{E}+00$ & $0.000 \mathrm{E}+00$ & --- & $\operatorname{DFREQ}(2,1,6)$ \\
\hline $0.000 \mathrm{E}+00$ & $0.000 \mathrm{E}+00$ & --- & $\operatorname{DFREQ}(2,2,6)$ \\
\hline $0.000 \mathrm{E}+00$ & $0.000 \mathrm{E}+00$ & --- & $\operatorname{DFREQ}(2,3,6)$ \\
\hline $0.000 \mathrm{E}+00$ & $0.000 \mathrm{E}+00$ & --- & $\operatorname{DFREQ}(2,4,6)$ \\
\hline $0.000 \mathrm{E}+00$ & $0.000 \mathrm{E}+00$ & --- & $\operatorname{DFREQ}(2,5,6)$ \\
\hline $0.000 \mathrm{E}+00$ & $0.000 \mathrm{E}+00$ & --- & $\operatorname{DFREQ}(2,6,6)$ \\
\hline $0.000 \mathrm{E}+00$ & $0.000 \mathrm{E}+00$ & --- & $\operatorname{DFREQ}(3,1,6)$ \\
\hline $0.000 \mathrm{E}+00$ & $0.000 \mathrm{E}+00$ & --- & $\operatorname{DFREQ}(3,2,6)$ \\
\hline $0.000 \mathrm{E}+00$ & $0.000 \mathrm{E}+00$ & --- & $\operatorname{DFREQ}(3,3,6)$ \\
\hline $0.000 \mathrm{E}+00$ & $0.000 \mathrm{E}+00$ & --- & $\operatorname{DFREQ}(3,4,6)$ \\
\hline $0.000 \mathrm{E}+00$ & $0.000 \mathrm{E}+00$ & --- & $\operatorname{DFREQ}(3,5,6)$ \\
\hline $0.000 \mathrm{E}+00$ & $0.000 \mathrm{E}+00$ & -- & $\operatorname{DFREQ}(3,6,6)$ \\
\hline
\end{tabular}

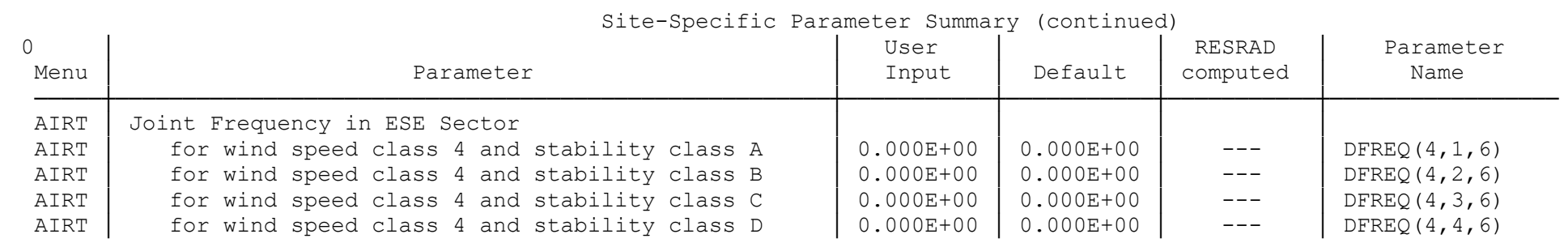


AIRT for wind speed class 4 and stability class E AIRT

AIRT

AIRT

AIRT

AIRT

AIRT

AIRT

AIRT

AIRT

AIRT

AIRT

AIRT

AIRT

AIRT

AIRT

AIRT

AIRT

AIRT

AIRT

AIRT

AIRT

AIRT

AIRT

AIRT

AIRT

AIRT

AIRT

AIRT

AIRT

AIRT

AIRT

AIRT

for wind speed class 4 and stability class F

Joint Frequency in ESE Sector

for wind speed class 5 and stability class A for wind speed class 5 and stability class B for wind speed class 5 and stability class C for wind speed class 5 and stability class D for wind speed class 5 and stability class $\mathrm{E}$ for wind speed class 5 and stability class F

Joint Frequency in ESE Sector

for wind speed class 6 and stability class A for wind speed class 6 and stability class B for wind speed class 6 and stability class C for wind speed class 6 and stability class D for wind speed class 6 and stability class E for wind speed class 6 and stability class F

Joint Frequency in SE Sector

for wind speed class 1 and stability class A for wind speed class 1 and stability class B for wind speed class 1 and stability class C for wind speed class 1 and stability Class D for wind speed class 1 and stability class $\mathrm{E}$ for wind speed class 1 and stability class F

Joint Frequency in SE Sector

for wind speed class 2 and stability class $A$ for wind speed class 2 and stability class B for wind speed class 2 and stability class C for wind speed class 2 and stability class D for wind speed class 2 and stability class E for wind speed class 2 and stability class F

Joint Frequency in SE Sector

for wind speed class 3 and stability class A for wind speed class 3 and stability class B for wind speed class 3 and stability class C for wind speed class 3 and stability class D for wind speed class 3 and stability class E for wind speed class 3 and stability class F RESRAD-OFFSITE, Version 4.0 .3 beta $\mathrm{T}^{1} \frac{1}{2}$ Limit $=30$ days Parent Dose Report

Title : Landfill Scenario

File : LANDFILL-PADUCAH-AUTHORIZED-RELEASE-REV.ROF

\begin{tabular}{|c|c|c|c|}
\hline $0.000 \mathrm{E}+00$ & $0.000 \mathrm{E}+00$ & --- & $\operatorname{DFREQ}(4,5,6)$ \\
\hline $0.000 \mathrm{E}+00$ & $0.000 \mathrm{E}+00$ & --- & $\operatorname{DFREQ}(4,6,6)$ \\
\hline $0.000 \mathrm{E}+00$ & $0.000 \mathrm{E}+00$ & --- & $\operatorname{DFREQ}(5,1,6)$ \\
\hline $0.000 \mathrm{E}+00$ & $0.000 \mathrm{E}+00$ & --- & $\operatorname{DFREQ}(5,2,6)$ \\
\hline $0.000 \mathrm{E}+00$ & $0.000 \mathrm{E}+00$ & --- & $\operatorname{DFREQ}(5,3,6)$ \\
\hline $0.000 \mathrm{E}+00$ & $0.000 \mathrm{E}+00$ & --- & $\operatorname{DFREQ}(5,4,6)$ \\
\hline $0.000 \mathrm{E}+00$ & $0.000 \mathrm{E}+00$ & --- & $\operatorname{DFREQ}(5,5,6)$ \\
\hline $0.000 \mathrm{E}+00$ & $0.000 \mathrm{E}+00$ & --- & $\operatorname{DFREQ}(5,6,6)$ \\
\hline $0.000 \mathrm{E}+00$ & $0.000 \mathrm{E}+00$ & --- & $\operatorname{DFREQ}(6,1,6)$ \\
\hline $0.000 \mathrm{E}+00$ & $0.000 \mathrm{E}+00$ & --- & DFREQ $(6,2,6)$ \\
\hline $0.000 \mathrm{E}+00$ & $0.000 \mathrm{E}+00$ & --- & $\operatorname{DFREQ}(6,3,6)$ \\
\hline $0.000 \mathrm{E}+00$ & $0.000 \mathrm{E}+00$ & --- & $\operatorname{DFREQ}(6,4,6)$ \\
\hline $0.000 \mathrm{E}+00$ & $0.000 \mathrm{E}+00$ & --- & $\operatorname{DFREQ}(6,5,6)$ \\
\hline $0.000 \mathrm{E}+00$ & $0.000 \mathrm{E}+00$ & --- & $\operatorname{DFREQ}(6,6,6)$ \\
\hline $0.000 \mathrm{E}+00$ & $0.000 \mathrm{E}+00$ & --- & $\operatorname{DFREQ}(1,1,7)$ \\
\hline $0.000 \mathrm{E}+00$ & $0.000 \mathrm{E}+00$ & --- & DFREQ $(1,2,7)$ \\
\hline $0.000 \mathrm{E}+00$ & $0.000 \mathrm{E}+00$ & --- & $\operatorname{DFREQ}(1,3,7)$ \\
\hline $0.000 \mathrm{E}+00$ & $1.000 \mathrm{E}-01$ & --- & $\operatorname{DFREQ}(1,4,7)$ \\
\hline $0.000 \mathrm{E}+00$ & $2.000 \mathrm{E}-01$ & --- & $\operatorname{DFREQ}(1,5,7)$ \\
\hline $0.000 \mathrm{E}+00$ & $7.000 \mathrm{E}-01$ & --- & $\operatorname{DFREQ}(1,6,7)$ \\
\hline $0.000 \mathrm{E}+00$ & $0.000 \mathrm{E}+00$ & --- & $\operatorname{DFREQ}(2,1,7)$ \\
\hline $0.000 \mathrm{E}+00$ & $0.000 \mathrm{E}+00$ & --- & DFREQ $(2,2,7)$ \\
\hline $0.000 \mathrm{E}+00$ & $0.000 \mathrm{E}+00$ & --- & $\operatorname{DFREQ}(2,3,7)$ \\
\hline $0.000 \mathrm{E}+00$ & $0.000 \mathrm{E}+00$ & --- & DFREQ $(2,4,7)$ \\
\hline $0.000 \mathrm{E}+00$ & $0.000 \mathrm{E}+00$ & --- & $\operatorname{DFREQ}(2,5,7)$ \\
\hline $0.000 \mathrm{E}+00$ & $0.000 \mathrm{E}+00$ & --- & $\operatorname{DFREQ}(2,6,7)$ \\
\hline $0.000 \mathrm{E}+00$ & $0.000 \mathrm{E}+00$ & --- & $\operatorname{DFREQ}(3,1,7)$ \\
\hline $0.000 \mathrm{E}+00$ & $0.000 \mathrm{E}+00$ & --- & DFREQ $(3,2,7)$ \\
\hline $0.000 \mathrm{E}+00$ & $0.000 \mathrm{E}+00$ & --- & $\operatorname{DFREQ}(3,3,7)$ \\
\hline $0.000 \mathrm{E}+00$ & $0.000 \mathrm{E}+00$ & --- & $\operatorname{DFREQ}(3,4,7)$ \\
\hline $0.000 \mathrm{E}+00$ & $0.000 \mathrm{E}+00$ & --- & $\operatorname{DFREQ}(3,5,7)$ \\
\hline $0.000 \mathrm{E}+00$ & $0.000 \mathrm{E}+00$ & --- & $\operatorname{DFREQ}(3,6,7)$ \\
\hline
\end{tabular}

Site-Specific Parameter Summary (continued)

\begin{tabular}{l|l|l|l|l|l}
0 & \multicolumn{4}{|c}{ Site-Specific Parameter Summary (continued) } & User \\
Menu & Parameter & Input & Default & $\begin{array}{c}\text { RESRAD } \\
\text { computed }\end{array}$ & $\begin{array}{c}\text { Parameter } \\
\text { Name }\end{array}$ \\
\hline
\end{tabular}


AIRT | Joint Frequency in SE Sector

AIRT for wind speed class 4 and stability class A

AIRT for wind speed class 4 and stability class B

AIRT for wind speed class 4 and stability class $C$

AIRT for wind speed class 4 and stability class D

AIRT for wind speed class 4 and stability class E

AIRT for wind speed class 4 and stability class F

AIRT Joint Frequency in SE Sector

AIRT for wind speed class 5 and stability class A

AIRT for wind speed class 5 and stability class B

AIRT for wind speed class 5 and stability class $C$

AIRT for wind speed class 5 and stability class D

AIRT for wind speed class 5 and stability class $E$

AIRT for wind speed class 5 and stability class F

AIRT Joint Frequency in SE Sector

AIRT for wind speed class 6 and stability class A

AIRT for wind speed class 6 and stability class B

AIRT for wind speed class 6 and stability class C

AIRT for wind speed class 6 and stability class D

AIRT for wind speed class 6 and stability class E

AIRT for wind speed class 6 and stability class F

T AIRT Joint Frequency in SSE Sector

AIRT for wind speed class 1 and stability class A

AIRT for wind speed class 1 and stability class $B$

AIRT for wind speed class 1 and stability class C

AIRT for wind speed class 1 and stability class D

AIRT for wind speed class 1 and stability class E

AIRT for wind speed class 1 and stability class F

AIRT Joint Frequency in SSE Sector

AIRT for wind speed class 2 and stability class A

AIRT for wind speed class 2 and stability class B

AIRT for wind speed class 2 and stability class C

AIRT for wind speed class 2 and stability class D

AIRT for wind speed class 2 and stability class E

AIRT for wind speed class 2 and stability class F

AIRT Joint Frequency in SSE Sector

AIRT for wind speed class 3 and stability class A

AIRT for wind speed class 3 and stability class B

AIRT for wind speed class 3 and stability class C

AIRT for wind speed class 3 and stability class D

AIRT for wind speed class 3 and stability class $E$

AIRT for wind speed class 3 and stability class $F$

1RESRAD-OFFSITE, Version 4.0 .3 beta $\mathrm{T}^{1} / 2$ Limit $=30$ days

Parent Dose Report

Title : Landfill Scenario

File : LANDFILL-PADUCAH-AUTHORIZED-RELEASE-REV.ROF

\begin{tabular}{|c|c|c|c|}
\hline $0.000 \mathrm{E}+00$ & $0.000 \mathrm{E}+00$ & --- & $\operatorname{DFREQ}(4,1,7)$ \\
\hline $0.000 \mathrm{E}+00$ & $0.000 \mathrm{E}+00$ & --- & $\operatorname{DFREQ}(4,2,7)$ \\
\hline $0.000 \mathrm{E}+00$ & $0.000 \mathrm{E}+00$ & --- & $\operatorname{DFREQ}(4,3,7)$ \\
\hline $0.000 \mathrm{E}+00$ & $0.000 \mathrm{E}+00$ & --- & $\operatorname{DFREQ}(4,4,7)$ \\
\hline $0.000 \mathrm{E}+00$ & $0.000 \mathrm{E}+00$ & --- & $\operatorname{DFREQ}(4,5,7)$ \\
\hline $0.000 \mathrm{E}+00$ & $0.000 \mathrm{E}+00$ & --- & $\operatorname{DFREQ}(4,6,7)$ \\
\hline $0.000 \mathrm{E}+00$ & $0.000 \mathrm{E}+00$ & --- & $\operatorname{DFREQ}(5,1,7)$ \\
\hline $0.000 \mathrm{E}+00$ & $0.000 \mathrm{E}+00$ & --- & $\operatorname{DFREQ}(5,2,7)$ \\
\hline $0.000 \mathrm{E}+00$ & $0.000 \mathrm{E}+00$ & --- & $\operatorname{DFREQ}(5,3,7)$ \\
\hline $0.000 \mathrm{E}+00$ & $0.000 \mathrm{E}+00$ & --- & $\operatorname{DFREQ}(5,4,7)$ \\
\hline $0.000 \mathrm{E}+00$ & $0.000 \mathrm{E}+00$ & --- & $\operatorname{DFREQ}(5,5,7)$ \\
\hline $0.000 \mathrm{E}+00$ & $0.000 \mathrm{E}+00$ & --- & $\operatorname{DFREQ}(5,6,7)$ \\
\hline $0.000 \mathrm{E}+00$ & $0.000 \mathrm{E}+00$ & --- & $\operatorname{DFREQ}(6,1,7)$ \\
\hline $0.000 \mathrm{E}+00$ & $0.000 \mathrm{E}+00$ & --- & $\operatorname{DFREQ}(6,2,7)$ \\
\hline $0.000 \mathrm{E}+00$ & $0.000 \mathrm{E}+00$ & --- & $\operatorname{DFREQ}(6,3,7)$ \\
\hline $0.000 \mathrm{E}+00$ & $0.000 \mathrm{E}+00$ & --- & $\operatorname{DFREQ}(6,4,7)$ \\
\hline $0.000 \mathrm{E}+00$ & $0.000 \mathrm{E}+00$ & --- & $\operatorname{DFREQ}(6,5,7)$ \\
\hline $0.000 \mathrm{E}+00$ & $0.000 \mathrm{E}+00$ & --- & $\operatorname{DFREQ}(6,6,7)$ \\
\hline $0.000 \mathrm{E}+00$ & $0.000 \mathrm{E}+00$ & --- & $\operatorname{DFREQ}(1,1,8)$ \\
\hline $0.000 \mathrm{E}+00$ & $0.000 \mathrm{E}+00$ & --- & $\operatorname{DFREQ}(1,2,8)$ \\
\hline $0.000 \mathrm{E}+00$ & $0.000 \mathrm{E}+00$ & --- & $\operatorname{DFREQ}(1,3,8)$ \\
\hline $0.000 \mathrm{E}+00$ & $1.000 \mathrm{E}-01$ & --- & $\operatorname{DFREQ}(1,4,8)$ \\
\hline $0.000 \mathrm{E}+00$ & $2.000 \mathrm{E}-01$ & --- & $\operatorname{DFREQ}(1,5,8)$ \\
\hline $0.000 \mathrm{E}+00$ & $7.000 \mathrm{E}-01$ & --- & $\operatorname{DFREQ}(1,6,8)$ \\
\hline $0.000 \mathrm{E}+00$ & $0.000 \mathrm{E}+00$ & --- & $\operatorname{DFREQ}(2,1,8)$ \\
\hline $0.000 \mathrm{E}+00$ & $0.000 \mathrm{E}+00$ & --- & $\operatorname{DFREQ}(2,2,8)$ \\
\hline $0.000 \mathrm{E}+00$ & $0.000 \mathrm{E}+00$ & --- & $\operatorname{DFREQ}(2,3,8)$ \\
\hline $0.000 \mathrm{E}+00$ & $0.000 \mathrm{E}+00$ & --- & $\operatorname{DFREQ}(2,4,8)$ \\
\hline $0.000 \mathrm{E}+00$ & $0.000 \mathrm{E}+00$ & --- & $\operatorname{DFREQ}(2,5,8)$ \\
\hline $0.000 \mathrm{E}+00$ & $0.000 \mathrm{E}+00$ & & $\operatorname{DFREQ}(2,6,8)$ \\
\hline $0.000 \mathrm{E}+00$ & $0.000 \mathrm{E}+00$ & --- & $\operatorname{DFREQ}(3,1,8)$ \\
\hline $0.000 \mathrm{E}+00$ & $0.000 \mathrm{E}+00$ & --- & $\operatorname{DFREQ}(3,2,8)$ \\
\hline $0.000 \mathrm{E}+00$ & $0.000 \mathrm{E}+00$ & --- & $\operatorname{DFREQ}(3,3,8)$ \\
\hline $0.000 \mathrm{E}+00$ & $0.000 \mathrm{E}+00$ & --- & $\operatorname{DFREQ}(3,4,8)$ \\
\hline $0.000 \mathrm{E}+00$ & $0.000 \mathrm{E}+00$ & --- & $\operatorname{DFREQ}(3,5,8)$ \\
\hline $0.000 \mathrm{E}+00$ & $0.000 \mathrm{E}+00$ & --- & $\operatorname{DFREQ}(3,6,8)$ \\
\hline
\end{tabular}

06/11/2020 11:17 Page 31

DFREQ $(3,5,8)$ 


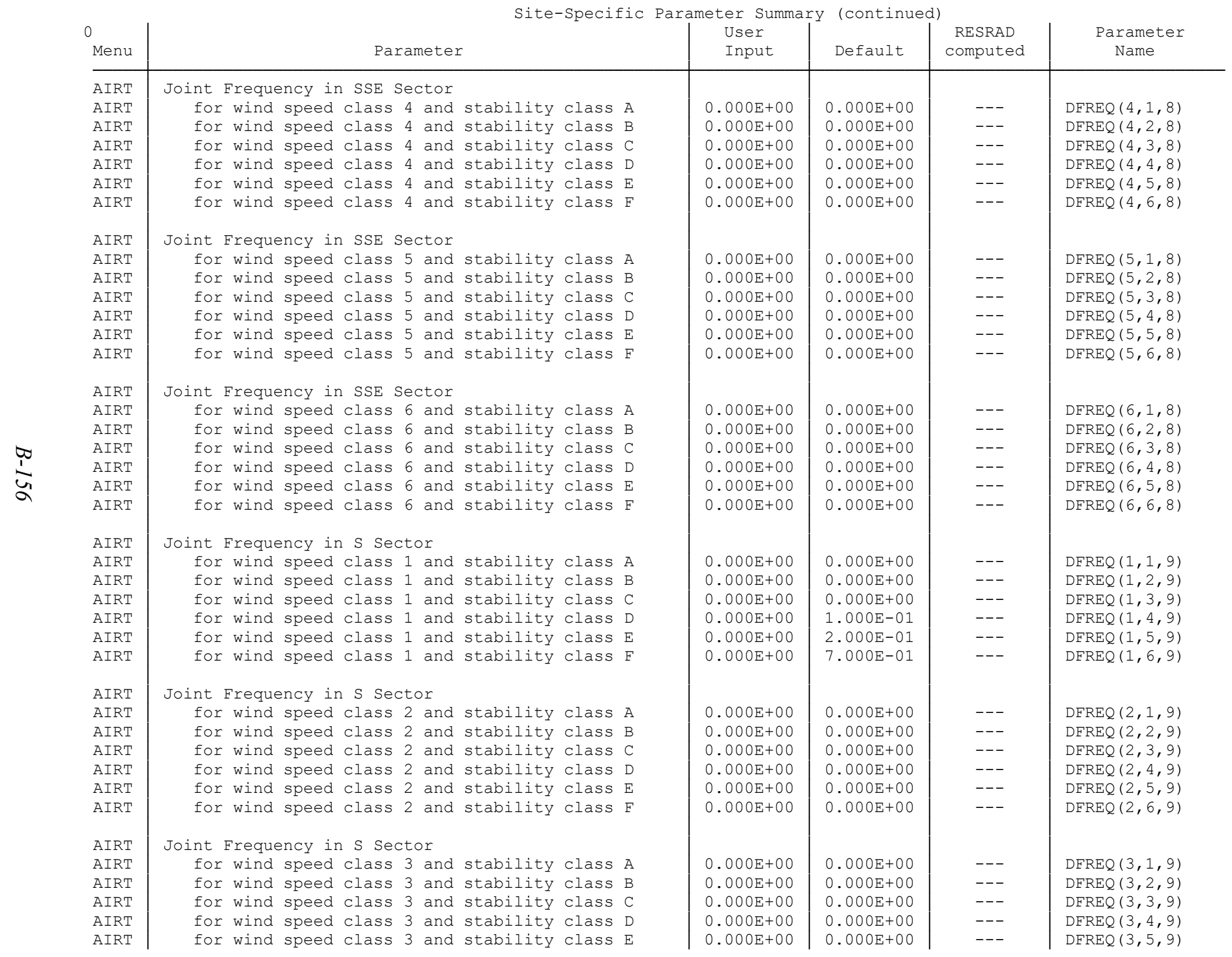


AIRT for wind speed class 3 and stability class F 1RESRAD-OFFSITE, Version 4.0 .3 beta T1/2 Limit $=30$ days Parent Dose Report

Title : Landfill Scenario

File : LANDFILL-PADUCAH-AUTHORIZED-RELEASE-REV.ROF \begin{tabular}{cc|c|c|c|}
$\left|\begin{array}{c}0.000 \mathrm{E}+00 \\
06 / 11 / 2020\end{array}\right| \begin{array}{ll}0.000 \mathrm{E}+00 \\
11: 17\end{array}$ Page & --- & $\mathbf{D F R E Q}(3,6,9)$
\end{tabular}

Site-Specific Parameter Summary (continued)

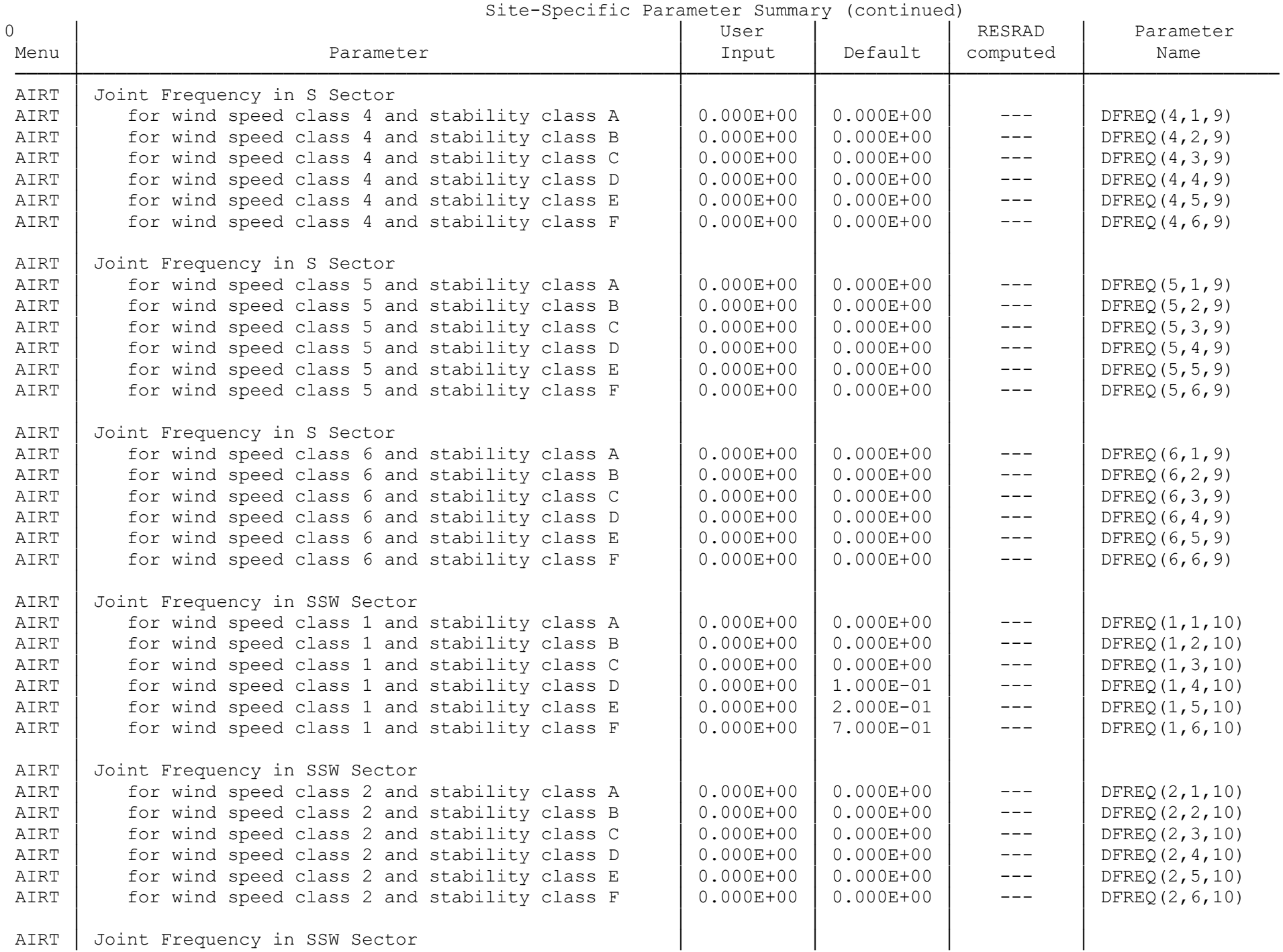



AIRT for wind speed class 3 and stability class A AIRT AIRT AIRT for wind speed class 3 and stability class B for wind speed class 3 and stability class C for wind speed class 3 and stability class D for wind speed class 3 and stability class E for wind speed class 3 and stability class F 1RESRAD-OFFSITE, Version 4.0 .3 beta $T^{1 / 2}$ Limit $=30$ days Parent Dose Report

Title : Landfill Scenario

File : LANDFILL-PADUCAH-AUTHORIZED-RELEASE-REV.ROF

\begin{tabular}{|l|l|l|l}
$0.000 \mathrm{E}+00$ & $0.000 \mathrm{E}+00$ & --- & $\operatorname{DFREQ}(3,1,10)$ \\
$0.000 \mathrm{E}+00$ & $0.000 \mathrm{E}+00$ & --- & $\operatorname{DFREQ}(3,2,10)$ \\
$0.000 \mathrm{E}+00$ & $0.000 \mathrm{E}+00$ & --- & $\operatorname{DFREQ}(3,3,10)$ \\
$0.000 \mathrm{E}+00$ & $0.000 \mathrm{E}+00$ & -- & $\operatorname{DFREQ}(3,4,10)$ \\
$0.000 \mathrm{E}+00$ & $0.000 \mathrm{E}+00$ & --- & $\operatorname{DFREQ}(3,5,10)$ \\
$0.000 \mathrm{E}+00$ & $0.000 \mathrm{E}+00$ & --- & $\operatorname{DFREQ}(3,6,10)$
\end{tabular}

$\operatorname{DFREQ}(3,5,10)$

06/11/2020 11:17 Page 33

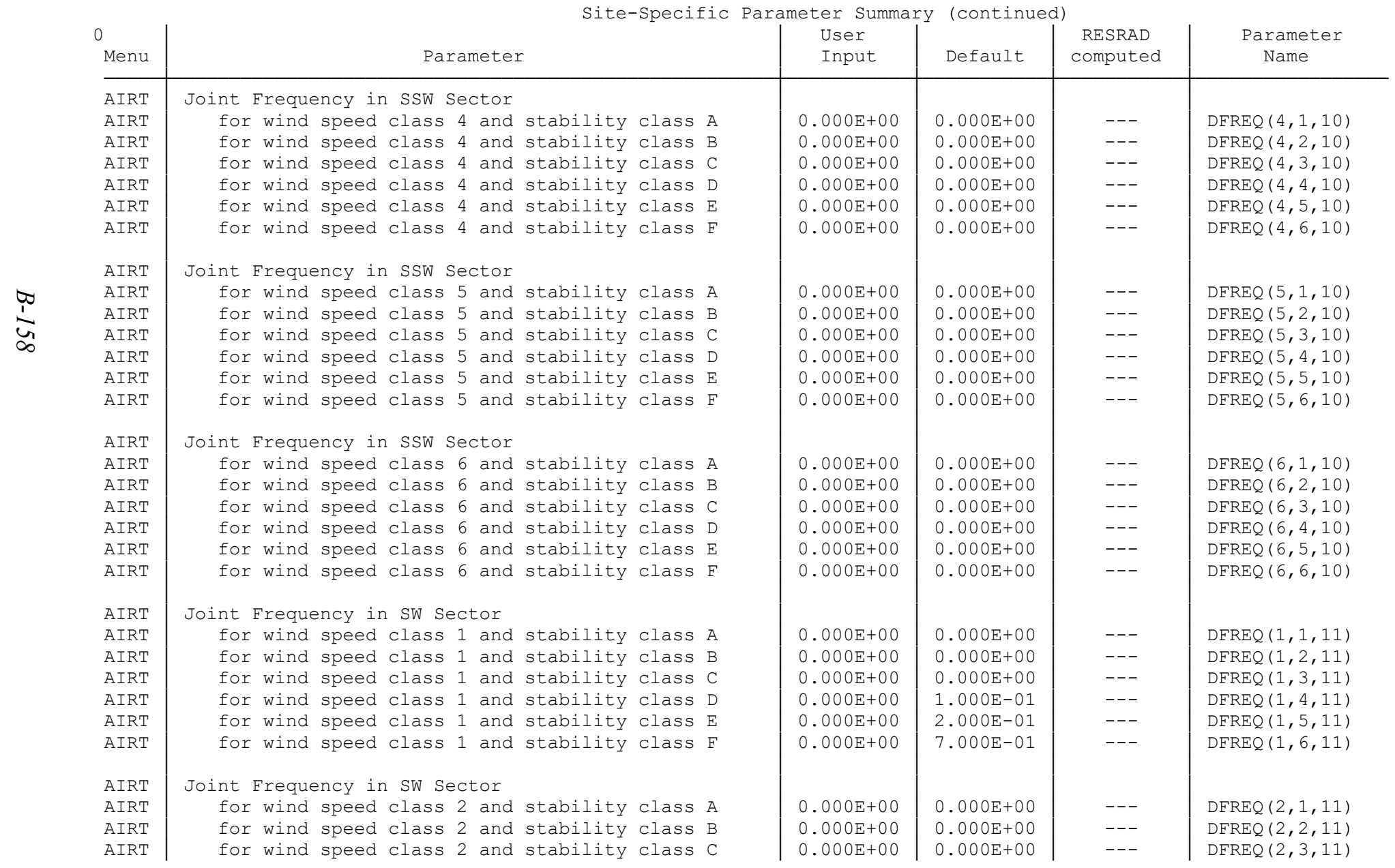


AIRT for wind speed class 2 and stability class D AIRT for wind speed class 2 and stability class $\mathrm{E}$ Parent Dose Report

Title : Landfill Scenario

File : LANDFILL-PADUCAH-AUTHORIZED-RELEASE-REV.ROF RESRAD-OFFSITE, Version 4.0 .3 beta T1/2 Limit = 30 days

\begin{tabular}{|l|l|l|l}
$0.000 \mathrm{E}+00$ & $0.000 \mathrm{E}+00$ & --- & $\operatorname{DFREQ}(2,4,11)$ \\
$0.000 \mathrm{E}+00$ & $0.000 \mathrm{E}+00$ & -- & $\operatorname{DFREQ}(2,5,11)$ \\
$0.000 \mathrm{E}+00$ & $0.000 \mathrm{E}+00$ & -- & $\operatorname{DFREQ}(2,6,11)$ \\
& & & \\
$0.000 \mathrm{E}+00$ & $0.000 \mathrm{E}+00$ & --- & $\operatorname{DFREQ}(3,1,11)$ \\
$0.000 \mathrm{E}+00$ & $0.000 \mathrm{E}+00$ & --- & $\operatorname{DFREQ}(3,2,11)$ \\
$0.000 \mathrm{E}+00$ & $0.000 \mathrm{E}+00$ & -- & $\operatorname{DFREQ}(3,3,11)$ \\
$0.000 \mathrm{E}+00$ & $0.000 \mathrm{E}+00$ & -- & $\operatorname{DFREQ}(3,4,11)$ \\
$0.000 \mathrm{E}+00$ & $0.000 \mathrm{E}+00$ & --- & $\operatorname{DFREQ}(3,5,11)$ \\
$0.000 \mathrm{E}+00$ & $0.000 \mathrm{E}+00$ & --- & $\operatorname{DFREQ}(3,6,11)$
\end{tabular}

Site-Specific Parameter Summary (continued)

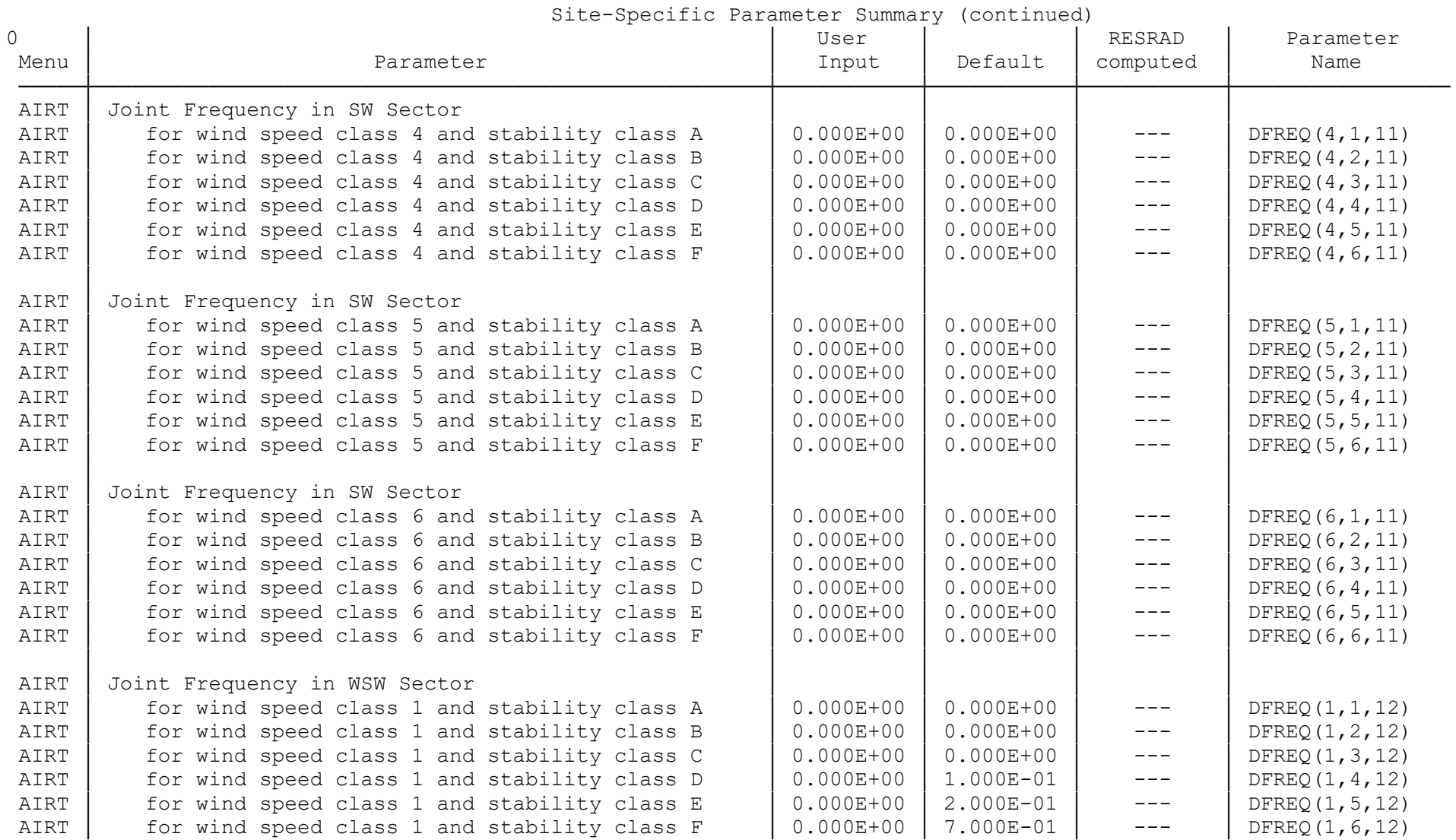




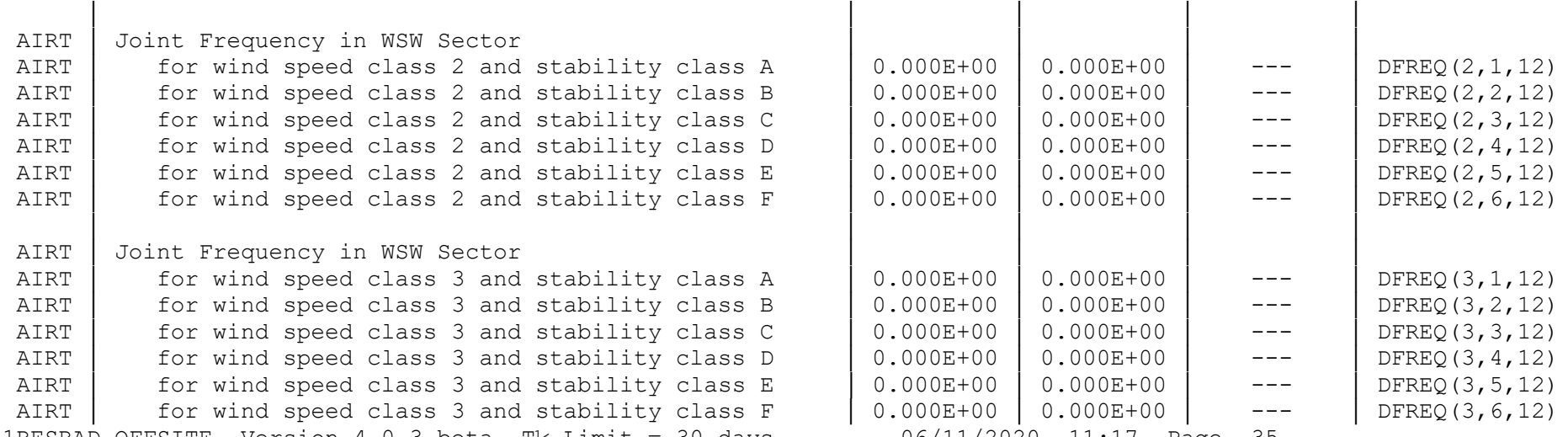

1RESRAD-OFFSITE, Version 4.0 .3 beta $\mathrm{T}^{1 / 2} \mathrm{Limit}=30$ days Parent Dose Report

Title : Landfill Scenario

File: LANDFILL-PADUCAH-AUTHORIZED-RELEASE-REV.ROF

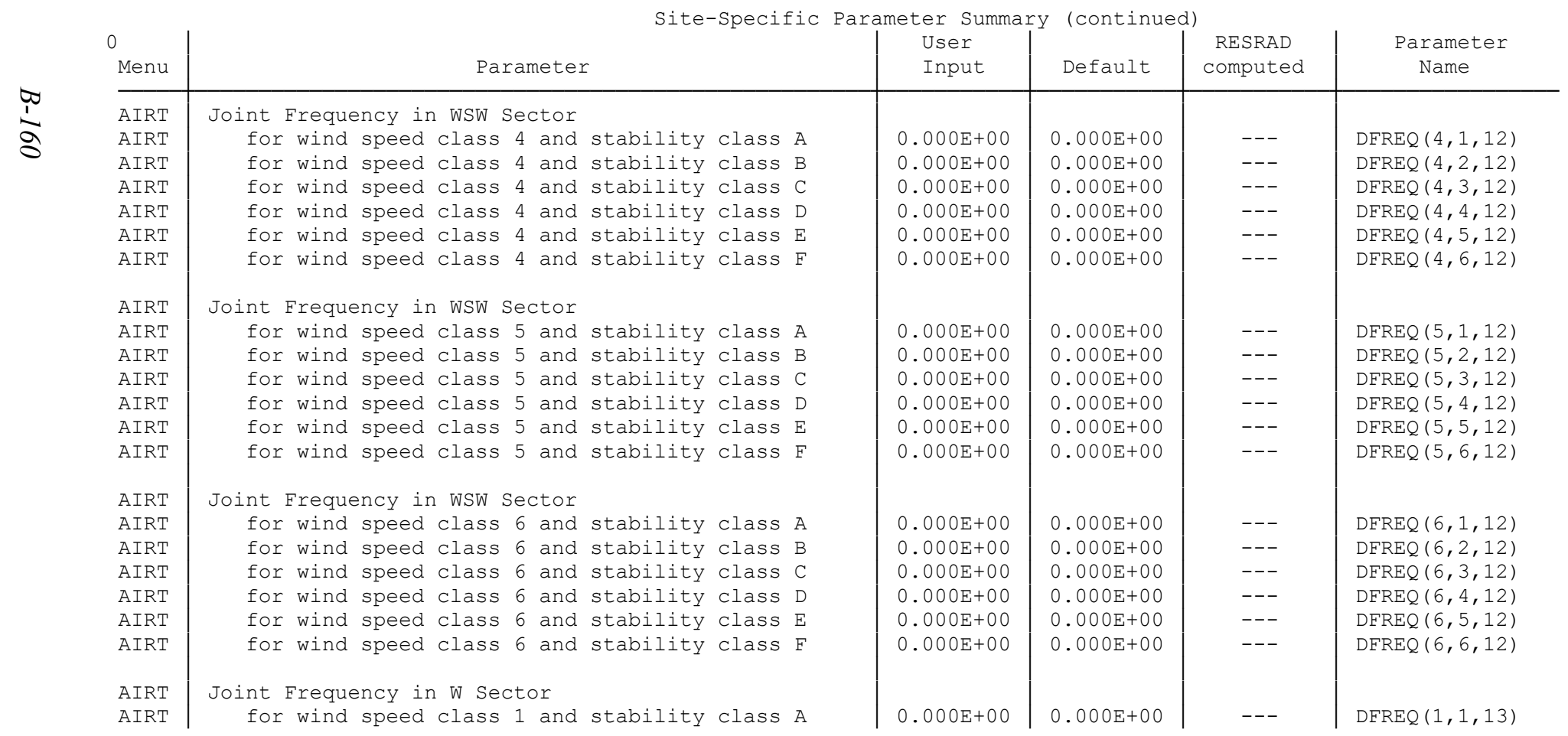




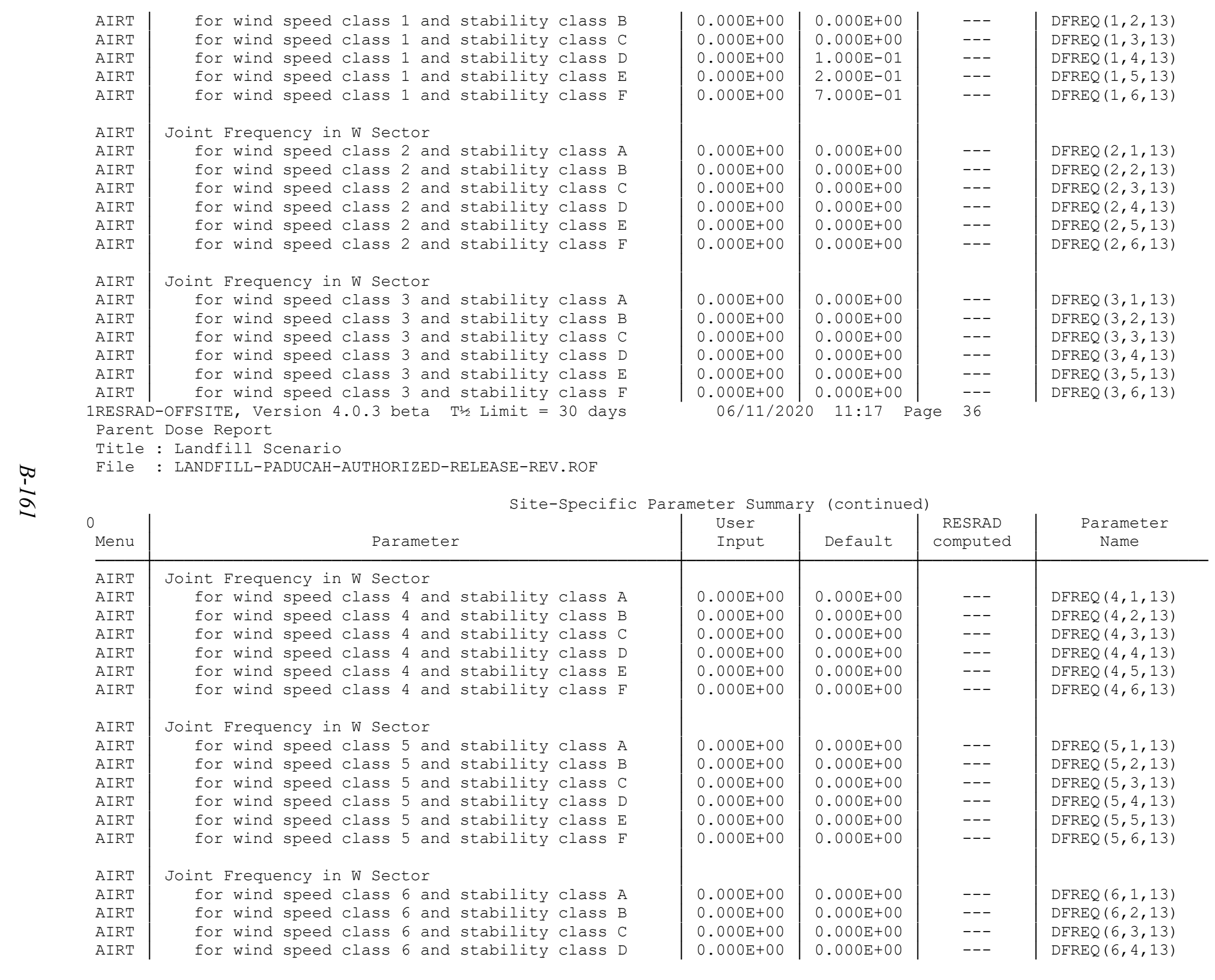


AIRT for wind speed class 6 and stability class E AIRT

AIRT

AIRT

AIRT

AIRT

AIRT

AIRT

AIRT

AIRT

AIRT

AIRT

AIRT

AIRT

AIRT

AIRT

AIRT

AIRT

AIRT

for wind speed class 6 and stability class F

Joint Frequency in wNW Sector

for wind speed class 1 and stability class A for wind speed class 1 and stability class B for wind speed class 1 and stability class D for wind speed class 1 and stability class $\mathrm{E}$ for wind speed class 1 and stability class F

Joint Frequency in WNW Sector

for wind speed class 2 and stability class A for wind speed class 2 and stability class C for wind speed class 2 and stability class D for wind speed class 2 and stability class E for wind speed class 2 and stability class F

Joint Frequency in WNW Sector

for wind speed class 3 and stability class A for wind speed class 3 and stability class C for wind speed Class 3 and stability Class D for wind speed class 3 and stability class E for wind speed class 3 and stability class $F$ 1RESRAD-OFFSITE, Version 4.0 .3 beta $T^{1} \frac{1}{2}$ Limit $=30$ days Parent Dose Report

Title : Landfill Scenario

File : LANDFILL-PADUCAH-AUTHORIZED-RELEASE-REV.ROF for wind speed class 1 and stability class $C$ for wind speed class 2 and stability class B for wind speed class 3 and stability class B

\begin{tabular}{|c|c|c|c|}
\hline $0.000 \mathrm{E}+00$ & $0.000 \mathrm{E}+00$ & --- & $\operatorname{DFREQ}(6,5,13)$ \\
\hline $0.000 \mathrm{E}+00$ & $0.000 \mathrm{E}+00$ & --- & $\operatorname{DFREQ}(6,6,13)$ \\
\hline $0.000 \mathrm{E}+00$ & $0.000 \mathrm{E}+00$ & --- & $\operatorname{DFREQ}(1,1,14)$ \\
\hline $0.000 \mathrm{E}+00$ & $0.000 \mathrm{E}+00$ & --- & $\operatorname{DFREQ}(1,2,14)$ \\
\hline $0.000 \mathrm{E}+00$ & $0.000 \mathrm{E}+00$ & --- & $\operatorname{DFREQ}(1,3,14)$ \\
\hline $0.000 \mathrm{E}+00$ & $1.000 \mathrm{E}-01$ & --- & $\operatorname{DFREQ}(1,4,14)$ \\
\hline $0.000 \mathrm{E}+00$ & $2.000 \mathrm{E}-01$ & --- & $\operatorname{DFREQ}(1,5,14)$ \\
\hline $0.000 \mathrm{E}+00$ & $7.000 \mathrm{E}-01$ & --- & $\operatorname{DFREQ}(1,6,14)$ \\
\hline $0.000 \mathrm{E}+00$ & $0.000 \mathrm{E}+00$ & --- & $\operatorname{DFREQ}(2,1,14)$ \\
\hline $0.000 \mathrm{E}+00$ & $0.000 \mathrm{E}+00$ & --- & $\operatorname{DFREQ}(2,2,14)$ \\
\hline $0.000 \mathrm{E}+00$ & $0.000 \mathrm{E}+00$ & --- & $\operatorname{DFREQ}(2,3,14)$ \\
\hline $0.000 \mathrm{E}+00$ & $0.000 \mathrm{E}+00$ & --- & $\operatorname{DFREQ}(2,4,14)$ \\
\hline $0.000 \mathrm{E}+00$ & $0.000 \mathrm{E}+00$ & --- & $\operatorname{DFREQ}(2,5,14)$ \\
\hline $0.000 \mathrm{E}+00$ & $0.000 \mathrm{E}+00$ & --- & $\operatorname{DFREQ}(2,6,14)$ \\
\hline $0.000 \mathrm{E}+00$ & $0.000 \mathrm{E}+00$ & --- & $\operatorname{DFREQ}(3,1,14)$ \\
\hline $0.000 \mathrm{E}+00$ & $0.000 \mathrm{E}+00$ & --- & DFREQ $(3,2,14)$ \\
\hline $0.000 \mathrm{E}+00$ & $0.000 \mathrm{E}+00$ & --- & $\operatorname{DFREQ}(3,3,14)$ \\
\hline $0.000 \mathrm{E}+00$ & $0.000 \mathrm{E}+00$ & --- & $\operatorname{DFREQ}(3,4,14)$ \\
\hline $0.000 \mathrm{E}+00$ & $0.000 \mathrm{E}+00$ & --- & $\operatorname{DFREQ}(3,5,14)$ \\
\hline $0.000 \mathrm{E}+00$ & $0.000 \mathrm{E}+00$ & --- & $\operatorname{DFREQ}(3,6,14)$ \\
\hline
\end{tabular}

Site-Specific Parameter Summary (continued)

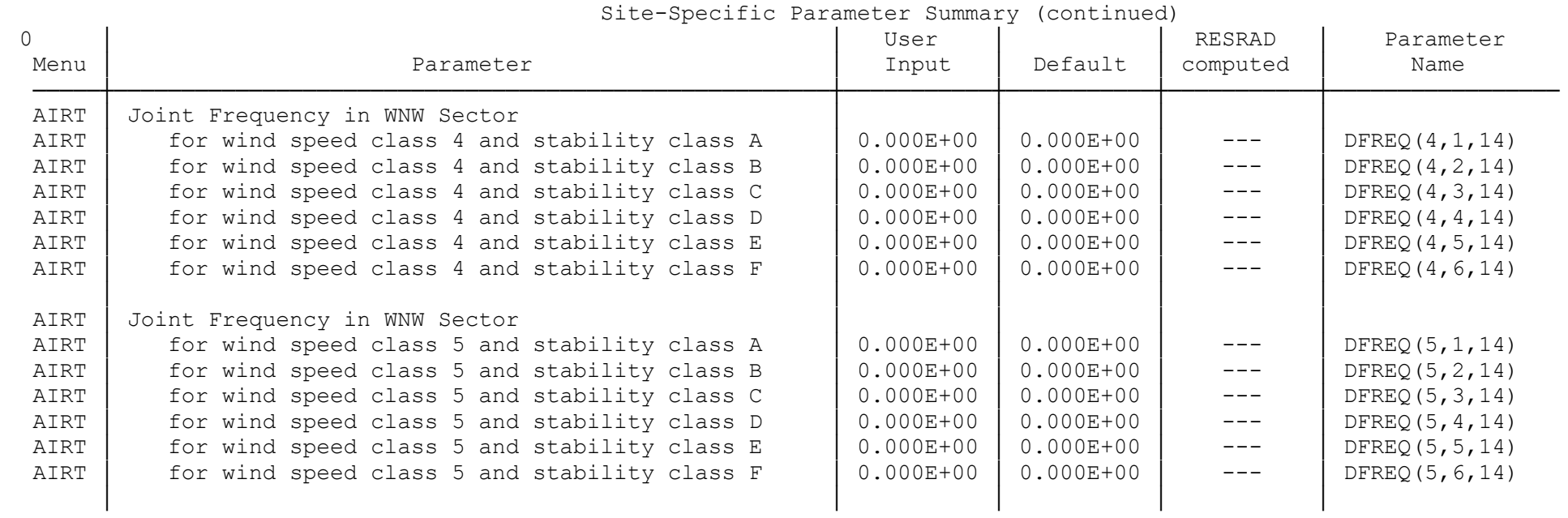


AIRT | Joint Frequency in WNW Sector

AIRT for wind speed class 6 and stability class A

AIRT for wind speed class 6 and stability class B

AIRT for wind speed class 6 and stability class C

AIRT for wind speed class 6 and stability class D

AIRT for wind speed class 6 and stability class E

AIRT for wind speed class 6 and stability class F

AIRT Joint Frequency in NW Sector

AIRT for wind speed class 1 and stability class A

AIRT for wind speed class 1 and stability class B

AIRT for wind speed class 1 and stability class C

AIRT for wind speed class 1 and stability class D

AIRT for wind speed class 1 and stability class $E$

AIRT for wind speed class 1 and stability class F

AIRT Joint Frequency in NW Sector

AIRT for wind speed class 2 and stability class A

AIRT for wind speed class 2 and stability class B

AIRT for wind speed class 2 and stability class C

AIRT for wind speed class 2 and stability class D

AIRT for wind speed class 2 and stability class $E$

AIRT for wind speed class 2 and stability class F

$\rightarrow \quad$ AIRT Joint Frequency in NW Sector

AIRT for wind speed class 3 and stability class A

AIRT for wind speed class 3 and stability class $B$

AIRT for wind speed class 3 and stability class $C$

AIRT for wind speed class 3 and stability class D

AIRT for wind speed class 3 and stability class $E$ AIRT for wind speed class 3 and stability class F 1RESRAD-OFFSITE, Version 4.0 .3 beta $\mathrm{T}^{\frac{1}{2}}$ Limit $=30$ days Parent Dose Report

Title : Landfill Scenario

File : LANDFILL-PADUCAH-AUTHORIZED-RELEASE-REV.ROF

\begin{tabular}{|c|c|c|c|}
\hline $0.000 \mathrm{E}+00$ & $0.000 \mathrm{E}+00$ & --- & $\operatorname{DFREQ}(6,1,14)$ \\
\hline $0.000 \mathrm{E}+00$ & $0.000 \mathrm{E}+00$ & --- & $\operatorname{DFREQ}(6,2,14)$ \\
\hline $0.000 \mathrm{E}+00$ & $0.000 \mathrm{E}+00$ & --- & $\operatorname{DFREQ}(6,3,14)$ \\
\hline $0.000 \mathrm{E}+00$ & $0.000 \mathrm{E}+00$ & --- & $\operatorname{DFREQ}(6,4,14)$ \\
\hline $0.000 \mathrm{E}+00$ & $0.000 \mathrm{E}+00$ & --- & $\operatorname{DFREQ}(6,5,14)$ \\
\hline $0.000 \mathrm{E}+00$ & $0.000 \mathrm{E}+00$ & --- & $\operatorname{DFREQ}(6,6,14)$ \\
\hline $0.000 \mathrm{E}+00$ & $0.000 \mathrm{E}+00$ & --- & $\operatorname{DFREQ}(1,1,15)$ \\
\hline $0.000 \mathrm{E}+00$ & $0.000 \mathrm{E}+00$ & --- & $\operatorname{DFREQ}(1,2,15)$ \\
\hline $0.000 \mathrm{E}+00$ & $0.000 \mathrm{E}+00$ & --- & $\operatorname{DFREQ}(1,3,15)$ \\
\hline $0.000 \mathrm{E}+00$ & $1.000 \mathrm{E}-01$ & --- & $\operatorname{DFREQ}(1,4,15)$ \\
\hline $0.000 \mathrm{E}+00$ & $2.000 \mathrm{E}-01$ & --- & $\operatorname{DFREQ}(1,5,15)$ \\
\hline $0.000 \mathrm{E}+00$ & $7.000 \mathrm{E}-01$ & --- & $\operatorname{DFREQ}(1,6,15)$ \\
\hline $0.000 \mathrm{E}+00$ & $0.000 \mathrm{E}+00$ & --- & $\operatorname{DFREQ}(2,1,15)$ \\
\hline $0.000 \mathrm{E}+00$ & $0.000 \mathrm{E}+00$ & --- & $\operatorname{DFREQ}(2,2,15)$ \\
\hline $0.000 \mathrm{E}+00$ & $0.000 \mathrm{E}+00$ & --- & $\operatorname{DEREQ}(2,3,15)$ \\
\hline $0.000 \mathrm{E}+00$ & $0.000 \mathrm{E}+00$ & --- & $\operatorname{DEREQ}(2,4,15)$ \\
\hline $0.000 \mathrm{E}+00$ & $0.000 \mathrm{E}+00$ & --- & DFREQ $(2,5,15)$ \\
\hline $0.000 \mathrm{E}+00$ & $0.000 \mathrm{E}+00$ & --- & $\operatorname{DFREQ}(2,6,15)$ \\
\hline $0.000 \mathrm{E}+00$ & $0.000 \mathrm{E}+00$ & --- & $\operatorname{DFREQ}(3,1,15)$ \\
\hline $0.000 \mathrm{E}+00$ & $0.000 \mathrm{E}+00$ & --- & $\operatorname{DFREQ}(3,2,15)$ \\
\hline $0.000 \mathrm{E}+00$ & $0.000 \mathrm{E}+00$ & --- & $\operatorname{DFREQ}(3,3,15)$ \\
\hline $0.000 \mathrm{E}+00$ & $0.000 \mathrm{E}+00$ & --- & $\operatorname{DFREQ}(3,4,15)$ \\
\hline $0.000 \mathrm{E}+00$ & $0.000 \mathrm{E}+00$ & --- & $\operatorname{DFREQ}(3,5,15)$ \\
\hline $0.000 \mathrm{E}+00$ & $0.000 \mathrm{E}+00$ & --- & $\operatorname{DFREQ}(3,6,15)$ \\
\hline
\end{tabular}

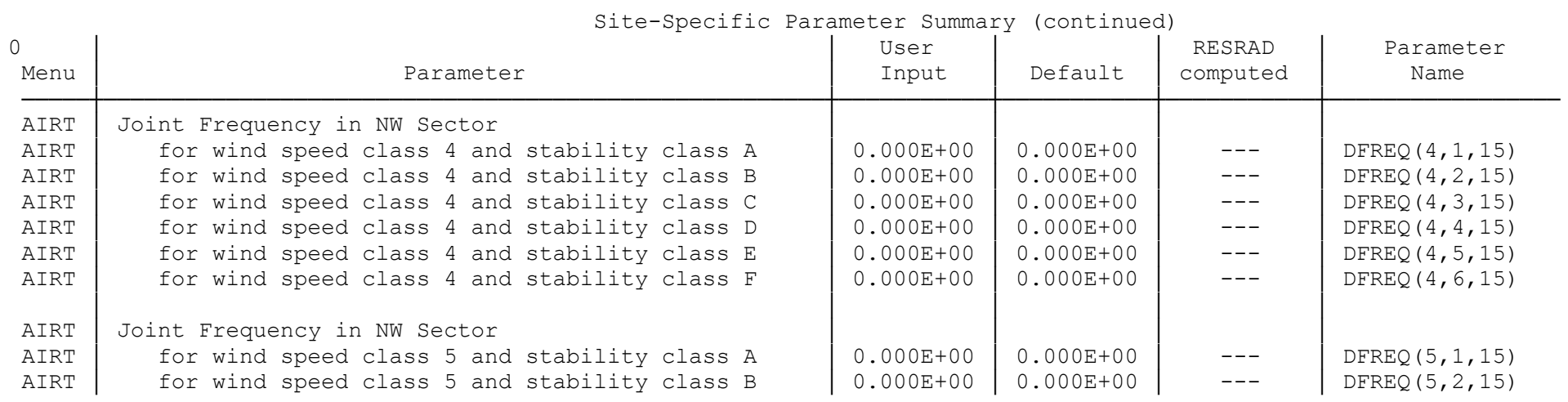




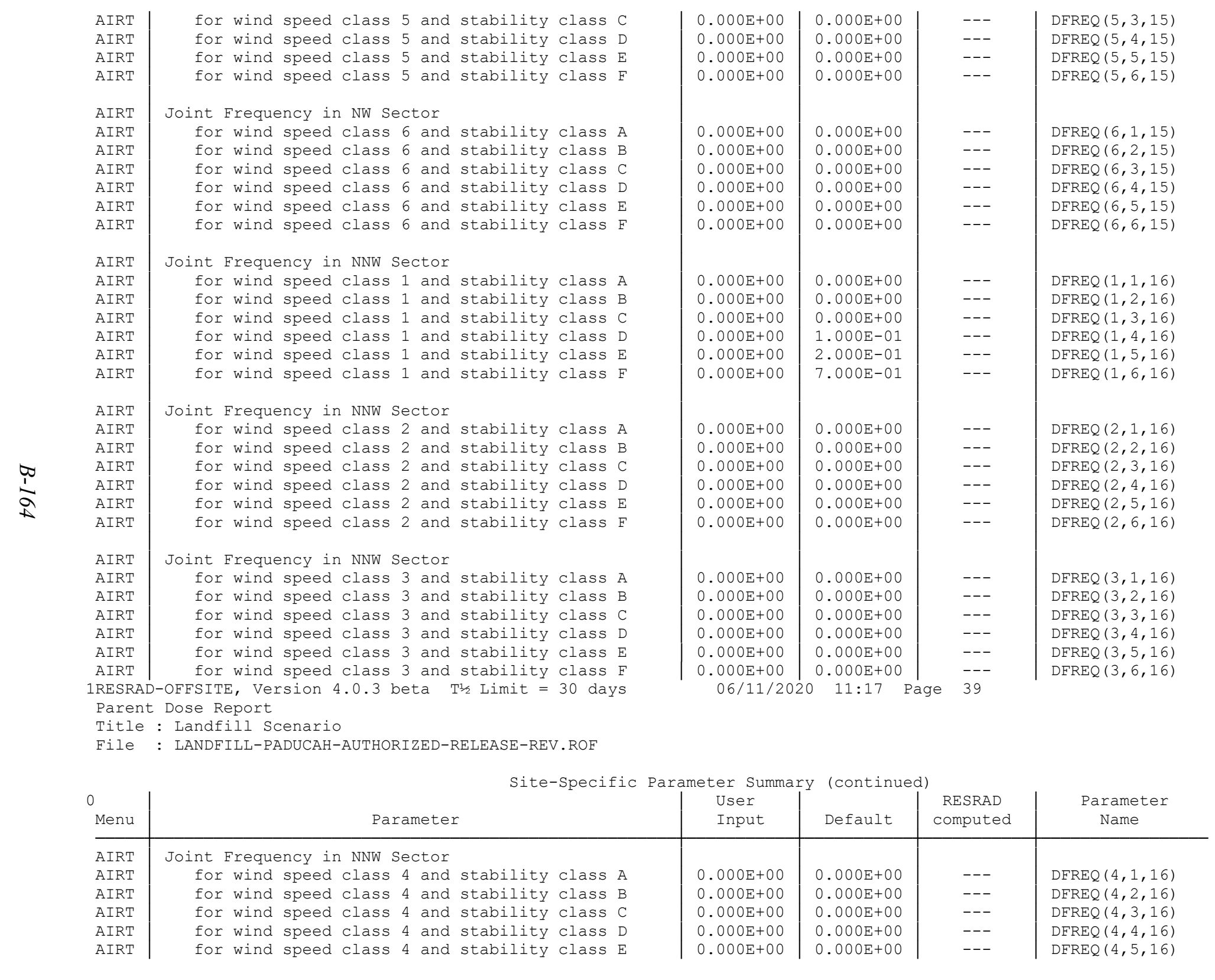




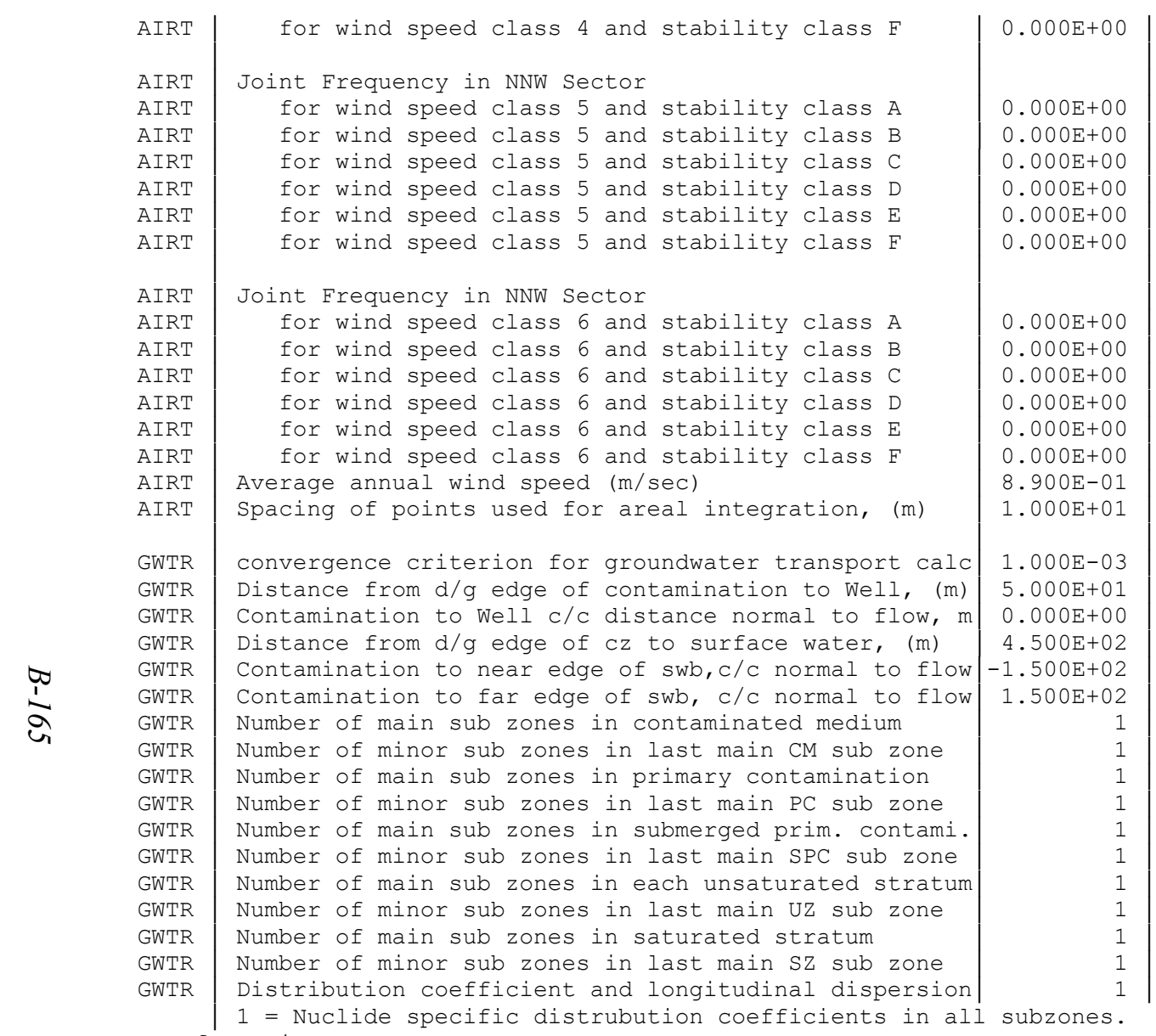

\begin{tabular}{|c|c|c|}
\hline $0.000 \mathrm{E}+00$ & --- & $\operatorname{DFREQ}(4,6,16)$ \\
\hline $0.000 \mathrm{E}+00$ & --- & $\operatorname{DFREQ}(5,1,16)$ \\
\hline $0.000 \mathrm{E}+00$ & --- & $\operatorname{DFREQ}(5,2,16)$ \\
\hline $0.000 \mathrm{E}+00$ & --- & $\operatorname{DFREQ}(5,3,16)$ \\
\hline $0.000 \mathrm{E}+00$ & --- & $\operatorname{DFREQ}(5,4,16)$ \\
\hline $0.000 \mathrm{E}+00$ & --- & $\operatorname{DFREQ}(5,5,16)$ \\
\hline $0.000 \mathrm{E}+00$ & --- & DFREQ $(5,6,16)$ \\
\hline $0.000 \mathrm{E}+00$ & --- & $\operatorname{DFREQ}(6,1,16)$ \\
\hline $0.000 \mathrm{E}+00$ & --- & $\operatorname{DFREQ}(6,2,16)$ \\
\hline $0.000 \mathrm{E}+00$ & --- & $\operatorname{DFREQ}(6,3,16)$ \\
\hline $0.000 \mathrm{E}+00$ & --- & $\operatorname{DFREQ}(6,4,16)$ \\
\hline $0.000 \mathrm{E}+00$ & --- & $\operatorname{DFREQ}(6,5,16)$ \\
\hline $0.000 \mathrm{E}+00$ & --- & $\operatorname{DFREQ}(6,6,16)$ \\
\hline $8.900 \mathrm{E}-01$ & --- & WIND \\
\hline $1.000 \mathrm{E}+01$ & --- & ATGRID \\
\hline $1.000 \mathrm{E}-03$ & --- & EPS \\
\hline $1.000 \mathrm{E}+02$ & --- & OFFLPAQW \\
\hline $0.000 \mathrm{E}+00$ & --- & OFFLNAQW \\
\hline $4.500 \mathrm{E}+02$ & --- & OFFLPAQS \\
\hline$-1.500 \mathrm{E}+02$ & --- & OFFLNAQSN \\
\hline $1.500 \mathrm{E}+02$ & --- & OFFLNAQSF \\
\hline 1 & --- & NPCM \\
\hline 1 & --- & NPCMF \\
\hline 1 & --- & NPCZ \\
\hline 1 & --- & NPCZF \\
\hline 1 & --- & NSPCZ \\
\hline 1 & --- & NSPCZF \\
\hline 1 & --- & NPSS \\
\hline 1 & --- & NPSSF \\
\hline 1 & --- & NAQS \\
\hline 1 & --- & NAQSF \\
\hline 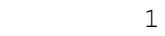 & --- & \\
\hline
\end{tabular}

transformation.

GWTR | Retardation factor flag for groundwater transport |

Longitudinal dispersion in all but the subzone of

$0=$ (total porosity + distribution coefficient*dry

\begin{tabular}{c|c|}
0 & 0 \\
ty) / total porosity & --
\end{tabular}

IRESRAD-OFFSITE, Version 4.0 .3 beta T1/2 Limit $=30$ days $\quad 06 / 111 / 2020 \quad 11: 17$ Page 40

Parent Dose Report

Title : Landfill Scenario

File : LANDFILL-PADUCAH-AUTHORIZED-RELEASE-REV.ROF

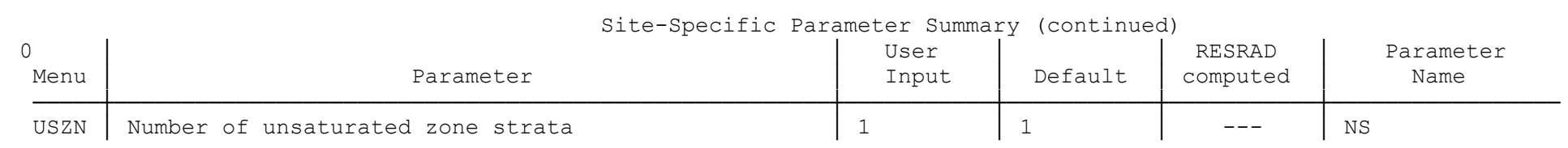


USZN | Unsat. zone 1, thickness (m)

USZN Unsat. zone 1 , soil density $(\mathrm{g} / \mathrm{cm} * * 3)$

USZN Unsat. zone 1, total porosity

USZN Unsat. zone 1, effective porosity

USZN Unsat. zone 1, field capacity

USZN Unsat. zone 1, hydraulic conductivity (m/yr)

USZN Unsat. zone 1, soil-specific b parameter

USZN Unsat. zone 1, longitudinal dispersivity (m)

SZNE Well pump intake depth (m below water table)

Depth of aquifer contributing to surface water body Thickness of saturated zone $(\mathrm{m})$

\begin{tabular}{l|l} 
SZNE & Thickness of saturated zone $(\mathrm{m})$ \\
SZNE & Density of saturated zone $(\mathrm{g} / \mathrm{cm} * * 3)$
\end{tabular}

\begin{tabular}{l|l} 
SZNE & Density of saturated zone $(\mathrm{g} / \mathrm{cm}$ \\
SZNE & Saturated zone total porosity
\end{tabular}

SZNE Saturated zone effective porosity

SZNE Saturated zone hydraulic conductivity (m/yr)

SZNE Saturated zone hydraulic gradient to well

SZNE Satur. zone hydraulic gradient to surface water body

SZNE longitudinal dispersivity to well (m)

SZNE longitudinal dispersivity to $\operatorname{SWB}(\mathrm{m})$

SZNE lateral (horizontal) dispersivity to well (m)

SZNE lateral (horizontal) dispersivity to SWB (m)

SZNE lateral (vertical) dispersivity to well (m)

SZNE lateral (vertical) dispersivity to SWB (m)

$\rightarrow \quad$ SZNE Irrigation rate over aquifer to well (m/yr)

SZNE Evapotranspiration coefficient over aquifer to well

SZNE Evapotranspiration coefficient over aquifer to SWB

SZNE Runoff coefficient over aquifer to well

SZNE Runoff coefficient over aquifer to SWB

SZNE Concentration of mobile colloids in the aquifer

SZNE Water - Soil Distribution coefficient of colloids

SZNE Water - Mobile Colloids Distribution coefficient

WTRU Drinking water intake (L/yr)

WTRU Fraction of drinking water from surface water

WTRU Fraction of drinking water from well water

WTRU Fraction of household water from surface water

WTRU Fraction of household water from well water

WTRU Livestock water intake for meat 1 (L/day)

WTRU Fraction of livestock water 1 from surface water

WTRU Fraction of livestock water 1 from well water

WTRU Livestock water intake for milk (L/day)

WTRU Fraction of dairy cow water from surface water

WTRU Fraction of dairy cow water from well water

WTRU Irrigation rate in Agricultural Area 1 (m/yr)

1RESRAD-OFFSITE, Version 4.0 .3 beta $\mathrm{T}^{1 / 2}$ Limit $=30$ days

Parent Dose Report

Title : Landfill Scenario

File : LANDFILL-PADUCAH-AUTHORIZED-RELEASE-REV.ROF

\begin{tabular}{|c|c|c|c|}
\hline $4.000 \mathrm{E}+00$ & $4.000 \mathrm{E}+00$ & --- & $\mathrm{H}(1)$ \\
\hline $1.500 \mathrm{E}+00$ & $1.500 \mathrm{E}+00$ & --- & DENSUZ (1) \\
\hline $4.000 \mathrm{E}-01$ & $4.000 \mathrm{E}-01$ & --- & TPUZ (1) \\
\hline $2.000 \mathrm{E}-01$ & $2.000 \mathrm{E}-01$ & --- & EPUZ (1) \\
\hline $3.000 \mathrm{E}-01$ & $3.000 \mathrm{E}-01$ & --- & FCUZ (1) \\
\hline $1.000 \mathrm{E}+01$ & $1.000 \mathrm{E}+01$ & --- & $\operatorname{HCUZ}(1)$ \\
\hline $5.300 \mathrm{E}+00$ & $5.300 \mathrm{E}+00$ & --- & BUZ (1) \\
\hline $1.000 \mathrm{E}-01$ & $1.000 \mathrm{E}-01$ & --- & ALPHALU (1) \\
\hline $1.000 \mathrm{E}+01$ & $1.000 \mathrm{E}+01$ & --- & DWIBWT \\
\hline $5.000 \mathrm{E}+00$ & $5.000 \mathrm{E}+00$ & --- & DPTHAQSW \\
\hline $1.000 \mathrm{E}+02$ & $1.000 \mathrm{E}+02$ & --- & DPTHAQ \\
\hline $1.500 \mathrm{E}+00$ & $1.500 \mathrm{E}+00$ & --- & DENSAQ \\
\hline $4.000 \mathrm{E}-01$ & $4.000 \mathrm{E}-01$ & --- & TPSZ \\
\hline $2.000 \mathrm{E}-01$ & $2.000 \mathrm{E}-01$ & --- & EPSZ \\
\hline $1.000 \mathrm{E}+02$ & $1.000 \mathrm{E}+02$ & --- & HCSZ \\
\hline $2.000 \mathrm{E}-02$ & $2.000 \mathrm{E}-02$ & --- & HGW \\
\hline $2.000 \mathrm{E}-02$ & $2.000 \mathrm{E}-02$ & --- & HGSW \\
\hline $3.000 \mathrm{E}+00$ & $3.000 \mathrm{E}+00$ & --- & ALPHALOW \\
\hline $1.000 \mathrm{E}+01$ & $1.000 \mathrm{E}+01$ & --- & ALPHALOSW \\
\hline $4.000 \mathrm{E}-01$ & $4.000 \mathrm{E}-01$ & --- & ALPHATW \\
\hline $1.000 \mathrm{E}+00$ & $1.000 \mathrm{E}+00$ & --- & ALPHATSW \\
\hline $2.000 \mathrm{E}-02$ & $2.000 \mathrm{E}-02$ & --- & ALPHAVW \\
\hline $6.000 \mathrm{E}-02$ & $6.000 \mathrm{E}-02$ & --- & ALPHAVSW \\
\hline not used & $0.000 \mathrm{E}+00$ & --- & RIAQW \\
\hline not used & $0.000 \mathrm{E}+00$ & --- & RIAQSW \\
\hline not used & $1.000 \mathrm{E}+00$ & --- & EVAPTRAQW \\
\hline not used & $1.000 \mathrm{E}+00$ & --- & EVAPTRAQSW \\
\hline not used & $1.000 \mathrm{E}+00$ & --- & RUNOFFAQW \\
\hline not used & $1.000 \mathrm{E}+00$ & --- & RUNOFFAQSW \\
\hline $0.000 \mathrm{E}+00$ & $0.000 \mathrm{E}+00$ & --- & $\mathrm{CCOL}$ \\
\hline $0.000 \mathrm{E}+00$ & $0.000 \mathrm{E}+00$ & --- & K1Col \\
\hline $0.000 \mathrm{E}+00$ & $0.000 \mathrm{E}+00$ & --- & $\mathrm{K} 3 \mathrm{Col}$ \\
\hline $5.100 \mathrm{E}+02$ & $5.100 \mathrm{E}+02$ & --- & DWI \\
\hline $0.000 \mathrm{E}+00$ & $0.000 \mathrm{E}+00$ & --- & FSWD \\
\hline $1.000 \mathrm{E}+00$ & $1.000 \mathrm{E}+00$ & --- & FWWD \\
\hline $0.000 \mathrm{E}+00$ & $0.000 \mathrm{E}+00$ & --- & FSWHH \\
\hline $1.000 \mathrm{E}+00$ & $1.000 \mathrm{E}+00$ & --- & FWWHH \\
\hline $5.000 \mathrm{E}+01$ & $5.000 \mathrm{E}+01$ & --- & LWI (1) \\
\hline $0.000 \mathrm{E}+00$ & $0.000 \mathrm{E}+00$ & --- & FSWLV (1) \\
\hline $1.000 \mathrm{E}+00$ & $1.000 \mathrm{E}+00$ & --- & FWWLV (1) \\
\hline $1.600 \mathrm{E}+02$ & $1.600 \mathrm{E}+02$ & --- & LWI (2) \\
\hline $0.000 \mathrm{E}+00$ & $0.000 \mathrm{E}+00$ & --- & FSWLV (2) \\
\hline $1.000 \mathrm{E}+00$ & $1.000 \mathrm{E}+00$ & --- & FWWLV (2) \\
\hline $2.000 \mathrm{E}-01$ & $2.000 \mathrm{E}-01$ & --- & RIRRIG (1) \\
\hline
\end{tabular}




\begin{tabular}{|c|c|c|c|c|c|}
\hline $\begin{array}{l}0 \\
\text { Menu }\end{array}$ & Site-Specific Par & $\begin{array}{l}\text { ameter Sumn } \\
\begin{array}{|c}\text { User } \\
\text { Input }\end{array}\end{array}$ & $\left.\right|_{\text {Default }} ^{\text {ry (continue }}$ & $\begin{array}{l}\text { RESRAD } \\
\text { computed }\end{array}$ & $\begin{array}{c}\text { Parameter } \\
\text { Name }\end{array}$ \\
\hline WTRU & Fraction of irrigation water 1 from surface water & $0.000 \mathrm{E}+00$ & $0.000 \mathrm{E}+00$ & --- & $\operatorname{FSWIR}(1)$ \\
\hline WTRU & Fraction of irrigation water 1 from well water & $1.000 \mathrm{E}+00$ & $1.000 \mathrm{E}+00$ & --- & FWWIR (1) \\
\hline WTRU & Irrigation rate in Agricultural Area 2 (m/yr) & $2.000 \mathrm{E}-01$ & $2.000 \mathrm{E}-01$ & --- & RIRRIG (2) \\
\hline WTRU & Fraction of irrigation water 2 from surface water & $0.000 \mathrm{E}+00$ & $0.000 \mathrm{E}+00$ & --- & FSWIR (2) \\
\hline WTRU & Fraction of irrigation water 2 from well water & $1.000 \mathrm{E}+00$ & $1.000 \mathrm{E}+00$ & --- & FWWIR (2) \\
\hline WTRU & Irrigation rate in Agricultural Area 3 (m/yr) & $2.000 \mathrm{E}-01$ & $2.000 \mathrm{E}-01$ & --- & RIRRIG (3) \\
\hline WTRU & Fraction of irrigation water 3 from surface water & $0.000 \mathrm{E}+00$ & $0.000 \mathrm{E}+00$ & --- & FSWIR (3) \\
\hline WTRU & Fraction of irrigation water 3 from well water & $1.000 \mathrm{E}+00$ & $1.000 \mathrm{E}+00$ & --- & FWWIR (3) \\
\hline WTRU & Irrigation rate in Agricultural Area 4 (m/yr) & $2.000 \mathrm{E}-01$ & $2.000 \mathrm{E}-01$ & --- & RIRRIG (4) \\
\hline WTRU & Fraction of irrigation water 4 from surface water & $0.000 \mathrm{E}+00$ & $0.000 \mathrm{E}+00$ & --- & FSWIR (4) \\
\hline WTRU & Fraction of irrigation water 4 from well water & $1.000 \mathrm{E}+00$ & $1.000 \mathrm{E}+00$ & --- & FWWIR (4) \\
\hline WTRU & Irrigation rate in offsite dwelling site (m/yr) & $2.000 \mathrm{E}-01$ & $2.000 \mathrm{E}-01$ & --- & RIRRIGDWELL \\
\hline WTRU & Fraction of irrigation water from surface water & $0.000 \mathrm{E}+00$ & $0.000 \mathrm{E}+00$ & --- & FSWIRDWELL \\
\hline WTRU & Fraction of irrigation water from well water & $1.000 \mathrm{E}+00$ & $1.000 \mathrm{E}+00$ & --- & FWWIRDWELL \\
\hline WTRU & Well pumping rate $(\mathrm{m} * * 3 / \mathrm{yr})$ & $5.100 \mathrm{E}+03$ & $5.100 \mathrm{E}+03$ & --- & UW \\
\hline SWBY & Surface area of water in surface water body, $m^{* * 2}$ & $9.000 \mathrm{E}+04$ & $9.000 \mathrm{E}+04$ & --- & ALAKE \\
\hline SWBY & Volume of surface water body, $m * * 3$ & $1.500 \mathrm{E}+05$ & $1.500 \mathrm{E}+05$ & --- & VLAKE \\
\hline SWBY & Potential evaporation, m/y & $1.000 \mathrm{E}+00$ & $1.000 \mathrm{E}+00$ & --- & EVAPOT \\
\hline SWBY & Stream outflow as a fraction of seepage+stm outflows & $9.983 \mathrm{E}-01$ & $9.983 \mathrm{E}-01$ & --- & FSTMFLOW \\
\hline SWBY & Use inflow ratio for outflow ratio, 1 yes, 0 no & 1 & 1 & --- & FSTMFLOWIN \\
\hline SWBY & Settling velocity of suspended sediments, $\mathrm{cm} / \mathrm{s}$ & $1.000 \mathrm{E}-01$ & $1.000 \mathrm{E}-01$ & --- & Vsettle \\
\hline SWBY & Dry bulk density of bottom sediments, $\mathrm{g} / \mathrm{cm} * * 3$ & $1.500 \mathrm{E}+00$ & $1.500 \mathrm{E}+00$ & --- & Rhobsed \\
\hline SWBY & Thickness of bottom sediment absorbing nuclides, $m$ & $5.000 \mathrm{E}-02$ & $5.000 \mathrm{E}-02$ & --- & ThickSed \\
\hline SWBY & Number of distinct catchments & 1 & 1 & --- & $\mathrm{NCATCH}$ \\
\hline SWBY & Catchment 1 , smaller $\mathrm{X}$ coordinate $(\mathrm{m})$ & $-1.450 \mathrm{E}+03$ & $-1.450 \mathrm{E}+03$ & --- & $\operatorname{CATCHXY~}(1,1)$ \\
\hline SWBY & Catchment 1 , larger X coordinate $(\mathrm{m})$ & $1.550 \mathrm{E}+03$ & $1.550 \mathrm{E}+03$ & --- & САTCHXY $(2,1)$ \\
\hline SWBY & Catchment 1 , smaller $\mathrm{Y}$ coordinate $(\mathrm{m})$ & $-2.450 \mathrm{E}+03$ & $-2.450 \mathrm{E}+03$ & --- & $\operatorname{CATCHXY~}(3,1)$ \\
\hline SWBY & Catchment 1, larger Y coordinate (m) & $5.500 \mathrm{E}+02$ & $5.500 \mathrm{E}+02$ & --- & CATCHXY $(4,1)$ \\
\hline SWBY & Catchment 1 , area, $m^{* * 2}$ & $9.000 \mathrm{E}+06$ & $9.000 \mathrm{E}+06$ & --- & AREACA (1) \\
\hline SWBY & Catchment 1, runoff coefficient & $2.000 \mathrm{E}-01$ & $2.000 \mathrm{E}-01$ & --- & RUNOFFCA (1) \\
\hline SWBY & Catchment 1, soil erodibility factor, tons/acre & $4.000 \mathrm{E}-01$ & $4.000 \mathrm{E}-01$ & --- & ERODIBILITYCA (1) \\
\hline SWBY & Catchment 1, slope-length-steepness factor & $4.000 \mathrm{E}-01$ & $4.000 \mathrm{E}-01$ & --- & SLPLENSTPCA (1) \\
\hline SWBY & Catchment 1 , Cover and management factor & $3.000 \mathrm{E}-03$ & $3.000 \mathrm{E}-03$ & --- & CRPMANGCA (1) \\
\hline SWBY & Catchment 1, support practice factor & $1.000 \mathrm{E}+00$ & $1.000 \mathrm{E}+00$ & --- & CONVPRACCA (1) \\
\hline SWBY & Catchment 1 , sediment delivery ratio & $2.121 \mathrm{E}-01$ & $2.121 \mathrm{E}-01$ & --- & $\operatorname{SDRCA}(1)$ \\
\hline SWBY & Catchment 1, use SRD - Area correlation, 1 yes, 0 no & 1 & 1 & --- & SDRACOR \\
\hline SWBY & Catchment 1 , deposited radionuclide delivery ratio & $2.000 \mathrm{E}-02$ & $2.000 \mathrm{E}-02$ & --- & $\operatorname{DDRCA}(1)$ \\
\hline SWBY & Compute atmospheric deposition on catchment & yes & no & --- & ComputeDep \\
\hline SWBY & Convergence criterion for deposition calculations & $1.000 \mathrm{E}-03$ & $1.000 \mathrm{E}-03$ & --- & ConvCritatm \\
\hline INGE & Fish consumption (kg/yr) & $5.400 \mathrm{E}+00$ & $5.400 \mathrm{E}+00$ & --- & DFI (1) \\
\hline INGE & Fraction of Fish from affected area & $5.000 \mathrm{E}-01$ & $5.000 \mathrm{E}-01$ & --- & FFISH (1) \\
\hline INGE & Other Aquatic food consumption ( $\mathrm{kg} / \mathrm{yr}$ ) & $9.000 \mathrm{E}-01$ & $9.000 \mathrm{E}-01$ & --- & $\operatorname{DFI}(2)$ \\
\hline INGE & Fraction of Aquatic food from affected area & $5.000 \mathrm{E}-01$ & $5.000 \mathrm{E}-01$ & --- & FFISH (2) \\
\hline INGE & Non-Leafy vegetables consumption ( $\mathrm{kg} / \mathrm{yr}$ ) & $1.600 \mathrm{E}+02$ & $1.600 \mathrm{E}+02$ & --- & DVI (1) \\
\hline INGE & Fraction of vegetable 1 from affected area & $5.000 \mathrm{E}-01$ & $5.000 \mathrm{E}-01$ & --- & FVEG (1) \\
\hline
\end{tabular}


Parent Dose Report

Title : Landfill Scenario

File : LANDFILL-PADUCAH-AUTHORIZED-RELEASE-REV.ROF

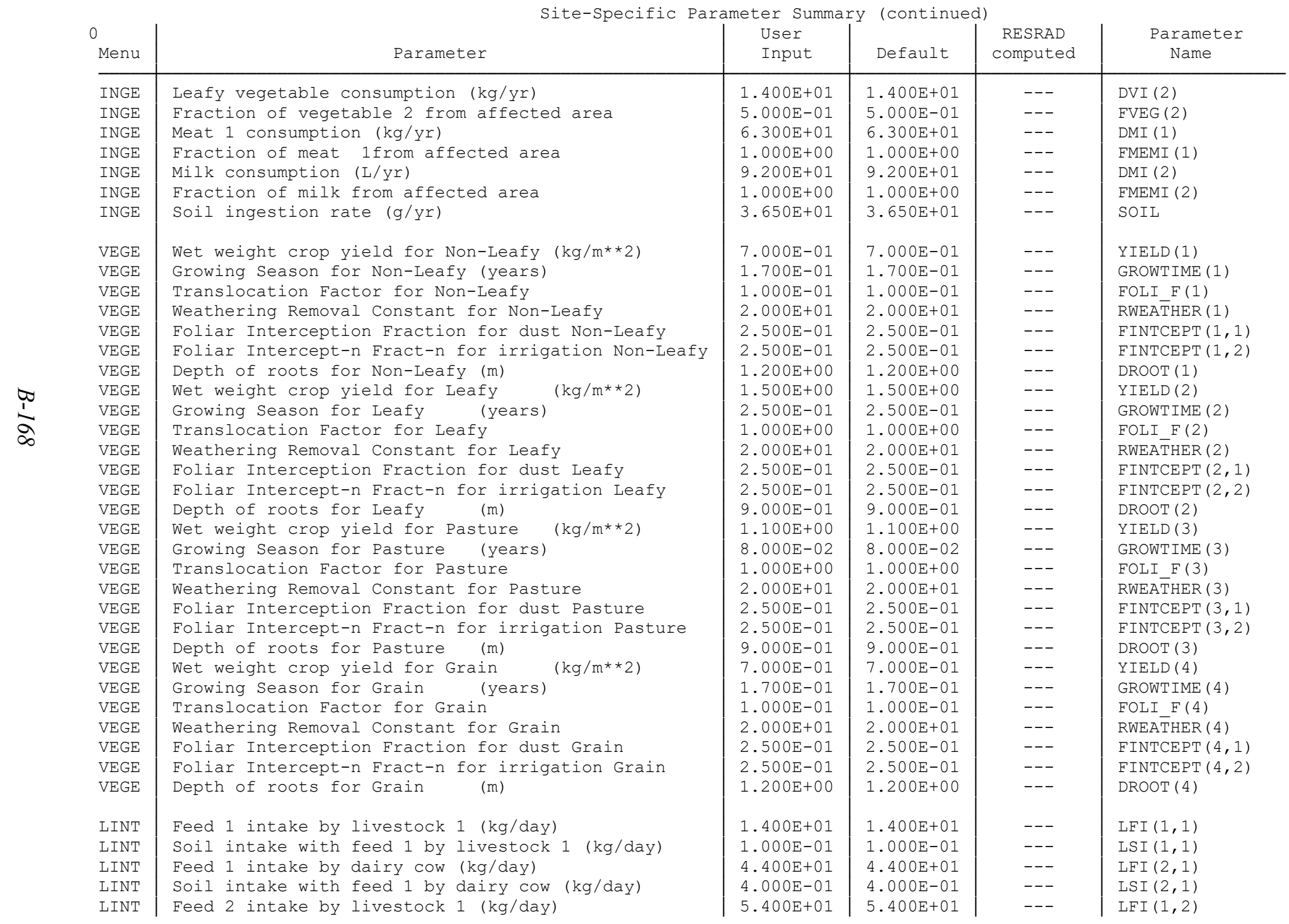


LINT Soil intake with feed 2 by livestock 1 ( $\mathrm{kg} /$ day)

LINT Feed 2 intake by dairy cow (kg/day)

LINT Soil intake with feed 2 by dairy cow (kg/day)

IRESRAD-OFFSITE, Version 4.0 .3 beta $T^{1 / 2}$ Limit $=30$ days

Parent Dose Report

Title : Landfill Scenario

File : LANDFILL-PADUCAH-AUTHORIZED-RELEASE-REV.ROF

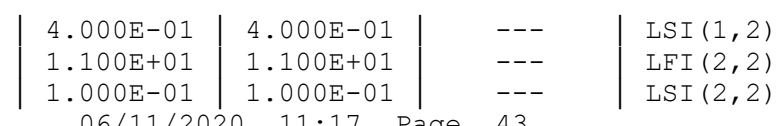

$06 / 11 / 2020 \cdot 11: 17$ Page 43

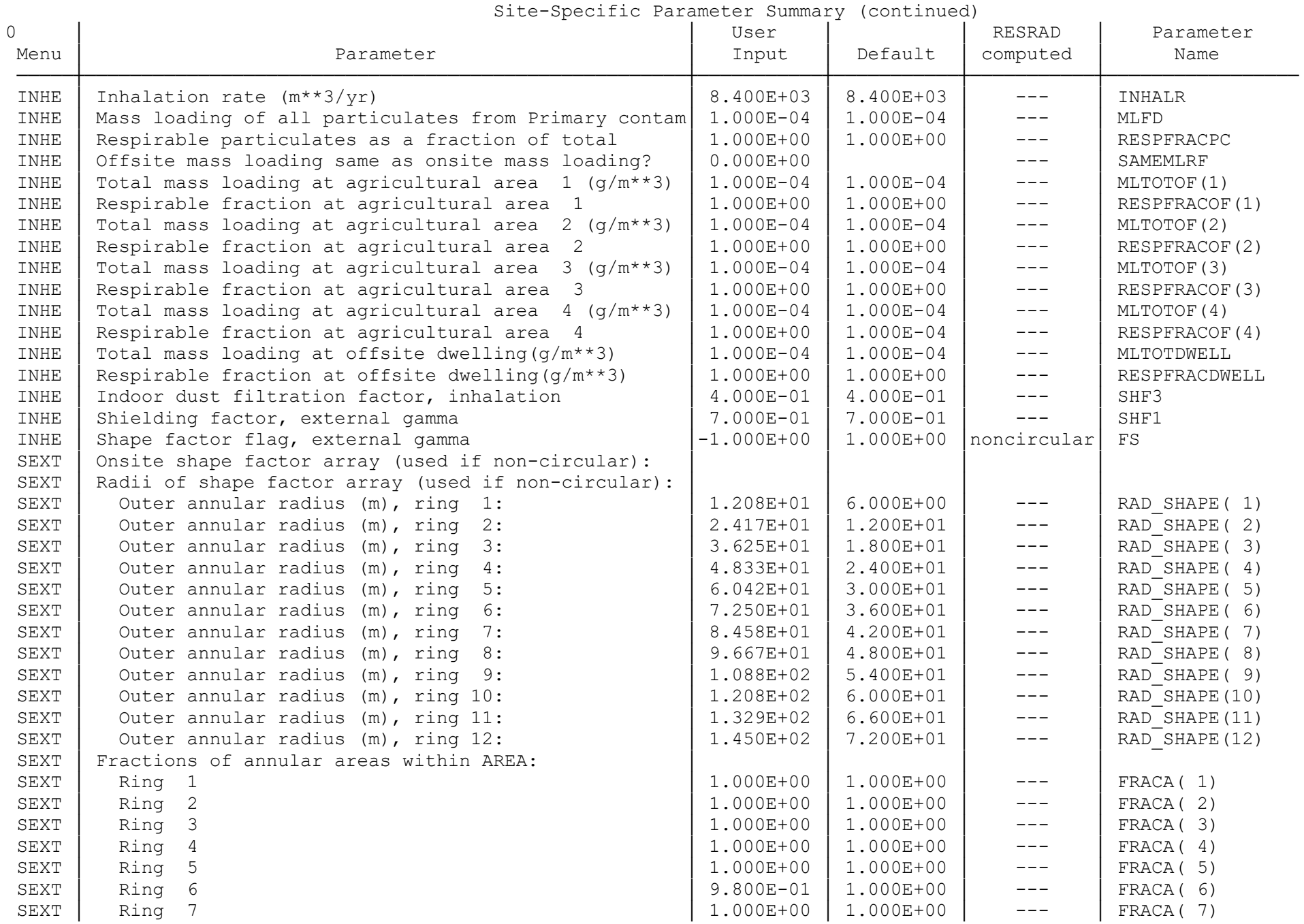




\begin{tabular}{l|lr} 
SEXT & Ring & 8 \\
SEXT & Ring & 9 \\
SEXT & Ring & 10 \\
SEXT & Ring & 11 \\
SEXT & Ring & 12
\end{tabular}

1RESRAD-OFFSITE, Version 4.0 .3 beta $\mathrm{T}^{\frac{1}{2}}$ Limit $=30$ days

Parent Dose Report

Title : Landfill Scenario

File : LANDFILL-PADUCAH-AUTHORIZED-RELEASE-REV.ROF

\begin{tabular}{|l|l|l|l}
$1.000 \mathrm{E}+00$ & $1.000 \mathrm{E}+00$ & --- & FRACA $(8)$ \\
$7.800 \mathrm{E}-01$ & $7.700 \mathrm{E}-01$ & -- & FRACA $(9)$ \\
$3.600 \mathrm{E}-01$ & $3.700 \mathrm{E}-01$ & -- & FRACA (10) \\
$1.600 \mathrm{E}-01$ & $1.700 \mathrm{E}-01$ & -- & FRACA $(11)$ \\
$3.400 \mathrm{E}-02$ & $3.100 \mathrm{E}-02$ & -- & FRACA (12)
\end{tabular}

FRACA (11)

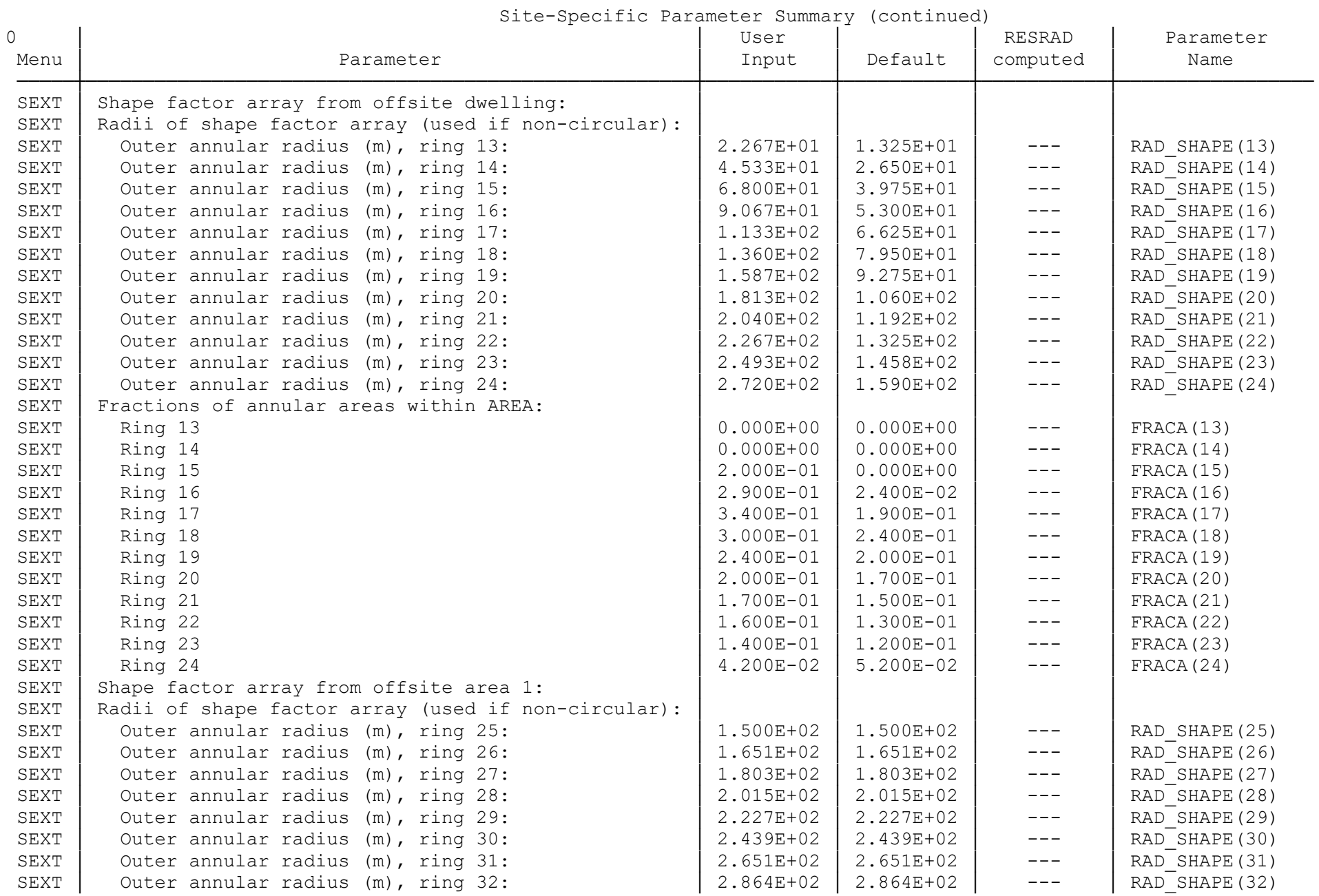


SEXT Outer annular radius (m), ring 33:

SEXT Outer annular radius (m), ring 34:

SEXT Outer annular radius (m), ring 35:

SEXT Outer annular radius (m), ring 36:

RESRAD-OFFSITE, Version 4.0 .3 beta $\mathrm{T}^{1 / 2}$ Limit $=30$ days

Parent Dose Report

Title : Landfill Scenario

File : LANDFILL-PADUCAH-AUTHORIZED-RELEASE-REV.ROF

\begin{tabular}{|l|l|l|l}
$3.076 \mathrm{E}+02$ & $3.076 \mathrm{E}+02$ & --- & RAD_SHAPE (33) \\
$3.288 \mathrm{E}+02$ & $3.288 \mathrm{E}+02$ & --- & RAD_SHAPE (34) \\
$3.500 \mathrm{E}+02$ & $3.500 \mathrm{E}+02$ & --- & RAD_SHAPE (35) \\
$3.640 \mathrm{E}+02$ & $3.640 \mathrm{E}+02$ & --- & RAD_SHAPE (36) \\
0
\end{tabular}

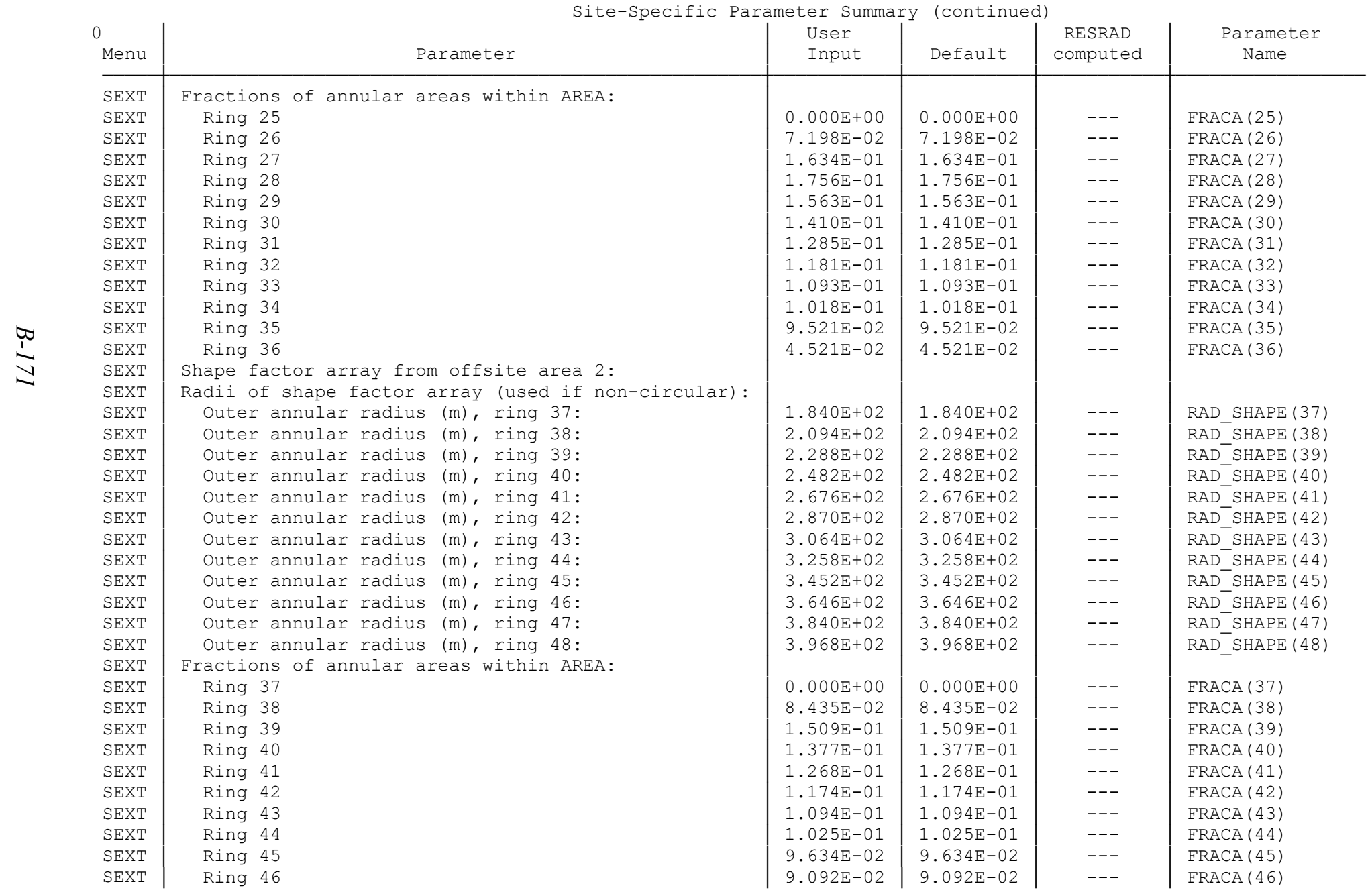


\begin{tabular}{l|l} 
SEXT & Ring 47
\end{tabular}

SEXT Ring 48

1RESRAD-OFFSITE, Version 4.0 .3 beta $\mathrm{T}^{1 / 2}$ Limit $=30$ days

Parent Dose Report

Title : Landfill Scenario

File : LANDFILL-PADUCAH-AUTHORIZED-RELEASE-REV.ROF

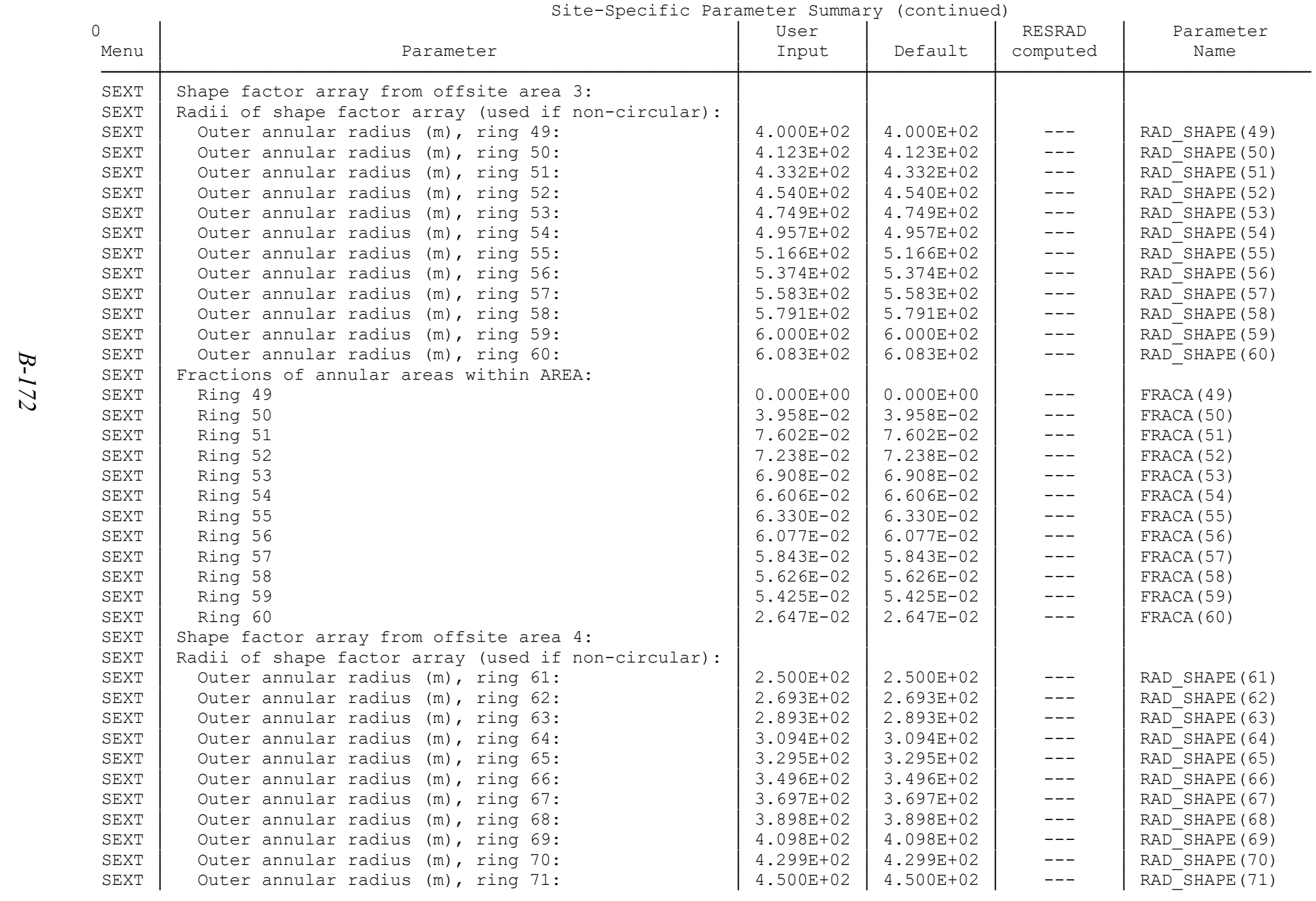

\begin{tabular}{|l|l|l|l}
$8.609 \mathrm{E}-02$ & $8.609 \mathrm{E}-02$ & --- & FRACA (47) \\
$4.124 \mathrm{E}-02$ & $4.124 \mathrm{E}-02$ & --- & FRACA (48)
\end{tabular}

$06 / 11 / 2020 \quad 11: 17$ Page 46 
SEXT Outer annular radius (m), ring 72 :

RESRAD-OFFSITE, Version 4.0 .3 beta T1/2 Limit $=30$ days

Parent Dose Report

Title : Landfill Scenario

File : LANDFILL-PADUCAH-AUTHORIZED-RELEASE-REV.ROF

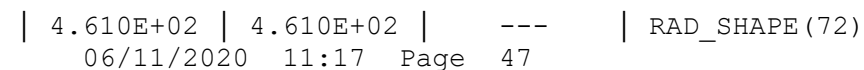

Site-Specific Parameter Summary (continued)

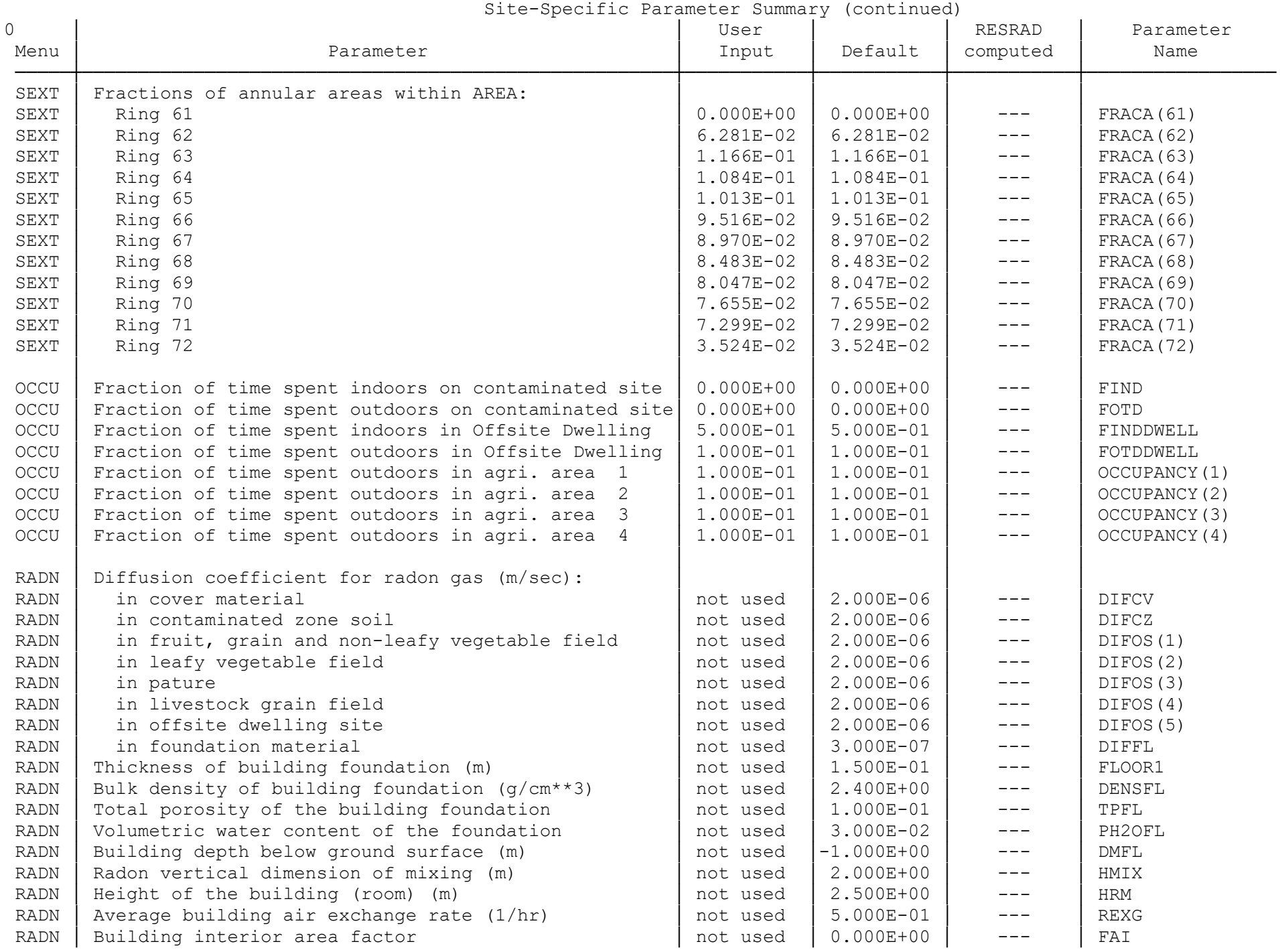


RADN $\mid$ Emanating power of $\mathrm{Rn}-222$ gas

RADN Emanating power of Rn-220 gas

C14 C-14 evasion layer thickness in soil (m)

C14 Vertical dimension of mixing for vegetation (m)

C14 C-14 evasion flux rate from soil ( $1 / \mathrm{sec}$ )

IRESRAD-OFFSITE, Version 4.0 .3 beta $T^{\frac{1}{2}}$ Limit $=30$ days

Parent Dose Report

Title : Landfill Scenario

File : LANDFILL-PADUCAH-AUTHORIZED-RELEASE-REV.ROF

\begin{tabular}{|l|l|l|l|} 
not used & $2.500 \mathrm{E}-01$ & --- & EMANA (1) \\
not used & $1.500 \mathrm{E}-01$ & -- & EMANA (2) \\
& & & \\
not used & $3.000 \mathrm{E}-01$ & --- & DMC \\
not used & $1.000 \mathrm{E}+00$ & --- & HMIXV \\
not used & $7.000 \mathrm{E}-07$ & -- & C14EVSN \\
&
\end{tabular}

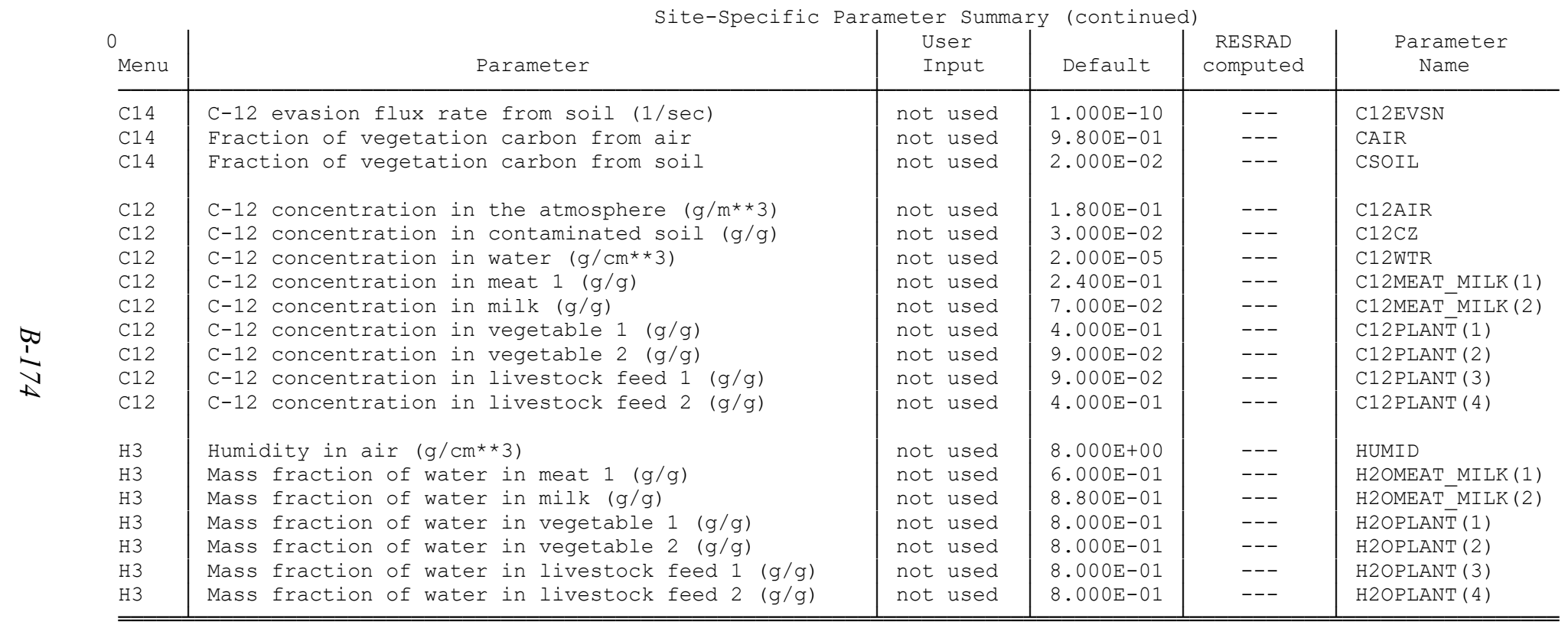

Summary of Pathway Selections

\begin{tabular}{c|c} 
Pathway & User Selection \\
\hline $1--$ external gamma & active \\
$2--$ inhalation (w/o radon) & active \\
$3--$ plant ingestion & active \\
$4--$ meat ingestion & active \\
$5--$ milk ingestion & active \\
$6--$ aquatic foods & active \\
$7--$ drinking water & active
\end{tabular}




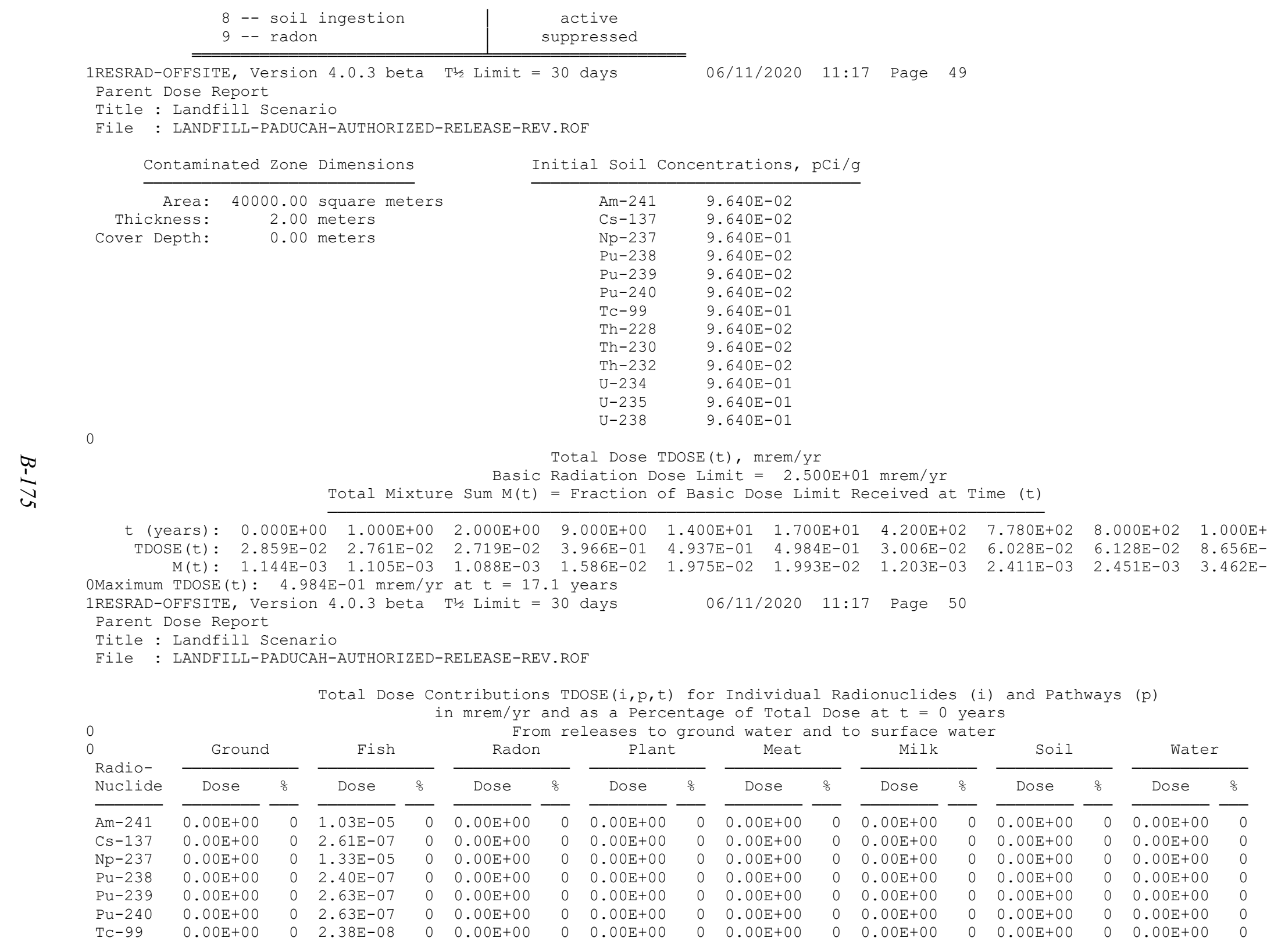




\begin{tabular}{|c|c|c|c|c|c|c|c|c|c|c|c|c|c|c|c|}
\hline Th-228 & $0.00 \mathrm{E}+00$ & 0 & $1.60 \mathrm{E}-08$ & 0 & $0.00 \mathrm{E}+00$ & 0 & $0.00 \mathrm{E}+00$ & 0 & $0.00 \mathrm{E}+00$ & 0 & $0.00 \mathrm{E}+00$ & 0 & $0.00 \mathrm{E}+00$ & 0 & $0.00 \mathrm{E}+00$ \\
\hline Th-230 & $0.00 \mathrm{E}+00$ & 0 & $3.56 \mathrm{E}-08$ & 0 & $0.00 \mathrm{E}+00$ & 0 & $0.00 \mathrm{E}+00$ & 0 & $0.00 \mathrm{E}+00$ & 0 & $0.00 \mathrm{E}+00$ & 0 & $0.00 \mathrm{E}+00$ & 0 & $0.00 \mathrm{E}+00$ \\
\hline Th-232 & $0.00 \mathrm{E}+00$ & 0 & $1.52 \mathrm{E}-06$ & 0 & $0.00 \mathrm{E}+00$ & 0 & $0.00 \mathrm{E}+00$ & 0 & $0.00 \mathrm{E}+00$ & 0 & $0.00 \mathrm{E}+00$ & 0 & $0.00 \mathrm{E}+00$ & 0 & $0.00 \mathrm{E}+00$ \\
\hline $\mathrm{U}-234$ & $0.00 \mathrm{E}+00$ & 0 & $2.36 \mathrm{E}-06$ & 0 & $0.00 \mathrm{E}+00$ & 0 & $0.00 \mathrm{E}+00$ & 0 & $0.00 \mathrm{E}+00$ & 0 & $0.00 \mathrm{E}+00$ & 0 & $0.00 \mathrm{E}+00$ & 0 & $0.00 \mathrm{E}+00$ \\
\hline $\mathrm{U}-235$ & $0.00 \mathrm{E}+00$ & 0 & $2.28 \mathrm{E}-06$ & 0 & $0.00 \mathrm{E}+00$ & 0 & $0.00 \mathrm{E}+00$ & 0 & $0.00 \mathrm{E}+00$ & 0 & $0.00 \mathrm{E}+00$ & 0 & $0.00 \mathrm{E}+00$ & 0 & $0.00 \mathrm{E}+00$ \\
\hline $\mathrm{U}-238$ & $0.00 \mathrm{E}+00$ & 0 & $2.33 E-06$ & 0 & $0.00 \mathrm{E}+00$ & 0 & $0.00 \mathrm{E}+00$ & 0 & $0.00 \mathrm{E}+00$ & 0 & $0.00 \mathrm{E}+00$ & 0 & $0.00 \mathrm{E}+00$ & 0 & $0.00 \mathrm{E}+00$ \\
\hline & 00 & 0 & $3.32 \mathrm{E}-05$ & 0 & $0.00 \mathrm{E}+00$ & 0 & . & 0 & . & 0 & $0.00 \mathrm{E}+00$ & 0 & $0.00 \mathrm{E}+00$ & 0 & $0.00 \mathrm{E}+00$ \\
\hline
\end{tabular}

Total Dose Contributions TDOSE (i,p,t) for Individual Radionuclides (i) and Pathways (p) in mrem/yr and as a Percentage of Total Dose at $t=0$ years

$0 \quad$ Directly from primary contamination and from release to atmosphere (Inhalation excludes radon) Radio- Ground Inhalation Radon Plant

$\frac{\text { Nuclide }}{\text { Am-241 }} \frac{\text { Dose }}{1.59 \mathrm{E}-05} \frac{\circ}{0} \frac{\text { Dose }}{7.09 \mathrm{E}-04} \frac{\%}{2} \frac{\text { Dose }}{0.00 \mathrm{E}+00} \frac{\frac{\circ}{0}}{\frac{0}{1.32 \mathrm{E}-06}}-$

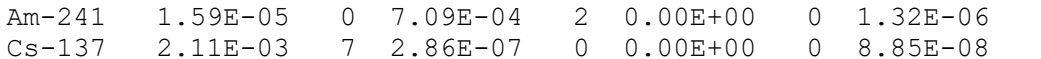

$\mathrm{Np}-237 \quad 6.00 \mathrm{E}-03 \quad 21 \quad 3.71 \mathrm{E}-03 \quad 13 \quad 0.00 \mathrm{E}+00 \quad 0 \quad 7.49 \mathrm{E}-06$

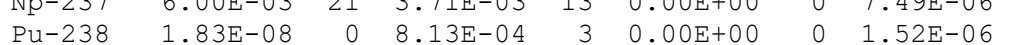

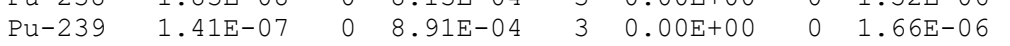

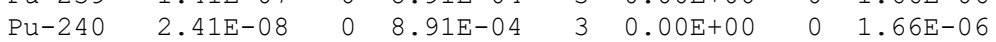

$\begin{array}{llllllll}\mathrm{TC}-99 & 4.05 \mathrm{E}-07 & 0 & 6.79 \mathrm{E}-07 & 0 & 0.00 \mathrm{E}+00 & 0 & 4.87 \mathrm{E}-08\end{array}$

$\begin{array}{rrrrrrrr}T h-228 & 4.62 \mathrm{E}-03 & 16 & 2.72 \mathrm{E}-04 & 1 & 0.00 \mathrm{E}+00 & 0 & 8.01 \mathrm{E}-07 \\ \mathrm{Th}-230 & 1.92 \mathrm{E}-06 & 0 & 7.42 \mathrm{E}-04 & 3 & 0.00 \mathrm{E}+00 & 0 & 1.40 \mathrm{E}-06\end{array}$

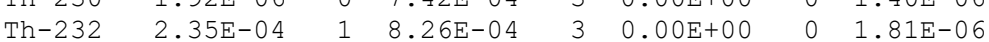

$\mathrm{U}-234 \quad 1.56 \mathrm{E}-06 \quad 0 \quad 6.97 \mathrm{E}-0420.00 \mathrm{E}+00-0 \quad 3.25 \mathrm{E}-06$

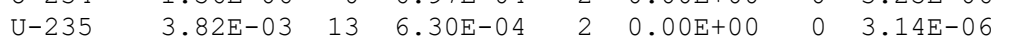

$\mathrm{U}-238$

Total

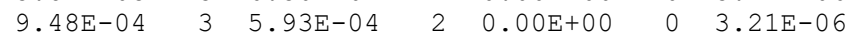

$2.74 \mathrm{E}-05$

Meat

Milk

All Pathways*

1RESRAD-OFFSITE, Version 4.0 .3 beta $\mathrm{T}^{1 / 2} \mathrm{Limit}=30$ days

Parent Dose Report

Title : Landfill Scenario

File : LANDFILL-PADUCAH-AUTHORIZED-RELEASE-REV.ROF

Total Dose Contributions TDOSE (i,p,t) for Individual Radionuclides (i) and Pathways (p) in mrem/yr and as a Percentage of Total Dose at $t=1$ years

\begin{tabular}{|c|c|c|c|c|c|c|c|c|c|c|c|c|c|c|c|c|}
\hline \multirow{2}{*}{$\begin{array}{l}\text { Radio- } \\
\text { Nuclide }\end{array}$} & \multicolumn{2}{|c|}{ Ground } & \multicolumn{2}{|l|}{ Fish } & \multicolumn{2}{|c|}{$\begin{array}{l}\text { From } \\
\text { Radon }\end{array}$} & \multicolumn{2}{|c|}{$\begin{array}{l}\text { ses to } 9 \\
\text { Plant }\end{array}$} & \multicolumn{2}{|c|}{$\begin{array}{l}\text { water and } t \\
\text { Meat }\end{array}$} & \multicolumn{2}{|c|}{$\begin{array}{c}\text { o surface wate } \\
\text { Milk }\end{array}$} & \multicolumn{2}{|l|}{ Soil } & \multicolumn{2}{|c|}{ Water } \\
\hline & Dose & $\%$ & Dose & $\frac{\circ}{0}$ & Dose & $\frac{\circ}{0}$ & Dose & $\%$ & Dose & $\%$ & Dose & $\%$ & Dose & $\%$ & Dose & $\frac{\circ}{0}$ \\
\hline & & 0 & & 0 & & 0 & & 0 & & 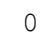 & & & & 0 & & \\
\hline $\mathrm{Cs}-1$ & $.00 \mathrm{E}+00$ & 0 & $50 \mathrm{E}-07$ & 0 & $0 \mathrm{E}+00$ & 0 & $0 \mathrm{E}+$ & 0 & JOE & 0 & 0 & 0 & 0 & 0 & 00 & \\
\hline $\mathrm{Np}-2$ & $0 \mathrm{E}+00$ & 0 & & 0 & ل & 0 & & 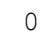 & & - & & 0 & & - & & \\
\hline $\mathrm{Pu}-23$ & $O F+0$ & 列 & -07 & 0 & S & 0 & r & 0 & (2) & 0 & & & & 0 & 0 & \\
\hline $\mathrm{Pu}-239$ & $00 \mathrm{E}+00$ & 0 & $7.58 \mathrm{E}-07$ & 0 & $0.00 \mathrm{E}+00$ & 0 & $0.00 \mathrm{E}+00$ & 0 & $0.00 \mathrm{E}+00$ & 0 & $0.00 \mathrm{E}+00$ & 0 & $0.00 \mathrm{E}+00$ & 0 & $0.00 \mathrm{E}+00$ & \\
\hline $\mathrm{Pu}-240$ & $.00 \mathrm{E}+00$ & 0 & $7.58 \mathrm{E}-07$ & 0 & $0.00 \mathrm{E}+00$ & 0 & $0.00 \mathrm{E}+00$ & 0 & $0.00 \mathrm{E}+00$ & 0 & $0.00 \mathrm{E}+00$ & 0 & $0.00 \mathrm{E}+00$ & 0 & $0.00 \mathrm{E}+00$ & \\
\hline & & 知 & $\mathrm{E}-08$ & 0 & $0 \mathrm{E}+00$ & & $\begin{array}{lll}0 & 0\end{array}$ & & $.00 \mathrm{E}+00$ & & $0=0$ & & $965-10$ & & & \\
\hline
\end{tabular}




\begin{tabular}{|c|c|c|c|c|c|c|c|c|c|c|c|c|c|c|c|c|}
\hline Th-228 & $0.00 \mathrm{E}+00$ & 0 & $3.60 \mathrm{E}-08$ & 0 & $0.00 \mathrm{E}+00$ & 0 & $0.00 \mathrm{E}+00$ & 0 & $0.00 \mathrm{E}+00$ & 0 & $0.00 \mathrm{E}+00$ & 0 & $0.00 \mathrm{E}+00$ & 0 & $0.00 \mathrm{E}+00$ & 0 \\
\hline Th-230 & $0.00 \mathrm{E}+00$ & 0 & $1.27 \mathrm{E}-07$ & 0 & $0.00 \mathrm{E}+00$ & 0 & $0.00 \mathrm{E}+00$ & 0 & $0.00 \mathrm{E}+00$ & 0 & $0.00 \mathrm{E}+00$ & 0 & $0.00 \mathrm{E}+00$ & 0 & $0.00 \mathrm{E}+00$ & 0 \\
\hline Th-232 & $0.00 \mathrm{E}+00$ & 0 & $6.08 \mathrm{E}-06$ & 0 & $0.00 \mathrm{E}+00$ & 0 & $0.00 \mathrm{E}+00$ & 0 & $0.00 \mathrm{E}+00$ & 0 & $0.00 \mathrm{E}+00$ & 0 & $0.00 \mathrm{E}+00$ & 0 & $0.00 \mathrm{E}+00$ & 0 \\
\hline $\mathrm{U}-234$ & $0.00 \mathrm{E}+00$ & 0 & $3.56 \mathrm{E}-06$ & 0 & $0.00 \mathrm{E}+00$ & 0 & $0.00 \mathrm{E}+00$ & 0 & $0.00 \mathrm{E}+00$ & 0 & $0.00 \mathrm{E}+00$ & 0 & $0.00 \mathrm{E}+00$ & 0 & $0.00 \mathrm{E}+00$ & 0 \\
\hline U-235 & $0.00 \mathrm{E}+00$ & 0 & $3.44 \mathrm{E}-06$ & 0 & $0.00 \mathrm{E}+00$ & 0 & $0.00 \mathrm{E}+00$ & 0 & $0.00 \mathrm{E}+00$ & 0 & $0.00 \mathrm{E}+00$ & 0 & $0.00 \mathrm{E}+00$ & 0 & $0.00 \mathrm{E}+00$ & 0 \\
\hline $\mathrm{U}-238$ & $0.00 \mathrm{E}+00$ & 0 & $3.52 \mathrm{E}-06$ & 0 & $0.00 \mathrm{E}+00$ & 0 & $0.00 \mathrm{E}+00$ & 0 & $0.00 \mathrm{E}+00$ & 0 & $0.00 \mathrm{E}+00$ & 0 & $0.00 \mathrm{E}+00$ & 0 & $0.00 \mathrm{E}+00$ & 0 \\
\hline & $98 \mathrm{E}-14$ & 0 & $6.33 E-05$ & 0 & $0.00 \mathrm{E}+00$ & 0 & $9.26 \mathrm{E}-11$ & 0 & $0.00 \mathrm{E}+00$ & 0 & $0.00 \mathrm{E}+00$ & 0 & $1.96 \mathrm{E}-14$ & 0 & נר & 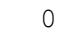 \\
\hline
\end{tabular}

Total Dose Contributions TDOSE $(i, p, t)$ for Individual Radionuclides (i) and Pathways (p) in mrem/yr and as a Percentage of Total Dose at $t=1$ years

$0 \quad$ Directly from primary contamination and from release to atmosphere (Inhalation excludes radon) Radio- Ground Inhalation Radon Plant

\begin{tabular}{|c|c|c|c|c|c|c|c|c|}
\hline & & & & & & & & \\
\hline Nuclide & Dose & 웅 & Dose & 응 & Dose & 응 & Dose & $\frac{\circ}{0}$ \\
\hline & $1.58 \mathrm{E}-05$ & 0 & $7.02 \mathrm{E}-04$ & 3 & $0.00 \mathrm{E}+00$ & 0 & $1.31 \mathrm{E}-06$ & 0 \\
\hline
\end{tabular}

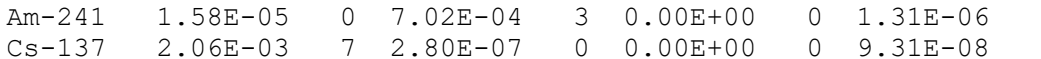

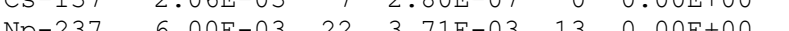

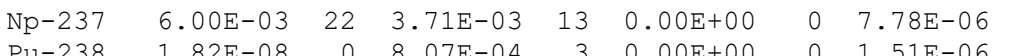

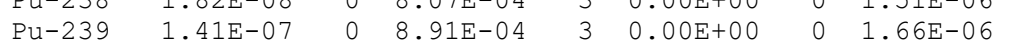

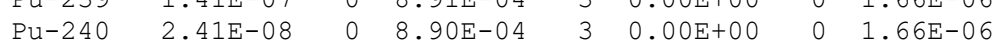

$\begin{array}{llllllll}\mathrm{TC}-99 & 1.86 \mathrm{E}-07 & 0 & 3.12 \mathrm{E}-07 & 0 & 0.00 \mathrm{E}+00 & 0 & 2.78 \mathrm{E}-08\end{array}$

$\begin{array}{llllllll}\text { Th-228 } & 3.22 \mathrm{E}-03 & 12 & 1.90 \mathrm{E}-04 & 1 & 0.00 \mathrm{E}+00 & 0 & 5.58 \mathrm{E}-07\end{array}$

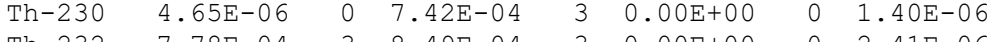

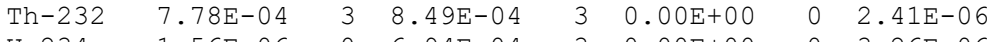

$\begin{array}{llllllll}\mathrm{U}-234 & 1.56 \mathrm{E}-06 & 0 & 6.94 \mathrm{E}-04 & 3 & 0.00 \mathrm{E}+00 & 0 & 3.26 \mathrm{E}-06\end{array}$

$\begin{array}{llllllll}\mathrm{U}-235 & 3.81 \mathrm{E}-03 & 14 & 6.28 \mathrm{E}-04 & 2 & 0.00 \mathrm{E}+00 & 0 & 3.15 \mathrm{E}-06\end{array}$

$\mathrm{U}-238$

Total

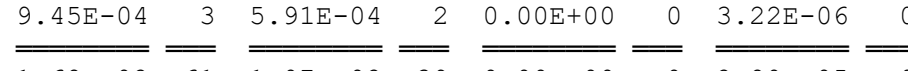

0 * Sum of dose from all releases and from primary contamination.

1RESRAD-OFFSITE, Version 4.0 .3 beta $T^{1} \frac{1}{2}$ Limit $=30$ days

Parent Dose Report

Title : Landfill Scenario

File : LANDFILL-PADUCAH-AUTHORIZED-RELEASE-REV.ROF

Total Dose Contributions TDOSE (i,p,t) for Individual Radionuclides (i) and Pathways (p) in mrem/yr and as a Percentage of Total Dose at $t=2$ years

\begin{tabular}{|c|c|c|c|c|c|c|c|c|c|c|c|c|c|c|c|c|}
\hline \multirow{2}{*}{$\begin{array}{l}\text { Radio- } \\
\text { Nuclide }\end{array}$} & \multicolumn{2}{|c|}{ Ground } & \multicolumn{2}{|l|}{ Fish } & \multicolumn{2}{|c|}{$\begin{array}{l}\text { From } \\
\text { Radon }\end{array}$} & \multicolumn{2}{|c|}{$\begin{array}{l}\text { ises to grour } \\
\text { Plant }\end{array}$} & \multicolumn{2}{|c|}{$\begin{array}{l}\text { d water and t } \\
\text { Meat }\end{array}$} & \multicolumn{2}{|l|}{ surf } & \multicolumn{2}{|l|}{ Soil } & \multicolumn{2}{|c|}{ Water } \\
\hline & Dose & $\div$ & Dose & $\frac{\circ}{0}$ & Dose & $\div$ & Dose & $\div$ & Dose & $\div$ & Dose & $\div$ & Dose & $\frac{\circ}{0}$ & Dose & \% \\
\hline$A m-241$ & & 0 & & 0 & & 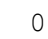 & & 0 & & 0 & & & & 0 & & \\
\hline $\mathrm{Cs}-13$ & $00 \mathrm{E}+00$ & 0 & $21 \mathrm{E}-06$ & 0 & $00 \mathrm{E}+00$ & 0 & $0 \mathrm{E}+00$ & 0 & OUE- & 0 & 0 & 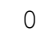 & D & 0 & 00 & \\
\hline $\mathrm{Np}-2$ & $0 E+00$ & 0 & $54 \mathrm{E}-05$ & 0 & $0 \mathrm{E}+$ & 0 & & 0 & & - & & & & 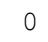 & & \\
\hline $\mathrm{Pu}-238$ & $\mathrm{OF}+\mathrm{O} \mathrm{O}$ & 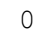 & 06 & 0 & م & 0 & & 0 & & 0 & & & & 0 & & \\
\hline $\mathrm{Pu}-239$ & $00 \mathrm{E}+00$ & 0 & $1.19 \mathrm{E}-06$ & 0 & $0.00 \mathrm{E}+00$ & 0 & $0.00 \mathrm{E}+00$ & 0 & $0.00 \mathrm{E}+00$ & 0 & 00 & 0 & $0.00 \mathrm{E}$ & 0 & $0.00 \mathrm{E}+00$ & \\
\hline $\mathrm{Pu}-240$ & $.00 \mathrm{E}+00$ & 0 & $1.19 \mathrm{E}-06$ & 0 & $0.00 \mathrm{E}+00$ & 0 & $0.00 \mathrm{E}+00$ & 0 & $0.00 \mathrm{E}+00$ & 0 & $0.00 \mathrm{E}+00$ & 0 & $0.00 \mathrm{E}+00$ & 0 & $0.00 \mathrm{E}+00$ & \\
\hline & & 0 & $00 \mathrm{E}-09$ & 0 & $0 F+0$ & & & & $32 F-08$ & & & & & & & \\
\hline
\end{tabular}




\begin{tabular}{|c|c|c|c|c|c|c|c|c|c|c|c|c|c|c|c|c|}
\hline Th-228 & $0.00 \mathrm{E}+00$ & 0 & $4.24 \mathrm{E}-08$ & 0 & $0.00 \mathrm{E}+00$ & 0 & $0.00 \mathrm{E}+00$ & 0 & $0.00 \mathrm{E}+00$ & 0 & $0.00 \mathrm{E}+00$ & 0 & $0.00 \mathrm{E}+00$ & 0 & $0.00 \mathrm{E}+00$ & 0 \\
\hline Th-230 & $0.00 \mathrm{E}+00$ & 0 & $2.50 \mathrm{E}-07$ & 0 & $0.00 \mathrm{E}+00$ & 0 & $0.00 \mathrm{E}+00$ & 0 & $0.00 \mathrm{E}+00$ & 0 & $0.00 \mathrm{E}+00$ & 0 & $0.00 \mathrm{E}+00$ & 0 & $0.00 \mathrm{E}+00$ & 0 \\
\hline Th-232 & $0.00 \mathrm{E}+00$ & 0 & $1.10 \mathrm{E}-05$ & 0 & $0.00 \mathrm{E}+00$ & 0 & $0.00 \mathrm{E}+00$ & 0 & $0.00 \mathrm{E}+00$ & 0 & $0.00 \mathrm{E}+00$ & 0 & $0.00 \mathrm{E}+00$ & 0 & $0.00 \mathrm{E}+00$ & 0 \\
\hline $\mathrm{U}-234$ & $0.00 \mathrm{E}+00$ & 0 & $3.58 \mathrm{E}-06$ & 0 & $0.00 \mathrm{E}+00$ & 0 & $0.00 \mathrm{E}+00$ & 0 & $0.00 \mathrm{E}+00$ & 0 & $0.00 \mathrm{E}+00$ & 0 & $0.00 \mathrm{E}+00$ & 0 & $0.00 \mathrm{E}+00$ & 0 \\
\hline U-235 & $0.00 \mathrm{E}+00$ & 0 & $3.47 E-06$ & 0 & $0.00 \mathrm{E}+00$ & 0 & $0.00 \mathrm{E}+00$ & 0 & $0.00 \mathrm{E}+00$ & 0 & $0.00 \mathrm{E}+00$ & 0 & $0.00 \mathrm{E}+00$ & 0 & $0.00 \mathrm{E}+00$ & 0 \\
\hline $\mathrm{U}-238$ & $0.00 \mathrm{E}+00$ & 0 & $3.54 \mathrm{E}-06$ & 0 & $0.00 \mathrm{E}+00$ & 0 & $0.00 \mathrm{E}+00$ & 0 & $0.00 \mathrm{E}+00$ & 0 & $0.00 \mathrm{E}+00$ & 0 & $0.00 \mathrm{E}+00$ & 0 & $0.00 \mathrm{E}+00$ & 0 \\
\hline & $1.15 \mathrm{E}-10$ & 0 & $7.62 \mathrm{E}-05$ & 0 & $0.00 \mathrm{E}+00$ & 0 & $8.56 \mathrm{E}-06$ & 0 & $6.32 \mathrm{E}-08$ & 0 & $5.02 \mathrm{E}-06$ & 0 & $1.14 \mathrm{E}-10$ & 0 & 2.7 & 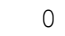 \\
\hline
\end{tabular}

Total Dose Contributions TDose $(i, p, t)$ for Individual Radionuclides (i) and Pathways (p) in mrem/yr and as a Percentage of Total Dose at $t=2$ years

0 Directly from primary contamination and from release to atmosphere (Inhalation excludes radon) Radio- Ground Inhalation Radon Plant

$\frac{\text { Nuclide }}{\text { Am-241 }} \frac{\text { Dose }}{1.56 \mathrm{E}-05} \frac{\%}{0} \frac{\text { Dose }}{6.95 \mathrm{E}-04} \frac{\frac{\circ}{3}}{\frac{\text { Dose }}{0.00 \mathrm{E}+00} \frac{\%}{0} \frac{\text { Dose }}{1.30 \mathrm{E}-06}}-$

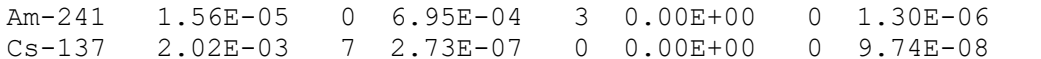

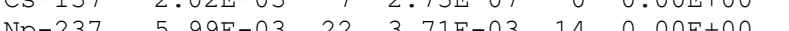

$\begin{array}{llllllll}\mathrm{Np}-237 & 5.99 \mathrm{E}-03 & 22 & 3.71 \mathrm{E}-03 & 14 & 0.00 \mathrm{E}+00 & 0 & 8.06 \mathrm{E}-06\end{array}$

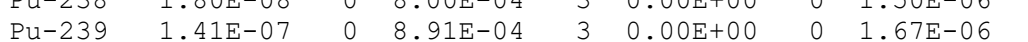

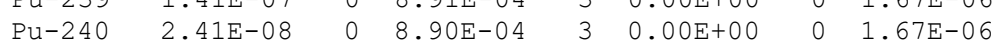

$\begin{array}{llllllll}\mathrm{TC}-99 & 8.54 \mathrm{E}-08 & 0 & 1.43 \mathrm{E}-07 & 0 & 0.00 \mathrm{E}+00 & 0 & 1.28 \mathrm{E}-08\end{array}$

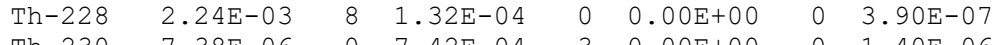

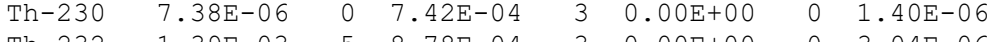

$\begin{array}{llllllll}\mathrm{Th}-232 & 1.39 \mathrm{E}-03 & 5 & 8.78 \mathrm{E}-04 & 3 & 0.00 \mathrm{E}+00 & 0 & 3.04 \mathrm{E}-06\end{array}$

$\begin{array}{llllllll}\mathrm{U}-234 & 1.55 \mathrm{E}-06 & 0 & 6.92 \mathrm{E}-04 & 3 & 0.00 \mathrm{E}+00 & 0 & 3.26 \mathrm{E}-06\end{array}$

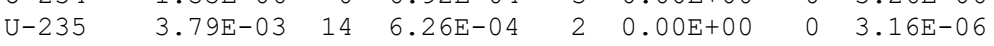

$\mathrm{U}-238$

Total

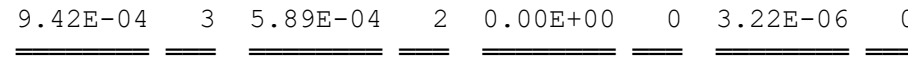

$0 *$ Sum of dose from all releases and from primary contamination.

1RESRAD-OFFSITE, Version 4.0 .3 beta $T^{1} \frac{1}{2}$ Limit $=30$ days

Parent Dose Report

Title : Landfill Scenario

File : LANDFILL-PADUCAH-AUTHORIZED-RELEASE-REV.ROF

Total Dose Contributions TDOSE (i,p,t) for Individual Radionuclides (i) and Pathways (p) in mrem/yr and as a Percentage of Total Dose at $t=9$ years

\begin{tabular}{|c|c|c|c|c|c|c|c|c|c|c|c|c|c|c|c|c|}
\hline \multirow{2}{*}{$\begin{array}{l}\text { Radio- } \\
\text { Nuclide }\end{array}$} & \multicolumn{2}{|c|}{ Ground } & \multicolumn{2}{|l|}{ ish } & \multicolumn{2}{|c|}{$\begin{array}{l}\text { From } \\
\text { Radon }\end{array}$} & \multicolumn{2}{|l|}{$\begin{array}{l}\text { es to } \\
\text { Plant }\end{array}$} & \multicolumn{2}{|c|}{$\begin{array}{l}\text { water and } t \\
\text { Meat }\end{array}$} & \multicolumn{2}{|c|}{$\begin{array}{c}\text { o surface wate } \\
\text { Milk }\end{array}$} & \multicolumn{2}{|l|}{ Soil } & \multicolumn{2}{|c|}{ Water } \\
\hline & Dose & $\%$ & Dose & $\div$ & Dose & $\%$ & Dose & $\%$ & Dose & $\%$ & Dose & $\%$ & Dose & 응 & Dose & $\%$ \\
\hline $\mathrm{Am}-$ & $.00 \mathrm{E}+00$ & 0 & 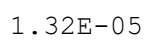 & 0 & ) & 0 & 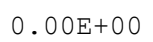 & 0 & 0 & 0 & 0 & 0 & 0 & 0 & 00 & \\
\hline $\mathrm{Cs}_{-}$ & $00 \mathrm{E}+00$ & 0 & 06 & 0 & $.00 \mathrm{E}$ & 0 & $0.00 \mathrm{~F}$ & 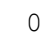 & 0.00 & 0 & 0.00 & 0 & 0.00 & 0 & 0 & \\
\hline & & 0 & & 0 & & 0 & & 0 & & & & & & & & \\
\hline $\mathrm{Pu}-238$ & $0 \mathrm{E}+00$ & 0 & $7 \mathrm{E}-06$ & 0 & $0 \mathrm{E}+$ & 0 & $10 \mathrm{E}$ & 0 & OE & 0 & $O F$ & 0 & 0 & 0 & 00 & \\
\hline $\mathrm{Pu}-239$ & $00 \mathrm{E}+00$ & 0 & $3.01 \mathrm{E}-06$ & 0 & $0.00 \mathrm{E}+00$ & 0 & $0.00 \mathrm{E}+00$ & 0 & $0.00 \mathrm{E}+00$ & 0 & $0.00 \mathrm{E}+00$ & 0 & $0.00 \mathrm{E}+00$ & 0 & $0.00 \mathrm{E}+00$ & \\
\hline $\mathrm{Pu}-240$ & $.00 \mathrm{E}+00$ & 0 & $3.01 \mathrm{E}-06$ & 0 & $0.00 \mathrm{E}+00$ & 0 & $0.00 \mathrm{E}+00$ & 0 & $0.00 \mathrm{E}+00$ & ( & $0.00 \mathrm{E}+00$ & 0 & $0.00 \mathrm{E}+00$ & 0 & $0.00 \mathrm{E}+00$ & \\
\hline & & & & & $00 E+00$ & & $02 E-01$ & 86 & $.33 E-03$ & & $4.70 E-02$ & & (e) & & $2.18 \mathrm{E}-01$ & \\
\hline
\end{tabular}

$\begin{array}{lllllllllllllllllll}\mathrm{TC}-99 & 1.20 \mathrm{E}-06 & 0 & 3.45 \mathrm{E}-11 & 0 & 0.00 \mathrm{E}+00 & 0 & 1.02 \mathrm{E}-01 & 26 & 1.33 \mathrm{E}-03 & 0 & 4.70 \mathrm{E}-02 & 12 & 1.19 \mathrm{E}-06 & 0 & 2.18 \mathrm{E}-01 & 55\end{array}$ 


\begin{tabular}{|c|c|c|c|c|c|c|c|c|c|c|c|c|c|c|c|c|}
\hline Th-228 & $0.00 \mathrm{E}+00$ & 0 & $1.26 \mathrm{E}-08$ & 0 & $0.00 \mathrm{E}+00$ & 0 & $0.00 \mathrm{E}+00$ & 0 & $0.00 \mathrm{E}+00$ & 0 & $0.00 \mathrm{E}+00$ & 0 & $0.00 \mathrm{E}+00$ & 0 & $0.00 \mathrm{E}+00$ & 0 \\
\hline Th-230 & $0.00 \mathrm{E}+00$ & 0 & $1.85 \mathrm{E}-06$ & 0 & $0.00 \mathrm{E}+00$ & 0 & $0.00 \mathrm{E}+00$ & 0 & $0.00 \mathrm{E}+00$ & 0 & $0.00 \mathrm{E}+00$ & 0 & $0.00 \mathrm{E}+00$ & 0 & $0.00 \mathrm{E}+00$ & 0 \\
\hline Th-232 & $0.00 \mathrm{E}+00$ & 0 & $3.92 \mathrm{E}-05$ & 0 & $0.00 \mathrm{E}+00$ & 0 & $0.00 \mathrm{E}+00$ & 0 & $0.00 \mathrm{E}+00$ & 0 & $0.00 \mathrm{E}+00$ & 0 & $0.00 \mathrm{E}+00$ & 0 & $0.00 \mathrm{E}+00$ & 0 \\
\hline$U-234$ & $0.00 \mathrm{E}+00$ & 0 & $3.50 \mathrm{E}-06$ & 0 & $0.00 \mathrm{E}+00$ & 0 & $0.00 \mathrm{E}+00$ & 0 & $0.00 \mathrm{E}+00$ & 0 & $0.00 \mathrm{E}+00$ & 0 & $0.00 \mathrm{E}+00$ & 0 & $0.00 \mathrm{E}+00$ & 0 \\
\hline $\mathrm{U}-235$ & $0.00 \mathrm{E}+00$ & 0 & $3.42 \mathrm{E}-06$ & 0 & $0.00 \mathrm{E}+00$ & 0 & $0.00 \mathrm{E}+00$ & 0 & $0.00 \mathrm{E}+00$ & 0 & $0.00 \mathrm{E}+00$ & 0 & $0.00 \mathrm{E}+00$ & 0 & $0.00 \mathrm{E}+00$ & 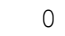 \\
\hline $\mathrm{U}-238$ & $0.00 \mathrm{E}+00$ & 0 & $3.46 \mathrm{E}-06$ & 0 & $0.00 \mathrm{E}+00$ & 0 & $0.00 \mathrm{E}+00$ & 0 & $0.00 \mathrm{E}+00$ & 0 & $0.00 \mathrm{E}+00$ & 0 & $0.00 \mathrm{E}+00$ & 0 & $0.00 \mathrm{E}+00$ & 0 \\
\hline 工 & 6 & 0 & 4 & 0 & $0.00 \mathrm{E}+00$ & 0 & 01 & 26 & 1.33 & 0 & $4.70 \mathrm{E}-02$ & 12 & $1.19 \mathrm{E}-06$ & 0 & $2.18 \mathrm{E}-01$ & 55 \\
\hline
\end{tabular}

Total Dose Contributions TDOSE $(i, p, t)$ for Individual Radionuclides (i) and Pathways (p) in mrem/yr and as a Percentage of Total Dose at $t=9$ years

Directly from primary contamination and from release to atmosphere (Inhalation excludes radon)

\begin{tabular}{|c|c|c|c|c|c|c|c|c|c|c|c|c|c|c|c|c|}
\hline \multirow{2}{*}{$\begin{array}{l}\text { Radio- } \\
\text { Nuclide }\end{array}$} & & \multicolumn{2}{|l|}{ halati } & \multicolumn{2}{|c|}{ Radon } & \multicolumn{2}{|c|}{ Plant } & \multicolumn{2}{|l|}{ Meat } & \multicolumn{2}{|l|}{ Milk } & \multicolumn{2}{|l|}{ Soil } & \multicolumn{2}{|l|}{$1 \mathrm{~Pa}$} \\
\hline & Dose & 응 & Dose & 응 & Dose & 응 & Dose & 응 & Dose & 웅 & Dose & $\%$ & Dose & $\div$ & Dose & 응 \\
\hline$A m-241$ & $.46 \mathrm{E}-05$ & 0 & $6.49 \mathrm{E}-04$ & 0 & $0.00 \mathrm{E}+00$ & 0 & $1.22 \mathrm{E}-06$ & 0 & $2.78 \mathrm{E}-09$ & 0 & $2.51 \mathrm{E}-10$ & 0 & $8.06 \mathrm{E}-09$ & 0 & $6.78 \mathrm{E}-04$ & 0 \\
\hline $\mathrm{Cs}-137$ & $.72 \mathrm{E}-03$ & 0 & $2.32 \mathrm{E}-07$ & 0 & $0.00 \mathrm{E}+00$ & 0 & $1.21 \mathrm{E}-07$ & 0 & $1.49 \mathrm{E}-07$ & 0 & $7.13 \mathrm{E}-08$ & 0 & $7.19 \mathrm{E}-10$ & 0 & $1.72 \mathrm{E}-03$ & 0 \\
\hline $\mathrm{Np}-237$ & $5.97 \mathrm{E}-03$ & 2 & $3.69 \mathrm{E}-03$ & 1 & $0.00 \mathrm{E}+00$ & 0 & $9.97 \mathrm{E}-06$ & 0 & $4.31 \mathrm{E}-07$ & 0 & $4.26 \mathrm{E}-09$ & 0 & 7. $33 \mathrm{E}-08$ & 0 & $9.71 \mathrm{E}-03$ & 2 \\
\hline $\mathrm{Pu}-238$ & $1.71 \mathrm{E}-08$ & 0 & $7.57 \mathrm{E}-04$ & 0 & $0.00 \mathrm{E}+00$ & 0 & $1.44 \mathrm{E}-06$ & 0 & $6.75 \mathrm{E}-09$ & 0 & $1.50 \mathrm{E}-10$ & 0 & $1.47 \mathrm{E}-08$ & 0 & $7.61 \mathrm{E}-04$ & 0 \\
\hline $\mathrm{Pu}-239$ & $1.41 \mathrm{E}-07$ & 0 & $8.90 \mathrm{E}-04$ & 0 & $0.00 \mathrm{E}+00$ & 0 & $1.69 \mathrm{E}-06$ & 0 & $7.91 \mathrm{E}-09$ & 0 & $1.75 \mathrm{E}-10$ & 0 & $1.72 \mathrm{E}-08$ & 0 & $8.95 \mathrm{E}-04$ & 0 \\
\hline $\mathrm{Pu}-240$ & $2.41 \mathrm{E}-08$ & 0 & $8.89 \mathrm{E}-04$ & 0 & $0.00 \mathrm{E}+00$ & 0 & $1.69 \mathrm{E}-06$ & 0 & $7.90 \mathrm{E}-09$ & 0 & $1.75 \mathrm{E}-10$ & 0 & $1.72 \mathrm{E}-08$ & 0 & $8.94 \mathrm{E}-04$ & 0 \\
\hline TC-99 & $3.68 \mathrm{E}-10$ & 0 & $3.85 \mathrm{E}-07$ & 0 & $0.00 \mathrm{E}+00$ & 0 & $5.51 \mathrm{E}-11$ & 0 & $2.28 \mathrm{E}-13$ & 0 & $3.59 \mathrm{E}-12$ & 0 & $2.91 \mathrm{E}-15$ & 0 & $3.68 \mathrm{E}-01$ & 93 \\
\hline Th-228 & $.78 \mathrm{E}-04$ & 0 & $1.05 \mathrm{E}-05$ & 0 & $0.00 \mathrm{E}+00$ & 0 & $3.13 \mathrm{E}-08$ & 0 & $1.47 \mathrm{E}-10$ & 0 & $1.63 \mathrm{E}-11$ & 0 & $3.19 \mathrm{E}-10$ & 0 & $1.88 \mathrm{E}-04$ & 0 \\
\hline Th -230 & $2.62 \mathrm{E}-05$ & 0 & $7.43 \mathrm{E}-04$ & 0 & $0.00 \mathrm{E}+00$ & 0 & $1.44 \mathrm{E}-06$ & 0 & $8.13 \mathrm{E}-09$ & 0 & $1.91 \mathrm{E}-09$ & 0 & $1.46 \mathrm{E}-08$ & 0 & $7.72 \mathrm{E}-04$ & 0 \\
\hline Th-232 & $5.23 \mathrm{E}-03$ & 1 & $1.07 \mathrm{E}-03$ & 0 & $0.00 \mathrm{E}+00$ & 0 & $7.33 \mathrm{E}-06$ & 0 & $2.23 \mathrm{E}-07$ & 0 & $3.92 \mathrm{E}-07$ & 0 & $5.08 \mathrm{E}-08$ & 0 & $6.35 \mathrm{E}-03$ & 2 \\
\hline $\mathrm{U}-234$ & $1.53 \mathrm{E}-06$ & 0 & $6.77 \mathrm{E}-04$ & 0 & $0.00 \mathrm{E}+00$ & 0 & $3.28 \mathrm{E}-06$ & 0 & $5.11 \mathrm{E}-08$ & 0 & $1.99 \mathrm{E}-07$ & 0 & $2.72 \mathrm{E}-08$ & 0 & $6.85 \mathrm{E}-04$ & 0 \\
\hline $\mathrm{U}-235$ & $3.71 \mathrm{E}-03$ & 1 & $6.15 \mathrm{E}-04$ & 0 & $0.00 \mathrm{E}+00$ & 0 & $3.18 \mathrm{E}-06$ & 0 & $5.17 \mathrm{E}-08$ & 0 & $1.93 \mathrm{E}-07$ & 0 & $2.63 \mathrm{E}-08$ & 0 & $4.33 E-03$ & 1 \\
\hline $\mathrm{U}-238$ & $9.21 \mathrm{E}-04$ & 0 & $5.76 \mathrm{E}-04$ & 0 & $0.00 \mathrm{E}+00$ & 0 & $3.23 \mathrm{E}-06$ & 0 & $5.04 \mathrm{E}-08$ & 0 & $1.97 \mathrm{E}-07$ & 0 & $2.68 \mathrm{E}-08$ & 0 & $1.50 \mathrm{E}-03$ & 0 \\
\hline & $8 \mathrm{E}-02$ & 4 & $06 \mathrm{E}-02$ & 3 & $0.00 \mathrm{E}+00$ & 0 & $3.46 \mathrm{E}-05$ & 0 & $9.90 \mathrm{E}-07$ & 0 & $1.06 \mathrm{E}-06$ & 0 & $2.77 \mathrm{E}-07$ & 0 & $3.97 \mathrm{E}-01$ & 100 \\
\hline
\end{tabular}

Total

$1.78 \mathrm{E}-02 \quad 4 \quad 1.06 \mathrm{E}-02$

$0.00 \mathrm{E}+00$

3. $46 \mathrm{E}-05$

$9.90 \mathrm{E}-07$

$01.06 \mathrm{E}-06$

$2.77 \mathrm{E}-07$

$3.97 \mathrm{E}-01 \quad 100$

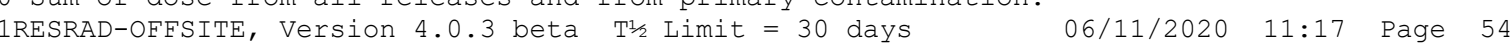

Parent Dose Report

Title : Landfill Scenario

File : LANDFILL-PADUCAH-AUTHORIZED-RELEASE-REV.ROF

Total Dose Contributions TDOSE (i,p,t) for Individual Radionuclides (i) and Pathways (p) in mrem/yr and as a Percentage of Total Dose at $t=14$ years

\begin{tabular}{|c|c|c|c|c|c|c|c|c|c|c|c|c|c|c|c|c|}
\hline \multirow{2}{*}{$\begin{array}{l}0 \\
\text { Radio- } \\
\text { Nuclide }\end{array}$} & \multicolumn{2}{|c|}{ Ground } & \multicolumn{2}{|l|}{ Fish } & \multicolumn{2}{|c|}{$\begin{array}{l}\text { From } \\
\text { Radon }\end{array}$} & \multicolumn{2}{|c|}{$\begin{array}{l}\text { ses to grour } \\
\text { Plant }\end{array}$} & \multicolumn{2}{|c|}{$\begin{array}{l}\text { water and } t \\
\text { Meat }\end{array}$} & \multicolumn{2}{|c|}{$\begin{array}{l}\text { - surface water } \\
\text { Milk }\end{array}$} & \multicolumn{2}{|l|}{ Soil } & \multicolumn{2}{|c|}{ Water } \\
\hline & Dose & $\%$ & Dose & $\%$ & Dose & $\%$ & Dose & $\%$ & Dose & $\%$ & Dose & $\%$ & Dose & $\%$ & Dose & $\%$ \\
\hline & & 0 & & 0 & & 0 & & 0 & م $\cap \cap F+\cap$ & 0 & & 0 & & 0 & & \\
\hline & $\mathrm{JEt}+$ & 0 & $0 E^{-}-$ & 0 & 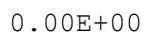 & 0 & UI & 0 & 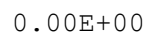 & 0 & & & & c & & \\
\hline $\mathrm{Np}-237$ & $00 \mathrm{E}+00$ & 0 & $0 E-05$ & 0 & $0 E+00$ & 0 & $0 E+00$ & 0 & $00 \mathrm{E}+00$ & 0 & $.00 \mathrm{E}+00$ & U & $0.00 \mathrm{E}+00$ & 0 & $.00 \mathrm{E}+00$ & \\
\hline $\mathrm{Pu}-238$ & $00 \mathrm{E}+00$ & 0 & $.93 E-06$ & 0 & 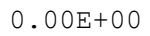 & 0 & & 0 & & 0 & & 0 & & 0 & & \\
\hline $\mathrm{Pu}$ & (2) & 0 & & 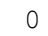 & & 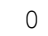 & & 0 & & 0 & & 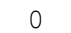 & & 1 & & \\
\hline $\mathrm{Pu}-240$ & $.00 \mathrm{E}+00$ & 0 & $3.57 \mathrm{E}-06$ & 0 & $0.00 \mathrm{E}+00$ & 0 & $.00 \mathrm{E}+00$ & 0 & $0.00 \mathrm{E}+00$ & 0 & $0.00 \mathrm{E}+00$ & 0 & $0.00 \mathrm{E}+00$ & 0 & $0.00 \mathrm{E}+00$ & \\
\hline & & & & & & & & & & & & & & & & \\
\hline
\end{tabular}

$\begin{array}{llllllllllllllllllll}\mathrm{TC}-99 & 1.53 \mathrm{E}-06 & 0 & 7.04 \mathrm{E}-13 & 0 & 0.00 \mathrm{E}+00 & 0 & 1.29 \mathrm{E}-01 & 26 & 1.70 \mathrm{E}-03 & 0 & 5.92 \mathrm{E}-02 & 12 & 1.52 \mathrm{E}-06 & 0 & 2.74 \mathrm{E}-01 & 55\end{array}$ 


\begin{tabular}{|c|c|c|c|c|c|c|c|c|c|c|c|c|c|c|c|c|}
\hline Th-228 & $0.00 \mathrm{E}+00$ & 0 & $3.08 \mathrm{E}-09$ & 0 & $0.00 \mathrm{E}+00$ & 0 & $0.00 \mathrm{E}+00$ & 0 & $0.00 \mathrm{E}+00$ & 0 & $0.00 \mathrm{E}+00$ & 0 & $0.00 \mathrm{E}+00$ & 0 & $0.00 \mathrm{E}+00$ & 0 \\
\hline Th-230 & $0.00 \mathrm{E}+00$ & 0 & $3.67 \mathrm{E}-06$ & 0 & $0.00 \mathrm{E}+00$ & 0 & $0.00 \mathrm{E}+00$ & 0 & $0.00 \mathrm{E}+00$ & 0 & $0.00 \mathrm{E}+00$ & 0 & $0.00 \mathrm{E}+00$ & 0 & $0.00 \mathrm{E}+00$ & 0 \\
\hline Th-232 & $0.00 \mathrm{E}+00$ & 0 & $5.49 \mathrm{E}-05$ & 0 & $0.00 \mathrm{E}+00$ & 0 & $0.00 \mathrm{E}+00$ & 0 & $0.00 \mathrm{E}+00$ & 0 & $0.00 \mathrm{E}+00$ & 0 & $0.00 \mathrm{E}+00$ & 0 & $0.00 \mathrm{E}+00$ & 0 \\
\hline$U-234$ & $0.00 \mathrm{E}+00$ & 0 & $3.45 \mathrm{E}-06$ & 0 & $0.00 \mathrm{E}+00$ & 0 & $0.00 \mathrm{E}+00$ & 0 & $0.00 \mathrm{E}+00$ & 0 & $0.00 \mathrm{E}+00$ & 0 & $0.00 \mathrm{E}+00$ & 0 & $0.00 \mathrm{E}+00$ & 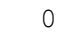 \\
\hline $\mathrm{U}-235$ & $0.00 \mathrm{E}+00$ & 0 & $3.40 \mathrm{E}-06$ & 0 & $0.00 \mathrm{E}+00$ & 0 & $0.00 \mathrm{E}+00$ & 0 & $0.00 \mathrm{E}+00$ & 0 & $0.00 \mathrm{E}+00$ & 0 & $0.00 \mathrm{E}+00$ & 0 & $0.00 \mathrm{E}+00$ & 0 \\
\hline $\mathrm{U}-238$ & $0.00 \mathrm{E}+00$ & 0 & $3.40 \mathrm{E}-06$ & 0 & $0.00 \mathrm{E}+00$ & 0 & $0.00 \mathrm{E}+00$ & 0 & $0.00 \mathrm{E}+00$ & 0 & $0.00 \mathrm{E}+00$ & 0 & $0.00 \mathrm{E}+00$ & 0 & $0.00 \mathrm{E}+00$ & 0 \\
\hline 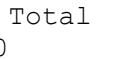 & $1.53 \mathrm{E}-06$ & 0 & 4 & 0 & 0.00 & 0 & 1 & 26 & -03 & 0 & $92 \mathrm{E}-02$ & 12 & 06 & 0 & 2 & 55 \\
\hline
\end{tabular}

Total Dose Contributions TDOSE $(i, p, t)$ for Individual Radionuclides (i) and Pathways (p) in mrem/yr and as a Percentage of Total Dose at $t=14$ years

$0 \quad$ Directly from primary contamination and from release to atmosphere (Inhalation excludes radon)

\begin{tabular}{|c|c|c|c|c|c|c|c|c|c|c|c|c|c|c|c|c|}
\hline \multirow{2}{*}{$\begin{array}{l}\text { Radio- } \\
\text { Nuclide }\end{array}$} & \multicolumn{2}{|c|}{ S } & \multicolumn{2}{|l|}{ halat } & \multicolumn{2}{|c|}{ Radon } & \multicolumn{2}{|c|}{ Plant } & \multicolumn{2}{|l|}{ Meat } & \multicolumn{2}{|l|}{ Milk } & \multicolumn{2}{|l|}{ Soil } & \multicolumn{2}{|c|}{1 Pathways* } \\
\hline & Dose & 응 & Dose & 응 & Dose & 웅 & Dose & 웅 & Dose & 웅 & Dose & $\frac{\circ}{0}$ & Dose & 웅 & Dose & $\div$ \\
\hline$A m-241$ & $1.39 \mathrm{E}-05$ & 0 & $6.18 \mathrm{E}-04$ & 0 & $0.00 \mathrm{E}+00$ & 0 & $1.17 \mathrm{E}-06$ & 0 & $2.69 \mathrm{E}-09$ & 0 & $2.41 \mathrm{E}-10$ & 0 & $9.55 \mathrm{E}-09$ & 0 & $6.45 \mathrm{E}-04$ & 0 \\
\hline $\mathrm{Cs}-137$ & $1.53 \mathrm{E}-03$ & 0 & $2.07 \mathrm{E}-07$ & 0 & $0.00 \mathrm{E}+00$ & 0 & $1.33 \mathrm{E}-07$ & 0 & $1.62 \mathrm{E}-07$ & 0 & $7.09 \mathrm{E}-08$ & 0 & $9.77 \mathrm{E}-10$ & 0 & $.53 \mathrm{E}-03$ & 0 \\
\hline $\mathrm{Np}-237$ & $5.95 \mathrm{E}-03$ & 1 & $3.68 \mathrm{E}-03$ & 1 & $0.00 \mathrm{E}+00$ & 0 & $1.13 \mathrm{E}-05$ & 0 & $4.90 \mathrm{E}-07$ & 0 & $4.55 \mathrm{E}-09$ & 0 & $1.09 \mathrm{E}-07$ & 0 & $9.68 \mathrm{E}-03$ & 2 \\
\hline $\mathrm{Pu}-238$ & $1.65 \mathrm{E}-08$ & 0 & $7.27 \mathrm{E}-04$ & 0 & $0.00 \mathrm{E}+00$ & 0 & $1.40 \mathrm{E}-06$ & 0 & $6.86 \mathrm{E}-09$ & 0 & $1.49 \mathrm{E}-10$ & 0 & $2.14 \mathrm{E}-08$ & 0 & $7.32 \mathrm{E}-04$ & 0 \\
\hline $\mathrm{Pu}-239$ & $1.41 \mathrm{E}-07$ & 0 & $8.89 \mathrm{E}-04$ & 0 & $0.00 \mathrm{E}+00$ & 0 & $1.70 \mathrm{E}-06$ & 0 & $8.36 \mathrm{E}-09$ & 0 & $1.80 \mathrm{E}-10$ & 0 & $2.61 \mathrm{E}-08$ & 0 & $8.95 \mathrm{E}-04$ & 0 \\
\hline $\mathrm{Pu}-240$ & $2.41 \mathrm{E}-08$ & 0 & $8.88 \mathrm{E}-04$ & 0 & $0.00 \mathrm{E}+00$ & 0 & $1.70 \mathrm{E}-06$ & 0 & $8.35 \mathrm{E}-09$ & 0 & $1.80 \mathrm{E}-10$ & 0 & $2.61 \mathrm{E}-08$ & 0 & $8.94 \mathrm{E}-04$ & 0 \\
\hline TC-99 & $7.51 \mathrm{E}-12$ & 0 & $4.89 \mathrm{E}-07$ & 0 & $0.00 \mathrm{E}+00$ & 0 & $1.12 \mathrm{E}-12$ & 0 & $4.65 \mathrm{E}-15$ & 0 & $7.34 \mathrm{E}-14$ & 0 & $5.94 \mathrm{E}-17$ & 0 & $4.64 \mathrm{E}-01$ & 94 \\
\hline Th-228 & $2.91 \mathrm{E}-05$ & 0 & $.71 \mathrm{E}-06$ & 0 & $0.00 \mathrm{E}+00$ & 0 & $5.17 \mathrm{E}-09$ & 0 & $2.54 \mathrm{E}-11$ & 0 & $2.74 \mathrm{E}-12$ & 0 & $7.97 \mathrm{E}-11$ & 0 & $3.08 \mathrm{E}-05$ & 0 \\
\hline Th-230 & $3.95 E-05$ & 0 & $7.43 \mathrm{E}-04$ & 0 & $0.00 \mathrm{E}+00$ & 0 & $1.47 \mathrm{E}-06$ & 0 & $1.03 \mathrm{E}-08$ & 0 & $2.85 \mathrm{E}-09$ & 0 & $2.25 \mathrm{E}-08$ & 0 & $7.88 \mathrm{E}-04$ & 0 \\
\hline Th-232 & $6.80 \mathrm{E}-03$ & 1 & $1.15 \mathrm{E}-03$ & 0 & $0.00 \mathrm{E}+00$ & 0 & $9.90 \mathrm{E}-06$ & 0 & $3.19 \mathrm{E}-07$ & 0 & $5.22 \mathrm{E}-07$ & 0 & $8.79 \mathrm{E}-08$ & 0 & $8.02 \mathrm{E}-03$ & 2 \\
\hline $\mathrm{U}-234$ & $1.52 \mathrm{E}-06$ & 0 & $6.66 \mathrm{E}-04$ & 0 & $0.00 \mathrm{E}+00$ & 0 & $3.27 E-06$ & 0 & $5.23 \mathrm{E}-08$ & 0 & $2.00 \mathrm{E}-07$ & 0 & $3.71 \mathrm{E}-08$ & 0 & -04 & 0 \\
\hline$U-235$ & $3.65 \mathrm{E}-03$ & 1 & $6.07 \mathrm{E}-04$ & 0 & $0.00 \mathrm{E}+00$ & 0 & $3.18 \mathrm{E}-06$ & 0 & $5.44 \mathrm{E}-08$ & 0 & $1.94 \mathrm{E}-07$ & 0 & $3.61 \mathrm{E}-08$ & 0 & $4.26 \mathrm{E}-03$ & 1 \\
\hline $\mathrm{U}-238$ & $9.06 \mathrm{E}-04$ & 0 & $5.67 \mathrm{E}-04$ & 0 & $0.00 \mathrm{E}+00$ & 0 & $3.23 \mathrm{E}-06$ & 0 & $5.17 \mathrm{E}-08$ & 0 & $1.98 \mathrm{E}-07$ & 0 & $3.67 \mathrm{E}-08$ & 0 & $1.48 \mathrm{E}-03$ & 0 \\
\hline & $39 E-02$ & 4 & $.05 E-02$ & 2 & $0.00 \mathrm{E}+00$ & 0 & $3.84 \mathrm{E}-05$ & 0 & $1.17 \mathrm{E}-06$ & 0 & $1.19 \mathrm{E}-06$ & 0 & $4.14 \mathrm{E}-07$ & 0 & $4.94 \mathrm{E}-01$ & 100 \\
\hline
\end{tabular}

Total

$1.89 \mathrm{E}-02$

$1.05 \mathrm{E}-02$

$0.00 \mathrm{E}+00$

$3.84 \mathrm{E}-05$

0

IRESRAD-OFFSITE, Version 4.0 .3 beta T1/2 Limit $=30$ days $\quad 06 / 11 / 2020 \quad 11: 17 \quad$ Page 55

Parent Dose Report

Title : Landfill Scenario

File : LANDFILL-PADUCAH-AUTHORIZED-RELEASE-REV.ROF

Total Dose Contributions TDOSE (i,p,t) for Individual Radionuclides (i) and Pathways (p) in $\mathrm{mrem} / \mathrm{yr}$ and as a Percentage of Total Dose at $t=17$ years

\begin{tabular}{|c|c|c|c|c|c|c|c|c|c|c|c|c|c|c|c|c|}
\hline \multirow{2}{*}{$\begin{array}{l}0 \\
0 \\
\text { Radio- } \\
\text { Nuclide }\end{array}$} & \multicolumn{2}{|c|}{ Ground } & \multicolumn{2}{|l|}{ Fish } & \multicolumn{2}{|c|}{$\begin{array}{l}\text { Fror } \\
\text { Radon }\end{array}$} & \multicolumn{2}{|l|}{ Plant } & \multicolumn{2}{|l|}{ Meat } & \multicolumn{4}{|c|}{ Soil } & \multicolumn{2}{|l|}{ Water } \\
\hline & Dose & $\%$ & Dose & $\%$ & Dose & $\%$ & Dose & $\%$ & Dose & $\%$ & Dose & $\frac{\circ}{0}$ & Dose & $\frac{\circ}{0}$ & Dose & $\therefore$ \\
\hline $\mathrm{Am}-$ & +00 & 0 & 5 & 0 & $E+00$ & 0 & 0 & 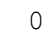 & 0 & 0 & 0.00 & 0 & 00 & 0 & 0. & \\
\hline $\mathrm{Cs}-13$ & $.00 \mathrm{E}+00$ & 0 & $3.96 \mathrm{E}-06$ & 0 & $.00 \mathrm{E}+00$ & 0 & $.00 \mathrm{E}+00$ & 0 & $0.00 \mathrm{E}$ & 0 & 0.001 & 0 & $0.00 \mathrm{E}+00$ & 0 & 0.00 & \\
\hline & & 0 & & , & & 0 & & 0 & & & & & & & & \\
\hline Pu-238 & $00 \mathrm{E}+00$ & 0 & $3.02 \mathrm{E}-06$ & 0 & $00 \mathrm{E}+00$ & 0 & $00 \mathrm{E}+00$ & 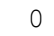 & $O O E$ & 0 & $\mathrm{OOE}$ & 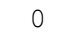 & $00 \mathrm{E}$ & 0 & $00 \mathrm{E}+00$ & \\
\hline $\mathrm{Pu}-239$ & $.00 \mathrm{E}+00$ & 0 & $3.77 \mathrm{E}-06$ & 0 & $0.00 \mathrm{E}+00$ & 0 & $0.00 \mathrm{E}+00$ & 0 & $0.00 \mathrm{E}+00$ & 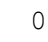 & $0.00 \mathrm{E}+00$ & 0 & $0.00 \mathrm{E}+00$ & 0 & $0.00 \mathrm{E}+00$ & \\
\hline $\mathrm{Pu}-240$ & $0.00 \mathrm{E}+00$ & 0 & $3.76 \mathrm{E}-06$ & 0 & $0.00 \mathrm{E}+00$ & 0 & $0.00 \mathrm{E}+00$ & 0 & $0.00 \mathrm{E}+00$ & 0 & $0.00 \mathrm{E}+00$ & 0 & $0.00 \mathrm{E}+00$ & 0 & $0.00 \mathrm{E}+00$ & \\
\hline
\end{tabular}

$\begin{array}{lllllllllllllllll}\mathrm{TC}-99 & 1.54 \mathrm{E}-06 & 0 & 6.81 \mathrm{E}-14 & 0 & 0.00 \mathrm{E}+00 & 0 & 1.31 \mathrm{E}-01 & 26 & 1.71 \mathrm{E}-03 & 0 & 5.98 \mathrm{E}-02 & 12 & 1.53 \mathrm{E}-06 & 0 & 2.76 \mathrm{E}-01 & 55\end{array}$ 


\begin{tabular}{|c|c|c|c|c|c|c|c|c|c|c|c|c|c|c|c|c|}
\hline Th-228 & $0.00 \mathrm{E}+00$ & 0 & $1.24 \mathrm{E}-09$ & 0 & $0.00 \mathrm{E}+00$ & 0 & $0.00 \mathrm{E}+00$ & 0 & $0.00 \mathrm{E}+00$ & 0 & $0.00 \mathrm{E}+00$ & 0 & $0.00 \mathrm{E}+00$ & 0 & $0.00 \mathrm{E}+00$ & 0 \\
\hline Th-230 & $0.00 \mathrm{E}+00$ & 0 & $4.98 \mathrm{E}-06$ & 0 & $0.00 \mathrm{E}+00$ & 0 & $0.00 \mathrm{E}+00$ & 0 & $0.00 \mathrm{E}+00$ & 0 & $0.00 \mathrm{E}+00$ & 0 & $0.00 \mathrm{E}+00$ & 0 & $0.00 \mathrm{E}+00$ & 0 \\
\hline Th-232 & $0.00 \mathrm{E}+00$ & 0 & $6.33 \mathrm{E}-05$ & 0 & $0.00 \mathrm{E}+00$ & 0 & $0.00 \mathrm{E}+00$ & 0 & $0.00 \mathrm{E}+00$ & 0 & $0.00 \mathrm{E}+00$ & 0 & $0.00 \mathrm{E}+00$ & 0 & $0.00 \mathrm{E}+00$ & 0 \\
\hline $\mathrm{U}-234$ & $0.00 \mathrm{E}+00$ & 0 & $3.41 \mathrm{E}-06$ & 0 & $0.00 \mathrm{E}+00$ & 0 & $0.00 \mathrm{E}+00$ & 0 & $0.00 \mathrm{E}+00$ & 0 & $0.00 \mathrm{E}+00$ & 0 & $0.00 \mathrm{E}+00$ & 0 & $0.00 \mathrm{E}+00$ & 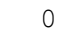 \\
\hline $\mathrm{U}-235$ & $0.00 \mathrm{E}+00$ & 0 & $3.39 \mathrm{E}-06$ & 0 & $0.00 \mathrm{E}+00$ & 0 & $0.00 \mathrm{E}+00$ & 0 & $0.00 \mathrm{E}+00$ & 0 & $0.00 \mathrm{E}+00$ & 0 & $0.00 \mathrm{E}+00$ & 0 & $0.00 \mathrm{E}+00$ & 0 \\
\hline $\mathrm{U}-238$ & $0.00 \mathrm{E}+00$ & 0 & $3.37 \mathrm{E}-06$ & 0 & $0.00 \mathrm{E}+00$ & 0 & $0.00 \mathrm{E}+00$ & 0 & $0.00 \mathrm{E}+00$ & 0 & $0.00 \mathrm{E}+00$ & 0 & $0.00 \mathrm{E}+00$ & 0 & $0.00 \mathrm{E}+00$ & 0 \\
\hline 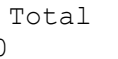 & 6 & 0 & 4 & 0 & 0.00 & 0 & 1 & 26 & -03 & 0 & $98 \mathrm{E}-02$ & 12 & 06 & 0 & 2 & 55 \\
\hline
\end{tabular}

Total Dose Contributions TDOSE $(i, p, t)$ for Individual Radionuclides (i) and Pathways (p) in mrem/yr and as a Percentage of Total Dose at $t=17$ years

$0 \quad$ Directly from primary contamination and from release to atmosphere (Inhalation excludes radon)

Radio- Ground Inhalation Radon $\quad$ Plant Meat Milk Pathways

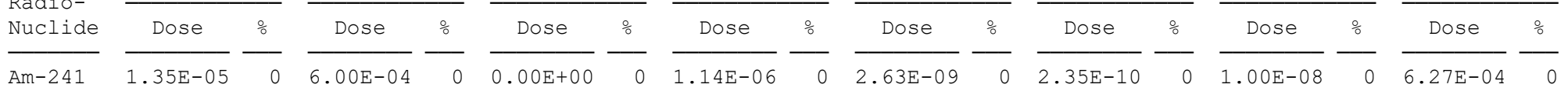

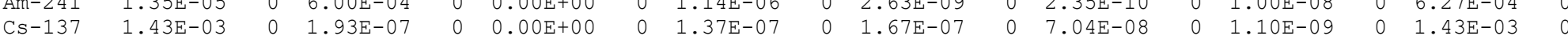

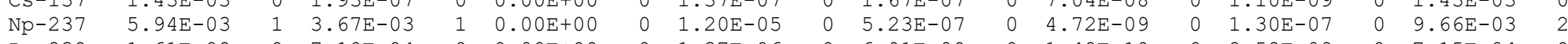

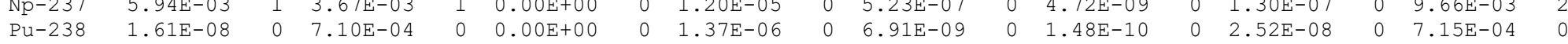

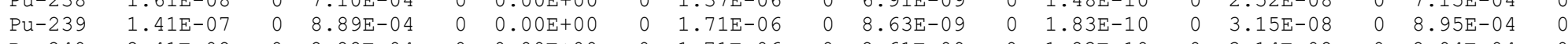

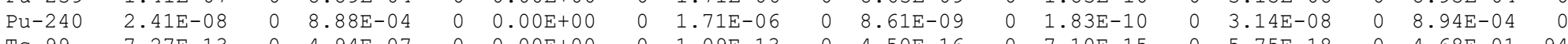

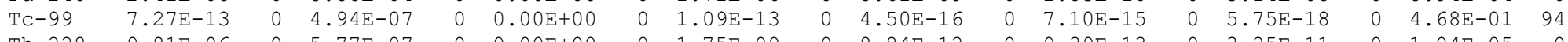

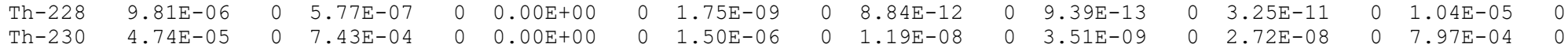

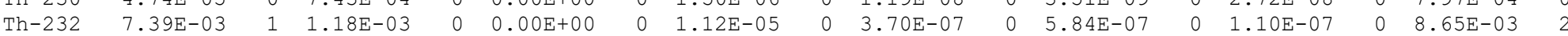

$\begin{array}{lllllllllllllllll}\mathrm{U}-234 & 1.51 \mathrm{E}-06 & 0 & 6.60 \mathrm{E}-04 & 0 & 0.00 \mathrm{E}+00 & 0 & 3.26 \mathrm{E}-06 & 0 & 5.29 \mathrm{E}-08 & 0 & 2.00 \mathrm{E}-07 & 0 & 4.20 \mathrm{E}-08 & 0 & 6.68 \mathrm{E}-04 & 0\end{array}$

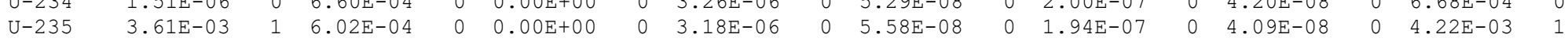

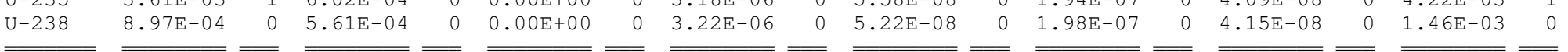

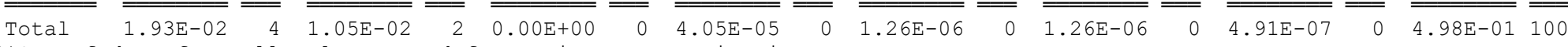
0 Sum of dose from all releases and from primary contamination.

1RESRAD-OFFSITE, Version 4.0 .3 beta T1/2 Limit $=30$ days $\quad 06 / 11 / 202011: 17$ Page 56

Parent Dose Report

Title : Landfill Scenario

File : LANDFILL-PADUCAH-AUTHORIZED-RELEASE-REV.ROF

Total Dose Contributions TDOSE (i,p,t) for Individual Radionuclides (i) and Pathways (p) in mrem/yr and as a Percentage of Total Dose at $t=420$ years

\begin{tabular}{|c|c|c|c|c|c|c|c|c|c|c|c|c|c|c|c|c|}
\hline \multirow{2}{*}{$\begin{array}{l}\text { Radio- } \\
\text { Nuclide }\end{array}$} & \multicolumn{2}{|c|}{ Ground } & \multicolumn{2}{|l|}{ Fish } & \multicolumn{2}{|c|}{$\begin{array}{l}\text { From } \\
\text { Radon }\end{array}$} & \multicolumn{2}{|c|}{$\begin{array}{l}\text { ses to grou } \\
\text { Plant }\end{array}$} & \multicolumn{2}{|c|}{$\begin{array}{l}\text { water and } t \\
\text { Meat }\end{array}$} & \multicolumn{2}{|c|}{ water } & \multicolumn{2}{|l|}{ Soil } & \multicolumn{2}{|c|}{ Water } \\
\hline & Dose & $\div$ & Dose & $\frac{\circ}{0}$ & Dose & $\div$ & Dose & $\div$ & Dose & $\frac{\circ}{0}$ & Dose & $\div$ & Dose & $\frac{\circ}{0}$ & Dose & 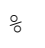 \\
\hline & & 0 & & 0 & & 0 & $94 \mathrm{~F}-03$ & 6 & 1. $40 F-1$ & c & 2.01E-06 & 0 & $.28 \mathrm{E}-$ & 0 & & \\
\hline & U⿻一𠃋十 & 0 & $U \mathrm{~L}^{-}$ & 0 & OL & 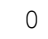 & 0 & 0 & 00 & 0 & & & D & & & \\
\hline Np-237 & $38 \mathrm{E}-18$ & 0 & $0 E-05$ & 0 & $0 \mathrm{E}+00$ & 0 & $0 E-15$ & 0 & $3.65 \mathrm{E}-16$ & - & $34 \mathrm{E}-15$ & 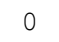 & $5.79 \mathrm{E}-18$ & 0 & $1.83 \mathrm{E}-14$ & \\
\hline $\mathrm{Pu}-238$ & $43 E-20$ & 0 & $1.33 \mathrm{E}-07$ & 0 & $0.00 \mathrm{E}+00$ & 0 & & 0 & & & & 0 & & 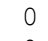 & $.09 E-15$ & \\
\hline $\mathrm{Pu}-239$ & $39 \mathrm{E}-18$ & 0 & $6 \mathrm{E}-06$ & 0 & $\pi$ & ( & & 0 & & 0 & & 0 & & 0 & 6 & \\
\hline $\mathrm{Pu}-240$ & $8.16 \mathrm{E}-23$ & 0 & $3.84 \mathrm{E}-06$ & 0 & $0.00 \mathrm{E}+00$ & 0 & $4.70 \mathrm{E}-18$ & 0 & $2.29 \mathrm{E}-19$ & 0 & $1.47 \mathrm{E}-18$ & 0 & $3.60 \mathrm{E}-21$ & 0 & $1.15 \mathrm{E}-17$ & \\
\hline & & & & & & & & & & & & & & & & \\
\hline
\end{tabular}




\begin{tabular}{|c|c|c|c|c|c|c|c|c|c|c|c|c|c|c|c|c|}
\hline Th-228 & $0.00 \mathrm{E}+00$ & 0 & $0.00 \mathrm{E}+00$ & 0 & $0.00 \mathrm{E}+00$ & 0 & $0.00 \mathrm{E}+00$ & 0 & $0.00 \mathrm{E}+00$ & 0 & $0.00 \mathrm{E}+00$ & 0 & $0.00 \mathrm{E}+00$ & 0 & $0.00 \mathrm{E}+00$ & 0 \\
\hline Th-230 & $0.00 \mathrm{E}+00$ & 0 & $2.34 \mathrm{E}-04$ & 1 & $0.00 \mathrm{E}+00$ & 0 & $0.00 \mathrm{E}+00$ & 0 & $0.00 \mathrm{E}+00$ & 0 & $0.00 \mathrm{E}+00$ & 0 & $0.00 \mathrm{E}+00$ & 0 & $0.00 \mathrm{E}+00$ & 0 \\
\hline Th-232 & $0.00 \mathrm{E}+00$ & 0 & $3.13 \mathrm{E}-04$ & 1 & $0.00 \mathrm{E}+00$ & 0 & $0.00 \mathrm{E}+00$ & 0 & $0.00 \mathrm{E}+00$ & 0 & $0.00 \mathrm{E}+00$ & 0 & $0.00 \mathrm{E}+00$ & 0 & $0.00 \mathrm{E}+00$ & 0 \\
\hline $\mathrm{U}-234$ & $8.04 \mathrm{E}-15$ & 0 & $4.11 \mathrm{E}-06$ & 0 & $0.00 \mathrm{E}+00$ & 0 & $2.26 \mathrm{E}-10$ & 0 & $1.10 \mathrm{E}-11$ & 0 & $7.04 \mathrm{E}-11$ & 0 & $1.93 \mathrm{E}-13$ & 0 & $5.50 \mathrm{E}-10$ & 0 \\
\hline $\mathrm{U}-235$ & $8.19 \mathrm{E}-10$ & 0 & $2.48 \mathrm{E}-06$ & 0 & $0.00 \mathrm{E}+00$ & 0 & $9.10 \mathrm{E}-08$ & 0 & $2.92 \mathrm{E}-10$ & 0 & $1.01 \mathrm{E}-09$ & 0 & $7.94 \mathrm{E}-11$ & 0 & $2.21 \mathrm{E}-07$ & 0 \\
\hline $\mathrm{U}-238$ & $3.88 \mathrm{E}-12$ & 0 & $8.86 \mathrm{E}-07$ & 0 & $0.00 \mathrm{E}+00$ & 0 & $2.23 \mathrm{E}-10$ & 0 & $1.09 \mathrm{E}-11$ & 0 & $6.97 \mathrm{E}-11$ & 0 & $1.91 \mathrm{E}-13$ & 0 & $5.45 \mathrm{E}-10$ & 0 \\
\hline & -06 & 0 & $93 E-04$ & 2 & $00 \mathrm{E}+\mathrm{C}$ & 0 & 94 & 6 & .40 & 0 & $02 \mathrm{E}-06$ & 0 & $28 \mathrm{E}-06$ & 0 & $.72 \mathrm{E}-03$ & \\
\hline
\end{tabular}

Total Dose Contributions TDOSE $(i, p, t)$ for Individual Radionuclides (i) and Pathways (p) in mrem/yr and as a Percentage of Total Dose at $t=420$ years

$0 \quad$ Directly from primary contamination and from release to atmosphere (Inhalation excludes radon)

\begin{tabular}{|c|c|c|c|c|c|c|c|c|c|c|c|c|c|c|c|c|}
\hline \multirow{2}{*}{$\begin{array}{l}\text { Radio- } \\
\text { Nuclide }\end{array}$} & \multicolumn{2}{|c|}{ Ground } & \multicolumn{2}{|c|}{ Inhalation } & \multicolumn{2}{|c|}{ Radon } & \multicolumn{2}{|c|}{ Plant } & \multicolumn{2}{|l|}{ Meat } & \multicolumn{2}{|l|}{$\operatorname{Milk}$} & \multicolumn{2}{|l|}{ Soil } & \multicolumn{2}{|c|}{ All Pathways } \\
\hline & Dose & $\div$ & Dose & 응 & Dose & $\%$ & Dose & $\div$ & Dose & \% & Dose & $\div$ & Dose & $\div$ & Dose & $\%$ \\
\hline$m-241$ & $2.70 \mathrm{E}-07$ & 0 & $.87 \mathrm{E}-05$ & 0 & $.00 \mathrm{E}+00$ & 0 & $2.16 \mathrm{E}-08$ & 0 & $5.54 \mathrm{E}-11$ & 0 & $4.51 \mathrm{E}-12$ & 0 & $2.30 \mathrm{E}-10$ & 0 & $6.71 \mathrm{E}-03$ & 22 \\
\hline Cs-137 & $1.30 \mathrm{E}-07$ & 0 & $1.73 \mathrm{E}-11$ & 0 & $0.00 \mathrm{E}+00$ & 0 & $1.60 \mathrm{E}-10$ & 0 & $1.89 \mathrm{E}-10$ & 0 & $5.13 \mathrm{E}-11$ & 0 & $2.15 \mathrm{E}-12$ & 0 & $1.31 \mathrm{E}-07$ & 0 \\
\hline $\mathrm{Np}-237$ & $4.61 \mathrm{E}-03$ & 15 & $2.83 \mathrm{E}-03$ & 9 & $0.00 \mathrm{E}+00$ & 0 & $3.24 \mathrm{E}-05$ & 0 & $1.46 \mathrm{E}-06$ & 0 & $9.07 \mathrm{E}-09$ & 0 & $7.40 \mathrm{E}-07$ & 0 & $7.51 \mathrm{E}-03$ & 25 \\
\hline $\mathrm{Pu}-238$ & $9.46 \mathrm{E}-10$ & 0 & $2.86 \mathrm{E}-05$ & 0 & $0.00 \mathrm{E}+00$ & 0 & $8.90 \mathrm{E}-08$ & 0 & $1.22 \mathrm{E}-09$ & 0 & $1.96 \mathrm{E}-11$ & 0 & $1.98 \mathrm{E}-08$ & 0 & $2.88 \mathrm{E}-05$ & 0 \\
\hline $\mathrm{Pu}-239$ & $1.38 \mathrm{E}-07$ & 0 & $8.54 \mathrm{E}-04$ & 3 & $0.00 \mathrm{E}+00$ & 0 & $2.65 \mathrm{E}-06$ & 0 & $3.63 \mathrm{E}-08$ & 0 & $4.92 \mathrm{E}-10$ & 0 & $5.90 \mathrm{E}-07$ & 0 & $8.61 \mathrm{E}-04$ & 3 \\
\hline $\mathrm{Pu}-240$ & $2.38 \mathrm{E}-08$ & 0 & $8.26 \mathrm{E}-04$ & 3 & $0.00 \mathrm{E}+00$ & 0 & $2.56 \mathrm{E}-06$ & 0 & $3.51 \mathrm{E}-08$ & 0 & $4.76 \mathrm{E}-10$ & 0 & $5.71 \mathrm{E}-07$ & 0 & $8.33 \mathrm{E}-04$ & 3 \\
\hline ТC-99 & $0.00 \mathrm{E}+00$ & 0 & $0.00 \mathrm{E}+00$ & 0 & $0.00 \mathrm{E}+00$ & 0 & $0.00 \mathrm{E}+00$ & 0 & $0.00 \mathrm{E}+00$ & 0 & $0.00 \mathrm{E}+00$ & 0 & $0.00 \mathrm{E}+00$ & 0 & $9.81 \mathrm{E}-45$ & 0 \\
\hline Th-228 & $2.94 \mathrm{E}-44$ & 0 & $0.00 \mathrm{E}+00$ & 0 & $0.00 \mathrm{E}+00$ & 0 & $0.00 \mathrm{E}+00$ & 0 & $0.00 \mathrm{E}+00$ & 0 & $0.00 \mathrm{E}+00$ & 0 & $0.00 \mathrm{E}+00$ & 0 & $2.94 \mathrm{E}-44$ & 0 \\
\hline Th-230 & $6.79 \mathrm{E}-04$ & 2 & $7.58 \mathrm{E}-04$ & 3 & $0.00 \mathrm{E}+00$ & 0 & $5.90 \mathrm{E}-06$ & 0 & $4.33 E-07$ & 0 & $1.29 \mathrm{E}-07$ & 0 & $7.32 \mathrm{E}-07$ & 0 & $1.68 \mathrm{E}-03$ & 6 \\
\hline Th-232 & $8.92 \mathrm{E}-03$ & 30 & $1.26 \mathrm{E}-03$ & 4 & $0.00 \mathrm{E}+00$ & 0 & $1.28 \mathrm{E}-04$ & 0 & $4.90 \mathrm{E}-06$ & P & $4.99 \mathrm{E}-06$ & 0 & $2.70 \mathrm{E}-06$ & $h$ & $1.06 \mathrm{E}-02$ & 35 \\
\hline $\mathrm{U}-234$ & $1.01 \mathrm{E}-05$ & 0 & $1.88 \mathrm{E}-04$ & 1 & $0.00 \mathrm{E}+00$ & 0 & $9.92 \mathrm{E}-07$ & 0 & $2.18 \mathrm{E}-08$ & 0 & $5.84 \mathrm{E}-08$ & 0 & $3.14 \mathrm{E}-08$ & 0 & $2.03 \mathrm{E}-04$ & 1 \\
\hline $\mathrm{U}-235$ & $9.71 \mathrm{E}-04$ & 3 & $2.54 \mathrm{E}-04$ & 1 & $0.00 \mathrm{E}+00$ & 0 & $1.18 \mathrm{E}-06$ & 0 & $5.23 \mathrm{E}-08$ & 0 & $5.52 \mathrm{E}-08$ & 0 & $2.58 \mathrm{E}-08$ & 0 & $1.23 \mathrm{E}-03$ & 4 \\
\hline $\mathrm{U}-238$ & $2.36 \mathrm{E}-04$ & 1 & $1.47 \mathrm{E}-04$ & 0 & $0.00 \mathrm{E}+00$ & 0 & $8.94 \mathrm{E}-07$ & 0 & $1.59 \mathrm{E}-08$ & 0 & $5.61 \mathrm{E}-08$ & 0 & $2.13 \mathrm{E}-08$ & 0 & $3.85 \mathrm{E}-04$ & 1 \\
\hline oldt & $1.54 \mathrm{E}-02$ & 51 & $7.17 \mathrm{E}-03$ & 24 & +00 & 0 & -04 & 1 & $5 E-06$ & 0 & $30 E-06$ & 0 & $.43 E-06$ & 0 & $.01 \mathrm{E}-02$ & 0 \\
\hline
\end{tabular}

* Sum of dose from all releases and from primary contamination.

IRESRAD-OFFSITE, Version 4.0 .3 beta T1/2 Limit $=30$ days $\quad 06 / 11 / 2020 \quad 11: 17$ Page 57

Parent Dose Report

Title : Landfill Scenario

File : LANDFILL-PADUCAH-AUTHORIZED-RELEASE-REV.ROF

Total Dose Contributions TDOSE (i,p,t) for Individual Radionuclides (i) and Pathways (p) in mrem/yr and as a Percentage of Total Dose at $t=778$ years

\begin{tabular}{|c|c|c|c|c|c|c|c|c|c|c|c|c|c|c|c|c|}
\hline \multirow{2}{*}{$\begin{array}{l}\text { Radio- } \\
\text { Nuclide }\end{array}$} & \multicolumn{2}{|c|}{ Ground } & \multicolumn{2}{|l|}{ Fish } & \multicolumn{2}{|c|}{$\begin{array}{l}\text { From } \\
\text { Radon }\end{array}$} & \multicolumn{2}{|c|}{$\begin{array}{l}\text { ses to grour } \\
\text { Plant }\end{array}$} & \multicolumn{2}{|c|}{$\begin{array}{l}\text { water and } t \\
\text { Meat }\end{array}$} & \multicolumn{2}{|c|}{ wate } & \multicolumn{2}{|l|}{ Soil } & \multicolumn{2}{|c|}{ Water } \\
\hline & Dose & $\div$ & Dose & $\frac{\circ}{0}$ & Dose & $\div$ & Dose & $\%$ & Dose & $\frac{\circ}{0}$ & Dose & $\frac{\circ}{0}$ & Dose & $\frac{\circ}{0}$ & Dose & \\
\hline & & 0 & & 0 & & 0 & $05 \mathrm{~F}-02$ & 17 & $58 \mathrm{E}-\mathrm{C}$ & c & $1.09 \mathrm{E}-$ & 0 & $.41 \mathrm{E}-$ & 0 & & \\
\hline & $U E+00$ & 0 & $4 \mathrm{E}-\mathrm{-}-\mathrm{s}$ & 0 & OL & 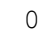 & D & 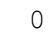 & 00 & 0 & & & & & & \\
\hline Np-237 & $.35 E-10$ & 0 & $8 E-05$ & 0 & $0 \mathrm{E}+00$ & 0 & $6.61 \mathrm{E}-08$ & 0 & $3.24 \mathrm{E}-09$ & 0 & $05 \mathrm{E}-08$ & s & $1.60 \mathrm{E}-10$ & 0 & $1.59 \mathrm{E}-07$ & \\
\hline $\mathrm{Pu}-238$ & $.93 E-13$ & 0 & $85 \mathrm{E}-09$ & 0 & $20 F+0$ & $P_{1}>$ & & 0 & & & & 0 & & 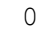 & & \\
\hline $\mathrm{Pu}-$ & $52 \mathrm{E}-13$ & 0 & -06 & 0 & & ( & & 0 & & 0 & & 0 & & 0 & 2 & \\
\hline $\mathrm{Pu}-240$ & $1.98 \mathrm{E}-15$ & 0 & $3.58 \mathrm{E}-06$ & 0 & $0.00 \mathrm{E}+00$ & 0 & $3.75 \mathrm{E}-11$ & 0 & $1.84 \mathrm{E}-12$ & 0 & $1.16 \mathrm{E}-11$ & 0 & $8.74 E-14$ & 0 & $9.05 E-11$ & \\
\hline & & & & & & & & & & & & & & & & \\
\hline
\end{tabular}




\begin{tabular}{|c|c|c|c|c|c|c|c|c|c|c|c|c|c|c|c|c|}
\hline Th-228 & $0.00 \mathrm{E}+00$ & 0 & $0.00 \mathrm{E}+00$ & 0 & $0.00 \mathrm{E}+00$ & 0 & $0.00 \mathrm{E}+00$ & 0 & $0.00 \mathrm{E}+00$ & 0 & $0.00 \mathrm{E}+00$ & 0 & $0.00 \mathrm{E}+00$ & 0 & $0.00 \mathrm{E}+00$ & 0 \\
\hline Th-230 & $2.56 \mathrm{E}-09$ & 0 & $3.06 \mathrm{E}-04$ & 1 & $0.00 \mathrm{E}+00$ & 0 & $6.18 \mathrm{E}-09$ & 0 & $8.58 \mathrm{E}-10$ & 0 & $2.60 \mathrm{E}-09$ & 0 & $3.70 \mathrm{E}-11$ & 0 & $1.18 \mathrm{E}-08$ & 0 \\
\hline Th-232 & $4.50 \mathrm{E}-41$ & 0 & $3.19 \mathrm{E}-04$ & 1 & $0.00 \mathrm{E}+00$ & 0 & $3.85 \mathrm{E}-40$ & 0 & $5.24 \mathrm{E}-41$ & 0 & $1.86 \mathrm{E}-40$ & 0 & $4.22 \mathrm{E}-43$ & 0 & $8.64 E-40$ & 0 \\
\hline U-234 & $2.77 \mathrm{E}-08$ & 0 & $6.58 \mathrm{E}-06$ & 0 & $0.00 \mathrm{E}+00$ & 0 & $2.43 E-04$ & 0 & $1.19 \mathrm{E}-05$ & 0 & $7.53 \mathrm{E}-05$ & 0 & $5.96 \mathrm{E}-07$ & 0 & $5.86 \mathrm{E}-04$ & 1 \\
\hline U-235 & $4.93 E-05$ & 0 & $1.19 \mathrm{E}-06$ & 0 & $0.00 \mathrm{E}+00$ & 0 & $2.97 \mathrm{E}-04$ & 0 & $5.39 \mathrm{E}-05$ & 0 & $7.31 \mathrm{E}-05$ & 0 & $7.66 \mathrm{E}-07$ & 0 & $7.08 \mathrm{E}-04$ & 1 \\
\hline $\mathrm{U}-238$ & $1.20 \mathrm{E}-05$ & 0 & $2.73 \mathrm{E}-07$ & 0 & $0.00 \mathrm{E}+00$ & 0 & $2.41 \mathrm{E}-04$ & 0 & $1.18 \mathrm{E}-05$ & 0 & $7.47 \mathrm{E}-05$ & 0 & $5.90 \mathrm{E}-07$ & 0 & $5.81 \mathrm{E}-04$ & 1 \\
\hline$t$ & $7.66 \mathrm{E}-05$ & 0 & $.64 E-04$ & 1 & $.00 \mathrm{E}+00$ & 0 & $1.13 \mathrm{E}-02$ & 19 & $1.54 \mathrm{E}-04$ & 0 & $2.34 \mathrm{E}-04$ & 0 & $E-05$ & 0 & 2 & 5 \\
\hline
\end{tabular}

Total Dose Contributions TDOSE (i,p,t) for Individual Radionuclides (i) and Pathways (p) in mrem/yr and as a Percentage of Total Dose at $t=778$ years

Directly from primary contamination and from release to atmosphere (Inhalation excludes radon)

\begin{tabular}{|c|c|c|c|c|c|c|c|c|c|c|c|c|c|c|c|c|}
\hline \multirow{2}{*}{$\begin{array}{l}\text { Radio- } \\
\text { Nuclide }\end{array}$} & \multicolumn{2}{|c|}{ Ground } & \multicolumn{2}{|c|}{ Inhalation } & \multicolumn{2}{|c|}{ Radon } & \multicolumn{2}{|c|}{ Plant } & \multicolumn{2}{|l|}{ Meat } & \multicolumn{2}{|l|}{ Milk } & \multicolumn{2}{|l|}{ Soil } & \multicolumn{2}{|c|}{ All Pathways* } \\
\hline & Dose & $\%$ & Dose & $\%$ & Dose & $\%$ & Dose & $\%$ & Dose & $\%$ & Dose & $\%$ & Dose & $\frac{0}{0}$ & Dose & $\%$ \\
\hline$A m-241$ & $2.04 \mathrm{E}-08$ & 0 & $.08 \mathrm{E}-04$ & 0 & $0.00 \mathrm{E}+00$ & 0 & $7.26 \mathrm{E}-10$ & 0 & $5.65 \mathrm{E}-12$ & 0 & $1.58 \mathrm{E}-13$ & 0 & $8.81 E-12$ & 0 & $3.62 \mathrm{E}-02$ & 60 \\
\hline $\mathrm{Cs}-137$ & $3.34 \mathrm{E}-11$ & 0 & $4.38 \mathrm{E}-15$ & 0 & $0.00 \mathrm{E}+00$ & 0 & $6.74 \mathrm{E}-14$ & 0 & $7.97 \mathrm{E}-14$ & 0 & $2.12 \mathrm{E}-14$ & 0 & $9.26 \mathrm{E}-16$ & 0 & $3.37 E-11$ & 0 \\
\hline $\mathrm{Np}-237$ & $3.66 \mathrm{E}-03$ & 6 & $2.25 E-03$ & 4 & $0.00 \mathrm{E}+00$ & 0 & $2.64 \mathrm{E}-05$ & 0 & $1.19 \mathrm{E}-06$ & 0 & $7.40 \mathrm{E}-09$ & 0 & $6.07 \mathrm{E}-07$ & 0 & $5.96 \mathrm{E}-03$ & 10 \\
\hline $\mathrm{Pu}-238$ & $5.99 \mathrm{E}-10$ & 0 & $1.65 \mathrm{E}-06$ & 0 & $0.00 \mathrm{E}+00$ & 0 & $6.26 \mathrm{E}-09$ & 0 & $1.02 \mathrm{E}-10$ & 0 & $2.45 \mathrm{E}-12$ & 0 & $1.78 \mathrm{E}-09$ & 0 & $1.67 \mathrm{E}-06$ & 0 \\
\hline $\mathrm{Pu}-239$ & $1.34 \mathrm{E}-07$ & 0 & $8.22 \mathrm{E}-04$ & 1 & $0.00 \mathrm{E}+00$ & 0 & $3.11 \mathrm{E}-06$ & 0 & $5.06 \mathrm{E}-08$ & 0 & $6.49 \mathrm{E}-10$ & 0 & $8.87 \mathrm{E}-07$ & 0 & $8.30 \mathrm{E}-04$ & 1 \\
\hline $\mathrm{Pu}-240$ & $2.30 E-08$ & 0 & $7.74 \mathrm{E}-04$ & 1 & $0.00 \mathrm{E}+00$ & 0 & $2.92 \mathrm{E}-06$ & 0 & $4.76 \mathrm{E}-08$ & 0 & $6.12 \mathrm{E}-10$ & 0 & $8.36 \mathrm{E}-07$ & 0 & $7.82 \mathrm{E}-04$ & 1 \\
\hline $\mathrm{TC}-99$ & $0.00 \mathrm{E}+00$ & 0 & $0.00 \mathrm{E}+00$ & 0 & $0.00 \mathrm{E}+00$ & 0 & $0.00 \mathrm{E}+00$ & 0 & $0.00 \mathrm{E}+00$ & 0 & $0.00 \mathrm{E}+00$ & 0 & $0.00 \mathrm{E}+00$ & 0 & $9.81 E-45$ & 0 \\
\hline Th-228 & $2.94 E-44$ & 0 & $0.00 \mathrm{E}+00$ & 0 & $0.00 \mathrm{E}+00$ & 0 & $0.00 \mathrm{E}+00$ & 0 & $0.00 \mathrm{E}+00$ & 0 & $0.00 \mathrm{E}+00$ & 0 & $0.00 \mathrm{E}+00$ & 0 & 2.94 & 0 \\
\hline Th-230 & $8.69 \mathrm{E}-04$ & 1 & $7.62 \mathrm{E}-04$ & 1 & $0.00 \mathrm{E}+00$ & 0 & $8.51 \mathrm{E}-06$ & 0 & $6.49 \mathrm{E}-07$ & 0 & $1.92 \mathrm{E}-07$ & 0 & 1. $32 \mathrm{E}-06$ & 0 & $1.95 \mathrm{E}-03$ & 3 \\
\hline Th-232 & $9.09 \mathrm{E}-03$ & 15 & $1.26 \mathrm{E}-03$ & 2 & $0.00 \mathrm{E}+00$ & 0 & $2.26 \mathrm{E}-04$ & 0 & $8.71 \mathrm{E}-06$ & 0 & $8.68 \mathrm{E}-06$ & 0 & $4.94 \mathrm{E}-06$ & 0 & $1.09 \mathrm{E}-02$ & 18 \\
\hline $\mathrm{U}-234$ & $1.83 E-05$ & 0 & $7.34 \mathrm{E}-05$ & 0 & $0.00 \mathrm{E}+00$ & 0 & $4.46 \mathrm{E}-07$ & 0 & $1.75 \mathrm{E}-08$ & 0 & $2.10 \mathrm{E}-08$ & 0 & $3.07 \mathrm{E}-08$ & 0 & $1.02 \mathrm{E}-03$ & 2 \\
\hline $\mathrm{U}-235$ & $3.02 \mathrm{E}-04$ & 1 & $1.06 \mathrm{E}-04$ & 0 & $0.00 \mathrm{E}+00$ & 0 & $4.41 \mathrm{E}-07$ & 0 & $2.54 \mathrm{E}-08$ & 0 & $1.69 \mathrm{E}-08$ & 0 & 9.15 & 0 & 1.59 & 3 \\
\hline $\mathrm{U}-238$ & $7.18 \mathrm{E}-05$ & 0 & $4.66 \mathrm{E}-05$ & 0 & $0.00 \mathrm{E}+00$ & 0 & $2.73 \mathrm{E}-07$ & 0 & $4.84 \mathrm{E}-09$ & 0 & $1.71 \mathrm{E}-08$ & 0 & $6.51 \mathrm{E}-09$ & 0 & $1.04 \mathrm{E}-03$ & 2 \\
\hline & & 23 & $20 E-03$ & 10 & $00 \mathrm{E}+00$ & 0 & $4=$ & 0 & 1.0 & 0 & $8.935-06$ & 0 & $8.63 \mathrm{E}-06$ & 0 & $6.03 \mathrm{E}-02$ & 100 \\
\hline
\end{tabular}

Total

$1.40 \mathrm{E}-02 \quad 23 \quad 6.20 \mathrm{E}-03 \quad 10$

$\begin{array}{lll}0.00 \mathrm{E}+00 & 0 & 2.68 \mathrm{E}-04 \\ \text { from primary contamination. }\end{array}$

1RESRAD-OFFSITE, Version 4.0 .3 beta $T^{1 / 2}$ Limit $=30$ days $\quad 06 / 11 / 2020 \quad 11: 17$ Page 58

Parent Dose Report

Title : Landfill Scenario

File : LANDFILL-PADUCAH-AUTHORIZED-RELEASE-REV.ROF

Total Dose Contributions TDOSE (i,p,t) for Individual Radionuclides (i) and Pathways (p) in mrem/yr and as a Percentage of Total Dose at $t=800$ years

\begin{tabular}{|c|c|c|c|c|c|c|c|c|c|c|c|c|c|c|c|c|}
\hline \multirow{2}{*}{$\begin{array}{l}0 \\
0 \\
\text { Radio- } \\
\text { Nuclide }\end{array}$} & \multicolumn{2}{|c|}{ Ground } & \multicolumn{2}{|l|}{ Fish } & \multicolumn{2}{|c|}{$\begin{array}{l}\text { From } \\
\text { Radon }\end{array}$} & \multicolumn{2}{|c|}{$\begin{array}{l}\text { es to } 9 \\
\text { Plant }\end{array}$} & \multicolumn{2}{|c|}{$\begin{array}{l}\text { water and } t \\
\text { Meat }\end{array}$} & \multicolumn{2}{|c|}{$\begin{array}{l}\text { o surface wate } \\
\text { Milk }\end{array}$} & \multicolumn{2}{|l|}{ Soil } & \multicolumn{2}{|l|}{ Water } \\
\hline & Dose & $\%$ & Dose & $\%$ & Dose & $\%$ & Dose & $\%$ & Dose & $\%$ & Dose & $\%$ & Dose & $\%$ & Dose & \\
\hline & & 0 & & 0 & & 0 & 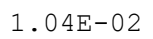 & 17 & $775 \mathrm{~F}-05$ & 0 & & 0 & & 0 & & \\
\hline & $0 E+00$ & 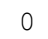 & $5 E-14$ & 0 & UE & ( & 0 & 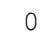 & 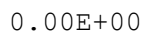 & 0 & & & & c & & \\
\hline Np-237 & $18 \mathrm{E}-10$ & 0 & $34 E-05$ & 0 & $0 \mathrm{E}+00$ & 0 & $91 \mathrm{E}-08$ & 0 & $4.87 \mathrm{E}-09$ & 0 & 8 & S & $46 \mathrm{E}-10$ & 0 & $.39 \mathrm{E}-07$ & \\
\hline $\mathrm{Pu}-238$ & $4.40 \mathrm{E}-13$ & 0 & $6.62 \mathrm{E}-09$ & 0 & $0 \mathrm{E}+00$ & 0 & $6 \mathrm{E}-09$ & 0 & $1.94 \mathrm{E}-10$ & 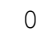 & & 0 & & 0 & $.54 \mathrm{E}-09$ & \\
\hline $\mathrm{Pu}-239$ & $81 \mathrm{E}-13$ & 0 & $3.80 E-06$ & & 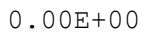 & 0 & $36 \mathrm{E}-12$ & - & & & & & 50 & 0 & -12 & \\
\hline $\mathrm{Pu}-240$ & $3.00 \mathrm{E}-15$ & 0 & $3.57 \mathrm{E}-06$ & 0 & $0.00 \mathrm{E}+00$ & 0 & $.57 \mathrm{E}-11$ & 0 & $2.74 \mathrm{E}-12$ & 0 & $.73 \mathrm{E}-11$ & 0 & $1.32 \mathrm{E}-13$ & 0 & $.34 \mathrm{E}-10$ & \\
\hline & & & & & & & & & & & & $c_{0}>$ & & & & \\
\hline
\end{tabular}




\begin{tabular}{|c|c|c|c|c|c|c|c|c|c|c|c|c|c|c|c|c|}
\hline Th-228 & $0.00 \mathrm{E}+00$ & 0 & $0.00 \mathrm{E}+00$ & 0 & $0.00 \mathrm{E}+00$ & 0 & $0.00 \mathrm{E}+00$ & 0 & $0.00 \mathrm{E}+00$ & 0 & $0.00 \mathrm{E}+00$ & 0 & $0.00 \mathrm{E}+00$ & 0 & $0.00 \mathrm{E}+00$ & 0 \\
\hline Th-230 & $5.00 \mathrm{E}-09$ & 0 & $3.08 \mathrm{E}-04$ & 1 & $0.00 \mathrm{E}+00$ & 0 & $1.17 \mathrm{E}-08$ & 0 & $1.63 \mathrm{E}-09$ & 0 & $4.89 E-09$ & 0 & $7.38 \mathrm{E}-11$ & 0 & $2.22 \mathrm{E}-08$ & 0 \\
\hline Th-232 & $4.50 \mathrm{E}-41$ & 0 & $3.19 \mathrm{E}-04$ & 1 & $0.00 \mathrm{E}+00$ & 0 & $3.85 E-40$ & 0 & $5.24 \mathrm{E}-41$ & 0 & $1.86 \mathrm{E}-40$ & 0 & $4.22 \mathrm{E}-43$ & 0 & $8.63 E-40$ & 0 \\
\hline U-234 & $3.96 \mathrm{E}-08$ & 0 & $6.70 \mathrm{E}-06$ & 0 & $0.00 \mathrm{E}+00$ & 0 & $3.37 \mathrm{E}-04$ & 1 & $1.66 \mathrm{E}-05$ & 0 & $1.04 \mathrm{E}-04$ & 0 & $8.43 E-07$ & 0 & $8.12 \mathrm{E}-04$ & 1 \\
\hline U-235 & $6.98 \mathrm{E}-05$ & 0 & $1.14 \mathrm{E}-06$ & 0 & $0.00 \mathrm{E}+00$ & 0 & $4.14 \mathrm{E}-04$ & 1 & $7.65 \mathrm{E}-05$ & 0 & $1.01 \mathrm{E}-04$ & 0 & $1.09 E-06$ & 0 & $9.88 \mathrm{E}-04$ & 2 \\
\hline $\mathrm{U}-238$ & $1.69 \mathrm{E}-05$ & 0 & $2.55 \mathrm{E}-07$ & 0 & $0.00 \mathrm{E}+00$ & 0 & $3.35 \mathrm{E}-04$ & 1 & $1.64 \mathrm{E}-05$ & 0 & $1.04 \mathrm{E}-04$ & 0 & $8.35 E-07$ & 0 & $8.06 \mathrm{E}-04$ & 1 \\
\hline ta & -04 & 0 & $.66 \mathrm{E}-04$ & 1 & $.00 \mathrm{E}+00$ & 0 & $.15 \mathrm{E}-02$ & 19 & $1.85 \mathrm{E}-04$ & 0 & 04 & 1 & -05 & 0 & 2 & 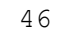 \\
\hline
\end{tabular}

Total Dose Contributions TDose $(i, p, t)$ for Individual Radionuclides (i) and Pathways (p)

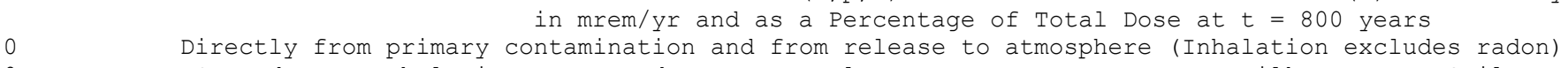

\begin{tabular}{|c|c|c|c|c|c|c|c|c|c|c|c|c|c|c|c|c|}
\hline \multirow{2}{*}{$\begin{array}{l}\text { Radio- } \\
\text { Nuclide }\end{array}$} & \multicolumn{2}{|c|}{ Ground } & \multicolumn{2}{|c|}{ Inhalation } & \multicolumn{2}{|c|}{ Radon } & \multicolumn{2}{|c|}{ Plant } & \multicolumn{2}{|c|}{ Meat } & \multicolumn{2}{|c|}{ Milk } & \multicolumn{2}{|c|}{ Soil } & \multicolumn{2}{|c|}{ All Pathways* } \\
\hline & Dose & $\%$ & Dose & $\%$ & Dose & $\%$ & Dose & $\%$ & Dose & $\%$ & Dose & $\%$ & Dose & $\%$ & Dose & $\%$ \\
\hline$A m-241$ & $1.87 \mathrm{E}-08$ & 0 & $1.07 \mathrm{E}-04$ & 0 & $0.00 \mathrm{E}+00$ & 0 & $6.01 \mathrm{E}-10$ & 0 & $5.30 \mathrm{E}-12$ & 0 & 1. $32 \mathrm{E}-13$ & 0 & $7.48 \mathrm{E}-12$ & 0 & $3.61 \mathrm{E}-02$ & 59 \\
\hline $\mathrm{Cs}-137$ & $2.01 E-11$ & 0 & $2.63 E-15$ & 0 & $0.00 \mathrm{E}+00$ & 0 & $4.14 \mathrm{E}-14$ & 0 & $4.90 \mathrm{E}-14$ & 0 & $1.30 \mathrm{E}-14$ & 0 & $5.70 \mathrm{E}-16$ & 0 & $2.03 \mathrm{E}-11$ & 0 \\
\hline $\mathrm{Np}-237$ & $3.61 \mathrm{E}-03$ & 6 & $2.21 \mathrm{E}-03$ & 4 & $0.00 \mathrm{E}+00$ & 0 & $2.60 \mathrm{E}-05$ & 0 & $1.17 \mathrm{E}-06$ & 0 & $7.30 \mathrm{E}-09$ & 0 & $5.99 \mathrm{E}-07$ & 0 & $5.87 \mathrm{E}-03$ & 10 \\
\hline $\mathrm{Pu}-238$ & $6.09 \mathrm{E}-10$ & 0 & $1.38 \mathrm{E}-06$ & 0 & $0.00 \mathrm{E}+00$ & 0 & $5.30 \mathrm{E}-09$ & 0 & $8.71 \mathrm{E}-11$ & 0 & $2.18 \mathrm{E}-12$ & 0 & $1.52 \mathrm{E}-09$ & 0 & $1.41 \mathrm{E}-06$ & 0 \\
\hline $\mathrm{Pu}-239$ & $1.34 \mathrm{E}-07$ & 0 & $8.20 \mathrm{E}-04$ & 1 & $0.00 \mathrm{E}+00$ & 0 & $3.13 \mathrm{E}-06$ & 0 & $5.12 \mathrm{E}-08$ & 0 & $6.56 \mathrm{E}-10$ & 0 & $9.01 \mathrm{E}-07$ & 0 & $8.28 \mathrm{E}-04$ & 1 \\
\hline $\mathrm{Pu}-240$ & $2.29 \mathrm{E}-08$ & 0 & $7.71 \mathrm{E}-04$ & 1 & $0.00 \mathrm{E}+00$ & 0 & $2.94 \mathrm{E}-06$ & 0 & $4.82 \mathrm{E}-08$ & 0 & $6.17 \mathrm{E}-10$ & 0 & $8.47 \mathrm{E}-07$ & 0 & $7.79 \mathrm{E}-04$ & 1 \\
\hline TC-99 & $0.00 \mathrm{E}+00$ & 0 & $0.00 \mathrm{E}+00$ & 0 & $0.00 \mathrm{E}+00$ & 0 & $0.00 \mathrm{E}+00$ & 0 & $0.00 \mathrm{E}+00$ & 0 & $0.00 \mathrm{E}+00$ & 0 & $0.00 \mathrm{E}+00$ & 0 & $9.81 \mathrm{E}-45$ & 0 \\
\hline Th-228 & $2.94 E-44$ & 0 & $0.00 \mathrm{E}+00$ & 0 & $0.00 \mathrm{E}+00$ & 0 & $0.00 \mathrm{E}+00$ & 0 & $0.00 \mathrm{E}+00$ & 0 & $0.00 \mathrm{E}+00$ & 0 & $0.00 \mathrm{E}+00$ & 0 & $2.94 \mathrm{E}-44$ & 0 \\
\hline Th-230 & $8.75 E-04$ & 1 & $7.63 \mathrm{E}-04$ & 1 & $0.00 \mathrm{E}+00$ & 0 & $8.65 \mathrm{E}-06$ & 0 & $6.60 \mathrm{E}-07$ & 0 & $1.95 \mathrm{E}-07$ & 0 & $1.35 \mathrm{E}-06$ & 0 & $1.96 \mathrm{E}-03$ & 3 \\
\hline Th-232 & $9.10 \mathrm{E}-03$ & 15 & $1.26 \mathrm{E}-03$ & 2 & $0.00 \mathrm{E}+00$ & 0 & $2.32 \mathrm{E}-04$ & 0 & $8.94 \mathrm{E}-06$ & 0 & $8.90 \mathrm{E}-06$ & 0 & $5.07 \mathrm{E}-06$ & 0 & $1.09 \mathrm{E}-02$ & 18 \\
\hline $\mathrm{U}-234$ & $1.87 \mathrm{E}-05$ & 0 & $7.06 \mathrm{E}-05$ & 0 & $0.00 \mathrm{E}+00$ & 0 & $4.31 \mathrm{E}-07$ & 0 & $1.76 \mathrm{E}-08$ & 0 & $1.99 \mathrm{E}-08$ & 0 & $3.11 \mathrm{E}-08$ & 0 & $1.37 \mathrm{E}-03$ & 2 \\
\hline $\mathrm{U}-235$ & $2.81 E-04$ & 0 & $1.02 \mathrm{E}-04$ & 0 & $0.00 \mathrm{E}+00$ & 0 & $4.14 \mathrm{E}-07$ & 0 & $2.41 \mathrm{E}-08$ & 0 & $1.57 \mathrm{E}-08$ & 0 & $8.58 \mathrm{E}-09$ & 0 & $2.04 \mathrm{E}-03$ & 3 \\
\hline $\mathrm{U}-238$ & $6.68 \mathrm{E}-05$ & 0 & $4.40 \mathrm{E}-05$ & 0 & $0.00 \mathrm{E}+00$ & 0 & $2.54 \mathrm{E}-07$ & 0 & $4.50 E-09$ & 0 & $1.59 \mathrm{E}-08$ & 0 & $6.06 \mathrm{E}-09$ & 0 & $1.39 \mathrm{E}-03$ & 2 \\
\hline & & 23 & $5 E-03$ & 10 & +00 & 0 & -04 & 0 & -05 & 0 & $E-06$ & 0 & $.82 \mathrm{E}-06$ & 0 & 02 & 00 \\
\hline
\end{tabular}

$\begin{array}{cccccccc}\text { Total } & 1.39 \mathrm{E}-02 & 23 & 6.15 \mathrm{E}-03 & 10 & 0.00 \mathrm{E}+00 & 0 & 2.74 \mathrm{E}-04 \\ 0 * \text { Sum of dose from all releases and from primary contamination. }\end{array}$

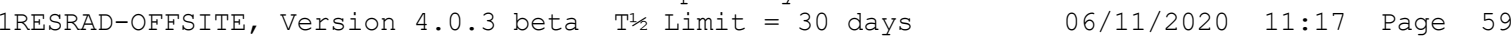

Parent Dose Report

Title : Landfill Scenario

File : LANDFILL-PADUCAH-AUTHORIZED-RELEASE-REV.ROF

Total Dose Contributions TDOSE (i,p,t) for Individual Radionuclides (i) and Pathways (p) in mrem/yr and as a Percentage of Total Dose at $t=1000$ years

\begin{tabular}{|c|c|c|c|c|c|c|c|c|c|c|c|c|c|c|c|c|}
\hline \multirow{2}{*}{$\begin{array}{l}0 \\
\text { Radio- } \\
\text { Nuclide }\end{array}$} & \multicolumn{2}{|c|}{ Ground } & \multicolumn{2}{|l|}{ Fish } & \multicolumn{2}{|c|}{$\begin{array}{l}\text { From } \\
\text { Radon }\end{array}$} & \multicolumn{2}{|c|}{$\begin{array}{l}\text { es to } g \\
\text { Plant }\end{array}$} & \multicolumn{2}{|c|}{$\begin{array}{l}\text { water and } \\
\text { Meat }\end{array}$} & \multicolumn{2}{|c|}{$\begin{array}{c}\text { o surface wate } \\
\text { Milk }\end{array}$} & \multicolumn{2}{|l|}{ Soil } & \multicolumn{2}{|l|}{ Water } \\
\hline & Dose & $\%$ & Dose & \% & Dose & $\%$ & Dose & $\div$ & Dose & 웅 & Dose & $\%$ & Dose & $\%$ & Dose & $\%$ \\
\hline $\mathrm{Am}$ & 05 & 0 & 10 & 0 & $.00 \mathrm{E}+00$ & 0 & -03 & 10 & 6.40 & 0 & 9.201 & 0 & 1.20 & 0 & $2.15 \mathrm{E}-02$ & 25 \\
\hline $\mathrm{Cs}-137$ & $0.00 \mathrm{E}+00$ & 0 & $7.86 \mathrm{E}-16$ & 0 & $.00 \mathrm{E}+00$ & 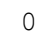 & $0.00 \mathrm{E}+00$ & 0 & $0.00 \mathrm{E}+00$ & 0 & $0.00 \mathrm{E}+00$ & 0 & $0.00 \mathrm{E}+00$ & 0 & $0.00 \mathrm{E}+00$ & 0 \\
\hline Np-237 & $5.82 \mathrm{E}-09$ & 0 & $2.06 \mathrm{E}-05$ & 0 & $0.00 \mathrm{E}+00$ & 0 & $1.50 \mathrm{E}-06$ & 0 & $7.36 \mathrm{E}-08$ & 0 & $4.63 E-07$ & 0 & $4.38 E-09$ & 0 & $3.60 \mathrm{E}-06$ & 0 \\
\hline $\mathrm{Pu}-238$ & $6.82 \mathrm{E}-12$ & 0 & $1.55 \mathrm{E}-09$ & 0 & $0.00 \mathrm{E}+00$ & 0 & $4.70 \mathrm{E}-08$ & 0 & $2.31 \mathrm{E}-09$ & 0 & $1.46 \mathrm{E}-08$ & 0 & $1.30 \mathrm{E}-10$ & 0 & $1.13 \mathrm{E}-07$ & 0 \\
\hline $\mathrm{Pu}-239$ & $6.22 \mathrm{E}-12$ & 0 & $3.71 E-06$ & 0 & $0.00 \mathrm{E}+00$ & 0 & $3.53 \mathrm{E}-11$ & 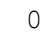 & $7.02 \mathrm{E}-12$ & 0 & $8.33 \mathrm{E}-12$ & 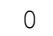 & $1.01 \mathrm{E}-13$ & ) & $8.40 \mathrm{E}-11$ & 0 \\
\hline $\mathrm{Pu}-240$ & $4.87 \mathrm{E}-14$ & 0 & $3.44 \mathrm{E}-06$ & 0 & $0.00 \mathrm{E}+00$ & 0 & $7.95 \mathrm{E}-10$ & 0 & $3.91 \mathrm{E}-11$ & 0 & $2.46 \mathrm{E}-10$ & 0 & $2.15 E-12$ & 0 & $1.91 \mathrm{E}-09$ & 0 \\
\hline & & & & & & & & 0 & & & & & & & & \\
\hline
\end{tabular}




\begin{tabular}{|c|c|c|c|c|c|c|c|c|c|c|c|c|c|c|c|c|}
\hline Th-228 & $0.00 \mathrm{E}+00$ & 0 & $0.00 \mathrm{E}+00$ & 0 & $0.00 \mathrm{E}+00$ & 0 & $0.00 \mathrm{E}+00$ & 0 & $0.00 \mathrm{E}+00$ & 0 & $0.00 \mathrm{E}+00$ & 0 & $0.00 \mathrm{E}+00$ & 0 & $0.00 \mathrm{E}+00$ & 0 \\
\hline Th-230 & $4.07 \mathrm{E}-07$ & 0 & $3.25 \mathrm{E}-04$ & 0 & $0.00 \mathrm{E}+00$ & 0 & $8.13 \mathrm{E}-07$ & 0 & $1.14 \mathrm{E}-07$ & 0 & $3.20 \mathrm{E}-07$ & 0 & $6.83 \mathrm{E}-09$ & 0 & $1.44 \mathrm{E}-06$ & 0 \\
\hline Th-232 & $4.50 \mathrm{E}-41$ & 0 & $3.19 \mathrm{E}-04$ & 0 & $0.00 \mathrm{E}+00$ & 0 & $3.84 \mathrm{E}-40$ & 0 & $5.23 E-41$ & 0 & $1.85 \mathrm{E}-40$ & 0 & $4.22 \mathrm{E}-43$ & 0 & $8.62 \mathrm{E}-40$ & 0 \\
\hline $\mathrm{U}-234$ & $4.37 \mathrm{E}-07$ & 0 & $7.63 \mathrm{E}-06$ & 0 & $0.00 \mathrm{E}+00$ & 0 & $2.79 \mathrm{E}-03$ & 3 & $1.37 \mathrm{E}-04$ & 0 & $8.63 \mathrm{E}-04$ & 1 & $7.91 \mathrm{E}-06$ & 0 & $6.70 \mathrm{E}-03$ & 8 \\
\hline $\mathrm{U}-235$ & $6.58 \mathrm{E}-04$ & 1 & $7.02 \mathrm{E}-07$ & 0 & $0.00 \mathrm{E}+00$ & 0 & $3.67 \mathrm{E}-03$ & 4 & $7.59 \mathrm{E}-04$ & 1 & $8.39 \mathrm{E}-04$ & 1 & $1.10 \mathrm{E}-05$ & 0 & $8.72 \mathrm{E}-03$ & 10 \\
\hline U-238 & $1.59 \mathrm{E}-04$ & 0 & $1.34 \mathrm{E}-07$ & 0 & $0.00 \mathrm{E}+00$ & 0 & $2.77 \mathrm{E}-03$ & 3 & $1.36 \mathrm{E}-04$ & 0 & $8.57 \mathrm{E}-04$ & 1 & $7.83 \mathrm{E}-06$ & 0 & $6.66 \mathrm{E}-03$ & 8 \\
\hline & $8.31 E-04$ & 1 & $6.80 \mathrm{E}-04$ & 1 & $0.00 \mathrm{ET}=0$ & 0 & $1.81 \mathrm{E}-\mathrm{UL}$ & 21 & $1.10 E-03$ & 1 & $2.57 \mathrm{E}-03$ & 3 & $3.87 \mathrm{E}-05$ & 0 & $4.36 \mathrm{E}-02$ & \\
\hline
\end{tabular}

Total Dose Contributions TDOSE $(i, p, t)$ for Individual Radionuclides (i) and Pathways (p) in mrem/yr and as a Percentage of Total Dose at $t=1000$ years

$0 \quad$ Directly from primary contamination and from release to atmosphere (Inhalation excludes radon) Ground Plant Meat Milk All Pathways*

\begin{tabular}{|c|c|c|c|c|c|c|c|c|}
\hline de & Dose & $\%$ & Dose & $\%$ & Dose & $\%$ & Dose & $\div$ \\
\hline$A m-241$ & $1.20 \mathrm{E}-08$ & 0 & $9.16 \mathrm{E}-05$ & 0 & $0.00 \mathrm{E}+00$ & 0 & $1.52 \mathrm{E}-10$ & . \\
\hline Cs -137 & $1.98 \mathrm{E}-13$ & 0 & $2.58 \mathrm{E}-17$ & 0 & $0.00 \mathrm{E}+00$ & 0 & $4.80 \mathrm{E}-16$ & 0 \\
\hline $\mathrm{Np}-237$ & $3.17 \mathrm{E}-03$ & 4 & $1.95 \mathrm{E}-03$ & 2 & $0.00 \mathrm{E}+00$ & 0 & $2.29 \mathrm{E}-05$ & \\
\hline $\mathrm{Pu}-238$ & $7.04 \mathrm{E}-10$ & 0 & $2.82 \mathrm{E}-07$ & 0 & $0.00 \mathrm{E}+00$ & 0 & $1.16 \mathrm{E}-09$ & 0 \\
\hline $\mathrm{Pu}-239$ & $1.32 \mathrm{E}-07$ & 0 & $8.03 E-04$ & 1 & $0.00 \mathrm{E}+00$ & 0 & $3.28 \mathrm{E}-06$ & \\
\hline $\mathrm{Pu}-240$ & $2.24 \mathrm{E}-08$ & 0 & $7.43 \mathrm{E}-04$ & 1 & $0.00 \mathrm{E}+00$ & 0 & $3.03 \mathrm{E}-06$ & \\
\hline TC-99 & $0.00 \mathrm{E}+00$ & 0 & $0.00 \mathrm{E}+00$ & 0 & $0.00 \mathrm{E}+00$ & 0 & $0.00 \mathrm{E}+00$ & \\
\hline Th-228 & $2.94 \mathrm{E}-44$ & 0 & $0.00 \mathrm{E}+00$ & 0 & $0.00 \mathrm{E}+00$ & 0 & $0.00 \mathrm{E}+00$ & \\
\hline Th-230 & $9.19 \mathrm{E}-04$ & 1 & $7.64 \mathrm{E}-04$ & 1 & $0.00 \mathrm{E}+00$ & 0 & $9.82 \mathrm{E}-06$ & $v$ \\
\hline Th-232 & $9.19 \mathrm{E}-03$ & 11 & $1.26 \mathrm{E}-03$ & 1 & $0.00 \mathrm{E}+00$ & 0 & $2.85 \mathrm{E}-04$ & \\
\hline $\mathrm{U}-234$ & $2.15 \mathrm{E}-05$ & 0 & $6.93 \mathrm{E}-05$ & 0 & $0.00 \mathrm{E}+00$ & 0 & $3.46 \mathrm{E}-07$ & \\
\hline $\mathrm{U}-235$ & $1.46 \mathrm{E}-04$ & 0 & $9.38 \mathrm{E}-05$ & 0 & $0.00 \mathrm{E}+00$ & 0 & $2.35 \mathrm{E}-07$ & 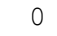 \\
\hline $\mathrm{U}-238$ & $3.44 \mathrm{E}-05$ & 0 & $4.21 \mathrm{E}-05$ & 0 & $0.00 \mathrm{E}+00$ & 0 & $1.31 \mathrm{E}-07$ & 0 \\
\hline
\end{tabular}

Total $1.35 \mathrm{E}-02 \quad \overline{16} \quad \overline{5.82 \mathrm{E}-03}$ $0.00 \mathrm{E}+00$ $3.25 \mathrm{E}-04$

$\overline{\frac{\text { Dose }}{3.79 \mathrm{E}-12} \frac{\%}{0} \frac{\text { Dose }}{3.74 \mathrm{E}-14} \frac{\%}{0}}$
Soil Dose \% $2.60 \mathrm{E}-12 \quad 0 \quad 3.06 \mathrm{E}-02 \quad 35$ $\begin{array}{lllllll}5.68 \mathrm{E}-16 & 0 & 1.50 \mathrm{E}-16 & 0 & 6.66 \mathrm{E}-18 & 0 & 2.00 \mathrm{E}-13\end{array}$ $1.03 \mathrm{E}-06-0 \quad 6.43 \mathrm{E}-09-0 \quad 5.28 \mathrm{E}-07 \quad 0 \quad 5.17 \mathrm{E}-03$

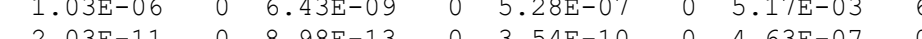

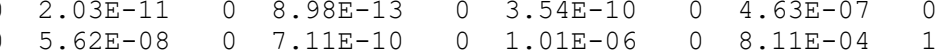
$\begin{array}{llllllll}5.62 \mathrm{E}-08 & 0 & 7.11 \mathrm{E}-10 & 0 & 1.01 \mathrm{E}-06 & 0 & 8.11 \mathrm{E}-04 & 1 \\ 5.20 \mathrm{E}-08 & 0 & 6.58 \mathrm{E}-10 & 0 & 9.33 \mathrm{E}-07 & 0 & 7.51 \mathrm{E}-04 & 1\end{array}$

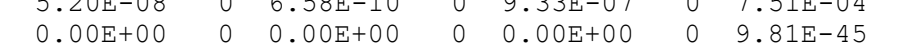
$\begin{array}{llllllllll}0 & 0.00 \mathrm{E}+00 & 0 & 0.00 \mathrm{E}+00 & 0 & 0.00 \mathrm{E}+00 & 0 & 2.94 \mathrm{E}-44 & 0\end{array}$ $\begin{array}{lllllllll}0 & 7.47 \mathrm{E}-07 & 0 & 2.21 \mathrm{E}-07 & 0 & 1.67 \mathrm{E}-06 & 0 & 2.02 \mathrm{E}-03 & 2\end{array}$

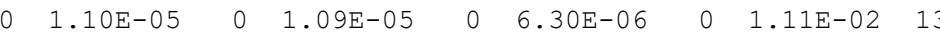

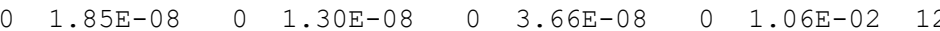

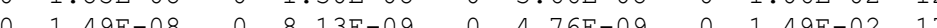
$0 *$ Sum of dose from all releases and from primary contamination. 1RESRAD-OFFSITE, Version 4.0 .3 beta $T^{1 / 2}$ Limit $=30$ days

Parent Dose Report

Title : Landfill Scenario

File : LANDFILL-PADUCAH-AUTHORIZED-RELEASE-REV.ROF

Dose/Source Ratios Summed Over All Pathways

Parent and Progeny Principal Radionuclide Contributions Indicated Fraction

$A m-241$

Am-241

Am-241

$\mathrm{Am}-241$

Am-241 $1.000 \mathrm{E}+00$

\section{$\mathrm{Np}-237+\mathrm{D}$}

$\overline{7.642 \mathrm{E}-03} \overline{7.609 \mathrm{E}-03} \overline{7.535 \mathrm{E}-03} \overline{7.033 \mathrm{E}-03} \overline{6.696 \mathrm{E}-03} \overline{6.501 \mathrm{E}-03} \overline{6.956 \mathrm{E}-02} \overline{3.755 \mathrm{E}-01} \frac{.747 \mathrm{E}-01}{3.170 \mathrm{E}-01}$ $\mathrm{U}-233-1.000 \mathrm{E}+00$ Th-229+D $1.000 \mathrm{E}+00$ Th $-229+\mathrm{D}$ $\begin{array}{llllllllll}1.629 \mathrm{E}-09 & 4.870 \mathrm{E}-09 & 8.080 \mathrm{E}-09 & 2.965 \mathrm{E}-08 & 4.413 \mathrm{E}-08 & 5.245 \mathrm{E}-08 & 2.852 \mathrm{E}-07 & 2.559 \mathrm{E}-06 & 2.793 \mathrm{E}-06 & 4.746 \mathrm{E}-06\end{array}$ Am-241

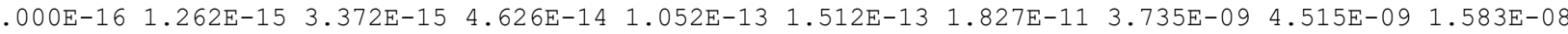

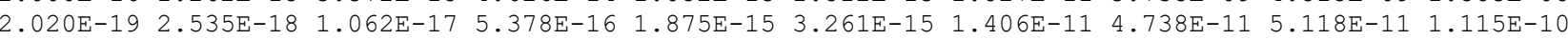
$\begin{array}{lllllllllll}7.642 \mathrm{E}-03 & 7.609 \mathrm{E}-03 & 7.535 \mathrm{E}-03 & 7.033 \mathrm{E}-03 & 6.696 \mathrm{E}-03 & 6.501 \mathrm{E}-03 & 6.956 \mathrm{E}-02 & 3.755 \mathrm{E}-01 & 3.747 \mathrm{E}-01 & 3.170 \mathrm{E}-01\end{array}$

$\mathrm{Np}-237+\mathrm{D} \quad \mathrm{NP}-237+\mathrm{D}$

$\mathrm{Np}-237+\mathrm{D}$

Th-229+D

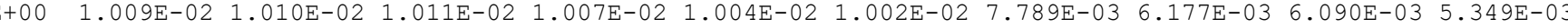

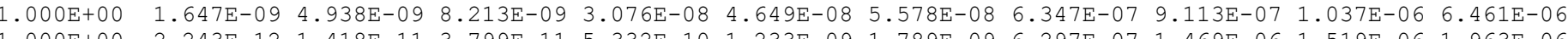

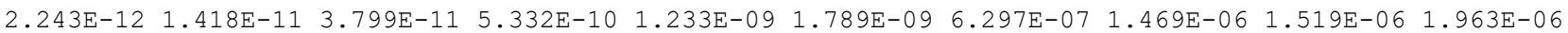


Parent Dose Report

$\begin{array}{lllllllllllll}1.009 \mathrm{E}-02 & 1.010 \mathrm{E}-02 & 1.011 \mathrm{E}-02 & 1.007 \mathrm{E}-02 & 1.004 \mathrm{E}-02 & 1.002 \mathrm{E}-02 & 7.790 \mathrm{E}-03 & 6.180 \mathrm{E}-03 & 6.093 \mathrm{E}-03 & 5.358 \mathrm{E}-03\end{array}$ $\begin{array}{llllllllll} & \end{array}$ $.000 \mathrm{E}+00 \quad 1.035 \mathrm{E}-093.097 \mathrm{E}-09 \quad 5.137 \mathrm{E}-09 \quad 1.876 \mathrm{E}-08 \quad 2.783 \mathrm{E}-08 \quad 3.302 \mathrm{E}-08 \quad 9.485 \mathrm{E}-08 \quad 1.391 \mathrm{E}-07 \quad 1.856 \mathrm{E}-07 \quad 1.857 \mathrm{E}-06$

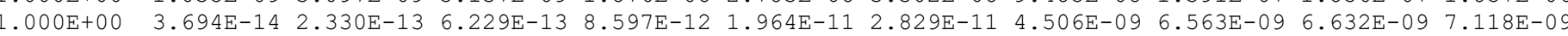
$\begin{array}{lllllllllll}1.000 \mathrm{E}+00 & 3.835 \mathrm{E}-17 & 4.816 \mathrm{E}-16 & 2.018 \mathrm{E}-15 & 1.022 \mathrm{E}-13 & 3.565 \mathrm{E}-13 & 6.198 \mathrm{E}-13 & 2.381 \mathrm{E}-09 & 5.791 \mathrm{E}-09 & 5.965 \mathrm{E}-09 & 7.339 \mathrm{E}-09\end{array}$ $\begin{array}{lllllllllll}1.000 \mathrm{E}+00 & 6.121 \mathrm{E}-21 & 1.473 \mathrm{E}-19 & 9.353 \mathrm{E}-19 & 1.561 \mathrm{E}-16 & 7.964 \mathrm{E}-16 & 1.637 \mathrm{E}-15 & 4.237 \mathrm{E}-11 & 1.133 \mathrm{E}-10 & 1.170 \mathrm{E}-10 & 1.482 \mathrm{E}-10\end{array}$

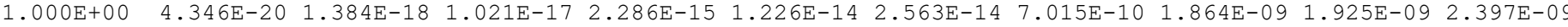
$\begin{array}{llllllllll}8.455 \mathrm{E}-03 & 8.392 \mathrm{E}-03 & 8.330 \mathrm{E}-03 & 7.894 \mathrm{E}-03 & 7.591 \mathrm{E}-03 & 7.413 \mathrm{E}-03 & 2.991 \mathrm{E}-04 & 1.738 \mathrm{E}-05 & 1.465 \mathrm{E}-05 & 4.806 \mathrm{E}-06\end{array}$

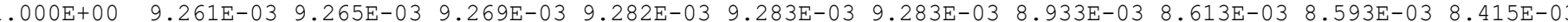
$\begin{array}{llllllllllll}1.000 \mathrm{E}+00 & 2.278 \mathrm{E}-12 & 6.825 \mathrm{E}-12 & 1.136 \mathrm{E}-11 & 4.266 \mathrm{E}-11 & 6.458 \mathrm{E}-11 & 7.755 \mathrm{E}-11 & 1.008 \mathrm{E}-0 & 1.253 \mathrm{E}-09 & 1.283 \mathrm{E}-09 & 2.339 \mathrm{E}-09\end{array}$ $\begin{array}{lllllllllll}1.000 \mathrm{E}+00 & 4.701 \mathrm{E}-17 & 2.972 \mathrm{E}-16 & 7.955 \mathrm{E}-16 & 1.110 \mathrm{E}-14 & 2.555 \mathrm{E}-14 & 3.696 \mathrm{E}-14 & 9.077 \mathrm{E}-12 & 2.854 \mathrm{E}-11 & 3.545 \mathrm{E}-11 & 3.554 \mathrm{E}-10\end{array}$ $\begin{array}{lllllllllll}1.000 \mathrm{E}+00 & 4.701 \mathrm{E}-17 & 2.972 \mathrm{E}-16 & 7.955 \mathrm{E}-16 & 1.110 \mathrm{E}-14 & 2.555 \mathrm{E}-14 & 3.696 \mathrm{E}-14 & 9.077 \mathrm{E}-12 & 2.854 \mathrm{E}-11 & 3.545 \mathrm{E}-11 & 3.554 \mathrm{E}-10 \\ 1.000 \mathrm{E}+00 & 1.943 \mathrm{E}-18 & 2.417 \mathrm{E}-17 & 1.003 \mathrm{E}-16 & 4.804 \mathrm{E}-15 & 1.612 \mathrm{E}-14 & 2.740 \mathrm{E}-14 & 3.125 \mathrm{E}-11 & 5.842 \mathrm{E}-11 & 5.963 \mathrm{E}-11 & 8.107 \mathrm{E}-11\end{array}$ $\begin{array}{lllllllllll}9.261 \mathrm{E}-03 & 9.265 \mathrm{E}-03 & 9.269 \mathrm{E}-03 & 9.282 \mathrm{E}-03 & 9.283 \mathrm{E}-03 & 9.283 \mathrm{E}-03 & 8.933 \mathrm{E}-03 & 8.613 \mathrm{E}-03 & 8.593 \mathrm{E}-03 & 8.415 \mathrm{E}-03\end{array}$ $\begin{array}{lllllllllll}4.950 \mathrm{E}-08 & 4.583 \mathrm{E}-10 & 4.585 \mathrm{E}-10 & 4.587 \mathrm{E}-10 & 4.590 \mathrm{E}-10 & 4.589 \mathrm{E}-10 & 4.588 \mathrm{E}-10 & 4.280 \mathrm{E}-10 & 4.014 \mathrm{E}-10 & 3.998 \mathrm{E}-10 & 3.855 \mathrm{E}-10\end{array}$

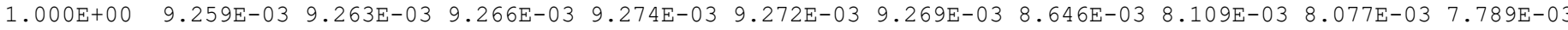
$\begin{array}{lllllllllll}1.000 \mathrm{E}+00 & 1.003 \mathrm{E}-11 & 3.010 \mathrm{E}-11 & 5.010 \mathrm{E}-11 & 1.880 \mathrm{E}-10 & 2.844 \mathrm{E}-10 & 3.414 \mathrm{E}-10 & 4.343 \mathrm{E}-09 & 6.534 \mathrm{E}-09 & 7.258 \mathrm{E}-09 & 3.623 \mathrm{E}-08\end{array}$

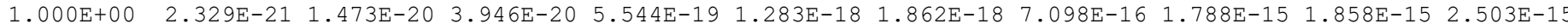

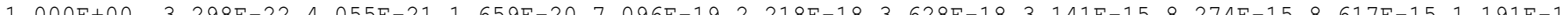

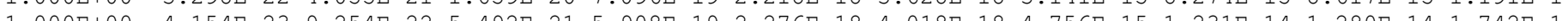

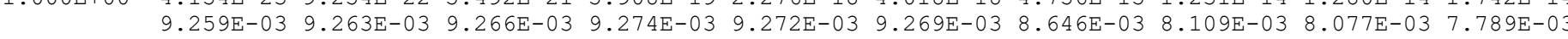

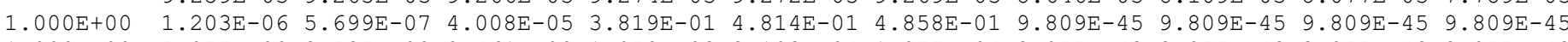

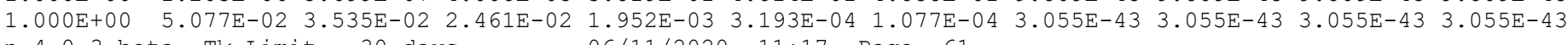

Dose/Source Ratios Summed Over All Pathways Parent and Progeny Principal Radionuclide Contributions Indicated

Parent Product Thread DSR (j,t) (mrem/yr)/(pCi/g)

Th-230

Th-230

Th -230

Th -230

Th -230

$0 \mathrm{Th}-232$

Th -232

Th-232

Th -232

OU-234

$\mathrm{U}-234$

$\mathrm{U}-234$

$\mathrm{U}-234$

$\mathrm{U}-234$

$\mathrm{U}-234$

OU $-235+\mathrm{D}$

$\mathrm{U}-235+\mathrm{D}$

$\mathrm{U}-235+\mathrm{D}$ Fraction

Th-230 $1.000 \mathrm{E}+00$
$\mathrm{Ra}-226+\mathrm{D} 1.000 \mathrm{E}+00$ Pb-210+D $1.000 \mathrm{E}+00$ $\begin{array}{ll}\mathrm{Pb}-210+\mathrm{D} & 1.000 \mathrm{E}+00 \\ \mathrm{PO}-210 & 1.000 \mathrm{E}+00\end{array}$ Th-232 Tha-228+D $\operatorname{sDSR}(j)$ $\mathrm{U}-234$ Th-230 Ra $-226+\mathrm{D}$ $\mathrm{Pb}-210+\mathrm{D}$ Po- 210 $\Sigma \mathrm{DSR}(j)$ $\mathrm{U}-235+\mathrm{D}$ $\mathrm{Pa}-231$ Ac $-227+D$ $\begin{array}{lllllllllll}0.000 \mathrm{E}+00 & 1.000 \mathrm{E}+00 & 2.000 \mathrm{E}+00 & 9.000 \mathrm{E}+00 & 1.400 \mathrm{E}+01 & 1.700 \mathrm{E}+01 & 4.200 \mathrm{E}+02 & 7.780 \mathrm{E}+02 & 8.000 \mathrm{E}+02 & 1.000 \mathrm{E}+03\end{array}$ $\overline{7.720 \mathrm{E}-03} \overline{7.721 \mathrm{E}-03} \overline{7.722 \mathrm{E}-03} \overline{7.727 \mathrm{E}-03} \overline{7.730 \mathrm{E}-03} \overline{7.732 \mathrm{E}-03} \overline{7.814 \mathrm{E}-03} \overline{7.835 \mathrm{E}-03} \overline{7.836 \mathrm{E}-03} \overline{7.846 \mathrm{E}-03}$ $\begin{array}{llllllllll}1.438 \mathrm{E}-05 & 4.313 \mathrm{E}-05 & 7.181 \mathrm{E}-05 & 2.705 \mathrm{E}-04 & 4.101 \mathrm{E}-04 & 4.930 \mathrm{E}-04 & 7.144 \mathrm{E}-03 & 9.142 \mathrm{E}-03 & 9.211 \mathrm{E}-03 & 9.703 \mathrm{E}-03\end{array}$ $\begin{array}{lllllllllll}3.256 \mathrm{E}-09 & 2.232 \mathrm{E}-08 & 6.027 \mathrm{E}-08 & 7.714 \mathrm{E}-07 & 1.689 \mathrm{E}-06 & 2.377 \mathrm{E}-06 & 1.396 \mathrm{E}-04 & 1.857 \mathrm{E}-04 & 1.873 \mathrm{E}-04 & 1.992 \mathrm{E}-04\end{array}$

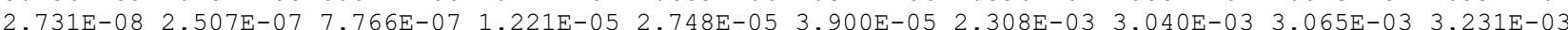

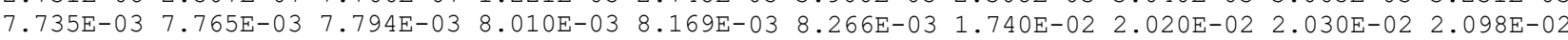
$\begin{array}{lllllllllll}1.000 \mathrm{E}+00 & 8.488 \mathrm{E}-03 & 8.489 \mathrm{E}-03 & 8.490 \mathrm{E}-03 & 8.496 \mathrm{E}-03 & 8.500 \mathrm{E}-03 & 8.502 \mathrm{E}-03 & 8.623 \mathrm{E}-03 & 8.674 \mathrm{E}-03 & 8.677 \mathrm{E}-03 & 8.704 \mathrm{E}-03 \\ 1.000 \mathrm{E}+00 & 2.117 \mathrm{E}-03 & 6.060 \mathrm{E}-03 & 9.557 \mathrm{E}-03 & 2.508 \mathrm{E}-02 & 3.038 \mathrm{E}-02 & 3.235 \mathrm{E}-02 & 4.111 \mathrm{E}-02 & 4.293 \mathrm{E}-02 & 4.303 \mathrm{E}-02 & 4.399 \mathrm{E}-02\end{array}$

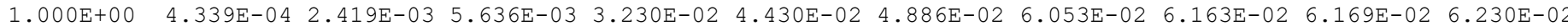
$\begin{array}{lllllllllllllllll}1.104 \mathrm{E}-02 & 1.697 \mathrm{E}-02 & 2.368 \mathrm{E}-02 & 6.587 \mathrm{E}-02 & 8.319 \mathrm{E}-02 & 8.971 \mathrm{E}-02 & 1.103 \mathrm{E}-01 & 1.132 \mathrm{E}-01 & 1.134 \mathrm{E}-01 & 1.150 \mathrm{E}-01\end{array}$ $\begin{array}{llllllllll}7.303 \mathrm{E}-04 & 7.292 \mathrm{E}-04 & 7.268 \mathrm{E}-04 & 7.103 \mathrm{E}-04 & 6.987 \mathrm{E}-04 & 6.918 \mathrm{E}-04 & 1.815 \mathrm{E}-04 & 1.008 \mathrm{E}-03 & 1.373 \mathrm{E}-03 & 1.094 \mathrm{E}-02\end{array}$

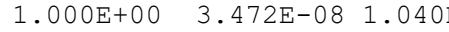
$\begin{array}{lllllllllll}1.000 \mathrm{E}+00 & 4.862 \mathrm{E}-11 & 3.075 \mathrm{E}-10 & 8.236 \mathrm{E}-10 & 1.151 \mathrm{E}-08 & 2.654 \mathrm{E}-08 & 3.842 \mathrm{E}-08 & 1.025 \mathrm{E}-05 & 1.914 \mathrm{E}-05 & 1.955 \mathrm{E}-05 & 2.285 \mathrm{E}-05\end{array}$ $\begin{array}{lllllllllll}1.000 \mathrm{E}+00 & 8.715 \mathrm{E}-15 & 1.143 \mathrm{E}-13 & 4.811 \mathrm{E}-13 & 2.274 \mathrm{E}-11 & 7.640 \mathrm{E}-11 & 1.303 \mathrm{E}-10 & 1.897 \mathrm{E}-07 & 3.800 \mathrm{E}-07 & 3.891 \mathrm{E}-07 & 4.710 \mathrm{E}-07\end{array}$ $\begin{array}{lllllllllll}1.000 \mathrm{E}+00 & 6.664 \mathrm{E}-14 & 1.161 \mathrm{E}-12 & 5.658 \mathrm{E}-12 & 3.457 \mathrm{E}-10 & 1.208 \mathrm{E}-09 & 2.086 \mathrm{E}-09 & 3.141 \mathrm{E}-06 & 6.241 \mathrm{E}-06 & 6.384 \mathrm{E}-06 & 7.458 \mathrm{E}-06\end{array}$

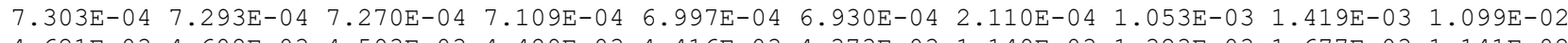

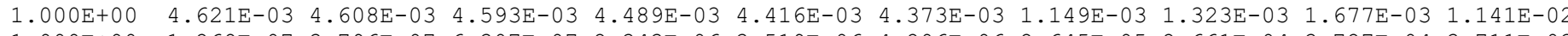
$\begin{array}{lllllllllll}1.000 \mathrm{E}+00 & 1.268 \mathrm{E}-07 & 3.796 \mathrm{E}-07 & 6.307 \mathrm{E}-07 & 2.343 \mathrm{E}-06 & 3.519 \mathrm{E}-06 & 4.206 \mathrm{E}-06 & 2.645 \mathrm{E}-05 & 2.661 \mathrm{E}-04 & 3.727 \mathrm{E}-04 & 3.711 \mathrm{E}-03\end{array}$

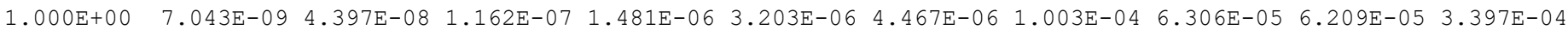




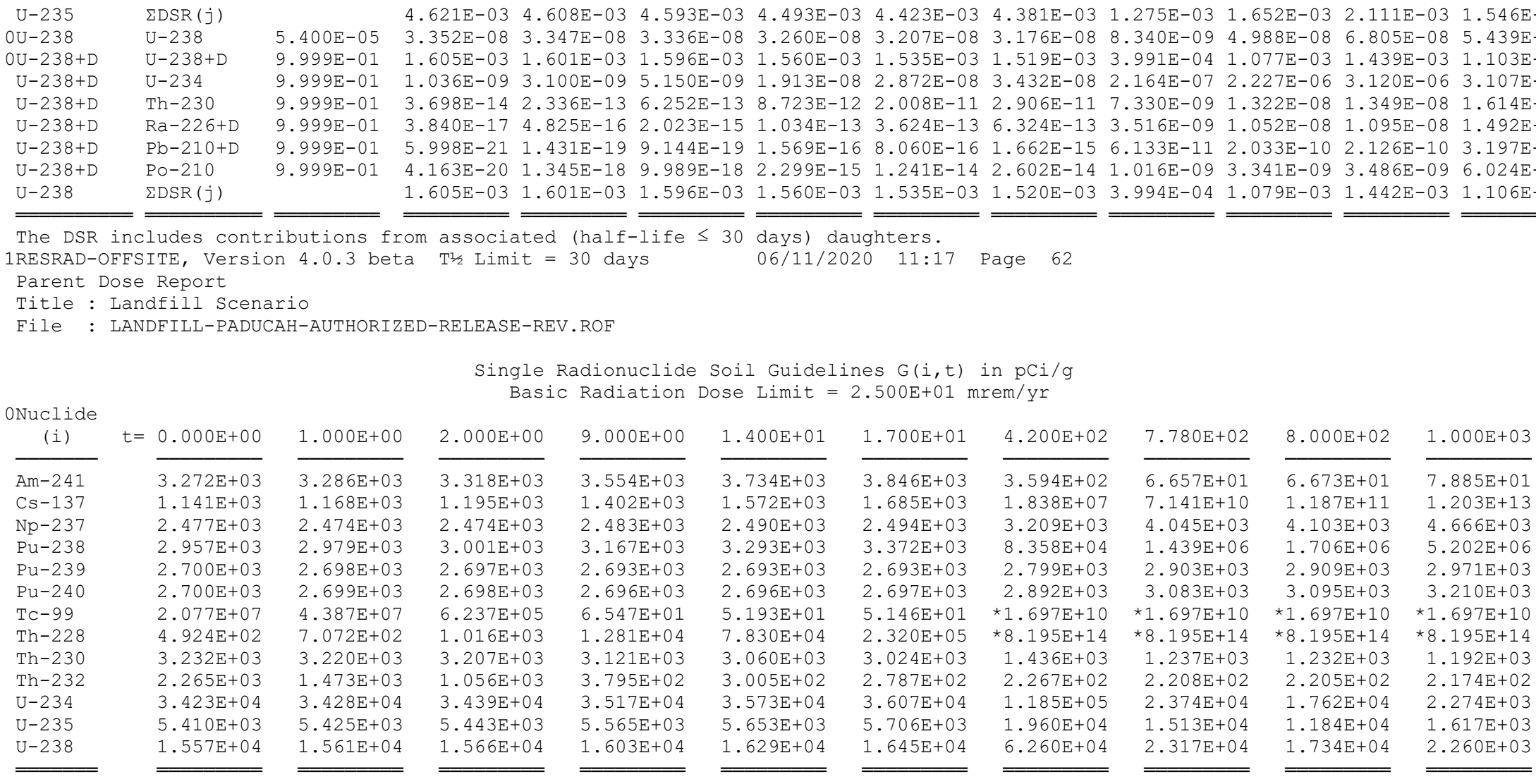

*At specific activity limit

Summed Dose/Source Ratios DSR (i,t) in (mrem/yr)/(pCi/g)

and Single Radionuclide Soil Guidelines $\mathrm{G}(i, t)$ in $\mathrm{pCi} / \mathrm{g}$

at tmin = time of minimum single radionuclide soil guideline

and at $\operatorname{tmax}=$ time of maximum total dose $=17.1$ years

\begin{tabular}{|c|c|c|c|c|c|c|}
\hline $\begin{array}{l}\text { Nuclide } \\
\text { (i) }\end{array}$ & $\begin{array}{l}\text { Initial } \\
(\mathrm{pCi} / \mathrm{g})\end{array}$ & $\begin{array}{c}\operatorname{tmin} \\
(\text { years) }\end{array}$ & $\operatorname{DSR}(i, \operatorname{tmin})$ & $\begin{array}{c}\mathrm{G}(\mathrm{i}, \operatorname{tmin}) \\
(\mathrm{pCi} / \mathrm{g})\end{array}$ & $\operatorname{DSR}(i, t \max )$ & $\begin{array}{c}\mathrm{G}(\mathrm{i}, \mathrm{tmax}) \\
(\mathrm{pCi} / \mathrm{g})\end{array}$ \\
\hline & 2 & 779 & $5-01$ & 6.65 & 6.49 & 3.8 \\
\hline Cs -137 & 9.6 & 0 & & +03 & 02 & +03 \\
\hline $\mathrm{Np}-237$ & $9.640 \mathrm{E}-01$ & 1.51 & $1.011 \mathrm{E}-02$ & $2.474 \mathrm{E}+03$ & $1.002 \mathrm{E}-02$ & $2.494 \mathrm{E}+03$ \\
\hline $\mathrm{Pu}-238$ & $9.640 \mathrm{E}-02$ & 0 & $8.455 \mathrm{E}-03$ & $2.957 \mathrm{E}+03$ & $E-03$ & $3.375 \mathrm{E}+03$ \\
\hline $\mathrm{Pu}-239$ & 9.6 & 13.6 & 03 & 2.6 & 03 & 2.6 \\
\hline $\mathrm{Pu}-240$ & $9.640 \mathrm{E}-02$ & 9.05 & $9.274 \mathrm{E}-03$ & $2.696 \mathrm{E}+03$ & $9.269 \mathrm{E}-03$ & $2.697 \mathrm{E}+03$ \\
\hline $\mathrm{Tc}-99$ & $9.640 \mathrm{E}-01$ & 17.1 & $4.858 \mathrm{E}-01$ & $5.146 \mathrm{E}+01$ & $4.858 \mathrm{E}-01$ & $5.146 \mathrm{E}+07$ \\
\hline
\end{tabular}




\begin{tabular}{llrrrrr} 
Th-228 & $9.640 \mathrm{E}-02$ & 0 & $5.077 \mathrm{E}-02$ & $4.924 \mathrm{E}+02$ & $1.036 \mathrm{E}-04$ & $2.412 \mathrm{E}+05$ \\
Th-230 & $9.640 \mathrm{E}-02$ & 1030 & $2.107 \mathrm{E}-02$ & $1.187 \mathrm{E}+03$ & $8.269 \mathrm{E}-03$ & $3.023 \mathrm{E}+03$ \\
Th-232 & $9.640 \mathrm{E}-02$ & 1030 & $1.152 \mathrm{E}-01$ & $2.170 \mathrm{E}+02$ & $8.990 \mathrm{E}-02$ & $2.781 \mathrm{E}+02$ \\
$\mathrm{U}-234$ & $9.640 \mathrm{E}-01$ & 1030 & $1.361 \mathrm{E}-02$ & $1.836 \mathrm{E}+03$ & $6.928 \mathrm{E}-04$ & $3.609 \mathrm{E}+04$ \\
$\mathrm{U}-235$ & $9.640 \mathrm{E}-01$ & 1030 & $1.946 \mathrm{E}-02$ & $1.284 \mathrm{E}+03$ & $4.380 \mathrm{E}-03$ & $5.708 \mathrm{E}+03$ \\
$\mathrm{U}-238$ & $9.640 \mathrm{E}-01$ & 1030 & $1.371 \mathrm{E}-02$ & $1.823 \mathrm{E}+03$ & $1.519 \mathrm{E}-03$ & $1.646 \mathrm{E}+04$ \\
\hline
\end{tabular}

1RESRAD-OFFSITE, Version 4.0 .3 beta $\mathrm{T}^{\frac{1}{2}}$ Limit $=30$ days Parent Dose Report

Title : Landfill Scenario

File: LANDFILL-PADUCAH-AUTHORIZED-RELEASE-REV.ROF

Individual Nuclide Dose Summed Over All Pathways

Parent Nuclide and Thread Fraction Indicated

ONuclide Parent THF(i)

$1.0005+00$

$\mathrm{Am}-241 \mathrm{Am}-241 \quad 1.000 \mathrm{E}+00$

$0 \mathrm{~Np}-237 \mathrm{Am}-241 \quad 1.000 \mathrm{E}+00$

$\mathrm{Np}-237 \mathrm{~Np}-237 \quad 1.000 \mathrm{E}+00$

Np-237 $\mathrm{DDOSE}(j):$

OU-233 Am-241 $1.000 \mathrm{E}+00$

$\begin{array}{lll}\mathrm{U}-233 & \mathrm{~Np}-237 & 1.000 \mathrm{E}+00\end{array}$

$\mathrm{U}-233 \quad \mathrm{DOSE}(j):$

$0 \mathrm{Th}-229 \quad \mathrm{Am}-241 \quad 1.000 \mathrm{E}+00$

Th-229 Np-237 $1.000 \mathrm{E}+00$

Th-229 $\mathrm{DDOSE}(j):$

OCs $-137 \mathrm{Cs}-137$ 1.000E+00

$0 \mathrm{Pu}-238 \mathrm{Pu}-238 \quad 1.840 \mathrm{E}-09$

$\mathrm{Pu}-238 \mathrm{Pu}-238 \quad 1.000 \mathrm{E}+00$

$\mathrm{Pu}-238 \quad \Sigma \mathrm{DOSE}(j)$ :

OU-234 Pu-238 $1.000 \mathrm{E}+00$

$\begin{array}{lll}\mathrm{U}-234 & \mathrm{U}-234 & 1.000 \mathrm{E}+0\end{array}$

$\mathrm{U}-234$ U-238 9.999E-01

$\mathrm{U}-234 \quad \mathrm{DOSE}(j)$ :

0 Th-230 Pu-238 1.000E+00

$\mathrm{Th}-230 \mathrm{Th}-230 \quad 1.000 \mathrm{E}+00$

Th-230 U-234 $1.000 \mathrm{E}+00$

Th-230 U-238 9.999E-01

Th-230 EDOSE $(j)$ :

ORa-226 Pu-238 1.000E+00

$\mathrm{Ra}-226 \mathrm{Th}-230 \quad 1.000 \mathrm{E}+00$

$\mathrm{Ra}-226 \mathrm{U}-234 \quad 1.000 \mathrm{E}+00$

$\mathrm{Ra}-226 \mathrm{U}-238 \quad 9.999 \mathrm{E}-01$

$\operatorname{Ra}-226 \quad \operatorname{DOSE}(j):$

$0 \mathrm{~Pb}-210 \mathrm{Pu}-238$ 1.000E+00

$\mathrm{Pb}-210 \mathrm{Th}-230 \quad 1.000 \mathrm{E}+00$

$\mathrm{Pb}-210 \mathrm{U}-234 \quad 1.000 \mathrm{E}+00$

$\mathrm{Pb}-210 \quad \mathrm{U}-238 \quad 9.999 \mathrm{E}-01$

$\mathrm{Pb}-210 \quad \Sigma \mathrm{DOSE}(j)$ :
$\operatorname{DOSE}(j, t), \mathrm{mrem} / \mathrm{yr}$

$t=0.000 \mathrm{E}+00 \quad 1.000 \mathrm{E}+00 \quad 2.000 \mathrm{E}+00 \quad 9.000 \mathrm{E}+00 \quad 1.400 \mathrm{E}+01 \quad 1.700 \mathrm{E}+01 \quad 4.200 \mathrm{E}+02 \quad 7.780 \mathrm{E}+02 \quad 8.000 \mathrm{E}+02 \quad 1.000 \mathrm{E}+03$

$\begin{array}{lllllllllllll}7.367 \mathrm{E}-04 & 7.335 \mathrm{E}-04 & 7.263 \mathrm{E}-04 & 6.780 \mathrm{E}-04 & 6.455 \mathrm{E}-04 & 6.267 \mathrm{E}-04 & 6.706 \mathrm{E}-03 & 3.620 \mathrm{E}-02 & 3.612 \mathrm{E}-02 & 3.056 \mathrm{E}-02\end{array}$

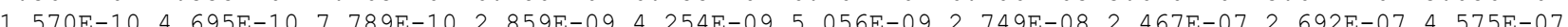
$9.730 \mathrm{E}-03 \quad 9.741 \mathrm{E}-03 \quad 9.742 \mathrm{E}-03 \quad 9.707 \mathrm{E}-03 \quad 9.679 \mathrm{E}-03 \quad 9.663 \mathrm{E}-03 \quad 7.508 \mathrm{E}-03 \quad 5.955 \mathrm{E}-03 \quad 5.871 \mathrm{E}-03 \quad 5.157 \mathrm{E}-03$

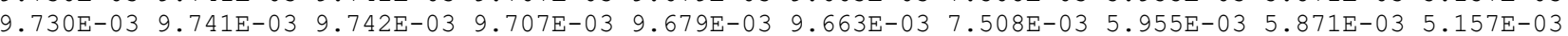

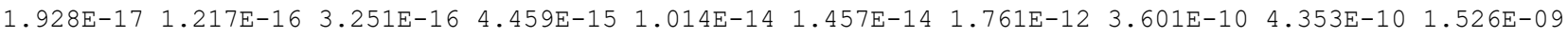
1.588E-09 $4.760 \mathrm{E}-09 \quad 7.917 \mathrm{E}-09 \quad 2.965 \mathrm{E}-08 \quad 4.482 \mathrm{E}-08 \quad 5.377 \mathrm{E}-08 \quad 6.118 \mathrm{E}-07 \quad 8.785 \mathrm{E}-07 \quad 9.993 \mathrm{E}-07 \quad 6.228 \mathrm{E}-06$

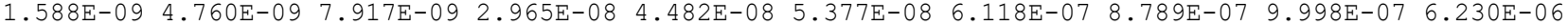
$\begin{array}{llllllllll}948 \mathrm{E}-20 & 2.444 \mathrm{E}-19 & 1.023 \mathrm{E}-18 & 5.184 \mathrm{E}-17 & 1.807 \mathrm{E}-16 & 3.143 \mathrm{E}-16 & 1.356 \mathrm{E}-12 & 4.567 \mathrm{E}-12 & 4.934 \mathrm{E}-12 & 1.075 \mathrm{E}-11\end{array}$ $\begin{array}{lllllllll} & \end{array}$ $\begin{array}{lllllllllll}2.162 \mathrm{E}-12 & 1.367 \mathrm{E}-11 & 3.662 \mathrm{E}-11 & 5.141 \mathrm{E}-10 & 1.189 \mathrm{E}-09 & 1.724 \mathrm{E}-09 & 6.070 \mathrm{E}-07 & 1.416 \mathrm{E}-06 & 1.465 \mathrm{E}-06 & 1.893 \mathrm{E}-06\end{array}$ $2.112 \mathrm{E}-032.064 \mathrm{E}-03 \quad 2.018 \mathrm{E}-03 \quad 1.719 \mathrm{E}-03 \quad 1.533 \mathrm{E}-03 \quad 1.431 \mathrm{E}-03 \quad 1.311 \mathrm{E}-07 \quad 3.375 \mathrm{E}-112.031 \mathrm{E}-112.003 \mathrm{E}-13$

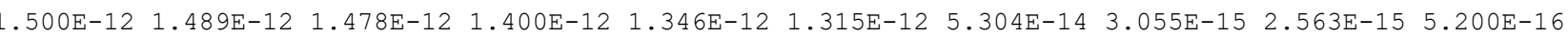

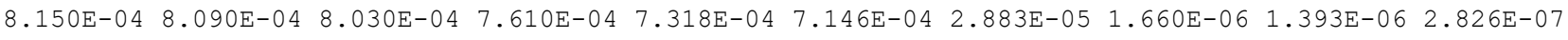

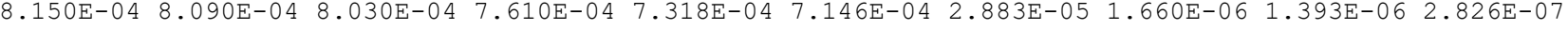
$\begin{array}{llllllllll}9.982 \mathrm{E}-11 & 2.986 \mathrm{E}-10 & 4.952 \mathrm{E}-10 & 1.808 \mathrm{E}-09 & 2.683 \mathrm{E}-09 & 3.183 \mathrm{E}-09 & 9.143 \mathrm{E}-09 & 1.341 \mathrm{E}-08 & 1.789 \mathrm{E}-08 & 1.790 \mathrm{E}-07\end{array}$ $\begin{array}{llllllllll}7.040 \mathrm{E}-04 & 7.029 \mathrm{E}-04 & 7.006 \mathrm{E}-04 & 6.847 \mathrm{E}-04 & 6.735 \mathrm{E}-04 & 6.669 \mathrm{E}-04 & 1.749 \mathrm{E}-04 & 9.716 \mathrm{E}-04 & 1.324 \mathrm{E}-03 & 1.055 \mathrm{E}-02\end{array}$

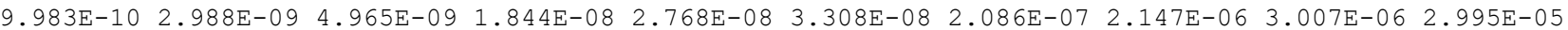
$\begin{array}{llllllllll}7.040 \mathrm{E}-04 & 7.029 \mathrm{E}-04 & 7.006 \mathrm{E}-04 & 6.847 \mathrm{E}-04 & 6.735 \mathrm{E}-04 & 6.669 \mathrm{E}-04 & 1.752 \mathrm{E}-04 & 9.738 \mathrm{E}-04 & 1.327 \mathrm{E}-03 & 1.058 \mathrm{E}-02\end{array}$

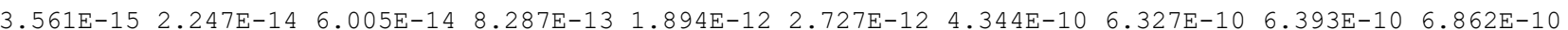

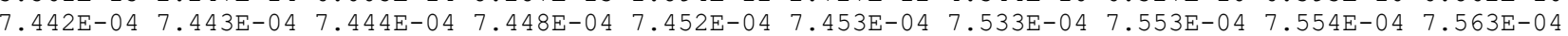
$\begin{array}{llllllllll}3.347 \mathrm{E}-08 & 1.003 \mathrm{E}-07 & 1.668 \mathrm{E}-07 & 6.268 \mathrm{E}-07 & 9.491 \mathrm{E}-07 & 1.140 \mathrm{E}-06 & 1.533 \mathrm{E}-05 & 1.891 \mathrm{E}-05 & 1.903 \mathrm{E}-05 & 2.005 \mathrm{E}-05\end{array}$ $\begin{array}{llllllllll}3.565 \mathrm{E}-14 & 2.252 \mathrm{E}-13 & 6.027 \mathrm{E}-13 & 8.409 \mathrm{E}-12 & 1.936 \mathrm{E}-11 & 2.801 \mathrm{E}-11 & 7.066 \mathrm{E}-09 & 1.274 \mathrm{E}-08 & 1.300 \mathrm{E}-08 & 1.556 \mathrm{E}-08\end{array}$

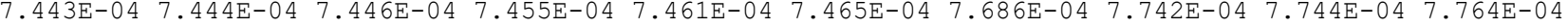
$\begin{array}{llllllllll}3.697 \mathrm{E}-18 & 4.643 \mathrm{E}-17 & 1.945 \mathrm{E}-16 & 9.857 \mathrm{E}-15 & 3.436 \mathrm{E}-14 & 5.975 \mathrm{E}-14 & 2.295 \mathrm{E}-10 & 5.582 \mathrm{E}-10 & 5.750 \mathrm{E}-10 & 7.075 \mathrm{E}-10\end{array}$

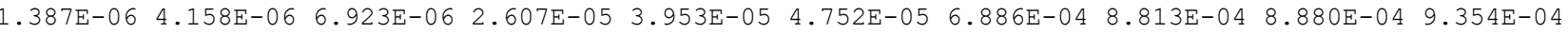
$\begin{array}{llllllllll}4.687 \mathrm{E}-11 & 2.964 \mathrm{E}-10 & 7.940 \mathrm{E}-10 & 1.110 \mathrm{E}-08 & 2.558 \mathrm{E}-08 & 3.704 \mathrm{E}-08 & 9.885 \mathrm{E}-06 & 1.845 \mathrm{E}-05 & 1.885 \mathrm{E}-05 & 2.203 \mathrm{E}-05\end{array}$ $\begin{array}{llllllllll}3.701 \mathrm{E}-17 & 4.651 \mathrm{E}-16 & 1.951 \mathrm{E}-15 & 9.965 \mathrm{E}-14 & 3.494 \mathrm{E}-13 & 6.096 \mathrm{E}-13 & 3.390 \mathrm{E}-09 & 1.015 \mathrm{E}-08 & 1.056 \mathrm{E}-08 & 1.438 \mathrm{E}-08\end{array}$

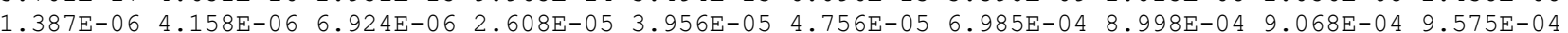

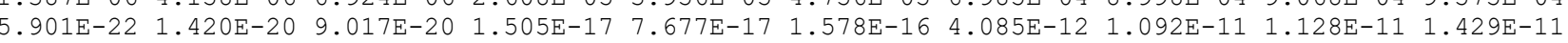

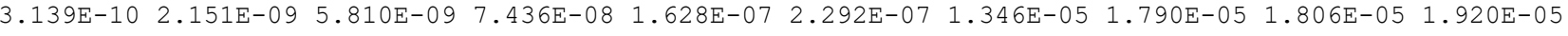
$\begin{array}{llllllllll}8.401 \mathrm{E}-15 & 1.102 \mathrm{E}-13 & 4.638 \mathrm{E}-13 & 2.192 \mathrm{E}-11 & 7.365 \mathrm{E}-11 & 1.256 \mathrm{E}-10 & 1.829 \mathrm{E}-07 & 3.663 \mathrm{E}-07 & 3.751 \mathrm{E}-07 & 4.541 \mathrm{E}-07\end{array}$ $\begin{array}{llllllllll}5.782 \mathrm{E}-21 & 1.380 \mathrm{E}-19 & 8.815 \mathrm{E}-19 & 1.513 \mathrm{E}-16 & 7.770 \mathrm{E}-16 & 1.602 \mathrm{E}-15 & 5.912 \mathrm{E}-11 & 1.960 \mathrm{E}-10 & 2.050 \mathrm{E}-10 & 3.082 \mathrm{E}-10\end{array}$ 3.139E-10 2.152E-09 5.811E-09 $7.439 \mathrm{E}-08 \quad 1.629 \mathrm{E}-07 \quad 2.293 \mathrm{E}-07 \quad 1.364 \mathrm{E}-05 \quad 1.826 \mathrm{E}-05 \quad 1.843 \mathrm{E}-05 \quad 1.966 \mathrm{E}-05$ 
Parent Dose Report

Title : Landfill Scenario

File: LANDFILL-PADUCAH-AUTHORIZED-RELEASE-REV.ROF

Individual Nuclide Dose Summed Over All Pathways

Parent Nuclide and Thread Fraction Indicated

ONuclide Parent THF(i)

$\mathrm{PO}-210 \mathrm{Pu}-238 \quad 1.000 \mathrm{E}+00$

$\begin{array}{lll}\mathrm{Po}-210 & \mathrm{Th}-230 & 1.000 \mathrm{E}+00\end{array}$

$\mathrm{PO}-210 \mathrm{U}-234 \quad 1.000 \mathrm{E}+00$

$\mathrm{PO}-210 \mathrm{U}-238 \quad 9.999 \mathrm{E}-01$

Po-210 $\mathrm{DDOSE}(j):$

OPu-239 Pu-239 1.000E+00

OU-235 Pu-239 $1.000 \mathrm{E}+00$

$\mathrm{U}-235$ U-235 $1.000 \mathrm{E}+00$

U-235 $\quad \mathrm{DOSE}(j):$

$0 \mathrm{~Pa}-231 \mathrm{Pu}-2391.000 \mathrm{E}+00$

$\mathrm{Pa}-231 \mathrm{U}-235 \quad 1.000 \mathrm{E}+00$

$\mathrm{Pa}-231 \quad \mathrm{DDOSE}(j):$

OAC-227 Pu-239 1.000E +00

$\mathrm{AC}-227 \quad \mathrm{U}-235 \quad 1.000 \mathrm{E}+00$

$\mathrm{AC}-227 \quad \mathrm{DOSE}(j):$

OPu-240 $\mathrm{Pu}-240 \quad 4.950 \mathrm{E}-08$

$\mathrm{Pu}-240 \quad \mathrm{Pu}-240 \quad 1.000 \mathrm{E}+00$

$\mathrm{Pu}-240$ $\mathrm{DDOSE}(j):$

OU-236 Pu-240 1.000E+00

$0 \mathrm{Th}-232$ Pu-240 $1.000 \mathrm{E}+00$

Th-232 Th-232 1.000E+00

Th-232 $\mathrm{DDOSE}(j)$ :

ORa-228 Pu-240 1.000E+00

$\mathrm{Ra}-228 \mathrm{Th}-232 \quad 1.000 \mathrm{E}+00$

$\mathrm{Ra}-228 \quad \Sigma \mathrm{DOSE}(j)$ :

0Th-228 Pu-240 1.000E+00

Th-228 Th-228 1.000E+00

Th-228 Th-232 1.000E+00

Th-228 $\Sigma$ DOSE $(j):$

OTC-99 TC-99 1.000E+00

OU-238 U-238 $5.400 \mathrm{E}-05$

$\mathrm{U}-238 \mathrm{U}-238 \quad 9.999 \mathrm{E}-01$

$\mathrm{U}-238 \quad \Sigma \mathrm{DOSE}(j)$ $\operatorname{DOSE}(j, t), \mathrm{mrem} / \mathrm{yr}$

$t=0.000 \mathrm{E}+00 \quad 1.000 \mathrm{E}+00 \quad 2.000 \mathrm{E}+00 \quad 9.000 \mathrm{E}+00 \quad 1.400 \mathrm{E}+01 \quad 1.700 \mathrm{E}+01 \quad 4.200 \mathrm{E}+02 \quad 7.780 \mathrm{E}+02 \quad 8.000 \mathrm{E}+02 \quad 1.000 \mathrm{E}+03$

$\begin{array}{lllllllllll}4.189 \mathrm{E}-21 & 1.334 \mathrm{E}-19 & 9.844 \mathrm{E}-19 & 2.204 \mathrm{E}-16 & 1.182 \mathrm{E}-15 & 2.470 \mathrm{E}-15 & 6.762 \mathrm{E}-11 & 1.797 \mathrm{E}-10 & 1.856 \mathrm{E}-10 & 2.311 \mathrm{E}-10\end{array}$

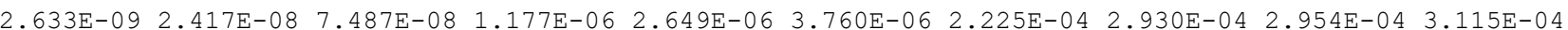
$6.424 \mathrm{E}-14 \quad 1.120 \mathrm{E}-12 \quad 5.455 \mathrm{E}-12 \quad 3.332 \mathrm{E}-10 \quad 1.164 \mathrm{E}-092011 \mathrm{E}-09 \quad 3.028 \mathrm{E}-06 \quad 6.016 \mathrm{E}-06 \quad 6.154 \mathrm{E}-067.189 \mathrm{E}-06$

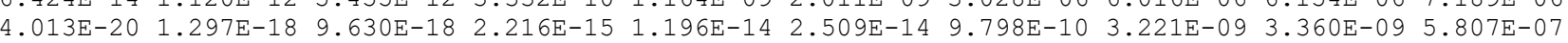
$\begin{array}{llllllllll}2.633 \mathrm{E}-09 & 2.417 \mathrm{E}-08 & 7.487 \mathrm{E}-08 & 1.177 \mathrm{E}-06 & 2.650 \mathrm{E}-06 & 3.762 \mathrm{E}-06 & 2.255 \mathrm{E}-04 & 2.990 \mathrm{E}-04 & 3.016 \mathrm{E}-04 & 3.193 \mathrm{E}-04\end{array}$

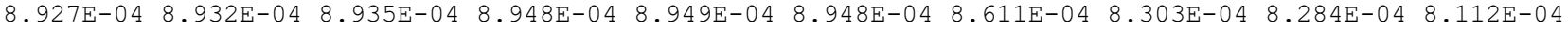
$\begin{array}{llllllllll}2.196 \mathrm{E}-13 & 6.579 \mathrm{E}-13 & 1.095 \mathrm{E}-12 & 4.113 \mathrm{E}-12 & 6.225 \mathrm{E}-12 & 7.476 \mathrm{E}-12 & 9.717 \mathrm{E}-11 & 1.208 \mathrm{E}-10 & 1.237 \mathrm{E}-10 & 2.255 \mathrm{E}-10\end{array}$

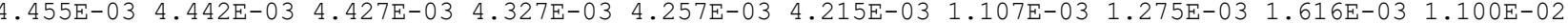

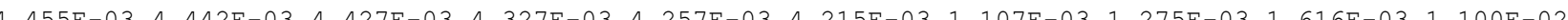

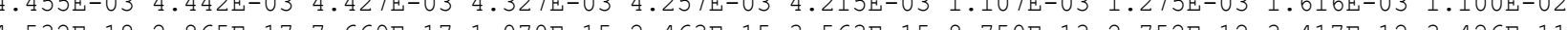

$.532 \mathrm{E}-182.865 \mathrm{E}-17.7 .669 \mathrm{E}-17 \mathrm{~g} 1.070 \mathrm{E}-15 \mathrm{2} 2.463 \mathrm{E}-153.5$

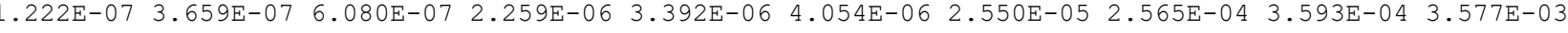
$\begin{array}{llllllllll}1.222 \mathrm{E}-07 & 3.659 \mathrm{E}-07 & 6.080 \mathrm{E}-07 & 2.259 \mathrm{E}-06 & 3.392 \mathrm{E}-06 & 4.054 \mathrm{E}-06 & 2.550 \mathrm{E}-05 & 2.565 \mathrm{E}-04 & 3.593 \mathrm{E}-04 & 3.577 \mathrm{E}-03\end{array}$ $\begin{array}{llllllllll}1.874 \mathrm{E}-19 & 2.330 \mathrm{E}-18 & 9.673 \mathrm{E}-18 & 4.631 \mathrm{E}-16 & 1.554 \mathrm{E}-15 & 2.642 \mathrm{E}-15 & 3.012 \mathrm{E}-12 & 5.632 \mathrm{E}-12 & 5.749 \mathrm{E}-12 & 7.815 \mathrm{E}-12\end{array}$ $\begin{array}{llllllllll}6.790 \mathrm{E}-09 & 4.239 \mathrm{E}-08 & 1.120 \mathrm{E}-07 & 1.428 \mathrm{E}-06 & 3.088 \mathrm{E}-06 & 4.306 \mathrm{E}-06 & 9.666 \mathrm{E}-05 & 6.079 \mathrm{E}-05 & 5.985 \mathrm{E}-05 & 3.275 \mathrm{E}-04\end{array}$

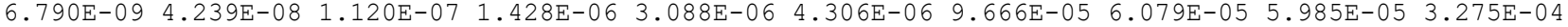
$4.418 \mathrm{E}-11$ 4.420E-11 $4.421 \mathrm{E}-11 \quad 4.425 \mathrm{E}-11 \quad 4.424 \mathrm{E}-11 \quad 4.423 \mathrm{E}-11 \quad 4.126 \mathrm{E}-11 \quad 3.870 \mathrm{E}-11 \quad 3.854 \mathrm{E}-11 \quad 3.717 \mathrm{E}-11$ $\begin{array}{llllllllll}8.926 \mathrm{E}-04 & 8.929 \mathrm{E}-04 & 8.932 \mathrm{E}-04 & 8.940 \mathrm{E}-04 & 8.938 \mathrm{E}-04 & 8.935 \mathrm{E}-04 & 8.335 \mathrm{E}-04 & 7.817 \mathrm{E}-04 & 7.786 \mathrm{E}-04 & 7.508 \mathrm{E}-04\end{array}$ $8.926 \mathrm{E}-04 \quad 8.929 \mathrm{E}-04 \quad 8.932 \mathrm{E}-04 \quad 8.940 \mathrm{E}-04 \quad 8.938 \mathrm{E}-04 \quad 8.935 \mathrm{E}-04 \quad 8.335 \mathrm{E}-04 \quad 7.817 \mathrm{E}-04 \quad 7.786 \mathrm{E}-04 \quad 7.508 \mathrm{E}-04$

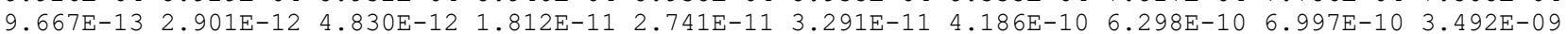

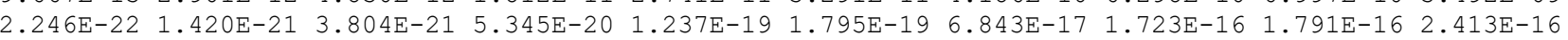
$\begin{array}{llllllllll}8.183 \mathrm{E}-04 & 8.184 \mathrm{E}-04 & 8.184 \mathrm{E}-04 & 8.190 \mathrm{E}-04 & 8.194 \mathrm{E}-04 & 8.196 \mathrm{E}-04 & 8.313 \mathrm{E}-04 & 8.362 \mathrm{E}-04 & 8.364 \mathrm{E}-04 & 8.390 \mathrm{E}-04\end{array}$ $\begin{array}{llllllllll}8.183 \mathrm{E}-04 & 8.184 \mathrm{E}-04 & 8.184 \mathrm{E}-04 & 8.190 \mathrm{E}-04 & 8.194 \mathrm{E}-04 & 8.196 \mathrm{E}-04 & 8.313 \mathrm{E}-04 & 8.362 \mathrm{E}-04 & 8.364 \mathrm{E}-04 & 8.390 \mathrm{E}-04\end{array}$

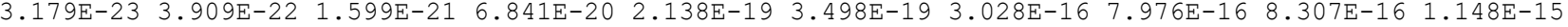

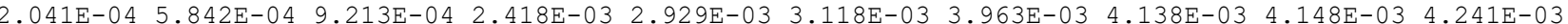

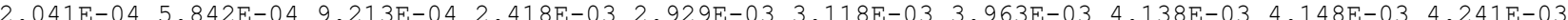
. . $4.894 \mathrm{E}-03$ 3.408E-03 $2.373 \mathrm{E}-031.882 \mathrm{E}-042.078 \mathrm{E}-05 \quad 1.039 \mathrm{E}-052.943 \mathrm{E}-442.943 \mathrm{E}-442.943 \mathrm{E}-442.943 \mathrm{E}-44$

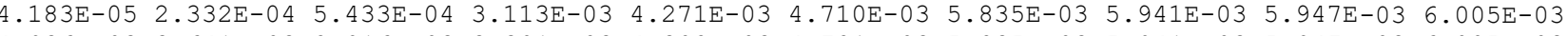

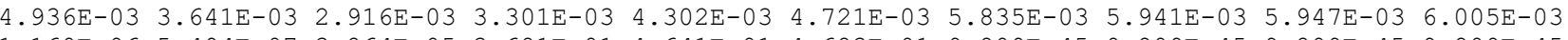
1.160E-06 5.494E-07 3.864E-05 3.681E-01 4.641E-01 4.683E-01 9.809E-45 9.809E-45 9.809E-45 9.809E-45 $\begin{array}{llllllllll}3.231 \mathrm{E}-08 & 3.226 \mathrm{E}-08 & 3.216 \mathrm{E}-08 & 3.143 \mathrm{E}-08 & 3.092 \mathrm{E}-08 & 3.061 \mathrm{E}-08 & 8.040 \mathrm{E}-09 & 4.809 \mathrm{E}-08 & 6.560 \mathrm{E}-08 & 5.243 \mathrm{E}-07\end{array}$ $\begin{array}{llllllllll}1.547 \mathrm{E}-03 & 1.544 \mathrm{E}-03 & 1.539 \mathrm{E}-03 & 1.504 \mathrm{E}-03 & 1.479 \mathrm{E}-03 & 1.465 \mathrm{E}-03 & 3.848 \mathrm{E}-04 & 1.038 \mathrm{E}-03 & 1.387 \mathrm{E}-03 & 1.063 \mathrm{E}-02\end{array}$ $\begin{array}{llllllllll}1.547 \mathrm{E}-03 & 1.544 \mathrm{E}-03 & 1.539 \mathrm{E}-03 & 1.504 \mathrm{E}-03 & 1.479 \mathrm{E}-03 & 1.465 \mathrm{E}-03 & 3.848 \mathrm{E}-04 & 1.038 \mathrm{E}-03 & 1.387 \mathrm{E}-03 & 1.063 \mathrm{E}-02\end{array}$

THF(i) is the thread fraction of the parent nuclide.

IRESRAD-OFFSITE, Version 4.0 .3 beta T1/2 Limit $=30$ days $\quad 06 / 11 / 2020 \quad 11: 17 \quad$ Page 65

Parent Dose Report

Title : Landfill Scenario

File : LANDFILL-PADUCAH-AUTHORIZED-RELEASE-REV.ROF 
ONuclide Parent THF(i)

\begin{tabular}{|c|c|c|}
\hline (j) & (i) & \\
\hline $\mathrm{Am}-241$ & $A m-241$ & $1.000 \mathrm{E}+00$ \\
\hline ONp-237 & $A m-241$ & $1.000 \mathrm{E}+00$ \\
\hline $\mathrm{Np}-237$ & Np-237 & $1.000 \mathrm{E}+00$ \\
\hline $\mathrm{Np}-237$ & $\Sigma S(j):$ & \\
\hline OU-233 & $A m-241$ & $1.000 \mathrm{E}+00$ \\
\hline $\mathrm{U}-233$ & $\mathrm{~Np}-237$ & $1.000 \mathrm{E}+00$ \\
\hline $\mathrm{U}-233$ & $\Sigma S(j):$ & \\
\hline 0 Th-229 & $A m-241$ & $1.000 \mathrm{E}+00$ \\
\hline Th-229 & $\mathrm{Np}-237$ & $1.000 \mathrm{E}+00$ \\
\hline Th-229 & $\Sigma S(j):$ & \\
\hline $0 \mathrm{Cs}-137$ & $\mathrm{Cs}-137$ & $1.000 \mathrm{E}+00$ \\
\hline $0 \mathrm{Pu}-238$ & $\mathrm{Pu}-238$ & $1.840 \mathrm{E}-09$ \\
\hline $\mathrm{Pu}-238$ & $\mathrm{Pu}-238$ & $1.000 \mathrm{E}+00$ \\
\hline $\mathrm{Pu}-238$ & $\Sigma S(j):$ & \\
\hline OU-234 & $\mathrm{Pu}-238$ & $1.000 \mathrm{E}+00$ \\
\hline $\mathrm{U}-234$ & $\mathrm{U}-234$ & $1.000 \mathrm{E}+00$ \\
\hline $\mathrm{U}-234$ & $\mathrm{U}-238$ & $9.999 \mathrm{E}-01$ \\
\hline$U-234$ & $\Sigma S(j):$ & \\
\hline 0 Th-230 & $\mathrm{Pu}-238$ & $1.000 \mathrm{E}+00$ \\
\hline Th-230 & Th -230 & $1.000 \mathrm{E}+00$ \\
\hline Th-230 & $U-234$ & $1.000 \mathrm{E}+00$ \\
\hline Th-230 & $\mathrm{U}-238$ & $9.999 \mathrm{E}-01$ \\
\hline Th-230 & $\Sigma S(j):$ & \\
\hline ORa-226 & $\mathrm{Pu}-238$ & $1.000 \mathrm{E}+00$ \\
\hline $\mathrm{Ra}-226$ & Th -230 & $1.000 \mathrm{E}+00$ \\
\hline $\mathrm{Ra}-226$ & $\mathrm{U}-234$ & $1.000 \mathrm{E}+00$ \\
\hline $\mathrm{Ra}-226$ & $\mathrm{U}-238$ & $9.999 \mathrm{E}-01$ \\
\hline$R a-226$ & $\Sigma S(j):$ & \\
\hline $0 \mathrm{~Pb}-210$ & $\mathrm{Pu}-238$ & م م \\
\hline $\mathrm{Pb}-210$ & Th -230 & $1.000 \mathrm{E}+00$ \\
\hline $\mathrm{Pb}-210$ & $\mathrm{U}-234$ & $1.000 \mathrm{E}+00$ \\
\hline $\mathrm{Pb}-210$ & $\mathrm{U}-238$ & $9.999 \mathrm{E}-01$ \\
\hline
\end{tabular}

$\mathrm{Pb}-210 \quad \mathrm{U}-238 \quad 9.999 \mathrm{E}-01$

$\mathrm{Pb}-210 \quad \mathrm{SS}(\mathrm{j}):$

$\mathrm{S}(j, t), \mathrm{pCi} / \mathrm{g}$

$t=0.000 \mathrm{E}+00 \quad 1.000 \mathrm{E}+00 \quad 2.000 \mathrm{E}+00 \quad 9.000 \mathrm{E}+00 \quad 1.400 \mathrm{E}+01 \quad 1.700 \mathrm{E}+01 \quad 4.200 \mathrm{E}+02 \quad 7.780 \mathrm{E}+02 \quad 8.000 \mathrm{E}+02 \quad 1.000 \mathrm{E}+03$

$\begin{array}{llllllllll}9.640 \mathrm{E}-02 & 9.546 \mathrm{E}-02 & 9.452 \mathrm{E}-02 & 8.822 \mathrm{E}-02 & 8.398 \mathrm{E}-02 & 8.154 \mathrm{E}-02 & 1.540 \mathrm{E}-03 & 4.532 \mathrm{E}-05 & 3.649 \mathrm{E}-05 & 5.090 \mathrm{E}-06\end{array}$ $\begin{array}{llllllllll}0.000 \mathrm{E}+00 & 3.106 \mathrm{E}-08 & 6.180 \mathrm{E}-08 & 2.681 \mathrm{E}-07 & 4.064 \mathrm{E}-07 & 4.860 \mathrm{E}-07 & 2.531 \mathrm{E}-06 & 2.048 \mathrm{E}-06 & 2.019 \mathrm{E}-06 & 1.775 \mathrm{E}-06\end{array}$ $\begin{array}{lllllllllll}9.640 \mathrm{E}-01 & 9.634 \mathrm{E}-01 & 9.628 \mathrm{E}-01 & 9.584 \mathrm{E}-01 & 9.553 \mathrm{E}-01 & 9.534 \mathrm{E}-01 & 7.342 \mathrm{E}-01 & 5.821 \mathrm{E}-01 & 5.739 \mathrm{E}-01 & 5.041 \mathrm{E}-01\end{array}$ $\begin{array}{lllllllllll}9.640 \mathrm{E}-01 & 9.634 \mathrm{E}-01 & 9.628 \mathrm{E}-01 & 9.584 \mathrm{E}-01 & 9.553 \mathrm{E}-01 & 9.534 \mathrm{E}-01 & 7.342 \mathrm{E}-01 & 5.821 \mathrm{E}-01 & 5.739 \mathrm{E}-01 & 5.041 \mathrm{E}-01\end{array}$ $\begin{array}{llllllllll}0.000 \mathrm{E}+00 & 6.816 \mathrm{E}-14 & 2.710 \mathrm{E}-13 & 5.308 \mathrm{E}-12 & 1.255 \mathrm{E}-11 & 1.826 \mathrm{E}-11 & 2.325 \mathrm{E}-09 & 2.762 \mathrm{E}-09 & 2.756 \mathrm{E}-09 & 2.619 \mathrm{E}-09\end{array}$ $\begin{array}{llllllllll}0.000 \mathrm{E}+00 & 4.207 \mathrm{E}-06 & 8.398 \mathrm{E}-06 & 3.727 \mathrm{E}-05 & 5.741 \mathrm{E}-05 & 6.929 \mathrm{E}-05 & 8.100 \mathrm{E}-04 & 8.329 \mathrm{E}-04 & 8.278 \mathrm{E}-04 & 7.673 \mathrm{E}-04\end{array}$ $0.000 \mathrm{E}+00 \quad 4.207 \mathrm{E}-06 \quad 8.398 \mathrm{E}-06 \quad 3.727 \mathrm{E}-05 \quad 5.741 \mathrm{E}-05 \quad 6.929 \mathrm{E}-05 \quad 8.100 \mathrm{E}-04 \quad 8.329 \mathrm{E}-04 \quad 8.278 \mathrm{E}-04 \quad 7.673 \mathrm{E}-04$

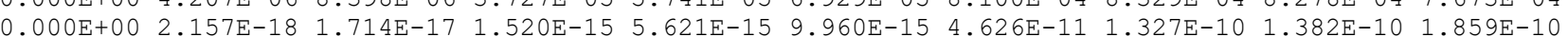

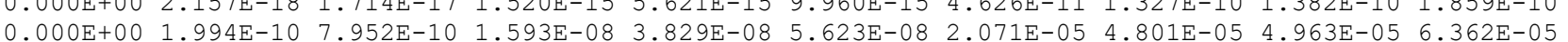

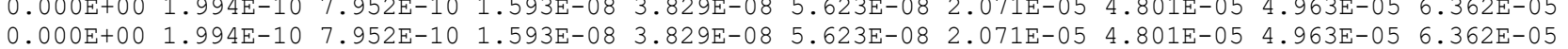
$\begin{array}{llllllllll}9.640 \mathrm{E}-02 & 9.419 \mathrm{E}-02 & 9.204 \mathrm{E}-02 & 7.828 \mathrm{E}-02 & 6.972 \mathrm{E}-02 & 6.505 \mathrm{E}-02 & 5.795 \mathrm{E}-06 & 1.463 \mathrm{E}-09 & 8.791 \mathrm{E}-10 & 8.590 \mathrm{E}-12\end{array}$

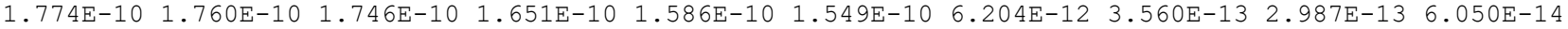

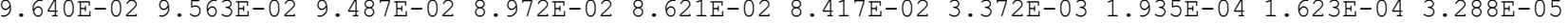

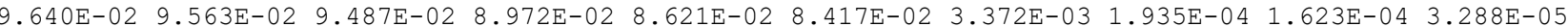

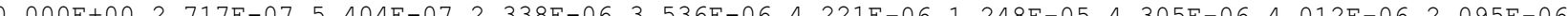
-01 $9.202 \mathrm{E}-01 \quad 9.11$ $\begin{array}{lllll} & \end{array}$ $\begin{array}{llllllllll}9.640 \mathrm{E}-01 & 9.608 \mathrm{E}-01 & 9.576 \mathrm{E}-01 & 9.356 \mathrm{E}-01 & 9.202 \mathrm{E}-01 & 9.111 \mathrm{E}-01 & 2.392 \mathrm{E}-01 & 7.288 \mathrm{E}-02 & 6.775 \mathrm{E}-02 & 3.488 \mathrm{E}-02\end{array}$

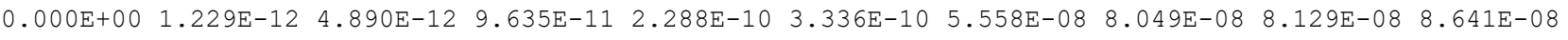
$\begin{array}{llllllllll}9.640 \mathrm{E}-02 & 9.640 \mathrm{E}-02 & 9.640 \mathrm{E}-02 & 9.639 \mathrm{E}-02 & 9.638 \mathrm{E}-02 & 9.638 \mathrm{E}-02 & 9.592 \mathrm{E}-02 & 9.552 \mathrm{E}-02 & 9.550 \mathrm{E}-02 & 9.527 \mathrm{E}-02\end{array}$

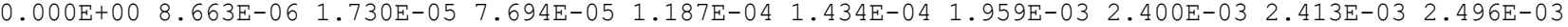

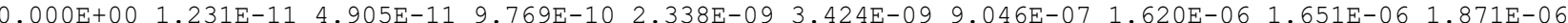

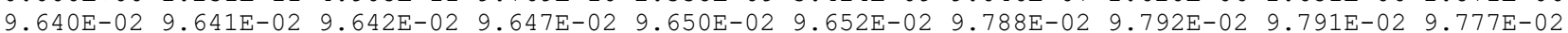

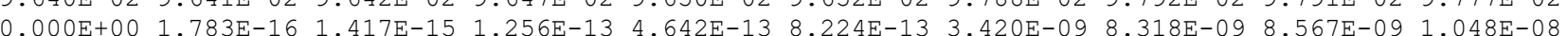

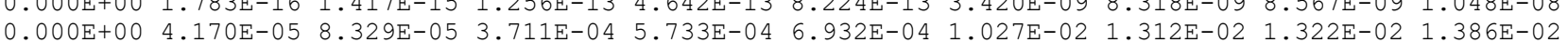
$\begin{array}{llllllllll}0.000 \mathrm{E}+00 & 4.170 \mathrm{E}-05 & 8.329 \mathrm{E}-05 & 3.711 \mathrm{E}-04 & 5.733 \mathrm{E}-04 & 6.932 \mathrm{E}-04 & 1.027 \mathrm{E}-02 & 1.312 \mathrm{E}-02 & 1.322 \mathrm{E}-02 & 1.386 \mathrm{E}-02 \\ 0.000 \mathrm{E}+00 & 1.881 \mathrm{E}-09 & 7.499 \mathrm{E}-09 & 1.495 \mathrm{E}-07 & 3.581 \mathrm{E}-07 & 5.248 \mathrm{E}-07 & 1.474 \mathrm{E}-04 & 2.749 \mathrm{E}-04 & 2.806 \mathrm{E}-04 & 3.234 \mathrm{E}-04\end{array}$ $\begin{array}{llllllllll}0.000 \mathrm{E}+00 & 1.881 \mathrm{E}-09 & 7.499 \mathrm{E}-09 & 1.495 \mathrm{E}-07 & 3.581 \mathrm{E}-07 & 5.248 \mathrm{E}-07 & 1.474 \mathrm{E}-04 & 2.749 \mathrm{E}-04 & 2.806 \mathrm{E}-04 & 3.234 \mathrm{E}-04 \\ 0.000 \mathrm{E}+00 & 1.785 \mathrm{E}-15 & 1.420 \mathrm{E}-14 & 1.269 \mathrm{E}-12 & 4.718 \mathrm{E}-12 & 8.386 \mathrm{E}-12 & 5.050 \mathrm{E}-08 & 1.511 \mathrm{E}-07 & 1.571 \mathrm{E}-07 & 2.076 \mathrm{E}-07\end{array}$ $\begin{array}{llllllllll}0.000 \mathrm{E}+00 & 1.785 \mathrm{E}-15 & 1.420 \mathrm{E}-14 & 1.269 \mathrm{E}-12 & 4.718 \mathrm{E}-12 & 8.386 \mathrm{E}-12 & 5.050 \mathrm{E}-08 & 1.511 \mathrm{E}-07 & 1.571 \mathrm{E}-07 & 2.076 \mathrm{E}-07 \\ 0.000 \mathrm{E}+00 & 4.171 \mathrm{E}-05 & 8.330 \mathrm{E}-05 & 3.713 \mathrm{E}-04 & 5.736 \mathrm{E}-04 & 6.937 \mathrm{E}-04 & 1.042 \mathrm{E}-02 & 1.340 \mathrm{E}-02 & 1.350 \mathrm{E}-02 & 1.419 \mathrm{E}-02\end{array}$ $0.000 \mathrm{E}+00 \quad 1.384 \mathrm{E}-18 \quad 2.183 \mathrm{E}-17 \quad 8.345 \mathrm{E}-15 \quad 4.666 \mathrm{E}-14 \quad 9.871 \mathrm{E}-14 \quad 2.837 \mathrm{E}-09 \quad 7.547 \mathrm{E}-09 \quad 7.794 \mathrm{E}-09 \quad 9.703 \mathrm{E}-09$ $\begin{array}{llllllllll}0.000 \mathrm{E}+00 & 6.432 \mathrm{E}-07 & 2.539 \mathrm{E}-06 & 4.737 \mathrm{E}-05 & 1.083 \mathrm{E}-04 & 1.545 \mathrm{E}-04 & 9.346 \mathrm{E}-03 & 1.231 \mathrm{E}-02 & 1.241 \mathrm{E}-02 & 1.308 \mathrm{E}-02\end{array}$ $\begin{array}{llllllll} & \end{array}$

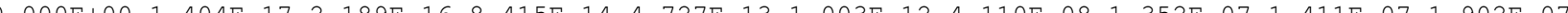

$\begin{array}{llllllllll}0.000 \mathrm{E}+00 & 6.433 \mathrm{E}-07 & 2.539 \mathrm{E}-06 & 4.738 \mathrm{E}-05 & 1.084 \mathrm{E}-04 & 1.546 \mathrm{E}-04 & 9.473 \mathrm{E}-03 & 1.256 \mathrm{E}-02 & 1.267 \mathrm{E}-02 & 1.339 \mathrm{E}-02\end{array}$ 1RESRAD-OFFSITE, Version 4.0 .3 beta T1/2 Limit $=30$ days $06 / 11 / 202011: 17$ Page 66

Parent Dose Report

Title : Landfill Scenario

File : LANDFILL-PADUCAH-AUTHORIZED-RELEASE-REV.ROF

Individual Nuclide Soil Concentration

Parent Nuclide and Thread Fraction Indicated

ONuclide Parent THF(i)

\begin{tabular}{|c|c|c|}
\hline (j) & (i) & \\
\hline Po-210 & $\mathrm{Pu}-238$ & .000 \\
\hline Po-210 & Th-230 & $1.000 \mathrm{E}$ \\
\hline Po-210 & $\mathrm{U}-234$ & $1.000 \mathrm{E}+$ \\
\hline Po-210 & $\mathrm{U}-238$ & $9.999 \mathrm{E}$ \\
\hline
\end{tabular}

$S(j, t), p C i / g$

$t=0.000 \mathrm{E}+00 \quad 1.000 \mathrm{E}+00 \quad 2.000 \mathrm{E}+00 \quad 9.000 \mathrm{E}+00 \quad 1.400 \mathrm{E}+01 \quad 1.700 \mathrm{E}+01 \quad 4.200 \mathrm{E}+02 \quad 7.780 \mathrm{E}+02 \quad 8.000 \mathrm{E}+02 \quad 1.000 \mathrm{E}+03$ $\overline{0.000 \mathrm{E}+00} \overline{3.860 \mathrm{E}-19} \overline{9.692 \mathrm{E}-18} \overline{6.626 \mathrm{E}-15} \overline{4.003 \mathrm{E}-14} \overline{8.686 \mathrm{E}-14} \frac{2.805 \mathrm{E}-09}{7.475 \mathrm{E}-09} \overline{7.719 \mathrm{E}-09} \overline{9.613 \mathrm{E}-09}$

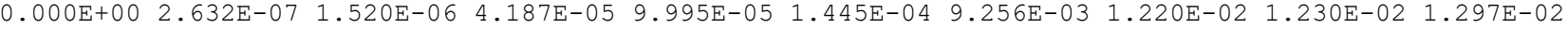

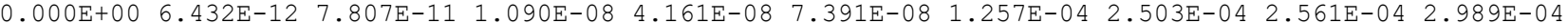
$0.000 \mathrm{E}+00 \quad 3.770 \mathrm{E}-18 \quad 9.706 \mathrm{E}-17 \quad 6.679 \mathrm{E}-14 \quad 4.054 \mathrm{E}-13 \quad 8.821 \mathrm{E}-13 \quad 4.062 \mathrm{E}-08 \quad 1.339 \mathrm{E}-07 \quad 1.397 \mathrm{E}-07 \quad 1.884 \mathrm{E}-07$ 


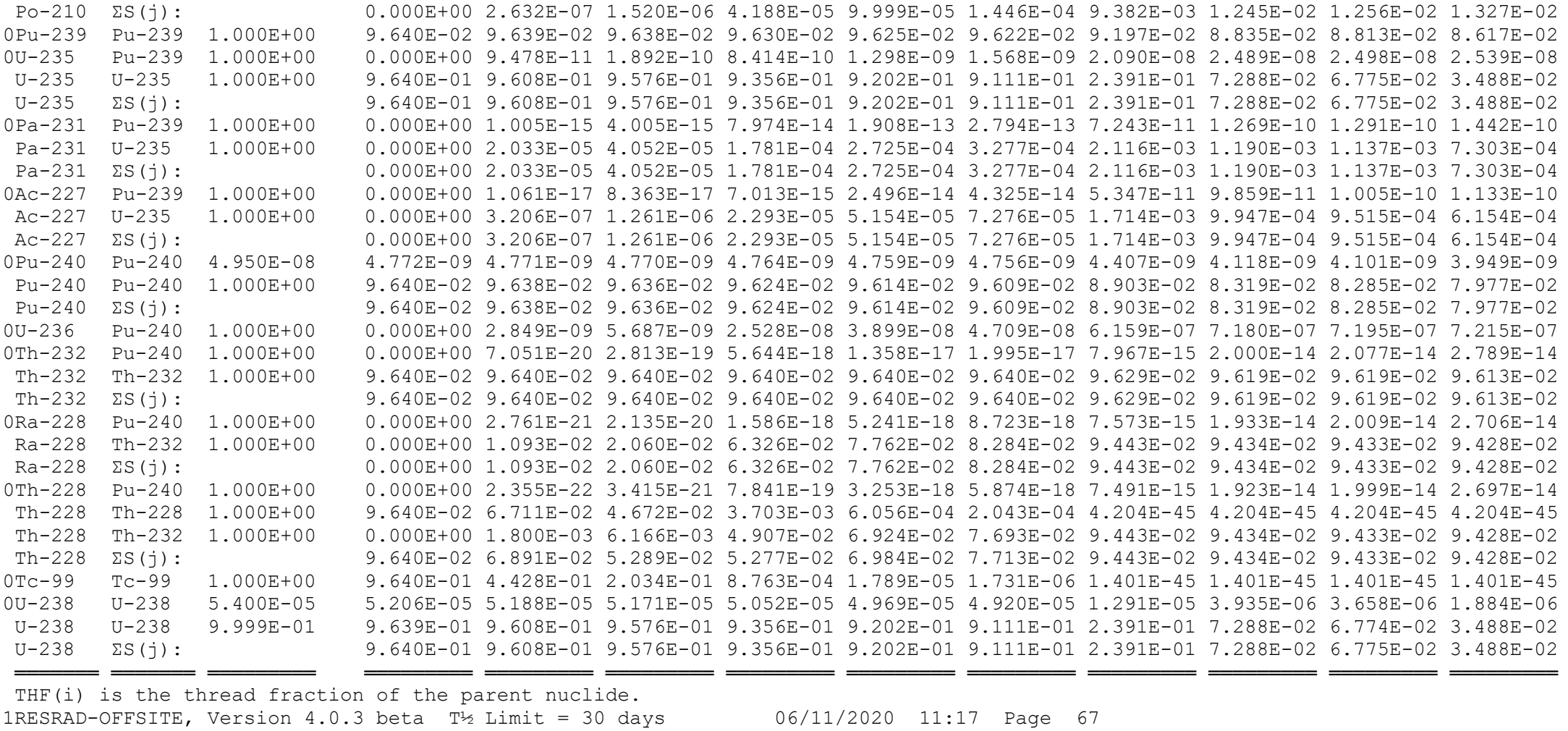

1RESRAD-OFFSITE, Version 4.0 .3 beta $T^{1 / 2}$ Limit $=30$ days

Parent Dose Report

Title : Landfill Scenario

File : LANDFILL-PADUCAH-AUTHORIZED-RELEASE-REV.ROF

Run Time Information

ResOCalc.EXE execution began at $11: 17$ on 06/11/2020

ResOCalc.EXE execution ended at 11:17 on 06/11/2020

ResOCalc.EXE execution time 23.338 seconds
06/11/2020 11:17 Page 67

Note: The dose used is from the time of peak total dose for an individual radionuclide (see Table 8 in the main report). 
This page intentionally blank.

B-192 



\section{Argonne $\mathbf{n}$}

\section{Environmental Science Division}

Argonne National Laboratory

9700 South Cass Avenue, Bldg. 240

Lemont, IL 60439-4832

www.anl.gov 